\title{
ఇUSGS
}

\section{Hawaiian Volcano Observatory Seismic Data, January to December 2006}

By Jennifer Nakata

Open-File Report 2007-1073

U.S. Department of the Interior

U.S. Geological Survey 


\section{U.S. Department of the Interior DIRK KEMPTHORNE, Secretary}

\section{U.S. Geological Survey Mark D. Myers, Director}

U.S. Geological Survey, Reston, Virginia 2007

For product and ordering information:

World Wide Web: http://www.usgs.gov/pubprod

Telephone: 1-888-ASK-USGS

For more information on the USGS - the Federal source for science about the Earth, its natural and living resources, natural hazards, and the environment: World Wide Web: http://www.usgs.gov

Telephone: 1-888-ASK-USGS

Any use of trade, product, or firm names is for descriptive purposes only and does not imply endorsement by the U.S. Government.

Although this report is in the public domain, permission must be secured from the individual copyright owners to reproduce any copyrighted material contained within this report. 
Introduction

Seismic Instrumentation

Figure 1 Map of Hawai $` i$ Island showing geographic and geologic features ......................... 5

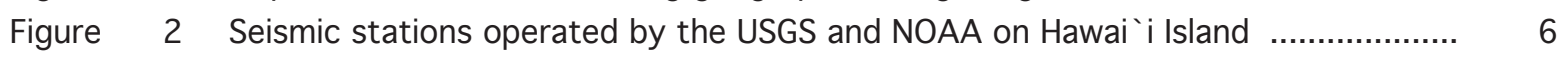

Figure $\quad 3$ Seismic network telemetry scheme on Hawai `i Island ............................................ 7

Figure $\quad 4 a$ Seismic network telemetry scheme at Kilauea summit ........................................... 8

Figure $\quad 4 \mathrm{~b}$ Broad-band telemetry scheme at Kilauea summit................................................... 8

Figure 5 Seismic network telemetry scheme on Maui Island .............................................. 9

Table 1 Seismic stations in Hawai $i$ operated by the USGS ............................................... 10

Table $\quad 2$ Seismic instrument types in use by HVO ......................................................... 12

Figure $\quad 6 \quad$ HVO system response curve of the four basic seismograph types ........................ 12

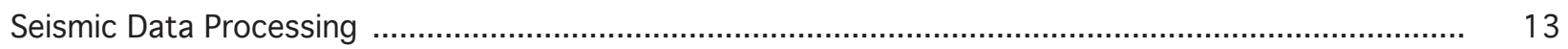

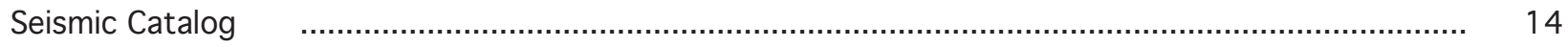

Table $\quad 3$ Coordinates of named regions used for classifying earthquakes .......................... 14

Figure 7 Earthquake classification, shallow for Kilauea and Mauna Loa ................................ 16

Figure 8 Earthquake classification, intermediate for Kỉlauea and Mauna Loa ....................... 17

Figure 9 Earthquake classification, crustal, for Hawai $i$ Island .......................................... 18

Figure 10 Earthquake classification, deep, for Hawai `i Island ............................................. 19

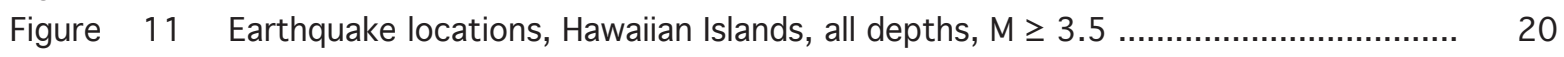

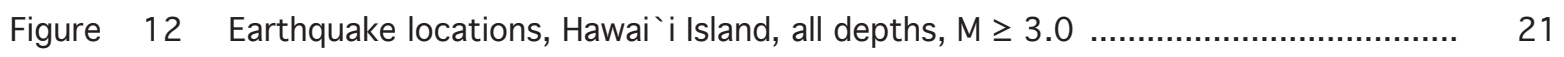

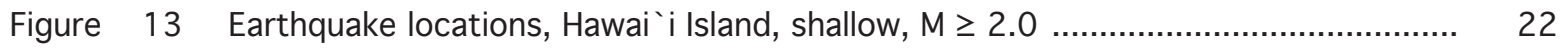

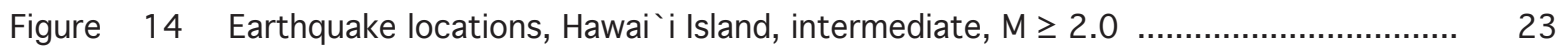

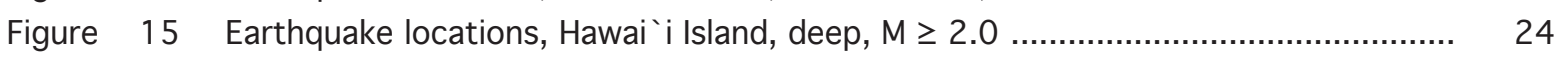

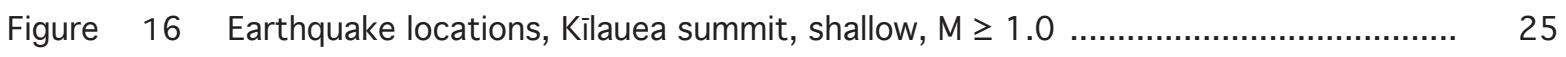

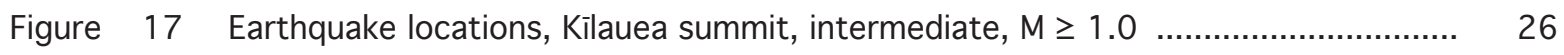

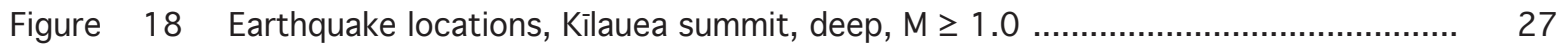

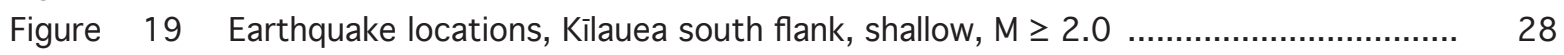

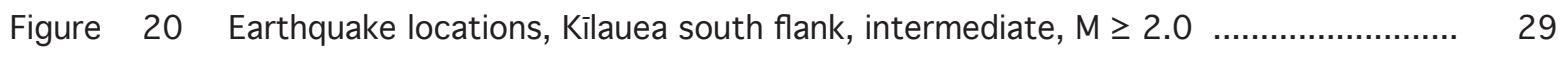

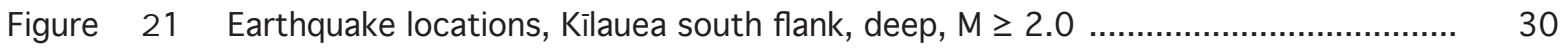

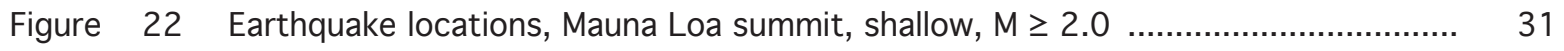

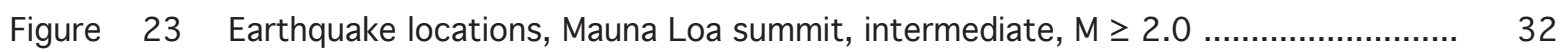

Figure 24 Earthquake locations, Mauna Loa summit, deep, $M \geq 2.0$.................................. 33

Table $\quad 4$ List of all located earthquakes ...................................................................... 34

Table 5 List of located earthquakes of magnitude 3.0 or greater .................................... 97 


\section{HAWAIIAN VOLCANO OBSERVATORY STAFF}

JAMES P. KAUAHIKAUA (SCIENTIST-IN-CHARGE)

STEVE R. BRANTLEY (DEPUTY SCIENTIST-IN-CHARGE)

\section{GEOLOGY}

C. CHRISTINA HELIKER*

RICHARD P. HOBLITT*

TIMOTHY R. ORR

DONALD A. SWANSON

FRANK A. TRUSDELL

\section{GEOPHYSICS}

JAMES P. KAUAHIKAUA

\section{SEISMOLOGY}

JENNIFER S. NAKATA

PAUL G. OKUBO

JEFF O. URIBE

DEFORMATION

KEVAN KAMABAYASHI

ASTA MIKLIUS

MICHAEL P. POLAND

MAURICE K. SAKO

GEOCHEMISTRY

TAMAR ELIAS

A. JEFFERSON SUTTON

\section{ELECTRONICS}

STEVEN K. FUKE BRUCE T. FURUKAWA

KENNETH T. HONMA

\section{COMPUTER}

WILFRED R. TANIGAWA*

\section{LIBRARY/PHOTO ARCHIVE}

T. JANE TAKAHASHI

ADMINISTRATION

PAULINE N. FUKUNAGA

MARIAN M. KAGIMOTO

MENDENHALL POSTDOCTRAL FELLOWSHIP

MARIE EDMONDS*

SCIENTIST EMERITUS

ROBERT Y. KOYANAGI

ARNOLD T. OKAMURA

SCEP

MARY L. MATHIS

JOSEPH L. NICHOLAS

CONTRACTS

Seismic :

L. GLADYS FORBES - record changing ADOLPH R. TEVES - record changing

CSAV Cooperative Employees

SARA E. ABRAHAM - Seismic+ LOREN ANTOLIK - Deformation+ DAVID WHILLDIN - Seismic RICHARD HERD - Deformation, Gas*

* Left in 2006

+ Arrived in 2006 


\section{INTRODUCTION}

The Hawaiian Volcano Observatory (HVO) summary presents seismic data gathered during the year. The seismic summary is offered without interpretation as a source of preliminary data. It is complete in the sense that most data for events of $\mathrm{M} \geq 1.5$ routinely gathered by the Observatory are included.

The HVO summaries have been published in various forms since 1956. Summaries prior to 1974 were issued quarterly, but cost, convenience of preparation and distribution, and the large quantities of data dictated an annual publication beginning with Summary 74 for the year 1974. Summary 86 (the introduction of CUSP at HVO) includes a description of the seismic instrumentation, calibration, and processing used in recent years. Beginning with 2004, summaries are simply identified by the year, rather than Summary number. The present summary includes background information on the seismic network and processing to allow use of the data and to provide an understanding of how they were gathered.

A report by Klein and Koyanagi (1980) tabulates instrumentation, calibration, and recording history of each seismic station in the network. It is designed as a reference for users of seismograms and phase data and includes and augments the information in the station table in this summary.

${ }^{1}$ Klein, F.W., and Koyanagi, R.Y., 1980, Hawaiian Volcano Observatory seismic network history, 1950-1979: U.S. Geological Survey Open-File Report 80-302, 84 p, 


\section{SEISMIC INSTRUMENTATION}

The network. The Hawaiian Volcano Observatory maintains an extensive telemetered seismic network on the Island of Hawai i. The standard HVO field sensors, 1-Hz geophones, are deployed as single-component, vertical-only units or as threecomponent combinations of one vertical and two orthogonal horizontal units. The 2006 network consisted of 48 station sites: 8 three-component, 2 six-component (which included a three-component Kinemetric Force-Balance accelerometer), 2 fourcomponent (Uwēkahuna included a low-gain vertical with a unity gain setting; 'Ainapō included a moderate-gain vertical with a 48db setting), 2 two-component (each site included a moderate-gain vertical with a 48db setting), 1 five-component (Āhua which included two moderate-gain horizontals with a 42db setting installed for experimental purpose) and 33 vertical-component-only sites. The coverage is most dense on and around Kilauea Volcano. During 1999, HVO added to the network three vertical-component-only sites on the Island of Maui. All seismic signals from the network are telemetered in real time to the Observatory for recording.

The Pacific Isunami Warning $\underline{\text { Center }}$ (NOAA) operates and maintains a network of stations on the islands of Hawai'i, Maui, and O'ahu. In 1999, radio links were established to share data, in real-time, between PTWC and HVO. PTWC signals from one O`ahu three-component station, and one Maui and four Hawai i vertical-component-only stations, were telemetered to the Observatory for recording.

Figure 1 is a map of selected geographic and geologic features. Figure 2 shows the sites of seismic stations operated by HVO and PTWC on the Island of Hawai i during 2006. Figure 3 indicates the telemetry scheme for the seismic stations on Hawai i Island, and figures $4 \mathrm{a}$ and $4 \mathrm{~b}$ are expanded views of the telemetry schemes at Kilauea summit: $4 \mathrm{a}$, HVO seismic stations and $4 \mathrm{~b}$, broadband network installed by Menlo Park and maintained by HVO. Figure 5 indicates the telemetry scheme for the seismic stations on Maui Island.

Table 1 lists seismic stations by site name, four-letter component codes, coordinates in degrees and minutes (Old Hawaiian Datum), elevation in meters, and other data, as described below, pertaining to each component. The list includes all the station components operated by HVO during 2006. All station names with field sensors installed at the site remained on the list, though operation may not have been continuous. Seismic station components operated by PTWC on the Islands of Hawai i, O`ahu and Maui are also listed. Phase times from PTWC stations, that are not telemetered to HVO, are used to supplement local earthquakes and earthquakes that occur within the Hawaiian Archipelago but distant from the Hawai $i$ Island network.

Instrumentation and recording. Each telemetered station's data channel has a voltage-controlled oscillator (VCO) for FM multiplex transmission to HVO via radio. These telemetering stations are all of Type 1, Earthquake Hazards Team (EHT) standard system used in USGS seismic networks (see table 2 for details). After discrimination at the receiver, the analog signals are converted to digital form as part of the routine computer location processing and archiving. Through July 2001, continuous signals from the telemetered network were saved on 4-mm digital-audio tape (DAT) recording units. Three DAT recorders ran in automatic rotation, as each $\sim 20$-hr tape was filled. Optic recordings are coded in table 1 as follows: $\mathrm{H}$ - Helicorder paper, and I - ink paper. DAT and paper records are archived at HVO.

Seismograph response and calibration. Response curve for the short-period seismograph type in use is given in figure 6 . The Type 1 curve gives the magnification of the standard EHT system from ground motion at the seismometer to the seismic trace, as would be seen on a $20 x$ Develocorder film viewer. The curve plots the unit response, which is multiplied by a constant but known factor, CAL, to get the response for an individual station. Individual CAL factors for Type 1 seismographs are Develocorder-equivalent peak-to-peak amplitudes, measured in millimeters, of a 100-microvolt 5 to $8-\mathrm{Hz}$ signal introduced to the preamp/VCO in place of the geophone at the field station. The calibration process is normally performed each time a station is visited for other required maintenance. Though Develocorder operations have ceased, calculations continue to be based on Develocorder equivalents. 


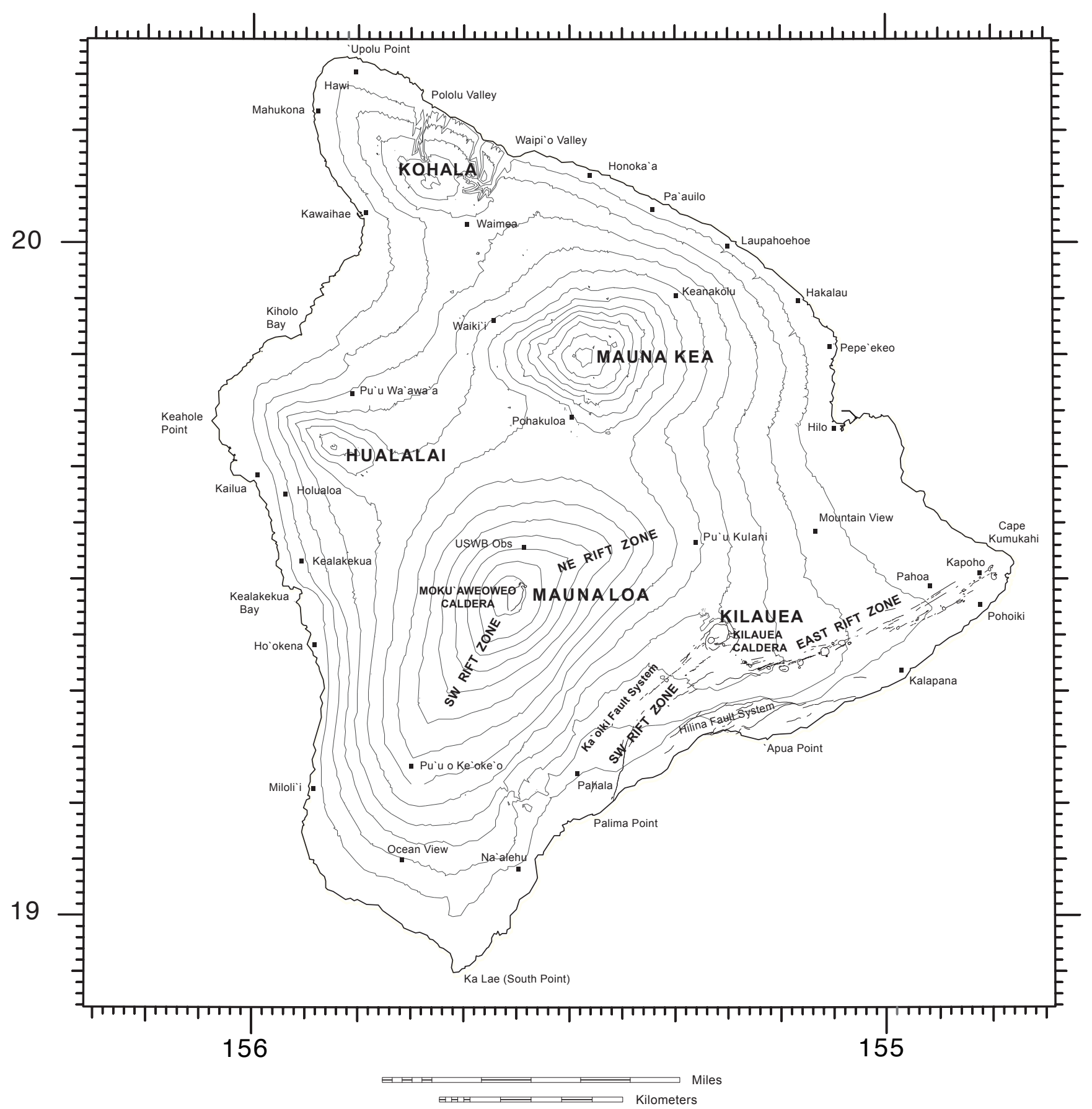

Figure 1. Map of the Island of Hawai i, showing principal settlements and selected geographic and geologic features. 


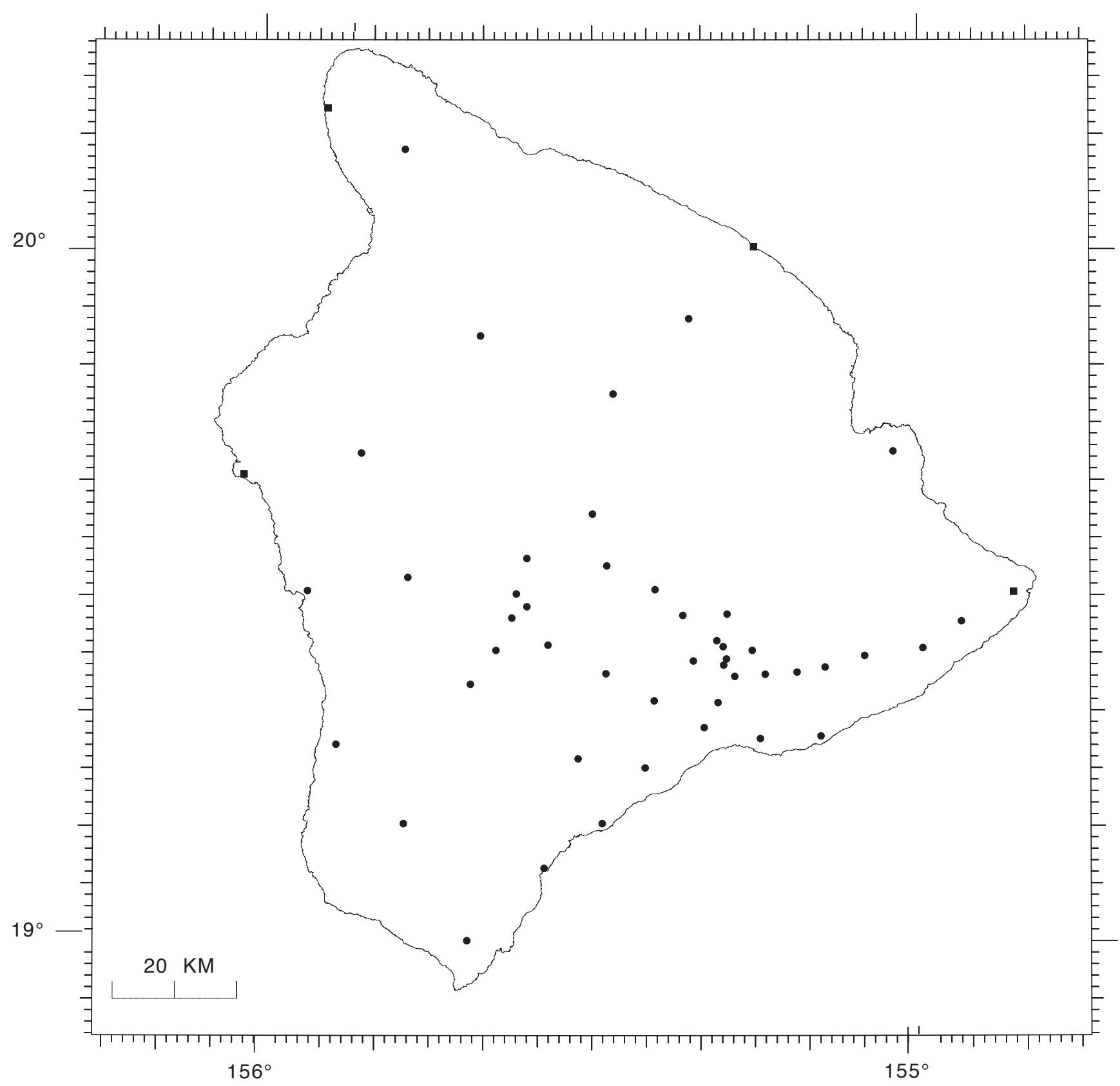

\section{- Network sites \\ - PTWC station sites}

Figure 2. The 2006 Hawaiian Volcano Observatory and PTWC seismic network on the Island of Hawai i. 


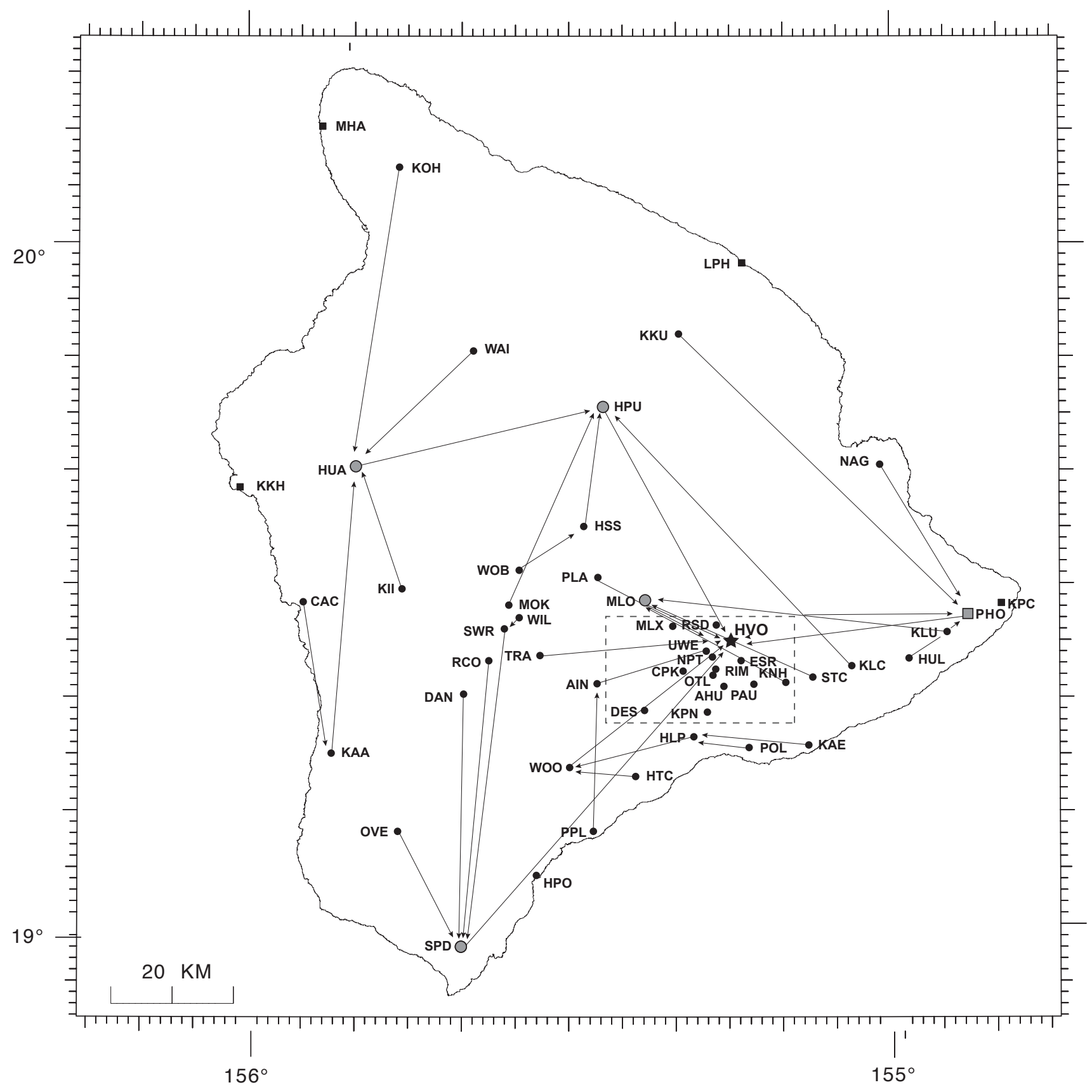

- Hawaiian Volcano Observatory

- $\quad$ Network sites

O Direct-to-Line 32 Channel

$\square \quad$ Direct-to-Line 32 Channel repeater sites

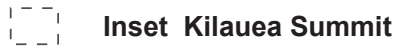

- PTWC station sites

Figure 3. Telemetry scheme for the 2006 Hawaiian Volcano Observatory seismic network on the Island of Hawai i. 

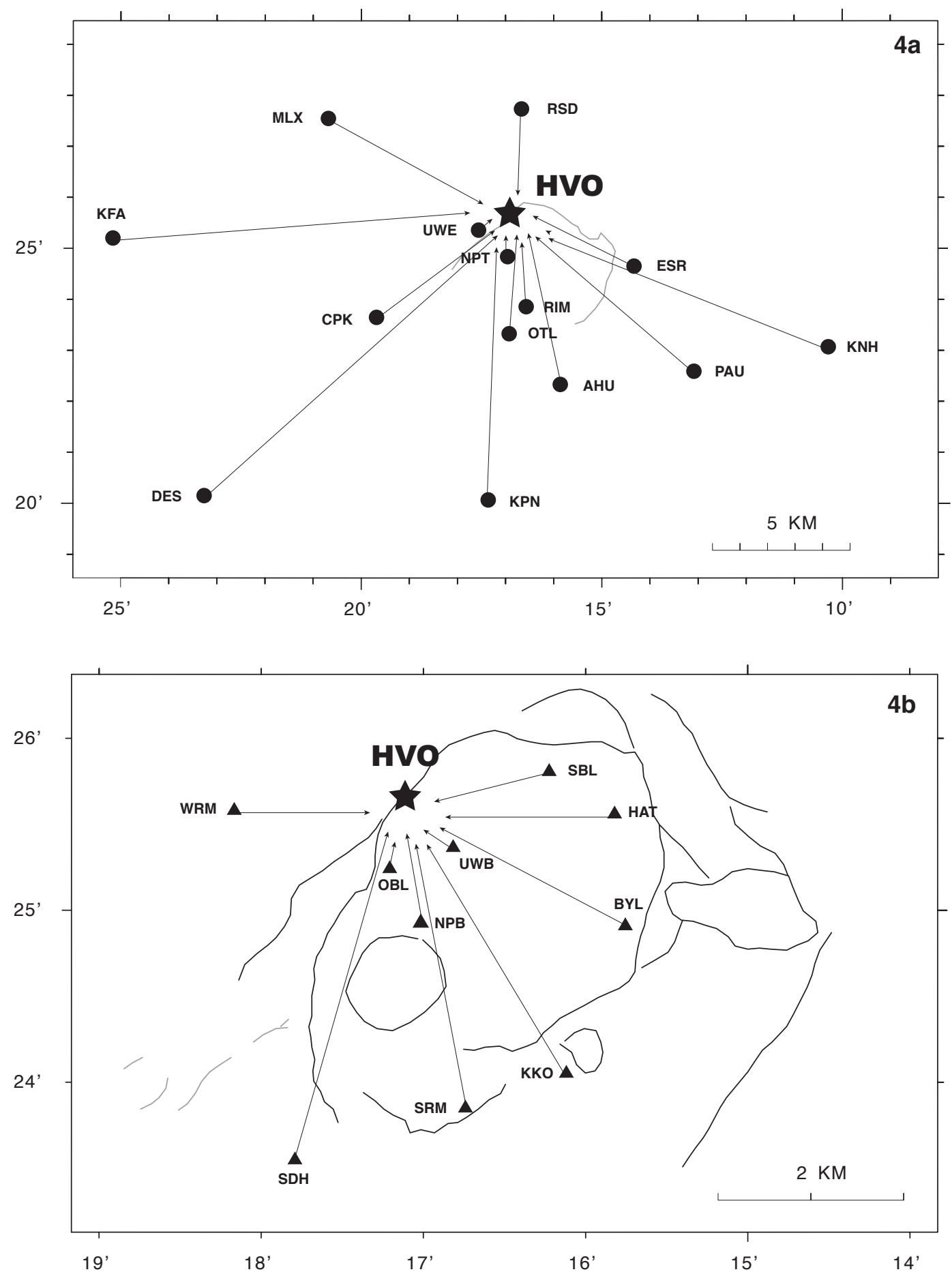

- Hawaiian Volcano Observatory

Network sites

A Broadband sites

Figure 4a. Expanded telemetry scheme for the 2006 Hawaiian Volcano Observatory seismic network at Kïlauea summit.

Figure 4b. Expanded telemetry scheme for the 2006 Menlo Park broadband seismic network at Kilauea summit. 


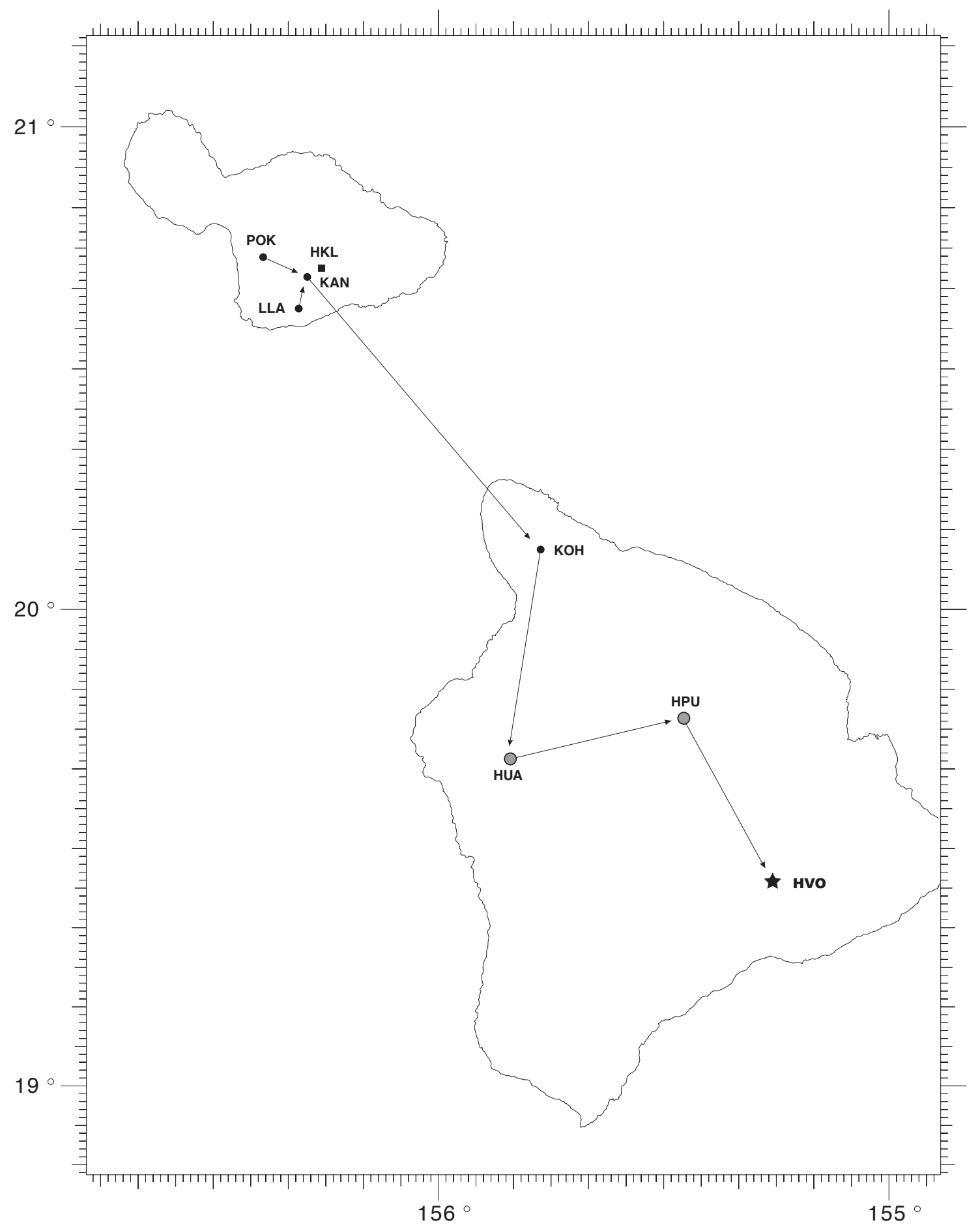

$\star \quad$ Hawaiian Volcano Observatory

- Network station sites

O Direct -to-Line 32 Channel

- $\quad$ PTWC station sites

Figure 5. Telemetry scheme for the 2006 Hawaiian Volcano Observatory and PTWC seismic network on the Island of Maui. The HVO stations were not in operation, thus produced no phase data for the 2006 catalog. 
Table 1. Seismic station sites and components in Hawai'i operated by the USGS in 2006.

\begin{tabular}{|c|c|c|c|c|c|c|c|c|c|c|c|c|}
\hline \multirow{2}{*}{ STATION NAME } & \multirow[t]{2}{*}{ CODE } & \multicolumn{2}{|c|}{--LAT--- } & \multicolumn{2}{|c|}{$---\mathrm{LON}---$} & \multirow{2}{*}{$\begin{array}{r}\text { ELEV } \\
(\mathrm{M})\end{array}$} & \multirow{2}{*}{$\begin{array}{c}\text { DELAY } \\
1\end{array}$} & \multirow{2}{*}{$\begin{array}{c}\text { DELAY } \\
2\end{array}$} & \multirow{2}{*}{ CAL } & \multicolumn{2}{|c|}{ SEIS } & \multirow{2}{*}{$\begin{array}{l}\text { OPTIC } \\
\text { RECORD }\end{array}$} \\
\hline & & & M & $\mathrm{D}$ & M & & & & & & & \\
\hline HUA & AHUV & 19 & 22.40 & 155 & 15.90 & 1070 & 10 & -0.13 & 2.6 & L5 & & I \\
\hline AHUA & AHUE & 19 & 22.40 & 155 & 15.90 & 1070 & -0.10 & -0.13 & 3.0 & E5 & MW & \\
\hline AHUA & AHUN & 19 & 22.40 & 155 & 15.90 & 1070 & -0.10 & -0.13 & 3.0 & E5 & MW & \\
\hline AHUA & $\mathrm{AH} 1 \mathrm{E}$ & 19 & 22.40 & 155 & 15.90 & 1070 & -0.10 & -0.13 & 1.0 & L5 & & \\
\hline AHUA & $\mathrm{AH} 1 \mathrm{~N}$ & 19 & 22.40 & 155 & 15.90 & 1070 & -0.10 & -0.13 & 1.0 & L5 & & \\
\hline AINAPO & AINV & 19 & 22.50 & 155 & 27.62 & 1524 & 0.13 & 0.17 & 6.8 & L5 & & \\
\hline AINAPO & AINE & 19 & 22.50 & 155 & 27.62 & 1524 & 0.13 & 0.17 & 3.0 & L5 & MW & \\
\hline AINAPO & AINN & 19 & 22.50 & 155 & 27.62 & 1524 & 0.13 & 0.17 & 3.0 & L5 & MW & \\
\hline AINAPO & AINZ & 19 & 22.50 & 155 & 27.62 & 1524 & 0.13 & 0.17 & 0.0 & L5 & & \\
\hline CAPTAIN COOK & $\mathrm{CACV}$ & 19 & 29.29 & 155 & 55.09 & 323 & 0.00 & -0.16 & 1.1 & L5 & & \\
\hline CONE PEAK & $\mathrm{CPKV}$ & 19 & 23.70 & 155 & 19.70 & 1038 & -0.26 & -0.07 & 6.0 & L5 & & \\
\hline DANDELION & DANV & 19 & 21.42 & 155 & 40.04 & 3003 & -0.27 & 0.03 & 4.3 & E5 & & \\
\hline DESERT & DESV & 19 & 20.20 & 155 & 23.30 & 815 & -0.29 & -0.13 & 4.5 & L5 & & I \\
\hline DIAMOND HEAD, O & OADHHZ & 21 & 16.12 & 157 & 48.25 & 137 & 0.00 & 0.00 & 0.0 & $\mathrm{~S} 1$ & & \\
\hline ESCAPE ROAD & ESRV & 19 & 24.68 & 155 & 14.33 & 1177 & -0.17 & -0.19 & 1.2 & L5 & & \\
\hline HEIHEIAHULU & HHAZ & 19 & 25.13 & 154 & 58.72 & 369 & -0.17 & -0.16 & 0.0 & F5 & & \\
\hline HEIHEIAHULU & HHAE & 19 & 25.13 & 154 & 58.72 & 369 & -0.17 & -0.16 & 0.0 & F5 & & \\
\hline HEIHEIAHULU & HHAN & 19 & 25.13 & 154 & 58.72 & 369 & -0.17 & -0.16 & 0.0 & F5 & & \\
\hline HALEAKALA, MAUI & I HKLZ & 20 & 42.63 & 156 & 15.55 & 3051 & 0.00 & 0.00 & 0.0 & S1 & & \\
\hline HILINA PALI & HLPV & 19 & 17.96 & 155 & 18.63 & 707 & 0.02 & 0.07 & 2.1 & L5 & & \\
\hline HONOLULU, OAHU & HONZ & 21 & 19.30 & 158 & 0.50 & 2 & 0.00 & 0.00 & 0.0 & $\mathrm{~S} 1$ & & \\
\hline HONOLULU, OAHU & HONE & 21 & 19.30 & 158 & 0.50 & 2 & 0.00 & 0.00 & 0.0 & $\mathrm{~S} 1$ & & \\
\hline HONOLULU, OAHU & HONN & 21 & 19.30 & 158 & 0.50 & 2 & 0.00 & 0.00 & 0.0 & $\mathrm{~S} 1$ & & \\
\hline HONUAPO & HPOZ & 19 & 5.34 & 155 & 33.23 & 15 & 0.00 & 0.00 & 0.0 & $\mathrm{~S} 1$ & & \\
\hline HALE POHAKU & HPUV & 19 & 46.72 & 155 & 27.54 & 3396 & 0.31 & 0.17 & 3.3 & L5 & & \\
\hline HUMUULA SHEEP $S^{\prime}$ & STHSAZ & 19 & 36.31 & 155 & 29.13 & 2445 & 0.20 & 0.35 & 0.0 & F5 & & \\
\hline HUMUULA SHEEP $S$ & STHSAN & 19 & 36.31 & 155 & 29.13 & 2445 & 0.20 & 0.35 & 0.0 & F5 & & \\
\hline HUMUULA SHEEP $S$ & STHSAE & 19 & 36.31 & 155 & 29.13 & 2445 & 0.20 & 0.35 & 0.0 & F5 & & \\
\hline HUMUULA SHEEP $S$ & STHSSV & 19 & 36.31 & 155 & 29.13 & 2445 & 0.20 & 0.35 & 4.0 & L5 & & \\
\hline HUMUULA SHEEP $S$ & STHSSE & 19 & 36.31 & 155 & 29.13 & 2445 & 0.20 & 0.35 & 3.0 & L5 & MW & \\
\hline HUMUULA SHEEP $S^{\prime}$ & STHSSN & 19 & 36.31 & 155 & 29.13 & 2445 & 0.20 & 0.35 & 3.0 & L5 & MW & \\
\hline HOT CAVES & HTCV & 19 & 14.33 & 155 & 24.02 & 381 & -0.16 & -0.07 & 2.3 & $\mathrm{E} 4$ & & \\
\hline HUALALAI & HUAV & 19 & 41.25 & 155 & 50.32 & 2189 & 0.67 & 0.38 & 2.8 & L5 & & \\
\hline HEIHEIAHULU & HULV & 19 & 25.13 & 154 & 58.72 & 369 & -0.17 & -0.16 & 1.6 & L5 & & $\mathrm{H}$ \\
\hline HEIHEIAHULU & HULE & 19 & 25.13 & 154 & 58.72 & 369 & -0.17 & -0.16 & 3.0 & E5 & MW & \\
\hline HEIHEIAHULU & HULN & 19 & 25.13 & 154 & 58.72 & 369 & -0.17 & -0.16 & 3.0 & L5 & MW & \\
\hline KAAPUNA & KAAV & 19 & 15.98 & 155 & 52.28 & 524 & -0.12 & -0.01 & 3.3 & E5 & & \\
\hline KAENA POINT & KAEV & 19 & 17.35 & 155 & 7.95 & 37 & -0.01 & 0.06 & 1.4 & L5 & & \\
\hline KANAHAU, MAUI & KANV & 20 & 41.60 & 156 & 17.84 & 2745 & 0.00 & 0.00 & 0.0 & L5 & & \\
\hline KANEKII & KIIV & 19 & 30.56 & 155 & 45.90 & 1841 & 0.15 & 0.37 & 3.0 & L5 & & \\
\hline KANEKII & KIIE & 19 & 30.56 & 155 & 45.90 & 1841 & 0.15 & 0.37 & 3.0 & L5 & MW & \\
\hline KANEKII & KIIN & 19 & 30.56 & 155 & 45.90 & 1841 & 0.15 & 0.37 & 3.0 & L5 & MW & \\
\hline KIPAPA, OAHU & KIPZ & 21 & 25.40 & 158 & 0.90 & 2 & 0.00 & 0.00 & 0.0 & S1 & & \\
\hline KAILUA, KONA & KKHZ & 19 & 39.40 & 156 & 1.12 & 1 & 0.00 & 0.00 & 0.0 & $\mathrm{~S} 1$ & & \\
\hline KEANAKOLU & KKUV & 19 & 53.39 & 155 & 20.58 & 1863 & 0.68 & 0.24 & 3.3 & L5 & & \\
\hline KALALUA CONE & $\mathrm{KLCV}$ & 19 & 24.35 & 155 & 4.08 & 659 & -0.25 & -0.30 & 3.4 & L5 & & \\
\hline PUU KALIU & KLUV & 19 & 27.48 & 154 & 55.26 & 271 & -0.17 & -0.30 & 3.4 & L5 & & \\
\hline KANE NUI O HAMO & O KNHV & 19 & 22.95 & 155 & 10.32 & 954 & -0.17 & -0.20 & 0.0 & L5 & & I \\
\hline KANE NUI O HAMO & $\mathrm{O} \mathrm{KNHZ}$ & 19 & 22.95 & 155 & 10.32 & 954 & -0.17 & -0.20 & 0.0 & L5 & & \\
\hline KOHALA & KOHV & 20 & 7.69 & 155 & 46.77 & 1166 & -0.03 & -0.17 & 6.3 & L5 & & \\
\hline KOHALA & KOHE & 20 & 7.69 & 155 & 46.77 & 1166 & -0.03 & -0.17 & 3.0 & L5 & MW & \\
\hline KOHALA & KOHN & 20 & 7.69 & 155 & 46.77 & 1166 & -0.03 & -0.17 & 3.0 & L5 & MW & \\
\hline KAPOHO CONE & $\mathrm{KPCZ}$ & 19 & 30.02 & 154 & 50.51 & 134 & 0.00 & 0.00 & 0.0 & $\mathrm{~S} 1$ & & \\
\hline KIPUKA NENE & KPNV & 19 & 20.10 & 155 & 17.40 & 924 & -0.11 & -0.08 & 3.5 & L5 & & \\
\hline
\end{tabular}




\begin{tabular}{|c|c|c|c|c|c|c|c|c|c|c|c|c|}
\hline \multirow[t]{2}{*}{ STATION NA } & \multirow[t]{2}{*}{ CODE } & \multicolumn{2}{|c|}{--LAT--- } & \multicolumn{2}{|c|}{$---\mathrm{LON}---$} & \multirow{2}{*}{$\begin{array}{r}\text { ELEV } \\
(\mathrm{M})\end{array}$} & DELAY & \multirow{2}{*}{$\begin{array}{c}\text { DELAY } \\
2\end{array}$} & \multirow[t]{2}{*}{ CAL } & \multirow{2}{*}{$\begin{array}{r}\text { SEIS } \\
\text { TYP }\end{array}$} & \multicolumn{2}{|c|}{ OPTIC } \\
\hline & & I & M & D & M & & 1 & & & & & $\mathrm{COB}$ \\
\hline LUALAILUA, MAUI & LLAV & 20 & 37.62 & 156 & 18.62 & 683 & 0.00 & 0.00 & 0.0 & L5 & & \\
\hline LAUPAHOEHOE & LPHZ & 19 & 59.82 & 155 & 14.58 & 1 & 0.00 & 0.00 & 0.0 & $\mathrm{~S} 1$ & & \\
\hline MAHUKONA & MHAZ & 20 & 11.27 & 155 & 54.18 & 1 & 0.00 & 0.00 & 0.0 & S1 & & \\
\hline MAUNA LOA & MLOV & 19 & 29.80 & 155 & 23.30 & 2010 & 0.03 & 0.08 & 5.6 & L5 & & I \\
\hline MAUNA LOA & MLOE & 19 & 29.80 & 155 & 23.30 & 2010 & 0.03 & 0.08 & 3.0 & L5 & MW & \\
\hline MAUNA LOA & MLON & 19 & 29.80 & 155 & 23.30 & 2010 & 0.03 & 0.08 & 3.0 & L5 & MW & \\
\hline MAUNA LOA $\mathrm{X}$ & MLXV & 19 & 27.60 & 155 & 20.70 & 1475 & 0.06 & 0.15 & 3.0 & L5 & & \\
\hline MOKUAWEOWEO & MOKV & 19 & 29.28 & 155 & 35.98 & 4104 & 0.15 & 0.16 & 4.2 & L5 & & $\mathrm{IH}$ \\
\hline NATIONAL GUARD & NAGV & 19 & 42.12 & 155 & 1.72 & 18 & 0.54 & 0.30 & 4.0 & R5 & & \\
\hline NATIONAL GUARD & NAGE & 19 & 42.12 & 155 & 1.72 & 18 & 0.54 & 0.30 & 3.0 & R5 & MW & \\
\hline NATIONAL GUARD & NAGN & 19 & 42.12 & 155 & 1.72 & 18 & 0.54 & 0.30 & 3.0 & R5 & MW & \\
\hline NORTH PIT & NPTV & 19 & 24.90 & 155 & 17.00 & 1115 & -0.30 & -0.18 & 3.0 & L5 & & $\mathrm{IH}$ \\
\hline NORTH PIT & NPTE & 19 & 24.90 & 155 & 17.00 & 1115 & -0.30 & -0.18 & 3.0 & L5 & MW & \\
\hline NORTH PIT & NPTN & 19 & 24.90 & 155 & 17.00 & 1115 & -0.30 & -0.18 & 3.0 & L5 & MW & \\
\hline OPANA, OAHU & OPAZ & 21 & 41.45 & 158 & 0.70 & 100 & 0.00 & 0.00 & 0.0 & $\mathrm{~S} 1$ & & \\
\hline OUTLET & OTLV & 19 & 23.38 & 155 & 16.94 & 1038 & -0.19 & -0.18 & 2.6 & L5 & & \\
\hline OUTLET & OTLZ & 19 & 23.38 & 155 & 16.94 & 1038 & -0.19 & -0.18 & 0.0 & L5 & & \\
\hline OCEANVIEW ESTATE & EOVEV & 19 & 9.21 & 155 & 45.92 & 1378 & 0.00 & 0.00 & 0.0 & L5 & & \\
\hline PAUAHI & PAUV & 19 & 22.62 & 155 & 13.10 & 994 & -0.21 & -0.24 & 2.9 & L5 & & \\
\hline PAUAHI & PAUE & 19 & 22.62 & 155 & 13.10 & 994 & -0.21 & -0.24 & 3.0 & L5 & MW & \\
\hline PAUAHI & PAUN & 19 & 22.62 & 155 & 13.10 & 994 & -0.21 & -0.24 & 3.0 & L5 & MW & \\
\hline PUU ULAULA & PLAV & 19 & 32.00 & 155 & 27.67 & 2992 & -0.03 & 0.13 & 6.3 & L5 & & I \\
\hline PUUOKALI, MAUI & POKV & 20 & 44.00 & 156 & 23.32 & 511 & 0.00 & 0.00 & 0.0 & L5 & & \\
\hline POLIOKEAWE PALI & POLV & 19 & 17.02 & 155 & 13.47 & 169 & -0.02 & 0.03 & 3.4 & E5 & & \\
\hline PUU PILI & PPLV & 19 & 9.50 & 155 & 27.87 & 35 & -0.15 & -0.15 & 1.4 & E5 & & \\
\hline RED CONE & $\mathrm{RCOV}$ & 19 & 24.36 & 155 & 37.79 & 3601 & 0.00 & 0.00 & 0.0 & L5 & & \\
\hline RIM & RIMV & 19 & 23.90 & 155 & 16.60 & 1128 & -0.21 & -0.13 & 0.0 & L5 & & $\Pi$ \\
\hline RAINSHED & RSDV & 19 & 27.78 & 155 & 16.68 & 1270 & 0.06 & 0.15 & 0.0 & L5 & & \\
\hline SOUTH POINT & SPDV & 18 & 58.94 & 155 & 40.24 & 250 & -0.17 & -0.22 & 0.0 & L5 & & \\
\hline SOUTH POINT & SPDE & 18 & 58.94 & 155 & 40.24 & 250 & -0.17 & -0.22 & 0.0 & L5 & MW & \\
\hline SOUTH POINT & SPDN & 18 & 58.94 & 155 & 40.24 & 250 & -0.17 & -0.22 & 0.0 & L5 & MW & \\
\hline STEAM CRACKS & STCV & 19 & 23.30 & 155 & 7.67 & 765 & -0.25 & -0.30 & 3.4 & L5 & & $\mathrm{H}$ \\
\hline SOUTHWEST RIFT & SWRV & 19 & 27.26 & 155 & 36.30 & 4048 & 0.01 & 0.04 & 5.6 & E5 & & \\
\hline TRAIL & TRAV & 19 & 24.91 & 155 & 32.96 & 3207 & 0.00 & 0.00 & 0.0 & L5 & & \\
\hline UWEKAHUNA & URAV & 19 & 25.40 & 155 & 17.60 & 1240 & -0.21 & 0.00 & 0.0 & R5 & & \\
\hline UWEKAHUNA & URAE & 19 & 25.40 & 155 & 17.60 & 1240 & -0.21 & 0.00 & 3.0 & R5 & MW & \\
\hline UWEKAHUNA & URAN & 19 & 25.40 & 155 & 17.60 & 1240 & -0.21 & 0.00 & 3.0 & R5 & MW & \\
\hline UWEKAHUNA & UUGZ & 19 & 25.40 & 155 & 17.60 & 1240 & 0.00 & 0.00 & 0.0 & Lo & & \\
\hline WAIKII & WAIV & 19 & 51.58 & 155 & 39.60 & 1433 & 0.20 & 0.35 & 0.0 & L5 & & \\
\hline WILKES CAMP & WILV & 19 & 28.15 & 155 & 35.02 & 4037 & 0.22 & 0.17 & 2.6 & E5 & & \\
\hline WILKES CAMP & WILE & 19 & 28.15 & 155 & 35.02 & 4037 & 0.22 & 0.17 & 3.0 & L5 & MW & \\
\hline WILKES CAMP & WILN & 19 & 28.15 & 155 & 35.02 & 4037 & 0.22 & 0.17 & 3.0 & L5 & MW & \\
\hline WAIMANALO RIDGE, & , WMRZ & 21 & 19.22 & 157 & 40.94 & 200 & 0.00 & 0.00 & 0.0 & $\mathrm{~S} 1$ & & \\
\hline WEATHER OBSERVAT & TWOBV & 19 & 32.31 & 155 & 35.01 & 3396 & 0.00 & 0.00 & 0.0 & E5 & & \\
\hline WOOD VALLEY & WOOV & 19 & 15.08 & 155 & 30.12 & 909 & -0.15 & -0.06 & 2.6 & E5 & & \\
\hline
\end{tabular}

During the year, there may have been outage periods that required station maintenance at certain sites. 
Table 2. Seismic instrument types

The codes in parentheses refer to the seismometer types listed in Table 1.

Type 1 (Codes E, L, R, and 4, 5) consists of:

a) Geophone - Electrotech EV-17 (E), Mark Products L4C (L) or Kinemetric Ranger SS1 (R).

$(\mathrm{L})$ and $(\mathrm{R})$ are 1.0-sec. period moving-magnet vertical- or horizontal- (E-W and N-S) component seismometers adjusted for an output of 0.5 volts $/ \mathrm{cm} / \mathrm{sec}$ and 0.8 , critically damped.

b) Preamp/VCO - USGS/OEVE Model J502, J512 (5) voltage-controlled oscillator. Three db points for bandpass filter at $0.1 \mathrm{~Hz}$ and $30 \mathrm{~Hz}$. Signals are transmitted on audio FM carrier over cable or FM radio link to HVO.

Code (W) - Wood-Anderson torsion seismograph.

Code (MW) - Horizontal-component seismograph based on a Type 1 system and modified to $3 \mathrm{x}$ a Wood-Anderson response.

Code (F) - Kinemetric Force-Balance Accelerometer (FBA23).

Code (S13) - Geotech, $1 \mathrm{~Hz}$ seismometer with A1 VCO operated by the Pacific Tsunami Warning Center.

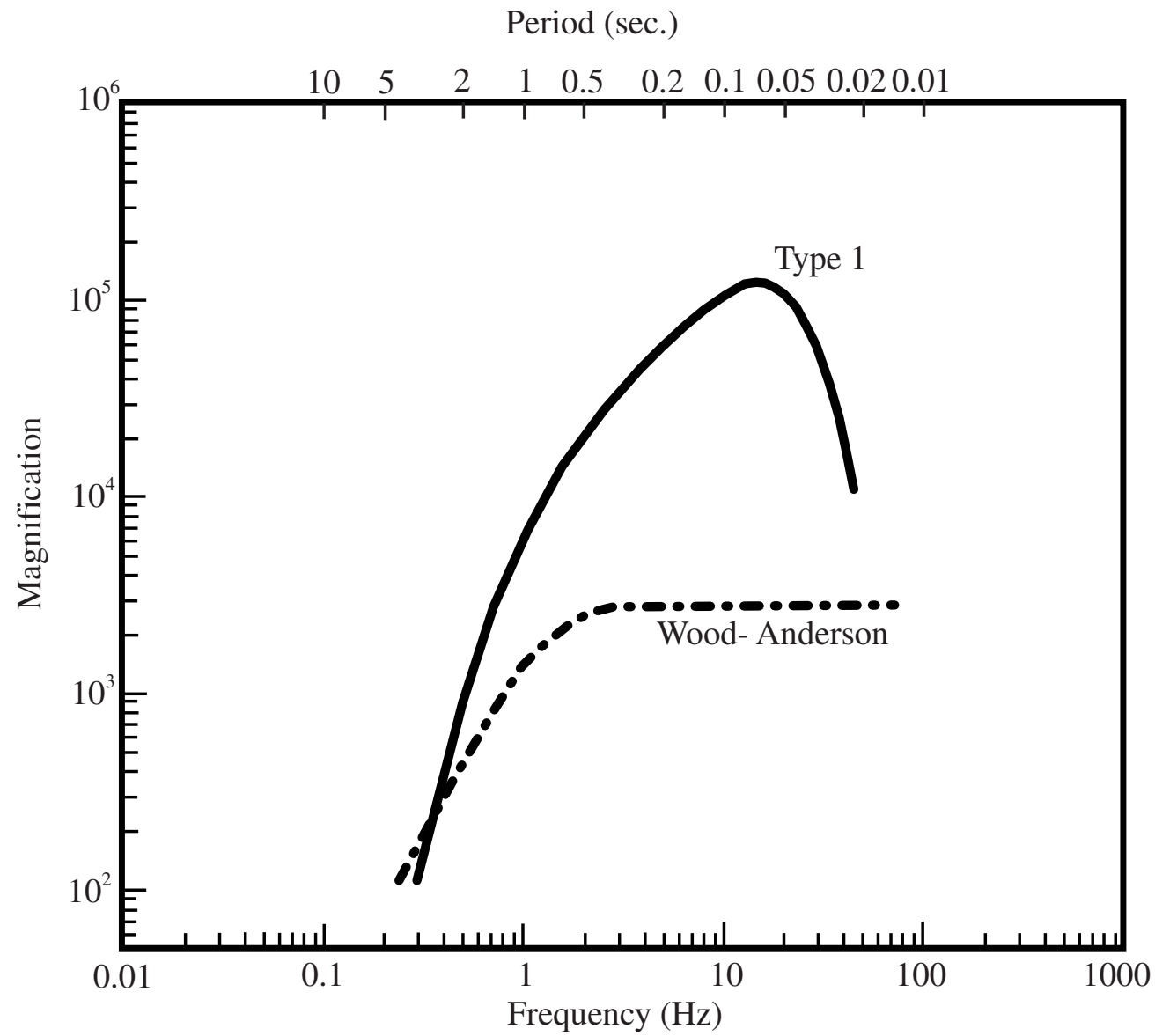

Figure 6. System-response curves for the Wood-Anderson torsion seismograph and for seismometers used by the Hawaiian Volcano Observatory. The Type 1 curve plots the unit response of the standard USGS microearthquake seismometer system as would be recorded on Develocorder film. This includes the geophone, all electronics including telemetry, Develocorder galvanometer, and projection of film by a $20 x$ viewer. The unit response curve is multiplied by constant but known factors (CAL) to obtain the responses for individual stations. 


\section{SEISMIC DATA PROCESSING}

Due to age and high cost of maintenance, Develocorder 'A' was discontinued on August 1, 1997. Daily count of classified micoearthquakes from source regions around Kilauea and Mauna Loa, and duration of tremor, were also discontinued. Coda duration, however, is measured in seconds from drum (ink or helicorder) records to determine a coda magnitude that is entered as an external magnitude in the final solution.

In 1986, HVO acquired a VAX 11-750 computer and adopted the CUSP (ㅁalifornia Institute of Technology USGS Seismic Processing) routine. Discriminated analog signals are converted to digital form, and detected events are saved in real time. Detected events are demultiplexed, and P-picks are made by the computer, producing a rough location. Events are examined by an analyst, on a graphics terminal, to refine computer P-picks and to time additional P- and S-phases for a preliminary location. Binary CUSP files are archived on magneto-optical media and translated into ASCII phase files. Locations and amplitude magnitudes are then determined, using the program HYPOINVERSE-2000 (Klein, 2002) ${ }^{2}$. Events are reworked and rerun, as needed, to produce a final solution. Magneto-optical copies of arrival times and output summary data are kept at HVO.

In July 1992, HVO acquired VAX workstations for timing earthquakes using a "generic" version of CUSP. In addition to timing $\mathrm{P}$ and $\mathrm{S}$ arrival signals, the VAX workstations are capable of measuring peak-to-peak amplitudes along with the associated period. This capability allowed the renewal of amplitude magnitude determinations from the network seismic stations. Amplitude data gathered from July 1992 to July 1997 became part of a test set to determine magnitude corrections for network stations. Results of newly determined magnitude corrections are detailed by Nakata and Okubo (1997) ${ }^{3}$.

HVO currently operates Earthworm software for the recording of all HVO seismic data including the import and export of seismic data from and to cooperating networks. HVO also utilizes the Earthworm processing system for rapid computation of earthquake products (locations, magnitude, spectrograms, helicorders, ShakeMaps, and recent earthquakes web pages). Analyst review of triggered events and seismic catalog generation is accomplished using the CUSP and HYPOINVERSE processing platforms.

The crustal model used is specified by velocities at four depth points. Velocity at any depth is given by linear interpolation between points and uses a homogeneous half-space, as listed below:

$\begin{array}{cr}\begin{array}{l}\text { VELOCITY } \\ (\mathrm{km} / \mathrm{sec})\end{array} & \begin{array}{c}\text { DEPTH } \\ (\mathrm{km})\end{array} \\ 1.9 & 0.0 \\ 6.5 & 4.6 \\ 6.9 & 15.0 \\ 8.3 & \geq 16.5\end{array}$

Two empirical sets of station delays or corrections were used in the HYPOINVERSE locations and are given in table 1. The delay models are separated by a circle of radius $34 \mathrm{~km}$, centered at $19^{\circ} 22^{\prime} \mathrm{N}$ and $155^{\circ} 10^{\prime} \mathrm{W}$. Delay model 1 is used for epicenters occurring within a circle of radius $31 \mathrm{~km}$ from the center. This region includes Kilauea and its south flank. A combination of the two delay models is used for epicenters that fall in a transition zone that is $6 \mathrm{~km}$ wide. Delay model 2 is applied to the rest of the island and offshore earthquakes. For a detailed description, refer to HYPOINVERSE-2000 (Klein, 2002).

Magnitudes for events are computed using recorded amplitudes on selected network vertical, Modified Wood-Anderson (MW) horizontal, and/or moderate and low gain stations. Amplitude readings are corrected to an equivalent Wood-Anderson amplitude using the curves of figure 6 and CAL factors listed in table 1.

Duration magnitude is determined by the length of signal, in seconds, read from drum recordings of Type 1 seismographs. This length of time is measured from the $\mathrm{P}$ arrival to the point where the earthquake signal has decayed to nearly the background noise level. Drum-recorded duration magnitude is calculated with a relationship equivalent to the develocorder viewer output.

${ }^{2}$ Klein, F.W., 2002, User's guide to HYPOINVERSE-2000, a Fortran Program to solve for earthquake locations and magnitudes: U.S. Geological Survey Open-File Report 02-171, 116 p.

${ }^{3}$ Nakata, J., and Okubo, P., 1997, Determination of station amplitude magnitude corrections for the Hawaiian Volcano Observatory telemetered seismograph network: Data from 1992-1997: U.S. Geological Survey Open-File Report 97-863, 73 p. 


\section{SEISMIC CATALOG}

The emphasis in both station coverage and detailed data analysis is on the highly active south half of the Island of Hawai i i. The set of well-recorded earthquakes located in the Hawai' i Island region is nearly complete above magnitude 2.0. Many smaller events are located in the densely instrumented Kilauea area. Substantial effort is made to locate earthquakes elsewhere within the Hawaiian Archipelago. Such coverage cannot be as complete as in south Hawai i, but nearly all events above magnitude 4.0 are located with limited precision.

Data presented in the seismic catalog are in three parts: (1) Maps showing computer-located hypocenters are given in figures 11-24. The location maps are of different scales and provide hypocenters with magnitude thresholds set at 1.0, 2.0, 3.0 , and 3.5, varying according to region. (2) The list of computer locations constitutes the bulk of this summary and is given in table 4. Each earthquake in the list is assigned a three-letter code based on its general location and depth. Figures 7-10 are maps of the regions used to assign the location codes. The latitude and longitude limits of rectangular regions are listed in table 3. When the listed coordinates overlap, precedence is given according to figures 7-10. (3) Table 5 re-lists the events in table 4 for which the preferred magnitude is 3.0 or larger. This list includes many of the earthquakes felt in Hawai $i$.

Table 3. Names and coordinates of regions used for classifying earthquakes.

All earthquakes locate in one of the following groups, identified by a numerical class or three-letter code:

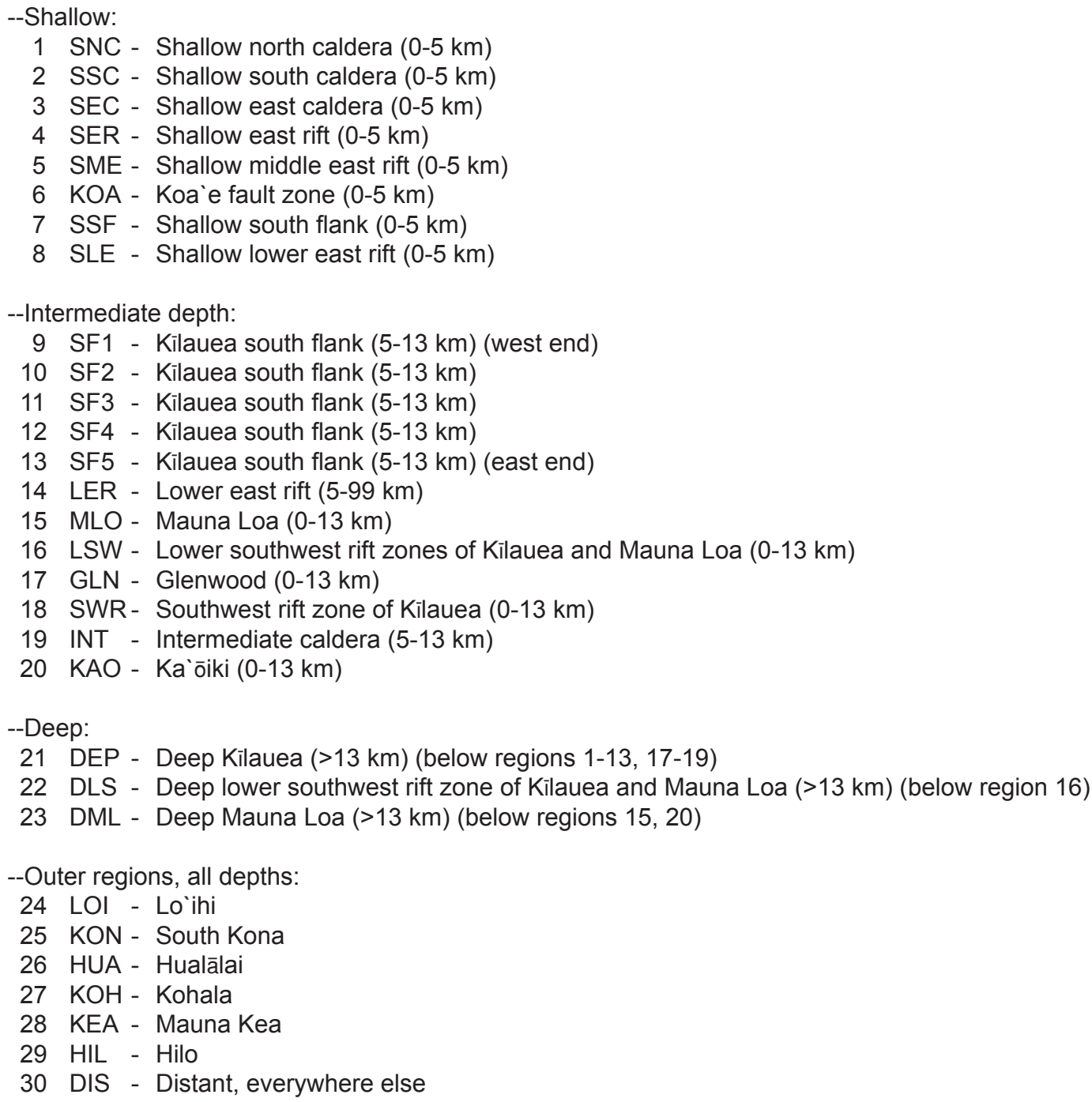


Table 3 (continued). The latitude and longitude limits of the regions are given below. If the coordinates overlap, precedence is given according to maps in figures 7-10.

\begin{tabular}{|c|c|c|c|c|c|}
\hline No. & Code & N. Lat. & S. Lat. & W. Lon. & E. Lon. \\
\hline 1 & SNC & 1928.0 & 1924.5 & 15519.0 & 15514.0 \\
\hline 2 & SSC & 1924.5 & 1922.0 & 15519.0 & 15516.5 \\
\hline 3 & SEC & 1924.5 & 1922.0 & 15516.5 & 15514.0 \\
\hline 4 & SER & 1926.0 & 1920.5 & 15514.0 & 15507.2 \\
\hline 5 & SME & 1926.0 & $1921.75-1920.0$ & 15507.2 & 15500.0 \\
\hline 6 & $\mathrm{KOA}$ & 1922.0 & 1920.5 & 15517.0 & 15514.0 \\
\hline 7 & SSF & 19 20.6-19 24.0 & 1910.0 & 15517.0 & 15500.0 \\
\hline 8 & SLE & 1932.0 & 1916.0 & 15500.0 & 15440.0 \\
\hline 9 & SF1 & 1922.0 & 1910.0 & 15517.0 & 15514.5 \\
\hline 10 & SF2 & 1926.0 & 1910.0 & 15514.5 & 15512.3 \\
\hline 11 & SF3 & 1926.0 & 1910.0 & 15512.3 & 15509.1 \\
\hline 12 & SF4 & 1926.0 & 1910.0 & 15509.1 & 15505.3 \\
\hline 13 & SF5 & 1926.0 & 1910.0 & 15505.3 & 15500.0 \\
\hline 14 & LER & 1932.0 & 1916.0 & 15500.0 & 15440.0 \\
\hline 15 & MLO & 1935.0 & 1919.0 & 15535.0 & 15519.0 \\
\hline 16 & LSW & 1919.0 & 1840.0 & 15543.0 & 15525.0 \\
\hline 17 & GLN & 1935.0 & 1926.0 & 15519.0 & 15500.0 \\
\hline 18 & SWR & 1922.0 & 1910.0 & 15525.0 & 15517.0 \\
\hline 19 & INT & 1928.0 & 1922.0 & 15519.0 & 15514.0 \\
\hline 20 & $\mathrm{KAO}$ & 1930.0 & 1919.0 & 15532.0 & 15519.0 \\
\hline 21 & DEP & 1935.0 & 1910.0 & 15525.0 & 15500.0 \\
\hline 22 & DLS & 1919.0 & 1840.0 & 15543.0 & 15525.0 \\
\hline 23 & DML & 1935.0 & 1919.0 & 15535.0 & 15519.0 \\
\hline 24 & LOI & 1910.0 & 1840.0 & 15525.0 & 15500.0 \\
\hline 25 & KON & 1939.0 & 1900.0 & 15620.0 & 15543.0 \\
\hline 26 & HUA & 1955.0 & 1939.0 & 15620.0 & 15543.0 \\
\hline 27 & $\mathrm{KOH}$ & 2025.0 & 1955.0 & 15620.0 & 15534.0 \\
\hline 28 & KEA & 2025.0 & 1935.0 & 15534.0 & 15440.0 \\
\hline 29 & HIL & 1947.0 & 1932.0 & 15509.0 & 15440.0 \\
\hline
\end{tabular}




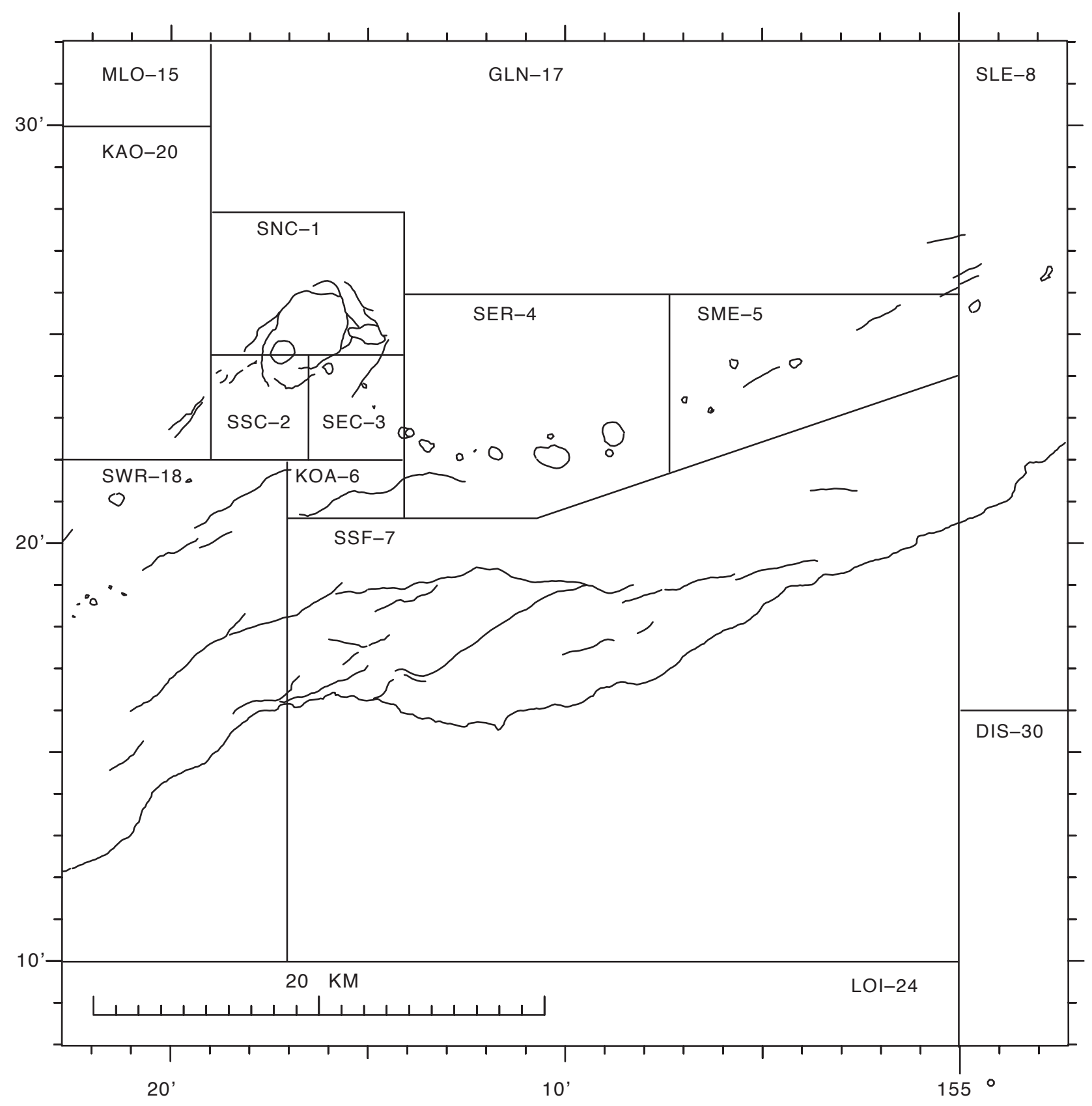

Figure 7. Earthquake classification, shallow (0-5 km deep), for Kilauea and the east flank of Mauna Loa. 


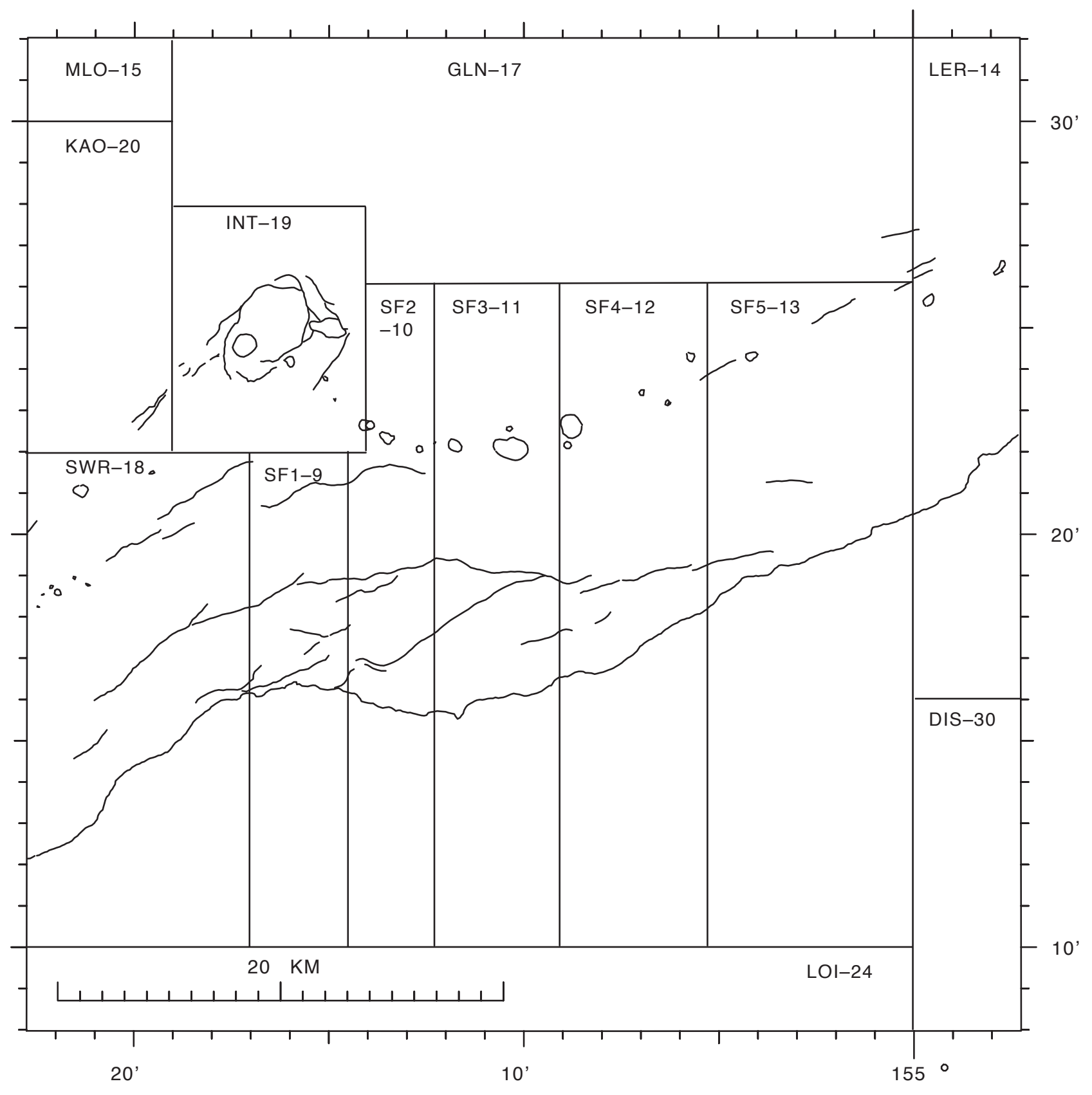

Figure 8. Earthquake classification, intermediate (5.1-13 km deep), for Killauea and the east flank of Mauna Loa. 


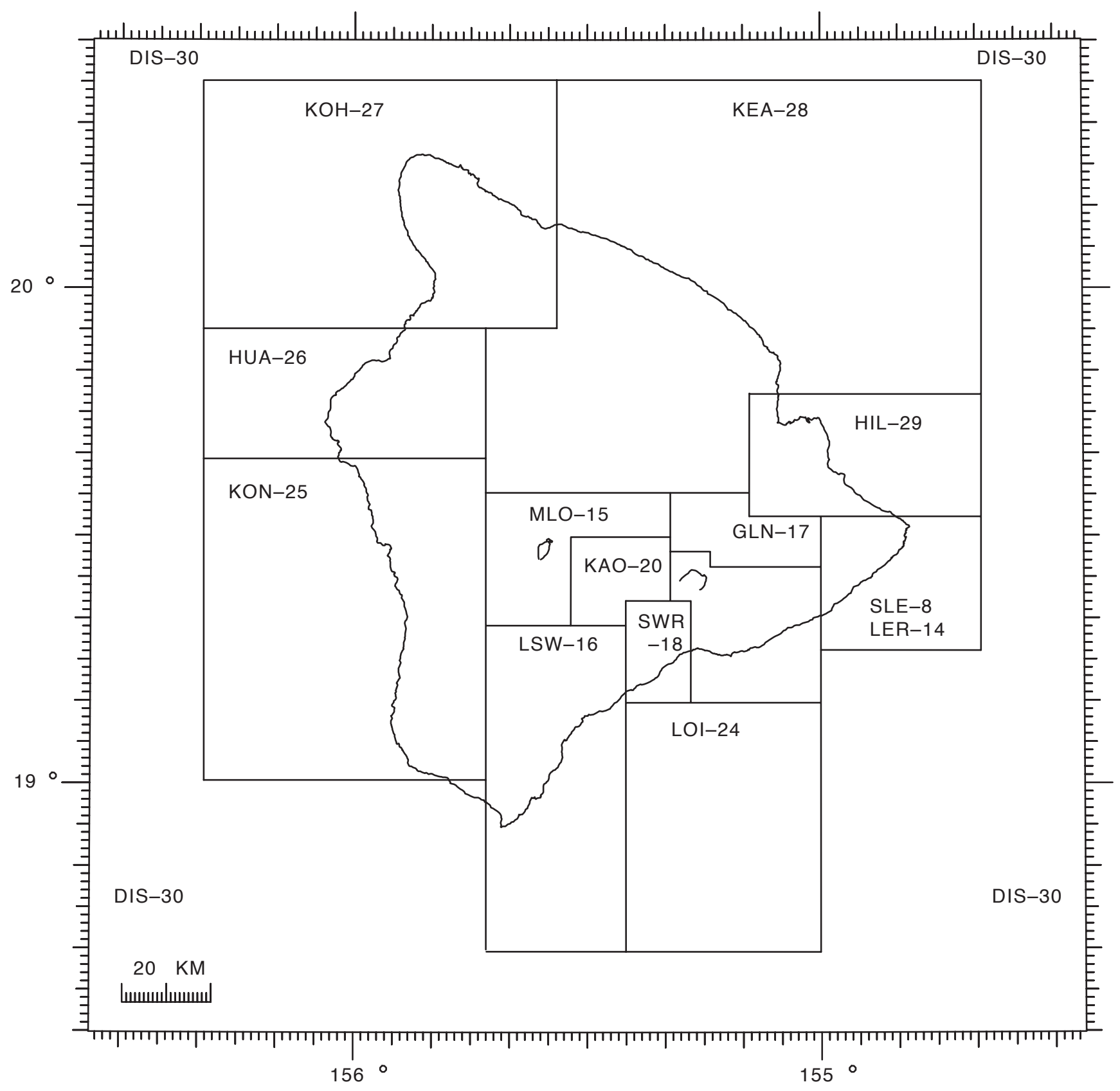

Figure 9. Earthquake classification, crustal (0-13 km deep), for the Island of Hawai' i. 


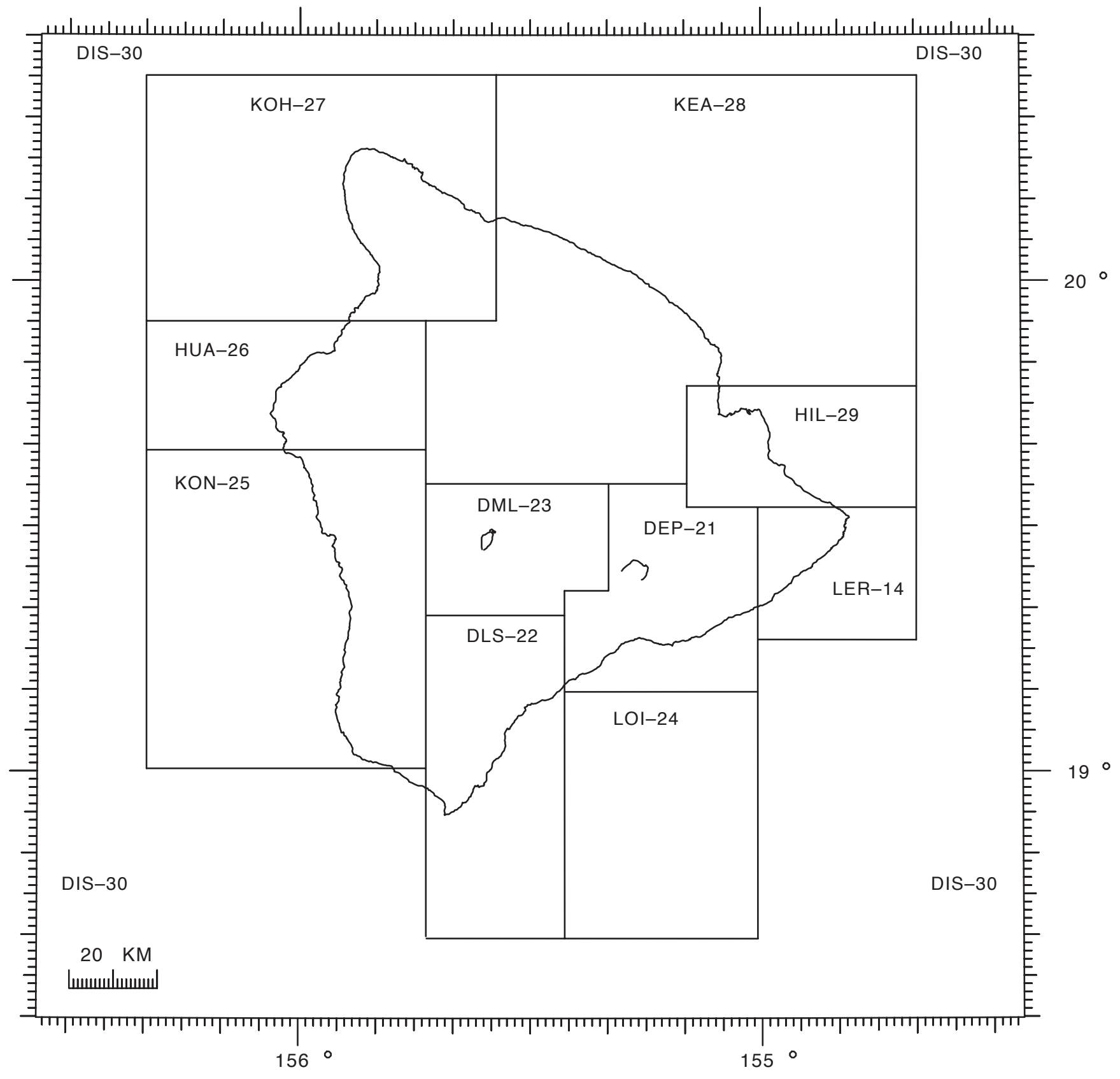

Figure 10. Earthquake classification, deep (greater than $13 \mathrm{~km}$ deep), for the Island of Hawai'i. 
Figure 11. 2006 earthquake locations, Hawiian Islands,

$0-60 \mathrm{~km}$ depth, $\mathrm{M} \geq 3.5$

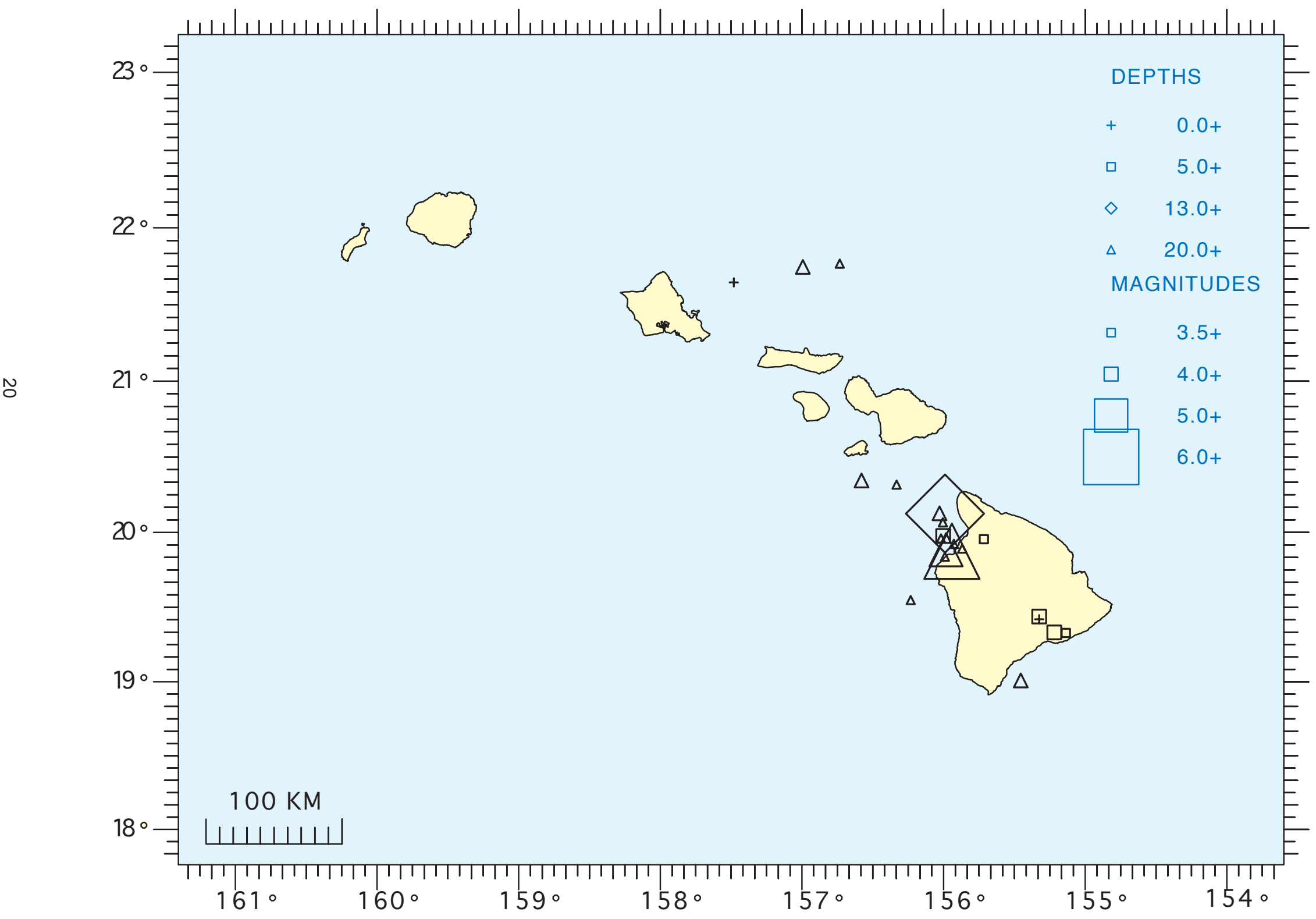


Figure 12. 2006 earthquake locations, Hawai i Island,

$$
0 \text { - } 60 \mathrm{~km} \text { depth, } M \geq 3.0
$$

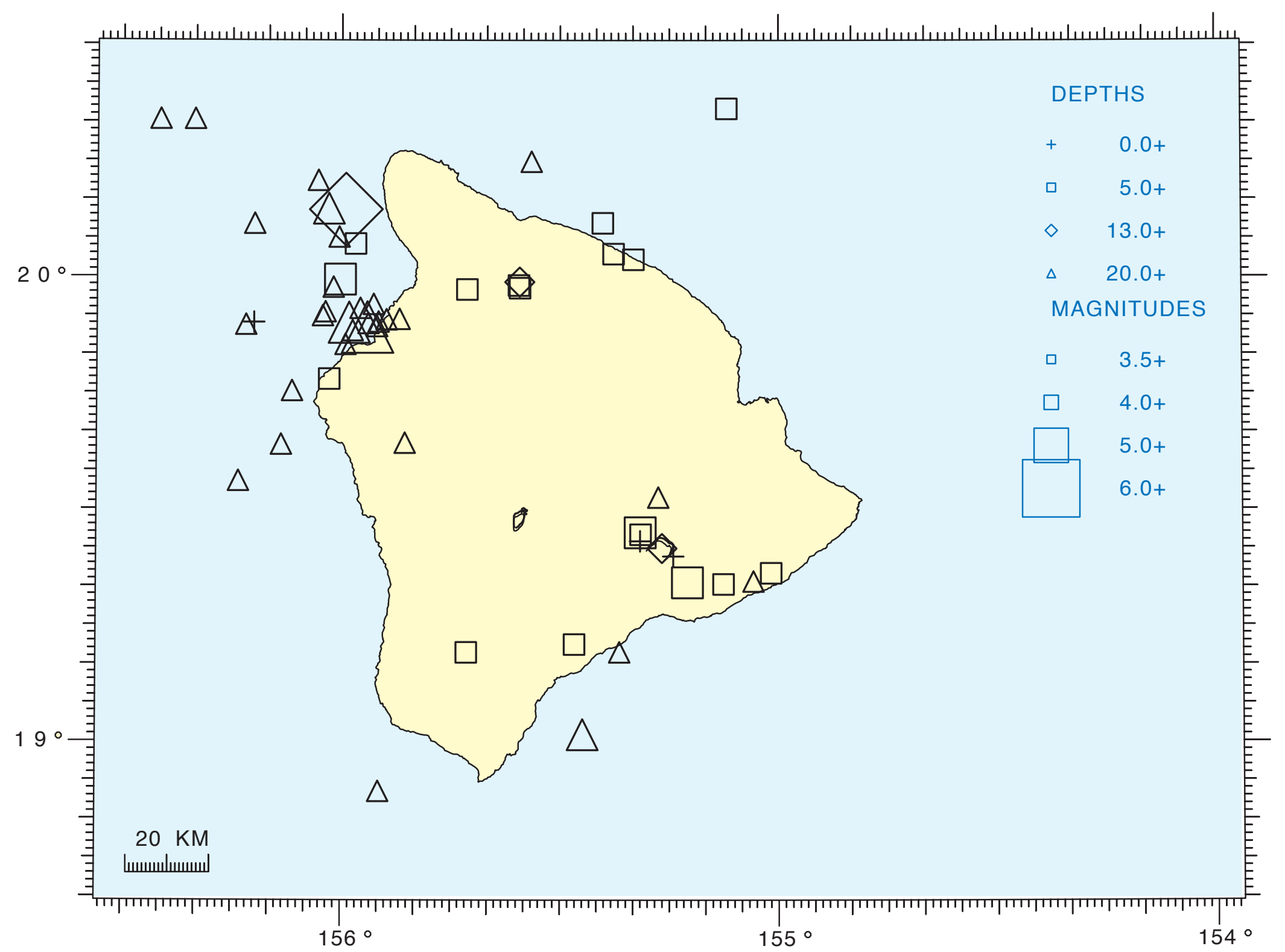


Figure 13. 2006 earthquake locations, Hawai i Island, shallow (0 - $5 \mathrm{~km}$ depth), $\mathrm{M} \geq 2.0$.

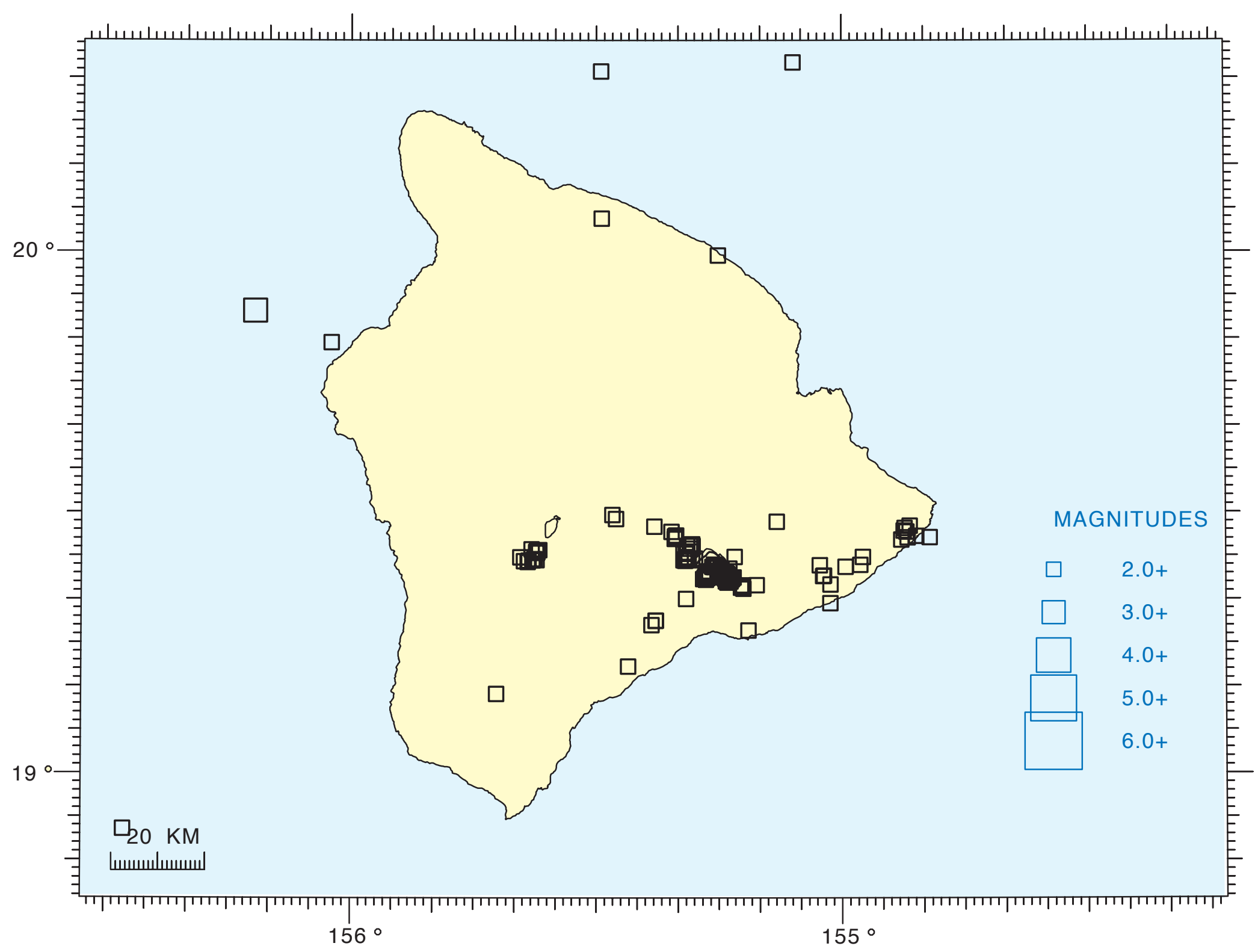


Figure 14. 2006 earthquake locations, Hawai i Island, intermediate (5.1 - $13 \mathrm{~km}$ depth), $\mathrm{M} \geq 2.0$.

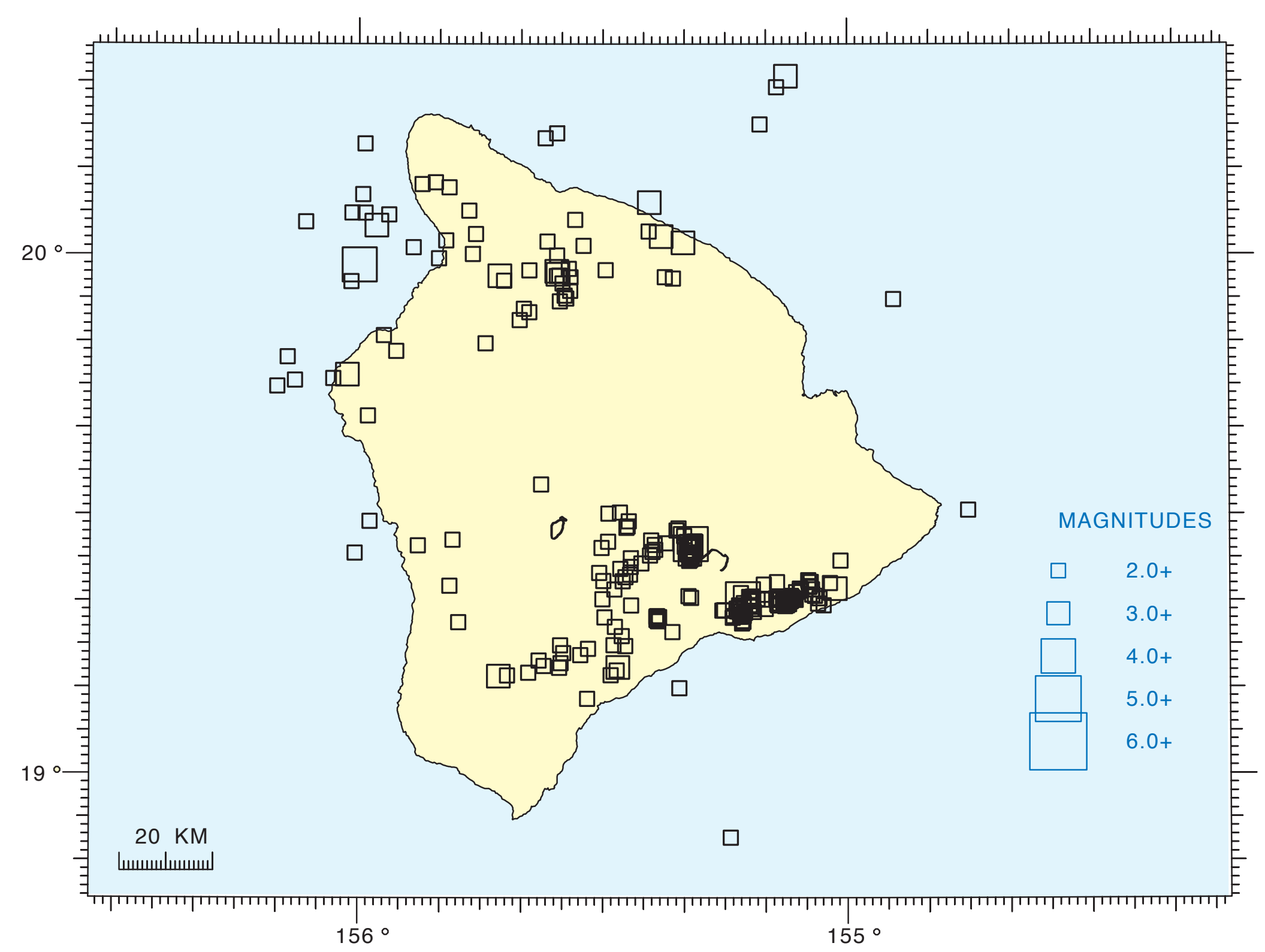


Figure 15. 2006 earthquake locations, Hawai i Island, deep (13.1 - 60 km depth), $M \geq 2.0$.

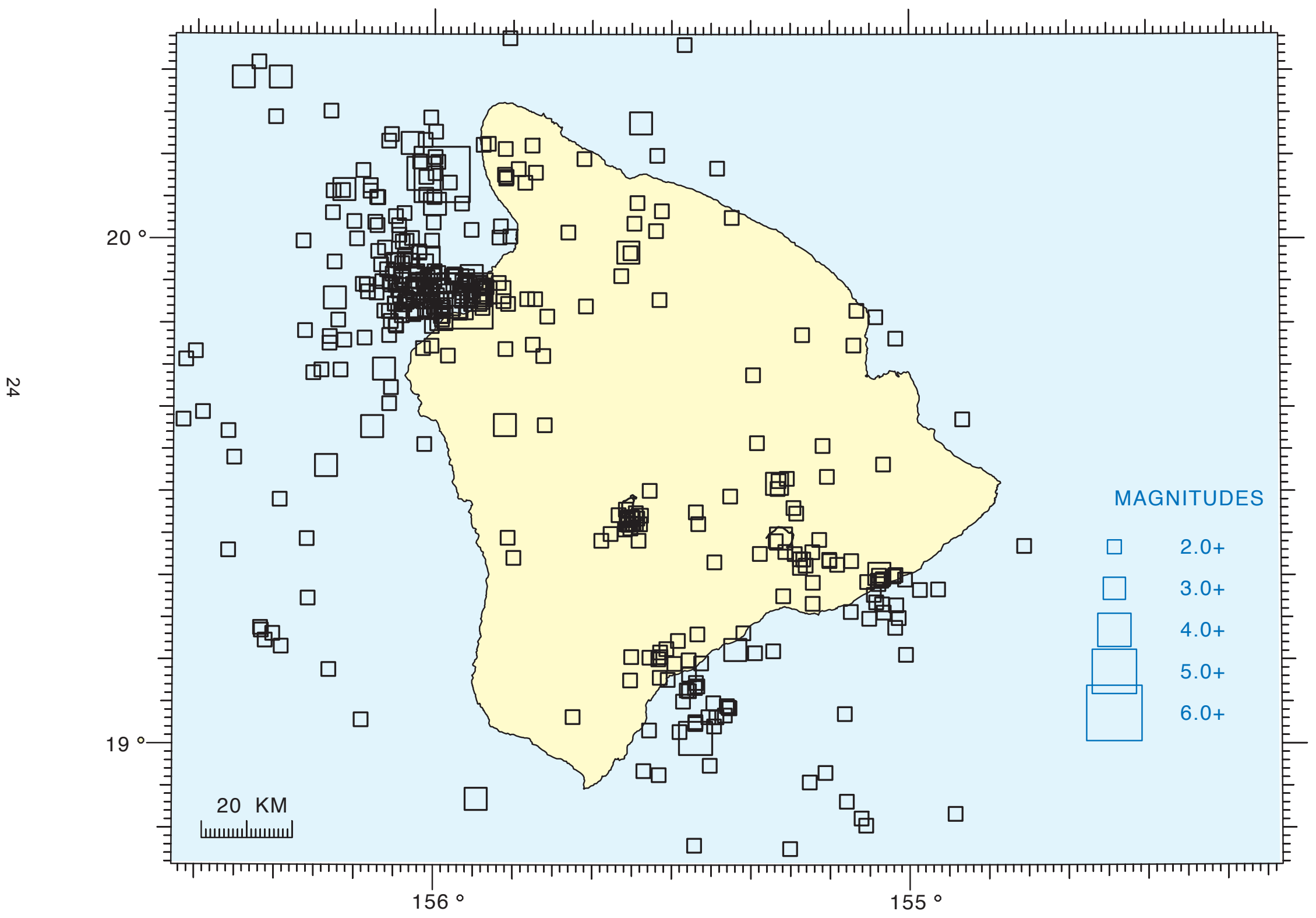


Figure 16. 2006 earthquake locations, Kilauea summit, shallow (0-5 km depth), $M \geq 1.0$.

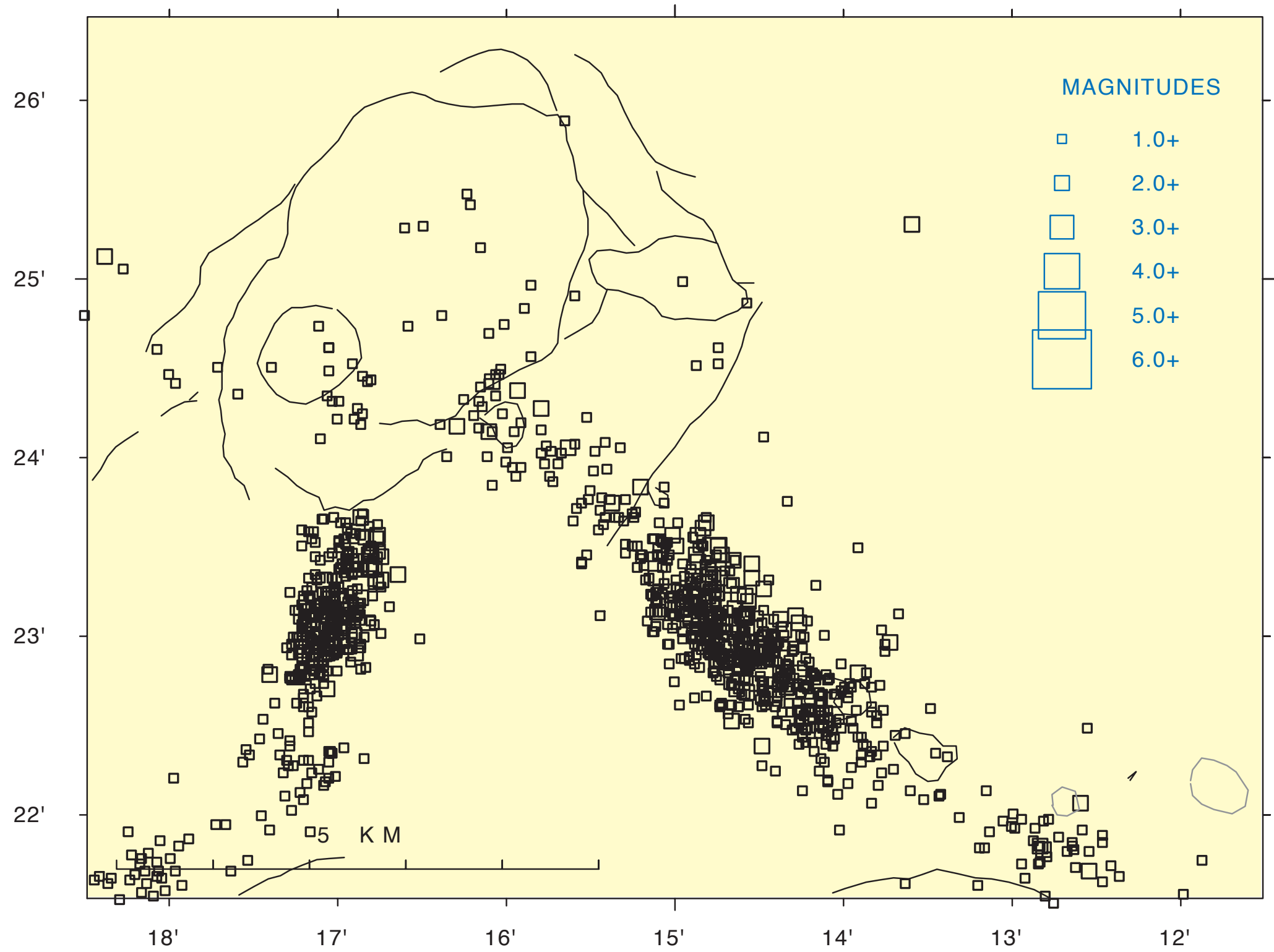


Figure 17. 2006 earthquake locations, Kïlauea summit, intermediate (5.1 - $13 \mathrm{~km}$ depth), $M \geq 1.0$.

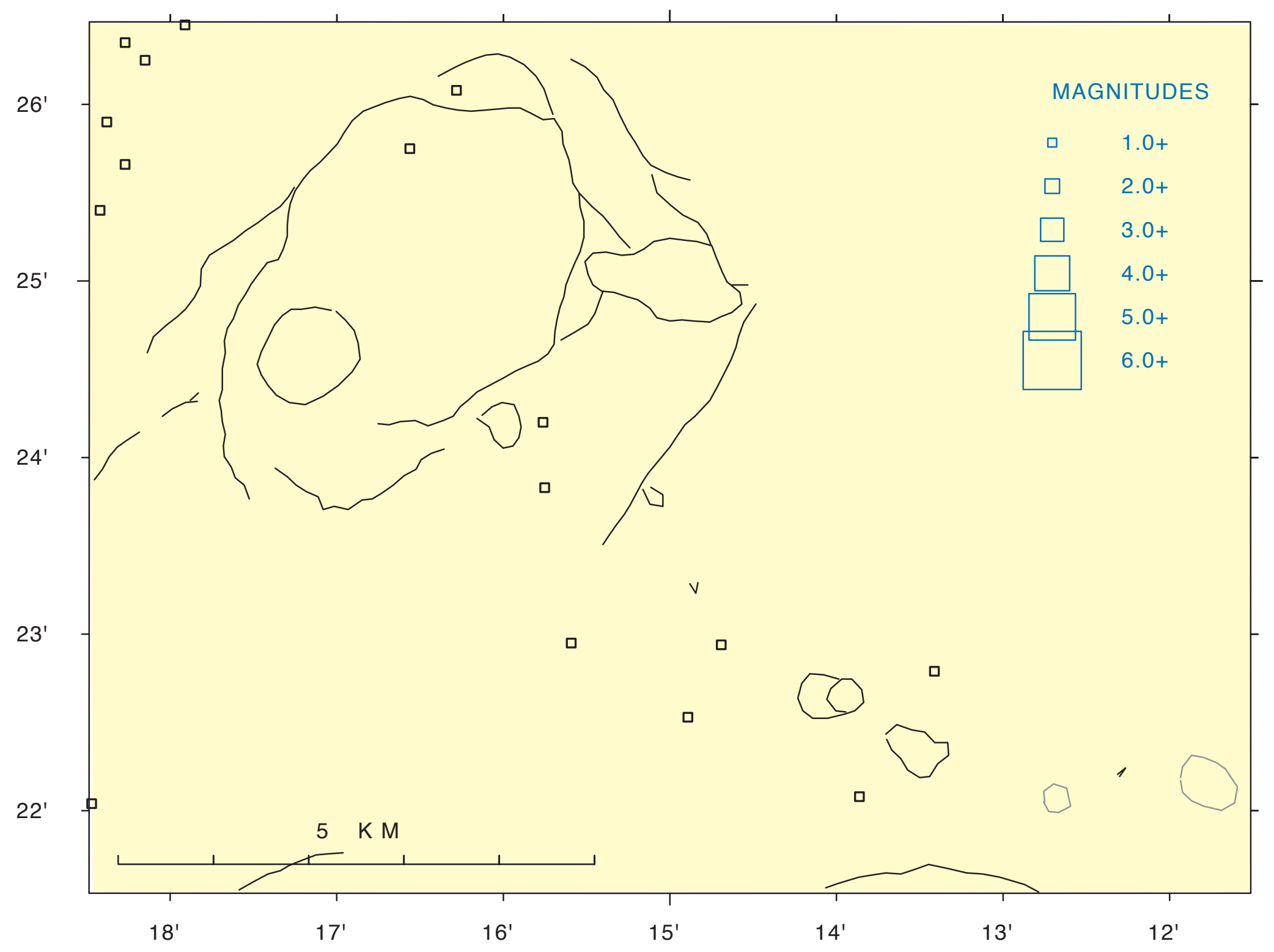


Figure 18. 2006 earthquake locations, Kïlauea summit, deep (13.1 - $60 \mathrm{~km}$ depth), $M \geq 1.0$.

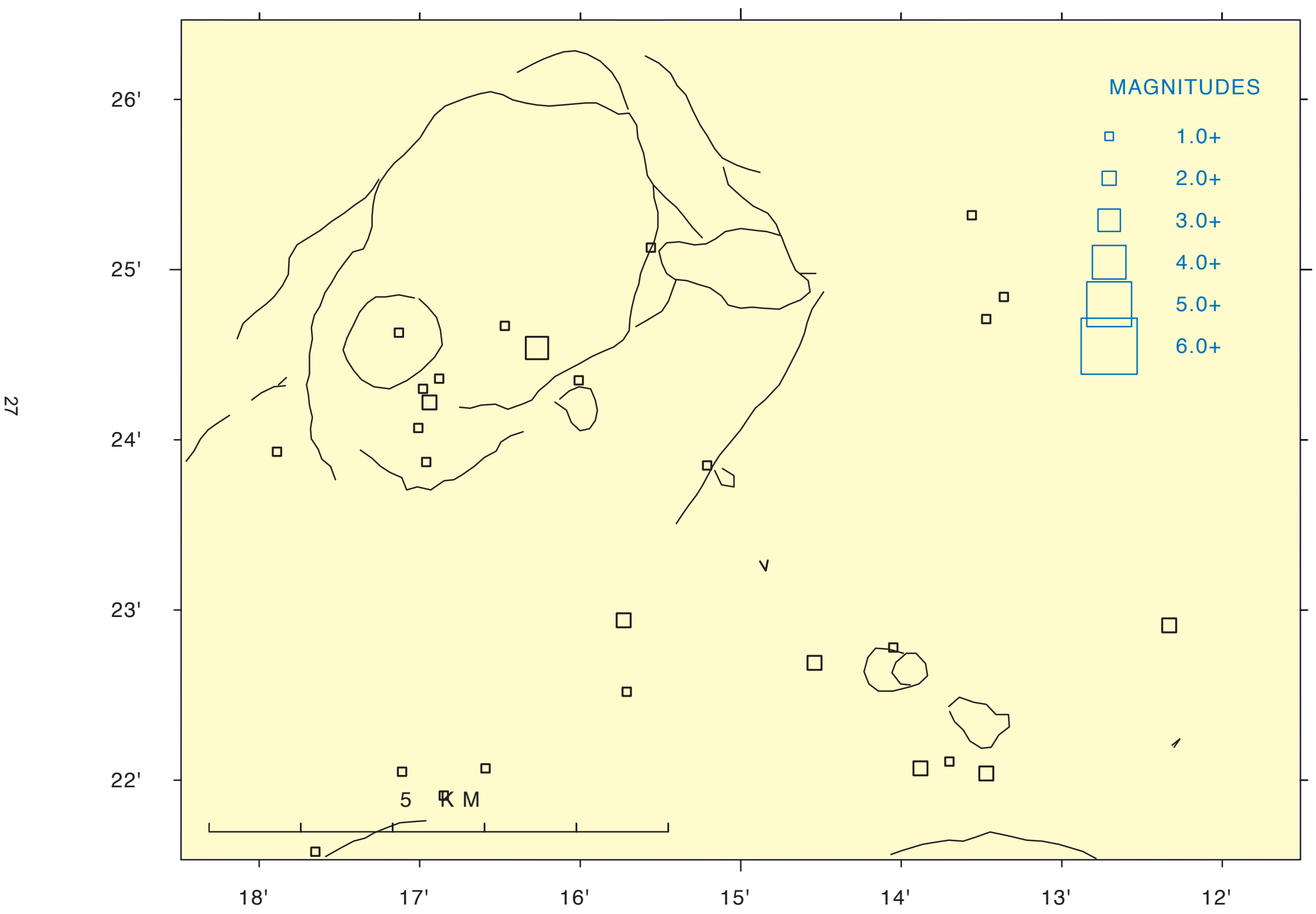


Figure 19. 2006 earthquake locations, Killauea south flank, shallow (0-5 km depth), $M \geq 2.0$.

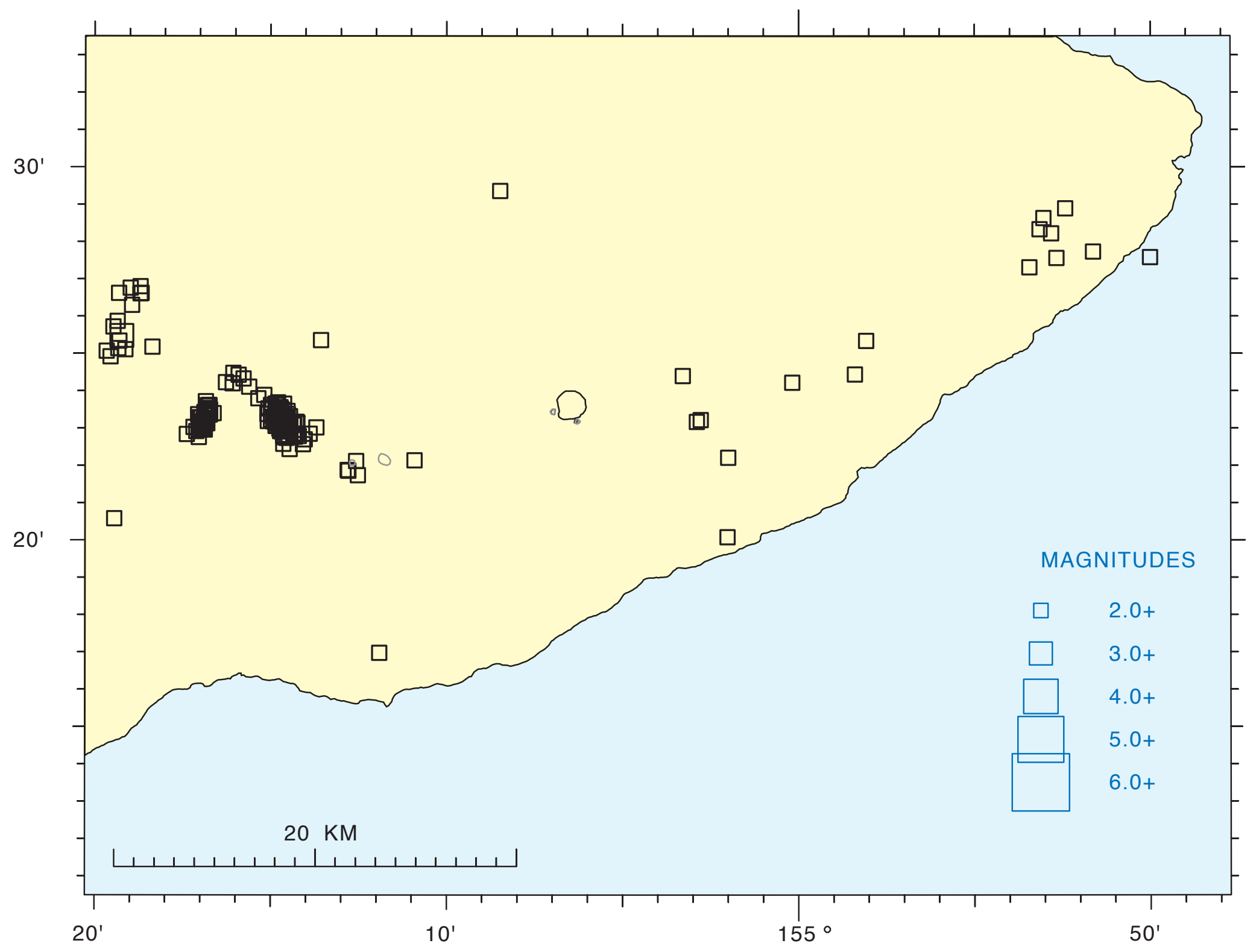


Figure 20. 2006 earthquake locations, Killauea south flank, intermediate (5.1 - $13 \mathrm{~km}$ depth), $\mathrm{M} \geq 2.0$.

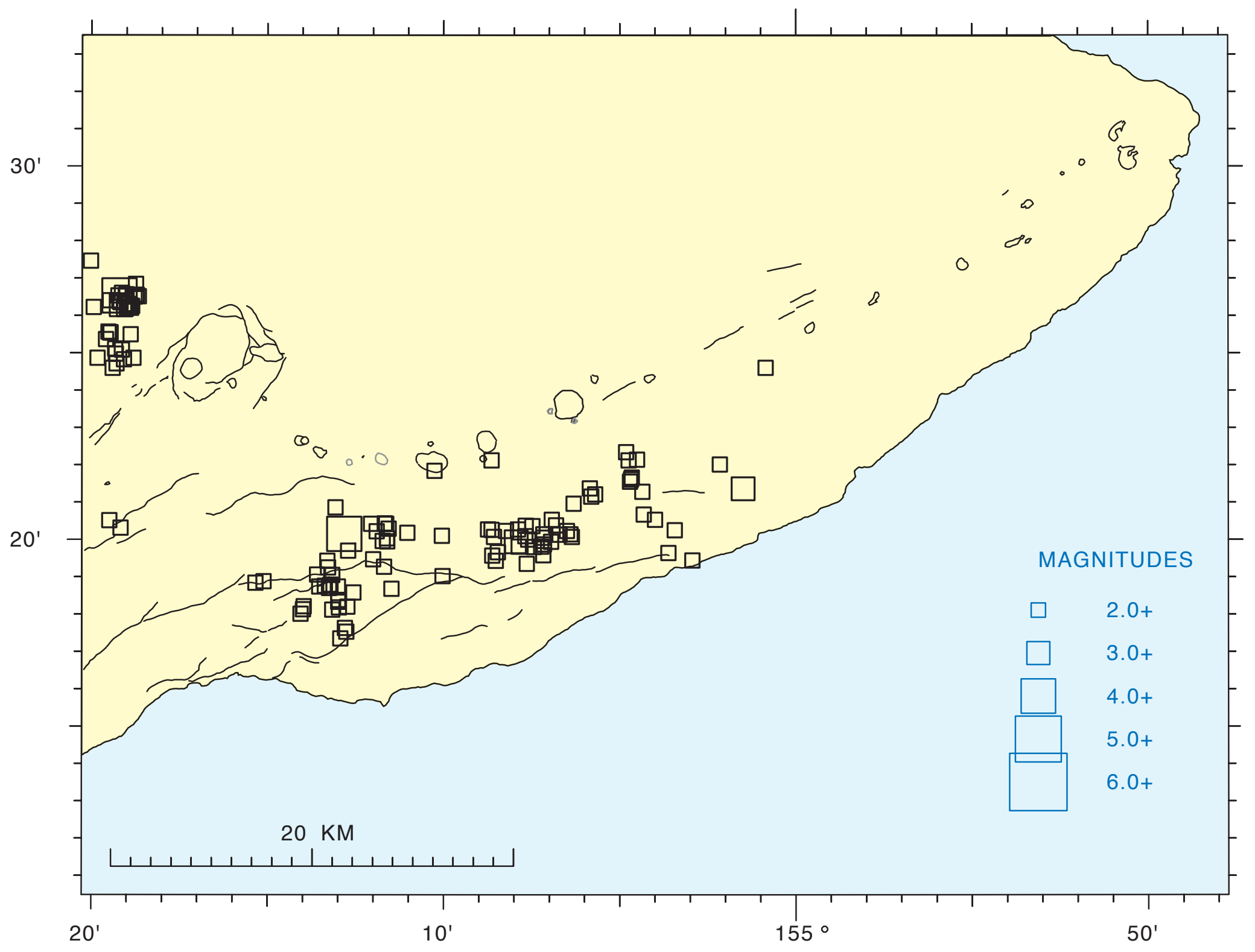


Figure 21. 2006 earthquake locations, Kïlauea south flank, deep (13.1 - $60 \mathrm{~km}$ depth), $\mathrm{M} \geq 2.0$.

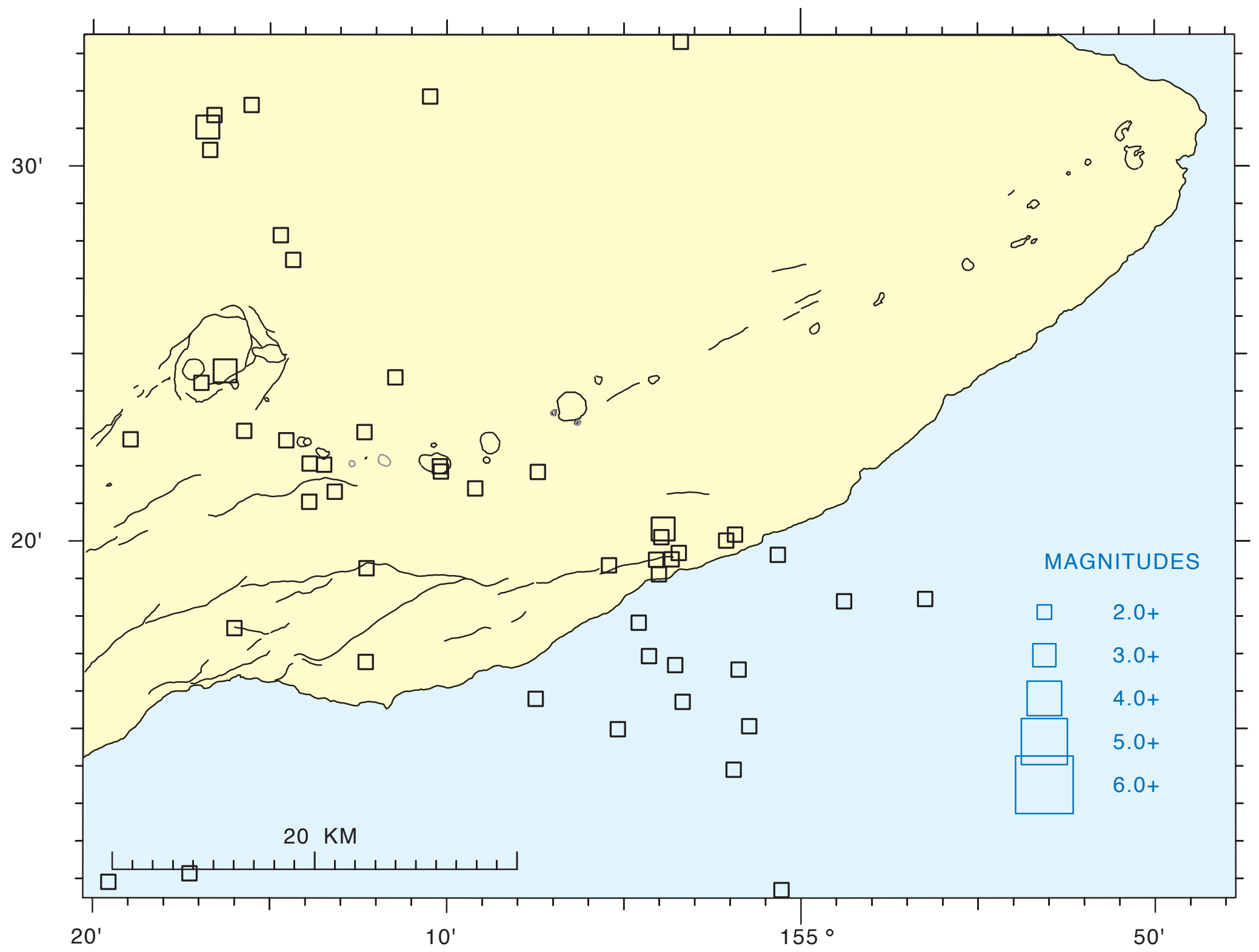


Figure 22. 2006 earthquake locations, Mauna Loa summit, shallow (0 - $5 \mathrm{~km}$ depth), $\mathrm{M} \geq 2.0$.

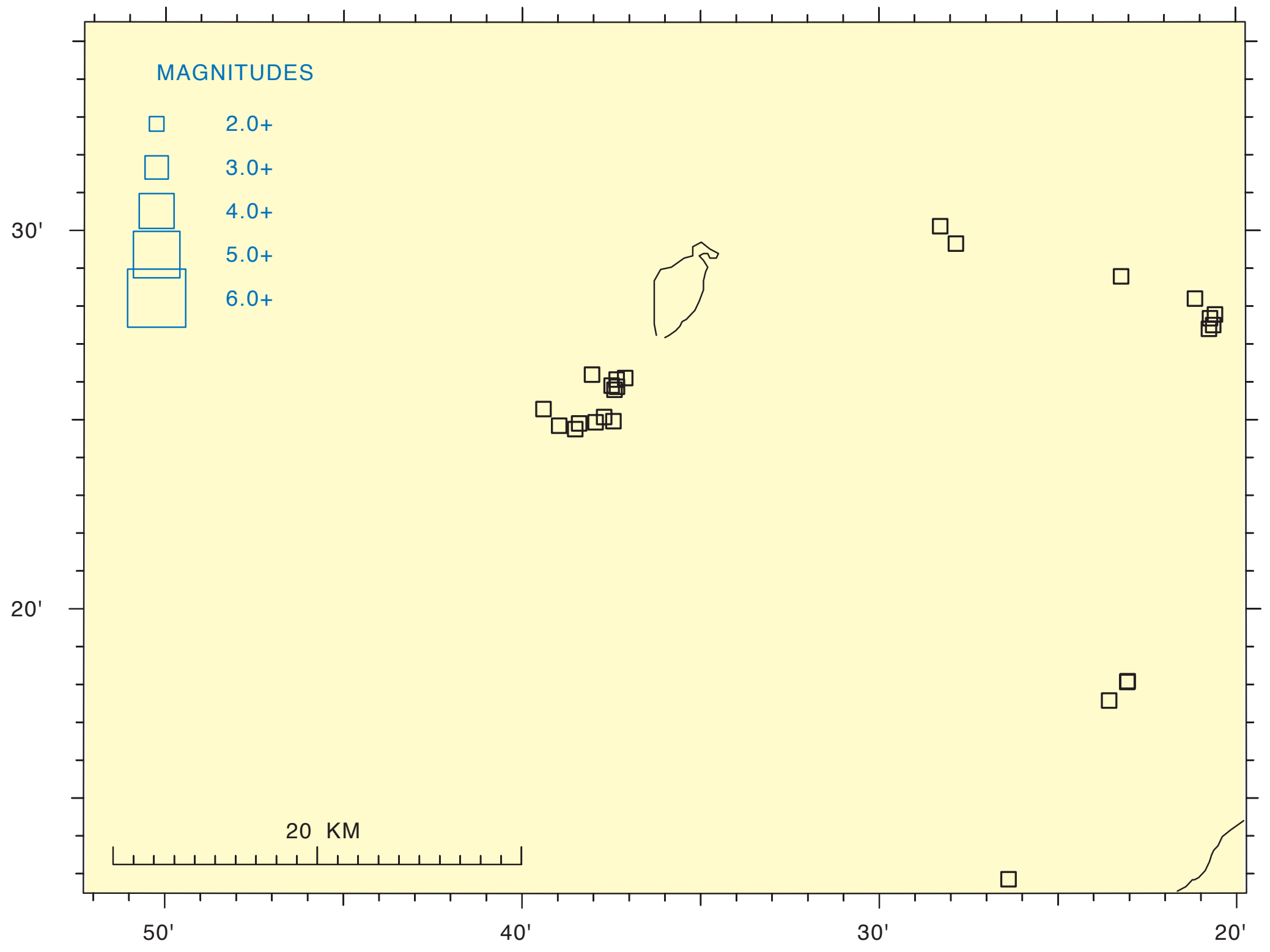


Figure 23. 2006 earthquake locations, Mauna Loa summit, intermediate (5.1 - $13 \mathrm{~km}$ depth), $M \geq 2.0$.

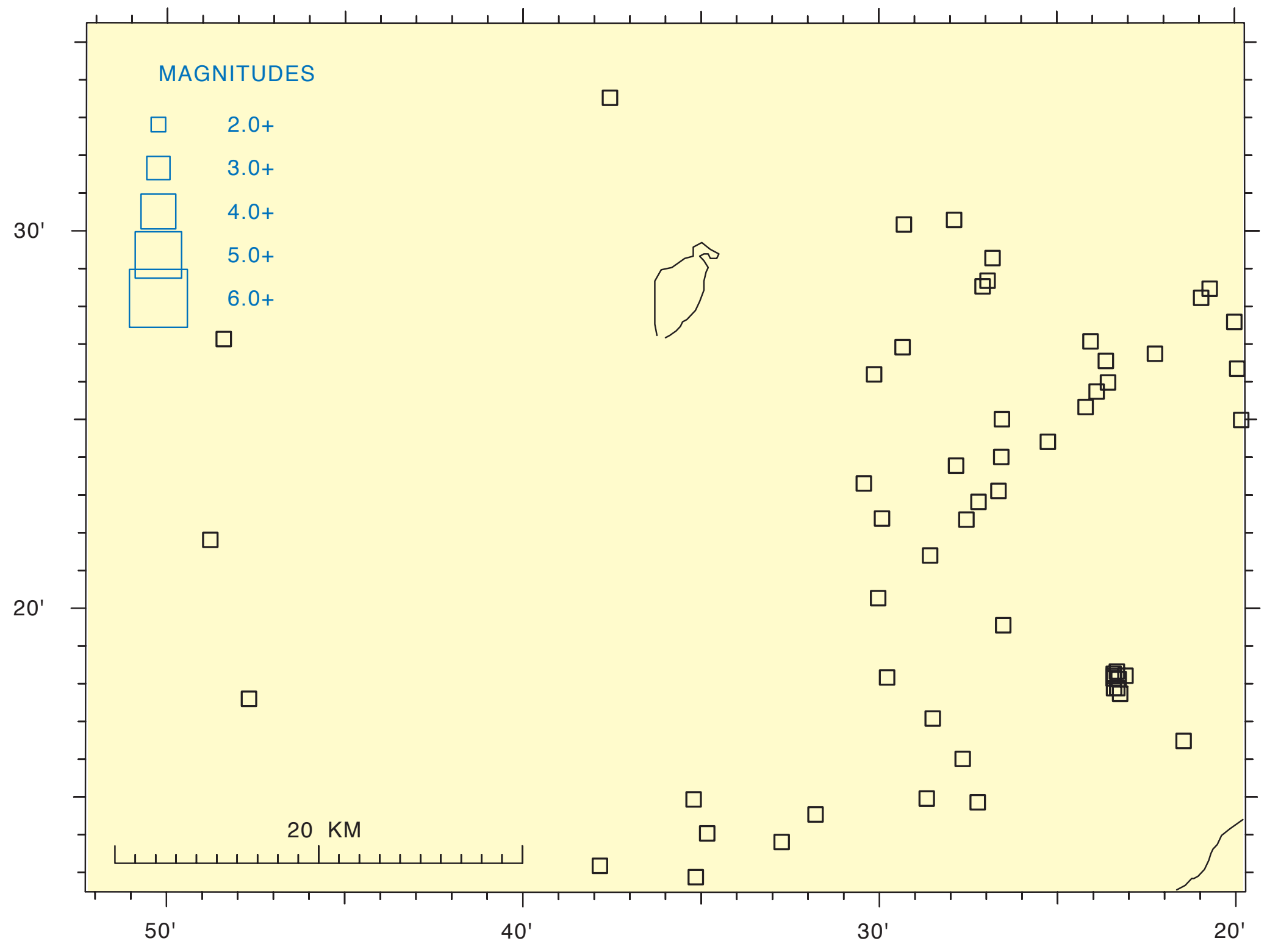


Figure 24. 2006 earthquake locations, Mauna Loa summit, deep (13.1 - $60 \mathrm{~km}$ depth), $\mathrm{M} \geq 2.0$.

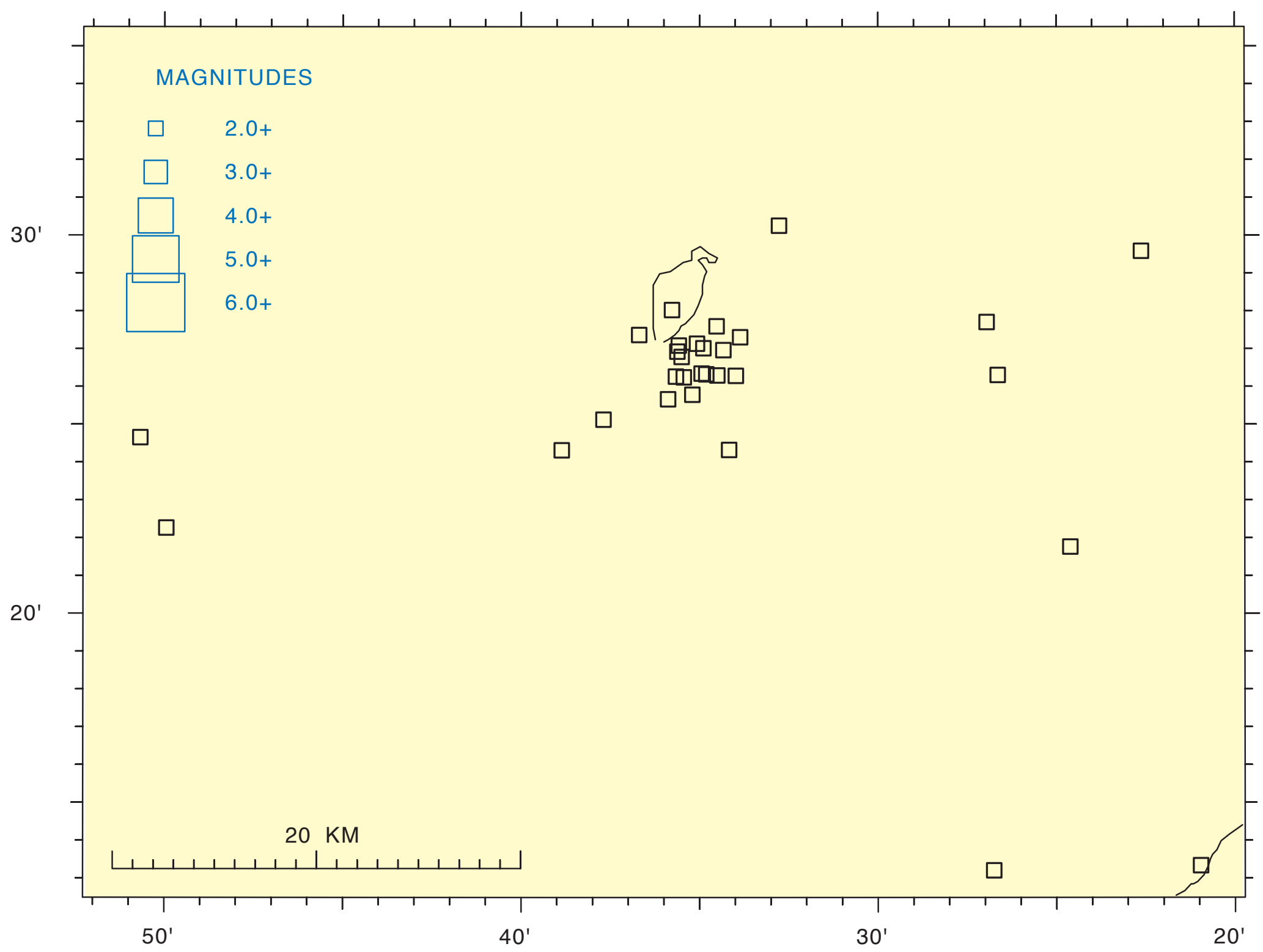


Table 4 is a chronological list of the 5,505 selected events successfully located during 2006. Summary data files are available

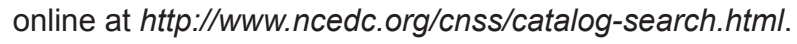

For each event in Table 2, the following data are presented:

ORIGIN TIME - in Hawaiian Standard Time: date, hour (HR), minute (MN), and second (SEC).

EPICENTER - in degrees and minutes of north latitude (LAT N) and west longitude (LON W) in Old Hawaiian Datum.

DEPTH - Depth of focus in kilometers.

NRD - Number of $P \& S$ readings with final weights $>0.1$.

NS - Number of $S$ readings with final weights $>0.1$

RMS SEC - Root mean square travel time residuals, in seconds.

ERH km - Standard error of the epicenter, in kilometers.

ERZ km - Standard error of depth of focus, in kilometers.

LOC REMKS - Remarks, three-letter code for geographic location of events. See Figures 7-10 for location of mnemonic code. Additional one-letter codes have the following meanings:

F felt

L long-period character

$\mathrm{T} \quad$ associated with harmonic tremor

B quarry or other blast

\# the location program had a convergence problem, which usually means that the depth may be unreliable.

- $\quad$ the depth was held fixed.

PREF MAG - The preferred magnitude chosen from the available magnitudes.

Preference set as: X-amplitude magnitude, if none

D-duration magnitude Develocorder equivalent, if none

U-external magnitude, usually calculated from drum records or from an external source.

AZ GAP - Largest azimuthal gap in degrees between azimuthally adjacent stations.

MIN DS - Distance to the nearest station, in kilometers.

Table 5 lists the 61 events of magnitude 3.0 or greater, selected from Table 4. 
--ORIGIN TIME (HST)-- -LAT N-- --LON W-- DEPTH N RMS ERH ERZ LOC

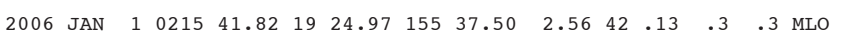
$\begin{array}{lllllllllllllll}2006 & \text { JAN } & 1 & 1048 & 9.85 & 19 & 20.25 & 155 & 7.38 & 6.42 & 41 & .11 & .4 & .9 & \text { SF } 4\end{array}$

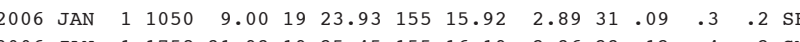
$\begin{array}{llllllll} & \end{array}$

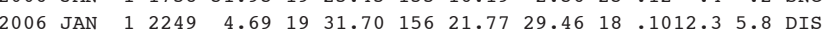

$\begin{array}{lllllllllllllll}2006 & \text { JAN } & 1 & 2254 & 29.15 & 19 & 22.89 & 155 & 16.90 & 2.55 & 32 & .07 & .2 & .2 & \text { SSC }\end{array}$ $\begin{array}{lllllllllllllll}2006 & \text { JAN } & 2 & 0018 & 30.63 & 19 & 24.23 & 155 & 15.89 & 3.50 & 19 & .07 & .4 & .4 & \text { SEC }\end{array}$ $\begin{array}{lllllllllllll}2006 & \text { JAN } & 2 & 0024 & 35.32 & 19 & 20.39 & 156 & 9.22 & 34.05 & 19 & .1010 .5 & 3.7 \\ \text { KON }\end{array}$ $\begin{array}{lllllllllllllll}2006 & \text { JAN } & 2 & 0127 & 2.78 & 19 & 23.00 & 155 & 16.94 & 2.81 & 25 & .10 & .3 & .2 & \text { SSC }\end{array}$ $\begin{array}{llllllllllllll}2006 & \text { JAN } & 2 & 0353 & 33.07 & 19 & 30.44 & 155 & 27.16 & 3.50 & 19 & .13 & .3 & .9 \\ \text { MLO }\end{array}$

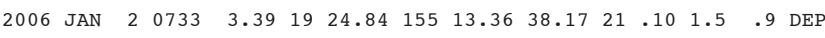

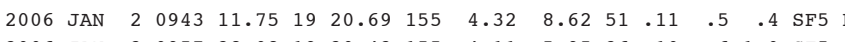

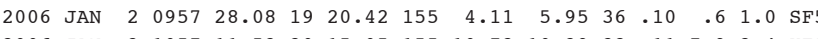

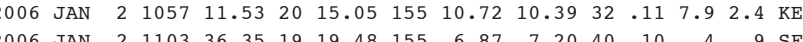

$\begin{array}{lllllllllllllll}2006 & \text { JAN } & 2 & 1419 & 53.64 & 19 & 34.23 & 155 & 40.91 & 8.83 & 22 & .10 & .6 & 1.6 & \text { MLO }\end{array}$ 2006 JAN $2 \begin{array}{llllllllllll}1433 & 22.52 & 19 & 33.78 & 155 & 41.16 & 9.62 & 24 & 09 & .5 & 1.4 & \text { MLO }\end{array}$

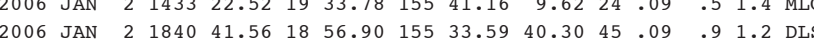

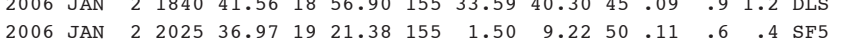
$\begin{array}{llllllllllllllll}2006 & \text { JAN } & 2 & 2149 & 59.78 & 19 & 10.64 & 155 & 44.28 & 13.10 & 27 & .14 & 1.7 & .8 & \text { KON }\end{array}$

$\begin{array}{llllllllllllll}2006 & \text { JAN } & 2 & 2152 & 8.51 & 19 & 29.78 & 155 & 48.09 & 10.49 & 22 & .14 & 1.1 & .6 \\ \text { KON }\end{array}$ $\begin{array}{lllllllllllllll}2006 & \text { JAN } & 3 & 0010 & 36.52 & 19 & 35.54 & 155 & 55.46 & 12.53 & 9 & .10 & 6.7 & 1.0 & \text { KON }\end{array}$

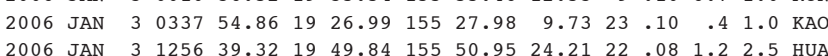

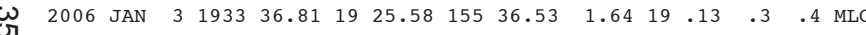

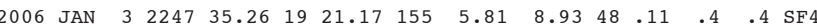

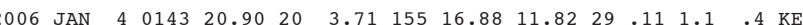

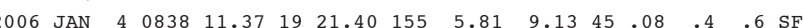
$\begin{array}{lllllllllllllll}2006 & \text { JAN } & 4 & 1022 & 15.85 & 19 & 24.52 & 155 & 38.06 & 3.21 & 19 & .12 & .4 & .5 & \text { MLO }\end{array}$ $\begin{array}{lllllllllllllll}2006 & \text { JAN } & 4 & 1228 & 6.74 & 19 & 19.58 & 155 & 8.22 & 8.59 & 45 & .10 & .4 & .6 & \mathrm{SF}\end{array}$

$\begin{array}{llllllllllllll}2006 & \text { JAN } & 4 & 1302 & 19.32 & 19 & 30.20 & 155 & 32.83 & 13.74 & 40 & .11 & .4 & .5\end{array}$

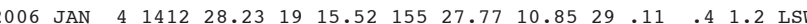
$\begin{array}{lllllllllllllll}2006 & \text { JAN } & 4 & 1447 & 4.86 & 20 & 1.17 & 155 & 55.39 & 26.59 & 35 & .10 & 1.1 & 1.9 & \text { кон }\end{array}$ $\begin{array}{lllllllllllllll}2006 & \text { JAN } & 4 & 2348 & 28.52 & 19 & 30.47 & 155 & 32.98 & 13.81 & 34 & .12 & .4 & .7 & \text { DM }\end{array}$ $\begin{array}{lllllllllllllll}2006 & \text { JAN } & 5 & 0101 & 50.71 & 19 & 21.72 & 155 & 17.61 & 2.75 & 18 & .10 & .3 & .4 & \text { SWR }\end{array}$

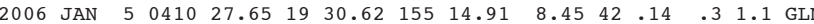

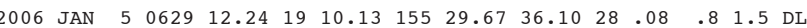

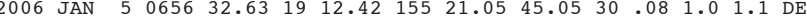

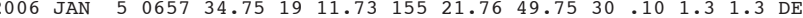
$\begin{array}{lllllllllllllll}2006 & \text { JAN } & 5 & 0729 & 46.31 & 19 & 48.93 & 155 & 35.97 & 14.42 & 26 & .11 & .9 & .6 & \text { KEA }\end{array}$

2006 JAN $5 \begin{array}{lllllllllllll}1003 & 26.75 & 19 & 26.08 & 155 & 19.63 & 6.03 & 21 & .07 & .4 & 1.0 & \text { KAO }\end{array}$

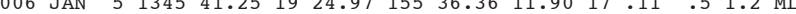

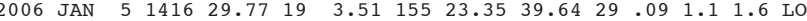

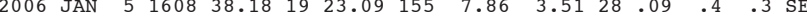

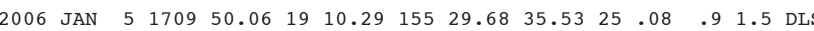
$2.5 \times \quad 99$ $1.4 \times \quad 132$ $1.7 \times 124$ $1.6 \times 30958$

$1.5 \times \quad 49 \quad 1$ $1.4 \times \quad 84$ $.0 \times 31845$ $1.4 \times 100-3$ $1.8 \times 263 \quad 2$ $2.5 \times 175$ $2.5 \times 31044$ $\begin{array}{lll}2.5 \times & 310 & 44 \\ 1.2 \times & 153 & 4\end{array}$ $1.3 \times \quad 96 \quad 11$ $\begin{array}{lll}1.3 \times & 126 & 10\end{array}$ $\begin{array}{lll}2.3 \times & 239 & 12\end{array}$ $\begin{array}{lll}3.1 \times & 193 & 7 \\ 1.6 \times 152 & 4\end{array}$ $\begin{array}{rrr}1.3 \times & 271 & 4 \\ 1.3 \times & 325 & 19\end{array}$ $\begin{array}{rrr}1.2 \times & 55 & 8 \\ 1.6 \times & 25 & 16\end{array}$ $1.6 \times 266 \quad 16$ $1.1 \times \quad 76 \quad 3$

$2.1 \times 150 \quad 5$ $1.8 \times 26520$ $1.7 \times \quad 147 \quad 5$ $1.4 \times \quad 98 \quad 1$ $\begin{array}{lll}1.7 \times & 115 & 4\end{array}$

$2.2 \times 104 \quad 5$ $1.5 \times \quad 98$ 2.3X $248 \quad 19$ $\begin{array}{lll}1.7 \times & 73 \quad 6\end{array}$ $1.4 \times 115 \quad 3$

$\begin{array}{lll}1.5 \mathrm{X} & 60 & 6\end{array}$ $\begin{array}{llll}1.7 \times & 112 & 3\end{array}$ $1.6 \times 22011$ $1.7 \times 227 \quad 13$ $1.6 \times 124 \quad 8$
---ORTGIN TIME (HST)-- -LAT N-- --LON W-- PEPTH N RMS ERH ERZ LOC PREF AZ MTN YEAR MON DA HRMN SEC DEG MIN DEG MIN KM RD SEC KM KM REMKS MAG GAP DS

$\begin{array}{llllllllllllllll}2006 & \text { JAN } & 5 & 1715 & 19.14 & 19 & 4.10 & 155 & 23.97 & 38.70 & 32 & .08 & 1.0 & 1.4 & \text { LOI }\end{array}$ $\begin{array}{llllllllllllllll}2006 & \text { JAN } & 5 & 2249 & 1.13 & 19 & 19.82 & 155 & 7.20 & 10.07 & 43 & .12 & .4 & .5 & \text { SF } 4\end{array}$ $\begin{array}{lllllllllllllll}2006 & \text { JAN } & 5 & 2338 & 57.89 & 19 & 21.83 & 155 & 4.82 & 8.69 & 46 & .11 & .4 & .4 & \text { SF5 }\end{array}$ $\begin{array}{llllllllllllllll}2006 & \text { JAN } & 5 & 2359 & 16.40 & 19 & 22.37 & 155 & 4.88 & 8.37 & 45 & .09 & .4 & .3 & \text { SF5 }\end{array}$ $\begin{array}{llllllllllllll}2006 & \text { JAN } & 6 & 0016 & 54.02 & 19 & 45.67 & 155 & 34.89 & 12.57 & 15 & .09 & .6 & 2.3 \\ \text { KEA }\end{array}$

$\begin{array}{lllllllllllllll}2006 & \text { JAN } & 6 & 0956 & 51.83 & 18 & 59.05 & 155 & 13.31 & 37.63 & 16 & .09 & 1.9 & 2.7 & \text { LOI }\end{array}$ $\begin{array}{llllllllllllllll}2006 & \text { JAN } & 6 & 1132 & 36.81 & 19 & 19.34 & 155 & 9.86 & 9.41 & 39 & .12 & .4 & .6 & \text { SF3 }\end{array}$ $\begin{array}{lllllllllllllll}2006 & \text { JAN } & 6 & 1731 & 30.92 & 19 & 19.95 & 155 & 11.98 & 5.83 & 32 & .10 & .4 & 1.0 & \text { SF3 }\end{array}$ 2006 JAN $\quad 6 \begin{array}{llllllllllllll} & 2153 & 24.98 & 19 & 23.56 & 155 & 17.11 & 2.68 & 16 & .03 & .3 & 2 & \text { SSC }\end{array}$ $\begin{array}{lllllllllllllll}2006 & \text { JAN } & 6 & 2317 & 1.26 & 19 & 25.97 & 155 & 19.40 & 6.39 & 33 & .11 & .4 & .8 & \text { KAO }\end{array}$

$\begin{array}{lllllllllllllll}2006 & \text { JAN } & 6 & 2335 & 3.31 & 19 & 26.13 & 155 & 18.69 & 7.43 & 22 & .11 & .5 & 1.0 & \text { INT }\end{array}$ $\begin{array}{lllllllllllllll}2006 & \text { JAN } & 7 & 0050 & 24.16 & 19 & 20.52 & 155 & 26.64 & 10.16 & 27 & .11 & .4 & .8 & \text { KAO }\end{array}$ 2006 JAN $7 \begin{array}{llllllllllll}0205 & 14.29 & 19 & 25.67 & 155 & 25.96 & 1.15 & 21 & .12 & .3 & .6 & \text { KAO }\end{array}$

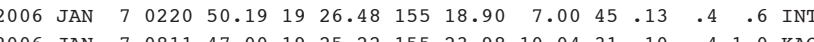

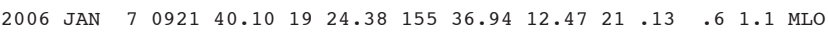
2006 JAN $7 \begin{array}{lllllllllllll} & 1317 & 47.06 & 19 & 11.27 & 155 & 42.76 & 7.29 & 42 & .13 & .5 & .9 & \text { ISW }\end{array}$

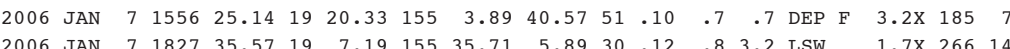

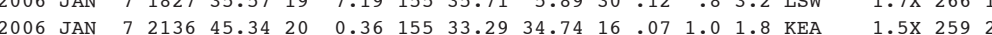

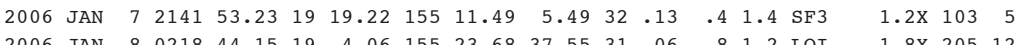

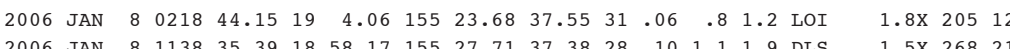
$\begin{array}{lllllllllllllllll}2006 & \text { JAN } 8 & 1138 & 35.39 & 18 & 58.17 & 155 & 27.71 & 37.38 & 28 & .10 & 1.1 & 1.9 & \text { DLS } & 1.5 \times & 268 & 21\end{array}$

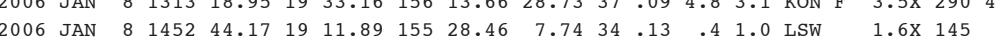

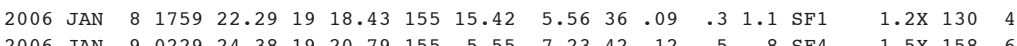

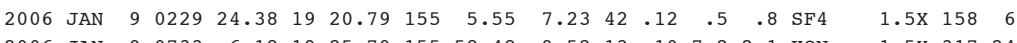

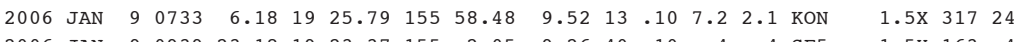

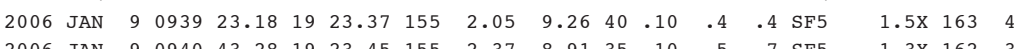
$\begin{array}{llllllllllllllllll}2006 & \text { JAN } & 9 & 1200 & 59.35 & 19 & 23.13 & 155 & 17.18 & 2.49 & 23 & .06 & .2 & .2 & \text { SSC } & 1.3 \mathrm{X} & 49 & 1\end{array}$

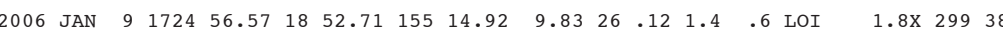

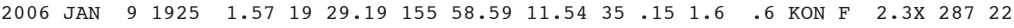

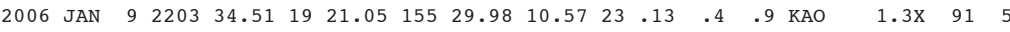

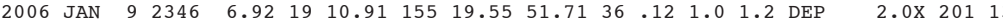

$\begin{array}{llllllllllllllll}2006 & \text { JAN } 10 & 1328 & 8.95 & 19 & 23.03 & 155 & 16.89 & 2.35 & 20 & .08 & .2 & .2 & \text { SSC } & 1.5 \mathrm{X} & 60\end{array}$

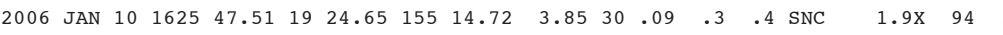

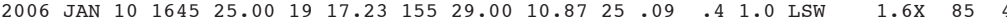

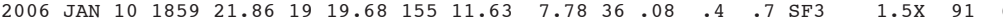
$\begin{array}{lllllllllllll}2006 & \text { JAN } 10 & 2156 & 37.66 & 19 & 42.68 & 155 & 44.52 & 14.39 & 18 & .08 & .8 & .4\end{array}$

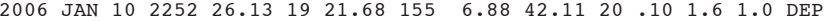

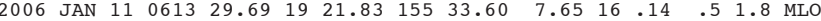

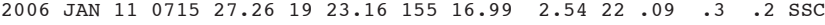
2006 JAN $110729 \begin{array}{lllllllllll}01.40 & 19 & 11.62 & 155 & 31.02 & 6.70 & 41 & .12 & .5 & .9 & \text { LSW }\end{array}$

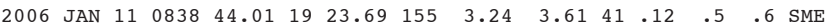

$1.1 \times 20019$

$1.8 \times 28914$ $1.2 \times 1146$ $1.3 \times \quad 47$ $1.9 \times 145 \quad 2$ 
---ORIGIN TIME (HST)-- -LAT N-- --LON W-- DEPTH N RMS ERH ERZ LOC YEAR MON DA HRMN SEC DEG MIN DEG MIN KM RD SEC KM KM REMKS

REF AZ MIN $\begin{array}{llllllllllllll}2006 & \text { JAN } 11 & 1044 & 24.62 & 19 & 11.54 & 155 & 37.16 & 0.45 & 28 & .11 & .8 & .4 & \text { LSW }\end{array}$

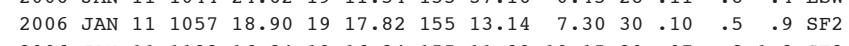

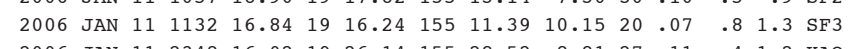

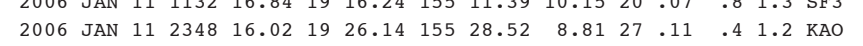

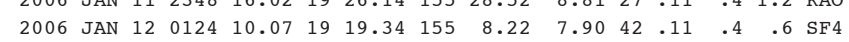

$\begin{array}{lllllllllllll}2006 & \text { JAN } 12 & 0429 & 24.68 & 20 & 20.63 & 155 & 29.74 & 1.02 & 47 & .12 & 2.0 & .6 \\ \text { KEA F }\end{array}$

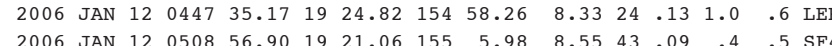

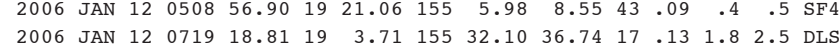

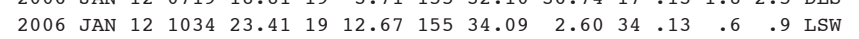

$\begin{array}{llllllllllllll}2006 & \text { JAN } 12 & 1349 & 48.60 & 19 & 19.20 & 155 & 11.35 & 5.35 & 33 & .09 & .4 & 1.4 & \text { SF3 }\end{array}$

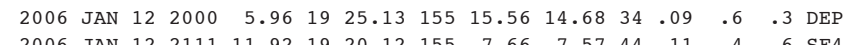

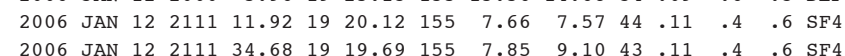
$\begin{array}{llllllllllllll}2006 & \text { JAN } 12 & 2113 & 37.74 & 19 & 20.29 & 155 & 7.52 & 7.01 & 31 & .12 & .5 & 1.0 & \text { SF } 4\end{array}$

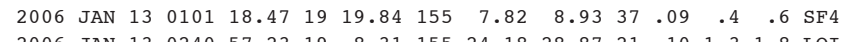

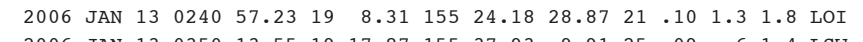

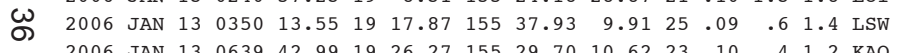

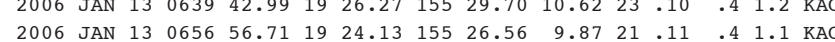

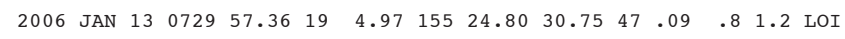

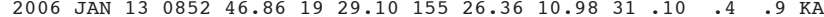
$\begin{array}{lllllllllllll}2006 & \text { JAN } 13 & 1216 & 7.58 & 19 & 23.79 & 155 & 29.73 & 10.67 & 35 & .09 & .4 & .9 \\ \text { KAО }\end{array}$

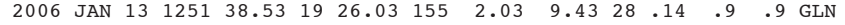

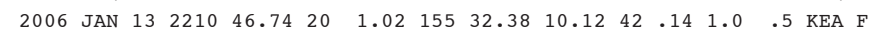

$\begin{array}{llllllllllllll}2006 & \text { JAN } 14 & 0407 & 46.43 & 19 & 28.75 & 155 & 26.19 & 6.23 & 19 & .11 & .4 & 1.6 & \text { KAO }\end{array}$ $\begin{array}{llllllllllllll}2006 & \text { JAN } & 14 & 0543 & 35.70 & 19 & 27.25 & 155 & 30.25 & 9.54 & 33 & .11 & .4 & 1.0 \\ 2006 & \text { KAO }\end{array}$

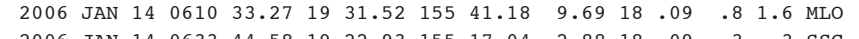
$\begin{array}{lllllllllllllll}2006 & \text { JAN } & 14 & 0633 & 44.58 & 19 & 22.93 & 155 & 17.04 & 2.88 & 18 & .09 & .3 & .3 & \text { SSC } \\ 2006 & \text { JAN } & 14 & 0651 & 13.58 & 19 & 25.84 & 155 & 37.44 & 2.49 & 19 & .09 & 3 & .4 & \text { MLO }\end{array}$

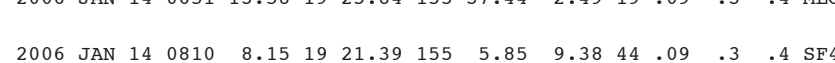

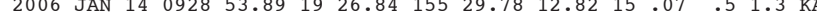

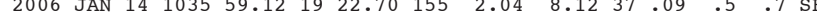
2006 JAN $14 \quad 1433 \begin{array}{lllllllllllll}48.82 & 19 & 11.69 & 155 & 27.75 & 8.61 & 44 & .11 & .4 & .9 & \text { LSN }\end{array}$ $\begin{array}{llllllllllllll}2006 & \text { JAN } 14 & 1441 & 32.86 & 19 & 25.98 & 155 & 37.21 & 3.06 & 17 & .08 & .4 & .5 & \text { MLO }\end{array}$

$\begin{array}{llllllllllllll}2006 & \text { JAN } 14 & 1807 & 15.66 & 19 & 21.28 & 155 & 5.85 & 9.47 & 42 & .10 & .4 & .4 & \text { SF } 4\end{array}$ $\begin{array}{llllllllllllll}2006 & \text { JAN } 14 & 2242 & 26.67 & 19 & 24.20 & 155 & 26.69 & 3.07 & 33 & .11 & .3 & .8 & \text { KAO }\end{array}$

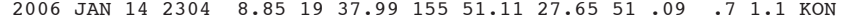

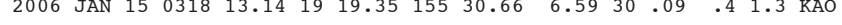

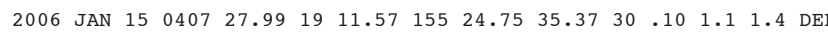

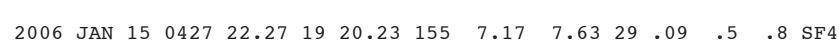

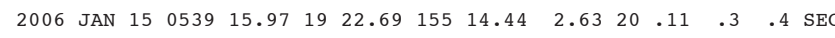

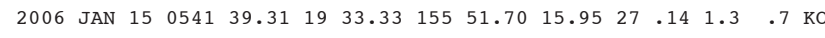
2006 JAN 15054435

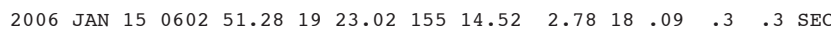

$1.6 \times 15214$ $\begin{array}{lll}1.5 \times & 107 & 2 \\ 1.2 \times & 214 & 4 \\ 1.3 \times & 63 & 7\end{array}$ $\begin{array}{rrr}1.2 \times & 214 & 4 \\ 1.3 \times & 63 & 7\end{array}$ $1.5 \times 115 \quad 4$

$2.3 \times 29853$ $1.6 \times 1951$ $\begin{array}{lll}1.5 \times & 148 & 5 \\ 1.6 \times & 296 & 21 \\ 1.4 \times & 210 & 8\end{array}$ $1.4 \times 210 \quad 8$

$1.2 \times 104 \quad 5$ $\begin{array}{lll}1.5 \times 110 & 2\end{array}$ $1.4 \times \quad 127 \quad 5$ $\begin{array}{lll}1.5 \times 126 & 4\end{array}$ $1.2 \times 129 \quad 5$

$\begin{array}{lll}1.3 \times & 145 \quad 5\end{array}$ $1.0 \times 28416$ $1.2 \times 112 \quad 8$ $1.3 \times \quad 66 \quad 6$ $1.3 \times \quad 60 \quad 4$

2.0X 19610 $\begin{array}{lll}1.4 \times & 65 & 6\end{array}$ $\begin{array}{lll}1.5 \times & 74 & 4\end{array}$ $\begin{array}{llll}1.3 \times & 174 & 5\end{array}$ $\begin{array}{lll}1.3 \times & 89 & 5\end{array}$ $1.3 \times \quad 75 \quad 9$ $.9 \times 169 \quad 8$ $1.2 \times \quad 82$ $1.0 \times \quad 91 \quad 3$

$1.7 \times 146 \quad 5$ $\begin{array}{lll}1.1 \times & 98 & 9\end{array}$ $1.5 \times 174 \quad 5$ $1.7 \times 111$ $1.3 \times \quad 89 \quad 3$ $\begin{array}{llll}1.5 \times 147 & 5\end{array}$ $1.5 \times \quad 59 \quad 4$ 3.2X $197 \quad 16$ $1.2 \times \quad 75 \quad 8$ $1.4 \times \quad 242 \quad 11$ $1.1 \times 137 \quad 5$ $1.4 \times \quad 82 \quad 2$ $\begin{array}{lll}1.5 \times & 225 & 11 \\ 1.3 \times & 119 & 2\end{array}$ $1.3 \times 119 \quad 2$ $1.2 \times \quad 81$
---ORIGIN TIME (HST)-- -LAT N-- --LON W-- DEPTH N RMS ERH ERZ LOC PREF AZ MIN YEAR MON DA HRMN SEC DEG MIN DEG MIN KM RD SEC KM KM REMRS MAG GAP DS

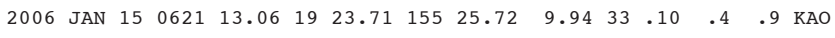

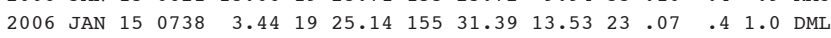

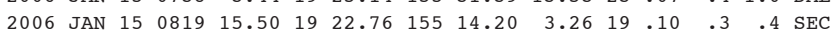

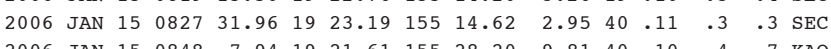

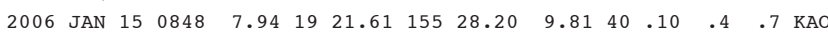

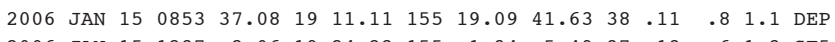

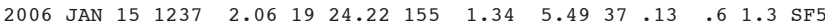

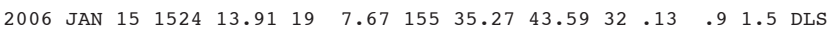

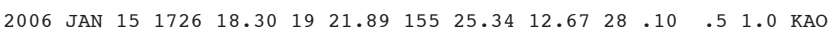
$\begin{array}{llllllllllllllll}2006 & \text { JAN } & 15 & 1937 & 9.71 & 19 & 22.30 & 155 & 13.93 & 3.50 & 17 & .07 & .5 & .4 & \text { SER }\end{array}$

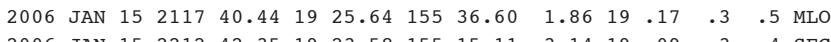

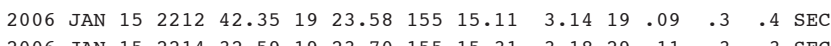

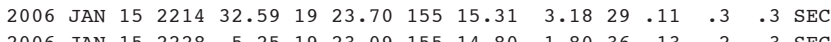

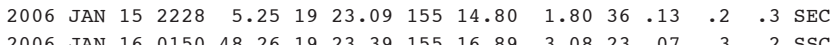

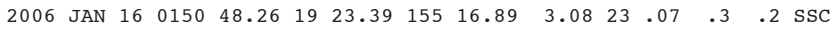

$\begin{array}{lllllllllllllll}2006 & \text { JAN } 16 & 0517 & 28.79 & 19 & 20.30 & 155 & 10.86 & 9.41 & 39 & .09 & .4 & .5 & \mathrm{SF} 3\end{array}$

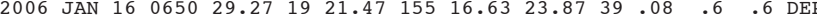

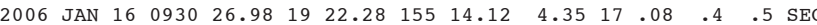

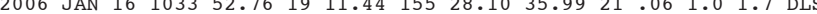
$\begin{array}{llllllllllllll}2006 & \text { JAN } & 16 & 1140 & 22.29 & 19 & 25.98 & 155 & 36.62 & 2.60 & 18 & .14 & .4 & .5\end{array}$

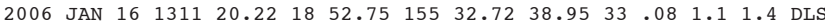
$\begin{array}{llllllllllll}2006 & \text { JAN } 16 & 1444 & 10.11 & 20 & 9.83 & 156 & 11.64 & 20.04 & 22 & .12 & 2.513 .4\end{array}$

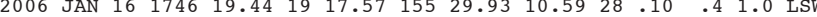

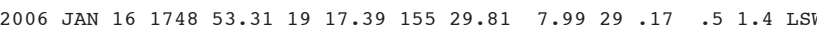

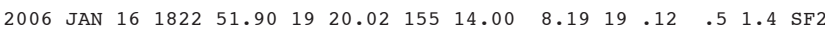

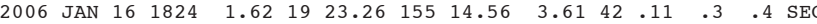

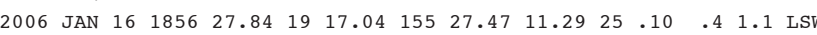

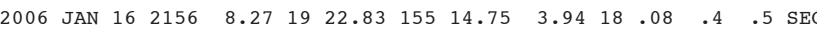

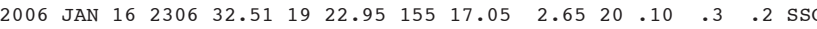

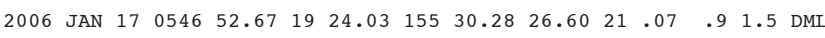

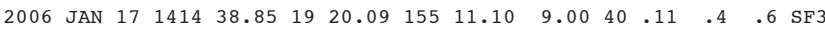
$\begin{array}{llllllllllllll}2006 & \text { JAN } 17 & 1423 & 36.35 & 19 & 24.66 & 155 & 38.19 & 3.11 & 17 & .13 & .4 & .4 & \text { MLO }\end{array}$

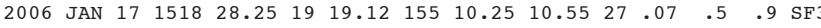
$\begin{array}{llllllllllllll}2006 & \text { JAN } 17 & 2007 & 50.84 & 19 & 23.54 & 155 & 16.78 & 3.00 & 34 & .10 & .3 & .2 & \text { SSC }\end{array}$

$\begin{array}{llllllllllllll}2006 & \text { JAN } 17 & 2120 & 35.46 & 19 & 23.44 & 155 & 16.95 & 2.84 & 25 & .07 & .3 & .2 & \text { SSC }\end{array}$

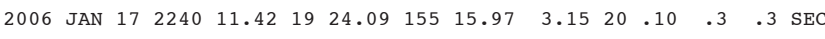
$\begin{array}{llllllllllllll}2006 & \text { JAN } 17 & 2310 & 39.07 & 19 & 25.21 & 155 & 19.13 & 8.53 & 23 & .12 & .5 & 1.0 & \text { KAO }\end{array}$

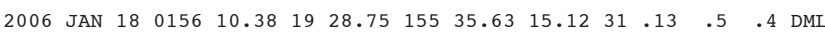

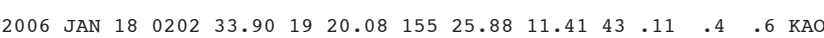

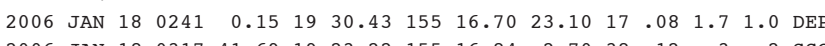

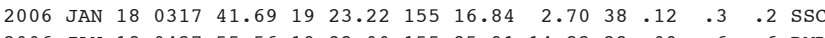

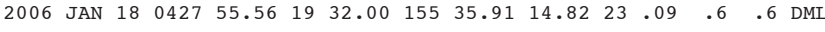

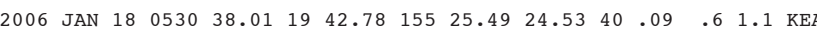

$1.3 \times \quad 60 \quad 4$ $1.3 \times \quad 73 \quad 3$ $\begin{array}{lll}1.4 \times & 87 & 2 \\ 2.1 \times & 50 & 3\end{array}$ $\begin{array}{lll}1.3 \times & 67 & 2\end{array}$

$1.5 \times 20113$ $1.7 \times 154 \quad 5$ $\begin{array}{rrrr}2.0 \times & 262 & 13 \\ 1.3 \times & 83 & 4 \\ 1.4 \times & 76 & 2\end{array}$ $\begin{array}{lll}1.3 \times & 76 & 2\end{array}$ $\begin{array}{lll}1.3 \times & 78 & 3 \\ 1.2 \times & 65 & 2\end{array}$ $\begin{array}{lll}1.2 \times & 65 & 2 \\ 1.8 \times & 60 & 2\end{array}$ $\begin{array}{lll}1.8 \mathrm{X} & 60 & 2 \\ 1.9 \mathrm{X} & 67 & 2\end{array}$ $1.4 \mathrm{X} \quad 46 \quad 0$

$\begin{array}{lll}1.3 \times & 82 & 5\end{array}$ $\begin{array}{lll}1.6 \mathrm{X} & 64 & 2\end{array}$ $1.2 \times \quad 91$ $1.2 \times 101$

1.9X $275 \quad 17$ $1.6 \times 34085$ $\begin{array}{rrr}1.4 \times & 107 & 5 \\ 1.3 \times & 97 & 4\end{array}$ $\begin{array}{lll}1.7 \times & 118 & 5\end{array}$ $\begin{array}{lll}2.2 \times & 47 & 3\end{array}$ $1.1 \times \quad 93 \quad 6$ $\begin{array}{lll}1.3 \times & 73 & 2\end{array}$ $\begin{array}{lll}1.3 \times & 49 & 1\end{array}$ $1.4 \times 134 \quad 5$

$\begin{array}{lll}1.4 \times \quad 85 & 5\end{array}$ $1.1 \times 100 \quad 1$ $1.5 \times 107 \quad 5$ $\begin{array}{lll}1.0 \times & 73 & 2\end{array}$ $1.5 \mathrm{X} \quad 45 \quad 0$ $1.1 \times \quad 79$ $\begin{array}{lll}1.1 \mathrm{x} & 82 & 3\end{array}$ $\begin{array}{lll}1.4 \mathrm{X} & 84 & 5 \\ 2.2 \mathrm{X} & 297 & 9\end{array}$ $\begin{array}{lll}2.2 \times 297 & 9 \\ 1.9 \times & 46 & 0\end{array}$ $\begin{array}{lll}1.9 \times & 46 & 0 \\ 1.0 \times & 126 & 5\end{array}$ $\begin{array}{rrr}1.0 \mathrm{X} & 126 & 5 \\ 1.5 \mathrm{X} & 68 & 8\end{array}$ 
---ORIGIN TIME (HST)-- -LAT N-- --LON W-- DEPTH N RMS ERH ERZ LOC YEAR MON DA HRMN SEC DEG MIN DEG MIN

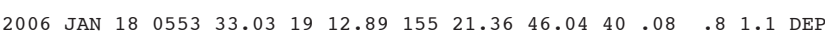

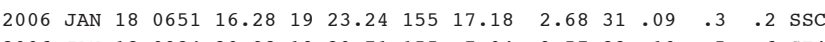

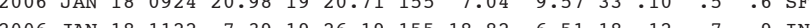

$\begin{array}{llllllllllllll}2006 & \text { JAN } 18 & 1137 & 25.56 & 19 & 28.27 & 155 & 58.04 & 37.70 & 29 & .12 & 1.7 & 1.1 & \text { KON }\end{array}$ $\begin{array}{lllllll}2006 & 0 & 0\end{array}$

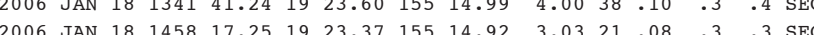

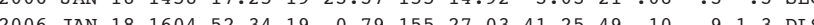

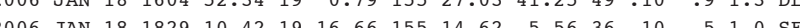

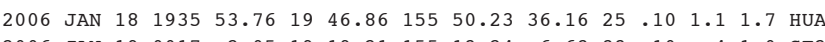
$\begin{array}{llllllllllllll}2006 & \text { JAN } 19 & 0017 & 2.05 & 19 & 19.21 & 155 & 13.24 & 6.63 & 33 & .10 & .4 & 1.0 & \text { SF2 }\end{array}$ $\begin{array}{llllllllllllll}2006 & \text { JAN } 19 & 0143 & 59.94 & 19 & 28.81 & 155 & 50.76 & 9.58 & 34 & .16 & .9 & .4 & \mathrm{KO}\end{array}$

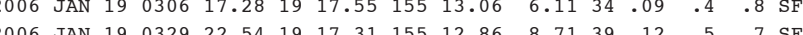

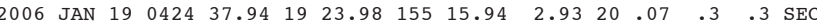

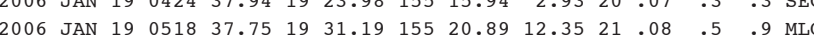

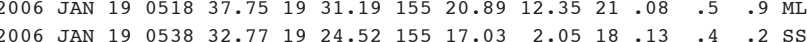

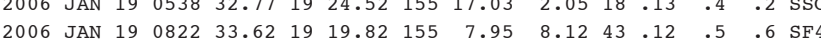
$\begin{array}{lllllllllllll} & 006 & & 0\end{array}$

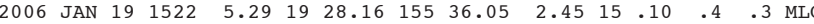

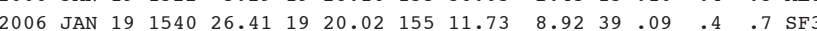

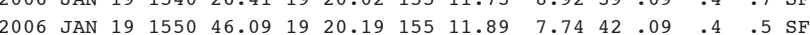

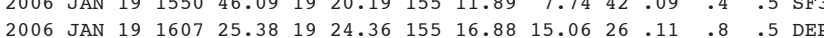

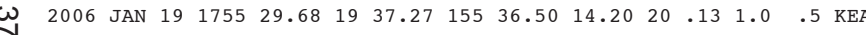

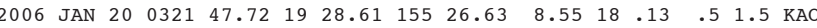

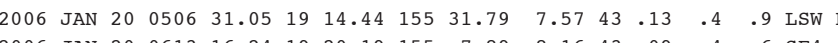

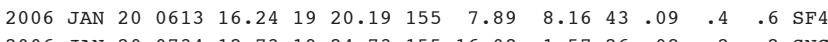

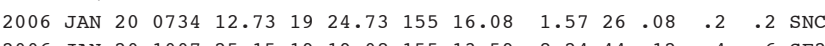
$\begin{array}{llllllllllllll}2006 & \text { JAN } 20 & 1007 & 25.15 & 19 & 19.08 & 155 & 13.59 & 8.24 & 44 & .12 & .4 & .6 & \text { SF2 }\end{array}$

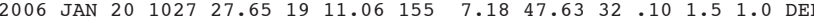

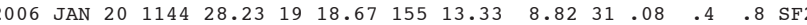

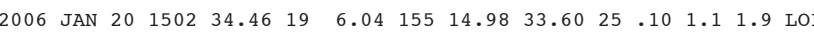
$\begin{array}{llllllllllllll}2006 & \text { JAN } 20 & 1534 & 11.04 & 19 & 23.01 & 155 & 17.01 & 2.73 & 42 & .10 & .2 & .2 & \text { SSC }\end{array}$ $\begin{array}{llllllllllllll}2006 & \text { JAN } 20 & 1633 & 6.43 & 19 & 27.83 & 155 & 29.32 & 10.89 & 19 & .13 & .5 & 1.6 & \text { KAO }\end{array}$

$\begin{array}{llllllllllllll}2006 & \text { JAN } 20 & 1657 & 2.52 & 19 & 15.93 & 155 & 7.44 & 38.14 & 15 & .07 & 2.5 & 1.6 & \text { DEP }\end{array}$

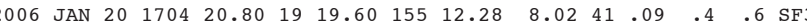

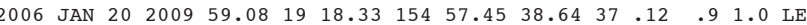

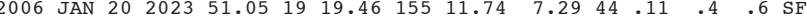
$\begin{array}{lllllllllllll}2006 & \text { JAN } 20 & 2225 & 22.44 & 19 & 25.31 & 155 & 19.12 & 6.61 & 22 & .07 & .4 & .9\end{array}$

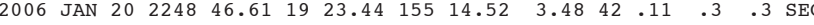
$\begin{array}{llllllllllllll}2006 & \text { JAN } 20 & 2313 & 20.42 & 19 & 28.68 & 156 & 41.53 & 35.80 & 34 & .10 & 7.7 & 2.7 & \text { DIS }\end{array}$

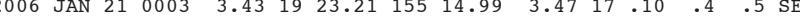

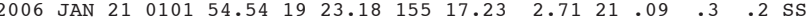

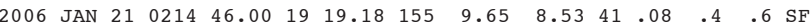

PREF AZ MIN MAG GAP DS $1.7 \times 16311$ $\begin{array}{rrr}1.7 \mathrm{X} & 48 & 0 \\ 1.6 \mathrm{X} & 135 & 5\end{array}$ $\begin{array}{lll}1.6 \times & 135 & 5 \\ 1.8 \times & 164 & 3\end{array}$ $1.8 \times 16403000$

$1.8 \times 31722$ $2.2 \times \quad 51 \quad 2$ $\begin{array}{lll}.3 \mathrm{X} & 74 & 2 \\ 1.7 & 214 & 16\end{array}$ $1.4 \times 199$ $1.6 \times 26331$ $1.0 \times \quad 76 \quad 4$ $\begin{array}{lll}1.0 \times & 76 & 4 \\ 1.8 \times & 195 & 9\end{array}$ $1.1 \times 125$ $1.3 \times 154$ $\begin{array}{lrr}1.2 x & 75 & 1 \\ 1.1 \times & 130 & 5\end{array}$ $1.1 \times 130 \quad 5$ $\begin{array}{rrr}1.3 \mathrm{X} & 81 & 1 \\ 1.6 \mathrm{X} & 121 & 5\end{array}$ $1.4 \times 181$ $\begin{array}{lll}1.1 \mathrm{x} & 81 & 2 \\ 1.9 \times & 83 & 5\end{array}$ $\begin{array}{lll}1.6 \mathrm{X} & 83 & 5 \\ 1.9 \mathrm{X} & 79 & 5 \\ 1.1 \times & 112 & 2\end{array}$ $\begin{array}{lll}1.9 \times & 112 & 5 \\ 1.1 \times & 112 & 2\end{array}$ $\begin{array}{lll}1.3 \times & 223 & 13\end{array}$

$\begin{array}{lll}1.2 \mathrm{X} & 85 & 6\end{array}$ $2.3 \times 126 \quad 3$ $1.8 \times 122 \quad 5$ 1.7X 1012 $2.3 \times \quad 69 \quad 4$

$1.9 \times 26816$ $1.2 \times \quad 81 \quad 3$ $1.6 \times 29620$ $2.3 \times \quad 38 \quad 1$ $\begin{array}{lll}1.3 \times & 78 & 8\end{array}$

$1.4 \times 317 \quad 11$ $1.5 \times \quad 86 \quad 5$ $1.6 \times 253 \quad 16$ $1.5 \times \quad 94 \quad 5$ $1.0 \mathrm{X} \quad 83 \quad 3$

$2.4 \mathrm{X} \quad 45 \quad 2$ 2.8X 29693 2.3X $71 \quad 2$ $1.3 \times \quad 74 \quad 1$ $1.3 \times 102$
---ORIGIN TTME (HST)-- -LAT N-- --LON W-- DEPTH N RMS ERH ERZ LOC $\begin{array}{llllllllllllll}2006 & \text { JAN } 21 & 0238 & 12.20 & 19 & 23.08 & 155 & 14.70 & 3.78 & 28 & .07 & .3 & .4 & \text { SEC }\end{array}$ $\begin{array}{llllllllll}2006 & & 4\end{array}$

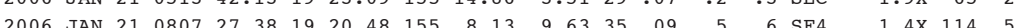

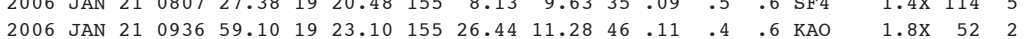

$\begin{array}{llllllllllllll}2006 & \text { JAN } 21 & 0938 & 14.29 & 19 & 23.11 & 155 & 26.16 & 11.01 & 33 & .10 & .4 & .9 & \text { KAO }\end{array}$

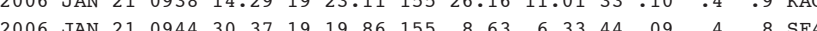
$\begin{array}{lllllllllll}2006 & 0.9 & \end{array}$ 2006 JAN $21163254.681919 .0415526 .4511 .4824 .06 \quad .10 \quad .4$ SEC

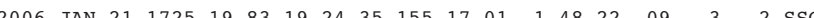
$\begin{array}{llllllllllllll}2006 & \text { JAN } 22 & 0750 & 9.68 & 19 & 31.57 & 155 & 54.74 & 13.78 & 28 & .11 & 1.0 & .8 & \text { KON }\end{array}$

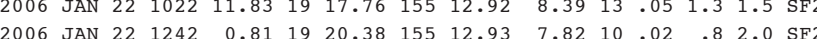

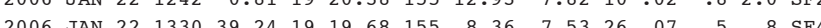

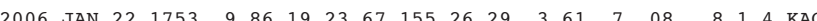

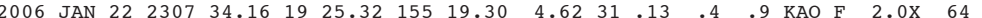

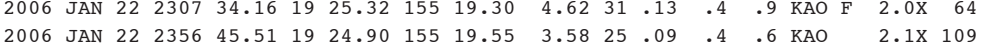

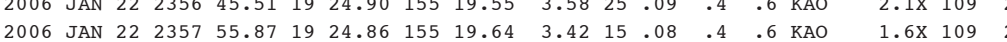

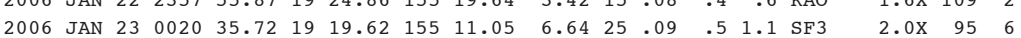

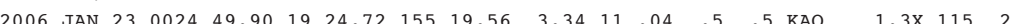

$\begin{array}{llllllllllllllllll}2006 & & & \end{array}$

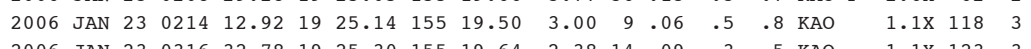

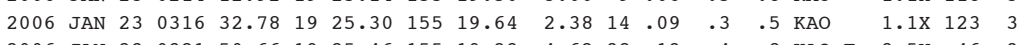

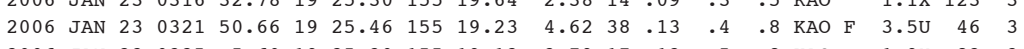

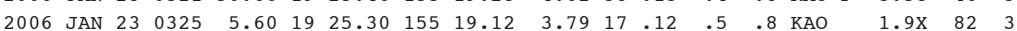

$\begin{array}{lllllllllllllllll}2006 & \text { JAN } 23 & 0328 & 36.40 & 19 & 25.38 & 155 & 19.16 & 4.15 & 14 & .12 & .4 & 1.0 & \mathrm{KAO} & 1.2 \mathrm{X} & 85 & 3\end{array}$

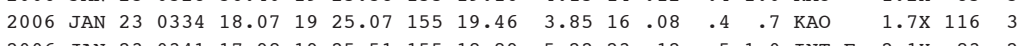

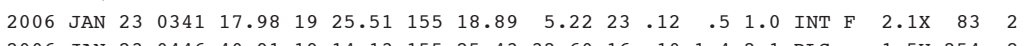

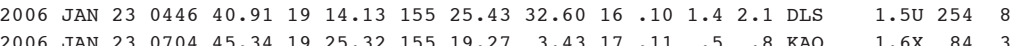

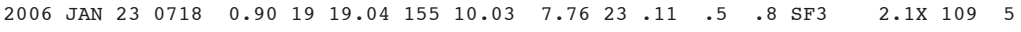

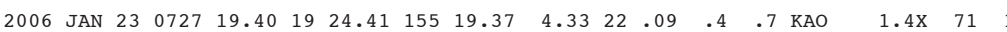

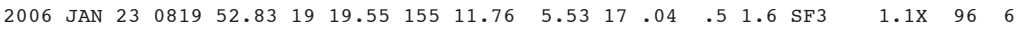

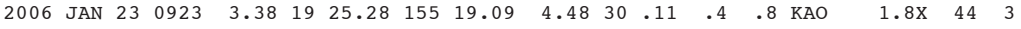

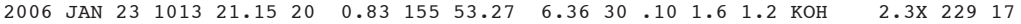

$\begin{array}{lllllllllllllllll}2006 & \text { JAN } 23 & 1113 & 1.33 & 19 & 24.94 & 155 & 15.57 & 3.04 & 13 & .04 & .4 & .4 & \text { SNC } & 1.4 \mathrm{X} & 158 & 2\end{array}$ $\begin{array}{llllllllllllllllll}2006 & \text { JAN } 23 & 1136 & 37.20 & 19 & 25.12 & 155 & 19.33 & 3.68 & 39 & .10 & .3 & .5 & \text { KAO } & F & 2.7 X & 45 & 3\end{array}$

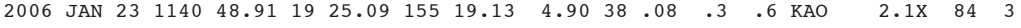

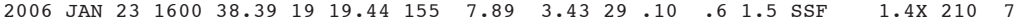

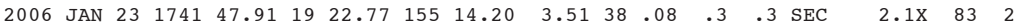

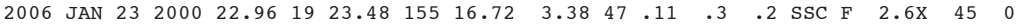
$\begin{array}{lllllllllllllll}2006 & \text { JAN } 24 & 0343 & 22.57 & 19 & 3.31 & 155 & 25.42 & 38.63 & 38 & .08 & .8 & 1.4 & \text { DLS } & 2.1 \mathrm{X} \\ 204 & 12\end{array}$

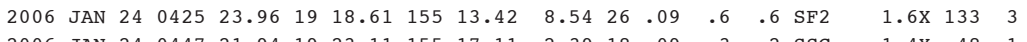

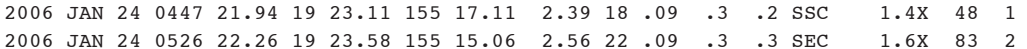




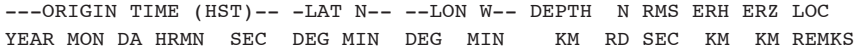

$\begin{array}{llllllllllllll}2006 & \text { JAN } 24 & 1333 & 21.64 & 19 & 23.56 & 155 & 15.05 & 3.51 & 19 & .07 & .3 & .4 & \text { SEC }\end{array}$ $\begin{array}{llllllllllllll}2006 & \text { JAN } 24 & 1343 & 32.20 & 19 & 25.07 & 155 & 18.89 & 7.68 & 34 & .10 & .4 & .7 & \text { INT }\end{array}$

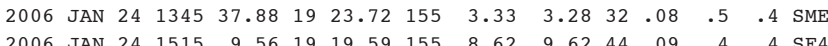
$\begin{array}{llllllllllllll}2006 & \text { JAN } 24 & 1515 & 9.56 & 19 & 19.59 & 155 & 8.62 & 9.62 & 44 & .09 & .4 & .4 & \text { SF } 4\end{array}$

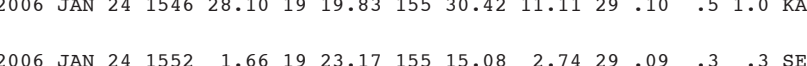

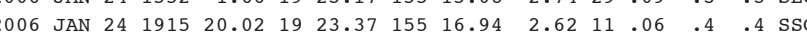

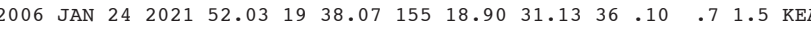
$\begin{array}{llllllllllllll}2006 & \text { JAN } 24 & 2138 & 52.28 & 19 & 19.37 & 155 & 7.64 & 8.37 & 42 & .08 & .4 & .4 & \mathrm{SF}\end{array}$

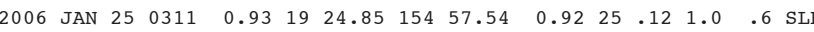

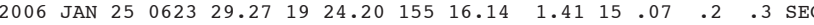

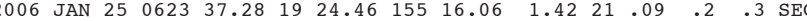

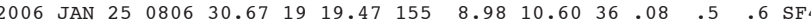

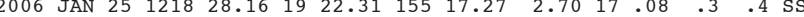

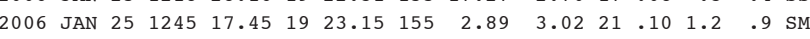

$\begin{array}{llllllllllllll}2006 & \text { JAN } 25 & 1247 & 51.90 & 19 & 24.78 & 155 & 15.99 & 1.45 & 16 & .10 & .3 & .4 & \text { SNC }\end{array}$

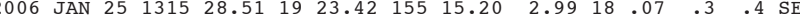

$\omega$

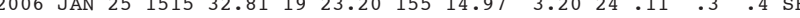

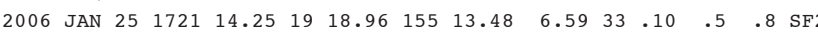

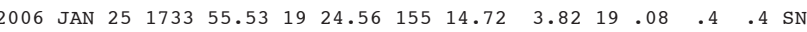
$\begin{array}{llllllllllllll}2006 & \text { JAN } 25 & 2043 & 17.67 & 19 & 23.27 & 155 & 15.12 & 3.06 & 17 & .08 & .4 & .4 & \text { SEC }\end{array}$

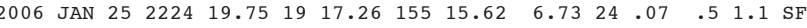

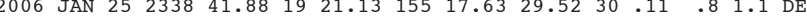

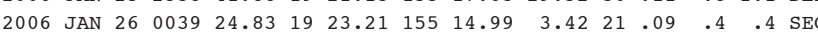

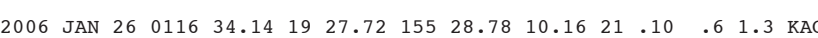

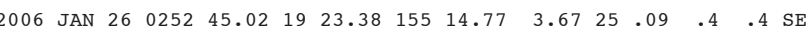

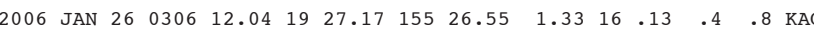

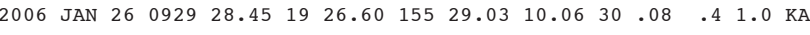

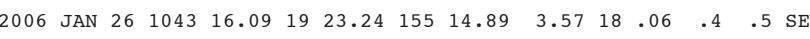

$\begin{array}{llllllllllllll}2006 & \text { JAN } 26 & 1455 & 1.61 & 19 & 23.74 & 155 & 15.42 & 2.60 & 17 & .07 & .3 & .4 & \text { SEC }\end{array}$

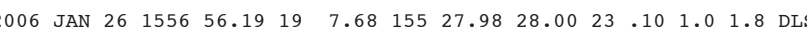

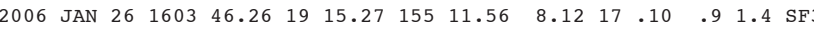
$\begin{array}{llllllllllllll}2006 & \text { JAN } 26 & 1657 & 29.17 & 19 & 23.70 & 155 & 15.22 & 2.68 & 15 & .06 & .4 & .4 & \text { SEC }\end{array}$

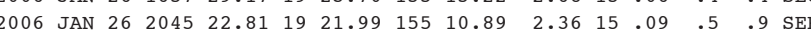

$\begin{array}{llllllllllllll}2006 & \text { JAN } 26 & 2045 & 59.18 & 19 & 25.29 & 155 & 39.46 & 3.04 & 23 & .07 & .8 & .7 & \text { MLO }\end{array}$

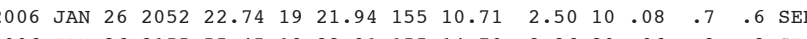

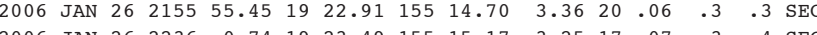

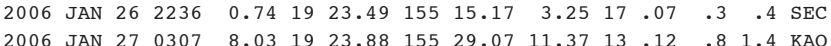

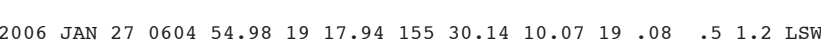

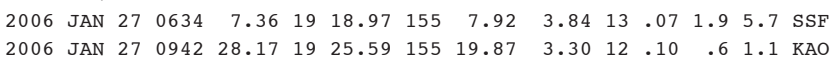
$\begin{array}{llllllllllllll}2006 & \text { JAN } 27 & 1139 & 17.59 & 19 & 18.27 & 155 & 29.88 & 9.85 & 17 & .09 & .6 & 1.4 & \text { LSW }\end{array}$ $\begin{array}{llllllllllllll}2006 & \text { JAN } 27 & 1156 & 22.68 & 19 & 18.38 & 155 & 30.39 & 10.63 & 11 & .06 & .8 & 1.5 & \text { LSW }\end{array}$
PREF AZ MIN $\begin{array}{lll}.4 \mathrm{X} & 83 \quad 2\end{array}$ $\begin{array}{lll}1.3 \times & 77 & 2\end{array}$ $\begin{array}{lll}1.8 \times 164 & 2\end{array}$ $2.2 \times 182 \quad 6$ $1.2 \times 1047$

$\begin{array}{lll}.8 \times \quad 60 & 2\end{array}$ $\begin{array}{llll}.9 \times & 144 & 0\end{array}$ $\begin{array}{lll}1.8 \mathrm{X} & 80 & 17\end{array}$ $2.0 \times 134 \quad 4$ $1.7 \times 194 \quad 6$

$1.1 \times \quad 82$ 2.3X $91 \quad 1$ $1.6 \times 112 \quad 4$ $1.2 \times \quad 59$ $2.0 \times 176 \quad 3$

$1.7 \times 105$ $1.5 \mathrm{x} \quad 64$ $\begin{array}{lll}2.3 \mathrm{X} & 54 & 3\end{array}$ $1.9 \mathrm{X} \quad 70 \quad 2$ 1.8X 113

$1.4 \times 129$ $\begin{array}{lll}1.3 \times & 74 \quad 2\end{array}$ $1.1 \times 188$ $\begin{array}{lll}1.7 \times & 60 & 2\end{array}$

$\begin{array}{lll}1.2 \times & 90 & 8\end{array}$ $2.1 \times \quad 66 \quad 3$ $1.4 \mathrm{x} \quad 62$ $1.8 \times \quad 85$ $.5 \times \quad 69 \quad 2$ $.0 \times \quad 98 \quad 2$ $\begin{array}{lll}1.4 \times \quad 266 & 3\end{array}$ $1.4 \times 282$ $1.6 \times \quad 93 \quad 2$ $1.8 \times 138$

$2.5 \times 210 \quad 3$ $1.1 \times 145$ $\begin{array}{lll}1.9 \times & 70 & 2 \\ 1.4 \times & 83 & 2\end{array}$ $\begin{array}{lll}1.4 \times & 83 & 2\end{array}$ $1.5 \times \quad 72 \quad 5$ $1.0 \times 247 \quad 8$ $\begin{array}{lll}1.2 \times 131 & 3 \\ 1.8 \times 104 & 6\end{array}$ $\begin{array}{lll}1.8 \times 104 & 6\end{array}$
---ORIGIN TIME (HST)--
YEAR MAN N-- --LON W-- DEPTH

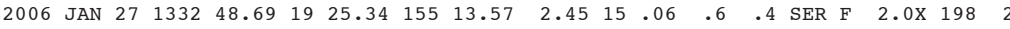

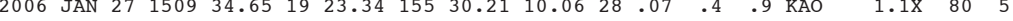

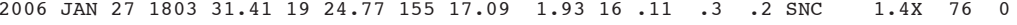

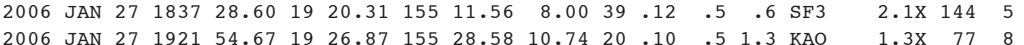

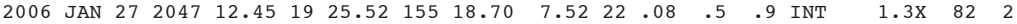

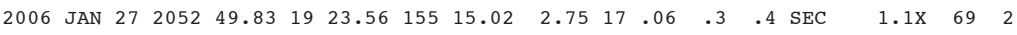

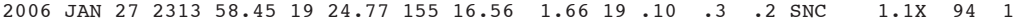

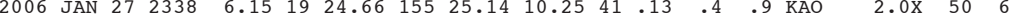
$\begin{array}{llllllllllllll}2006 & \text { JAN } 28 & 0013 & 41.89 & 19 & 25.75 & 155 & 19.02 & 8.65 & 23 & .10 & .6 & 1.0 & \text { INT }\end{array}$

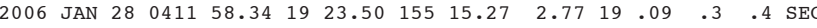

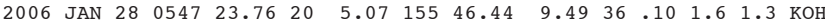

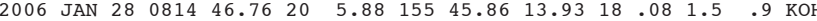

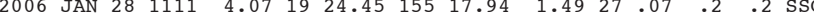
$\begin{array}{lllllllllllllll}2006 & \text { JAN } 28 & 1143 & 9.89 & 19 & 23.57 & 155 & 15.06 & 2.84 & 15 & .07 & .3 & .6 & \text { SEC }\end{array}$

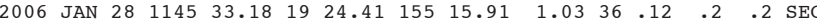
$\begin{array}{llllllllllllll}2006 & \text { JAN } 28 & 1147 & 24.74 & 19 & 24.00 & 155 & 15.75 & 2.83 & 17 & .08 & .3 & .4 & \text { SEC }\end{array}$

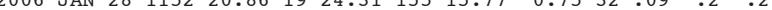

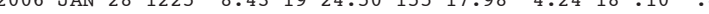

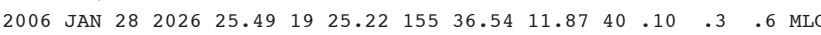

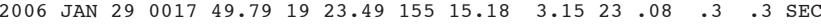

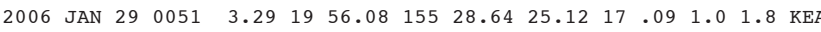
$\begin{array}{llllllllllllll}2006 & \text { JAN } 29 & 0131 & 58.85 & 19 & 11.31 & 155 & 28.40 & 8.87 & 40 & .11 & .4 & .8 & \text { LSW }\end{array}$

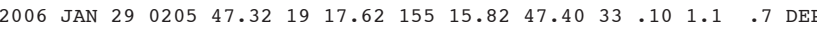
$\begin{array}{llllllllllllll}2006 & \text { JAN } 29 & 0253 & 28.24 & 19 & 21.64 & 155 & 8.93 & 2.40 & 24 & .10 & .5 & .5 & \text { SER }\end{array}$

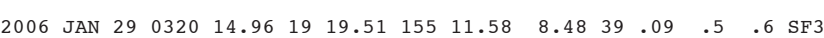
$\begin{array}{llllllllllllll}2006 & \text { JAN } 29 & 0506 & 26.20 & 19 & 24.83 & 155 & 16.36 & 1.48 & 24 & .11 & .2 & .2 & \text { SNC }\end{array}$

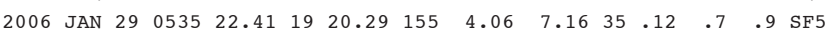

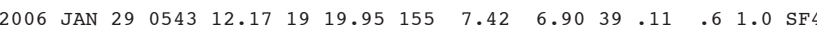
2006 JAN 290759

$\begin{array}{llllllllllllll}2006 & \text { JAN } 29 & 0835 & 47.37 & 19 & 23.29 & 155 & 30.61 & 10.88 & 32 & .10 & .4 & .9 & \text { KAO }\end{array}$ $\begin{array}{llllllllllllll}2006 & \text { JAN } 29 & 0929 & 58.78 & 19 & 19.65 & 155 & 11.46 & 8.48 & 43 & .08 & .4 & .6 & \mathrm{SF} 3\end{array}$ 2006 JAN $291050 \begin{array}{llllllllllll}27.64 & 19 & 19.61 & 155 & 8.56 & 7.01 & 26 & .09 & .6 & 1.1 & \text { SF4 }\end{array}$ $\begin{array}{llllllllllllll}2006 & \text { JAN } 29 & 1131 & 43.86 & 19 & 23.16 & 155 & 14.72 & 3.34 & 23 & .08 & .3 & .4 & \text { SEC }\end{array}$ $\begin{array}{llllllllllllll}2006 & \text { JAN } 29 & 1142 & 7.11 & 19 & 21.65 & 155 & 30.20 & 10.50 & 34 & .10 & .4 & .9 & \text { KAO }\end{array}$

$\begin{array}{lllllllllllll}2006 & \text { JAN } 29 & 1443 & 25.50 & 19 & 18.99 & 155 & 46.69 & 11.64 & 25 & .10 & .7 & .5 \\ \text { KoN }\end{array}$

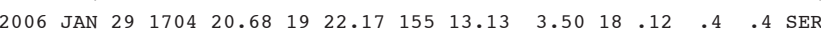

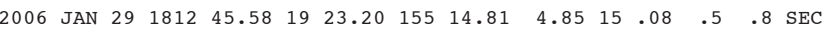

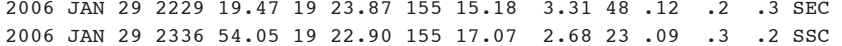

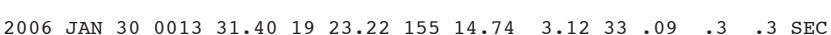

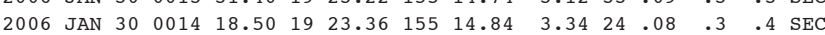
$\begin{array}{llllllllllllll}2006 & \text { JAN } 30 & 0014 & 18.50 & 19 & 23.36 & 155 & 14.84 & 3.34 & 24 & .08 & .3 & .4 & \text { SEC } \\ 2006 & \text { JAN } 30 & 0046 & 50.18 & 19 & 23.35 & 155 & 15.15 & 2.71 & 13 & .08 & .4 & .5 & \text { SEC }\end{array}$

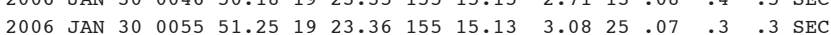

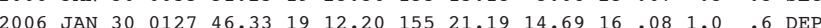

$1.2 \times \quad 89 \quad 3$

$1.3 \times \quad 61 \quad 2$ $2.1 \times 32347$ $\begin{array}{lll}1.5 \mathrm{X} & 59 & 2 \\ 1.3 \mathrm{X} & 98 & 2\end{array}$ $2.2 \times \quad 45 \quad 2$ $1.7 \times 113$ $\begin{array}{llll}2.2 \times & 82 & 2\end{array}$ $1.5 \times \quad 73 \quad 2$ $\begin{array}{llll}1.8 \times & 67 & 3\end{array}$ $\begin{array}{lll}1.5 \mathrm{X} & 64 \quad 2\end{array}$ $1.3 \times \quad 222 \quad 15$ $1.9 \times \quad 99$ $1.3 \times 154$ $1.3 \times 151$ $\begin{array}{ll}1.9 \times & 99\end{array}$ $1.5 \times 201$ $1.5 \times 187 \quad 6$ $\begin{array}{lll}1.5 \times \quad 83 & 5\end{array}$ $\begin{array}{lll}1.7 \times & 151 & 6\end{array}$ $1.3 \times 182$ $1.9 \times \quad 70 \quad 3$ $1.4 \times 107$
$1.2 \times 106$ $1.2 \times 106$ $1.5 \mathrm{X} \quad 49 \quad 1$

$2.0 \times \quad 68 \quad 3$ $\begin{array}{lll}1.7 \times \quad 71 & 3\end{array}$ $\begin{array}{lll}1.75 \times 102 & 2 \\ 1.8 \times & 55 & 2\end{array}$ 1.1X 19112 
---ORIGIN TIME (HST)-- -LAT N-- --LON W-- DEPTH N RMS ERH ERZ LOC

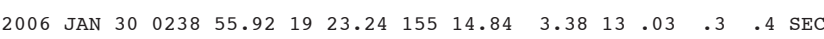

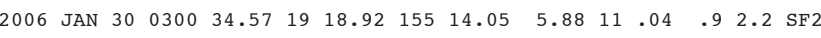

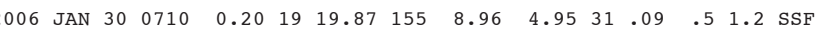

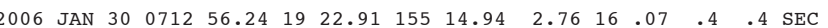

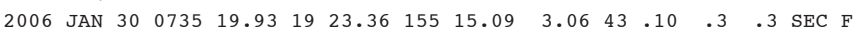

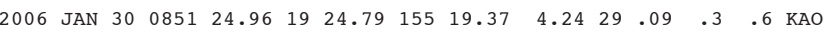

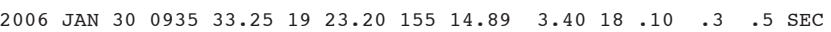

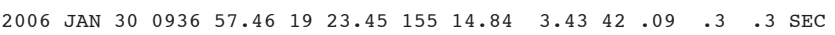

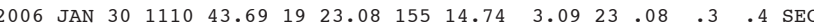

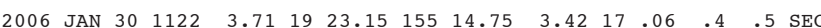

$\begin{array}{llllllllllllll}2006 & \text { JAN } 30 & 1238 & 9.88 & 19 & 20.29 & 155 & 6.74 & 8.48 & 44 & .08 & .4 & .6 & \text { SF } 4\end{array}$ $\begin{array}{llllllllllllll}2006 & \text { JAN } 30 & 1425 & 5.01 & 19 & 22.23 & 155 & 26.12 & 12.45 & 40 & .11 & .4 & .9 & \text { KAO }\end{array}$

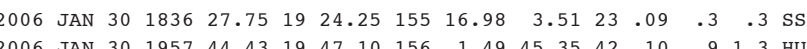

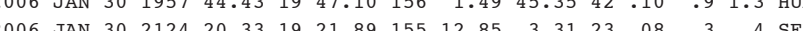

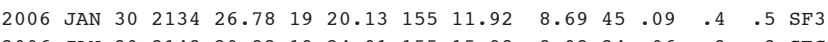

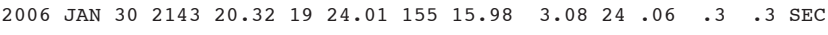

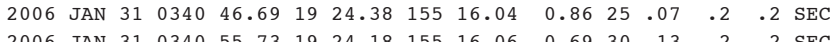
$\begin{array}{lllllllllllll}20 & .2 & .2 & \text { SEC }\end{array}$

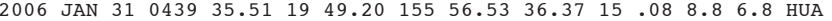

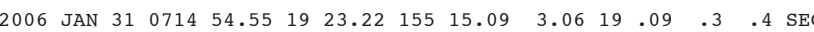

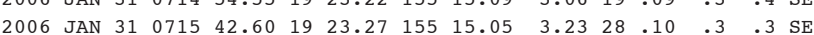

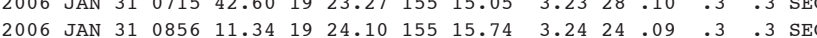

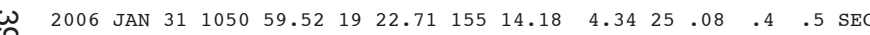

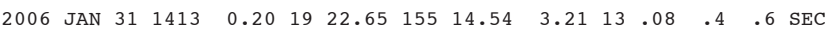

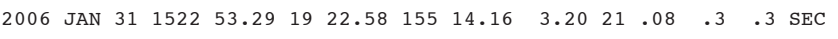

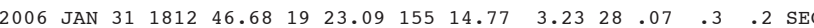

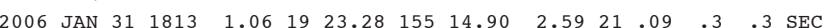

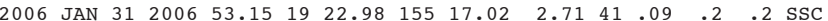

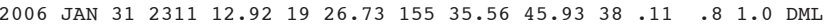

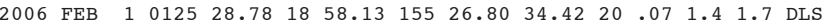
2006 FEB $110145 \quad 53.01 \quad 1926.80 \quad 155 \quad 35.77 \quad 40.18 \quad 16.06 \quad 1.3 \quad 2.0$ DML I

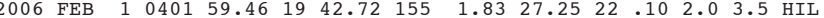
$\begin{array}{lllllllllllllll}2006 & \text { FEB } & 1 & 0447 & 28.12 & 19 & 22.66 & 155 & 14.70 & 2.95 & 19 & .10 & .3 & .3 & \text { SEC }\end{array}$

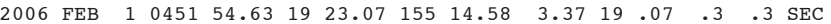
$\begin{array}{lllllllllllllll}2006 & \text { FEB } & 1 & 0608 & 44.57 & 19 & 19.54 & 155 & 12.23 & 5.39 & 16 & .07 & .6 & 2.0 & \mathrm{SF} 3\end{array}$ $\begin{array}{lllllllllllllll}2006 & \text { FEB } & 1 & 0833 & 44.60 & 19 & 22.89 & 155 & 14.72 & 2.93 & 16 & .08 & .3 & .4 & \text { SEC }\end{array}$

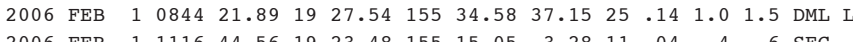
$\begin{array}{llllllllllllllll}2006 & \text { FEB } & 1 & 1116 & 44.56 & 19 & 23.48 & 155 & 15.05 & 3.28 & 11 & .04 & .4 & .6 & \text { SEC }\end{array}$

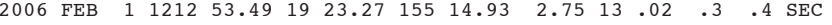

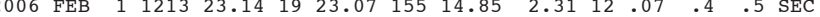
$\begin{array}{lllllllllllllll}2006 & \text { FEB } & 1 & 1216 & 5.56 & 19 & 23.16 & 155 & 14.98 & 2.94 & 14 & .06 & .3 & .4 & \mathrm{SEC}\end{array}$

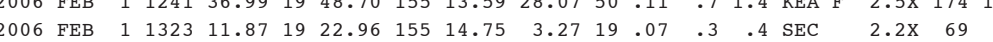

-ORTGIN TTME (HST)-- -LAT N-- --LON W-- DEPTH N RMS ERH ERZ LOC YEAR MON DA HPMN SEC DEG NTN DEG WTN DEPTH N RMS ERH ERZ LOC

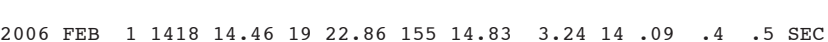
$\begin{array}{llllllllllllllll}2006 & \text { FEB } & 1 & 1433 & 30.83 & 19 & 23.30 & 155 & 15.02 & 3.19 & 12 & .05 & .4 & .5 & \text { SEC }\end{array}$ $\begin{array}{lllllllllllllll}2006 & \text { FEB } & 1 & 1433 & 30.83 & 19 & 23.30 & 155 & 15.02 & 3.19 & 12 & .05 & .4 & .5 & \text { SEC } \\ 2006 & \text { FEB } & 1 & 1524 & 32.34 & 19 & 23.27 & 155 & 14.93 & 2.60 & 9 & .03 & .4 & .6 & \text { SEC }\end{array}$

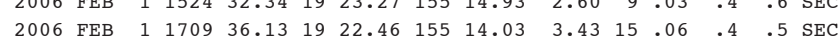

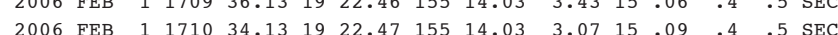

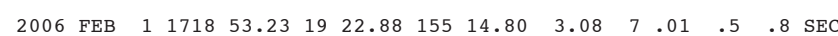

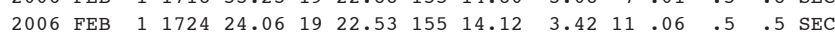

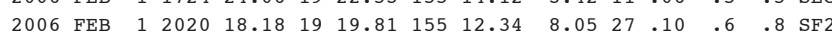

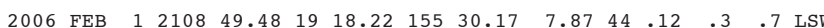
$\begin{array}{lllllllllllllll}2006 & \text { FEB } & 1 & 2243 & 36.69 & 19 & 37.99 & 155 & 8.12 & 14.77 & 34 & .10 & .5 & .6 & \text { HIL }\end{array}$

$\begin{array}{lllllllllllllll}2006 & \text { FEB } & 1 & 2327 & 24.15 & 19 & 23.08 & 155 & 14.76 & 3.40 & 19 & .07 & .3 & .4 & \text { SEC }\end{array}$ $\begin{array}{lllllllllllllll}2006 & \text { FEB } & 2 & 0015 & 33.73 & 19 & 23.06 & 155 & 14.93 & 2.87 & 15 & .08 & .4 & .4 & \text { SEC }\end{array}$

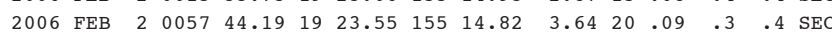
$\begin{array}{llllllllllllllll}2006 & \mathrm{FEB} & 2 & 0235 & 6.36 & 19 & 23.09 & 155 & 15.09 & 3.12 & 19 & .09 & .4 & .4 & \mathrm{SEC}\end{array}$

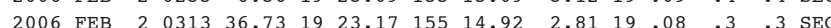

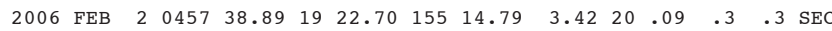
$\begin{array}{lllllllllllllll}2006 & \text { FEB } & 2 & 0513 & 20.69 & 19 & 20.43 & 155 & 6.57 & 9.40 & 43 & .09 & .5 & .5 & \mathrm{SF} 4\end{array}$ $\begin{array}{llllllllllllll}2006 & \text { FEB } & 2 & 0618 & 51.33 & 19 & 23.45 & 155 & 3.56 & 3.21 & 34 & .12 & .6 & .5\end{array}$ $\begin{array}{lllllllllllllll}2006 & \text { FEB } & 2 & 0641 & 34.75 & 19 & 15.68 & 155 & 29.74 & 42.35 & 33 & .07 & .8 & 1.2 & \text { DLS }\end{array}$ $\begin{array}{lllllllllllllll}2006 & \text { FEB } & 2 & 0808 & 48.21 & 19 & 23.54 & 155 & 15.20 & 3.26 & 22 & .10 & .3 & .4 & \text { SEC }\end{array}$

$\begin{array}{lllllllllllllll}2006 & \text { FEB } & 2 & 0826 & 52.17 & 19 & 22.94 & 155 & 17.16 & 2.34 & 24 & .09 & .2 & .2 & \mathrm{SSC}\end{array}$ $\begin{array}{lllllllllllllll}2006 & \text { FEB } & 2 & 0832 & 17.73 & 19 & 23.14 & 155 & 16.92 & 3.15 & 37 & .09 & .2 & .2 & \mathrm{SSC}\end{array}$ $\begin{array}{lllllllllllllll}2006 & \text { FEB } & 2 & 0833 & 2.13 & 19 & 23.16 & 155 & 17.13 & 2.68 & 17 & .06 & .3 & .3 & \text { SSC }\end{array}$

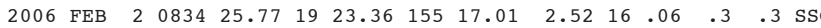
$\begin{array}{lllllllllllllll}2006 & \text { FEB } & 2 & 0836 & 35.06 & 19 & 23.08 & 155 & 16.98 & 2.77 & 21 & .06 & .3 & .2 & \text { SSC }\end{array}$

$\begin{array}{lllllllllllllll}2006 & \text { FEB } & 2 & 0838 & 5.07 & 19 & 24.54 & 155 & 17.37 & 1.64 & 25 & .08 & .2 & .1 & \mathrm{SSC}\end{array}$

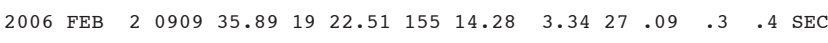

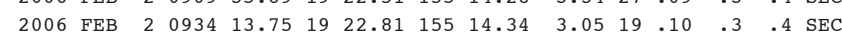
$\begin{array}{lllllllllllllll}2006 & \text { FEB } & 2 & 1011 & 35.58 & 19 & 9.83 & 155 & 16.87 & 44.32 & 27 & .11 & 1.4 & 1.6 & \text { LOI }\end{array}$

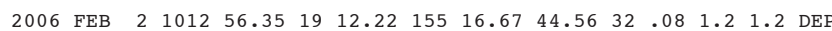

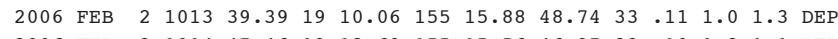
$\begin{array}{lllllllllllllll}2006 & \text { FEB } & 2 & 1014 & 47.46 & 19 & 12.69 & 155 & 15.56 & 46.97 & 33 & .10 & 1.3 & 1.1 & \text { DEP }\end{array}$ $\begin{array}{lllllllllllllll}2006 & \text { FEB } & 2 & 1015 & 22.29 & 19 & 12.41 & 155 & 16.26 & 48.01 & 32 & .13 & 1.4 & 1.2 & \text { DEP }\end{array}$

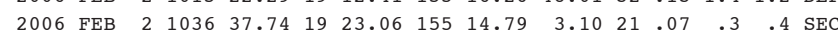

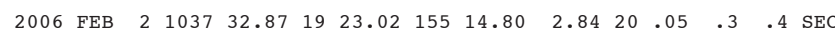

$\begin{array}{lllllllllllllll}2006 & \text { FEB } & 2 & 1116 & 4.50 & 19 & 22.51 & 155 & 5.01 & 9.85 & 37 & .09 & .5 & .4 & \text { SF5 }\end{array}$ $\begin{array}{lllllllllllllll}2006 & \text { FEB } & 2 & 1203 & 3.79 & 19 & 19.11 & 155 & 8.93 & 10.66 & 35 & .08 & .4 & .4 & \text { SF4 }\end{array}$ $\begin{array}{llllrlllllllllll}2006 & \text { FEB } & 2 & 1402 & 53.46 & 19 & 23.42 & 155 & 14.76 & 3.28 & 20 & .08 & .3 & .4 & \text { SEC } \\ 2006 & \text { FEB } & 2 & 1631 & 2.33 & 19 & 22.05 & 155 & 4.90 & 6.34 & 44 & .13 & .6 & .9 & \text { SF5 }\end{array}$ $\begin{array}{lllllllllllllll}2006 & \text { FEB } & 2 & 1633 & 56.52 & 19 & 23.17 & 155 & 16.91 & 2.94 & 29 & .09 & .3 & .2 & \text { SSC }\end{array}$

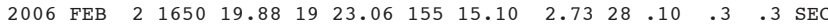

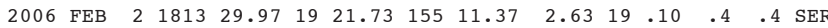

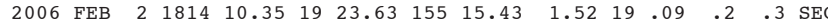
$\begin{array}{lllllllllllllll}2006 & \text { FEB } & 2 & 1815 & 0.73 & 19 & 23.54 & 155 & 14.81 & 3.70 & 24 & .09 & .3 & .4 & \mathrm{SEC}\end{array}$

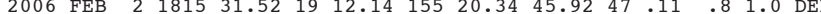

PREF AZ MIN 10 $1.6 \times 103 \quad 2$ $1.4 \times 107 \quad 2$ $1.4 \times$ 8 892

$.9 \times \quad 124 \quad 2$ $1.5 \times \quad 89 \quad 2$ $.5 \times 138 \quad 5$ $1.7 \mathrm{X} \quad 92 \quad 14$

$1.3 \times \quad 80 \quad 2$ $\begin{array}{lll}1.2 x & 74 & 2 \\ .4 \times & 70 & 2\end{array}$ $1.2 \times \quad 69$ $1.1 \times \quad 75 \quad 2$

$1.5 \times \quad 75 \quad 2$ $\begin{array}{lll}1.6 \times & 186 & 6 \\ 1.6 \times & 167 & 2\end{array}$ $1.6 \times 167 \quad 2$ $1.6 \times \quad 64 \quad 2$

$\begin{array}{lll}1.4 \times & 49 & 1 \\ 2.1 \times & 40 & 0\end{array}$ $\begin{array}{lll}2.1 \mathrm{x} & 40 & 0 \\ 1.4 \mathrm{x} & 66 & 1\end{array}$ $\begin{array}{lll}1.4 \mathrm{X} & 66 & 1 \\ 1.2 \mathrm{x} & 82 & 0\end{array}$ $1.3 \mathrm{x} \quad 47$

$\begin{array}{lll}1.7 \mathrm{X} & 43 & 1 \\ 1.8 \mathrm{X} & 86 & 2 \\ 1.6 \mathrm{X} & 85 & 2\end{array}$ $\begin{array}{lll}1.8 \mathrm{X} & 86 & 2 \\ 1.6 \mathrm{X} & 85 & 2 \\ 1.7 \mathrm{x} & 243 & 15\end{array}$ $\begin{array}{lll}1.7 \times & 243 & 15\end{array}$ $1.8 \times 224 \quad 10$

$\begin{array}{ll}1.8 \times 238 \quad 14 \\ 1.6 \times 245 & 9\end{array}$ $\begin{array}{lll}1.6 \times & 245 & 9 \\ 1.8 \times & 238 & 10\end{array}$ $\begin{array}{lll}1.8 \times & 238 \quad 10 \\ 1.6 \times & 72 & 2\end{array}$ $\begin{array}{lll}1.6 \mathrm{X} & 72 & 2 \\ 1.2 \mathrm{x} & 72 & 2\end{array}$

$\begin{array}{lll}1.3 \times & 170 \quad 4\end{array}$ $\begin{array}{rrr}1.5 \times & 187 & 7 \\ 1.4 \times & 72 & 2\end{array}$ $\begin{array}{rrr}.4 \times & 72 & 2 \\ .9 \times & 177 & 4\end{array}$ 1.6X $46 \quad 0$

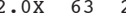
$\begin{array}{rrr}.1 \mathrm{X} & 134 & 3 \\ .1 \mathrm{x} & 87 & 2\end{array}$ $1.5 \times \quad 70 \quad 2$ $1.9 \times 17911$ 
---ORIGIN TIME (HST)-- -LAT N-- --LON W-- DEPTH N RMS ERH ERZ LOC PREF AZ MIN 11 YEAR MON DA HRMN SEC DEG MIN DEG MIN KM RD SEC KM KM REMKS MAG GAP DS

$\begin{array}{lllllllllllllll}2006 & \text { FEB } & 2 & 1900 & 45.54 & 19 & 23.48 & 155 & 15.11 & 2.36 & 20 & .09 & .3 & .4 & \text { SEC }\end{array}$

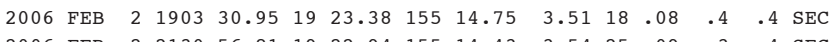

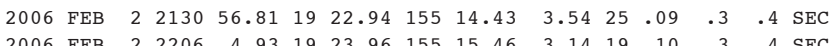
$\begin{array}{lllllllllllllll}2006 & \text { FEB } & 2 & 2206 & 4.93 & 19 & 23.96 & 155 & 15.46 & 3.14 & 19 & .10 & .3 & .4 & \text { SEC }\end{array}$ $\begin{array}{llllllllllllllll}2006 & \text { FEB } & 2 & 2258 & 44.39 & 19 & 22.84 & 155 & 14.60 & 3.52 & 18 & .04 & .3 & .3 & \text { SEC }\end{array}$

$\begin{array}{lllllllllllllll}2006 & \text { FEB } & 2 & 2323 & 35.88 & 19 & 22.66 & 155 & 14.11 & 3.92 & 27 & .09 & .4 & .4 & \mathrm{SEC}\end{array}$

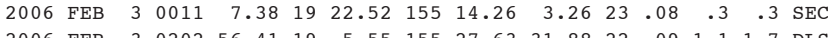

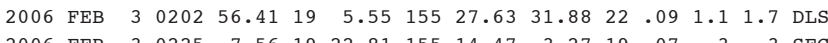

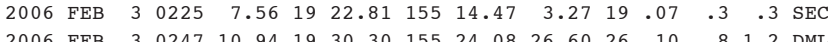

$\begin{array}{llllllllllllll}2006 & \text { FEB } & 3 & 0323 & 58.94 & 19 & 22.66 & 155 & 14.30 & 3.53 & 19.08 & .3 & .4 & \text { SEC }\end{array}$

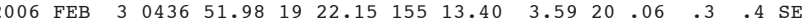
$\begin{array}{lllllllllllllll}2006 & F E B & 3 & 0529 & 16.64 & 19 & 20.64 & 155 & 7.52 & 8.71 & 41 & .09 & .6 & .6 & \mathrm{SF}\end{array}$

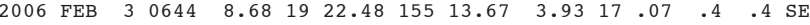
$\begin{array}{llllllllllllllll}2006 & \text { FEB } & 3 & 0734 & 8.29 & 19 & 23.13 & 155 & 14.39 & 2.76 & 20 & .11 & .4 & .4 & \text { SEC }\end{array}$

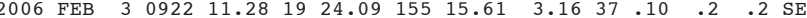

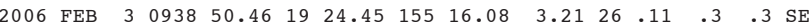

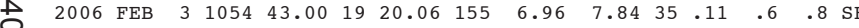

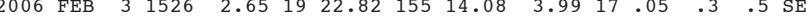
$\begin{array}{lllllllllllllll}2006 & \text { FEB } & 3 & 1613 & 23.63 & 19 & 22.75 & 155 & 13.93 & 4.06 & 20 & .08 & .3 & .4 & \text { SER }\end{array}$

$\begin{array}{lllllllllllllll}2006 & \text { FEB } & 3 & 1712 & 7.89 & 19 & 23.26 & 155 & 15.11 & 2.82 & 18 & .09 & .3 & .4 & \mathrm{SEC}\end{array}$

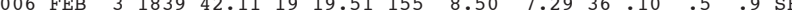

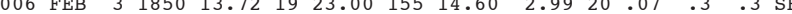

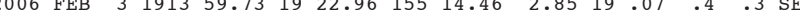

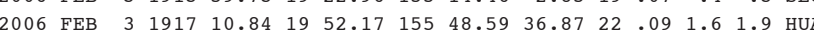

$\begin{array}{llllllllllllll}2006 & \text { FEB } & 3 & 2025 & 16.19 & 19 & 26.40 & 155 & 30.06 & 12.22 & 23 & .10 & .4 & 1.1 \\ \text { KAO }\end{array}$ $\begin{array}{llllllllllllllll}2006 & \text { FEB } & 3 & 2032 & 34.14 & 19 & 23.33 & 155 & 14.64 & 4.10 & 25 & .09 & .4 & .4 & \text { SEC }\end{array}$

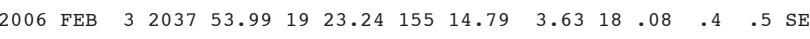

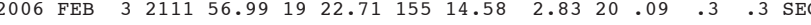

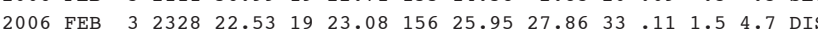

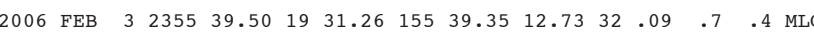
$\begin{array}{lllllllllllllll}2006 & \text { FEB } & 4 & 0014 & 6.00 & 19 & 22.93 & 155 & 14.69 & 2.74 & 19.10 & .3 & .3 & \mathrm{SEC}\end{array}$ $\begin{array}{lllllllllllllll}2006 & \text { FEB } & 4 & 0030 & 33.31 & 19 & 23.43 & 155 & 15.16 & 4.46 & 20 & .09 & .4 & .6 & \text { SEC }\end{array}$

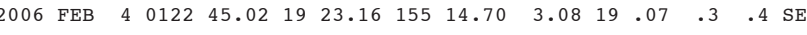
$\begin{array}{lllllllllllllll}2006 & \text { FEB } & 4 & 0158 & 26.43 & 19 & 27.38 & 155 & 19.50 & 1.84 & 24 & .11 & .3 & .6 & \text { KAO }\end{array}$

$\begin{array}{lllllllllllllll}2006 & \text { FEB } & 4 & 0219 & 50.95 & 19 & 13.16 & 155 & 32.63 & 10.68 & 29 & .11 & .5 & 1.2 & \text { LSW }\end{array}$ $\begin{array}{lllllllllllllll}2006 & \text { FEB } & 4 & 0220 & 38.27 & 19 & 23.48 & 155 & 17.03 & 2.96 & 20 & .09 & .3 & .2 & \mathrm{SSC}\end{array}$ $\begin{array}{lllllllllllllll}2006 & F E B & 4 & 0231 & 35.26 & 19 & 23.79 & 155 & 14.31 & 4.95 & 19 & .13 & .6 & .9 & \mathrm{SEC}\end{array}$ $\begin{array}{rrlllllllllllll}2006 & \text { FEB } & 4 & 0529 & 8.00 & 19 & 23.12 & 155 & 14.19 & 4.29 & 21 & .10 & .5 & .6 & \text { SEC } \\ 2006 & \text { FEB } & 4 & 0542 & 35.20 & 19 & 22.97 & 155 & 14.22 & 3.80 & 24 & .12 & .4 & .5 & \text { SEC }\end{array}$

$\begin{array}{lllllllllllllll}2006 & \text { FEB } & 4 & 0618 & 14.75 & 19 & 23.03 & 155 & 14.78 & 3.19 & 19.06 & .3 & .4 & \mathrm{SEC}\end{array}$ $\begin{array}{lllllllllllllll}2006 & \text { FEB } & 4 & 0620 & 55.63 & 19 & 24.55 & 155 & 14.85 & 4.40 & 23 & .11 & .4 & .6 & \text { SNC }\end{array}$

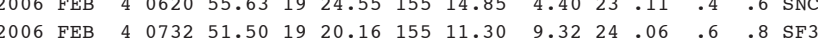

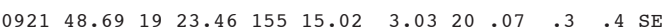
$\begin{array}{lllllllllllllll}2006 & \text { FEB } & 4 & 1007 & 47.51 & 19 & 24.18 & 155 & 15.93 & 2.94 & 16 & .05 & .3 & .4 & \text { SEC }\end{array}$
$1.3 \times \quad 60 \quad 2$ $1.3 \times \quad 813$ $\begin{array}{lll}.7 \mathrm{x} & 83 & 2 \\ .4 \mathrm{x} & 72 & 2 \\ 1.3 \mathrm{x} & 83 & 2\end{array}$ $1.3 \mathrm{X} \quad 83 \quad 2$

$1.7 \times \quad 84 \quad 2$ $1.5 \times \quad 86 \quad 2$ $1.5 \times 208 \quad 7$ $1.3 \times \quad 86 \quad 2$ $1.4 \times 120 \quad 2$

$\begin{array}{lll}2 \times \quad 88 & 2 \\ 5 \times & 03 & 1\end{array}$ $1.5 \times 103$ $1.9 \times 177 \quad 5$ $1.5 \times \quad 93 \quad 1$ $1.4 \mathrm{X} \quad 93 \quad 2$

$\begin{array}{lll}1 \times & 51 \quad 2\end{array}$ $\begin{array}{lll}.9 \mathrm{X} & 91 \quad 1\end{array}$ $1.5 \times 212 \quad 6$ $1.4 \mathrm{X} \quad 97 \quad 2$ $1.4 \times \quad 94$

$\begin{array}{lll}1.1 \times \quad 68 & 2\end{array}$ $1.4 \times 203$ $1.4 \times \quad 79 \quad 3$ $\begin{array}{lrr}1.5 \times & 82 & 2 \\ 1.4 \times & 274 & 16\end{array}$

$\begin{array}{llll}1.3 \times & 67 & 6\end{array}$ $\begin{array}{lll}1.8 \times & 72 & 3\end{array}$ $\begin{array}{lll}1.2 \times & 104 & 2\end{array}$ $1.0 \times \quad 83$ 2.3X 31875

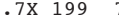
$\begin{array}{lll}1.2 \times & 76 & 2\end{array}$ $\begin{array}{lll}1.5 \mathrm{X} & 59 & 2\end{array}$ $1.3 \times \quad 82 \quad 3$ 1.6X 113 $\begin{array}{lll}1.4 \mathrm{X} & 46 & 0 \\ 1.5 \mathrm{X} & 104 & 2\end{array}$ $\begin{array}{lll}1.5 \times & 104 & 2 \\ 1.7 \times & 92 & 2\end{array}$ $\begin{array}{lll}1.7 x & 92 & 2 \\ 1.7 x & 84 & 2\end{array}$
---ORIGIN TIME (HST)-- -LAT N-- --LON W-- DEPTH N RMS ERH ERZ LOC PREF AZ MIN 12 YEAR MON DA HRMN SEC DEG MIN DEG MIN $\quad$ KM RD SEC KM KM REMKS MAG GAP DS

$\begin{array}{lllllllllllllll}2006 & \text { FEB } & 4 & 1050 & 46.74 & 19 & 22.21 & 155 & 13.95 & 3.61 & 18 & .12 & .4 & .5 & \text { SER }\end{array}$

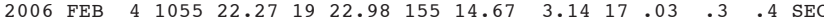

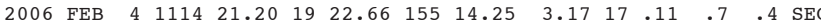

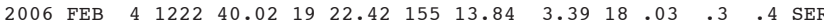
$\begin{array}{lllllllllllllllll}2006 & \text { FEB } & 4 & 1229 & 8.69 & 19 & 23.08 & 155 & 14.77 & 3.22 & 22 & .07 & .3 & .4 & \text { SEC }\end{array}$ $\begin{array}{llllllllllllllllll}2006 & \text { FEB } & 4 & 1231 & 30.61 & 19 & 23.29 & 155 & 14.86 & 2.50 & 13 & .02 & .3 & .5 & \text { SEC } & 1.3 \times & 76 & 2\end{array}$

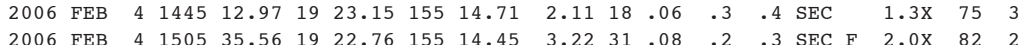

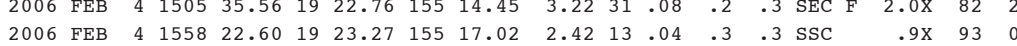

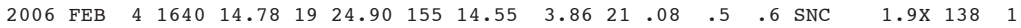

$\begin{array}{llllllllllllllllll}2006 & \text { FEB } & 4 & 1652 & 40.84 & 19 & 23.13 & 155 & 14.90 & 2.98 & 18 & .05 & .3 & .4 & \text { SEC } & 1.5 \mathrm{X} & 69 & 2\end{array}$

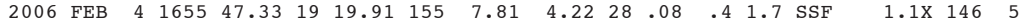

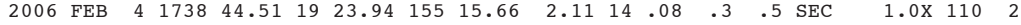
$\begin{array}{llllllllllllllllll}2006 & \text { FEB } & 4 & 1743 & 3.35 & 19 & 23.70 & 155 & 14.79 & 3.84 & 18 & .08 & .4 & .5 & \text { SEC } & 1.7 \mathrm{X} & 77 & 2\end{array}$

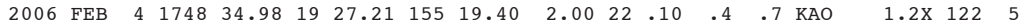

$\begin{array}{llllllllllllllllll}2006 & \text { FEB } & 4 & 1753 & 37.16 & 19 & 22.83 & 155 & 14.16 & 2.90 & 19 & .07 & .4 & .4 & \mathrm{SEC} & 1.5 \mathrm{X} & 89 & 2\end{array}$

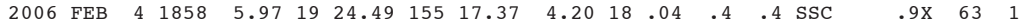

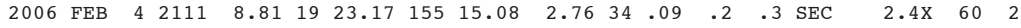

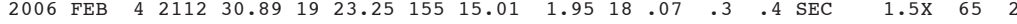

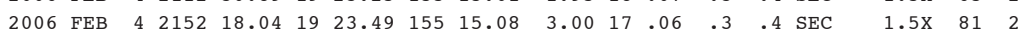

$\begin{array}{llllllllllllllllll}2006 & \text { FEB } & 4 & 2259 & 21.34 & 19 & 22.62 & 155 & 14.07 & 3.34 & 20 & .06 & .3 & .3 & \text { SEC } & 1.8 \mathrm{X} & 89 & 2\end{array}$ $\begin{array}{llllllllllllllllll}2006 & \text { FEB } & 4 & 2305 & 0.11 & 19 & 23.16 & 155 & 14.92 & 3.18 & 23 & .06 & .3 & .3 & \text { SEC } & 2.3 \mathrm{X} & 68 & 2\end{array}$

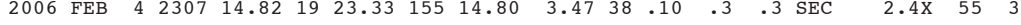

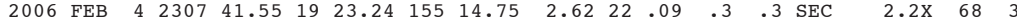

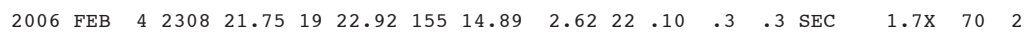

$\begin{array}{llllllllllllllllll}2006 & \text { FEB } & 5 & 0009 & 4.47 & 19 & 24.12 & 155 & 15.39 & 3.77 & 17 & .08 & .4 & .5 & \text { SEC } & 1.5 \times & 78 & 2\end{array}$

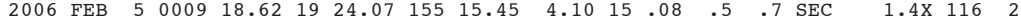

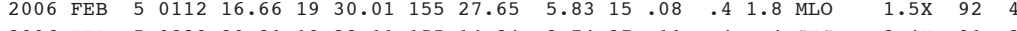

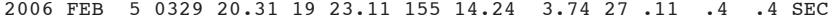

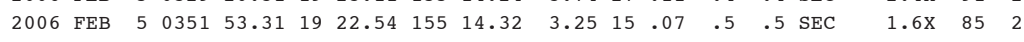

$\begin{array}{llllllllllllllllll}2006 & \text { FEB } & 5 & 0643 & 39.71 & 19 & 22.71 & 155 & 14.56 & 3.41 & 17 & .05 & .3 & .4 & \text { SEC } & 1.5 \mathrm{X} & 78 & 2\end{array}$ $\begin{array}{llllllllllllllllll}2006 & \text { FEB } & 5 & 0718 & 43.32 & 19 & 23.21 & 155 & 14.82 & 2.27 & 18 & .05 & .3 & .3 & \text { SEC } & 1.3 \mathrm{X} & 72 & 2\end{array}$

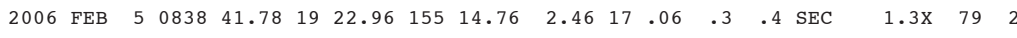

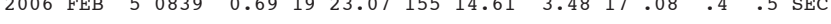
$\begin{array}{lllllllllllllll}2006 & \text { FEB } & 5 & 0841 & 27.63 & 19 & 23.28 & 155 & 14.73 & 2.50 & 19 & .11 & .3 & .4 & \mathrm{SEC}\end{array}$

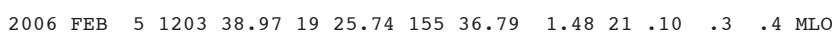

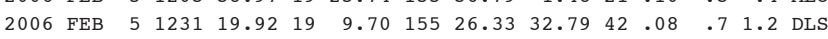

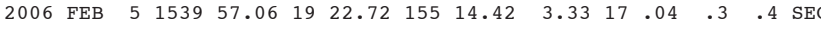

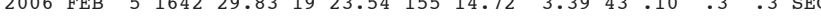

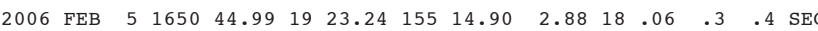

$\begin{array}{lllllllllllllll}2006 & \text { FEB } & 5 & 1723 & 12.78 & 19 & 22.63 & 155 & 14.20 & 3.00 & 21 & .07 & .3 & .3 & \mathrm{SEC}\end{array}$

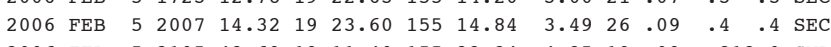

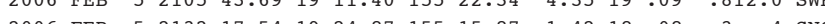

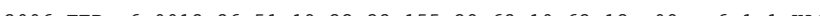

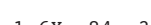
$2.1 \times 172 \quad 3$ $1.5 \times \quad 82 \quad 2$ $\begin{array}{lll}2.5 \mathrm{X} & 45 & 2 \\ 1.7 \mathrm{X} & 69 & 2\end{array}$ $1.7 \times \quad 86 \quad 2$ $2.1 \times \quad 52 \quad 2$ $\begin{array}{lll}1.5 \times & 185 & 10 \\ 1.4 \times & 111 & 2\end{array}$ $1.4 \times \quad 82 \quad 4$ 
---ORIGIN TIME (HST)-- -LAT N-- --LON W-- DEPTH N RMS ERH ERZ LOC YEAR MON DA HRMN SEC DEG MIN DEG MIN KM RD SEC KM KM REMKS

$\begin{array}{lllllllllllllll}2006 & \text { FEB } & 6 & 0041 & 15.24 & 19 & 24.93 & 155 & 19.86 & 6.43 & 25 & .11 & .5 & 1.0 & \text { KAO }\end{array}$

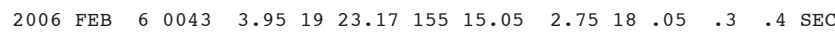
$\begin{array}{lllllllllllllll}2006 & \text { FEB } & 6 & 0154 & 36.04 & 19 & 28.58 & 155 & 26.95 & 7.65 & 43 & .13 & .4 & 1.0 & \text { KAO }\end{array}$

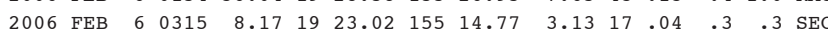
$\begin{array}{lllllllllllllll}2006 & \text { FEB } & 6 & 0316 & 41.42 & 19 & 10.98 & 155 & 22.00 & 1.39 & 18 & .05 & .8 & 1.0 & \text { SWR }\end{array}$

$\begin{array}{lllllllllllllll}2006 & \text { FEB } & 6 & 0356 & 35.39 & 19 & 13.12 & 155 & 18.36 & 7.14 & 45 & .12 & .5 & .8 & \text { SWR }\end{array}$ $\begin{array}{llllllllllllllll}2006 & \text { FEB } & 6 & 0448 & 36.61 & 19 & 8.55 & 155 & 21.28 & 11.14 & 24 & .14 & .9 & .7 & \text { LOI }\end{array}$ $\begin{array}{lllllllllllllll}2006 & \text { FEB } & 6 & 0449 & 25.21 & 19 & 22.71 & 155 & 14.03 & 3.86 & 14 & .05 & .4 & .5 & \text { SEC }\end{array}$ $\begin{array}{lllllllllllllll}2006 & \text { FEB } & 6 & 0535 & 36.52 & 19 & 23.58 & 155 & 15.10 & 2.86 & 13 & .05 & .3 & .4 & \text { SEC }\end{array}$ $\begin{array}{lllllllllllllll}2006 & \mathrm{FEB} & 6 & 0536 & 17.55 & 19 & 10.51 & 155 & 21.28 & 2.17 & 24 & .08 & .6 & 1.3 & \text { SWR }\end{array}$

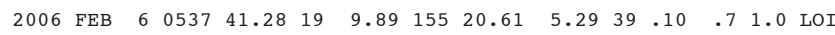
$\begin{array}{lllllllllllllll}2006 & \text { FEB } & 6 & 0726 & 47.87 & 19 & 22.81 & 155 & 14.73 & 3.13 & 18 & .09 & .4 & .4 & \text { SEC }\end{array}$ $\begin{array}{llllllllllllll}2006 & \text { FEB } & 6 & 0734 & 3.04 & 19 & 25.58 & 155 & 19.07 & 4.87 & 19 & .10 & .5 & 1.0 \\ \text { KAO }\end{array}$

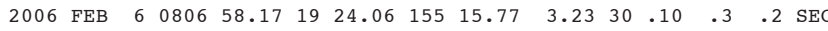

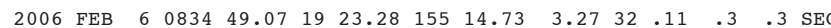

$\begin{array}{lllllllllllllll}2006 & \text { FEB } & 6 & 0911 & 1.58 & 19 & 27.47 & 155 & 18.85 & 4.76 & 21 & .09 & .5 & 1.5 & \text { SNC }\end{array}$

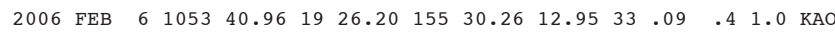

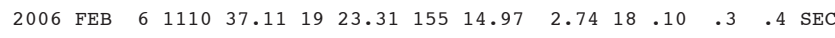
$\begin{array}{llllllllllllll}2006 & \text { FEB } & 6 & 1143 & 45.67 & 19 & 2.97 & 156 & 9.16 & 36.29 & 36 & .09 & 1.0 & 2.2 \\ \text { KON }\end{array}$ $\begin{array}{lllllllllllllll}2006 & \text { FEB } & 6 & 1158 & 7.52 & 19 & 23.42 & 155 & 15.03 & 3.64 & 17 & .08 & .4 & .5 & \mathrm{SEC}\end{array}$

$\begin{array}{lllllllllllllll}2006 & \text { FEB } & 6 & 1219 & 52.63 & 19 & 11.58 & 155 & 25.38 & 34.97 & 26 & .06 & .8 & 1.3 & \text { DLS }\end{array}$

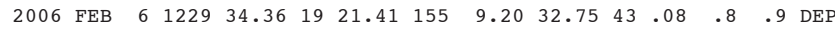

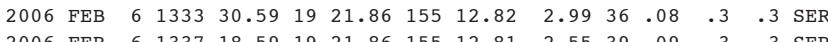

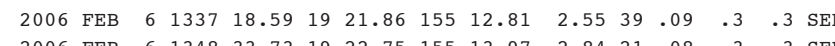

\begin{tabular}{llllllllllllll}
2006 & FEB & 6 & 1406 & 46.74 & 19 & 24.04 & 155 & 16.33 & 0.05 & 19 & .10 & .1 & .2 \\
\hline
\end{tabular}

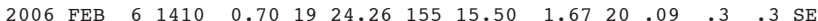

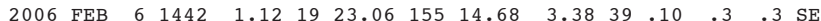

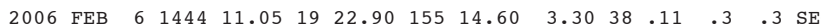

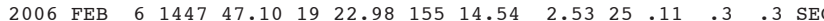

$\begin{array}{lllllllllllllll}2006 & \text { FEB } & 6 & 1517 & 46.20 & 19 & 21.66 & 155 & 18.51 & 3.32 & 20 & .07 & .3 & .6 & \text { SWR }\end{array}$

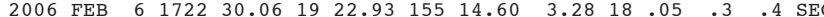

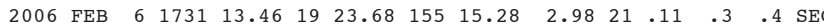
$\begin{array}{lllllllllllllll}2006 & \text { FEB } & 6 & 1857 & 3.74 & 19 & 22.44 & 155 & 14.20 & 2.92 & 19 & .10 & .5 & .4 & \text { SEC }\end{array}$ $\begin{array}{llllllllllllllll}2006 & \text { FEB } & 6 & 1930 & 28.63 & 19 & 14.11 & 155 & 23.58 & 34.57 & 24 & .10 & 1.1 & 1.5 & \text { DEP }\end{array}$

$\begin{array}{lllllllllllllll}2006 & \text { FEB } & 6 & 2036 & 45.05 & 19 & 21.39 & 155 & 18.64 & 1.14 & 20 & .08 & .2 & .4 & \text { SWR }\end{array}$

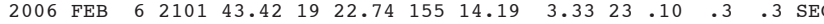
$\begin{array}{lllllllllllllll}2006 & \text { FEB } & 6 & 2104 & 14.71 & 19 & 22.62 & 155 & 13.74 & 4.32 & 18 & .09 & .5 & .6 & \text { SER }\end{array}$ $\begin{array}{lllllllllllllll}2006 & \text { FEB } & 6 & 2108 & 58.98 & 19 & 19.30 & 15 & 11.51 & 5.44 & 38 & .09 & .3 & 1.2 & \text { SF } 3\end{array}$ $\begin{array}{llllllllllllllll}2006 & \text { FEB } & 6 & 2215 & 6.01 & 19 & 18.45 & 155 & 13.52 & 7.34 & 36 & .12 & .4 & .9 & \text { SF2 }\end{array}$

$\begin{array}{llllllllllllll}2006 & \text { FEB } & 6 & 2327 & 38.04 & 19 & 46.52 & 156 & 0.27 & 34.36 & 15 & .09 & 5.5 & 4.2\end{array}$

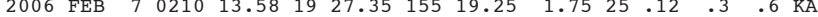

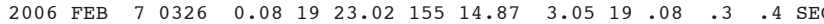

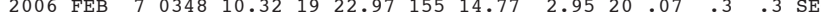
$\begin{array}{llllllllllllll}2006 & \text { FEB } & 7 & 0354 & 12.63 & 19 & 25.02 & 155 & 14.93 & 3.91 & 20 & .11 & .5 & .6\end{array}$
PREF AZ MIN 13 MAG GAP DS $1.3 \times \quad 79 \quad 2$ $1.5 \times \quad 71$ $\begin{array}{lll}2.1 \times & 47 & 7 \\ 1.4 x & 79 & 2 \\ 1.5 x & 203 & \end{array}$ $1.5 \times 203 \quad 11$

$1.9 \times 170 \quad 9$

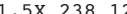
$\begin{array}{lrr}1.5 \times & 238 & 12 \\ 1.5 \times & 97 & 2\end{array}$ $\begin{array}{lll}1.5 \times & 97 & 2 \\ 1.2 \times & 95 & 2\end{array}$ $1.6 \times \quad 177 \quad 12$ $2.1 \times 18713$ $\begin{array}{rrr}2.1 \times & 187 & 13 \\ 1.6 x & 79 & 2\end{array}$ $\begin{array}{lll}1.7 \mathrm{X} & 86 & 3\end{array}$ $1.9 \times \quad 49 \quad 1$ $2.1 \times \quad 50$ $1.2 \times 128 \quad 4$ $\begin{array}{lll}1.5 \mathrm{X} & 66 & 8 \\ 1.3 \mathrm{x} & 73 & 2\end{array}$ $\begin{array}{rrr}1.5 \times & 66 & 8 \\ 2.3 \times & 73 & 2 \\ 1.5 \times & 286 & 42\end{array}$ $\begin{array}{lll}1.4 \times & 77 \quad 2\end{array}$

$\begin{array}{lll}1.5 \times & 165 & 6\end{array}$ $2.2 \times \quad 94 \quad 3$ $\begin{array}{lll}1.9 \times & 83 & 1\end{array}$ $2.1 \times \quad 58 \quad 1$ $1.7 \times \quad 94 \quad 2$

$\begin{array}{lll}1.2 \times & 107 \quad 1\end{array}$ $\begin{array}{lll}1.7 \times & 86 & 2\end{array}$ $2.1 \times \quad 50 \quad 2$ $\begin{array}{lll}2.0 \mathrm{x} & 49 & 2 \\ 1.5 \mathrm{x} & 81 & 3\end{array}$

$\begin{array}{lll}1.1 \times & 71 & 3\end{array}$ $\begin{array}{lll}1.2 \times & 84 & 2\end{array}$ $\begin{array}{lll}1.4 \times \quad 59 & 2\end{array}$ $\begin{array}{lll}1.3 \times & 87 \quad 2\end{array}$ $1.1 \times 20611$

$1.1 \times \quad 76 \quad 5$ $1.6 \times \quad 92 \quad 2$ $1.4 \times 102 \quad 1$ $1.3 \times 101 \quad 5$ $1.2 \times \quad 77 \quad 3$

$1.0 \times 28020$ $1.2 \times 126 \quad 5$ $\begin{array}{lll}1.5 \times & 70 & 2\end{array}$ $\begin{array}{lll}1.4 \mathrm{X} & 74 & 2\end{array}$ $1.2 \times 134$
--ORTGIN TIME (HST)-- -LAT N-- --LON W-- DEPTH N RMS ERH ERz LOC YEAR MON DA HRMN SEC DEG MIN DEG MIN KM RD SEC KM KM REMKS $\begin{array}{lllllllllllllll}2006 & \text { FEB } & 7 & 0425 & 32.69 & 19 & 20.92 & 155 & 4.50 & 5.74 & 31 & .10 & .5 & 1.2 & \text { SF5 }\end{array}$

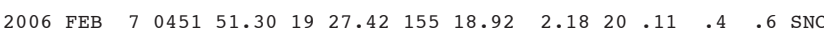

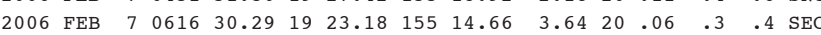

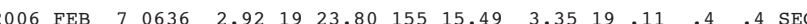
$\begin{array}{lllllllllllllll}2006 & \text { FEB } & 7 & 0658 & 17.43 & 19 & 49.39 & 155 & 37.45 & 28.67 & 23 & .09 & 1.1 & 1.9 & \mathrm{KEA}\end{array}$

$\begin{array}{lllllllllllllll}2006 & \text { FEB } & 7 & 0737 & 24.83 & 19 & 19.90 & 155 & 6.52 & 8.42 & 39 & .10 & .4 & .5 & \mathrm{SF} 4\end{array}$

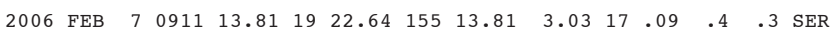

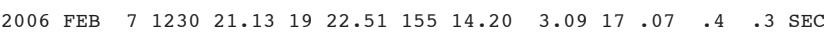

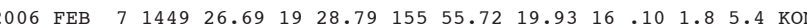
$\begin{array}{lllllllllllllll}2006 & \mathrm{FEB} & 7 & 1450 & 45.17 & 19 & 19.14 & 155 & 13.27 & 8.77 & 43 & .12 & .4 & .6 & \mathrm{SF} 2\end{array}$

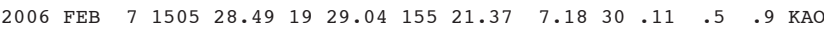
$\begin{array}{lllllllllllllll}2006 & \text { FEB } & 7 & 1518 & 18.77 & 19 & 23.03 & 155 & 14.40 & 3.44 & 20 & .08 & .3 & .4 & \text { SEC }\end{array}$

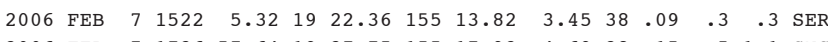
$\begin{array}{llllllllllllll}2006 & \text { FEB } & 7 & 1726 & 57.64 & 19 & 27.75 & 155 & 17.93 & 4.69 & 28 & .15 & .5 & 1.1\end{array}$ SNC

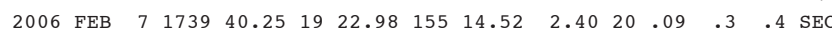

\begin{tabular}{llllllllllllll}
2006 & FEB & 7 & 1817 & 36.46 & 19 & 24.06 & 155 & 15.65 & 3.59 & 19 & .10 & .4 & .4 \\
\hline
\end{tabular} $\begin{array}{llllllllllllllll}2006 & \text { FEB } & 7 & 1830 & 51.09 & 19 & 45.84 & 154 & 48.78 & 36.00 & 31 & .12 & 1.1 & 1.3 & \text { HIL }\end{array}$ $\begin{array}{lllllllllllllll}2006 & \text { FEB } & 7 & 1907 & 3.29 & 19 & 22.57 & 155 & 14.12 & 3.49 & 21 & .05 & .3 & .3 & \text { SEC }\end{array}$

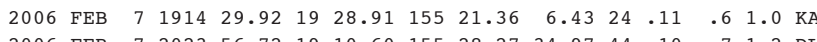

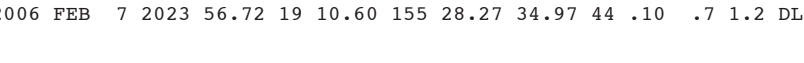

$\begin{array}{lllllllllllllll}2006 & \text { FEB } & 7 & 2100 & 42.98 & 20 & 9.55 & 155 & 35.96 & 37.14 & 40 & .09 & 1.0 & 1.9 & \text { кон }\end{array}$

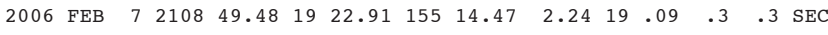
$\begin{array}{lllllllllllllll}2006 & \text { FEB } & 7 & 2111 & 56.86 & 19 & 22.87 & 155 & 14.31 & 2.41 & 20 & .12 & .3 & .3 & \text { SEC } \\ 2006 & \text { FEB } & 7 & 2134 & 16.45 & 19 & 22.68 & 155 & 14.47 & 2.87 & 18 & .10 & .4 & .3 & \text { SEC }\end{array}$

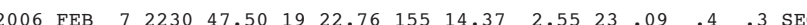

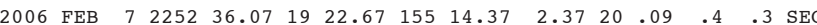
$\begin{array}{lllllllllllllll}2006 & \text { FEB } & 7 & 225 & 16.41 & 19 & 23.67 & 155 & 15.07 & 2.97 & 33 & .10 & .2 & .3 & \text { SEC }\end{array}$

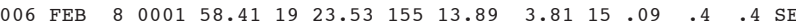
\begin{tabular}{llllllllllllll}
2006 & FEB & 8 & 0135 & 45.41 & 19 & 23.46 & 155 & 14.62 & 2.81 & 28 & .09 & .3 & .3 \\
\hline
\end{tabular} $\begin{array}{lllllllllllllll}2006 & \text { FEB } & 8 & 0142 & 52.77 & 19 & 22.71 & 155 & 14.02 & 3.61 & 17 & .08 & .5 & 4 & \text { SEC }\end{array}$

$\begin{array}{lllllllllllllll}2006 & \text { FEB } & 8 & 0322 & 15.73 & 19 & 23.36 & 155 & 14.52 & 3.70 & 37 & .11 & .3 & .4 & \mathrm{SEC}\end{array}$

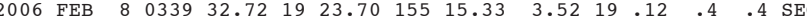

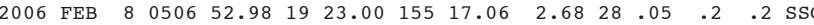
$\begin{array}{llllllllllllllll}2006 & \text { FEB } & 8 & 0649 & 55.24 & 19 & 24.56 & 155 & 38.25 & 3.60 & 17 & .11 & .4 & .4 & \text { MLO }\end{array}$ $\begin{array}{llllllllllllllll}2006 & \text { FEB } & 8 & 0735 & 0.97 & 19 & 24.07 & 155 & 15.71 & 3.77 & 20 & .11 & .4 & .5 & \text { SEC }\end{array}$

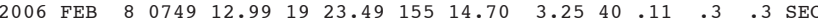

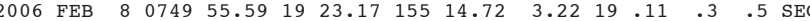

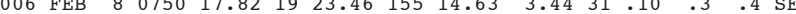

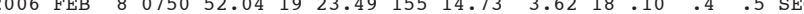

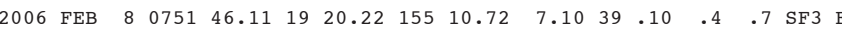

$\begin{array}{lllllllllllllll}2006 & \text { FEB } & 8 & 0804 & 44.91 & 19 & 22.68 & 155 & 14.03 & 3.44 & 37 & .09 & .3 & .3 & \text { SEC }\end{array}$

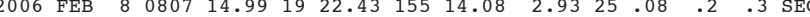

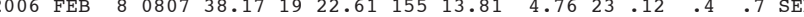

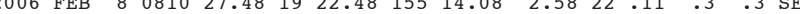
$\begin{array}{lllllllllllllll}2006 & \text { FEB } & 8 & 0840 & 17.66 & 19 & 24.28 & 155 & 16.00 & 2.98 & 16 & .07 & .3 & .3 & \text { SEC }\end{array}$
PREF AZ MIN MAG GAP DS

$1.4 \times 217 \quad 6$ $\begin{array}{rrr}1.0 \times 127 & 4 \\ 1.4 \times & 77 & 3 \\ 1.2 \times & 66 & 2\end{array}$ $\begin{array}{lll}1.4 \times & 77 & 3 \\ 1.2 \times & 66 & 2\end{array}$ $1.5 \times 26027$

$1.8 \times 154 \quad 5$ $1.5 \times 100 \quad 1$ $\begin{array}{lll}.3 \times & 137 \quad 2 \\ 1.2 \times & 279 & 31\end{array}$ $1.2 \times 27931$

$\begin{array}{lll}1.4 \times & 150 \quad 4\end{array}$ $\begin{array}{lll}1.4 \mathrm{X} & 85 & 2 \\ 2.0 \mathrm{x} & 51 & \end{array}$ $\begin{array}{rrr}2.0 \mathrm{x} & 51 & 1 \\ 1.2 \mathrm{x} & 139 & 2 \\ 1.2 \times & 86 & 3\end{array}$ $\begin{array}{lll}1.2 \times & 139 & 2 \\ 1.2 \times & 86 & 3\end{array}$

$1.2 \times \quad 77 \quad 2$ $\begin{array}{lll}1.6 \times \quad 274 \quad 24 & 24\end{array}$ $\begin{array}{rrrr}1.6 \times & 274 & 24 \\ 1.6 \times & 88 & 2\end{array}$ $\begin{array}{lrl}1.6 \times & 88 & 2 \\ 1.1 \times & 148 & 4\end{array}$ $1.9 \times \quad 90 \quad 2$

1.9X $237 \quad 19$ $\begin{array}{lll}1.4 \mathrm{X} & 87 \quad 2\end{array}$ $1.4 \mathrm{X} \quad 86 \quad 2$ $\begin{array}{lll}1.6 \times & 128 \quad 2\end{array}$ $\begin{array}{lll}1.7 \mathrm{X} & 84 & 2\end{array}$

$\begin{array}{lll}1.4 \times \quad 83 & 2\end{array}$ $1.8 \mathrm{X} \quad 54 \quad 2$ $1.5 \times 121 \quad 2$ $\begin{array}{lll}1.9 \times & 79 & 2 \\ 1.3 \mathrm{X} & 97 & 2\end{array}$

$2.0 \times \quad 45 \quad 2$ $\begin{array}{lll}1.4 \times & 61 & 2\end{array}$ $\begin{array}{lll}1.5 \mathrm{X} & 48 & 1\end{array}$ $\begin{array}{rrr}1.4 \times & 101 & 1 \\ 1.5 \times & 77 & 2\end{array}$

$2.1 \times \quad 52 \quad 2$ $1.1 \mathrm{x} \quad 82 \quad 3$ $2.0 \times \quad 72 \quad 2$ $\begin{array}{lll}1.6 \mathrm{X} & 95 & 2 \\ 1.4 \mathrm{X} & 83 & 5\end{array}$

$2.3 \times \quad 49 \quad 2$ $\begin{array}{lll}1.6 \times & 84 & 2\end{array}$ $\begin{array}{lll}1.8 \mathrm{X} & 96 & 1\end{array}$ $\begin{array}{lll}1.5 x & 85 & 1\end{array}$ 
---ORIGIN TIME (HST)-- -LAT N-- --LON W-- DEPTH N RMS ERH ERZ LOC YEAR MON DA HRMN SEC DEG MIN DEG MIN KM RD SEC KM KM REMKS

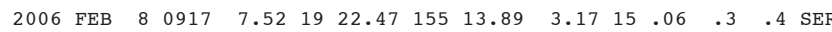

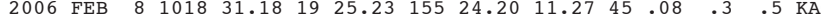

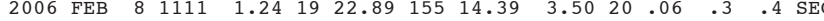
$\begin{array}{lllllllllllllll}2006 & \text { FEB } & 8 & 1128 & 12.64 & 19 & 23.16 & 155 & 14.55 & 3.23 & 47 & .11 & .3 & .3 & \text { SEC F }\end{array}$

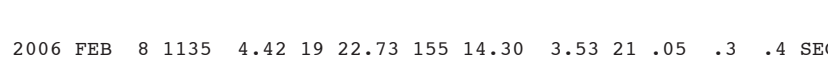

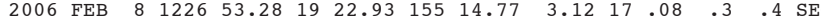
$\begin{array}{lllllllllllllll}2006 & \text { FEB } & 8 & 1240 & 10.78 & 19 & 25.21 & 15 & 16.13 & 1.51 & 14 & .06 & .3 & .4 & \text { SNC }\end{array}$

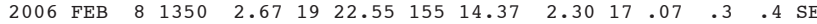

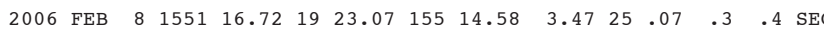

$\begin{array}{lllllllllllllll}2006 & \text { FEB } & 8 & 1615 & 36.83 & 19 & 22.83 & 155 & 14.66 & 2.88 & 19 & .10 & .3 & .4 & \text { SEC }\end{array}$ $\begin{array}{lllllllllllllll}2006 & \text { FEB } & 8 & 1818 & 53.21 & 19 & 26.53 & 155 & 19.79 & 2.74 & 25 & .12 & .4 & .9 & \text { KAO }\end{array}$

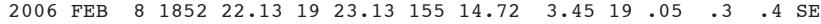

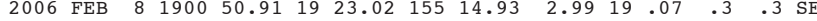
$\begin{array}{lllllllllllllll}2006 & \text { FEB } & 8 & 2130 & 29.47 & 19 & 26.50 & 155 & 19.68 & 3.04 & 25 & .10 & .4 & .8 & \text { KAO }\end{array}$

$\begin{array}{lllllllllllllll}2006 & \text { FEB } & 8 & 2152 & 55.68 & 19 & 23.34 & 155 & 14.88 & 3.15 & 21 & .07 & .3 & .3 & \text { SEC }\end{array}$ $\begin{array}{lllllllllllllll}2006 & \text { FEB } & 8 & 2223 & 18.60 & 19 & 23.15 & 155 & 14.48 & 4.09 & 19 & .08 & .4 & .5 & \mathrm{SEC}\end{array}$

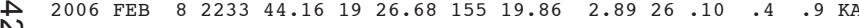
\begin{tabular}{llllllllllllll}
2006 & FEB & 8 & 2250 & 52.43 & 19 & 22.88 & 155 & 14.56 & 3.35 & 31 & .09 & .3 & .3 \\
\hline
\end{tabular} $\begin{array}{llllllllllllll}2006 & \text { FEB } & 9 & 0138 & 31.25 & 19 & 25.18 & 155 & 19.46 & 6.52 & 18 & .06 & .5 & 1.1\end{array}$

$\begin{array}{lllllllllllllll}2006 & \text { FEB } & 9 & 0603 & 9.18 & 19 & 23.36 & 155 & 15.02 & 1.50 & 14 & .08 & .3 & .5 & \text { SEC }\end{array}$

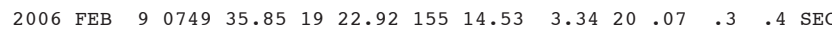

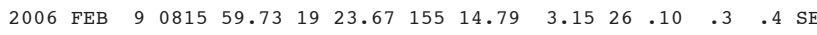

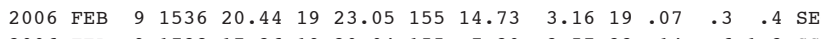

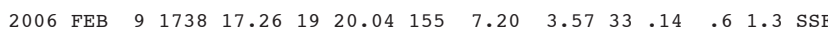

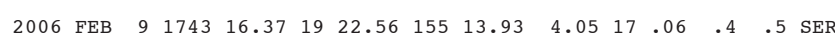

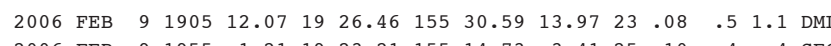
$\begin{array}{lllllllllllllll}2006 & \text { FEB } & 9 & 1955 & 1.21 & 19 & 23.21 & 155 & 14.73 & 3.41 & 25 & .10 & .4 & .4 & \mathrm{SEC}\end{array}$

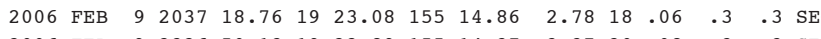
$\begin{array}{lllllllllllllll}2006 & \text { FEB } & 9 & 2326 & 50.13 & 19 & 22.89 & 155 & 14.37 & 3.37 & 20 & .08 & .3 & .3 & \text { SEC }\end{array}$

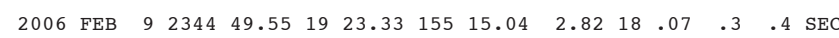

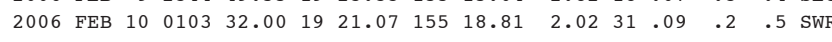

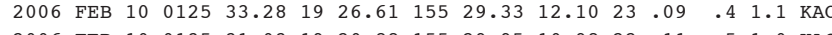

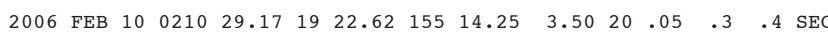

$\begin{array}{llllllllllllll}2006 & \text { FEB } 10 & 0330 & 17.85 & 19 & 22.92 & 155 & 14.68 & 3.33 & 30 & .07 & .2 & .3 & \text { SEC }\end{array}$

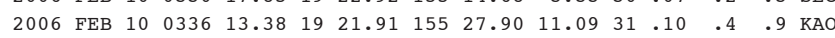

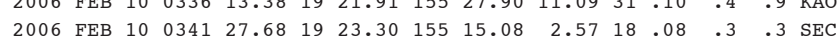

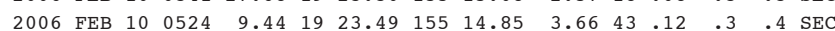

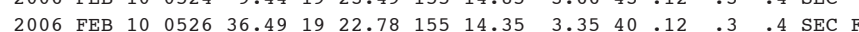

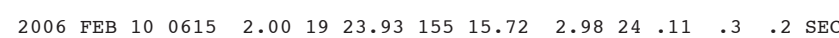

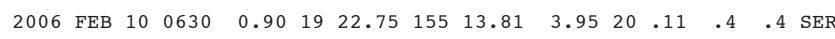

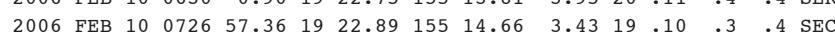

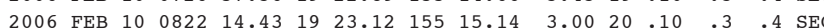
$\begin{array}{lllllllllllllll}2006 & \text { FEB } & 10 & 0919 & 56.69 & 19 & 22.20 & 155 & 13.77 & 3.05 & 19 & .09 & .3 & .4 & \text { SER }\end{array}$
PREF AZ MIN 15

$\begin{array}{lll}1.4 \times & 90 & 1\end{array}$ $\begin{array}{llll}.3 \times & 45 & 8\end{array}$ $\begin{array}{lll}1.4 \mathrm{X} & 85 & 2 \\ 2.9 \mathrm{x} & 47 & 3 \\ 2.4 \mathrm{x} & 74 & 2\end{array}$ $2.4 \times \quad 74 \quad 2$

$1.5 \times \quad 84 \quad 2$ $1.5 \times \quad 73 \quad 2$ $1.1 \times 118 \quad 2$ $1.4 \times \quad 85 \quad 2$ $1.9 \times \quad 79 \quad 3$

$\begin{array}{lll}1.6 \mathrm{X} & 81 & 2\end{array}$ $\begin{array}{lll}1.5 \times & 108 & 4\end{array}$ $\begin{array}{lll}1.6 \times & 75 & 2\end{array}$ $\begin{array}{lrr}1.7 \times & 107 & 4\end{array}$

$\begin{array}{lll}1.5 \mathrm{X} & 69 & 2\end{array}$ $\begin{array}{lll}1.7 \mathrm{X} & 83 & 3\end{array}$ $\begin{array}{lll}1.6 x & 89 & 5\end{array}$ $\begin{array}{lll}2.0 \mathrm{X} & 80 & 3\end{array}$ $1.3 \times \quad 64 \quad 3$

$\begin{array}{lll}1.0 \times & 76 \quad 2\end{array}$ $\begin{array}{lll}1.7 \times & 86 & 3 \\ 2.2 \times & 63 & 2\end{array}$ $\begin{array}{lll}2.2 \times & 63 & 2\end{array}$ $\begin{array}{lll}1.6 \mathrm{X} & 74 & 2\end{array}$ $\begin{array}{lll}1.4 \mathrm{X} & 95 & 1\end{array}$ $\begin{array}{lll}1.2 \mathrm{X} & 69 & 5 \\ 1.8 \mathrm{x} & 69 & 3\end{array}$ $\begin{array}{lll}1.8 \mathrm{X} & 69 & 3 \\ 1.4 \times & 77 & 2\end{array}$ $\begin{array}{lll}1.4 \times & 77 & 2 \\ 1.4 \times & 85 & 2\end{array}$ $1.5 \times-75 \quad 2$ $\begin{array}{llll}1.4 \times \quad 65 & & \end{array}$ $\begin{array}{lll}1.4 \mathrm{X} & 65 & 5 \\ 1.4 \mathrm{X} & 68 & 7\end{array}$ $\begin{array}{lll}1.4 \times & 68 & 7 \\ 1.1 \times & 92 & 5\end{array}$ $\begin{array}{lll}1.1 \times & 92 & 5 \\ 1.6 \times & 85 & 2\end{array}$

$\begin{array}{lll}1.9 \mathrm{X} & 71 \quad 2\end{array}$ $\begin{array}{lll}.2 \mathrm{X} & 84 & 1 \\ .4 \mathrm{x} & 75 & 2\end{array}$ $\begin{array}{lll}1.4 \times & 75 & 2 \\ 2.6 x & 55 & 2\end{array}$ $\begin{array}{lll}1.6 \times & 55 & 2\end{array}$

$\begin{array}{ll}1.6 \mathrm{X} & 73 \quad 2\end{array}$ $1.4 \times \quad 98 \quad 1$ $1.4 \times \quad 981$ $\begin{array}{lll}4 \times \quad 68 & 2\end{array}$ $1.5 \times \quad 96 \quad 1$
---ORIGIN TIME (HST)-- -LAT N-- --LON W-- DEPTH N RMS ERH ERZ LOC YEAR MON DA HRMN SEC DEG MIN DEG MIN KM RD SEC KM KM REMKS

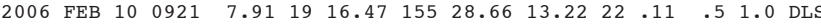

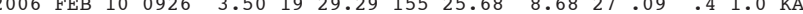

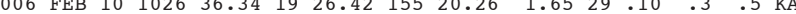

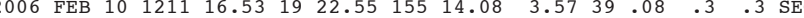
$\begin{array}{lllllllllllllll}2006 & \text { FEB } & 10 & 1322 & 38.02 & 19 & 13.60 & 155 & 25.65 & 35.65 & 27 & .12 & 1.0 & 1.6 & \text { DLS }\end{array}$

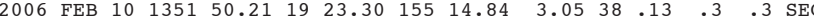

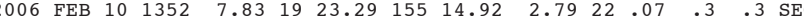

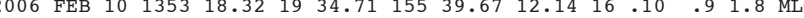
2006 FEB $10 \quad 1355 \quad 5.77 \begin{array}{lllllllll}19 & 22.38 & 155 & 13.43 & 3.70 & 19 & .11 & .4 & \end{array}$

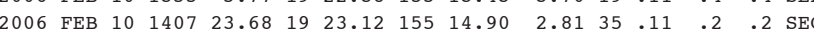

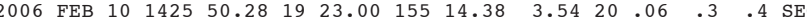

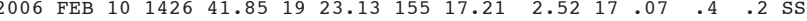
2006 FEB 10154125.951922 .8

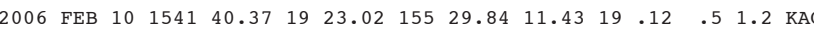

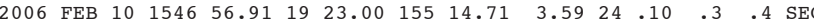

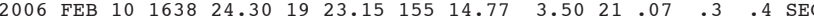

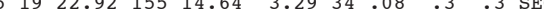

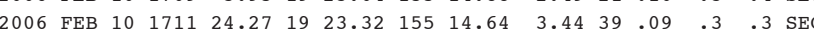

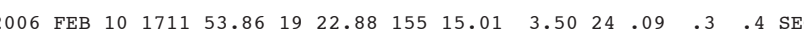

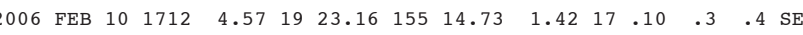

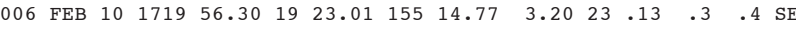

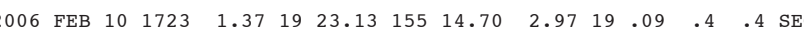

$\begin{array}{lllllllllllllll}2006 & \text { FEB } & 10 & 1836 & 53.72 & 19 & 23.17 & 155 & 14.83 & 3.37 & 20 & .09 & .4 & .4 & \mathrm{SEC}\end{array}$

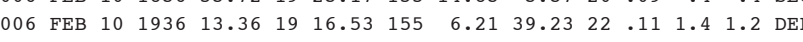
2006 FEB $10 \begin{array}{lllllllllllll}10 & 2106 & 36.12 & 19 & 24.09 & 155 & 15.30 & 3.75 & 19 & .12 & .4 & .5 & \mathrm{SEC}\end{array}$

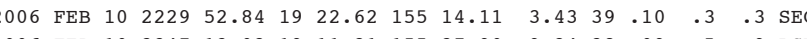

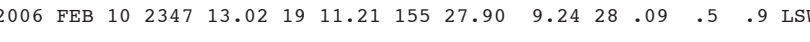

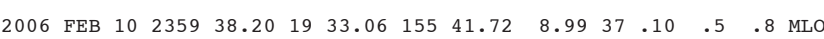
$\begin{array}{lllllllllllllll}2006 & \text { FEB } & 11 & 0051 & 49.27 & 19 & 17.56 & 155 & 19.62 & 11.44 & 39 & .10 & .4 & .7 & \text { SWR }\end{array}$

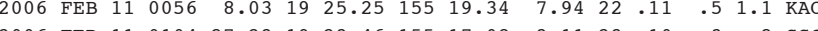
$\begin{array}{llllllll} & \end{array}$

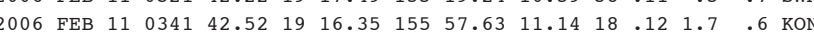

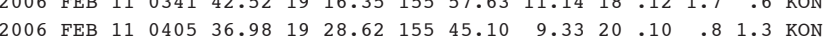

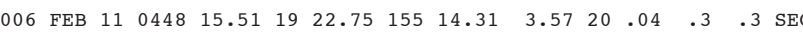

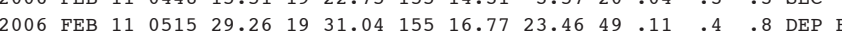

$\begin{array}{lllllllllllllll}2006 & \text { FEB } & 11 & 0538 & 54.88 & 19 & 22.97 & 155 & 14.48 & 3.57 & 31 & .11 & .4 & .4 & \text { SEC }\end{array}$

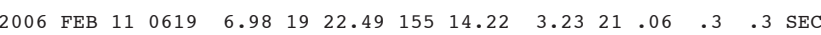

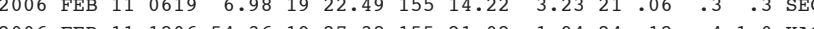
$\begin{array}{lllll}2006 & 0 & 0\end{array}$ $\begin{array}{lllllllllllll}2006 & & \end{array}$
PREF AZ MIN 16

$.5 \times \quad 92 \quad 4$ $1.3 \times 101 \quad 4$ $2.0 \times \quad 50 \quad 2$

$.1 \times \quad 56 \quad 2$ $1.7 \times \quad 68 \quad 2$ $1.1 \times 20512$ $\begin{array}{lll}1.3 \times & 99 & 1 \\ 2.0 \mathrm{X} & 64 & 2\end{array}$

$1.5 \times \quad 86 \quad 2$ $1.2 \mathrm{X} \quad 82 \quad 1$ $\begin{array}{lll}1.5 \mathrm{X} & 90 & 2\end{array}$ $1.7 \times \quad 92 \quad 2$ $\begin{array}{lll}1.5 \times & 79 & 4\end{array}$

$\begin{array}{lll}1.5 \mathrm{X} & 71 & 2\end{array}$ $\begin{array}{lll}1.6 \mathrm{X} & 79 & 2\end{array}$ $\begin{array}{lll}1.8 \times & 49 & 2\end{array}$ $2.1 \times \quad 51 \quad 3$

$\begin{array}{lll}1.6 \mathrm{X} & 67 & 2\end{array}$ $\begin{array}{lll}1.3 \mathrm{X} & 81 & 2 \\ 2.2 \mathrm{X} & 50 & 3\end{array}$ $2.2 \mathrm{X} \quad 50 \quad 3$ $\begin{array}{lll}1.9 \times & 85 & 2\end{array}$ $\begin{array}{lll}1.5 \times & 71 & 2\end{array}$ $\begin{array}{lll}1.5 \times & 300 & 13\end{array}$ $\begin{array}{lll}1.4 \mathrm{X} & 76 & 2 \\ 1.9 \mathrm{X} & 50 & 2\end{array}$ $\begin{array}{lll}1.9 \times & 50 & 2 \\ 1.3 \times & 133 & 3\end{array}$

$1.8 \times \quad 88 \quad 9$ $1.6 \times 130 \quad 2$ $\begin{array}{lll}1.0 \times & 83 & 3 \\ 1.5 \times & 47 & 0\end{array}$ $\begin{array}{lll}1.5 x & 47 & 0\end{array}$

$1.2 \times 163 \quad 1$

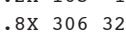
$\begin{array}{lrr}1.0 \times & 193 & 4 \\ 1.6 \times & 84 & 2\end{array}$ $\begin{array}{lll}1.6 \times & 84 & 2\end{array}$

$2.1 \times \quad 50 \quad 3$ $\begin{array}{lll}1.2 \mathrm{x} & 84 & 2 \\ 1.5 \mathrm{x} & 124 & 6\end{array}$ $\begin{array}{lll}1.5 \times 124 & 6 \\ 1.8 \times & 55 & 2\end{array}$ $\begin{array}{lll}1.1 \mathrm{X} & 111 \quad 5\end{array}$ 
--ORIGIN TIME (HST)-- -LAT N-- --LON W-- DEPTH N RMS ERH ERZ LOC

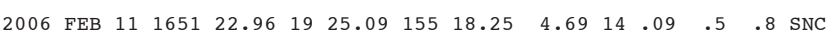
$\begin{array}{llllllllllllll}2006 & \text { FEB } & 11 & 1819 & 55.30 & 19 & 26.96 & 155 & 20.99 & 5.30 & 37 & .12 & .3 & 1.6\end{array}$

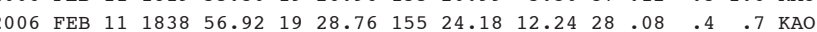
$\begin{array}{lllllllllllllll}2006 & \text { FEB } & 11 & 1843 & 55.94 & 19 & 27.36 & 155 & 20.92 & 4.54 & 23 & .11 & .5 & 3.2 & \text { KAO }\end{array}$

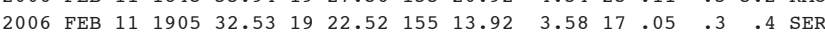

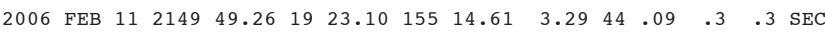
$\begin{array}{lllllllllllllll}2006 & \text { FEB } & 11 & 2230 & 48.86 & 19 & 23.15 & 155 & 14.58 & 3.74 & 38 & .10 & .3 & .4 & \text { SEC }\end{array}$

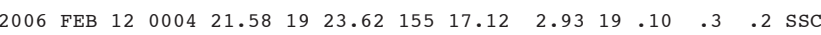

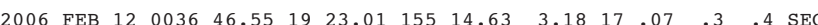

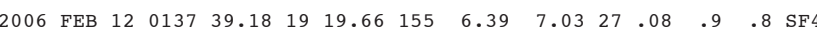

$\begin{array}{llllllllllllll}2006 & \text { FEB } 12 & 0140 & 58.53 & 19 & 18.56 & 155 & 5.82 & 7.00 & 17 & .05 & 1.6 & .9 & \text { SF } 4\end{array}$ $\begin{array}{lllllllllllllll}2006 & \text { FEB } & 12 & 0224 & 16.44 & 19 & 26.81 & 155 & 28.94 & 9.50 & 19 & .08 & .4 & 1.4 & \text { KAO }\end{array}$

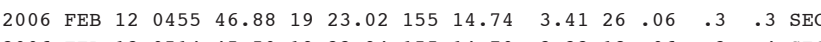
2006 FEB $12 \quad \begin{array}{llllllllllll}0514 & 45.50 & 19 & 22.94 & 155 & 14.70 & 3.32 & 18 & .06 & .3 & .4 & \text { SEC }\end{array}$

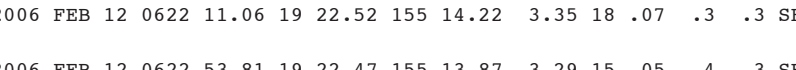

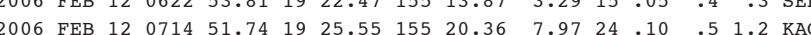

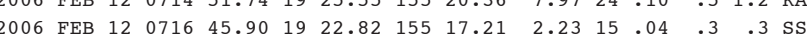

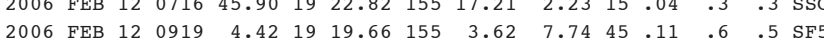

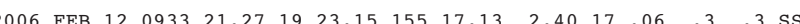

$\begin{array}{lllllllllllllll}2006 & \text { FEB } & 12 & 0941 & 6.79 & 19 & 23.26 & 155 & 14.78 & 3.40 & 28 & .08 & .3 & .3 & \mathrm{SEC}\end{array}$

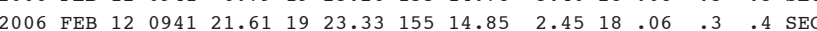
$\begin{array}{llllllllllllll}2006 & \text { FEB } 12 & 0942 & 6.33 & 19 & 23.24 & 155 & 14.92 & 3.22 & 18 & 07 & 3 & .4 & \text { SEC }\end{array}$

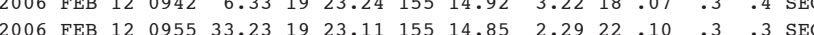

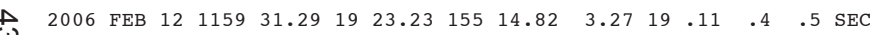

$\begin{array}{llllllllllllll}2006 & \text { FEB } 12 & 1203 & 20.60 & 19 & 23.55 & 155 & 15.27 & 3.08 & 21 & .09 & .3 & .4 & \mathrm{SEC}\end{array}$

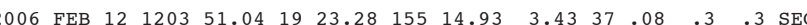

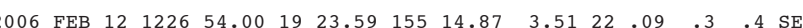

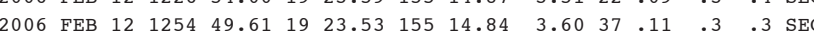
$\begin{array}{lllllllllllllll}2006 & \text { FEB } & 12 & 1507 & 34.00 & 19 & 22.72 & 155 & 14.12 & 3.49 & 19 & .08 & .3 & .4 & \text { SEC }\end{array}$

$\begin{array}{llllllllllllll}2006 & \text { FEB } 12 & 1606 & 21.77 & 19 & 22.56 & 155 & 14.23 & 3.55 & 27 & .09 & .3 & .4 & \mathrm{SEC}\end{array}$

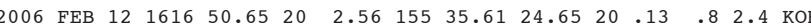

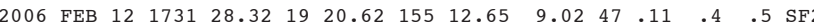

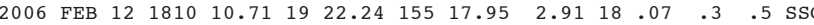

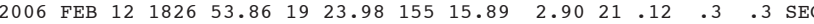

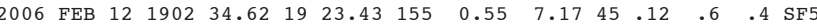

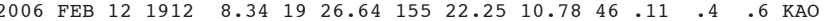

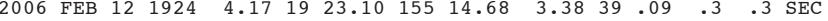

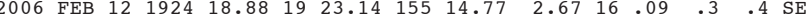

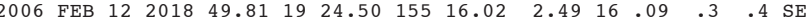

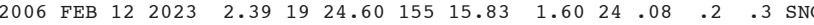

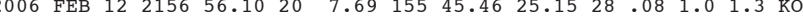

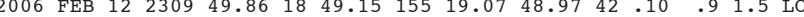

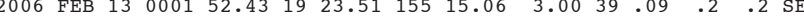

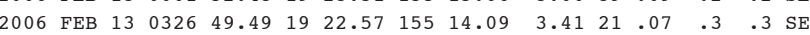

PREF AZ MIN $1.0 \times \quad 74 \quad 1$ $.8 \times \quad 50 \quad 6$ $1.3 \times 1230$ $1.3 \times 123-6$ $\begin{array}{llll}2.4 \mathrm{X} & 61 & 3\end{array}$

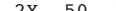
$\begin{array}{lll}2.2 \times & 50 & 3 \\ 1.0 \times & 55 & 1\end{array}$ $1.5 \times \quad 84 \quad 2$ $1.0 \times \quad 224$ $1.0 \times 2619$ $\begin{array}{lll}1.0 \mathrm{X} & 68 & 8 \\ 1.7 \mathrm{X} & 64 & 2\end{array}$ $\begin{array}{lll}1.7 \mathrm{X} & 64 & 2 \\ 1.5 \mathrm{X} & 75 & 2 \\ 1.3 \mathrm{x} & 86 & 2\end{array}$ $\begin{array}{lll}1.3 \mathrm{X} & 86 & 2\end{array}$ $\begin{array}{lll}1.4 \times & 91 & 1\end{array}$ $\begin{array}{lll}1.4 \mathrm{X} & 90 & 4\end{array}$ $\begin{array}{lrr}1.2 \times & 61 & 1 \\ 2.5 \times & 196 & 9\end{array}$ $1.3 \times \quad 66 \quad 1$ $2.1 \times \quad 57 \quad 3$ $\begin{array}{lll}1.8 \mathrm{X} & 70 & 3\end{array}$ $\begin{array}{lll}1.4 \mathrm{X} & 68 & 2\end{array}$ $\begin{array}{lll}1.4 \mathrm{X} & 68 & 2 \\ 1.5 \mathrm{x} & 77 & 2 \\ 1.3 \mathrm{X} & 72 & 2\end{array}$ 1.3X $72 \quad 2$

$\begin{array}{lll}1.4 \mathrm{X} & 55 \quad 2\end{array}$ $\begin{array}{lll}2.1 \mathrm{x} & 57 \quad 2\end{array}$ $\begin{array}{lll}1.4 \mathrm{X} & 67 & 2\end{array}$ $\begin{array}{lll}1.8 \times & 52 \quad 2\end{array}$ $1.3 \times \quad 89 \quad 2$

$\begin{array}{lll}1.7 \times \quad 86 & 2\end{array}$ $1.7 \times 186 \quad 22$ $1.9 \times 122 \quad 4$ $1.1 \times \quad 60 \quad 3$ $\begin{array}{lll}1.5 \times & 75 & 1\end{array}$

$1.7 \times 179 \quad 4$ $\begin{array}{lll}2.8 \times & 48 \quad 6\end{array}$ $2.1 \times \quad 62 \quad 3$ $\begin{array}{lll}1.7 \times & 93 & 2\end{array}$ $\begin{array}{lll}1.1 \mathrm{x} & 93 & 1\end{array}$
--ORTGIN TIME (HST)-- -LAT N-- --LON W-- DEPTH N RMS ERH ERZ LOC YEAR MON DA HRMN SEC DEG MIN DEG MIN KM RD SEC KM KM REMKS $\begin{array}{lllllllllllllll}2006 & \text { FEB } & 13 & 0520 & 7.40 & 19 & 20.32 & 155 & 19.25 & 1.38 & 26 & .09 & .3 & .5 & \text { SWR }\end{array}$

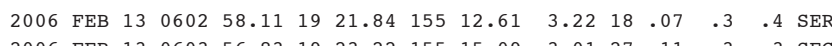

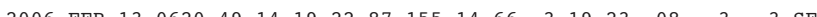
2006 FEB $130620499.141922 .87 \quad 155 \quad 14.66 \quad 3.1923 .08 \quad .3 \quad .3$ SEC

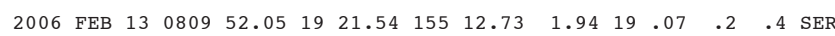
$\begin{array}{llllllllllll}2006 & .4 & .4 & \text { SER }\end{array}$

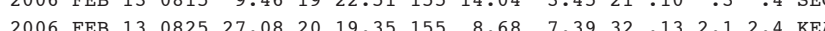

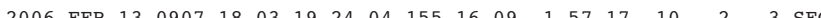

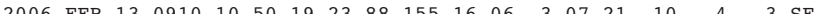

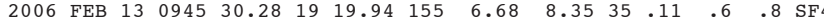
$\begin{array}{llllllllllll}2006 & .8 & \text { SF }\end{array}$

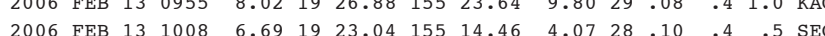
$\begin{array}{llllllllllll}2006 & & \end{array}$

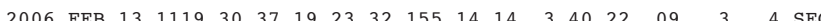

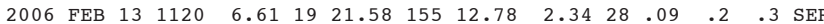

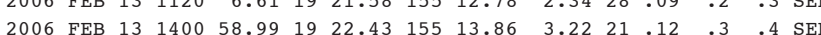

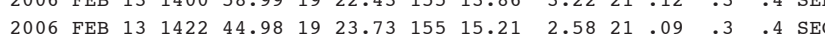
$\begin{array}{llllllllllllll}2006 & \text { FEB } & 13 & 1531 & 0.02 & 19 & 3.31 & 155 & 42.50 & 45.14 & 48 & 09 & 8 & 1.4\end{array}$

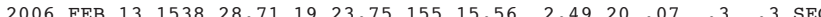

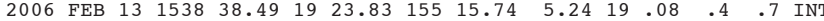

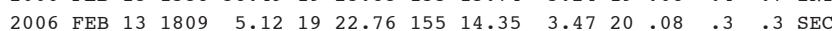

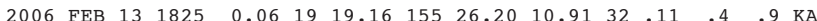

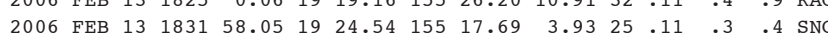

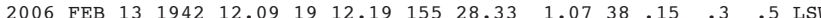

$\begin{array}{llllllllllllllllll}2006 & \text { FEB } & 13 & 2205 & 2.81 & 20 & 7.98 & 155 & 47.27 & 26.43 & 41 & .10 & 1.3 & 1.4 & \text { KOH F } & 2.3 \times & 292 & 1\end{array}$

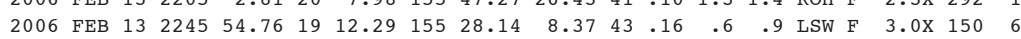

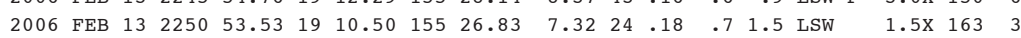

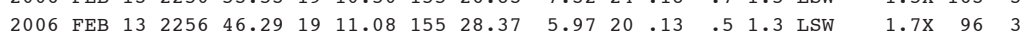

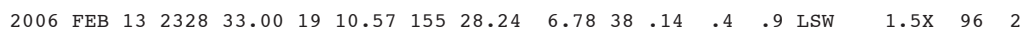

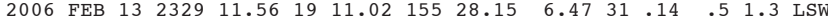
$\begin{array}{llllllllllllllll}2006 & \text { FEB } & 13 & 2329 & 24.72 & 19 & 11.57 & 155 & 28.11 & 1.25 & 24 & .13 & .4 & .5 & \text { LSW }\end{array}$

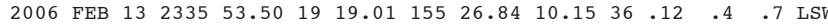

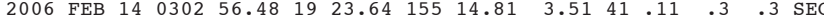
$\begin{array}{llllllllllllllll}2006 & \text { FEB } & 14 & 0317 & 2.81 & 19 & 23.54 & 155 & 14.97 & 3.22 & 29 & .08 & .3 & .3 & \text { SEC }\end{array}$

$\begin{array}{lllllllllllllll}2006 & \text { FEB } & 14 & 0432 & 47.26 & 19 & 23.25 & 155 & 15.01 & 2.94 & 21 & .08 & .3 & .4 & \mathrm{SEC}\end{array}$

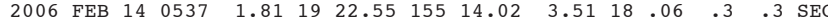

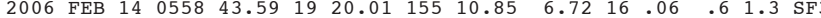

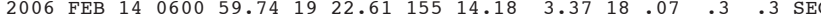

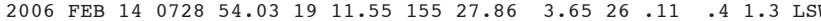

$\begin{array}{lllllllllllllll}2006 & \text { FEB } & 14 & 0756 & 19.98 & 19 & 22.80 & 155 & 14.62 & 2.89 & 21 & .08 & .3 & .4 & \mathrm{SEC}\end{array}$

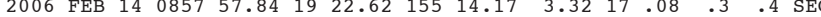

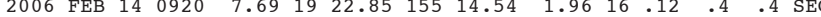

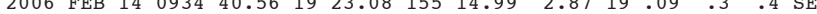

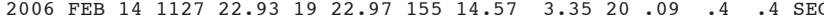

PREF AZ MIN

$1.2 \times \quad 78 \quad 4$ $\begin{array}{lll}1.1 \times & 97 & 2 \\ 1.5 \times & 59 & 2\end{array}$ $1.5 \times \quad 592$ $1.3 \times 209-6$

$1.4 \times 120 \quad 2$ $\begin{array}{rrr}1.6 \times & 89 & 2 \\ 2.1 \times & 310 & 52\end{array}$ $1.2 \times 113 \quad 1$ $6 \times 105$

$1.3 \times 217 \quad 6$ $1.2 \times \quad 76 \quad 5$ $\begin{array}{lll}1.8 \mathrm{X} & 83 & 3 \\ 1.2 \mathrm{x} & 79 & 3\end{array}$ $1.5 \times \quad 90 \quad 2$

$\begin{array}{lll}.8 \times & 80 & 2\end{array}$ $\begin{array}{lll}1.6 \mathrm{X} & 92 & 1 \\ 1.4 \mathrm{X} & 59 & 2\end{array}$ $\begin{array}{llll}2.4 \times & 179 & 9\end{array}$ $1.4 \times 100 \quad 2$

$\begin{array}{lll}1.5 \mathrm{X} & 69 & 2 \\ 1.5 \mathrm{x} & 85 & 2\end{array}$ $\begin{array}{lll}.5 x & 85 & 2 \\ 1.5 x & 123 & 5\end{array}$ $\begin{array}{rrr}1.5 \times & 85 & 2 \\ 1.8 \times & 47 & 1\end{array}$ \begin{tabular}{lll}
$.8 \mathrm{X}$ & 47 & 1 \\
\hline $\mathrm{X}$ & 99 & 5
\end{tabular} $\begin{array}{lll}.7 \times \quad 104 \quad 3 & 3\end{array}$ $\begin{array}{lll}1.5 \times & 105 \quad 4\end{array}$ $\begin{array}{lll}1.7 \mathrm{X} & 88 & 7\end{array}$ $\begin{array}{lll}2.5 X & 47 & 2\end{array}$ $\begin{array}{lll}2.2 \times & 57 \quad 2\end{array}$

$\begin{array}{lll}1.5 \mathrm{X} & 65 \quad 2\end{array}$ $\begin{array}{lll}1.7 X & 89 & 2\end{array}$ $\begin{array}{lll}1.2 \times & 178 \quad 5\end{array}$ $\begin{array}{rrr}1.3 x & 90 & 2 \\ 1.7 x & 113 & 4\end{array}$

$\begin{array}{lll}2.0 \times & 73 \quad 2\end{array}$ $\begin{array}{lll}1.6 \mathrm{X} & 87 & 2 \\ 1.4 \times & 85 & 3\end{array}$

$\begin{array}{lll}1.4 \times & 85 & 3\end{array}$

$\begin{array}{lll}.9 \mathrm{X} & 80 & 3\end{array}$ 
---ORIGIN TIME (HST)-- -LAT N-- --LON W-- DEPTH N RMS ERH ERZ LOC PREF AZ MIN 19 YEAR MON DA HRMN SEC DEG MIN DEG MIN KM RD SEC KM KM REMKS MAG GAP DS

$\begin{array}{lllllllllllllll}2006 & \text { FEB } & 14 & 1343 & 10.34 & 19 & 24.74 & 155 & 38.58 & 3.21 & 14 & .08 & .7 & .5 & \text { MLO }\end{array}$

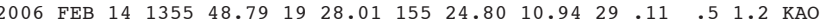

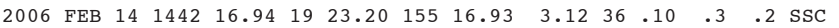

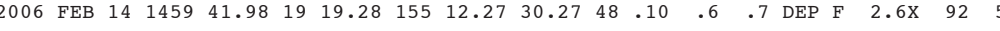

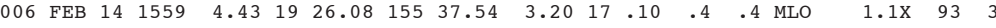

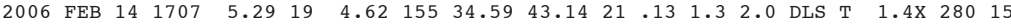

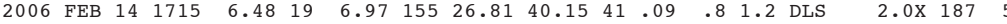

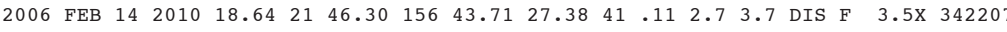

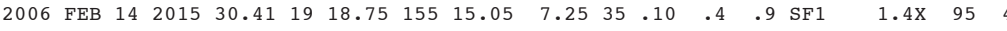

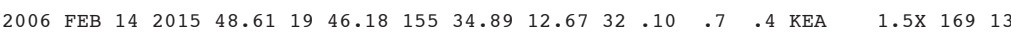

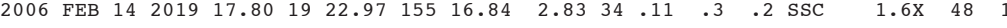

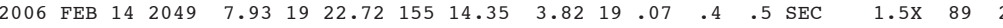

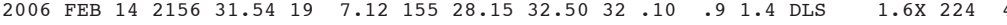

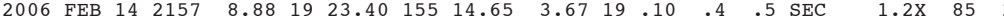

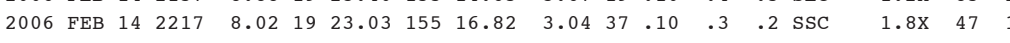

$\begin{array}{lllllllllllllllll}2006 & \text { FEB } 14 & 2255 & 5.33 & 19 & 24.31 & 155 & 25.26 & 11.63 & 42 & .12 & .4 & .7 & \text { KAO } & 2.2 \times & 47 & 5\end{array}$

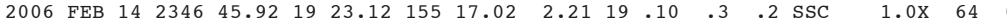

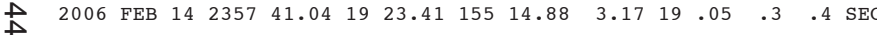

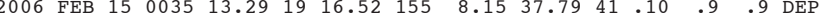
2006 FEB $15 \quad 023744.031937 .34 \quad 156 \quad 1.62 \quad 16.88 \quad 13.112 .515 .3$ KON $-1.1 \times 32430$

$\begin{array}{lllllllllllllll}2006 & \text { FEB } & 15 & 0254 & 13.46 & 19 & 22.54 & 155 & 14.10 & 3.42 & 19 & .07 & .3 & .3 & \mathrm{SEC}\end{array}$ $\begin{array}{lllllllllllllll}2006 & \text { FEB } & 15 & 0310 & 55.65 & 19 & 23.22 & 155 & 17.05 & 2.93 & 20 & .11 & .3 & .3 & \mathrm{SSC}\end{array}$

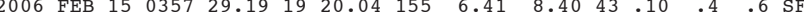
2006 FEB $150432 \quad 35.4119 \quad 1.78 \quad 155 \quad 13.97 \quad 28.76 \quad 22.13 \quad 1.6 \quad 3.0 \quad$ LO

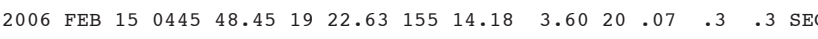

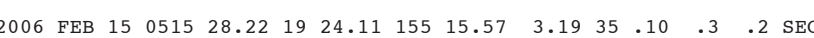

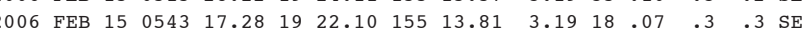
$\begin{array}{lllllllllllllll}2006 & \text { FEB } & 15 & 0656 & 20.26 & 19 & 30.58 & 155 & 26.27 & 3.01 & 22 & .14 & .4 & .7 & \text { MLO }\end{array}$

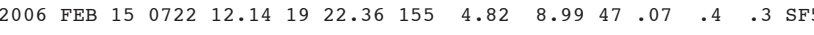

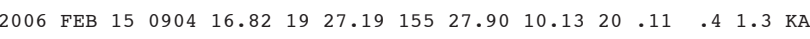

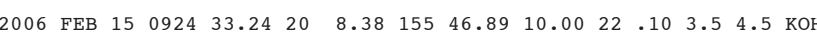

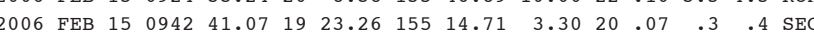

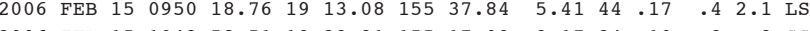

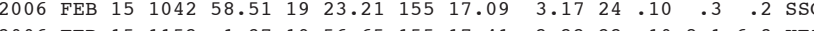

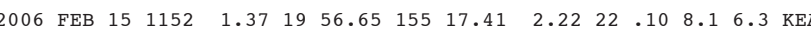

$\begin{array}{llllllllllllll}2006 & \text { FEB } & 15 & 1201 & 28.03 & 19 & 25.54 & 155 & 19.18 & 7.44 & 32 & .11 & .4 & .8 \\ \text { KAO }\end{array}$

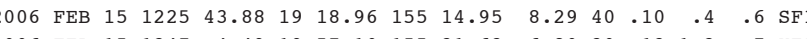

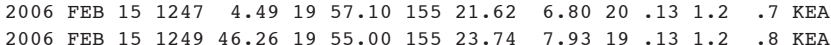

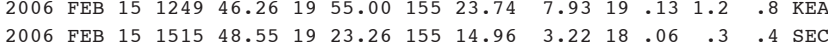

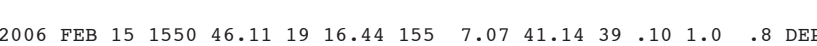

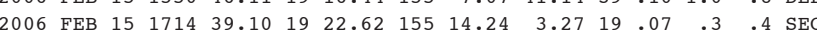

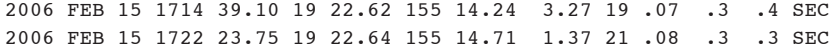

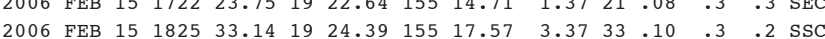
$\begin{array}{llllllllllllll}2006 & & \end{array}$
$1.3 \times \quad 90 \quad 2$ $1.1 \times \quad 48 \quad 0$ $1.5 \times 155 \quad 6$ $\begin{array}{rrr}1.4 \times & 308 & 28 \\ 1.5 \times & 86 & 2\end{array}$

$.8 \times \quad 80 \quad 2$ $1.4 \times \quad 62 \quad 2$ $1.3 \times 130 \quad 4$ $2.0 \times 147$ $.5 \times 29450$ $\begin{array}{lll}1.3 \times & 82 & 3\end{array}$ $2.3 \times 10914$ $1.5 \times \quad 61 \quad 0$ $\begin{array}{lll}1.2 \times & 87 & 3 \\ 1.5 \times & 89 & 4\end{array}$ $1.5 \times \quad 89 \quad 4$ $1.5 \times 31241$ $1.6 \times 28436$

$0.0 \times 22311$ $1.5 \mathrm{X} \quad 85 \quad 2$ $\begin{array}{lll}1.4 \mathrm{X} & 78 & 2 \\ 1.5 \mathrm{X} & 44 & 1\end{array}$ $\begin{array}{lll}1.5 \mathrm{X} & 44 & 1 \\ 1.4 \mathrm{X} & 79 & 2\end{array}$ $\begin{array}{llllllllllll}--- \text { ORIGIN TIME (HST)-- } & \text {-LAT N-- } & - \text { LON } & \text { W-- } & \text { DEPTH } & \text { N RMS } & \text { ERH } & \text { ERZ LOC } & \text { PREF AZ MIN } & 2 \\ \text { YEAR MON DA HRMN } & \text { SEC } & \text { DEG MIN } & \text { DEG } & \text { MIN } & \text { KM } & \text { RD } & \text { SEC } & \text { KM } & \text { KM REMKS } & \text { MAG } & \text { GAP DS }\end{array}$

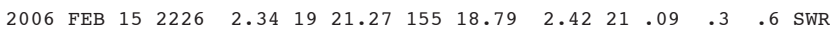

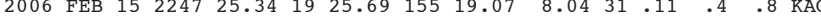

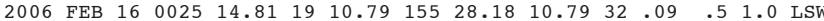
$\begin{array}{lllllllllllllll}2006 & \text { FEB } & 16 & 0059 & 21.98 & 19 & 20.38 & 155 & 6.70 & 8.93 & 40 & .10 & .4 & .4 & \mathrm{SF} 4\end{array}$ $\begin{array}{lllllllllllllll}2006 & \text { FEB } & 16 & 0408 & 29.67 & 19 & 46.18 & 155 & 21.21 & 32.07 & 40 & .12 & .6 & 1.2 & \text { KEA }\end{array}$ $1.5 \times 147 \quad 2$ $1.4 \times 146 \quad 6$ $1.8 \mathrm{X} 9811$

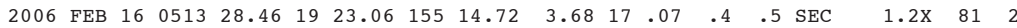

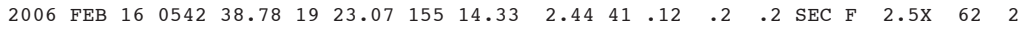

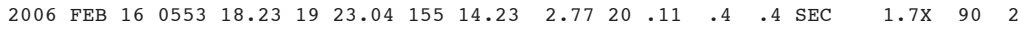

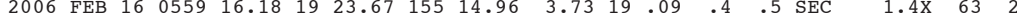

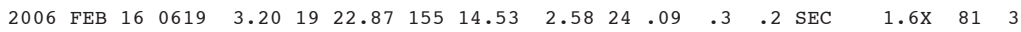

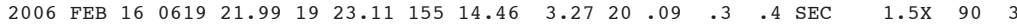

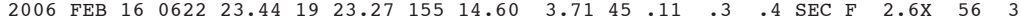

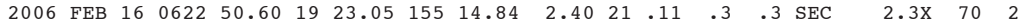

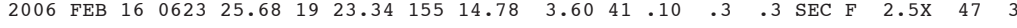

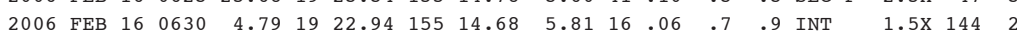

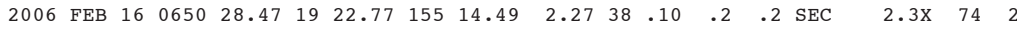
$\begin{array}{llllllllllllllllll}2006 & \text { FEB } & 16 & 0652 & 31.73 & 19 & 22.86 & 155 & 14.61 & 2.17 & 22 & .09 & .3 & .3 & \text { SEC } & 1.8 \mathrm{X} & 78 & 2\end{array}$

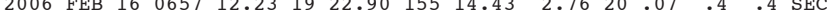

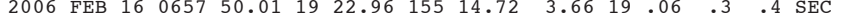

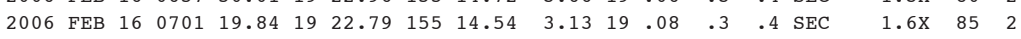

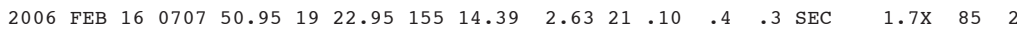

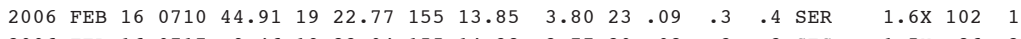

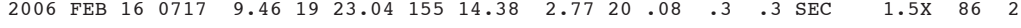

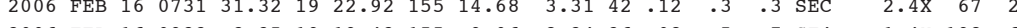

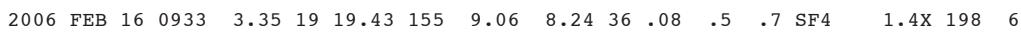

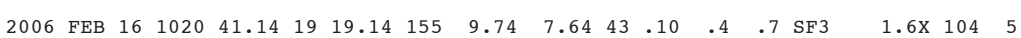

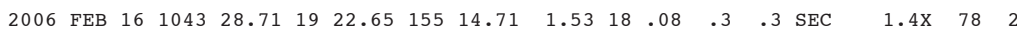

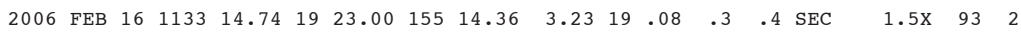

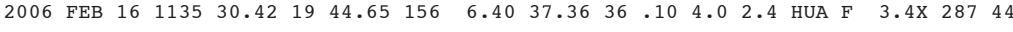

$\begin{array}{lllllllllllllll}2006 & \text { FEB } & 16 & 1236 & 23.68 & 19 & 22.76 & 155 & 14.34 & 3.60 & 19 & .08 & .4 & .4 & \text { SEC }\end{array}$

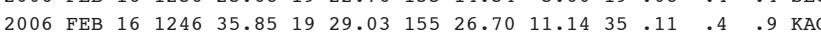

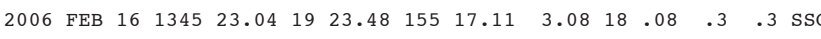

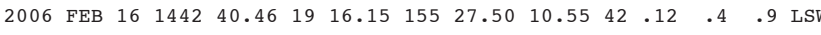
$\begin{array}{lllll}.08 & .3 & .4 \mathrm{SEC}\end{array}$ $\begin{array}{llllllllllllll}2006 & \text { FEB } & 16 & 1458 & 54.33 & 19 & 23.78 & 155 & 15.04 & 3.42 & 29 & .09 & .3 & .3 \\ \text { SEC }\end{array}$

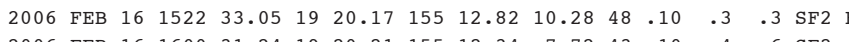

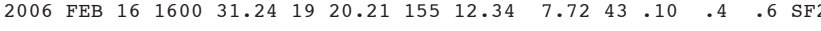

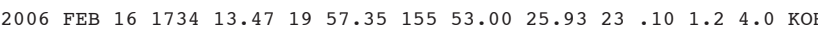

$\begin{array}{lllllllllllllll}2006 & \text { FEB } & 16 & 1814 & 25.70 & 19 & 32.68 & 155 & 53.55 & 19.69 & 18 & .11 & 1.8 & 4.9 & \mathrm{KON}\end{array}$

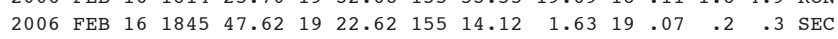

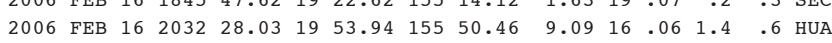

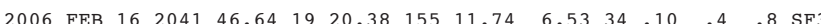

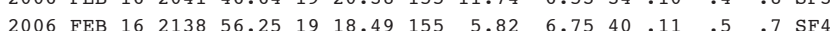

$1.4 \times \quad 89 \quad 2$ $1.5 \times \quad 716$ $1.3 \times \quad 810$ $\begin{array}{lll}1.8 \mathrm{X} & 97 & 5 \\ 1.4 \mathrm{X} & 60 & 2\end{array}$ $1.8 \times \quad 57 \quad 2$ $4.6 \mathrm{U} \quad 715$ $1.7 \times \quad 75 \quad 5$ $1.7 \times 28630$ $1.2 \times 31031$ $1.6 \times \quad 88 \quad 2$ $1.1 \times 29775$ $1.3 \times 226 \quad 4$ 
---ORIGIN TIME (HST)-- -LAT N-- --LON W-- DEPTH N RMS ERH ERZ LOC

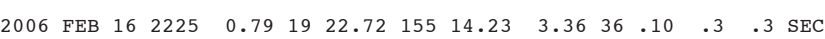

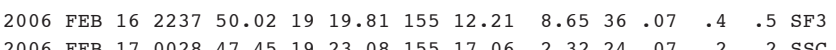

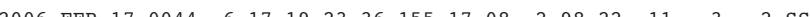

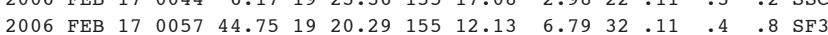

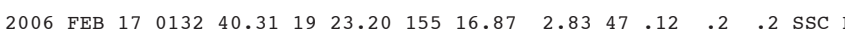

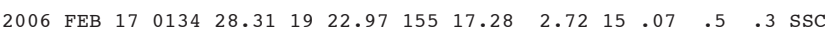

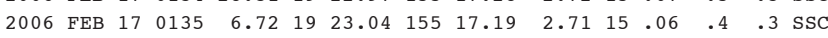

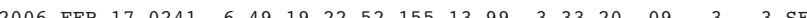

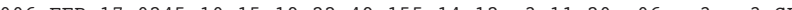

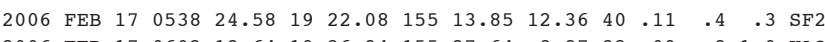

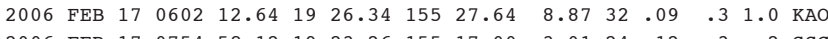

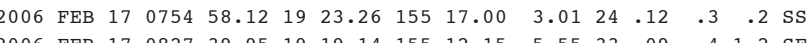

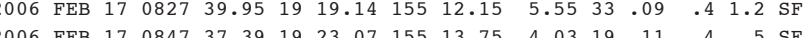

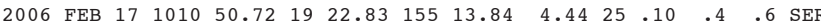

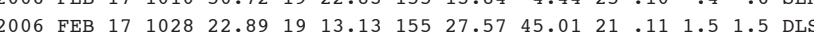

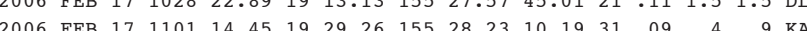

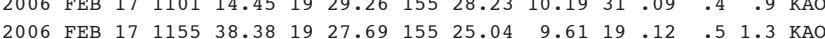

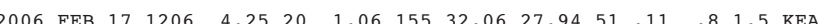

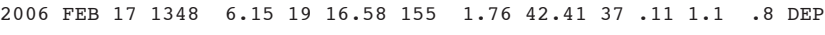

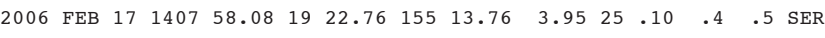

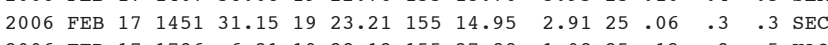

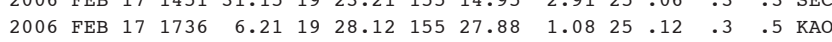

जे

$\begin{array}{lllllllllllllll}2006 & \text { FEB } & 17 & 1821 & 54.90 & 19 & 21.65 & 155 & 13.61 & 4.98 & 19 & .12 & .5 & .8 & \text { SER }\end{array}$

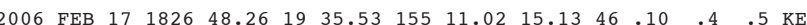

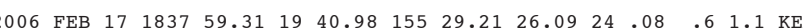

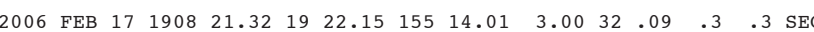

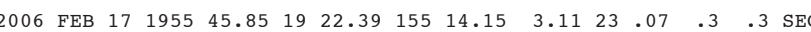

$\begin{array}{lllllllllllllll}2006 & \text { FEB } & 17 & 2039 & 43.39 & 19 & 30.62 & 155 & 54.39 & 12.72 & 25 & .14 & 1.1 & .6 & \text { KON }\end{array}$

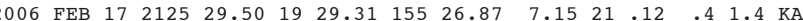

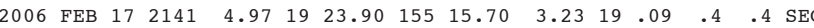

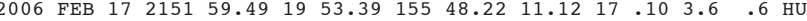

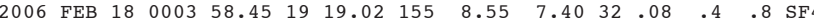

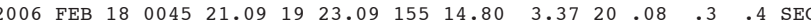

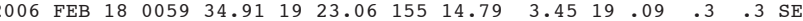

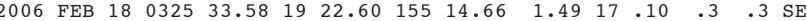

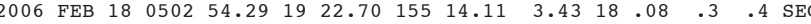

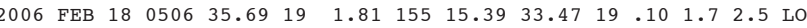

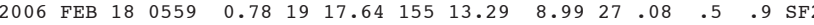

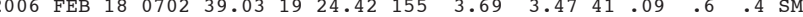

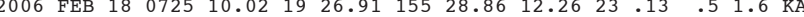

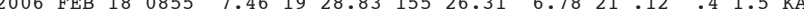

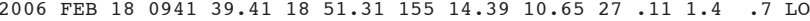

PREF AZ MIN

$1.7 \times \quad 82 \quad 2$ $.2 \times \quad 83 \quad 5$ $1.2 \times 18010$ $1.1 \times \quad 76$

$2.5 \times \quad 46 \quad 0$ $1.3 \times 175$ $1.4 \times \quad 90 \quad 2$ $1.2 \times \quad 88-2$

$1.3 \times \quad 52 \quad 2$ $\begin{array}{lll}1.3 \mathrm{X} & 61 & 7\end{array}$ $\begin{array}{lll}1.2 \mathrm{X} & 61 & 76 \\ 1.2 \mathrm{y} & 0\end{array}$ $\begin{array}{llll}1.2 \times & 166 & 5\end{array}$ $1.3 \times 106$

$\begin{array}{lrr}1.6 \mathrm{X} & 99 & 1 \\ 1.2 \mathrm{X} & 243 & 15\end{array}$ $\begin{array}{lrr}1.2 \times \quad 243 \quad 15 \\ 1.6 \times \quad 79 & 5\end{array}$ $\begin{array}{lll}1.6 x & 79 & 5 \\ 1.2 x & 61 & 5\end{array}$ $\begin{array}{rrr}1.2 x & 61 & 5 \\ 2.5 \times & 187 & 25\end{array}$ $2.1 \times \quad 255 \quad 15$ $1.8 \mathrm{X} \quad 99 \quad 1$ $\begin{array}{lll}1.5 \mathrm{X} & 68 & 2\end{array}$ $\begin{array}{lll}1.0 \times & 73 \quad 7\end{array}$ $2.1 \mathrm{X} \quad 50 \quad 3$

$1.5 \mathrm{x} \quad 56 \quad 2$ $\begin{array}{lll}2.1 \times & 79 & 17\end{array}$ $\begin{array}{lll}1.6 \times & 138 \quad 9\end{array}$ $\begin{array}{lll}1.9 \mathrm{X} & 67 & 2\end{array}$ $\begin{array}{lll}1.7 x & 89 & 2\end{array}$

$1.3 \times 22015$ $\begin{array}{lll}1.5 \mathrm{X} & 96 & 5 \\ 1.4 \mathrm{X} & 72 & 2\end{array}$ $1.3 \times 288 \quad 15$ $1.2 \times 105 \quad 3$

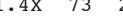
$\begin{array}{lll}.5 \times \quad 78 \quad 2 \\ 1.4 \times & 79 & 2\end{array}$ $\begin{array}{lll}1.4 \times & 79 & 2\end{array}$ $\begin{array}{lll}1.2 \times & 94 & 2\end{array}$ $1.4 \times 30826$

$1.1 \times 103$ $1.7 \times 151 \quad 1$ $1.2 \mathrm{X} \quad 69 \quad 8$ $1.2 \mathrm{X} \quad 90 \quad 6$ $1.8 \times 3094$
---ORTGIN TIME (HST)--- -LAT N-- --LON W-- DFPTH N RMS ERH FRZ LOC YEAR MON DA HRMN SEC DEG MIN DEG MIN KM RD SEC KM KM REM

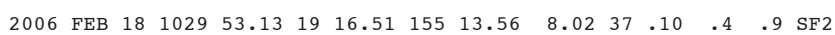

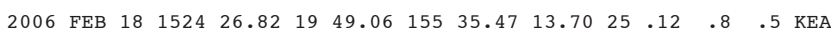

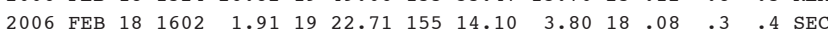

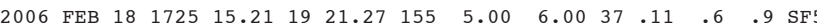

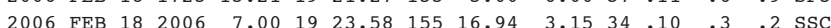

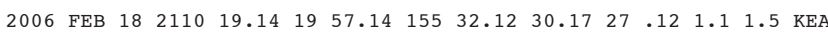

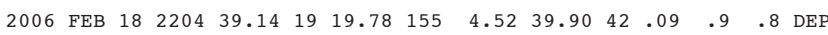

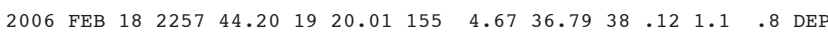
$\begin{array}{lllllllllllllll}2006 & \text { FEB } 18 & 2306 & 38.27 & 19 & 17.79 & 155 & 26.56 & 9.52 & 33 & .14 & .5 & .7 & \text { LSW }\end{array}$

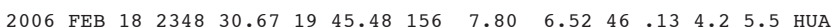

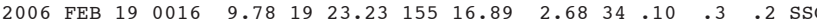

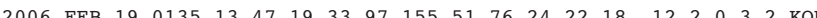

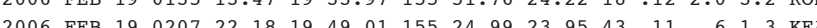
$\begin{array}{llllll}2006 & & \end{array}$

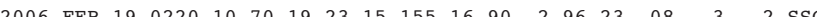

$\begin{array}{llllllllllllll}2006 & \text { FEB } 19 & 0426 & 31.58 & 19 & 22.31 & 155 & 30.38 & 10.87 & 33 & .08 & .4 & .9 & \text { KAO }\end{array}$

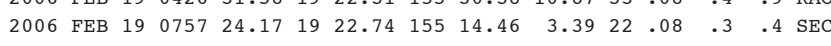

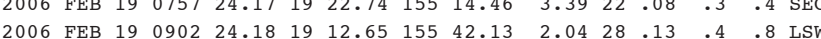

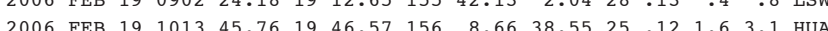

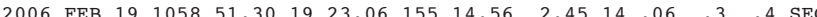

$\begin{array}{lllllllllllllll}2006 & \text { FEB } & 19 & 1258 & 28.04 & 19 & 25.25 & 155 & 18.93 & 6.90 & 24 & .08 & .4 & 8 & \text { INT }\end{array}$

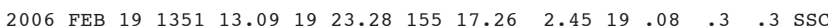

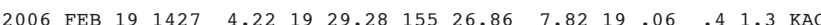

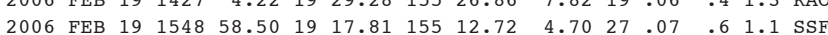

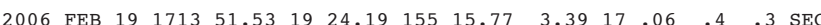

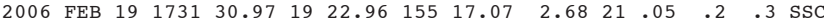

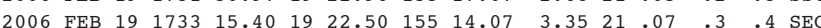

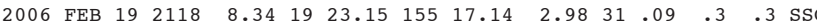

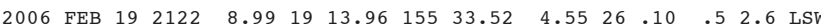

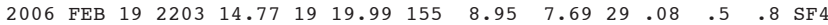

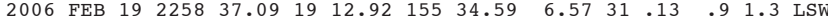

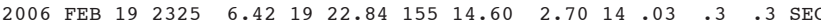

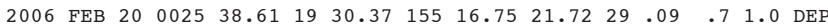

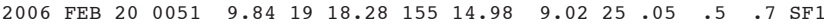

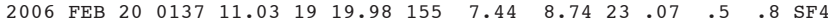

$\begin{array}{llllllllllllll}2006 & \text { FEB } 20 & 0419 & 22.79 & 19 & 23.06 & 155 & 2.50 & 8.19 & 32 & .10 & .6 & .4 & \text { SF5 }\end{array}$

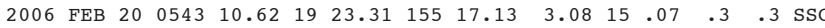

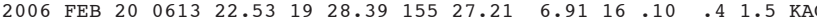
$\begin{array}{llllllllllllll}2006 & \text { FEB } 20 & 0651 & 47.77 & 19 & 19.81 & 155 & 12.42 & 8.45 & 21 & .05 & .5 & 1.0 & \text { SF2 }\end{array}$ $\begin{array}{llllllllllllll}2006 & \text { FEB } 20 & 0800 & 22.96 & 19 & 17.97 & 155 & 15.00 & 7.82 & 21 & .07 & .5 & 1.0 & \text { SF } 1\end{array}$

$\begin{array}{llllllllllllll}2006 & \text { FEB } 20 & 1002 & 22.44 & 19 & 18.36 & 155 & 12.84 & 8.90 & 19 & .05 & .5 & 1.1 & \mathrm{SF} 2\end{array}$

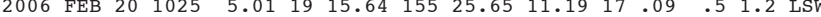

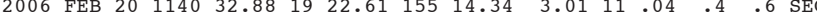

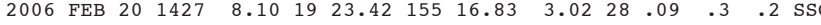

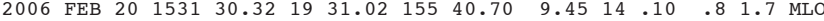

PREF AZ MIN 22 $.5 \times 162$ $1.6 \times 19026$ $\begin{array}{lll}1.2 \times & 95 & 2 \\ 1.3 \times & 159 & 6\end{array}$ $\begin{array}{lllll}.7 \times & 42 & 0\end{array}$

$1.7 \times 27621$ $\begin{array}{lll}1.7 \times & 189 & 8 \\ 1.8 \times & 180 & 8\end{array}$ $\begin{array}{rrr}1.2 \times \quad 96 & 7\end{array}$ $4 \times \quad 28963$ $1.7 \times \quad 46 \quad 0$ $1.3 \times 30529$

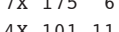
$.4 \times \quad 47 \quad 0$

$\begin{array}{lll}1.4 \times & 87 & 5\end{array}$ $\begin{array}{rrr}1.5 \times & 81 & 2 \\ 1.4 \times & 124 & 9\end{array}$ $2.0 \times 30050$ $2 \times \quad 80 \quad 3$ $\begin{array}{lll}1.2 x & 81 & 2 \\ 1.4 x & 48 & 1\end{array}$ $\begin{array}{rrr}.4 \mathrm{X} & 48 & 1 \\ .2 \mathrm{x} & 96 & 5 \\ 1.1 \mathrm{x} & & \end{array}$ $\begin{array}{lll}1.1 \times & 161 & 2\end{array}$ $1.1 \times 121 \quad 2$

$\begin{array}{lll}1.4 \mathrm{X} & 48 & 1 \\ 1.3 \mathrm{X} & 89 & 2\end{array}$ $\begin{array}{lll}.4 X & 89 & 2 \\ .7 X & 46 & 1\end{array}$ $1.6 \times 123 \quad 6$ $1.3 \times 140 \quad 5$

$2.0 \times \quad 228 \quad 9$ $\begin{array}{rrr}1.3 \times & 83 & 2 \\ 1.3 \times & 106 & 5\end{array}$ $1.2 \times 134 \quad 4$ $1.1 \times 155 \quad 5$

$\begin{array}{lll}1.5 \times & 163 & 4\end{array}$ $\begin{array}{lll}1.2 \times & 68 & 0\end{array}$ $\begin{array}{lll}1.0 \times & 75 \quad 7\end{array}$ $.9 \times 136 \quad 3$

$\begin{array}{lll}1.2 \times 103 & 3\end{array}$ $.9 \times 116 \quad 8$ $1.1 \times 133 \quad 2$ $\begin{array}{lll}1.9 \times & 39 & 0\end{array}$ $1.4 \mathrm{U} 1599$ 
---ORIGIN TIME (HST)-- -LAT N-- --LON W-- DEPTH N RMS ERH ERZ LOC PREF AZ MIN 23 YEAR MON DA HRMN SEC DEG MIN DEG MIN KM RD SEC KM KM REMKS MAG GAP DS

$\begin{array}{lllllllllllllll}2006 & \text { FEB } & 20 & 1544 & 17.88 & 19 & 20.87 & 155 & 29.85 & 10.91 & 33 & .12 & .4 & .9 & \text { KAO }\end{array}$ $\begin{array}{lllllllllllll}2006 & \text { FEB } 20 & 1544 & 50.43 & 19 & 20.69 & 155 & 30.01 & 11.41 & 29 & .10 & .4 & .9 \\ \text { KAO }\end{array}$

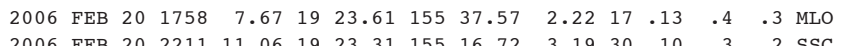

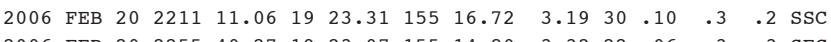

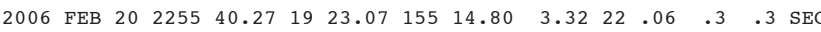

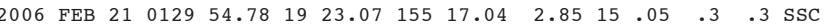

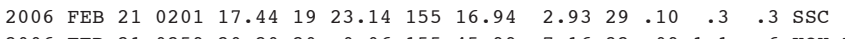

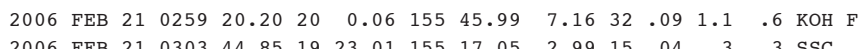

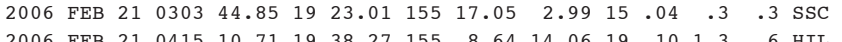

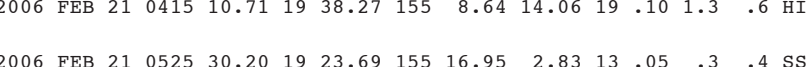

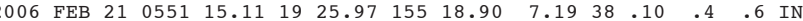

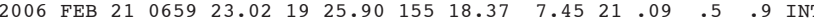

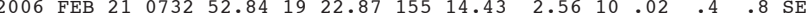

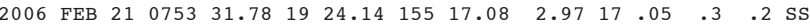

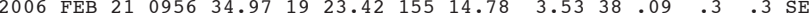

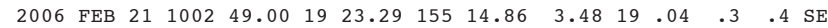

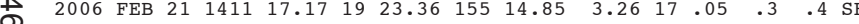

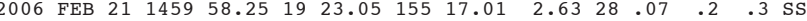

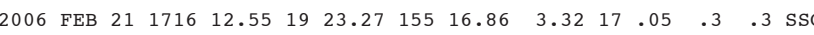

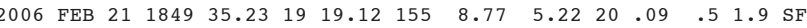

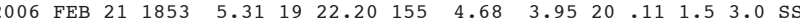

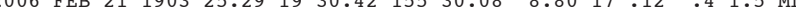

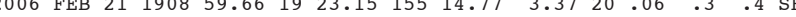

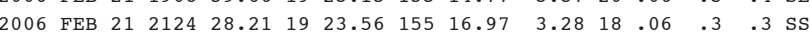

$\begin{array}{llllllllllllll}2006 & \text { FEB } 21 & 2340 & 3.31 & 19 & 22.16 & 155 & 17.20 & 2.86 & 12 & .06 & .4 & .6 & \text { SSC }\end{array}$

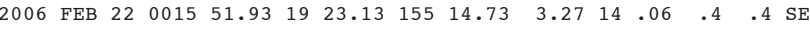

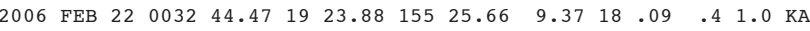

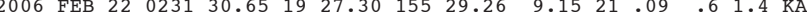

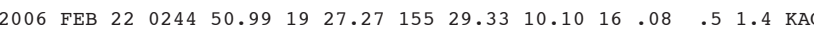

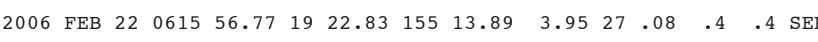

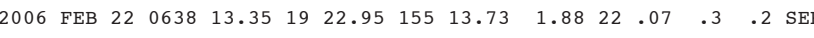

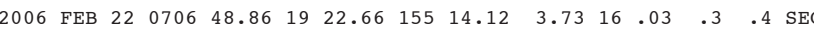
$\begin{array}{lllllllllllllll}2006 & \text { FEB } 22 & 0710 & 37.54 & 19 & 22.87 & 155 & 14.30 & 3.47 & 18 & .07 & .3 & .4 & \text { SEC }\end{array}$

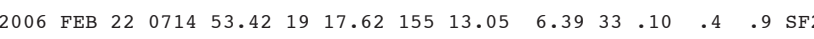

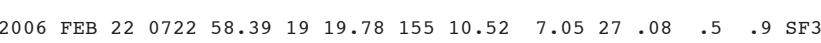

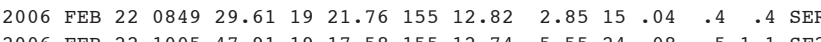
$\begin{array}{llllllllllllll}2006 & \text { FEB } 22 & 1005 & 47.91 & 19 & 17.58 & 155 & 12.74 & 5.55 & 24 & .08 & .5 & 1.1 & \text { SF } 2\end{array}$

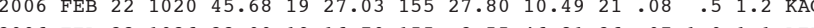

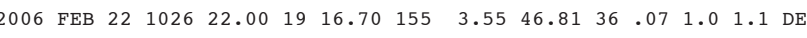

$\begin{array}{lllllllllllllll}2006 & \text { FEB } & 22 & 1145 & 57.19 & 19 & 17.35 & 155 & 12.73 & 5.70 & 15 & .04 & .6 & 1.2 & \mathrm{SF} 2\end{array}$ $\begin{array}{llllllllllllllll}2006 & \text { FEB } & 22 & 1641 & 2.84 & 19 & 25.46 & 155 & 18.46 & 5.69 & 14 & .07 & .4 & .8 & \text { INT }\end{array}$

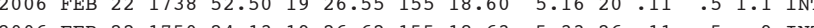

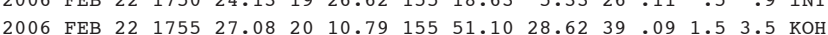

$1.3 \times \quad 91 \quad 5$ $.6 \times 1035$

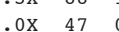
$1.8 \times \quad 72 \quad 2$

$.2 \times \quad 76 \quad 1$ $1.9 \times \quad 47 \quad 0$ $2.2 \times 294 \quad 40$ $\begin{array}{rrr}1.2 \mathrm{X} & 62 & 1 \\ 1.4 \mathrm{X} & 225 & 24\end{array}$

$1.0 \times \quad 70 \quad 1$ $.9 \times \quad 70 \quad 3$ $1.1 \mathrm{x} \quad 84 \quad 2$ $1.0 \times 151$ $1.2 \times \quad 73$

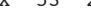
$\begin{array}{lll}.5 \mathrm{X} & 69 \quad 2\end{array}$ $1.3 \times \quad 77 \quad 3$ $.9 \times$
.97

$.4 \times 116$ $.0 \times 199 \quad 4$ $1.4 \times 1095$ $1.6 \mathrm{X} \quad 79 \quad 2$ $1.0 \times \quad 450$

$1.3 \times 115$ $\begin{array}{lll}1.3 \times & 81 \quad 2\end{array}$ $1.4 \mathrm{X} \quad 58$ $\begin{array}{llll}1.5 \times & 109 & 9\end{array}$ $.1 \times \quad 90 \quad 9$ $2.1 \mathrm{X} \quad 71 \quad 1$ $1.9 \mathrm{X} \quad 99 \quad 1$ $1.5 \times \quad 93$ $1.2 \mathrm{X} \quad 86 \quad 2$ $\begin{array}{lll}1.5 \times & 92 \quad 6\end{array}$ $1.3 \times 113 \quad 2$ $1.3 \times 1712$ $\begin{array}{lll}1.4 \times & 66 & 8 \\ 2.2 \times & 208 & 8\end{array}$ $.8 \times \quad 88 \quad 2$ $1.3 \times 101$ $\begin{array}{llll}1.5 \times 102 & 3\end{array}$
---ORIGIN TIME (HST)-- -LAT N-- --LON W-- DEPTH N RMS ERH ERZ LOC YEAR MON DA HRMN SEC DEG MIN DEG MIN KM RD SEC KM KM REMKS

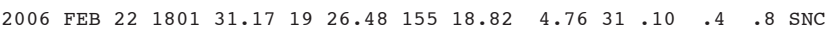

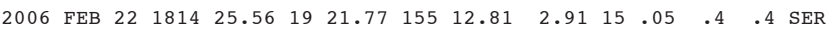

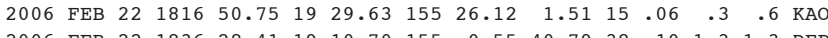

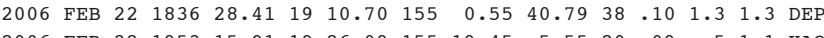

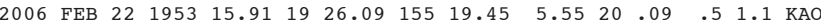

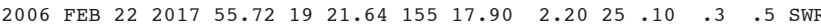

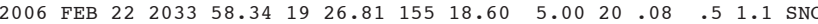

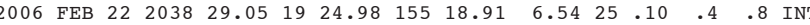

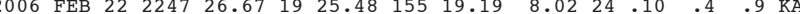

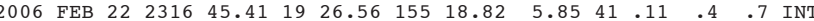

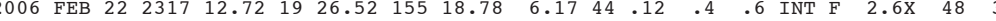

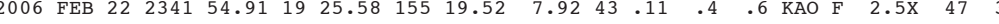

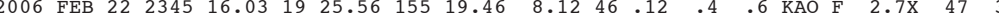

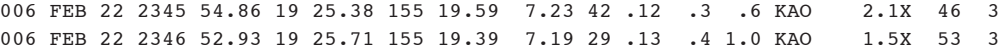

$\begin{array}{llllllllllllll}2006 & \text { FEB } & 22 & 2347 & 0.43 & 19 & 25.25 & 155 & 19.37 & 6.60 & 21 & .11 & .4 & 1.0 \\ \text { KAO }\end{array}$

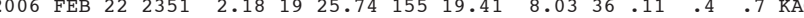

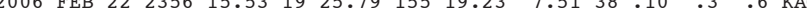

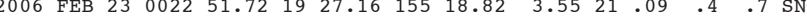
$\begin{array}{lllllllllllllll}2006 & \text { FEB } & 23 & 0032 & 43.30 & 19 & 25.36 & 155 & 19.37 & 7.70 & 22 & .10 & .5 & 1.0 & \mathrm{KAO}\end{array}$

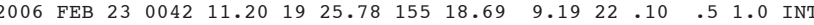

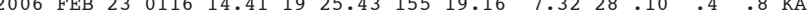
$\begin{array}{llllllllll} & & \end{array}$ 2006 FEB 230322

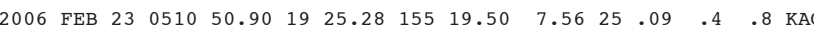

$\begin{array}{lllllllllllllll}2006 & \text { FEB } & 23 & 0553 & 50.43 & 19 & 22.81 & 155 & 14.17 & 3.63 & 25 & .11 & .3 & .4 & \text { SEC }\end{array}$ $\begin{array}{llllllllllllll}2006 & \text { FEB } & 23 & 0658 & 55.57 & 19 & 25.28 & 155 & 22.26 & 0.38 & 22 & .07 & .3 & .3 \\ \text { KAO }\end{array}$

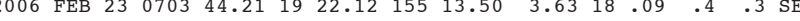
$\begin{array}{lllllllll} & .3 & \text { SER }\end{array}$

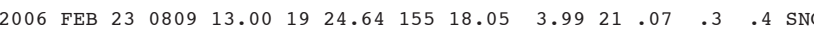

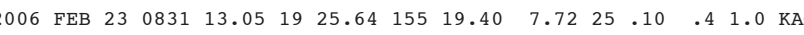

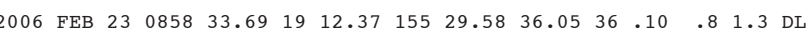

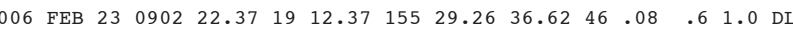

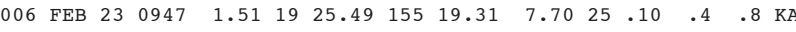

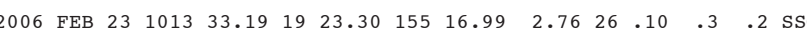

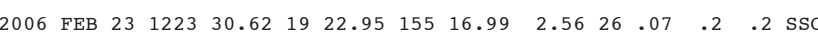

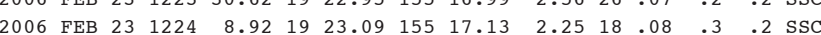
$\begin{array}{lllllllllllllll}2006 & \text { FEB } & 23 & 1224 & 8.92 & 19 & 23.09 & 155 & 17.13 & 2.25 & 18 & .08 & .3 & .2 & \text { SSC } \\ 2006 & \text { FEB } & 23 & 1236 & 48.15 & 19 & 22.99 & 155 & 14.60 & 2.33 & 17 & .04 & .3 & .4 & \text { SEC }\end{array}$ $\begin{array}{lllllllllllllll}2006 & \text { FEB } & 23 & 1236 & 48.15 & 19 & 22.99 & 155 & 14.60 & 2.33 & 17 & .04 & .3 & .4 & \text { SEC } \\ 2006 & \text { FEB } & 23 & 1249 & 0.72 & 19 & 22.64 & 155 & 14.60 & 2.32 & 23 & .10 & .3 & .3 & \text { SEC }\end{array}$

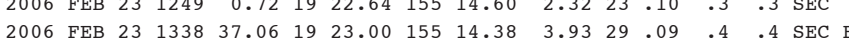

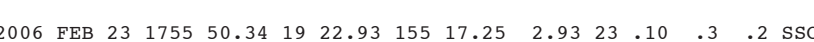

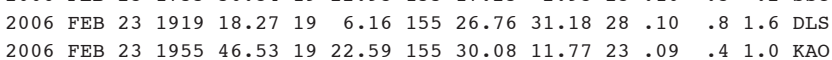

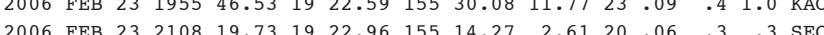

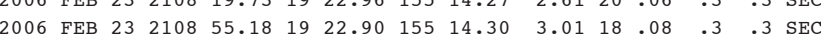

$\begin{array}{lll}.7 \times & 49 & 3 \\ .6 \mathrm{x} & 85 & 2 \\ 1.2 \times & 109 & 5\end{array}$ $1.2 \times 109 \quad 5$ $.2 \times 147 \quad 3$

$.6 \times \quad 54 \quad 4$ $.2 \times 108$ $77 \quad 2$

$1.7 \times \quad 84 \quad 3$ $1.5 \times \quad 92 \quad 3$ $1.8 \times \quad 91 \quad 3$ $1.0 \times 118 \quad 4$ $1.1 \times \quad 86 \quad 3$

$1.1 \times \quad 86 \quad 2$ $1.2 \times \quad 85 \quad 3$ $\begin{array}{lll}1.8 \mathrm{X} & 47 & 3\end{array}$ $\begin{array}{rrrr}1.6 \times & 246 & 14 \\ 1.1 \times & 84 & 3\end{array}$

$\begin{array}{lll}1.5 \mathrm{X} & 84 & 2\end{array}$ $1.1 \mathrm{x} \quad 76 \quad 5$ $\begin{array}{lll}1.3 \times & 101 & 1\end{array}$ $\begin{array}{lll}1.3 \mathrm{X} & 51 & 1 \\ 1.3 \times & 63 & \end{array}$ $1.3 \times \quad 63 \quad 2$

$\begin{array}{lll}1.0 \times \quad 90 \quad 3 \\ 1.9 \times & 09 & 5\end{array}$ $1.9 \times 109 \quad 5$ $2.3 \times \quad 83 \quad 5$ $1.2 \times 133 \quad 3$ $\begin{array}{lll}1.6 \mathrm{X} & 48 & 1\end{array}$ $\begin{array}{lll}1.5 \mathrm{X} & 66 & 1 \\ 1.2 \mathrm{x} & 85 & 3\end{array}$ $\begin{array}{lll}1.2 \times & 85 & 3\end{array}$ $\begin{array}{lll}.6 \mathrm{X} & 79 & 2 \\ .9 \mathrm{X} & 81 & 2\end{array}$

$\begin{array}{lll}1.3 \times & 50 & 1\end{array}$ $\begin{array}{rrr}1.3 \times & 192 & 6 \\ 1.3 \times & 82 & 4\end{array}$ $\begin{array}{lll}1.3 \times & 82 & 4 \\ 1.4 \mathrm{x} & 87 & 2\end{array}$ $\begin{array}{lll}1.4 \times & 87 & 2 \\ 1.2 \times & 92 & 2\end{array}$ 
--ORIGIN TIME (HST)-- -LAT N-- --LON W-- DEPTH N RMS ERH ERZ LOC YEAR MON DA HRMN SEC DEG MIN DEG MIN

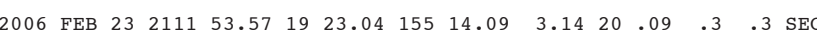

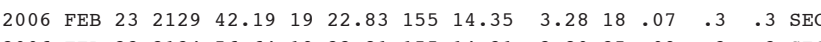
$\begin{array}{lllllllll}2006 & .3 & \text { SEC }\end{array}$

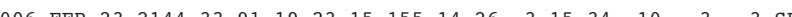

$\begin{array}{lllllllllllllll}2006 & \text { FEB } & 23 & 2153 & 30.68 & 19 & 23.07 & 155 & 16.99 & 2.72 & 19 & .05 & .3 & .2 & \text { SSC }\end{array}$

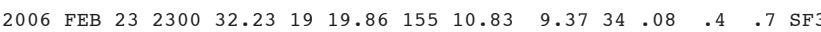

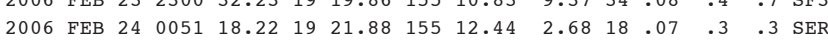

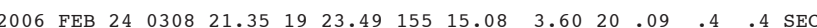

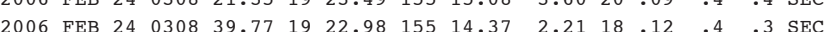

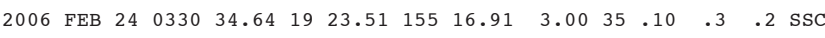

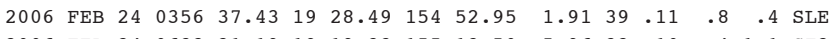

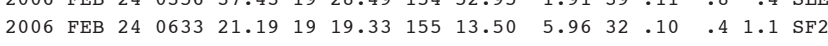

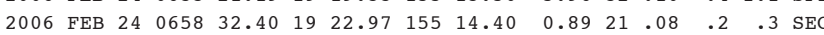
$\begin{array}{llllllllllllll}2006 & \text { FEB } & 24 & 0818 & 20.28 & 19 & 25.28 & 155 & 19.08 & 5.87 & 33 & .12 & .4 & .8\end{array}$

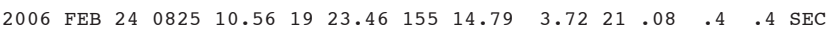

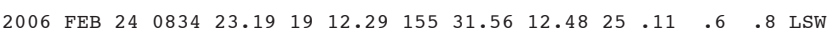

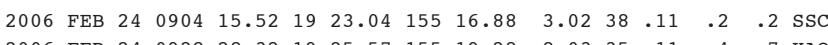

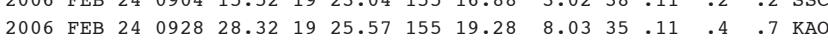

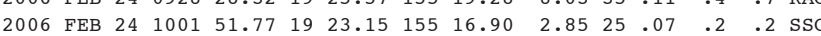

$\begin{array}{llllllllllllll}2006 & \text { FEB } 24 & 1038 & 52.10 & 18 & 47.64 & 155 & 15.15 & 44.46 & 40 & .09 & 1.1 & 2.2 & \text { LOI }\end{array}$

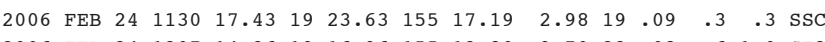

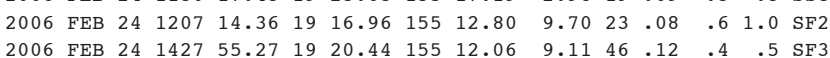

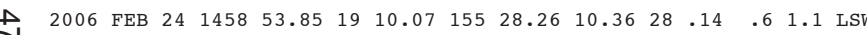

$\begin{array}{llllllllllllll}2006 & \text { FEB } 24 & 1543 & 25.10 & 19 & 18.58 & 155 & 15.32 & 8.28 & 35 & .10 & .5 & .8 & \text { SF } 1\end{array}$

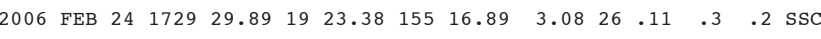

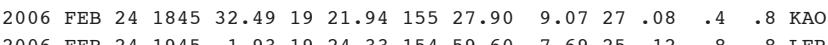

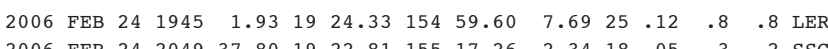
$\begin{array}{lllllllllllllll}2006 & \text { FEB } & 24 & 2049 & 37.80 & 19 & 22.81 & 155 & 17.26 & 2.34 & 18 & .05 & .3 & .2 & \text { SSC }\end{array}$

$\begin{array}{llllllllllllll}2006 & \text { FEB } & 24 & 2135 & 50.17 & 19 & 26.43 & 155 & 29.98 & 10.83 & 27 & .09 & .5 & 1.1 \\ \text { KAO }\end{array}$ $\begin{array}{lllllllllllll}2006 & \text { FEB } 25 & 0019 & 31.66 & 19 & 24.85 & 155 & 19.12 & 4.96 & 24 & .12 & .4 & .8 \\ \text { KAO }\end{array}$

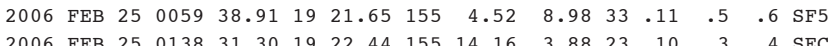

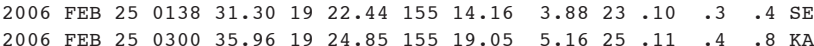

$\begin{array}{lllllllllllllll}2006 & \text { FEB } 25 & 0304 & 32.90 & 19 & 22.65 & 155 & 29.82 & 10.99 & 40 & .11 & .4 & .8 & \text { KAO }\end{array}$

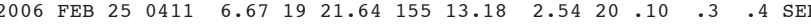

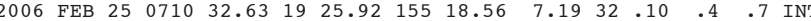

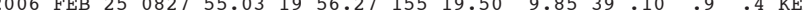

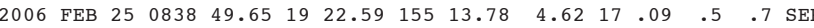

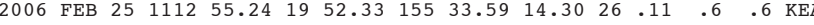

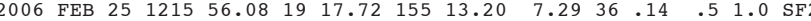

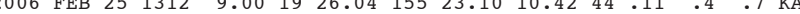

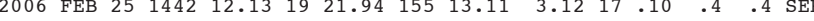

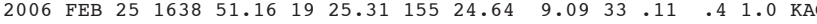

PREF AZ MIN 25 MAG GAP DS $\begin{array}{lll}1.5 \mathrm{X} & 94 \quad 2\end{array}$ $\begin{array}{lll}.4 \times & 85 & 2\end{array}$ $2.1 \times \quad 49$ $2.2 \times \quad 50 \quad 2$

\begin{tabular}{lll}
$1.3 \mathrm{X}$ & $62 \quad 1$ \\
\hline & $1.4 \times$ & 0
\end{tabular} $\begin{array}{lll}1.4 \times & 90 & 5 \\ 1.4 \times & 99 & 2\end{array}$ $1.4 \times \quad 99 \quad 2$ $\begin{array}{lll}1.2 \mathrm{X} & 86 \quad 2\end{array}$

$\begin{array}{lll}1.7 \times & 40 & 0\end{array}$ $1.6 \times 263 \quad 4$ $1.1 \times \quad 125 \quad 4$ $\begin{array}{lll}1.6 \times \quad 84 \quad 2 & 1.64\end{array}$ $\begin{array}{lll}1.6 \mathrm{x} & 84 & 2 \\ 1.5 \mathrm{x} & 82 & 3\end{array}$

$1.5 \times \quad 72 \quad 2$ $\begin{array}{llll}1.5 \times & 191 & 0\end{array}$ $2.2 \mathrm{x} \quad 39$ $1.4 \mathrm{X} \quad 50 \quad 3$ $\begin{array}{lll}1.7 \mathrm{X} & 37 \quad 0\end{array}$

2.2x 28146 $\begin{array}{lrr}1.2 \times & 76 & 1 \\ 1.3 \times & 253 & 1\end{array}$ $\begin{array}{rrr}1.3 \times & 253 & 1 \\ 2.3 \times & 74 & 4 \\ 1.4 x & \end{array}$ $\begin{array}{llll}2.3 \times & 74 & 4 \\ 1.4 \times & 169 & 1\end{array}$

1.3X $128 \quad 4$ $1.5 \mathrm{X} \quad 46 \quad 0$ $1.2 \times \quad 84 \quad 1$ $1.2 \times 189 \quad 2$ $1.0 \times \quad 51 \quad 1$
---ORIGIN TIME (HST)-- -LAT N-- --LON W-- DEPTH N RMS ERH ERZ LOC YEAR MON DA HRMN SEC DEG MIN DEG MIN KM RD SEC KM KM REM $\begin{array}{lllllllllllllll}2006 & \text { FEB } & 25 & 1657 & 10.50 & 19 & 20.45 & 155 & 11.63 & 8.39 & 43 & .09 & .4 & .6 & \text { SF3 }\end{array}$ $\begin{array}{lllllllllllllll}2006 & \text { FEB } & 25 & 1754 & 20.30 & 19 & 23.47 & 155 & 16.88 & 3.15 & 25 & .09 & .3 & .2 & \mathrm{SSC}\end{array}$

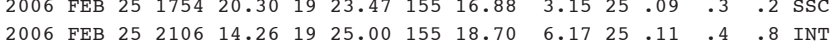

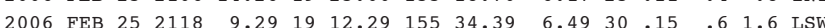

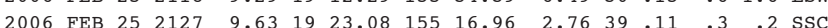

$\begin{array}{lllllllllllllll}2006 & \text { FEB } & 26 & 0307 & 43.32 & 19 & 19.35 & 155 & 8.97 & 7.05 & 30 & .09 & .4 & .9 & \text { SF4 }\end{array}$ $\begin{array}{lllllllllllllll}2006 & \text { FEB } & 26 & 0435 & 51.20 & 19 & 25.92 & 155 & 15.63 & 1.19 & 37 & .10 & .2 & .3 & \text { SNC }\end{array}$

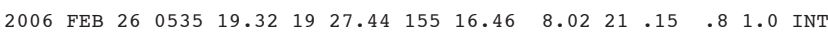
$\begin{array}{llllllllllllllll}2006 & \text { FFB } & 26 & 0542 & 31.66 & 19 & 22.99 & 155 & 30.62 & 10.94 & 19 & .06 & 4 & 1.0 & \text { KAO }\end{array}$

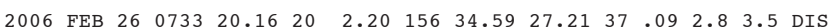

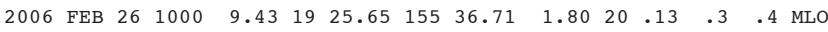

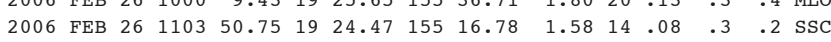

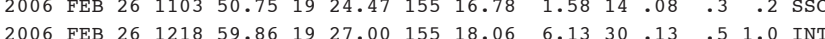

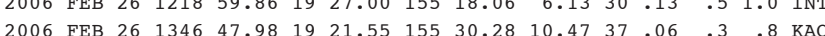
$\begin{array}{lllllllllllll}2006 & \text { FEB } & 26 & 1347 & 1.64 & 19 & 21.95 & 155 & 14.00 & 2.57 & 19 & 09 & 4\end{array}$

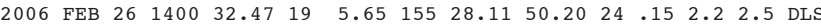

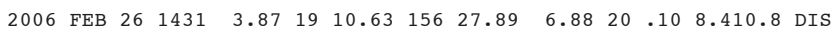

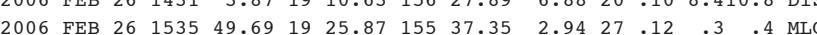

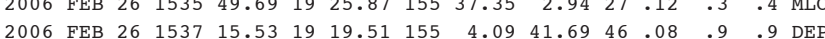

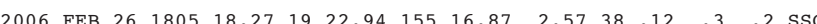

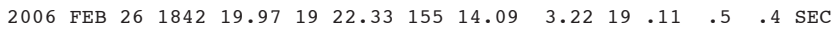

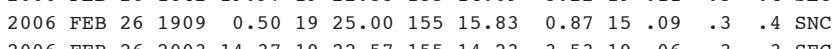

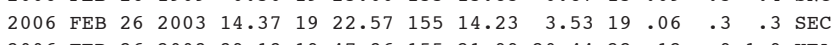

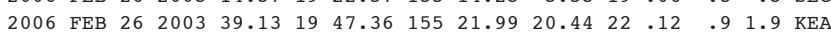

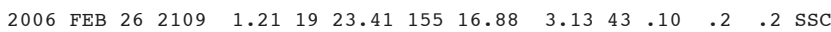

$\begin{array}{llllllllllllll}2006 & \text { FEB } & 26 & 2110 & 45.08 & 19 & 25.56 & 155 & 19.48 & 7.99 & 42 & .12 & .4 & .6 \\ \text { KAO }\end{array}$

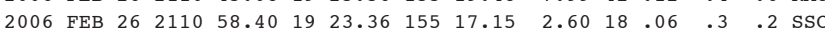

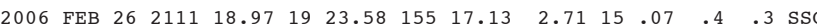

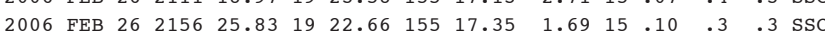

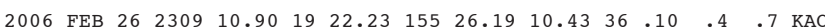

$\begin{array}{lllllllllllllll}2006 & \text { FEB } & 26 & 2320 & 12.38 & 19 & 27.06 & 155 & 18.60 & 4.27 & 22 & .12 & .4 & 1.0 & \text { SNC }\end{array}$

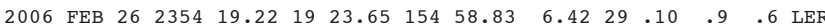

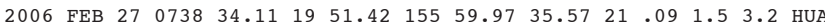
$\begin{array}{lllllllllllllll}2006 & \text { FEB } & 27 & 1209 & 14.43 & 19 & 15.17 & 155 & 35.82 & 2.33 & 41 & .13 & .4 & .6 & \text { LSW }\end{array}$ $\begin{array}{lllllllllllllll}2006 & \text { FEB } & 27 & 1243 & 23.11 & 19 & 19.51 & 155 & 11.52 & 7.57 & 35 & .09 & .4 & .7 & \text { SF3 }\end{array}$

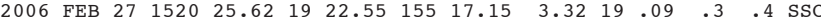

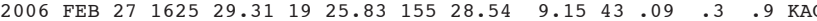

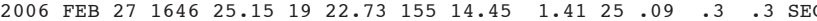

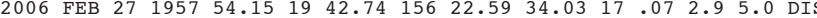

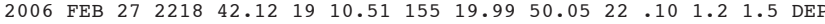

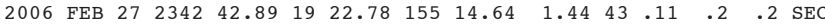
$\begin{array}{lllllllllllllll}2006 & \text { FEB } & 27 & 2344 & 13.07 & 19 & 20.49 & 155 & 14.09 & 5.16 & 15 & .11 & .8 & 1.5 & \text { SF2 }\end{array}$

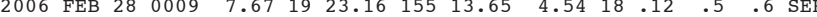

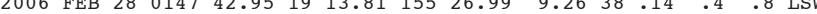

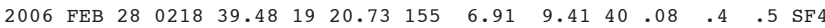

PREF AZ MIN 26 $\begin{array}{lll}1.6 \times & 44 & 0 \\ 1.1 \times & 75 & 2\end{array}$ $\begin{array}{lll}1.1 \times & 75 & 2 \\ 1.4 \times & 127 & 9\end{array}$ $\begin{array}{lll}1.4 \times & 127 & 9\end{array}$

$\begin{array}{lll}1.4 \times & 95 & 4\end{array}$ $\begin{array}{lll}1.8 \times & 67 & 3 \\ 1.0 \times & 174 & 1\end{array}$ $\begin{array}{lll}1.2 \times & 85 & 5\end{array}$ $2.6 \times 30284$ $\begin{array}{lll}1.3 \times & 81 & 3\end{array}$ $\begin{array}{lll}1.4 \times & 110 \quad 1\end{array}$ $\begin{array}{lll}1.4 \mathrm{X} & 83 & 3 \\ 1.5 \mathrm{X} & 62 & 5 \\ 1.5 \mathrm{x} & 151 & 2\end{array}$ $\begin{array}{lll}1.5 \times & 151 & 2\end{array}$ $1.3 \times 27528$ $\begin{array}{lll}1.7 \times & 332 & 82 \\ 1.3 \times & 91 & 3\end{array}$ $\begin{array}{rrr}1.3 \times & 91 & 3 \\ 2.9 x & 194 & 8 \\ 2.0 x & 48 & 1\end{array}$ $\begin{array}{lll}1.4 \times \quad 90 & 2\end{array}$ $\begin{array}{lll}1.1 \times 178 \quad 2 & 2\end{array}$ $1.2 \times \quad 89 \quad 2$ $\begin{array}{lll}1.6 \times 149 & 10\end{array}$ $2.6 \times \quad 44 \quad 0$ $\begin{array}{lll}2.2 \mathrm{X} & 47 & 3\end{array}$ $\begin{array}{lll}1.7 x & 71 & 0 \\ 1.3 x & 104 & 0\end{array}$ $\begin{array}{lll}1.3 \times & 104 & 0 \\ 1.2 \times & 147 & 2\end{array}$ $\begin{array}{rrr}1.2 \times & 147 & 2 \\ 1.5 \times & 62 & 3\end{array}$

$1.1 \times \quad 115 \quad 4$ $1.4 \times 204 \quad 3$ $1.7 \times 31146$ $\begin{array}{lll}1.8 \times & 105 & 10 \\ 1.6 \times & 162 & 6\end{array}$

$\begin{array}{lll}1.4 \mathrm{X} & 56 & 2 \\ 1.5 \mathrm{X} & 50 & 6\end{array}$ $\begin{array}{lll}1.5 \mathrm{X} & 50 & 6 \\ 1.7 \mathrm{x} & 77 & 2\end{array}$ $1.6 \times 33968$ $1.3 \times 25314$

$\begin{array}{lll}2.7 x & 73 \quad 2\end{array}$ $1.7 \times 1824$ $1.6 \times 116$ 1.6X $180 \quad 5$ 
---ORIGIN TIME (HST)-- -LAT N-- --LON W-- DEPTH N RMS ERH ERZ LOC PREF AZ MIN 27 YEAR MON DA HRMN SEC DEG MIN DEG MIN KM RD SEC KM KM REMKS MAG GAP DS

$\begin{array}{lllllllllllllll}2006 & \text { FEB } 28 & 0219 & 14.96 & 19 & 22.89 & 155 & 14.61 & 2.63 & 19 & .09 & .3 & .3 & \text { SEC }\end{array}$

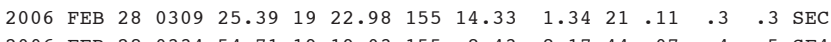

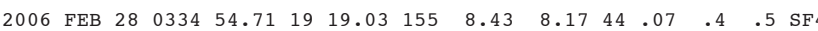

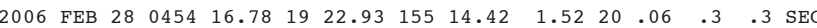

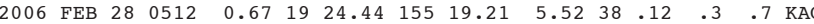

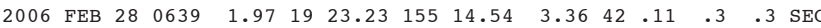

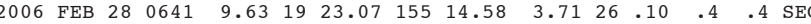

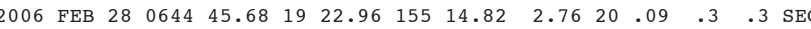

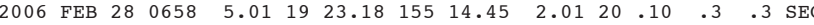

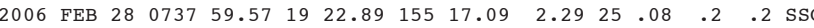

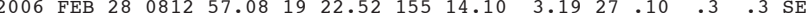

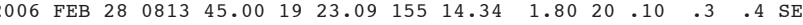

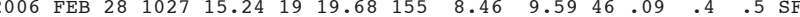

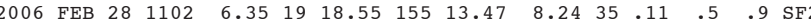

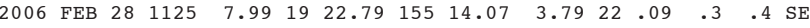

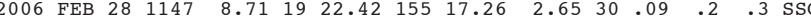

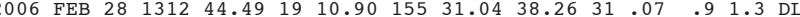

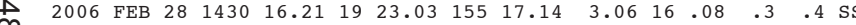

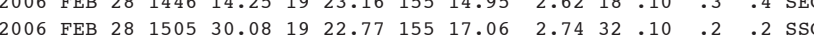

$\begin{array}{lllllllllllllll}2006 & \text { FEB } 28 & 1507 & 41.45 & 19 & 10.08 & 155 & 31.21 & 40.09 & 23 & .06 & .9 & 1.6 & \text { DLS }\end{array}$

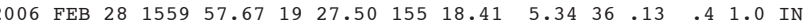

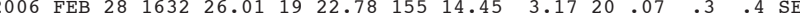

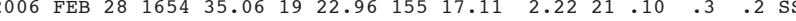

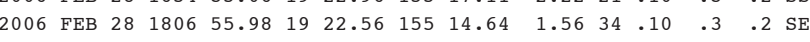

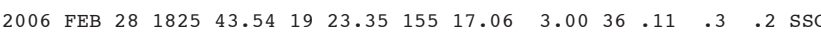
$\begin{array}{llllllllllllll}2006 & \text { FEB } 28 & 1843 & 16.76 & 19 & 10.49 & 155 & 31.02 & 38.00 & 35 & .07 & .8 & 1.1 & \text { DLS }\end{array}$

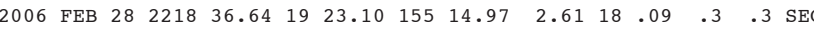

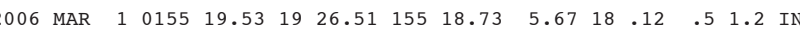
$\begin{array}{lllllllllllllll}2006 & \text { MAR } & 1 & 0213 & 2.02 & 19 & 26.61 & 155 & 18.63 & 5.77 & 29 & .11 & .4 & .8 & \text { IN }\end{array}$

$\begin{array}{lllllllllllllll}2006 & \text { MAR } & 1 & 0315 & 24.31 & 19 & 26.39 & 155 & 18.99 & 5.03 & 40 & .11 & .3 & .8 & \text { INT }\end{array}$

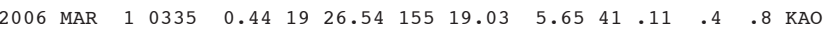

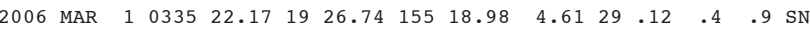

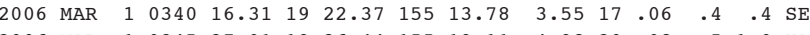

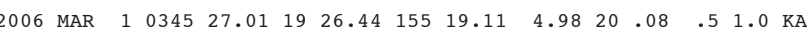

$\begin{array}{lllllllllllllll}2006 & \text { MAR } & 1 & 0414 & 48.48 & 19 & 22.87 & 155 & 17.19 & 2.52 & 19 & .06 & .2 & .3 & \text { SSC }\end{array}$

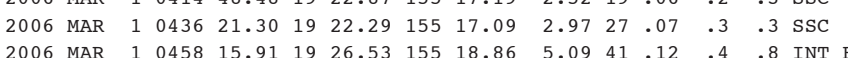
$\begin{array}{lllllllllllllll}2006 & \text { MAR } & 1 & 0458 & 15.91 & 19 & 26.53 & 155 & 18.86 & 5.09 & 41 & .12 & .4 & .8 & \text { INT }\end{array}$

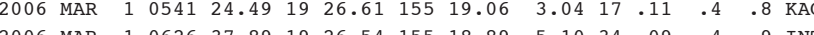
$\begin{array}{lllllllllllllll}2006 & \text { MAR } & 1 & 0626 & 37.89 & 19 & 26.54 & 155 & 18.89 & 5.10 & 34 & .09 & .4 & .9 & \text { IN }\end{array}$

$\begin{array}{lllllllllllllll}2006 & \text { MAR } & 1 & 0641 & 25.53 & 19 & 26.28 & 155 & 19.74 & 1.56 & 19 & .10 & .4 & .6 & \text { KAO }\end{array}$

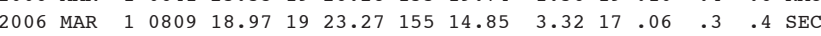

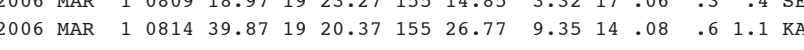

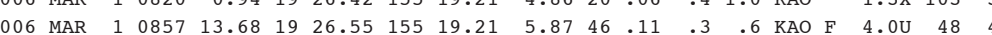

---ORIGIN TIME (HST)-- -LAT N-- --LON W-- DEPTH N RMS ERH ERZ LOC PREF AZ MIN 28 YEAR MON DA HRMN SEC DEG MIN DEG MIN $\quad$ KM RD SEC KM KM REMKS MAG GAP DS

$\begin{array}{llllllllllllllllll}2006 & \text { MAR } & 1 & 0858 & 59.94 & 19 & 26.60 & 155 & 18.67 & 4.71 & 43 & .12 & .3 & .8 & \text { SNC F } & 2.7 \mathrm{X} & 48 & 3\end{array}$

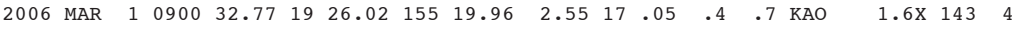

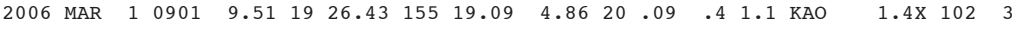
$\begin{array}{lllllllllllllllllll}2006 & \text { MAR } & 1 & 0903 & 5.85 & 19 & 26.57 & 155 & 18.72 & 5.35 & 36 & .11 & .4 & .8 & \text { INT } & 2.0 X & 50 & 3 \\ 2006\end{array}$

$\begin{array}{llllllllllllllllll}2006 & \text { MAR } & 1 & 0905 & 53.43 & 19 & 26.59 & 155 & 18.70 & 4.90 & 37 & .11 & .4 & .8 & \text { SNC } & 2.0 \mathrm{X} & 61 & 3\end{array}$

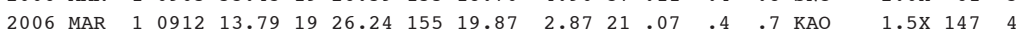

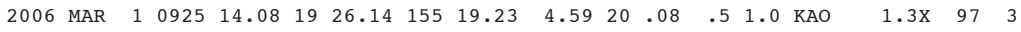

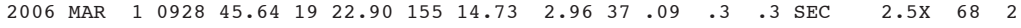

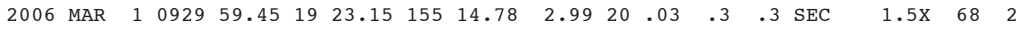

$\begin{array}{llllllllllllllllll}2006 & \text { MAR } & 1 & 0930 & 33.50 & 19 & 26.28 & 155 & 18.94 & 4.70 & 38 & .10 & .4 & .8 & \text { SNC } & 2.2 \mathrm{X} & 48 & 3\end{array}$

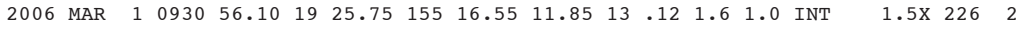

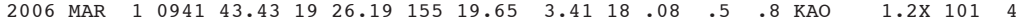
$\begin{array}{llllllllllllllllll}2006 & \text { MAR } & 1 & 0949 & 24.16 & 19 & 26.60 & 155 & 19.26 & 2.27 & 20 & .08 & .4 & .7 & \text { KAO } & 1.3 \times & 107 & 4\end{array}$

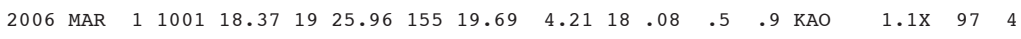

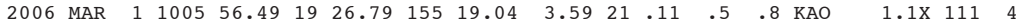

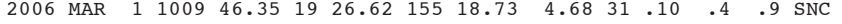

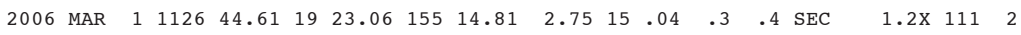

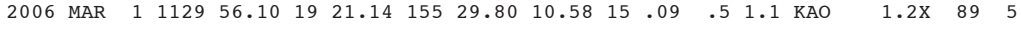

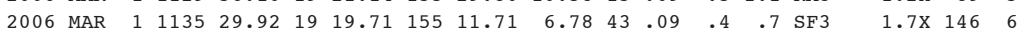

$\begin{array}{llllllllllllllllll}2006 & \text { MAR } & 1153 & 47.88 & 19 & 26.41 & 155 & 19.43 & 6.30 & 19 & .07 & .5 & 1.2 & \text { KAO } & 1.0 \times & 104 & 4\end{array}$ $\begin{array}{llllllllllllllllll}2006 & \text { MAR } & 1 & 1206 & 9.69 & 19 & 26.28 & 155 & 19.28 & 6.14 & 33 & .08 & .4 & .8 & \text { KAO } & 1.5 \times & 100 & 3\end{array}$ $\begin{array}{llllllllllllllllll}2006 & \text { MAR } & 1 & 1235 & 39.20 & 19 & 26.08 & 155 & 19.44 & 5.91 & 19 & .07 & .5 & 1.2 & \text { KAO } & 1.0 \mathrm{X} & 98 & 3\end{array}$

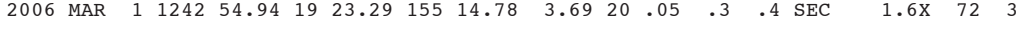

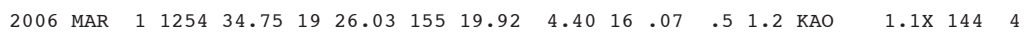

$\begin{array}{llllllllllllllllll}2006 & \text { MAR } & 1 & 1255 & 19.89 & 19 & 25.82 & 155 & 19.98 & 4.93 & 17 & .07 & .5 & 1.4 & \text { KAO } & 1.0 \times & 138 & 4\end{array}$ $\begin{array}{llllllllllllllllll}2006 & \text { MAR } & 1 & 1256 & 44.07 & 19 & 26.10 & 155 & 19.59 & 5.68 & 19 & .06 & .5 & 1.2 & \text { KAO } & 1.3 \times & 99 & 4\end{array}$

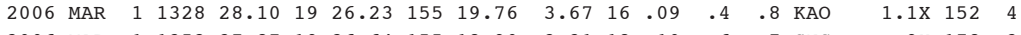

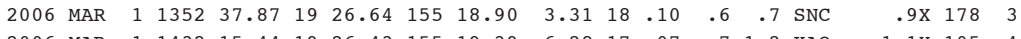

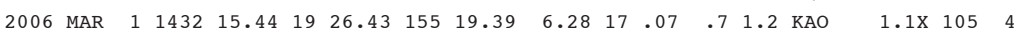

$\begin{array}{llllllllllllllllll}2006 & \text { MAR } & 1 & 1435 & 18.07 & 19 & 26.32 & 155 & 19.15 & 6.61 & 28 & .09 & .4 & .8 & \text { KAO } & 1.4 \times & 101 & 3\end{array}$

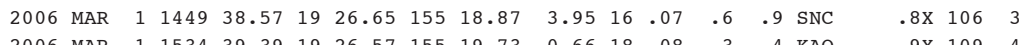

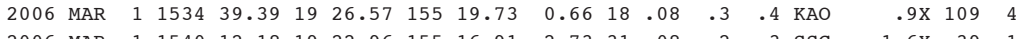

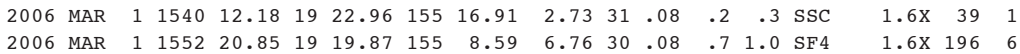

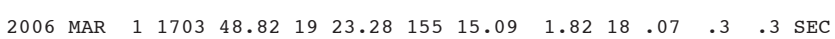

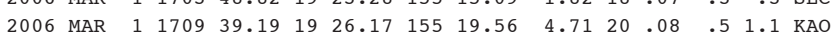

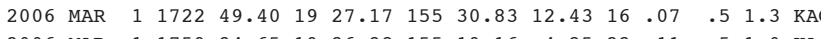

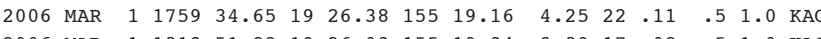
$\begin{array}{lllllllllllllll}2006 & \text { MAR } & 1 & 1813 & 51.32 & 19 & 26.08 & 155 & 19.84 & 3.89 & 17 & .08 & .5 & 1.0 & \text { KAO }\end{array}$

$\begin{array}{llllllllllllllll}2006 & \text { MAR } & 1 & 1816 & 13.76 & 19 & 26.24 & 155 & 19.74 & 4.33 & 16 & .07 & .6 & 1.2 & \text { KAO }\end{array}$

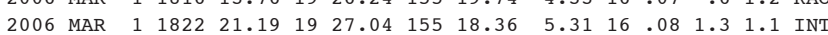

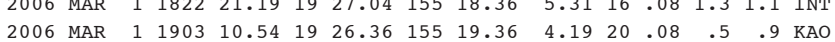

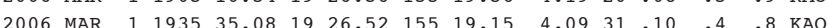

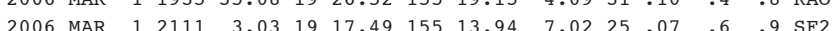

$1.4 \times 19280$ $1.1 \times 145 \quad 4$ $1.3 \times \quad 75 \quad 6$ $1.3 \times 102 \quad 3$ $1.2 \times 105$ $1.0 \times 168 \quad 3$ $1.2 \times 102 \quad 4$ $\begin{array}{rrr}1.6 \mathrm{X} & 90 & 3 \\ 1.1 \mathrm{x} & 126 & 1\end{array}$ 
---ORIGIN TIME (HST)-- -LAT N-- --LON W-- DEPTH N RMS ERH ERZ LOC PREF AZ MIN 29 YEAR MON DA HRMN SEC DEG MIN DEG MIN KM RD SEC KM KM REMKS MAG GAP DS

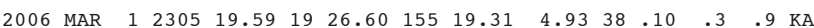

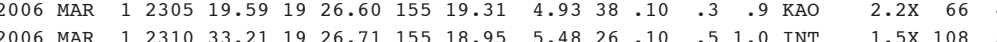

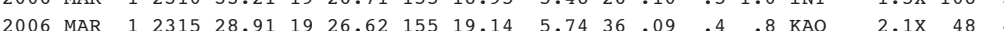

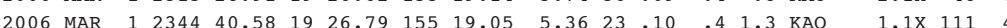

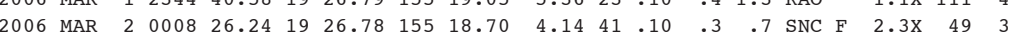

$\begin{array}{lllllllllllllllllll}2006 & \text { MAR } & 2 & 0015 & 24.53 & 19 & 26.86 & 155 & 18.74 & 5.68 & 34 & .14 & .4 & .9 & \text { INT } & \text { F } & 2.2 \mathrm{X} & 67 & 3\end{array}$

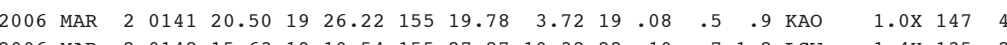

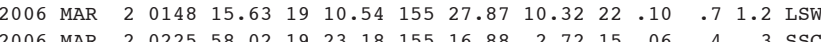

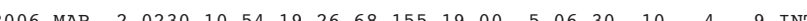

$\begin{array}{llllllllllllllllll}2006 & \text { MAR } & 2 & 0333 & 1.80 & 19 & 26.12 & 155 & 19.71 & 5.28 & 18 & .08 & .5 & 1.2 & \text { KAO } & 1.1 \times & 146 & 4\end{array}$

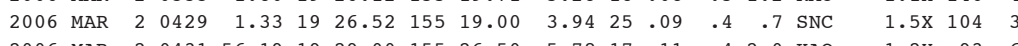

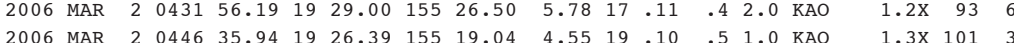

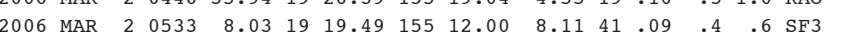

$\begin{array}{llllllllllllllllll}2006 & \text { MAR } & 2 & 0558 & 11.06 & 19 & 22.88 & 155 & 14.49 & 3.70 & 17 & .04 & .3 & .4 & \text { SEC } & 1.4 \mathrm{X} & 82 & 2\end{array}$

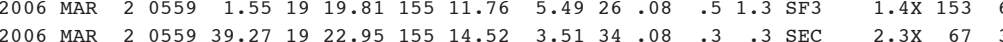

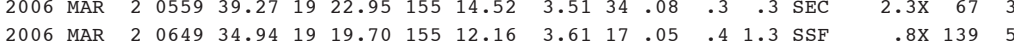

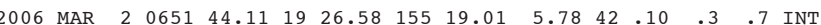

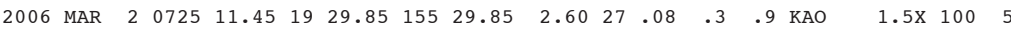

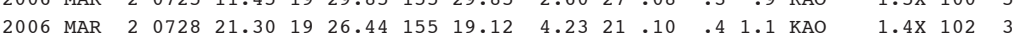
2006 MAR $2 \begin{array}{lllllllllllllll}0805 & 26.58 & 19 & 26.57 & 155 & 18.75 & 4.85 & 38 & .11 & .3 & .8 & \text { SNC } & 1.9 x & 68 & 3\end{array}$ $\begin{array}{llllllllllllllllll}2006 \text { MAR } & 2 & 0805 & 26.58 & 19 & 26.57 & 155 & 18.75 & 4.85 & 38 & .11 & .3 & .8 & \text { SNC } & 1.9 \mathrm{X} & 68 & 3 \\ 2006 & \text { MAR } & 2 & 0954 & 50.39 & 19 & 27.53 & 155 & 20.42 & 3.36 & 36 & .10 & .4 & 1.0 & \text { KAO } & 1.7 x & 50 & 6\end{array}$ $\begin{array}{lllllllllllll}2 & 1007 & 46.51 & 19 & 27.43 & 155 & 20.58 & 3.06 & 31 & .10 & .4 & 1.0 & \text { KAO }\end{array}$

$\begin{array}{lllllllllllllll}2006 & \text { MAR } & 2 & 1129 & 19.34 & 19 & 17.38 & 155 & 16.04 & 9.24 & 29 & .07 & .5 & .8 & \text { SF } 1\end{array}$ 2006 MAR $2 \begin{array}{lllllllllllll}2142 & 8.26 & 19 & 26.49 & 155 & 19.40 & 1.73 & 17 & .06 & .4 & .7 & \text { KAO }\end{array}$ $\begin{array}{llllllllllllllll}2006 & \text { MAR } & 2 & 1224 & 16.86 & 19 & 27.56 & 155 & 19.97 & 5.95 & 25 & .15 & .5 & 1.6 & \text { KAO }\end{array}$ $\begin{array}{llllllllllllllll}2006 & \text { MAR } & 2 & 1224 & 41.15 & 19 & 28.15 & 155 & 20.01 & 7.02 & 25 & .12 & .5 & 1.1 & \text { KAO } \\ 2006 & \text { MAR } & 2 & 1248 & 2.29 & 19 & 28.62 & 155 & 27.42 & 8.49 & 19 & .09 & .4 & 1.3 & \text { KAO }\end{array}$

$\begin{array}{lllllllllllllll}2006 & \text { MAR } & 2 & 1256 & 47.50 & 19 & 26.90 & 155 & 21.07 & 6.44 & 18 & .08 & .6 & 1.4 & \text { KAO }\end{array}$ $\begin{array}{lllllllllllllll}2006 & \text { MAR } & 2 & 1347 & 31.94 & 19 & 27.15 & 155 & 20.84 & 2.62 & 20 & .09 & .4 & .9 & \text { KAO }\end{array}$ $\begin{array}{lllllllllllllll}2006 & \text { MAR } & 2 & 1504 & 22.97 & 19 & 26.29 & 155 & 19.10 & 5.42 & 18 & .07 & .6 & 1.1 & \text { KAO }\end{array}$

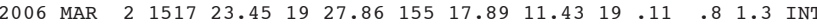
$\begin{array}{lllllllllllllll}2006 & \text { MAR } & 2 & 1537 & 5.15 & 19 & 25.63 & 155 & 19.31 & 4.58 & 17 & .06 & .6 & .8 & \text { KAO }\end{array}$

$\begin{array}{llllllllllllll}2006 & \text { MAR } & 2 & 1538 & 13.45 & 19 & 27.50 & 155 & 20.69 & 3.22 & 46 & .12 & .3 & .9 \\ \text { KAO F }\end{array}$ $\begin{array}{llllllllllllllll}2006 & \text { MAR } & 2 & 1544 & 30.34 & 19 & 27.58 & 155 & 21.21 & 0.67 & 27 & .07 & .4 & .4 & \text { KAO }\end{array}$ 2006 MAR $2 \begin{array}{lllllllllllll}1544 & 57.19 & 19 & 22.88 & 155 & 14.32 & 3.59 & 16 & .04 & .3 & .5 & \text { SEC }\end{array}$ $\begin{array}{llllllllllllllllll}2006 & \text { MAR } & 2 & 1550 & 11.75 & 19 & 27.68 & 155 & 20.75 & 2.43 & 22 & .07 & .5 & .8 & \text { KAO } & 1.7 x & 158 & 6\end{array}$

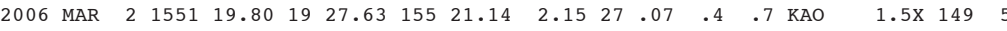
$\begin{array}{lllllllllllllllll}2006 & \text { MAR } & 2 & 1556 & 25.95 & 19 & 26.19 & 155 & 19.78 & 7.32 & 20 & .07 & .5 & 1.1 & \text { KAO } & 1.4 \times & 147\end{array}$ $\begin{array}{llllllllllllllllll}2006 & \text { MAR } & 2 & 1607 & 47.92 & 19 & 27.79 & 155 & 20.59 & 2.30 & 28 & .08 & .4 & .8 & \text { KAO } & 1.6 \times & 133 & 6\end{array}$ $\begin{array}{llllllllllllllllll}2006 & \text { MAR } & 2 & 1609 & 3.76 & 19 & 27.55 & 155 & 21.31 & 2.01 & 23 & .09 & .5 & .8 & \text { KAO } & 1.6 X & 153 & 5\end{array}$

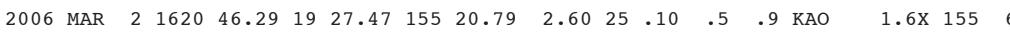

--ORTGIN TIME (HST)-- -LAT N-- --LON W-- DEPTH N RMS ERH ERZ LOC YEAR MON DA HRMN SEC DEG MIN DEG MIN KM RD SEC KM KM REMKS PREF AZ MIN 30 $\begin{array}{llllllllllllll}2006 & \text { MAR } & 2 & 1751 & 26.38 & 19 & 27.50 & 155 & 20.43 & 3.55 & 21 & .09 & .5 & 1.4 \\ \text { KAO }\end{array}$ $\begin{array}{lllllllllllllll}2006 & \text { MAR } & 2 & 1800 & 21.29 & 19 & 27.67 & 155 & 20.29 & 2.97 & 23 & .10 & .5 & 1.1 & \text { KAO }\end{array}$

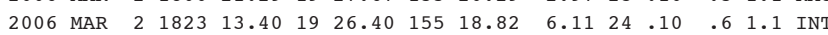

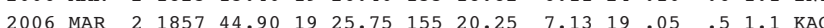
$\begin{array}{llllllllllllll}2006 & \text { MAR } & 2 & 1859 & 7.16 & 19 & 28.02 & 155 & 20.85 & 2.32 & 34 & .09 & .4 & .7 \\ \text { KAO }\end{array}$

$\begin{array}{lllllllllllllll}2006 & \text { MAR } & 2 & 1919 & 5.08 & 19 & 25.49 & 155 & 19.04 & 6.67 & 27 & .10 & .4 & .9 & \text { KAO }\end{array}$ $\begin{array}{llllllllllllll}2006 & \text { MAR } & 2 & 1921 & 16.62 & 19 & 25.19 & 155 & 19.02 & 6.48 & 19 & .05 & .4 & .9 \\ \text { KAO }\end{array}$ $\begin{array}{llllllllllllll}2006 & \text { MAR } & 2 & 1948 & 4.61 & 19 & 27.29 & 155 & 20.44 & 4.59 & 28 & .11 & .5 & 2.6 \\ \text { KAO }\end{array}$ $\begin{array}{lllllllllllllll}2006 & \text { MAR } & 2 & 1949 & 16.08 & 19 & 26.34 & 155 & 19.68 & 7.16 & 25 & .09 & .5 & 1.0 & \text { KAO }\end{array}$ $\begin{array}{llllllllllllll}2006 & \text { MAR } & 2 & 2115 & 57.43 & 19 & 27.40 & 155 & 20.81 & 1.74 & 40 & .11 & .3 & .5 \\ \text { KAO }\end{array}$

$\begin{array}{lllllllllllllll}2006 & \text { MAR } & 2 & 2152 & 22.60 & 19 & 26.07 & 155 & 19.89 & 7.11 & 32 & .08 & .4 & .8 & \text { KAO }\end{array}$

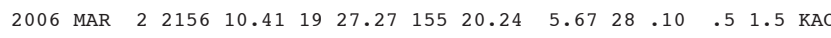
\begin{tabular}{llllllllllllll}
2006 & 2006 MAR & 2 & 2245 & 43.72 & 19 & 27.78 & 155 & 20.64 & 2.97 & 40 & .11 & .3 & .5 \\
\hline
\end{tabular}

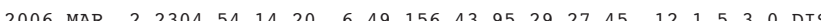

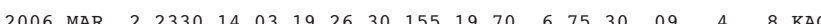

$\begin{array}{llllllllllllllll}2006 & \text { MAR } & 2 & 2345 & 24.98 & 19 & 26.24 & 155 & 19.94 & 7.36 & 40 & .11 & 4 & .7 & \text { KAO }\end{array}$

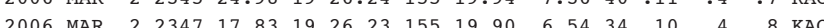

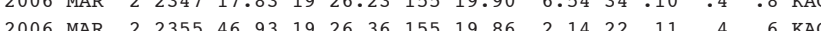

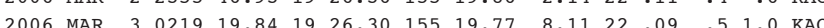

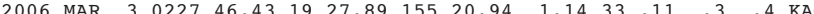

$\begin{array}{llllllllllllll}2006 & \text { MAR } & 3 & 0421 & 18.76 & 19 & 23.26 & 155 & 17.18 & 2.87 & 20 & .06 & 3 & .2 \\ \text { SSC }\end{array}$ $\begin{array}{lllllllllllllll}2006 & \text { MAR } & 3 & 0435 & 22.34 & 19 & 23.26 & 155 & 17.04 & 3.07 & 21 & .08 & 3 & .2 & \text { SSC }\end{array}$ 2006 MAR $3045652.02 \quad 19$

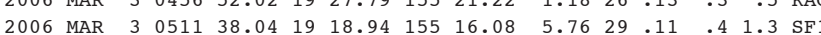
$\begin{array}{llllllllllllll}2006 & \text { MAR } & 3 & 0533 & 48.65 & 19 & 22.55 & 155 & 14.54 & 2.98 & 22 & 08 & 3 & 3\end{array}$

$\begin{array}{lllllllllllllll}2006 & \text { MAR } & 3 & 0614 & 10.22 & 19 & 10.49 & 155 & 40.10 & 9.85 & 26 & .14 & .7 & 1.3 & \text { LSW }\end{array}$ $\begin{array}{llllllllllllll}2006 & \text { MAR } & 3 & 0632 & 19.29 & 19 & 23.21 & 155 & 17.19 & 2.14 & 18 & .06 & .3 & .2 \\ \text { SSC }\end{array}$

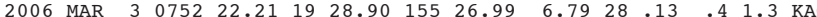
$\begin{array}{lllllllllllllll}2006 & \text { MAR } & 3 & 0811 & 25.65 & 19 & 28.36 & 155 & 20.71 & 5.83 & 44 & .12 & .4 & 1.1 & \text { KAO }\end{array}$

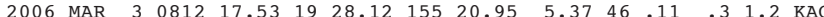

$\begin{array}{lllllllllllllll}2006 & \text { MAR } & 3 & 0813 & 0.61 & 19 & 28.98 & 155 & 20.85 & 7.80 & 24 & .09 & .5 & .9 & \text { KAO }\end{array}$ $\begin{array}{lllllllllllllll}2006 & \text { MAR } & 3 & 0832 & 49.67 & 19 & 22.85 & 154 & 59.86 & 6.11 & 22 & .07 & .7 & 1.1 & \text { LER }\end{array}$ $\begin{array}{llllllllllllll}2006 & \text { MAR } & 3 & 0856 & 3.92 & 19 & 27.58 & 155 & 21.00 & 1.79 & 20 & .06 & .3 & .6 \\ \text { KAO }\end{array}$

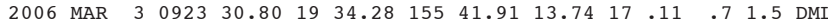
$\begin{array}{llllllllllllllll}2006 & \text { MAR } & 3 & 0950 & 39.79 & 19 & 27.48 & 155 & 21.38 & 6.34 & 24 & .10 & .4 & 1.4 & \text { KAO }\end{array}$

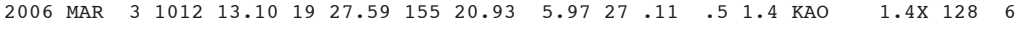

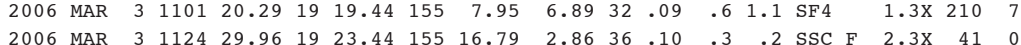

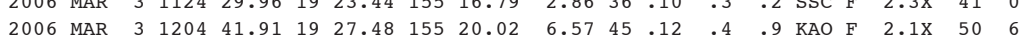

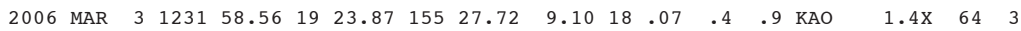

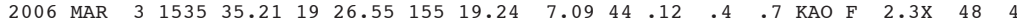
$\begin{array}{llllllllllllllllll}2006 & \text { MAR } & 3 & 1545 & 36.10 & 19 & 26.17 & 155 & 19.72 & 6.28 & 19 & .08 & .5 & 1.2 & \text { KAO } & 1.5 \times & 101 & 4\end{array}$

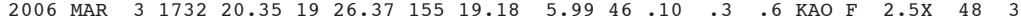

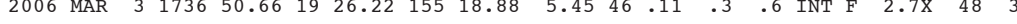

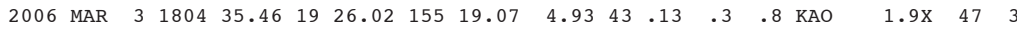


---ORIGIN TIME (HST)-- -LAT N-- --LON W-- DEPTH N RMS ERH ERZ LOC PREF AZ MIN 31 YEAR MON DA HRMN SEC DEG MIN DEG MIN KM RD SEC KM KM REMKS MAG GAP DS

$\begin{array}{llllllllllllll}2006 & \text { MAR } & 3 & 1813 & 49.47 & 19 & 25.82 & 155 & 19.56 & 4.10 & 22 & .11 & .4 & .9 \\ \text { KAO }\end{array}$ $\begin{array}{lllllllllllllll}2006 & \text { MAR } & 3 & 1823 & 1.26 & 19 & 18.71 & 155 & 3.57 & 41.34 & 31 & .10 & 1.5 & .8 & \text { DEP }\end{array}$ 2006 MAR $3 \begin{array}{llllllllllllll}1836 & 7.85 & 19 & 26.39 & 155 & 18.85 & 4.92 & 33 & .15 & .4 & .9 & \mathrm{SNC} \\ \end{array}$ $\begin{array}{llllllllllllllll}2006 & \text { MAR } & 3 & 1840 & 18.10 & 19 & 17.02 & 155 & 14.57 & 7.10 & 32 & .11 & .5 & .8 & \text { SF } 1 \\ 2006 & \text { MAR } & 3 & 1913 & 33.58 & 19 & 31.86 & 155 & 10.48 & 44.43 & 44 & .10 & .8 & .8 & \text { DFP }\end{array}$

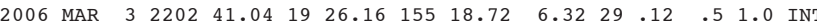

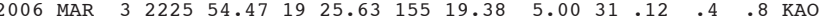
$\begin{array}{llllllllllllllll}2006 & \text { MAR } & 3 & 2322 & 57.88 & 19 & 26.02 & 155 & 19.02 & 5.07 & 36 & .12 & .4 & .8 & \text { INT }\end{array}$ 2006 MAR $3 \begin{array}{lllllllllllll}3 & 2329 & 3.07 & 19 & 11.47 & 155 & 28.41 & 9.11 & 27 & .13 & .6 & 1.3 & \text { LSW }\end{array}$ 2006 MAR $3 \begin{array}{lllllllllllllll}2339 & 0.96 & 19 & 27.84 & 155 & 21.85 & 6.19 & 33 & .12 & .4 & 1.1 & \mathrm{KAO}\end{array}$

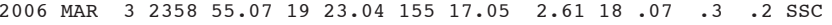

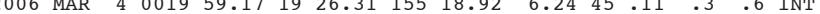

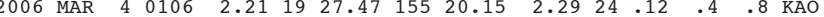

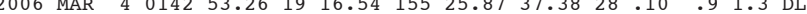

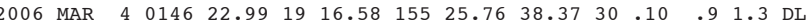

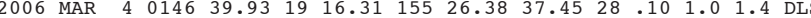

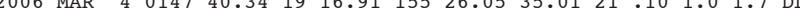

G 2006 MAR 4020 2006 MAR 40311 $\begin{array}{lllllllllllllll}2006 & \text { MAR } & 4 & 0318 & 58.40 & 19 & 23.12 & 155 & 16.78 & 3.07 & 19 & .11 & .4 & .3 & \text { SSC }\end{array}$

$\begin{array}{lllllllllllllll}2006 & \text { MAR } & 4 & 0328 & 4.28 & 19 & 25.71 & 155 & 19.61 & 5.48 & 19 & .10 & .5 & 1.3 & \text { KAO }\end{array}$

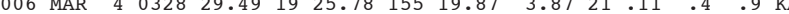

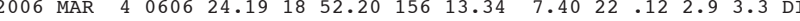

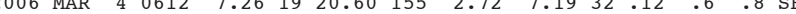

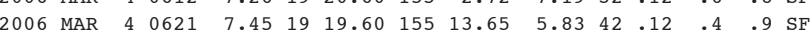

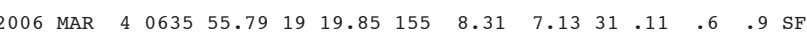
$\begin{array}{lllllllllllllll}2006 & \text { MAR } & 4 & 0700 & 47.99 & 19 & 23.26 & 155 & 14.99 & 3.27 & 19 & .08 & .3 & .4 & \text { SEC }\end{array}$

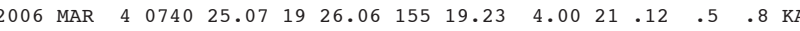

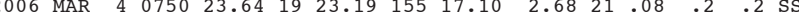
$\begin{array}{lllllllllllllll}2006 & \text { MAR } & 4 & 0757 & 35.57 & 19 & 25.83 & 155 & 19.10 & 7.37 & 21 & .08 & .5 & .9 & \text { KAO }\end{array}$

2006 MAR $4 \begin{array}{lllllllllllll}0828 & 43.31 & 19 & 23.57 & 155 & 16.97 & 3.12 & 30 & .08 & .3 & .2 & \text { SSC }\end{array}$

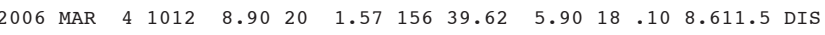
$\begin{array}{lllllllllllllll}2006 & \text { MAR } & 4 & 1128 & 55.44 & 19 & 26.22 & 155 & 18.86 & 4.95 & 32 & .11 & .4 & .8 & \text { SNC }\end{array}$

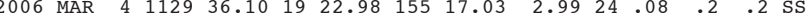
$\begin{array}{lllllllllllllll}2006 & \text { MAR } & 4 & 1215 & 2.47 & 19 & 25.80 & 155 & 19.72 & 4.86 & 24 & .09 & .4 & 1.0 & \text { KAO }\end{array}$

$\begin{array}{lllllllllllllll}2006 & \text { MAR } & 4 & 1221 & 56.34 & 19 & 27.34 & 155 & 17.40 & 7.44 & 21 & .11 & .6 & .9 & \text { INT }\end{array}$

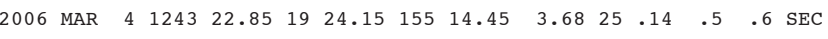

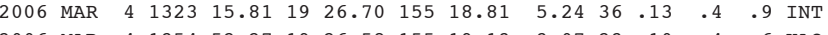
$\begin{array}{lllllllllllllll}2006 & \text { MAR } & 4 & 1354 & 52.27 & 19 & 26.58 & 155 & 19.13 & 3.07 & 23 & .10 & .4 & .6 & \text { KAO } \\ 2006 & \text { MAR } & 4 & 1511 & 54.58 & 19 & 25.70 & 155 & 19.47 & 5.00 & 40 & .11 & .3 & .9 & \text { KAO }\end{array}$

$\begin{array}{llllllllllllll}2006 & \text { MAR } & 4 & 1512 & 19.41 & 19 & 25.51 & 155 & 19.85 & 5.06 & 28 & .10 & .4 & .9 \\ \text { KAO }\end{array}$ $\begin{array}{llllllllllllll}2006 & \text { MAR } & 4 & 1528 & 48.86 & 19 & 26.11 & 155 & 19.24 & 5.64 & 33 & .12 & .4 & .9 \\ \text { KAO }\end{array}$ 2006 MAR $4 \begin{array}{llllllllllll}1533 & 35.57 & 19 & 25.76 & 155 & 19.40 & 5.83 & 42 & .12 & .4 & .8 & \text { KAO }\end{array}$

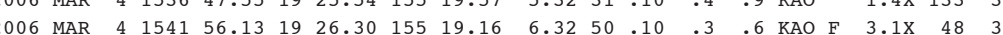

---ORIGIN TIME (HST)-- -LAT N-- --LON W-- DEPTH N RMS ERH ERZ LOC PREF AZ MIN 32 YEAR MON DA HRMN SEC DEG MIN DEG MIN KM RD SEC KM KM REMKS MAG GAP DS

$\begin{array}{llllllllllllllllll}2006 & \text { MAR } & 4 & 1545 & 19.24 & 19 & 25.46 & 155 & 19.60 & 4.25 & 40 & .12 & .3 & .7 & \text { KAO } & 2.0 \times & 46 & 3\end{array}$

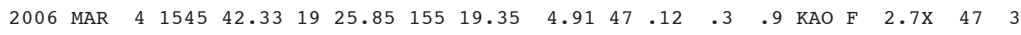
$\begin{array}{llllllllllllllllll}2006 & \text { MAR } & 4 & 1600 & 59.78 & 19 & 26.61 & 155 & 18.86 & 4.48 & 36 & .12 & .4 & .9 & \text { SNC } & 1.9 \mathrm{X} & 81 & 3\end{array}$

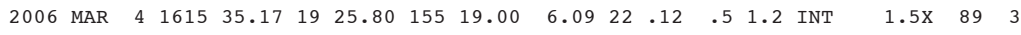

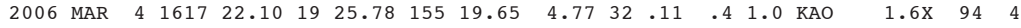

$\begin{array}{llllllllllllllllll}2006 & \text { MAR } & 4 & 1630 & 41.40 & 19 & 22.70 & 155 & 14.44 & 3.18 & 19 & .09 & .3 & .4 & \text { SEC } & 1.6 \mathrm{X} & 81 & 2\end{array}$

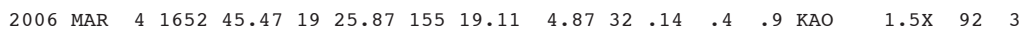
$\begin{array}{llllllllllllllllll}2006 & \text { MAR } & 4 & 1709 & 53.81 & 19 & 26.40 & 155 & 18.72 & 5.63 & 36 & .11 & .4 & .8 & \text { INT } & 1.8 \mathrm{X} & 76 & 3\end{array}$ $\begin{array}{llllllllllllllllll}2006 & \text { MAR } & 4 & 1713 & 22.58 & 19 & 26.43 & 155 & 18.74 & 5.93 & 38 & .11 & .4 & .8 & \text { INT } & 1.9 \mathrm{X} & 99 & 3\end{array}$

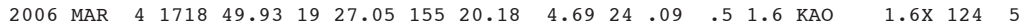

$\begin{array}{llllllllllllllllll}2006 & \text { MAR } & 4 & 1720 & 45.34 & 19 & 26.45 & 155 & 17.90 & 6.56 & 22 & .09 & .6 & .7 & \text { INT } & 1.3 \times & 110 & 2\end{array}$ $\begin{array}{llllllllllllllllll}2006 & \text { MAR } & 4 & 1721 & 31.19 & 19 & 25.89 & 155 & 19.58 & 3.26 & 21 & .12 & .4 & .7 & \text { KAO } & 1.1 \mathrm{x} & 95 & 4\end{array}$ $\begin{array}{llllllllllllllllll}2006 & \text { MAR } & 4 & 1747 & 26.52 & 19 & 26.37 & 155 & 18.69 & 6.08 & 39 & .11 & .4 & .7 & \text { INT } & 1.9 \mathrm{X} & 56 & 3\end{array}$

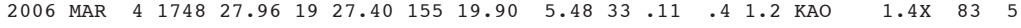

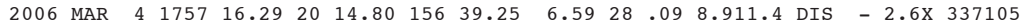

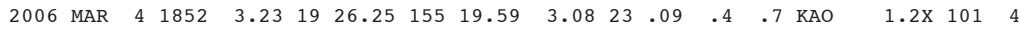

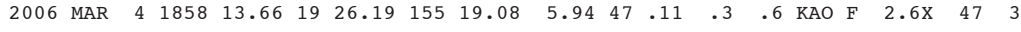

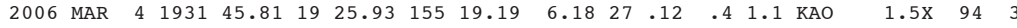

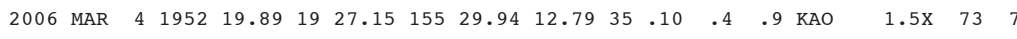

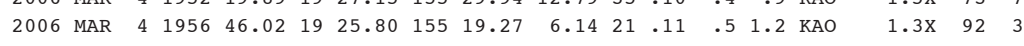

$\begin{array}{llllllllllllllllll}2006 & \text { MAR } & 4 & 2025 & 19.15 & 19 & 26.21 & 155 & 18.85 & 4.99 & 31 & .10 & .4 & .8 & \text { SNC } & 1.6 \mathrm{X} & 95 & 3\end{array}$ $\begin{array}{llllllllllllllllll}2006 & \text { MAR } & 4 & 2153 & 40.12 & 19 & 26.51 & 155 & 19.49 & 7.56 & 37 & .10 & .4 & .7 & \text { KAO } & 1.7 \mathrm{X} & 66 & 4\end{array}$

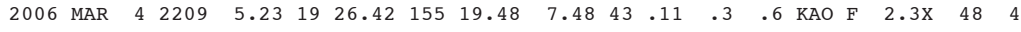

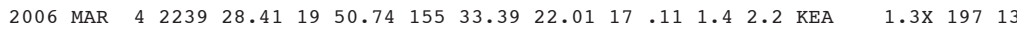

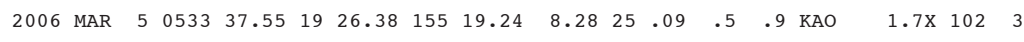

$\begin{array}{llllllllllllllllll}2006 & \text { MAR } & 5 & 0646 & 7.01 & 19 & 23.23 & 155 & 16.99 & 3.15 & 29 & .10 & .3 & .2 & \text { SSC } & 1.6 x & 47 & 0\end{array}$

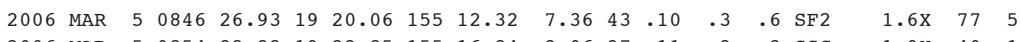
$\begin{array}{llllllllllllllllll}2006 & \text { MAR } & 5 & 0854 & 32.82 & 19 & 22.85 & 155 & 16.84 & 3.06 & 37 & .11 & .2 & .2 & \text { SSC } & 1.9 \mathrm{x} & 40 & 1\end{array}$

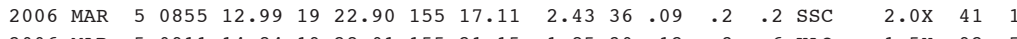

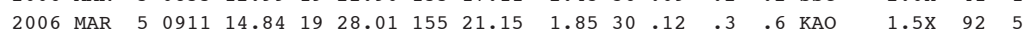

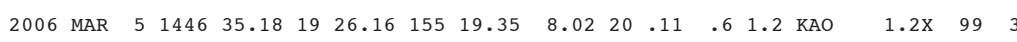

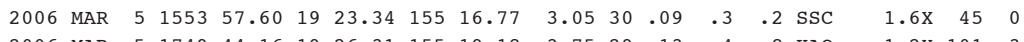
$\begin{array}{llllllllllllllllll}2006 & \text { MAR } & 5 & 1749 & 44.16 & 19 & 26.31 & 155 & 19.18 & 3.75 & 29 & .13 & .4 & .8 & \text { KAO } & 1.2 \mathrm{x} & 101 & 3\end{array}$

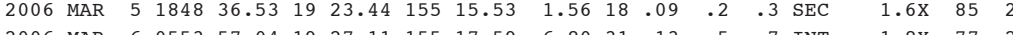
$\begin{array}{llllllllllllllllll}2006 & \text { MAR } & 6 & 0553 & 57.04 & 19 & 27.11 & 155 & 17.59 & 6.80 & 31 & .13 & .5 & .7 & \text { INT } & 1.8 \mathrm{X} & 77 & 2\end{array}$

$\begin{array}{llllllllllllllllll}2006 & \text { MAR } & 6 & 0555 & 4.85 & 19 & 28.20 & 155 & 21.20 & 2.29 & 39 & .11 & .3 & .5 & \text { KAO } & 2.0 \mathrm{X} & 69 & 5\end{array}$

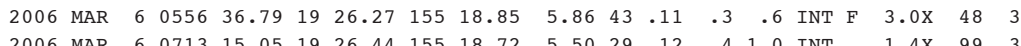

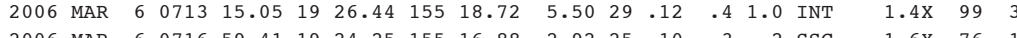

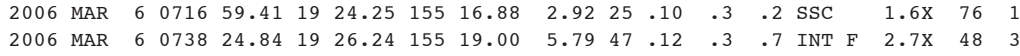

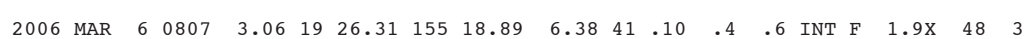

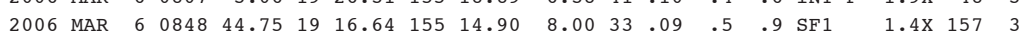

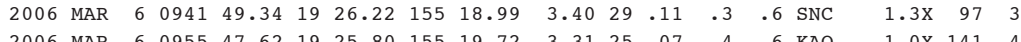

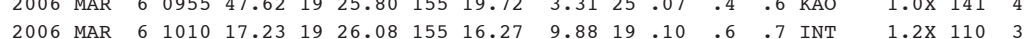


--ORIGIN TIME (HST)-- -LAT N-- --LON W-- DEPTH N RMS ERH ERZ LOC YEAR MON DA HRMN SEC DEG MIN DEG MIN KM RD SEC KM

$\begin{array}{llllllllllllll}2006 & \text { MAR } & 6 & 1019 & 29.25 & 19 & 23.38 & 155 & 16.62 & 3.49 & 36 & .11 & .3 & .2 \\ \text { SSC }\end{array}$ $\begin{array}{lllllllllllllll}2006 & \text { MAR } & 6 & 1021 & 18.29 & 19 & 23.04 & 155 & 17.06 & 2.54 & 21 & .09 & .2 & .2 & \text { SSC }\end{array}$

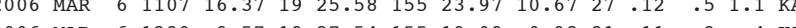
$\begin{array}{llllll} & \end{array}$

$\begin{array}{lllllllllllllll}2006 & \text { MAR } & 6 & 1617 & 40.60 & 19 & 26.12 & 155 & 18.82 & 4.97 & 38 & .13 & .4 & .8 & \text { SNC }\end{array}$ $\begin{array}{lllllllll}2006 & .4\end{array}$ $\begin{array}{llllllllllll}2006 & & \end{array}$

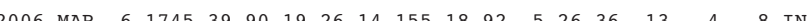

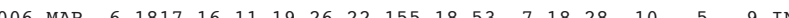

$\begin{array}{lllllllllllllll}2006 & \text { MAR } & 6 & 1819 & 11.94 & 19 & 34.06 & 155 & 43.29 & 8.50 & 23 & .12 & .9 & 1.4 & \text { KON }\end{array}$

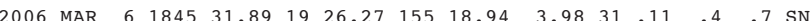

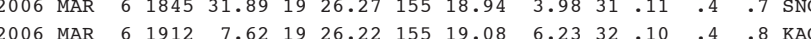

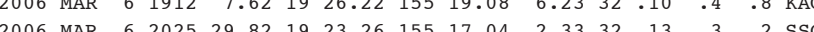

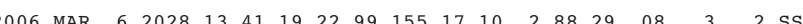

$\begin{array}{lllllllllllllll}2006 & \text { MAR } & 6 & 2029 & 1.70 & 19 & 23.18 & 155 & 17.04 & 2.57 & 19 & .07 & .3 & .2 & \text { SSC }\end{array}$

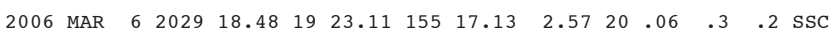

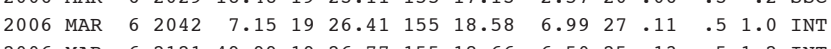

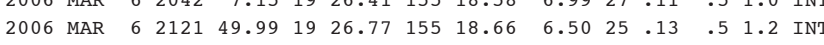

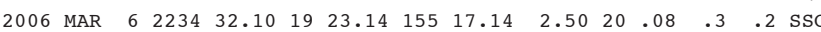

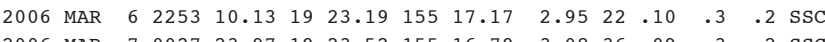

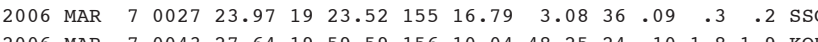

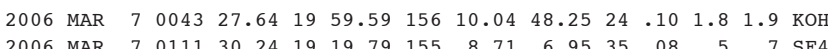
$\begin{array}{llllllllllllll}2006 & \text { MAR } & 7 & 0129 & 56.09 & 19 & 26.62 & 155 & 19.00 & 3.37 & 22 & .12 & .4 & .6\end{array}$

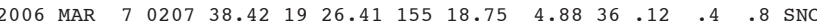

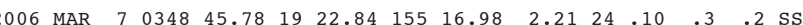

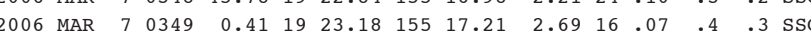

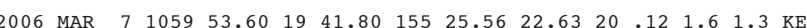
$\begin{array}{llllllllllllllll}2006 & \text { MAR } & 7 & 1116 & 30.08 & 19 & 23.59 & 155 & 16.81 & 3.09 & 44 & .11 & .2 & .2 & \text { SSC }\end{array}$

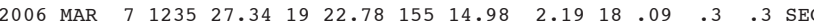

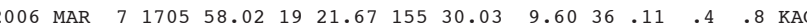
$\begin{array}{lllllllllllllll}2006 & \text { MAR } & 7 & 1825 & 23.29 & 19 & 19.31 & 155 & 8.79 & 8.65 & 39 & .09 & .4 & .6 & \mathrm{SF}\end{array}$ \begin{tabular}{llllllllllllll}
2006 & MAR & 7 & 2326 & 34.80 & 19 & 23.67 & 155 & 16.93 & 2.88 & 27 & .09 & .3 & .2 \\
\hline
\end{tabular} $\begin{array}{lllllllllllllll}2006 & \text { MAR } & 7 & 2344 & 18.73 & 19 & 19.82 & 155 & 11.71 & 4.85 & 29 & .11 & .5 & 1.8 & \text { SSF }\end{array}$

$\begin{array}{lllllllllllllll}2006 & \text { MAR } & 8 & 0226 & 52.89 & 19 & 11.42 & 155 & 28.43 & 34.56 & 38 & .06 & .7 & 1.1 & \text { DLS }\end{array}$

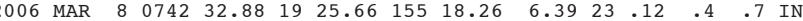
$\begin{array}{lllllllllllllll}2006 & \text { MAR } & 8 & 0848 & 59.20 & 19 & 23.03 & 155 & 17.24 & 2.44 & 20 & .07 & .2 & .3 & \text { SSC }\end{array}$ 2006 MAR $8 \begin{array}{llllllllllllll}0 & 0852 & 9.56 & 19 & 17.92 & 155 & 12.64 & 8.59 & 41 & .11 & .5 & .6 & \text { SF2 }\end{array}$ $\begin{array}{lllllllllllllll}2006 & \text { MAR } & 8 & 1010 & 36.30 & 19 & 12.94 & 155 & 33.80 & 5.88 & 31 & .14 & .6 & 2.3 & \text { LSW }\end{array}$

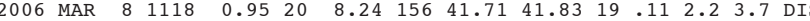

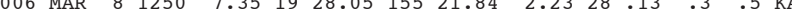

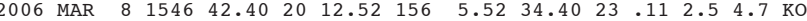

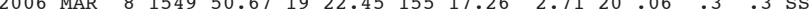

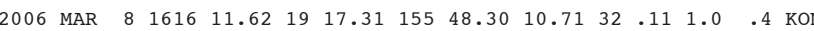

PREF AZ MIN 33 MAG GAP DS $2.0 \times \quad 84 \quad 1$ $1.6 \mathrm{x} \quad 70 \quad 1$ $1.0 \times 1318$ $1.0 \times 13124$

$1.9 \times \quad 54 \quad 3$ $1.9 \times \quad 59 \quad 3$ $1.8 \mathrm{X} \quad 94 \quad 3$

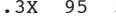
$1.3 \times \quad 92 \quad 2$ $\begin{array}{lll}1.7 \times & 224 & 8\end{array}$ $\begin{array}{lll}1.4 \mathrm{X} & 97 & 3 \\ 1.5 \mathrm{x} & 98 & 3\end{array}$ $\begin{array}{lll}1.5 \mathrm{X} & 98 & 3 \\ 1.9 \mathrm{X} & 45 & 0\end{array}$ $1.5 \times 48 \quad 1$

$\begin{array}{lll}1.3 \mathrm{X} & 47 & 0\end{array}$ $\begin{array}{lll}1.3 \mathrm{X} & 48 & 1\end{array}$ $\begin{array}{lll}1.3 \times & 97 & 3 \\ 1.2 \times & 107 & 3\end{array}$ $\begin{array}{lll}1.2 \times & 107 & 3\end{array}$ $\begin{array}{lll}1.4 \times & 48 \quad 1\end{array}$ $\begin{array}{lll}1.4 \mathrm{X} & 48 & 1 \\ 1.7 \mathrm{X} & 44 & 0\end{array}$ $\begin{array}{rrr}.7 \times & 44 & 0\end{array}$ $1.4 \times 1796$ $1.1 \times 114 \quad 3$

$1.6 \times \quad 58 \quad 3$ $1.5 \times \quad 49 \quad 1$ $1.1 \mathrm{x} \quad 88 \quad 1$ 1.5X 28912 $2.3 \times \quad 39 \quad 0$

$\begin{array}{lll}1.3 \mathrm{X} & 71 \quad 2\end{array}$ $1.4 \mathrm{X} \quad 62 \quad 4$ $1.5 \times 116 \quad 4$ $\begin{array}{lll}1.6 \times \quad 50 & 1\end{array}$ $1.2 \times 164 \quad 6$

$1.8 \times \quad 96 \quad 4$ $\begin{array}{lll}1.2 \mathrm{x} & 79 & 1 \\ 1.4 \mathrm{x} & 49 & 1\end{array}$ $1.4 \mathrm{X} \quad 49 \quad 1$ 2.0X $171 \quad 2$ 1.6x 2058

2.3× 32296 $1.3 \times 131 \quad 4$ 2.3X $318 \quad 63$ $1.4 \times \quad 54 \quad 2$ 1.6X 20516
--ORTGin TIME (HST)-- -LAT N-- --LON W-- DEPTH N RMS ERH ERz LOC YEAR MON DA HRMN SEC DEG MIN DEG MIN KM RD SEC KM KM REMKS $\begin{array}{lllllllllllllll}2006 & \text { MAR } & 8 & 1705 & 45.58 & 19 & 29.28 & 155 & 27.02 & 6.65 & 19 & .11 & .4 & 1.5 & \text { KAO }\end{array}$ $\begin{array}{lllllllllllllll}2006 & \text { MAR } & 8 & 2001 & 55.37 & 19 & 28.11 & 155 & 21.40 & 2.01 & 27 & .11 & .4 & .6 & \text { KAO }\end{array}$

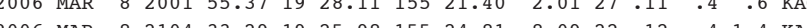
2006 MAR $8210433.291925 .9815524 .81 \quad 8.0922 .12 \quad .41 .4$ KAO

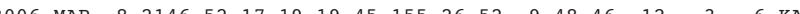

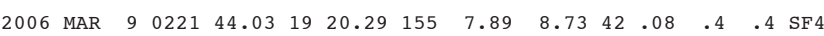
$\begin{array}{lllllllllllllll}2006 & \text { MAR } & 9 & 0339 & 49.62 & 19 & 23.39 & 155 & 23.60 & 13.64 & 29 & .08 & .4 & .6 & \text { DML }\end{array}$ $\begin{array}{lllllllllllllll}2006 & \text { MAR } & 9 & 0754 & 23.50 & 19 & 26.16 & 155 & 19.75 & 6.53 & 19 & .11 & .5 & 1.3 & \text { KAO }\end{array}$

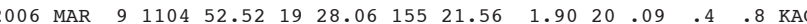

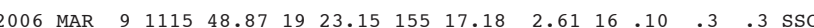

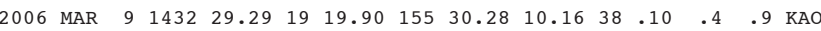

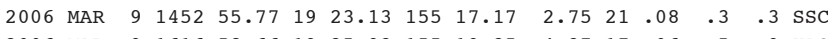
$\begin{array}{llllllllllllll}2006 & \text { MAR } & 9 & 1616 & 58.66 & 19 & 25.93 & 155 & 19.35 & 4.37 & 17 & .06 & .5 & .9 \\ \text { KAO }\end{array}$

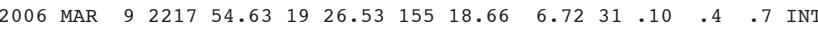

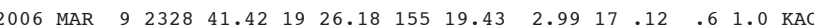

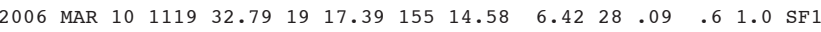

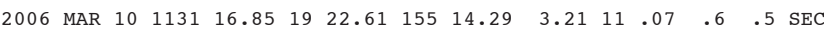

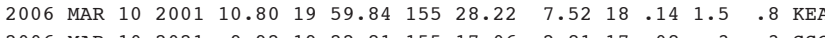

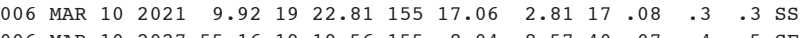
$\begin{array}{lllllllllllllll}006 & \text { MAR } 10 & 2027 & 55.16 & 19 & 19.56 & 155 & 8.04 & 8.57 & 40 & .07 & .4 & .5 & \text { SF } 4\end{array}$

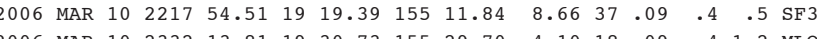

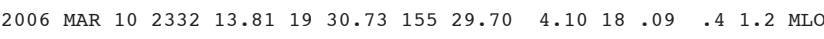
$\begin{array}{llllllllllllll}2006 & \text { MAR } & 10 & 2347 & 30.53 & 19 & 22.43 & 155 & 29.81 & 10.26 & 37 & .09 & .4 & .9 \\ 2006 & \text { KAO } \\ \text { MAR } & 11 & 0200 & 25.96 & 19 & 25.92 & 155 & 19.38 & 5.06 & 34 & .12 & .4 & .8 & \text { KAO }\end{array}$

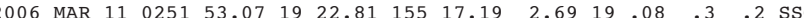

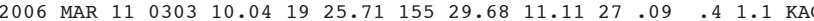

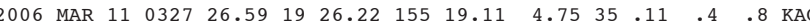

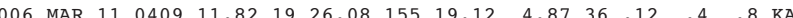

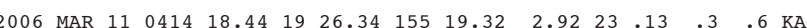

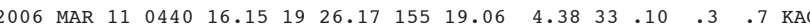

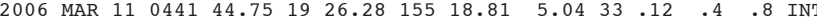

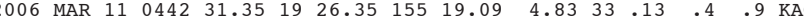

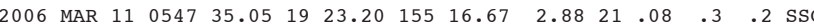

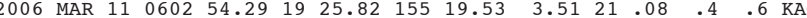

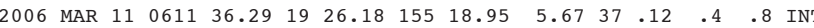

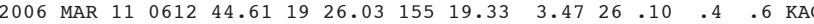

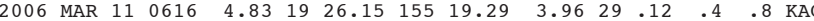

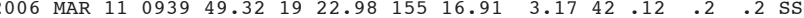

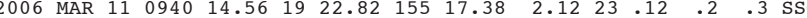

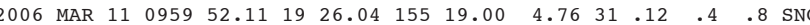

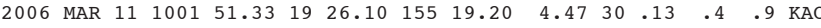

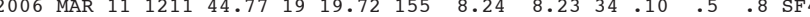

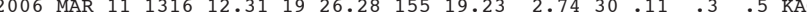

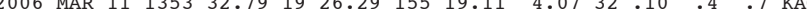
$\begin{array}{lllllllllllllll}2006 & \text { MAR } & 11 & 1355 & 32.04 & 19 & 25.51 & 155 & 19.02 & 6.86 & 33 & .11 & .4 & .8 & \text { KAO }\end{array}$
PREF AZ MIN 34 MAG GAP DS $1.3 \times \quad 93 \quad 5$ $\begin{array}{lll}.7 \times \quad 135 & 5 \\ 1 \times & 56 & 8\end{array}$ $\begin{array}{lll}1.2 \times 275 & 19\end{array}$ 1.2. 275 $2.2 \times 122 \quad 5$ $1.3 \times \quad 83 \quad 6$ $\begin{array}{lll}.2 \times 151 & 4 \\ 1.6 \times & 134 & 4\end{array}$ $1.3 \times \quad 67$

$\begin{array}{lll}1.7 x & 67 & 7\end{array}$ $\begin{array}{lll}1.5 \times \quad 48 & 1\end{array}$ $\begin{array}{lll}1.3 \times & 145 & 3\end{array}$ $2.0 \times \quad 48$ $1.4 \times 100 \quad 4$

1.6X $130 \quad 2$ $1.3 \times 174 \quad 2$ $\begin{array}{lll}1.6 \times & 296 & 18\end{array}$ $1.2 \times \quad 59 \quad 1$ 1.9X $120 \quad 4$

$1.3 \times \quad 96 \quad 5$ $\begin{array}{lll}1.2 \times & 108 \quad 4\end{array}$ $\begin{array}{lll}1.8 \times & 82 & 4\end{array}$ $\begin{array}{lll}1.4 \mathrm{X} & 73 & 3\end{array}$ $1.3 \mathrm{X} \quad 50 \quad 1$

$\begin{array}{lll}1.3 \times & 64 & 7\end{array}$ $\begin{array}{lll}1.8 \times & 76 & 3\end{array}$ $1.6 \times \quad 55 \quad 3$ $\begin{array}{rrr}1.6 x & 55 & 3 \\ 1.3 \times & 102 & 3 \\ 1.9 \times & 75 & 3\end{array}$

$\begin{array}{lll}1.4 \times \quad 57 & 3\end{array}$ $\begin{array}{lll}1.5 \times & 78 & 3\end{array}$ $1.5 \mathrm{x} \quad 51 \quad 1$ $\begin{array}{lll}1.1 \times & 94 & 3 \\ 1.78 & 56 & 3\end{array}$

$\begin{array}{lll}1.2 \times & 97 & 3 \\ & & \end{array}$ $\begin{array}{lll}1.3 \times & 98 & 3\end{array}$ $\begin{array}{lll}2.5 \times & 39 & 1\end{array}$ $\begin{array}{lll}2.2 \mathrm{X} & 75 & 1 \\ 1.4 \mathrm{X} & 94 & 3\end{array}$

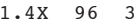
$1.6 \times 114 \quad 4$ $1.2 \times 101 \quad 3$ $1.3 \times 100 \quad 3$ 1.3X $85 \quad 2$ 


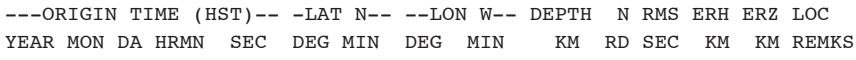

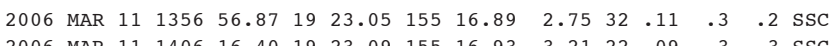

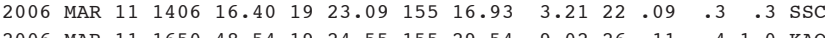

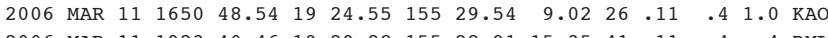

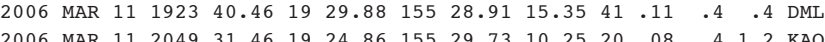

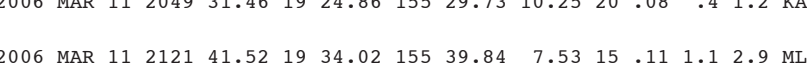

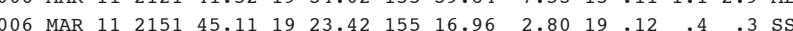

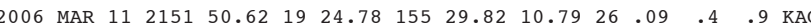

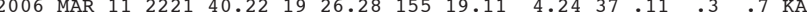

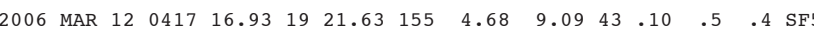

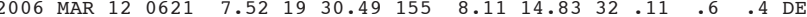

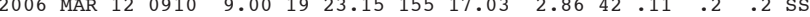

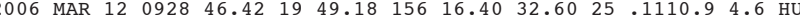

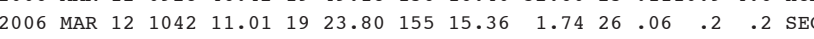

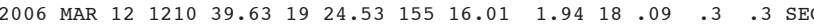

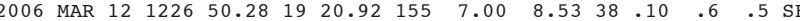

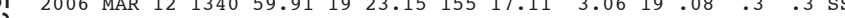

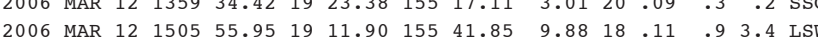

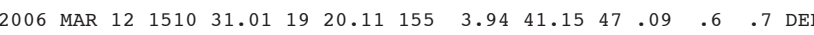

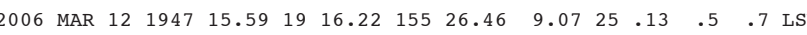
$\begin{array}{lllllllllllllll}2006 & \text { MAR } & 12 & 2044 & 45.54 & 19 & 20.25 & 155 & 8.23 & 8.77 & 44 & .12 & .5 & .5 & \text { SF } 4\end{array}$

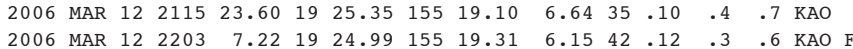

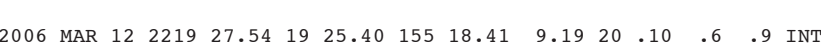

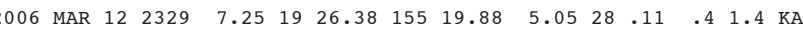

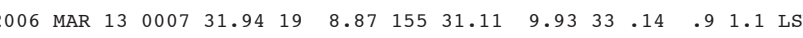
$\begin{array}{llllllllllllll}2006 & \text { MAR } 13 & 0206 & 37.29 & 19 & 25.17 & 155 & 19.12 & 7.28 & 27 & .08 & .4 & .7 & \text { KAO }\end{array}$

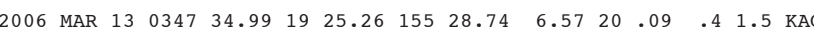

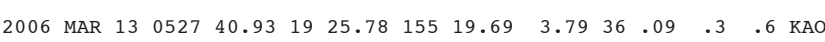

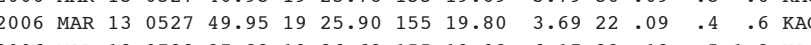

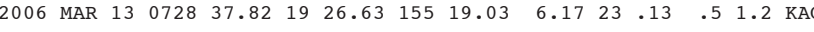

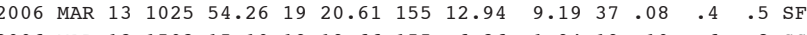

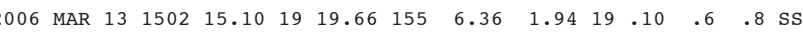

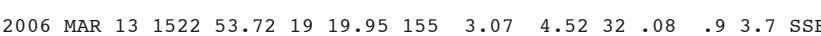

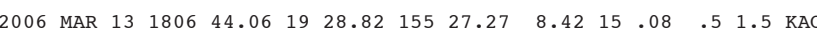

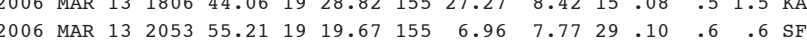
$\begin{array}{lllllllllllllll}2006 & \text { MAR } 14 & 0134 & 58.00 & 19 & 26.03 & 155 & 24.27 & 10.54 & 22 & .11 & .4 & 1.1 & \text { KAO } \\ 2006 & \text { MAR } & 14 & 0705 & 16.42 & 19 & 21.92 & 155 & 9.95 & 2.60 & 19 & .10 & .6 & .4 & \text { SER }\end{array}$

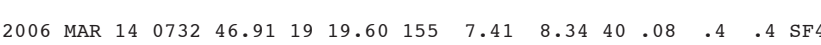

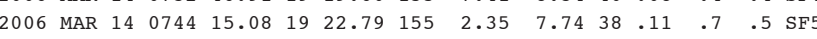

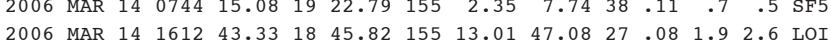

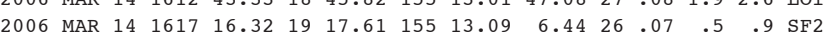
$\begin{array}{llllllllllllllll}2006 & \text { MAR } & 14 & 2242 & 11.38 & 19 & 20.38 & 155 & 13.10 & 6.02 & 28 & .11 & .5 & 1.0 & \text { SF2 }\end{array}$
PREF AZ MIN 35 $1.7 \times \quad 39 \quad 1$ $1.6 \times 61$ $\begin{array}{lll}2.0 \mathrm{X} & 59 & 4 \\ 1.2 \mathrm{x} & 68 & 6\end{array}$

$1.2 \times 221 \quad 11$ $1.3 \times \quad 66 \quad 0$ $1.5 \times \quad 55 \quad 6$ $1.8 \times \quad 58 \quad 3$ $2.4 \times 158 \quad 5$

$1.4 \times 121 \quad 13$ $1.7 \times 266$ $2.3 \times \quad 41 \quad 0$ $2.4 \times 32063$ $1.8 \times \quad 65 \quad 2$

$\begin{array}{lll}1.3 \times & 139 \quad 2\end{array}$ $1.6 \times 177 \quad 5$ $1.5 \times \quad 66$ $1.6 \times \quad 69$ $1.1 \times 136$

$2.5 \times 195$ $1.5 \times 166$ $2.2 \times 177$ $1.4 \times \quad 83$$$
\begin{array}{lll}
.4 \times \quad 92 & 1
\end{array}
$$
$\begin{array}{lll}.4 \times \quad 92 & 1\end{array}$ $1.2 \times 106 \quad 4$ $1.8 \times 26212$ $1.3 \times-63$

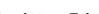
$1.5 \times \quad 99$ $\begin{array}{lll}1.5 \times & 106 & 3 \\ 1.6 \times & 65 & 4\end{array}$ $\begin{array}{rrr}1.6 \times & 65 & 4 \\ 1.1 \times & 197 & 7\end{array}$

$1.4 \times 216 \quad 8$ $.9 \times 108 \quad 6$ $1.1 \times 1725$ $\begin{array}{rrr}1.3 \times & 63 & 7 \\ 1.4 \times & 149 & 2\end{array}$

$1.7 \times 137 \quad 4$ $\begin{array}{llll}2.0 \times & 187 & 4 \\ 1.7 \times & 297 & 54\end{array}$ $1.7 \times 29754$ $\begin{array}{lll}1.4 \times 1 \times 126 & 4\end{array}$
---ORIGIN TIME (HST)-- -LAT N-- --LON W-- DEPTH N RMS ERH ERZ LOC YEAR MON DA HRMN SEC DEG MIN DEG MIN KM RD SEC KM KM REMKS

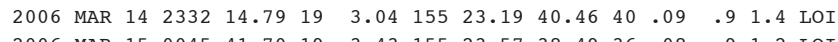

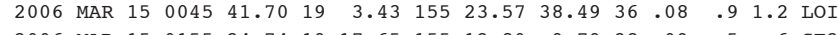

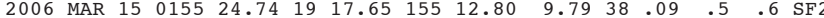

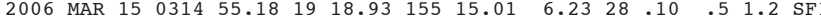

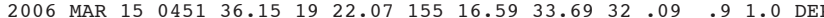

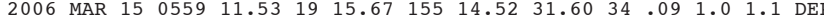
$\begin{array}{lllllllllllllll}2006 & \text { MAR } & 15 & 0726 & 47.74 & 19 & 18.09 & 155 & 13.02 & 4.04 & 22 & .07 & .9 & 1.4 & \text { SS }\end{array}$

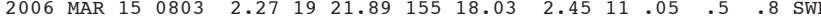

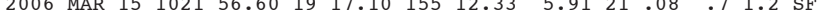
$\begin{array}{lllllllllllllll}2006 & \text { MAR } & 15 & 1050 & 49.14 & 19 & 18.20 & 155 & 12.97 & 10.07 & 42 & .09 & .4 & .5 & \text { SF2 }\end{array}$

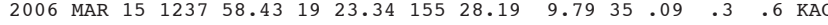

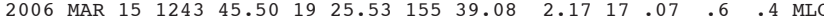
2006 MAR 151307

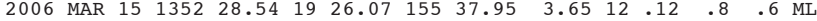

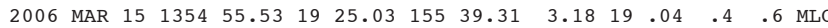

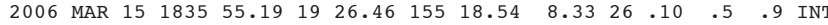

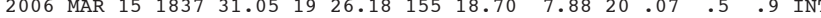

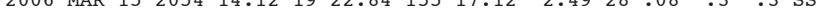

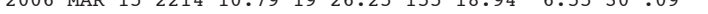

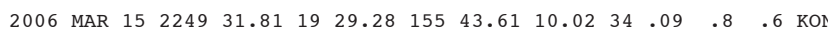

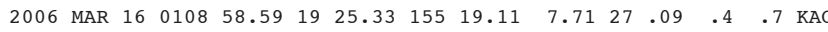

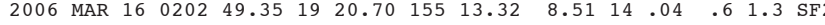

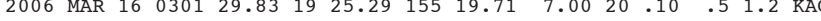

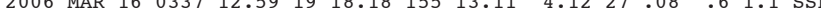

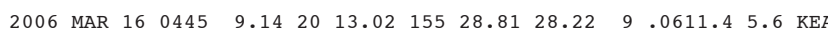

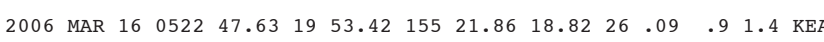

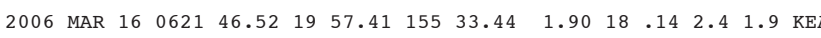

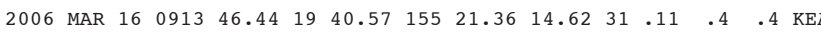

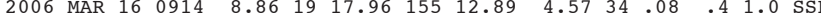
$.4 \quad .4 \quad \mathrm{SF} 2$

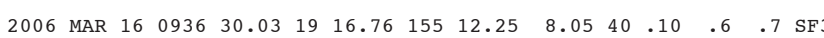

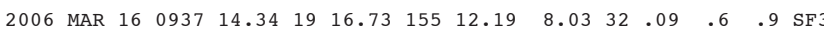

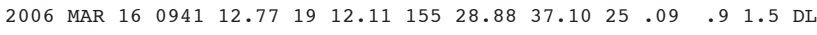

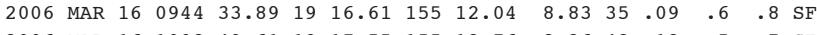
$\begin{array}{llllllllllllll}2006 & \text { MAR } 16 & 1008 & 49.61 & 19 & 17.55 & 155 & 12.76 & 8.26 & 42 & .12 & .5 & .7 & \text { SF2 }\end{array}$

$\begin{array}{lllllllllllllll}2006 & \text { MAR } 16 & 1017 & 30.32 & 19 & 17.28 & 155 & 12.53 & 6.60 & 35 & .13 & .6 & .9 & \text { SF2 }\end{array}$

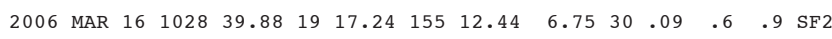

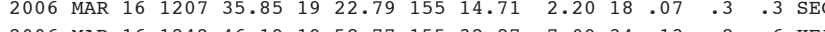

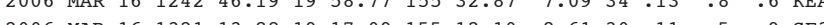

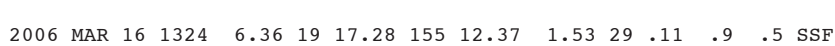

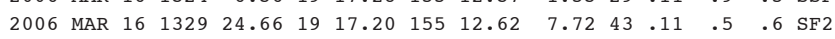
$\begin{array}{lllllllllllllll}2006 & \text { MAR } & 16 & 1329 & 24.66 & 19 & 17.20 & 155 & 12.62 & 7.72 & 43 & .11 & .5 & .6 & \text { SF2 } \\ 2006 & \text { MAR } & 16 & 1706 & 56.51 & 19 & 21.72 & 155 & 30.13 & 10.64 & 28 & .09 & .4 & .9 & \text { KAO }\end{array}$

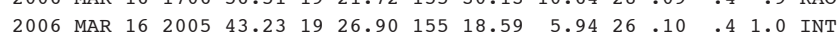
$\begin{array}{llllllllllllllll}2006 & \text { MAR } & 16 & 2011 & 33.98 & 19 & 22.33 & 155 & 17.54 & 3.89 & 18 & .07 & .4 & .5 & \text { SSC }\end{array}$
PREF AZ MIN 36

$.8 \times 22014$ $\begin{array}{rrr}.7 \times & 209 & 14 \\ 2.5 \times 175 & 9\end{array}$ $1.3 \times 113 \quad 4$ 1.6X $66 \quad 1$

$\begin{array}{lll}1.7 \times \quad 210 \quad 3 & 3\end{array}$

$.9 \times 158 \quad 2$

$2.5 \mathrm{X} 172 \quad 8$

$\begin{array}{lll}1.4 \mathrm{X} & 46 \quad 2\end{array}$ $1.1 \times 196 \quad 3$ $\begin{array}{lll}1.9 \times \quad 226 & 6\end{array}$ $1.3 \times 186 \quad 3$ 1.6X $121 \quad 3$

$1.1 \times \quad 75 \quad 3$ $\begin{array}{lll}1.1 \mathrm{X} & 94 & 2\end{array}$ $\begin{array}{lll}1.6 \times & 49 & 1\end{array}$ $\begin{array}{llll}1.4 \times \quad 126 & 3\end{array}$ $1.7 \times \quad 213 \quad 13$

$1.2 \times \quad 83 \quad 3$ $\begin{array}{lll}1.0 \times 119 \quad 4 & 4\end{array}$ $1.0 \times 123 \quad 3$ $\begin{array}{lll}.3 \times & 98 & 2\end{array}$ $.3 \times 231 \quad 2$

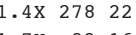
$\begin{array}{lll}1.7 \times \quad 82 & 16\end{array}$ $2.5 \times 172 \quad 8$

$1.9 \times 2092$ $\begin{array}{lll}1.5 \times 236 & 2 \\ 1.7 \times & 101 & 5\end{array}$ $\begin{array}{lll}1.7 \times & 101 & 5 \\ 1.5 \times & 234 & 3\end{array}$ $\begin{array}{lll}1.0 \times & 172 \quad 2\end{array}$

1.9x $206 \quad 2$ $1.2 \times \quad 237 \quad 2$
$1.3 \times$ $\begin{array}{lll}1.3 \times & 80 & 2 \\ 2.0 \times & 250 & 24\end{array}$ $1.3 \times 233 \quad 2$

$1.3 \times 220 \quad 2$ $\begin{array}{rrr}1.9 \times & 185 & 2 \\ 1.3 \times & 90 & 5\end{array}$ $\begin{array}{lll}1.3 \times & 90 & 5 \\ 1.2 x & 86 & 3\end{array}$ $1.2 \times \quad 56 \quad 2$ 
---ORIGIN TIME (HST)-- -LAT N-- --LON W-- DEPTH N RMS ERH ERZ LOC

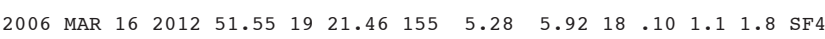
$\begin{array}{llllllllllllll}2006 & \text { MAR } 16 & 2013 & 40.47 & 19 & 21.69 & 155 & 18.05 & 2.61 & 21 & .07 & .3 & .6 & \text { SWR }\end{array}$

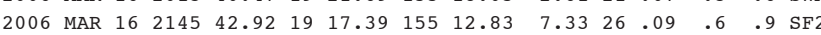

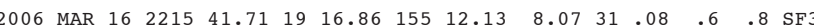

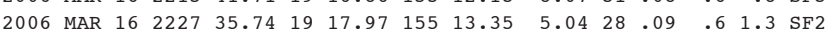

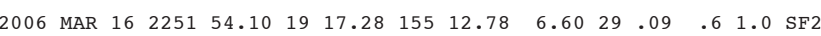

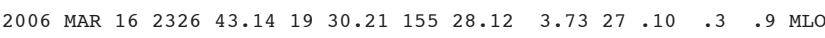
$\begin{array}{lllllllllllllll}2006 & \text { MAR } & 17 & 0334 & 30.49 & 19 & 17.26 & 155 & 12.38 & 8.22 & 37 & .12 & .5 & .6 & \text { SF2 }\end{array}$

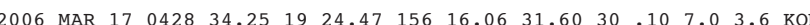

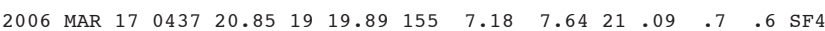

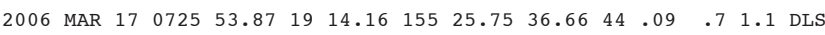

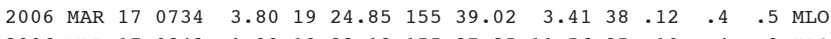

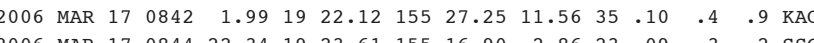

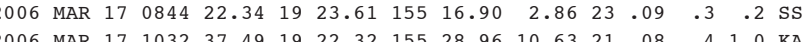

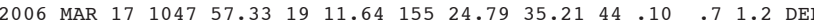

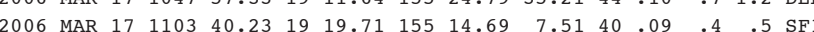
$\begin{array}{lllllllllllllll}2006 & \text { MAR } & 17 & 1121 & 47.13 & 19 & 22.07 & 155 & 27.18 & 11.67 & 28 & 11 & .5 & .5 & \text { SF }\end{array}$

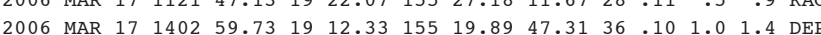

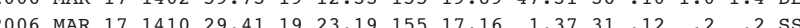

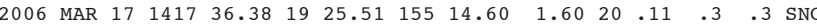

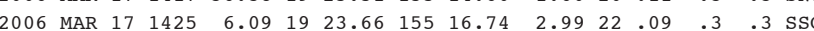
$\begin{array}{llllllllllllll}2006 & \text { MAR } & 17 & 1446 & 7.83 & 19 & 22.33 & 155 & 17.03 & 2.96 & 38 & .12 & .2 & .3 \\ \text { SSC }\end{array}$

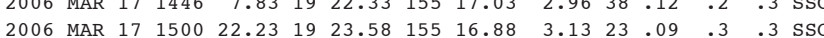

of 2006 MAR $17 \quad 1602 \quad 24.91 \quad 18 \quad 59.55 \quad 155 \quad 30.23 \quad 42.50 \quad 17 \quad 07 \quad 1.7 \quad 3.1 \quad$ DLS

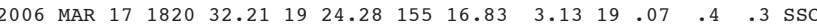

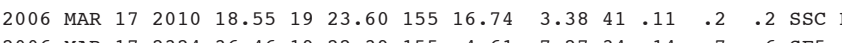

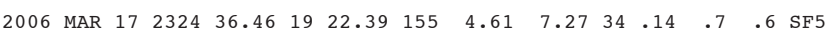

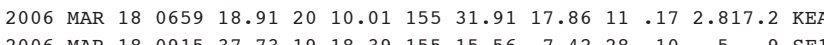

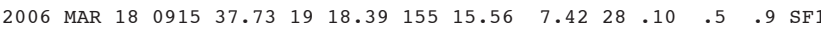

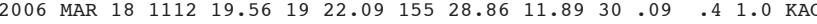

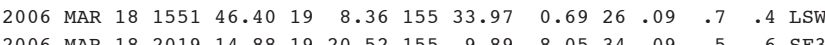

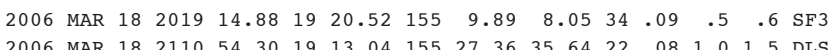

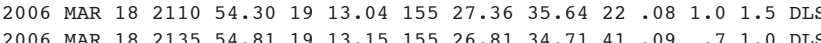

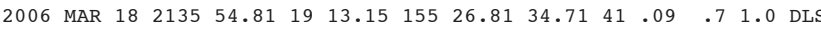

$\begin{array}{llllllllllllll}2006 & \text { MAR } 18 & 2259 & 25.20 & 19 & 13.25 & 155 & 26.92 & 34.79 & 37 & .09 & .7 & 1.1 & \text { DLS }\end{array}$

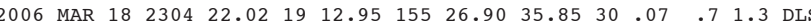
$\begin{array}{lllllllllllllll}2006 & \text { MAR } & 19 & 0057 & 5.43 & 19 & 22.74 & 155 & 26.66 & 10.76 & 23 & .08 & .4 & .9 & \text { KAO }\end{array}$ $\begin{array}{llllllllllllll}2006 & \text { MAR } & 19 & 0153 & 5.45 & 19 & 29.05 & 155 & 49.67 & 22.57 & 17 & .15 & 1.8 & 2.8 \\ 200 N\end{array}$ 2006 MAR $190240 \quad 13.73 \quad 19 \quad 21.04 \quad 155 \quad 55.52 \quad 11.37 \quad 13 \quad .1010 .8 \quad 2.7$ KON

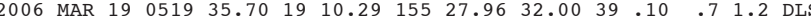

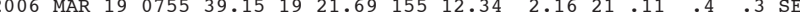

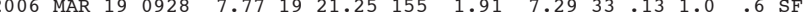

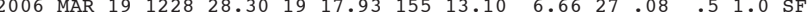
2006 MAR $19 \begin{array}{llllllllllllll}1320 & 41.67 & 19 & 16.90 & 155 & 28.33 & 9.06 & 38 & .13 & .4 & .7 & \text { LSW }\end{array}$
PREF AZ MIN $1.2 \times 220 \quad 5$ $1.2 \times \quad 65 \quad 4$ $1.3 \times 232$ $1.3 \times 232$ (2)

$1.1 \times 211 \quad 1$ $1.8 \times \quad 89 \quad 3$ $1.5 \times 196 \quad 2$ $2.4 \times 30460$ .3x 2126

$\begin{array}{lll}1.9 \times & 125 & 8 \\ 2.5 \times & 190 & 2\end{array}$ $\begin{array}{lll}2.5 \times & 190 \quad 2\end{array}$ $\begin{array}{lll}1.5 \times & 85 & 1 \\ 1.6 x & 44 & 0\end{array}$ $\begin{array}{lll}1.6 \times & 44 & 0 \\ 1.3 \mathrm{X} & 76 & 2\end{array}$

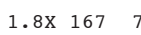
$\begin{array}{lll}1.5 \mathrm{X} & 86 & 5\end{array}$ $\begin{array}{lll}1.5 \mathrm{X} & 86 & 5 \\ 1.1 \mathrm{x} & 86 & 1\end{array}$ $1.8 \times 193 \quad 11$ $\begin{array}{lll}1.6 \mathrm{X} & 42 \quad 1\end{array}$

1.0X 1492 $1.4 \times \quad 56$ $1.9 \mathrm{X} \quad 61 \quad 2$ $\begin{array}{lll}1.6 \mathrm{X} & 76 & 0\end{array}$ $1.4 \times 29529$

$1.3 \times \quad 94 \quad 1$ $\begin{array}{lll}1.9 \times & 44 & 1\end{array}$ $\begin{array}{lll}1.5 \times & 175 & 4\end{array}$ $\begin{array}{rrr}2.1 \times & 317 & 36 \\ 1.0 x & 133 & 4\end{array}$

$\begin{array}{lll}1.5 \times & 77 \quad 2\end{array}$ $\begin{array}{llll}1.5 \times & 197 \quad 11\end{array}$ 1.5X $170 \quad 4$ $\begin{array}{lll}1.5 \times 117 & 6\end{array}$ $2.1 \times 124 \quad 7$

$\begin{array}{lll}1.8 \times & 121 & 7\end{array}$ $\begin{array}{llll}1.5 \times & 123 \quad 7\end{array}$ $\begin{array}{lll}1.3 \times & 76 \quad 2\end{array}$ $\begin{array}{lll}1.3 \times 280 & 22 \\ 1.2 \times 315 & 27\end{array}$

$1.9 \times 110 \quad 1$ $1.7 \times 122$ $1.7 \times 2117$ $\begin{array}{rrr}1.3 \times & 106 \\ 1.9 \times & 87\end{array}$
--ORTGIN TIME (HST)-- -LAT N-- --LON W-- DEPTH N RMS ERH ERZ LOC YEAR MON DA HRMN SEC DEG MIN DEG MIN KM RD SEC KM KM REMKS

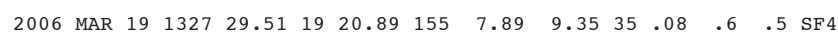
$\begin{array}{llllllllllllll}2006 & \text { MAR } 19 & 1429 & 9.00 & 19 & 19.48 & 155 & 11.80 & 5.12 & 26 & .08 & .5 & 1.6 & \mathrm{SF} 3\end{array}$

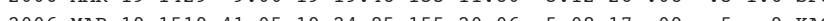
2006 MAR $19152740.271927 .0815520 .06 \quad 5.08 \quad 17.09 \quad .5 \quad .9$ KAO 2006 MAR $19214935.981927 .0815529 .78 \quad 8.93 \quad 34.12 \quad .41 .2$ kAO

$\begin{array}{llllllllllllll}2006 & \text { MAR } 19 & 2345 & 41.98 & 19 & 21.71 & 155 & 48.78 & 12.84 & 36 & .08 & .8 & .4 & \text { KON }\end{array}$

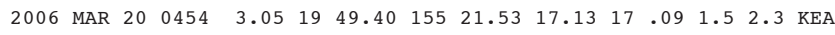

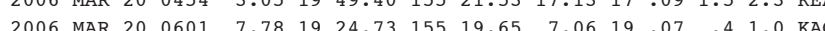

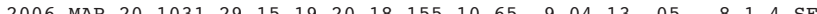

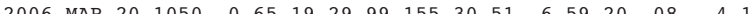

$\begin{array}{llllllllllllll}2006 & \text { MAR } 20 & 1112 & 20.65 & 19 & 23.30 & 155 & 17.00 & 2.94 & 28 & 07 & .3 & .2 & \text { SSC }\end{array}$

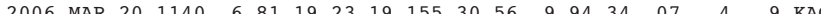
$\begin{array}{lllllllllll}2006 & .9 & \text { KAO }\end{array}$

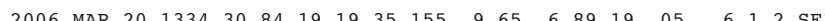

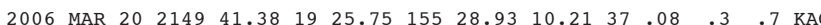

$\begin{array}{lllllllllllll}2006 & \text { MAR } 20 & 2311 & 56.63 & 19 & 22.93 & 155 & 26.32 & 12.25 & 23 & .09 & .4 & .9\end{array}$

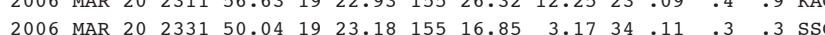

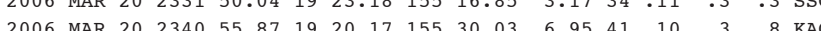

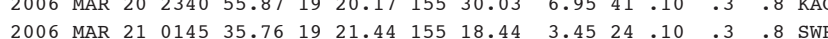

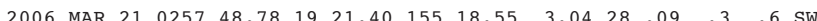

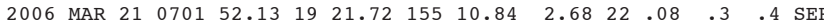

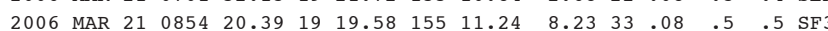

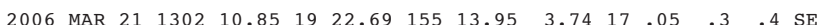

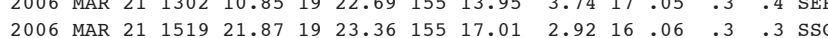

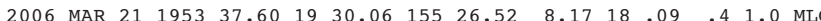

$\begin{array}{lllllllllllll}2006 & \text { MAR } 21 & 2012 & 24.70 & 19 & 32.57 & 155 & 42.18 & 5.64 & 20 & .15 & .7 & 3.8 \\ \text { MLO }\end{array}$

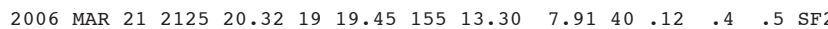

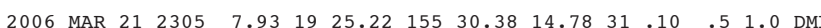

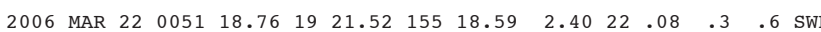

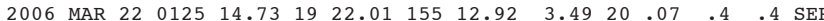

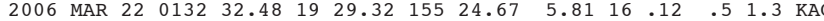
$\begin{array}{lllllllllllll}2006 & \text { MAR } 22 & 0235 & 13.76 & 19 & 26.80 & 155 & 22.71 & 10.02 & 27 & .09 & .4 & 1.0\end{array}$

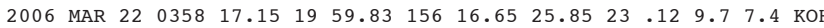

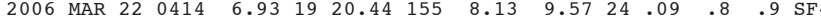

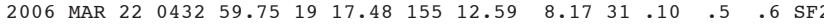

$\begin{array}{llllllllllllll}2006 & \text { MAR } 22 & 0438 & 31.70 & 19 & 57.55 & 155 & 42.67 & 8.93 & 39 & .08 & .9 & .7 & \text { KOH }\end{array}$

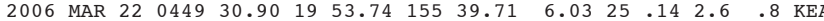

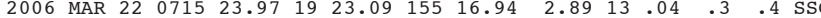

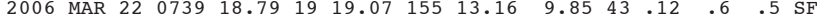
$\begin{array}{lllllllllllllll}2006 & \text { MAR } & 22 & 1237 & 7.96 & 19 & 22.82 & 155 & 14.15 & 3.58 & 16 & .07 & .3 & .4 & \text { SEC }\end{array}$

$\begin{array}{llllllllllllll}2006 & \text { MAR } 22 & 1538 & 49.61 & 19 & 13.08 & 155 & 32.36 & 8.13 & 21 & .10 & .4 & 1.3 & \text { LSW }\end{array}$

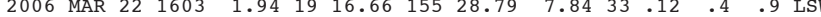

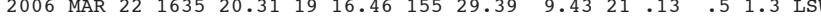

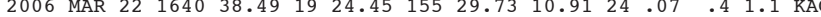
$\begin{array}{lllllllllllllll}2006 & \text { MAR } & 22 & 1641 & 53.98 & 19 & 24.52 & 155 & 29.83 & 11.07 & 27 & .09 & .5 & 1.0 & \text { KAO }\end{array}$
PREF AZ MIN 38 $.6 \times 170$ $.4 \times 148 \quad 5$ $\begin{array}{lll}.8 \times & 84 & 2 \\ .5 \times & 72 & 9\end{array}$ $1.7 \times \quad 167 \quad 6$ $2.1 \times 15515$ $.3 \times 28111$ $1.0 \times 155 \quad 5$ $1.3 \times 108 \quad 6$

$1.6 \times \quad 46 \quad 0$ $1.6 \times 82 \quad 55$ $1.7 x \quad 90 \quad 5$ $1.4 \times \quad 51 \quad 6$

$1.0 \times \quad 72 \quad 2$ $1.9 \times \quad 46 \quad 0$ $\begin{array}{lll}2.2 \mathrm{X} & 68 & 6 \\ 1.3 \mathrm{X} & 72 & 4\end{array}$ $1.4 \mathrm{X} \quad 74 \quad 5$

$\begin{array}{lll}1.5 \times & 141 & 2 \\ 1.6 \times & 166 & 6\end{array}$ $\begin{array}{rrrr}1.6 \times & 166 & 6 \\ 1.6 \times & 94 & 1\end{array}$ $\begin{array}{lll}1.6 \mathrm{X} & 94 & 1 \\ 1.1 \mathrm{X} & 67 & 0\end{array}$ $\begin{array}{lll}1.3 \mathrm{X} & 97 & 4\end{array}$

$\begin{array}{lll}1.3 \times & 114 & 7\end{array}$ $\begin{array}{lll}2.0 \mathrm{X} & 72 & 4 \\ 1.4 \mathrm{X} & 70 & 5\end{array}$ $\begin{array}{lll}1.1 \mathrm{x} & 73 \quad 4\end{array}$ 1.7x $111 \quad 1$

$1.1 \times 100 \quad 3$ $\begin{array}{rrr}.1 \times & 114 & 6 \\ .3 \times & 306 & 91\end{array}$ $1.3 \times 193 \quad 5$ 1.3X $204 \quad 2$

$3.5 \times 284 \quad 39$ $\begin{array}{rrr}.1 \times 26325 \\ .6 \times \quad 95 & 1\end{array}$ $\begin{array}{rrr}.6 \times & 95 & 1 \\ 2.5 \times & 175 & 7\end{array}$ $\begin{array}{lll}1.4 \times & 85 & 2\end{array}$

$\begin{array}{lll}1.6 \times & 124 & 5\end{array}$ $1.9 \mathrm{X} \quad 85 \quad 4$

$\begin{array}{lll}.4 \times & 813\end{array}$ $\begin{array}{lll}.6 \mathrm{x} & 71 & 5\end{array}$ 
---ORIGIN TIME (HST)-- -LAT N-- --LON W-- DEPTH N RMS ERH ERZ LOC PREF AZ MIN 39 YEAR MON DA HRMN SEC DEG MIN DEG MIN KM RD SEC KM KM REMKS MAG GAP DS

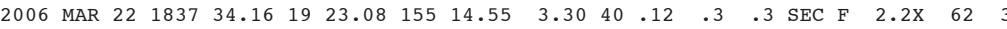

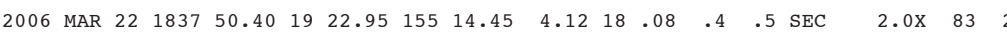

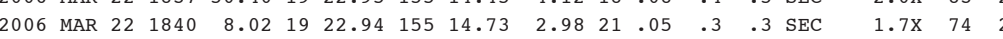

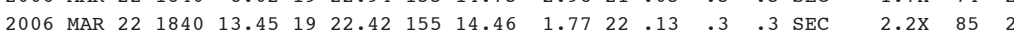

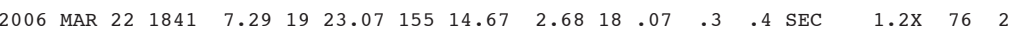

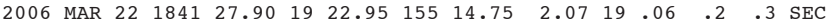

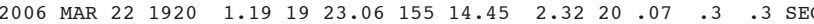

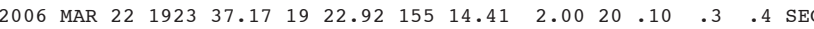

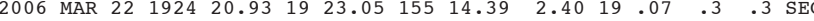

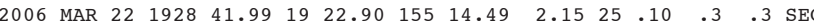

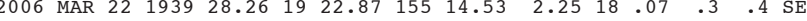

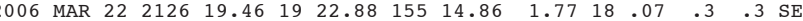

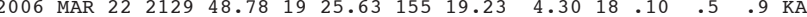

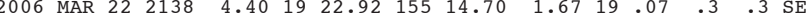
$\begin{array}{llllllllllllll}2006 & \text { MAR } 22 & 2340 & 47.16 & 19 & 20.95 & 155 & 10.63 & 8.61 & 22 & .08 & .5 & .5 & \text { SF } 3\end{array}$

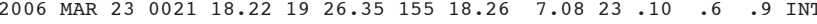

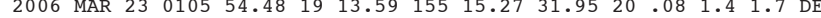

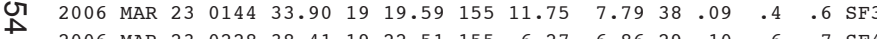
$\begin{array}{lllllllllllllll}2006 & \text { MAR } & 23 & 0228 & 38.41 & 19 & 22.51 & 155 & 6.27 & 6.86 & 29 & .10 & .6 & .7 & \text { SF } 4 \\ 2006 \text { MAR } & 23 & 0543 & 53.89 & 19 & 21.56 & 155 & 18.27 & 3.15 & 31 & 10 & .3 & .6 & \text { SWR }\end{array}$

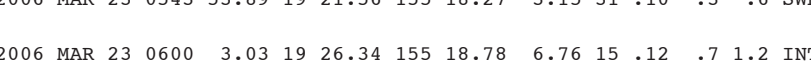
$\begin{array}{llllllllll} & \end{array}$

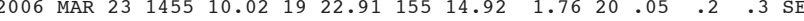

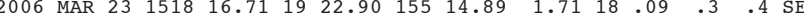

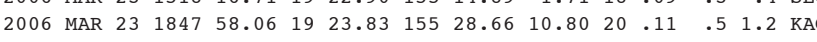

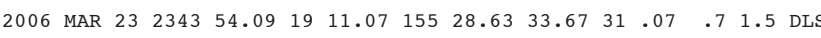

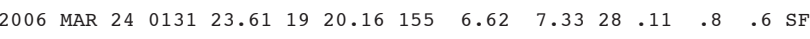

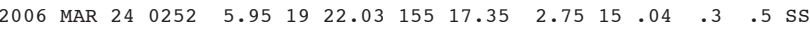

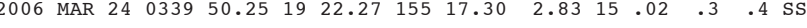

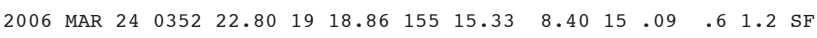

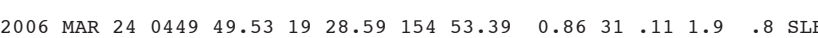

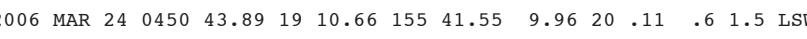
$\begin{array}{llllllllllllll}2006 & \text { MAR } 24 & 0516 & 5.88 & 19 & 15.14 & 155 & 34.07 & 8.20 & 29 & .15 & .6 & 1.8 & \text { LS }\end{array}$

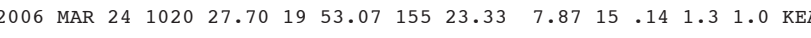

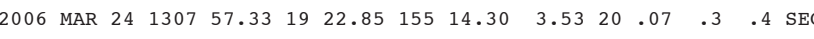

$\begin{array}{llllllllllllll}2006 & \text { MAR } 24 & 1328 & 40.95 & 19 & 23.14 & 155 & 16.97 & 2.92 & 26 & .09 & .3 & .2 & \text { SSC }\end{array}$ $\begin{array}{llllllllllllll}2006 & \text { MAR } 24 & 1631 & 40.28 & 19 & 21.88 & 155 & 4.73 & 6.60 & 19 & .12 & .8 & 1.2 & \text { SF5 }\end{array}$

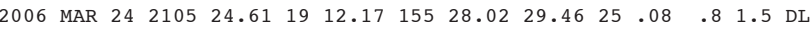

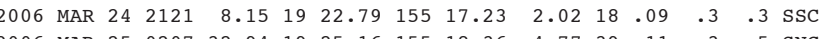

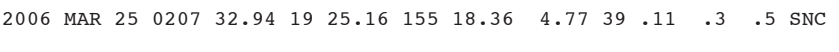

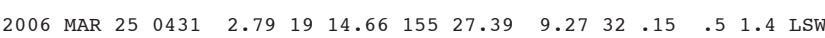

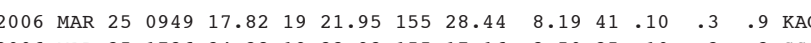

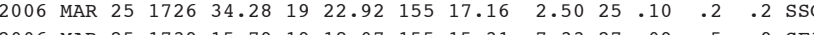

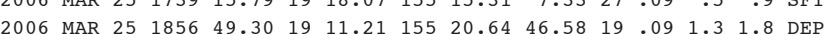

$1.5 \times \quad 74 \quad 2$ $1.6 \times \quad 79 \quad 2$ $1.8 \times \quad 79 \quad 2$ $\begin{array}{lll}1.5 \mathrm{x} & 80 & 2\end{array}$ $\begin{array}{lll}2.0 \times & 68 \quad 2\end{array}$

$\begin{array}{lll}1.5 \times & 81 & 3\end{array}$ $\begin{array}{lll}1.4 \mathrm{X} & 71 \quad 2\end{array}$ $\begin{array}{rrr}.9 \times & 93 & 3\end{array}$ $1.5 \mathrm{X} \quad 75 \quad 2$

$1.3 \times 165 \quad 3$

1.5X $91 \quad 2$ $1.4 \times 2507$ $1.5 \times \quad 92$ $1.2 \times \quad 184 \quad 3$ $2.0 \times \quad 55 \quad 4$

$.9 \times 105 \quad 3$ $1.2 \times 122 \quad 5$ $1.4 \times \quad 69$ $1.2 \times \quad 75$ $1.1 \times \quad 69 \quad 3$

$\begin{array}{ccc}1.5 \times \quad 88 & 3\end{array}$ $1.7 \times 176$ $\begin{array}{lll}1.0 \times \quad 62 & 3\end{array}$ $\begin{array}{lll}.1 \times & 61 \quad 2\end{array}$ $.8 \times 118 \quad 5$

$.2 \times 26920$ $1.5 \times 1168$ $1.6 \times 110 \quad 7$ $\begin{array}{lll}1.2 \times \quad 235 \quad 5 & 5\end{array}$ $1.4 \times \quad 85 \quad 2$

$\begin{array}{lll}1.5 \times & 47 & 0\end{array}$ $\begin{array}{llll}1.4 \times \quad 226 & 5\end{array}$ $1.6 \times 1065$ $\begin{array}{lll}1.3 \times & 61 & 1\end{array}$

$\begin{array}{lll}1.4 \times & 107 \quad 5\end{array}$ $\begin{array}{lll}2.0 \times & 64 \quad 2\end{array}$ $\begin{array}{lrr}1.4 \times & 49 & 1 \\ 1.1 \times & 114 & 4\end{array}$ $\begin{array}{lll}1.1 \times & 114 & 4 \\ 1.2 \times & 245 & 13\end{array}$
---ORIGIN TIME (HST)-- -LAT N-- --LON W-- DEPTH N RMS ERH ERZ LOC YEAR MON DA HRMN SEC DEG MIN DEG MIN KM RD SEC

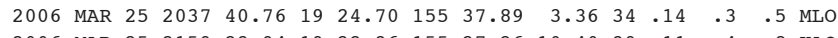

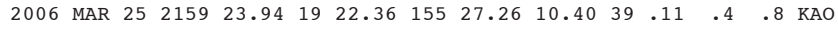

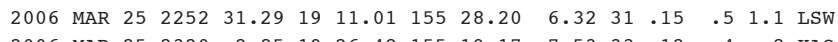

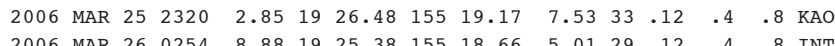

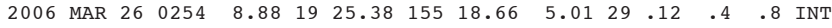

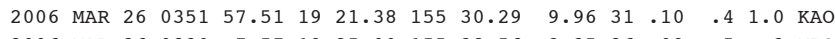

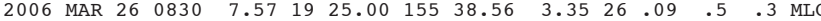

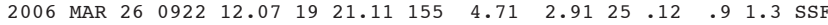

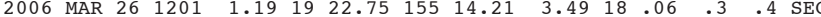
$\begin{array}{lllllllllllllll}2006 & \text { MAR } & 26 & 1259 & 54.22 & 19 & 20.09 & 155 & 7.11 & 8.14 & 27 & .07 & .5 & .9 & \text { SF4 }\end{array}$

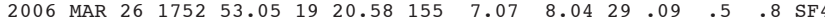
$\begin{array}{lllllllllllllll}2006 & \text { MAR } 26 & 2243 & 45.07 & 19 & 19.86 & 155 & 6.48 & 7.24 & 35 & .09 & .5 & .7 & \text { SF } 4\end{array}$

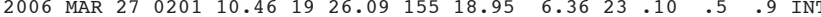

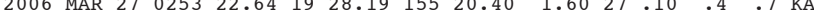

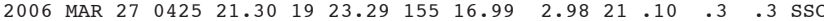

$\begin{array}{lllllllllllll}2006 & \text { MAR } 27 & 0703 & 7.38 & 19 & 18.72 & 155 & 47.22 & 11.69 & 30 & .09 & 1.0 & .4 \\ \text { KON }\end{array}$

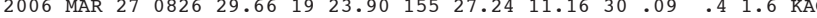
2006 MAR $27 \quad 1001 \quad 1.47 \quad 19$

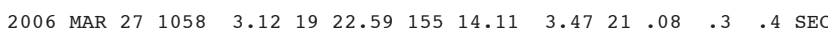

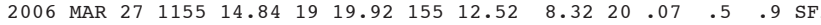

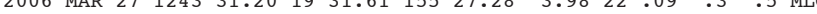

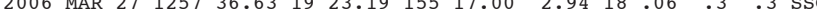

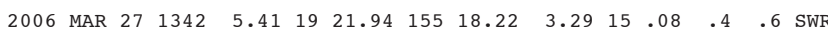

$\begin{array}{llllllllllllll}2006 & \text { MAR } 27 & 1533 & 17.80 & 19 & 18.11 & 155 & 14.76 & 6.57 & 19 & .12 & .9 & 1.3 & \text { SF }\end{array}$

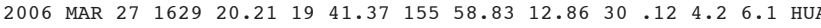

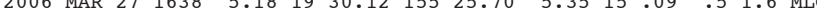

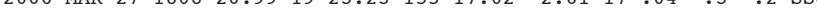

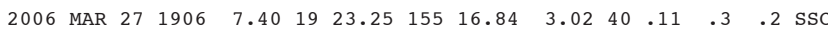

2006 MAR $27 \quad 1918 \quad 39.85 \quad 19 \quad 37.69 \begin{array}{llllllllll}155 & 21.22 & 11.38 & 18 & .11 & .6 & 1.6 & \text { KEA }\end{array}$

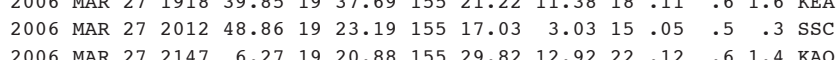

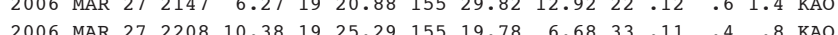

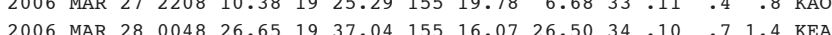
$\begin{array}{llllllllllllll}2006 & \text { MAR } 28 & 0052 & 31.18 & 19 & 25.29 & 155 & 20.04 & 6.64 & 20 & .10 & .5 & 1.3 & \text { KAO }\end{array}$

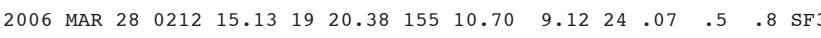

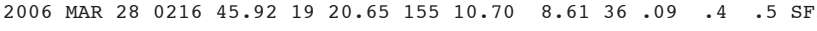

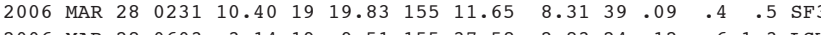

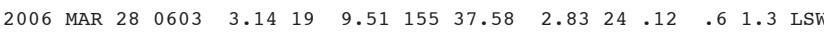

$\begin{array}{lllllllllllllll}2006 & \text { MAR } & 28 & 0828 & 52.28 & 19 & 19.36 & 155 & 13.03 & 7.72 & 35 & .08 & .4 & .6 & \text { SF2 }\end{array}$

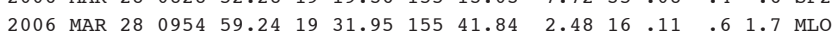

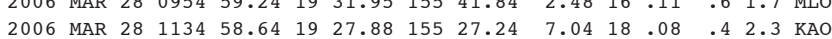

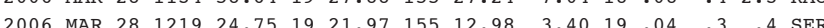

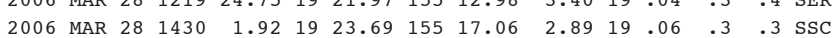
$\begin{array}{rrr}.0 \times & 96 & 1 \\ .4 \times & 65 & 1 \\ 1.18 & 145 & 3\end{array}$ $1.1 \times 145 \quad 3$ $1.4 \times \quad 63 \quad 3$ $1.1 \times \quad 79 \quad 2$

$\begin{array}{lrr}1.3 \times & 93 \quad 5 \\ 1.6 \times & 187 & 2\end{array}$ $\begin{array}{rrr}1.6 \times & 187 \quad 2 \\ 1.2 \times & 210 & 6\end{array}$ $\begin{array}{lll}1.3 \times & 87 & 2\end{array}$ $1.4 \times 163 \quad 5$

$1.6 \times 159 \quad 5$ $\begin{array}{lll}1.6 \times & 184 & 5\end{array}$ $\begin{array}{lll}1.2 \times & 72 & 3\end{array}$ $\begin{array}{lll}.2 x & 51 & 6 \\ .8 X & 46 & 0\end{array}$

$\begin{array}{lll}1.8 \times \quad 221 \quad 14 & \end{array}$ $\begin{array}{lll}1.4 \mathrm{X} & 61 \quad 10\end{array}$ $\begin{array}{lll}2.6 \mathrm{X} & 37 & 0\end{array}$ $\begin{array}{lll}1.9 x & 81 & 2\end{array}$ $\begin{array}{lll}1.7 \times \quad 85 & 2\end{array}$

$\begin{array}{lll}1.1 \times \quad 78 & 5\end{array}$ $\begin{array}{lll}1.2 \times & 135 & 1\end{array}$ $\begin{array}{lll}.4 \times \quad 92 & 3\end{array}$ $1.3 \times 465$ 3

\begin{tabular}{lll}
$.8 \times 145 \quad 3$ \\
\hline
\end{tabular} $2.1 \times 27246$ $\begin{array}{lll}.9 \times & 124 & 4\end{array}$ $\begin{array}{lll}.0 \times & 65 & 0\end{array}$ $2.6 \times \quad 46 \quad 0$

$\begin{array}{lll}.6 \times 154 & 14\end{array}$ $1.8 \times 150 \quad 0$ $\begin{array}{lll}1.1 \mathrm{x} & 78 & 9\end{array}$ $\begin{array}{lll}1.5 \times & 116 & 17\end{array}$

$.9 \times \quad 86 \quad 3$ $\begin{array}{lll}1.2 \times & 127 & 5 \\ 1.7 \times & 82 & 4\end{array}$ $\begin{array}{lll}1.7 x & 82 & 4\end{array}$ $\begin{array}{lll}1.8 \times & 87 & 6\end{array}$ $5 x-79$ $.9 \times 110 \quad 8$ $.9 \times 1108$ $\begin{array}{lll}.5 \mathrm{X} & 83 & 1\end{array}$ $\begin{array}{lll}.6 \times & 73 & 1\end{array}$ 
--ORIGIN TIME (HST)-- -LAT N-- --LON W-- DEPTH N RMS ERH ERZ LOC

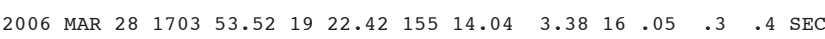
$\begin{array}{llllllllllllll}2006 & \text { MAR } 28 & 1859 & 37.83 & 19 & 20.36 & 155 & 10.78 & 7.98 & 39 & .12 & .5 & .6 & \mathrm{SF} 3\end{array}$

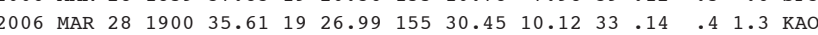
$\begin{array}{llllllllllllll}2006 & \text { MAR } & 28 & 2122 & 58.16 & 19 & 11.61 & 155 & 37.19 & 5.59 & 39.12 & .4 & 1.6 & \text { LSW }\end{array}$

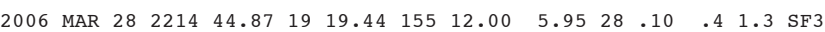

$\begin{array}{llllllllllllll}2006 & \text { MAR } 28 & 2312 & 15.77 & 19 & 18.48 & 155 & 14.81 & 7.05 & 21 & .06 & .5 & 1.1 & \mathrm{SF} 1\end{array}$ $\begin{array}{llllllllllllll}2006 & \text { MAR } 29 & 0228 & 19.60 & 19 & 26.31 & 155 & 29.33 & 9.29 & 36 & .11 & .4 & 1.2 & \text { KAO }\end{array}$ $\begin{array}{llllllllllllll}2006 & \text { MAR } 29 & 0432 & 58.44 & 19 & 22.96 & 155 & 17.09 & 2.80 & 21 & .08 & .3 & .2 & \text { SSC }\end{array}$ $\begin{array}{llllllllllllll}2006 & \text { MAR } 29 & 0433 & 37.54 & 19 & 22.98 & 155 & 16.92 & 3.27 & 44 & .11 & .2 & .2 & \text { SSC }\end{array}$

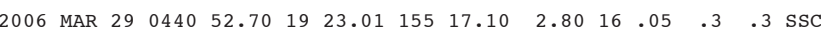

2006 MAR $29 \quad 0450 \quad 44.29 \quad 19 \quad 44.18 \quad 156 \quad 15.33 \quad 30.50 \quad 18.1011 .0 \quad 6.8$ HUA

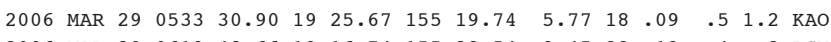
$\begin{array}{llllllllllllll}2006 & \text { MAR } 29 & 0619 & 48.66 & 19 & 16.74 & 155 & 28.54 & 9.45 & 38 & .12 & .4 & .8 & \text { LSW }\end{array}$ $\begin{array}{lllllllllllllll}2006 & \text { MAR } 29 & 0628 & 52.25 & 19 & 22.81 & 155 & 14.14 & 3.71 & 17 & .06 & .3 & .4 & \text { SEC }\end{array}$ $\begin{array}{llllllllllllll}2006 & \text { MAR } 29 & 0703 & 13.40 & 19 & 21.58 & 155 & 11.23 & 2.43 & 16 & .07 & .4 & .6 & \text { SER }\end{array}$

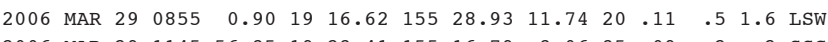
$\begin{array}{llllllllllllll}2006 & \text { MAR } 29 & 1145 & 56.35 & 19 & 23.41 & 155 & 16.79 & 3.06 & 35 & .09 & .2 & .2 & \text { SSC }\end{array}$

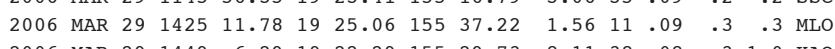

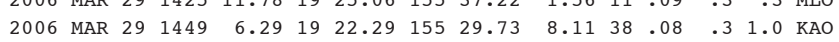

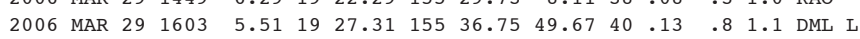

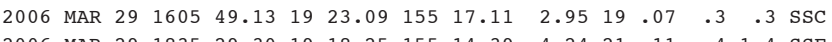

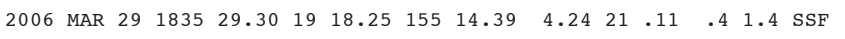

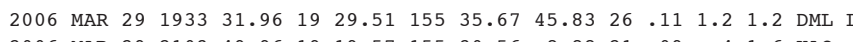

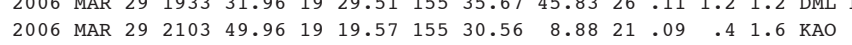

G 2006 MAR $292352 \quad 6.88 \quad 1925.42 \quad 155 \quad 33.61 \quad 40.29 \quad 21.14 \quad 1.1 \quad 2.0$ DML L

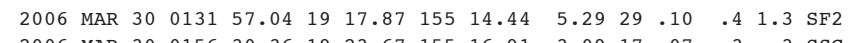

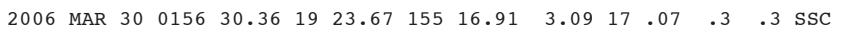

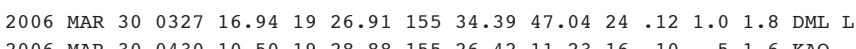

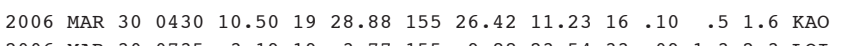

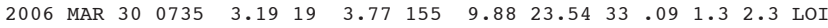

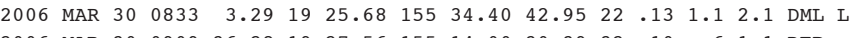

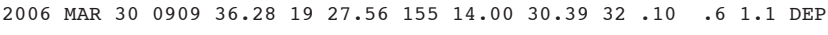

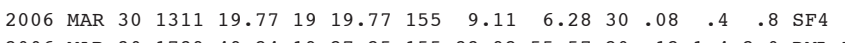
$\begin{array}{llllllllllllll}2006 & \text { MAR } 30 & 1739 & 49.24 & 19 & 27.25 & 155 & 33.92 & 55.57 & 20 & .12 & 1.4 & 2.0 & \text { DML L }\end{array}$

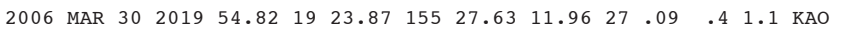

$\begin{array}{lllllllllllll}2006 & \text { MAR } 30 & 2359 & 38.99 & 19 & 25.16 & 155 & 29.97 & 11.17 & 37 & .11 & .4 & .8 \\ \text { KAO }\end{array}$ $\begin{array}{llllllllllllll}2006 & \text { MAR } 31 & 0042 & 39.08 & 19 & 18.53 & 155 & 13.28 & 6.10 & 30 & .10 & .4 & .8 & \text { SF2 }\end{array}$ $\begin{array}{lllllllllllllll}2006 & \text { MAR } & 31 & 0108 & 20.59 & 19 & 22.54 & 155 & 24.91 & 12.65 & 20 & .10 & .5 & .9 & \text { KAO }\end{array}$

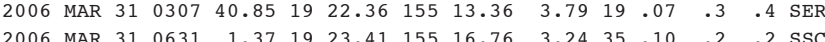

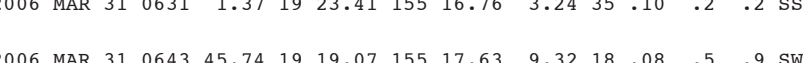
$\begin{array}{lllllllllllllll}2006 & \text { MAR } & 31 & 0643 & 45.74 & 19 & 19.07 & 155 & 17.63 & 9.32 & 18 & .08 & .5 & .9 & \text { SWR }\end{array}$

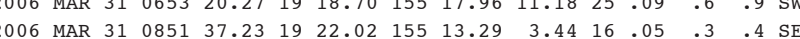

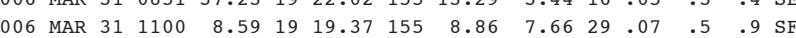

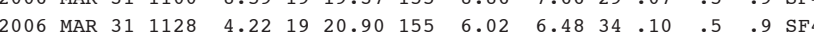

PREF AZ MIN

$1.3 \times \quad 90 \quad 2$ $1.8 \times \quad 81 \quad 5$ $1.6 \times 1908$ $1.2 \mathrm{x} 925$

$\begin{array}{lll}.7 \times & 124 \quad 4\end{array}$ $\begin{array}{lll}1.5 \times & 65 & 11\end{array}$ $\begin{array}{lll}1.4 \mathrm{X} & 48 \\ 2.8 \mathrm{X} & 47\end{array}$ $1.3 \times \quad 89$

2.1X 31774 $.9 \times \quad 99 \quad 4$ $\begin{array}{rrr}.6 \times & 86 & 4 \\ .4 \times & 90 & 2\end{array}$ $1.0 \times \quad 83 \quad 3$

$\begin{array}{lll}1.2 \mathrm{X} & 83 & 4\end{array}$ $2.3 \times \quad 39 \quad 0$ $.9 \mathrm{U} 126 \quad 2$ $\begin{array}{lll}1.3 \mathrm{X} & 60 & 12\end{array}$ $2.9 \times \quad 83$ $\begin{array}{lll}.5 \mathrm{X} & 64 & 1 \\ .2 \mathrm{U} & 92 & 3\end{array}$ $2.0 \times 125 \quad 1$ $\begin{array}{lll}1.0 \mathrm{x} & 76 & 8\end{array}$ $\begin{array}{lll}6 \mathrm{x} & 54 & 1\end{array}$

$1.0 \times 128 \quad 2$ $\begin{array}{lll}1.0 \times \quad 69 & 1\end{array}$ $\begin{array}{ccc}2.2 \mathrm{x} & 52 & 3 \\ 1.1 \mathrm{x} & 91 & 6 \\ 1.8 \mathrm{n} & 275 & 25\end{array}$ 1.8X $275 \quad 25$

$\begin{array}{lll}1.7 x & 73 \quad 3\end{array}$ $\begin{array}{rrr}1.8 \times & 70 & 5 \\ .3 \times & 109 & 5 \\ 2.3 \times & 69 & 3\end{array}$ $2.3 \times \quad 69 \quad 3$ $\begin{array}{lll}1.0 \times \quad 65 & 10\end{array}$
---ORIGIN TTME (HST)-- -LAT N-- --LON W-- DEPTH N RMS ERH ERZ LOC YEAR MON DA HRMN SEC DEG MIN DEG MIN KM RD SEC KM KM REM

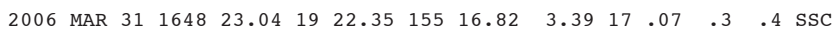
$\begin{array}{llllllllllllll}2006 & \text { MAR } 31 & 1732 & 24.90 & 19 & 26.10 & 155 & 24.21 & 12.09 & 30 & .10 & .5 & .7 & \text { KAO }\end{array}$

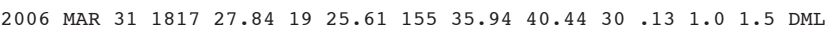
$\begin{array}{lllllllllllllll}2006 & \text { MAR } & 31 & 1938 & 9.83 & 19 & 21.34 & 155 & 18.81 & 3.67 & 15 & .10 & .5 & 1.1 & \text { SWR }\end{array}$

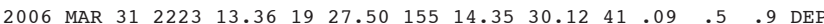

2006 APR $11013929.92 \quad 19 \quad 12.66 \quad 155 \quad 20.10 \quad 46.20 \quad 22.11 \quad 1.1 \quad 1.5$ DEP

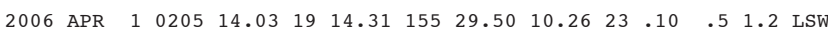
$\begin{array}{lllllllllllllll}2006 & \text { APR } & 1 & 0208 & 6.55 & 19 & 23.10 & 155 & 17.01 & 2.74 & 20 & .08 & .3 & .3 & \mathrm{SSC}\end{array}$ $\begin{array}{llllllllllllll}2006 & \text { APR } & 1 & 0258 & 42.87 & 19 & 29.10 & 155 & 26.70 & 9.02 & 34 & .11 & .4 & .9 \\ \text { KAO }\end{array}$ $\begin{array}{llllllllllllll}2006 & \mathrm{APR} & 1 & 0332 & 8.73 & 19 & 27.98 & 155 & 20.71 & 1.09 & 30 & .11 & .4 & .5 \\ \mathrm{KAO}\end{array}$

$\begin{array}{lllllllllllllll}2006 & \text { APR } & 1 & 0458 & 2.15 & 19 & 18.81 & 155 & 13.18 & 7.41 & 39 & .12 & .4 & .7 & \mathrm{SF} 2\end{array}$ $\begin{array}{lllllllllllllll}2006 & \text { APR } & 1 & 0502 & 37.72 & 19 & 18.40 & 155 & 12.99 & 8.14 & 37 & .11 & .4 & .6 & \mathrm{SF} 2\end{array}$

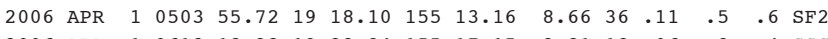

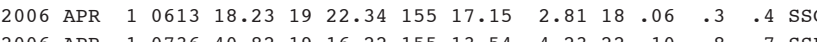
\begin{tabular}{lllllllllllll}
2006 APR & 1 & 0736 & 40.82 & 19 & 16.22 & 155 & 13.54 & 4.23 & 22 & .10 & .8 & .4 \\
\hline
\end{tabular} $\begin{array}{lllllllllllllll}2006 & \text { APR } & 1 & 0918 & 11.57 & 19 & 23.30 & 155 & 14.78 & 3.50 & 20 & .09 & .3 & .4 & \text { SEC }\end{array}$

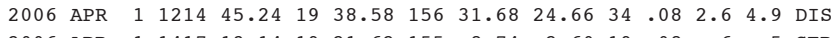

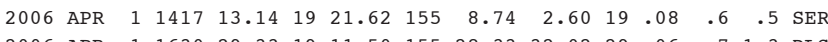

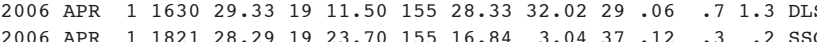

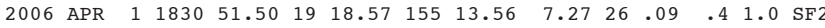
$\begin{array}{lllllllllllllll}2006 & \text { APR } & 1 & 2057 & 0.25 & 19 & 17.41 & 155 & 12.81 & 5.86 & 30 & .08 & 4 & 1.0 & \text { SF2 }\end{array}$

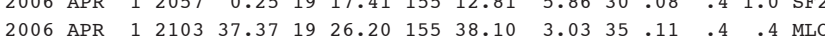

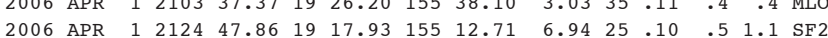

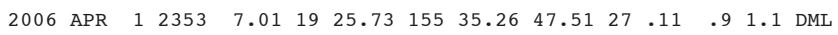

$\begin{array}{lllllllllllllll}2006 & \text { APR } & 2 & 0040 & 3.83 & 19 & 17.22 & 155 & 19.96 & 6.80 & 35 & .12 & .5 & 1.0 & \text { SWR }\end{array}$ $\begin{array}{lllllllllllllll}2006 & \text { APR } & 2 & 0126 & 17.59 & 19 & 30.70 & 155 & 48.37 & 10.70 & 20 & .10 & 1.0 & .7 & \text { KON }\end{array}$

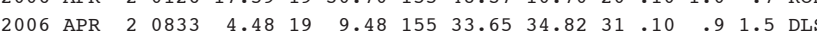
$\begin{array}{lllllllllllllll}2006 & \text { APR } & 2 & 1047 & 49.42 & 19 & 15.75 & 155 & 10.01 & 8.94 & 26 & .09 & .8 & 1.0 & \text { SF } 3\end{array}$

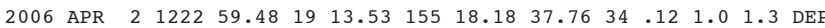

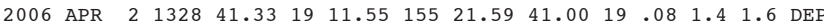
$\begin{array}{llllllllllllll}2006 & \text { APR } & 2 & 1738 & 13.66 & 19 & 22.72 & 155 & 30.39 & 13.76 & 17 & .08 & .5 & 1.4\end{array}$

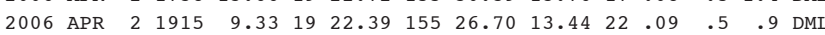

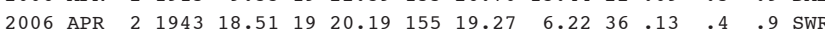

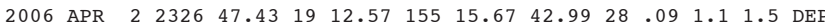

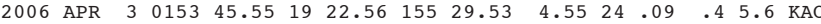
$\begin{array}{lllllllllllllll}2006 & \text { APR } & 3 & 0730 & 44.54 & 19 & 18.33 & 155 & 15.27 & 6.43 & 22 & .11 & .6 & 1.2 & \text { SF } 1\end{array}$

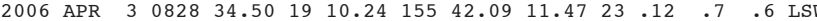
$\begin{array}{lllllllllllllllll}2006 & \text { APR } & 3 & 1014 & 31.36 & 19 & 23.11 & 155 & 23.87 & 13.55 & 23 & .07 & .6 & 1.0 & \text { DMI }\end{array}$ $\begin{array}{lllllllllllllll}2006 & \text { APR } & 3 & 1357 & 57.69 & 19 & 17.56 & 155 & 23.05 & 2.59 & 24 & .11 & .5 & .7 & \text { SWR }\end{array}$

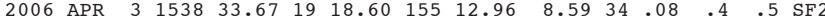

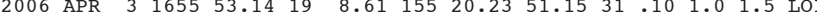
$\begin{array}{lllllllllllllll}2006 & \text { APR } & 3 & 1826 & 26.07 & 19 & 18.04 & 155 & 23.21 & 3.31 & 33 & .12 & .5 & .9 & \text { SWR }\end{array}$ 2006 APR $3 \quad \begin{array}{lllllllllllll}1845 & 16.81 & 19 & 19.84 & 155 & 12.74 & 5.52 & 21 & .11 & .5 & 1.2 & \text { SF2 }\end{array}$ $\begin{array}{llllllllllllllll}2006 & \text { APR } & 4 & 0109 & 49.94 & 19 & 27.42 & 155 & 14.12 & 31.34 & 37 & .10 & .6 & 1.0 & \text { DEP }\end{array}$
PREF AZ MIN 42 $\begin{array}{lll}2 \times & 85 & 5\end{array}$ $\begin{array}{lll}1.2 \times & 85 & 5 \\ 2 \times & 68 & 4\end{array}$

$1.4 \times 20810$ $1.3 \times \quad 90 \quad 2$ $\begin{array}{lll}.2 \mathrm{X} & 48 & 1 \\ 1.2 \mathrm{x} & 71 & 6\end{array}$ $2 \times 516$

$\begin{array}{lll}1.8 \times & 83 & 3\end{array}$ $2.0 \times \quad 97 \quad 3$ $\begin{array}{lll}.8 \times & 98 & 2 \\ 1.2 \times & 59 & 2\end{array}$ $\begin{array}{lll}1.3 \times & 213 & 1\end{array}$

$\begin{array}{lll}1.5 \times & 72 & 3\end{array}$ $\begin{array}{lll}2.2 \times & 329 & 81 \\ 1.4 \times & 167 & 3\end{array}$ $\begin{array}{rrr}.4 \times 167 & 3 \\ 4 \times & 99 & 4\end{array}$

$1.1 \times 127 \quad 3$ $\begin{array}{lll}.3 \times 149 & 1\end{array}$ $\begin{array}{lll}.2 \times 183 \quad 3 & 14\end{array}$ $\begin{array}{lll}.3 \times & 122 \quad 2 \\ 2.1 \times & 87 & 3\end{array}$

$1.3 \times 134 \quad 3$ $\begin{array}{lll}1.3 \times 279 & 22\end{array}$ $\begin{array}{lll}1.5 \times 122 & 10 \\ 1.2 \times & 253 & 6\end{array}$ $\begin{array}{lll}1.2 \times & 253 & 6 \\ 1.7 \times \quad 179 & 8\end{array}$ $\begin{array}{lll}1.2 \times \quad 240 \quad 13 & \end{array}$ $\begin{array}{lll}1.8 x & 84 & 6 \\ 1.2 x & 80 & 7\end{array}$ $\begin{array}{lll}1.9 \times & 54 & 4\end{array}$ $1.4 \times 208 \quad 9$

$\begin{array}{lll}1.1 \times \quad 79 & 12\end{array}$ $\begin{array}{llll}1.0 \times & 137 & 4\end{array}$ $\begin{array}{rrr}.5 \times 162 & 7 \\ .1 \times & 92 & 5\end{array}$ $\begin{array}{lll}1.1 \times & 157 \quad 5\end{array}$

$1.6 \times \quad 93 \quad 3$ $\begin{array}{lll}1.6 \times 191 & 17\end{array}$ $\begin{array}{lll}1.6 \times & 150 \quad 4\end{array}$ $\begin{array}{rrr}.9 \mathrm{X} & 76 & 5 \\ 1.6 \mathrm{X} & 69 & 5\end{array}$ 
---ORIGIN TIME (HST)-- -LAT N-- --LON W-- DEPTH N RMS ERH ERZ LOC PREF AZ MIN 43 YEAR MON DA HRMN SEC DEG MIN DEG MIN KM RD SEC KM KM REMKS MAG GAP DS

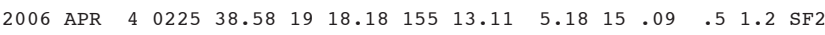

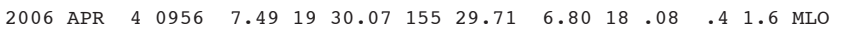

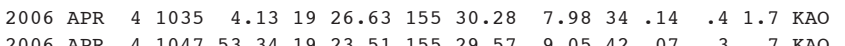
$\begin{array}{llllllllllllll}2006 & \text { APR } & 4 & 1047 & 53.34 & 19 & 23.51 & 155 & 29.57 & 9.05 & 42 & .07 & .3 & .7 \\ \text { KAO }\end{array}$ $\begin{array}{lllllllllllllllll}2006 & \mathrm{APR} & 4 & 1612 & 6.34 & 19 & 18.59 & 155 & 15.22 & 7.42 & 34 & .09 & .4 & .6 & \mathrm{KAO}\end{array}$ 2006 APR $4 \begin{array}{lllllllllllllll}1737 & 11.32 & 19 & 29.68 & 155 & 28.78 & 9.04 & 23 & .10 & .5 & 1.5 & \mathrm{KAO}\end{array}$

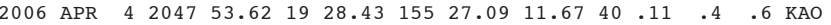

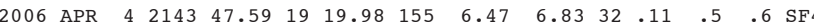
$\begin{array}{lllllllllllllll}2006 & \text { APR } & 4 & 2224 & 34.62 & 19 & 18.35 & 155 & 13.19 & 5.96 & 34 & .12 & .4 & .9 & \text { SF } 2\end{array}$

2006 APR $4 \begin{array}{lllllllllllll}2241 & 29.08 & 19 & 18.76 & 155 & 13.00 & 9.34 & 38 & .10 & .5 & .5 & \text { SF2 }\end{array}$

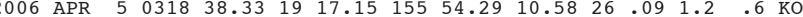

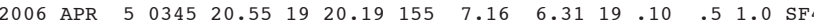

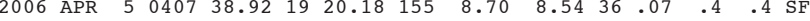

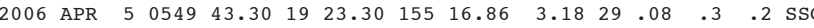

$\begin{array}{lllllllllllllll}2006 & \text { APR } & 5 & 1055 & 48.16 & 19 & 26.29 & 154 & 50.50 & 5.63 & 28 & .13 & 1.2 & .8 & \text { LER }\end{array}$

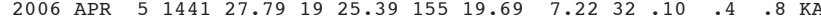

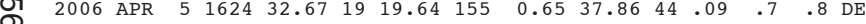
2006 APR $55 \begin{array}{lllllllllllll}2314 & 46.43 & 19 & 16.02 & 155 & 15.29 & 5.41 & 20 & .09 & .6 & 1.4 & \text { SF }\end{array}$

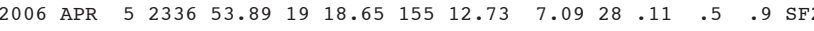

$\begin{array}{lllllllllllllll}2006 & \text { APR } & 6 & 0045 & 42.29 & 19 & 20.40 & 155 & 6.89 & 7.03 & 24 & .10 & .5 & 1.0 & \mathrm{SF} 4\end{array}$

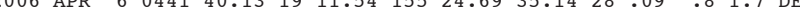

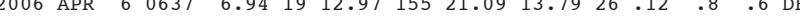

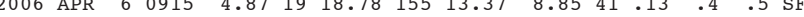
$\begin{array}{lllllllllllllll}2006 & \text { APR } & 6 & 0940 & 1.82 & 19 & 18.74 & 155 & 13.58 & 8.83 & 29 & .12 & .5 & .7 & \text { SF2 }\end{array}$

$\begin{array}{lllllllllllllll}2006 & \text { APR } & 6 & 1624 & 6.51 & 19 & 13.71 & 155 & 32.74 & 7.22 & 40 & .15 & .4 & 1.1 & \text { LSW }\end{array}$ 2006 APR $6 \begin{array}{lllllllllllll}1633 & 27.30 & 19 & 19.94 & 155 & 13.07 & 5.22 & 29 & .09 & .4 & 1.2 & \text { SF } 2\end{array}$

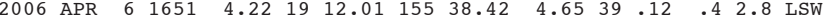
2006 APR $6 \begin{array}{llllllllllll}1711 & 43.28 & 19 & 46.13 & 156 & 1.37 & 7.60 & 44 & .12 & 1.0 & .7 & \text { HUA }\end{array}$ $\begin{array}{lllllllllllllll}2006 & \text { APR } & 6 & 1828 & 1.31 & 19 & 29.31 & 155 & 25.97 & 5.15 & 18 & .08 & .4 & 1.7 & \text { KAO }\end{array}$

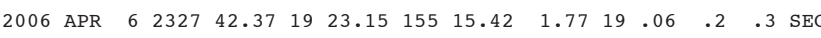
$\begin{array}{llllllllllllllll}2006 & \text { APR } & 7 & 0059 & 19.99 & 19 & 13.02 & 155 & 15.61 & 31.30 & 28 & .09 & .9 & 1.2 & \text { DE }\end{array}$ 2006 APR $7 \begin{array}{lllllllllllll} & 0231 & 39.22 & 19 & 24.43 & 155 & 30.04 & 8.85 & 27 & .08 & .4 & 1.0 & \text { KAO }\end{array}$

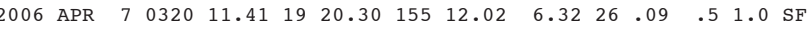

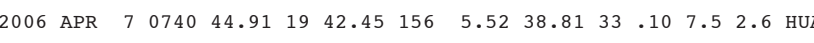

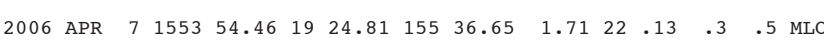
$\begin{array}{llllllllllllllll}2006 & \text { APR } & 7 & 1659 & 1.89 & 19 & 24.43 & 155 & 16.13 & 0.33 & 16 & .13 & .2 & .4 & \text { SEC }\end{array}$ $\begin{array}{lllllllllllllll}2006 & \text { APR } & 7 & 1748 & 26.00 & 19 & 32.54 & 155 & 43.98 & 0.58 & 26 & .13 & .8 & .5 & \text { кOI }\end{array}$

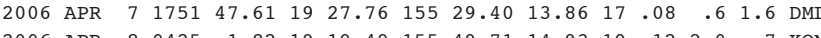
$\begin{array}{lllllllllllllll}2006 & \text { APR } & 8 & 0425 & 1.82 & 19 & 19.49 & 155 & 49.71 & 14.93 & 19 & .12 & 2.0 & .7 & \text { KON }\end{array}$

$\begin{array}{lllllllllllllll}2006 & \text { APR } & 8 & 0831 & 28.90 & 20 & 2.56 & 155 & 51.14 & 28.98 & 14 & .05 & 1.6 & 4.8 & \text { кон }\end{array}$

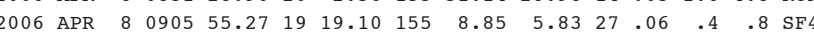

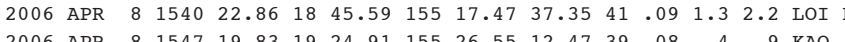

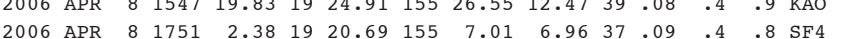

$.9 \times \quad 98 \quad 2$ $\begin{array}{rrr}.3 \times & 102 & 5 \\ 1.5 \times & 69 & 9\end{array}$ $\begin{array}{lll}.5 \times & 69 & 9 \\ 1.7 \times & 54 & 4\end{array}$ $1.4 \times 100 \quad 4$

$1.2 \times \quad 88 \quad 5$ $1.5 \times \quad 91 \quad 5$ $\begin{array}{lll}2.1 x & 47 & 7\end{array}$ $1.2 \times 182 \quad 5$ 1.6X 912

2.0X 1317

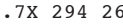
$.8 \times 160 \quad 5$ $\begin{array}{lll}.8 \times 119 & 5\end{array}$ $\begin{array}{lll}1.7 \times & 46 \quad 0\end{array}$

$1.5 \times 275 \quad 24$ $1.5 \times \quad 51 \quad 3$ 2.1X 25111 1.0X 205 $1.1 \times \quad 99 \quad 3$

$1.4 \times 165 \quad 6$ $1.4 \times 161$ $1.3 \times 172 \quad 10$ $\begin{array}{lll}2.3 \times & 79 & 3\end{array}$ $\begin{array}{lll}1.5 \mathrm{X} & 85 & 3\end{array}$ $2.0 \times 125 \quad 5$ $1.3 \times \quad 70$ $1.8 \mathrm{X} \quad 90 \quad 14$

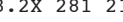
1.5 1015

$\begin{array}{lll}1.5 \times \quad 54 & 2\end{array}$ $1.3 \times 204 \quad 8$ $\begin{array}{lll}1.1 \times & 72 & 6\end{array}$ $1.3 \times \quad 76$ $2.6 \times 29941$ $\begin{array}{lll}1.6 \times & 97 \quad 2\end{array}$ $1.5 \times 136$ $\begin{array}{lll}1.5 \times 103 & 5 \\ 1.5 \times & 77 & 8\end{array}$ $\begin{array}{llr}1.5 \times & 77 & 8 \\ 1.6 \times & 249 & 17\end{array}$

$1.5 \times 29039$ $\begin{array}{lll}1.3 \times & 114 & 4\end{array}$ $\begin{array}{lll}2.8 \times & 284 & 47 \\ 2.3 \times & 51 & 10\end{array}$ $\begin{array}{rrr}2.3 \times & 51 & 10 \\ 1.9 \times & 159 & 5\end{array}$
---ORIGIN TIME (HST) -- -LAT N-- --LON W-- DEPTH N RMS ERH ERZ LOC YEAR MON DA HRMN SEC DEG MIN DEG MIN KM RD SEC KM KM REMKS

$\begin{array}{lllllllllllllll}2006 & \text { APR } & 8 & 1917 & 58.67 & 19 & 25.97 & 155 & 18.73 & 6.79 & 32 & .10 & .4 & .6 & \text { INT }\end{array}$ $\begin{array}{lllllllllllllll}2006 & \text { APR } & 8 & 2137 & 54.41 & 19 & 26.28 & 155 & 20.31 & 9.79 & 25 & .08 & .4 & .7 & \text { KAO }\end{array}$

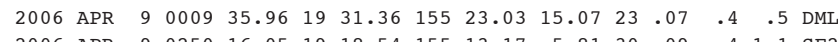
$\begin{array}{lllllllllllllll}2006 & \text { APR } & 9 & 0250 & 16.05 & 19 & 18.54 & 155 & 13.17 & 5.81 & 30 & .09 & .4 & 1.1 & \text { SF2 }\end{array}$ $\begin{array}{llllllllllllll}2006 & \text { APR } & 9 & 0438 & 44.80 & 19 & 27.47 & 155 & 23.86 & 10.95 & 22 & .10 & .4 & .9\end{array}$

$\begin{array}{lllllllllllllll}2006 & \text { APR } & 9 & 0705 & 34.33 & 19 & 19.84 & 155 & 9.58 & 10.50 & 26 & .07 & .5 & .6 & \mathrm{SF} 3\end{array}$ $\begin{array}{llllllllllllll}2006 & \text { APR } & 9 & 1332 & 49.72 & 19 & 25.21 & 155 & 19.36 & 6.39 & 38 & .14 & .4 & .7 \\ \text { KAO }\end{array}$

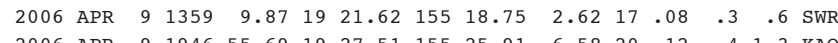
$\begin{array}{lllllllllllllll}2006 & \text { APR } & 9 & 1946 & 55.69 & 19 & 27.51 & 155 & 25.91 & 6.58 & 20 & .12 & .4 & 1.3 & \text { KAO }\end{array}$

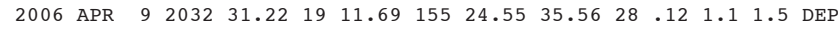

$\begin{array}{lllllllllllllll}2006 & \text { APR } & 9 & 2049 & 10.45 & 19 & 19.89 & 155 & 11.94 & 6.90 & 42 & .11 & .4 & .7 & \mathrm{SF}^{3}\end{array}$

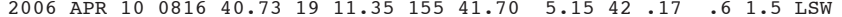

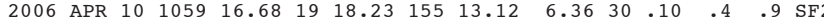

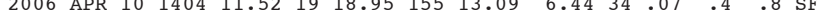

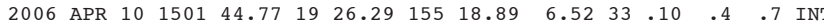

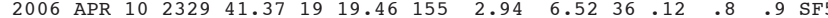

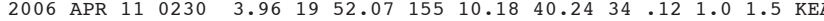

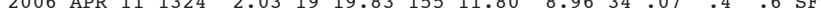

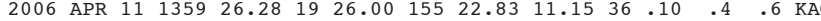

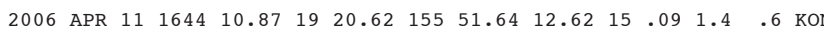

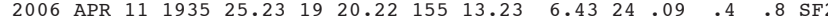

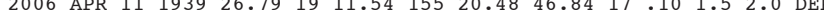

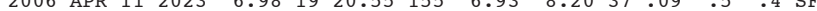

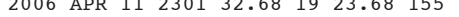

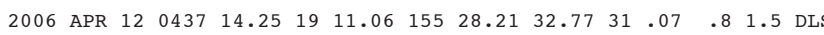

$\begin{array}{llllllllllllll}2006 & \text { APR } 12 & 0504 & 7.97 & 19 & 11.94 & 155 & 20.90 & 45.85 & 26 & .10 & 1.1 & 1.3 & \text { DEP }\end{array}$

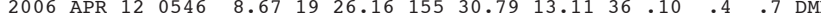

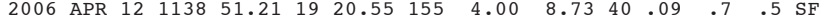
2006 APR $121343 \quad 36.531919 .95155$ 12.

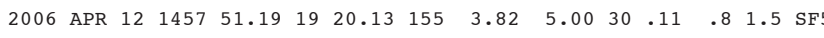

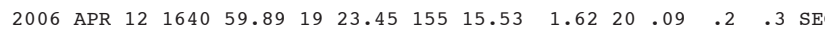

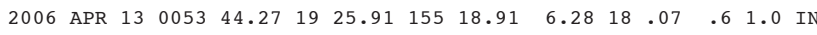

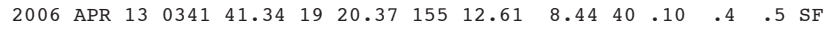

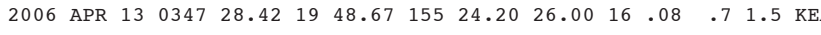

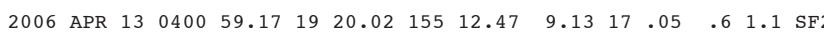

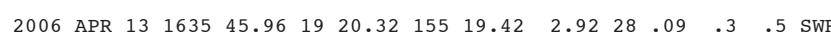

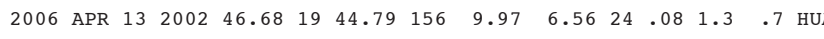

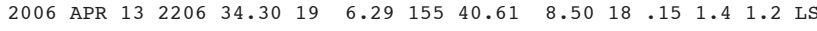

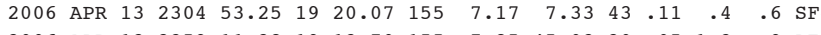
$\begin{array}{lllll}20 & .05 & 1.3 & .9 & \text { DEP }\end{array}$

$\begin{array}{lllllllllllllll}2006 & \text { APR } 14 & 0053 & 48.50 & 19 & 37.33 & 156 & 1.13 & 10.58 & 24 & .11 & 1.3 & .7 & \text { Kol }\end{array}$

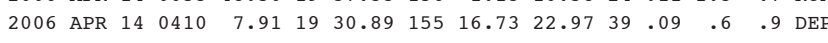

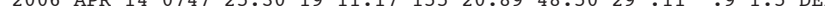

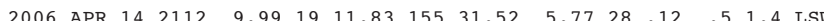
$\begin{array}{rrr}.8 \times & 57 & 2 \\ 1.5 \times & 66 & 5\end{array}$ $1.4 \times 115 \quad 3$ $1.1 \times \quad 88 \quad 3$ $1.5 \times \quad 75 \quad 4$

$.4 \times \quad 99 \quad 5$ $\begin{array}{lll}1.8 \times & 46 & 3\end{array}$ $\begin{array}{lll}1.3 \mathrm{X} & 85 & 4\end{array}$ $1.3 \times 20112$

$\begin{array}{lll}.6 \times & 84 \quad 5\end{array}$ $\begin{array}{lll}1.0 \times 103 & 8\end{array}$ $\begin{array}{lll}.8 \mathrm{X} & 51 & 3\end{array}$

$2.1 \times 245 \quad 9$ $\begin{array}{lll}1.8 \times & 223 \quad 18\end{array}$ $\begin{array}{lll}1.4 \mathrm{X} & 86 & 6\end{array}$ $\begin{array}{lll}1.6 \mathrm{X} & 47 & 7\end{array}$ $1.1 \times 28320$

$\begin{array}{lll}.8 \times & 66 & 4\end{array}$ $1.3 \times 242 \quad 16$ $2.0 \times 162 \quad 5$ $\begin{array}{ll}.3 \times & 96 \quad 2\end{array}$ $1.6 \times \quad 97 \quad 3$

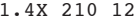
$\begin{array}{llll}2.0 \times \quad 65 & 4\end{array}$ $2.5 \times 225 \quad 7$

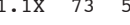
$\begin{array}{lll}1.5 \times & 230 & 8\end{array}$

$1.5 \times \quad 56 \quad 2$ $1.4 \mathrm{X} \quad 90 \quad 2$ $\begin{array}{lll}1.8 \times & 70 & 4\end{array}$ $\begin{array}{lll}1.2 \times 155 & 7 \\ 1.3 \times & 77 & 5\end{array}$ $1.3 \times 107 \quad 4$ $2.1 \times 29250$ $\begin{array}{lll}1.1 \times 300 & 25\end{array}$ $\begin{array}{lll}2.2 \times & 162 & 5\end{array}$ $1.7 \times 275 \quad 46$ $\begin{array}{lrr}1.7 x & 67 \quad 6\end{array}$ $\begin{array}{llll}1.6 \times & 174 & 13\end{array}$ $2.3 \times \quad 297 \quad 41$
$1.6 \times \quad 197 \quad 6$ 
---ORIGIN TIME (HST)-- -LAT N-- --LON W-- DEPTH N RMS ERH ERZ LOC $\begin{array}{llllllllllllll}2006 & \text { APR } 14 & 2357 & 23.43 & 19 & 20.36 & 155 & 8.16 & 6.35 & 31 & .12 & .5 & .9 & \text { SF4 }\end{array}$

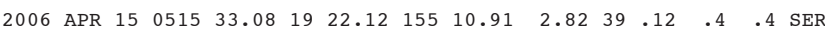

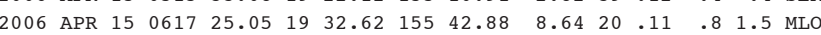
2006 APR $15 \quad \begin{array}{llllllllllll}0944 & 20.46 & 19 & 6.18 & 155 & 17.27 & 12.08 & 34 & .10 & .8 & .7 & \text { LOI }\end{array}$

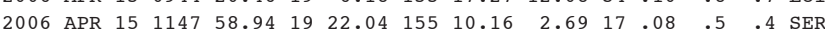

$\begin{array}{lllllllllllllll}2006 & \text { APR } & 15 & 1229 & 36.94 & 19 & 19.83 & 155 & 24.70 & 9.05 & 20 & .07 & .6 & 1.1 & \text { SWR }\end{array}$

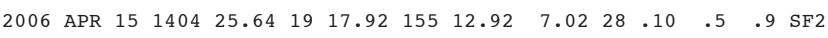

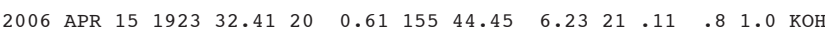

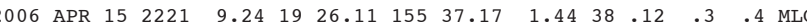

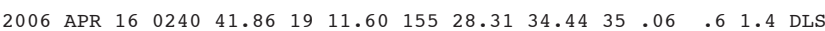

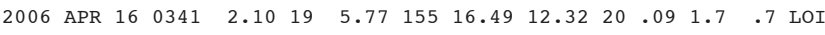

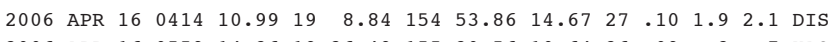

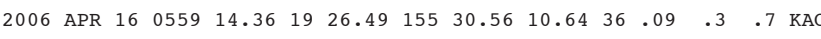

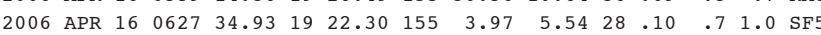

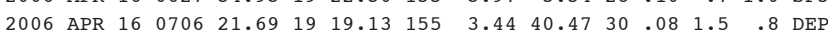

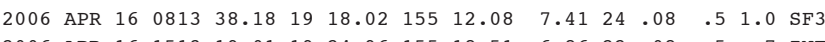

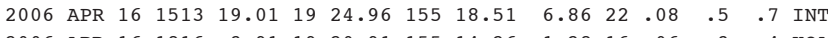
$\begin{array}{llllllllllllllll}2006 & \text { APR } & 16 & 1816 & 3.91 & 19 & 20.91 & 155 & 14.26 & 1.28 & 16 & .06 & .3 & .4 & \text { KOA } \\ 2006 & \text { APR } & 16 & 1829 & 23.47 & 19 & 15.63 & 155 & 31.51 & 10.97 & 37 & .12 & 4 & .9 & \end{array}$

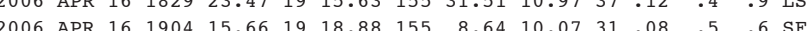

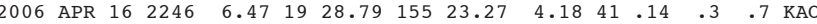

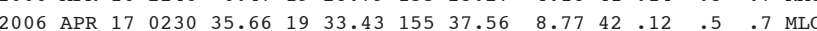
2006 APR $17 \quad \begin{array}{llllllllllll}0532 & 39.78 & 19 & 32.92 & 155 & 51.51 & 10.37 & 16 & .10 & 1.1 & .7 & \text { KON }\end{array}$ 2006 APR $17 \quad \begin{array}{llllllllllll}0 & 33.90 & 19 & 29.53 & 155 & 22.68 & 13.95 & 45 & 13 & 4 & 3 & 0\end{array}$

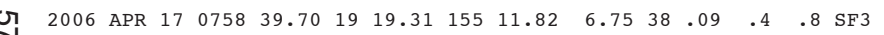

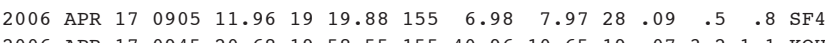

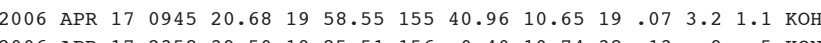
2006 APR $17 \begin{array}{llllllllllll}2358 & 39.50 & 19 & 25.51 & 156 & 0.40 & 10.74 & 38 & .13 & .9 & .5 & \text { KON }\end{array}$

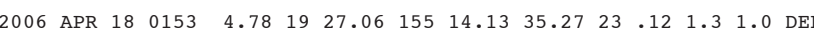

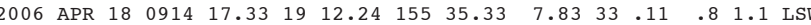

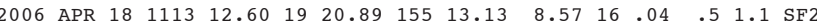

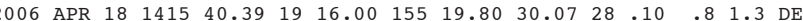

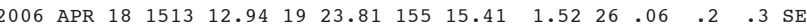

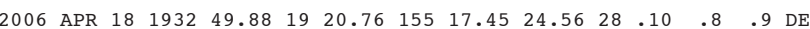

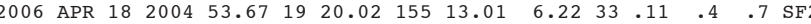

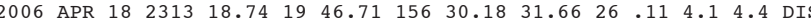

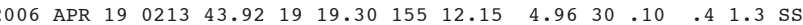

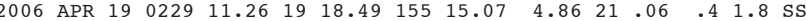

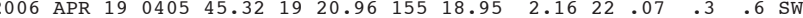

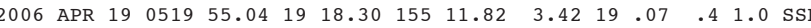

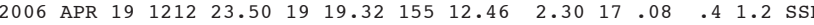

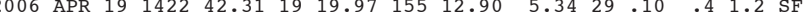

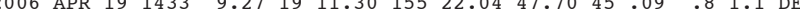

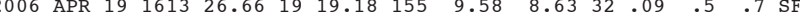

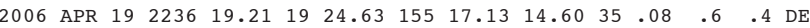
$1.3 \times 132$ $2.3 \times \quad 79$ $1.5 \times 214 \quad 20$ $1.5 \times 21420$

$.9 \times \quad 94 \quad 3$ $\begin{array}{lll}.3 \times & 115 & 2 \\ 1.5 \times & 146 & 14\end{array}$ $2.6 \times \quad 92$ $1.6 \times 100$

$1.2 \times 26421$ \#1.5X 28729 $\begin{array}{lll}1.5 \times & 68 & 8\end{array}$ $\begin{array}{lll}1.6 \times 220 & 4 \\ 1.6 \times 287 & 9\end{array}$

$1.3 \times 134 \quad 3$ $\begin{array}{lll}1.2 \times & 104 & 2 \\ 1.3 \times & 145 & 4 \\ 1.6 \times & 156 & 3\end{array}$ $1.6 \times 156 \quad 3$ 1.3X $118 \quad 3$

$\begin{array}{rrr}2.2 \times & 87 & 2 \\ 2.2 \times & 144 & 8\end{array}$ $\begin{array}{lll}2.2 \times & 144 & 8 \\ 2 \times & 282 & 8\end{array}$ $\begin{array}{lll}1.3 \times 282 & 28\end{array}$ $\begin{array}{lll}2.2 \mathrm{X} & 53 & 1 \\ 1.4 \mathrm{X} & 97 & 5\end{array}$

$1.3 \times 145 \quad 5$ $1.5 \times 256 \quad 36$ $2.2 \times 247 \quad 27$ $1.4 \times 1995$ 2.2X 22111

$1.3 \times \quad 60 \quad 3$ $1.5 \times 172$ $\begin{array}{lll}1.8 \mathrm{X} & 65 & 2\end{array}$ $1.3 \mathrm{X} \quad 68 \quad 4$ $\begin{array}{lll}1.3 \times & 70 \quad 5\end{array}$

$2.1 \times \quad 287 \quad 83$ $\begin{array}{rrr}1.2 \mathrm{X} & 93 & 5 \\ .9 \mathrm{X} & 123 & 4\end{array}$ $.0 \times \quad 86 \quad 5$ $\begin{array}{lrr}1.0 \times & 86 & 5 \\ 1.1 \mathrm{U} & 127 & 4\end{array}$
- ORTGIN TTME (HST)-- -LAT N-- --LON W-- DEPTH N RMS ERH ERZ LOC YEAR MON DA HRMN SEC DEG MIN DEG MIN DE RM SEC KM KM PEMKS

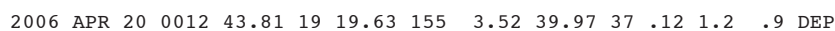

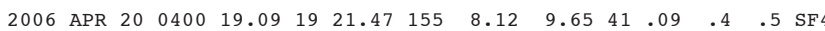

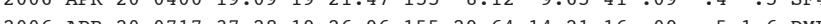

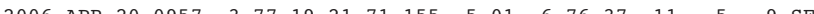

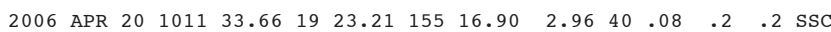

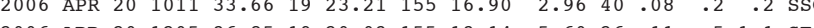

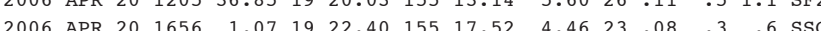

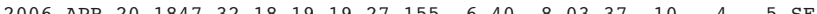
2006 APR 20200254.18

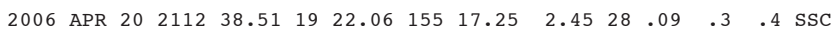
$\begin{array}{llllllllll}2006 & 0\end{array}$ $\begin{array}{lllllllllllll}2006 & 0\end{array}$ $\begin{array}{lllllllllll}2006 & 0\end{array}$

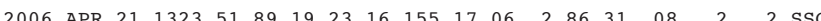

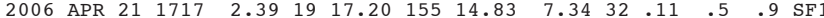
$\begin{array}{llllllllllllll}2006 & \text { APR } 21 & 1809 & 56.28 & 19 & 24.87 & 155 & 19.69 & 5.50 & 27 & 08 & .4 & .8 & \text { KAO }\end{array}$

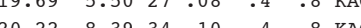

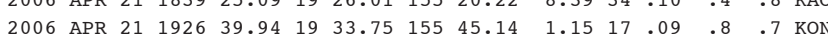

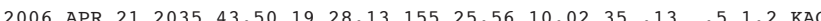

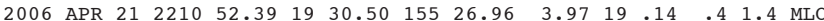

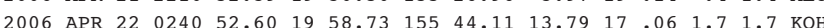

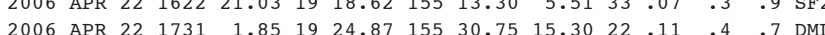

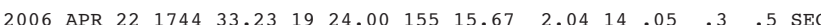

\begin{tabular}{lllllllllllll}
2006 & APR 22 & 1807 & 27.06 & 19 & 25.29 & 155 & 39.32 & 2.56 & 14 & .10 & .8 & .5 \\
\hline$L O$
\end{tabular}

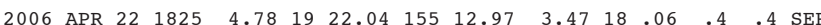

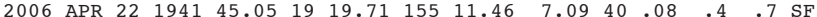

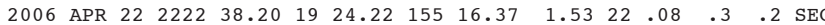

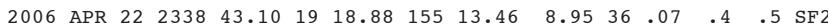

$\begin{array}{llllllllllllll}2006 & \text { APR } 22 & 2344 & 0.91 & 19 & 25.56 & 155 & 23.77 & 10.84 & 38 & .09 & .4 & .8 & \text { KAO }\end{array}$

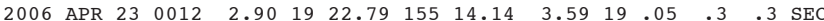

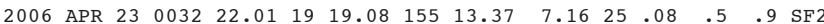

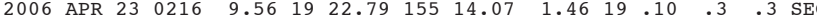

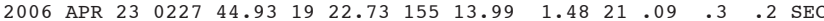

$\begin{array}{llllllllllllll}2006 & \text { APR } 23 & 0325 & 5.15 & 19 & 10.14 & 155 & 31.31 & 36.43 & 23 & .08 & .9 & 1.6 & \text { DLS }\end{array}$

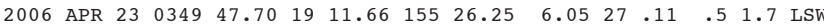

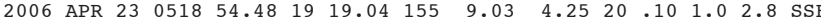

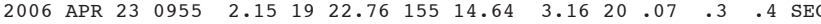

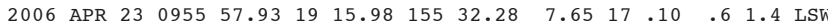

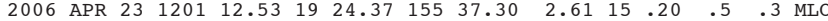
$\begin{array}{lllllllllllllll}2006 & \text { APR } 23 & 1307 & 34.71 & 19 & 13.56 & 155 & 37.52 & 2.08 & 22 & .11 & .6 & .9 & \text { LSW }\end{array}$

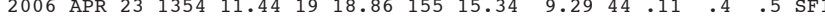

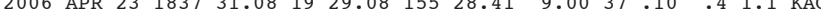

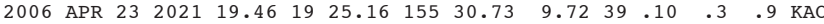

PREF AZ MIN 46 $.6 \times 198 \quad 9$ $\begin{array}{rrr}1.9 \times & 114 \quad 3 \\ 1.1 \times & 70 & 7\end{array}$ $\begin{array}{llll}1.1 \times & 70 & 7\end{array}$ $2.0 \times 156 \quad 5$ $\begin{array}{lll}2.5 \times & 37 \quad 0\end{array}$ $\begin{array}{lll}.1 \times & 68 & 5\end{array}$ $1.9 \times 166 \quad 4$ $\begin{array}{lll}1.9 \times & 166 \quad 47\end{array}$

$1.5 \mathrm{X} \quad 55 \quad 2$ $\begin{array}{lll}1.4 \times & 163 & 4 \\ 1.6 \times & 69 & 1\end{array}$ $\begin{array}{lll}1.3 \times \quad 238 \quad 8 & 8\end{array}$ $\begin{array}{lll}.6 \times \quad 48 \quad 0 & 0\end{array}$

$1.4 \times \quad 165 \quad 2$ $\begin{array}{lll}1.2 \mathrm{X} & 75 & 2 \\ 1.7 \mathrm{X} & 62 & 4\end{array}$ $\begin{array}{rrr}1.7 x & 62 & 4 \\ 1.3 \times & 134 & 6\end{array}$ $7 \times \quad 56 \quad 5$

$\begin{array}{lll}1.3 \times & 102 \quad 3 \\ 1.3 \times & 293 & 36\end{array}$ $\begin{array}{lll}1.3 \times \quad 29336 \\ 1.4 \times \quad 83 & 3\end{array}$ $\begin{array}{lll}1.4 \mathrm{X} & 83 & 3 \\ 1.1 \mathrm{x} & 55 & 4 \\ 1.2 \mathrm{x} & 113 & 2\end{array}$ $\begin{array}{lll}1.2 \times & 113 \quad 2\end{array}$

$1.0 \times 208 \quad 3$ $\begin{array}{lll}1.8 \mathrm{X} & 86 & 1 \\ 1.7 \mathrm{X} & 91 & 6\end{array}$ $\begin{array}{lll}1.8 \mathrm{X} & 80 & 1\end{array}$ $1.5 \times \quad 75 \quad 3$

$\begin{array}{ccc}1.9 \times & 46 \quad 8\end{array}$ $\begin{array}{rrr}1.4 \mathrm{X} & 90 & 2 \\ .8 \mathrm{X} & 75 & 4 \\ 1.7 \mathrm{X} & 92 & 2\end{array}$ $1.7 \times \quad 92 \quad 2$ $\begin{array}{lll}1.8 \mathrm{X} & 50 \quad 2\end{array}$

$\begin{array}{lll}1.8 \times & 117 & 6 \\ 1.6 \times & 145 & 5\end{array}$ $\begin{array}{lll}.6 \times 145 & 5 \\ .9 \times 107 & 4\end{array}$ $1.5 \times \quad 77 \quad 2$ $1.2 \mathrm{X} \quad 96 \quad 4$

$\begin{array}{lll}1.2 \times & 79 \quad 1\end{array}$ $1.4 \times 129 \quad 13$ $\begin{array}{lll}2.0 \times & 96 & 4\end{array}$ $\begin{array}{lll}.6 \times & 57 \quad 7\end{array}$ 
---ORIGIN TIME (HST)-- -LAT N-- --LON W-- DEPTH N RMS ERH ERZ LOC PREF AZ MIN 47 YEAR MON DA HRMN SEC DEG MIN DEG MIN KM RD SEC KM KM REMRS MAG GAP DS

$\begin{array}{llllllllllllll}2006 & \text { APR } 24 & 0108 & 38.44 & 19 & 23.41 & 155 & 30.53 & 9.10 & 24 & .08 & .4 & .9 & \text { KAO }\end{array}$ 2006 APR $24 \quad 0227 \quad 46.2918 \quad 56.72 \quad 155 \quad 13.63 \quad 51.97 \quad 18 \quad 10 \quad 2.7 \quad 3.5$ LOI

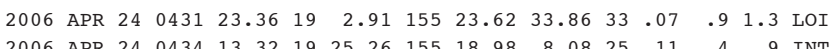

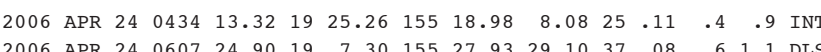

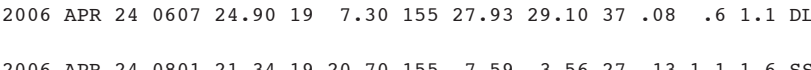

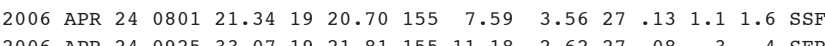

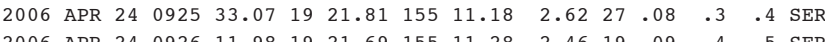

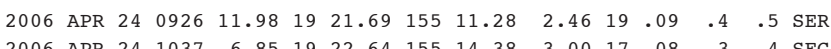

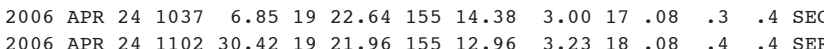

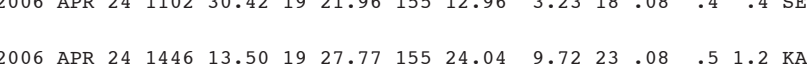

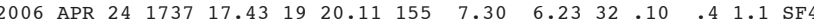

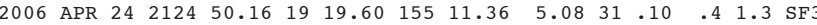

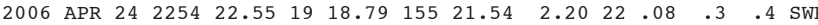

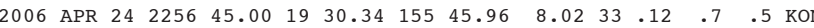

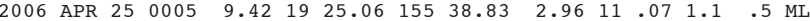

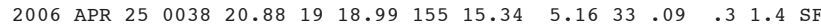

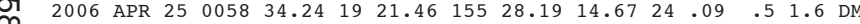

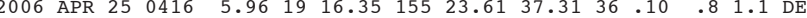

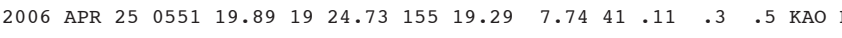

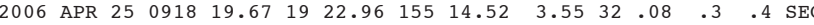

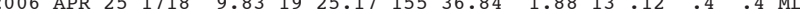

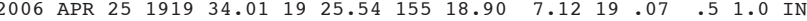

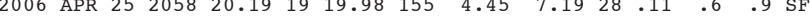

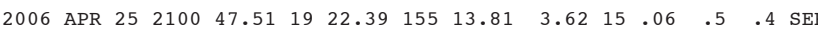

2006 APR $25 \quad 211027.43 \quad 1912.58 \quad 155 \quad 25.89 \quad 40.64 \quad 22 \quad .10 \quad 1.3 \quad 1.9$ DL

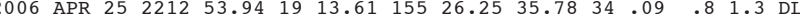

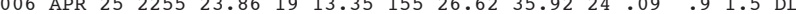

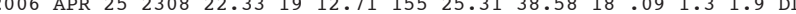

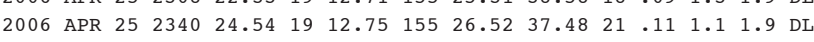

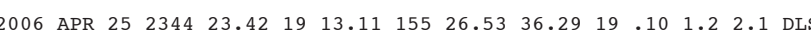

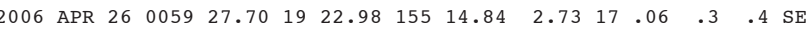

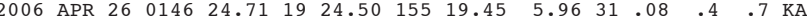

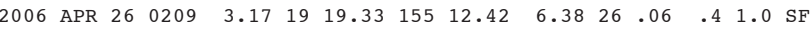

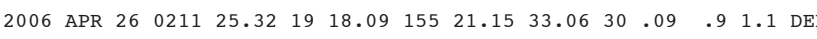

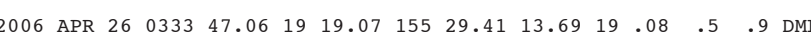
$\begin{array}{llllllllllllll}2006 & \text { APR } 26 & 0600 & 3.13 & 19 & 19.35 & 155 & 11.22 & 7.13 & 36 & .08 & .4 & .6 & \text { SF3 }\end{array}$

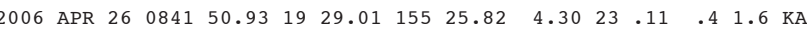

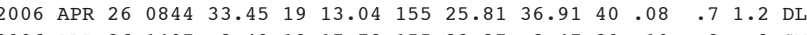

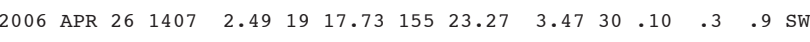

2006 APR $26 \quad 220751.4919 \quad 50.54 \quad 155 \quad 32.8921 .38 \quad 19.08 \quad 1.4 \quad 1.8$ KEA

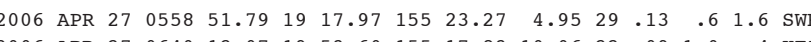

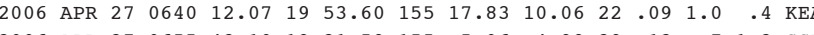

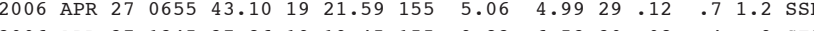

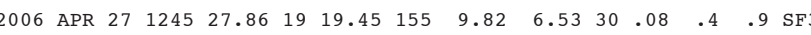

$\begin{array}{rrr}1.4 \mathrm{X} & 81 & 5 \\ 1.7 \times & 262 & 37\end{array}$ $1.7 \times 24014$ $\begin{array}{rrr}1.7 \times & 840 & 14 \\ 1.1 \times & 81\end{array}$ $\begin{array}{llll}1.8 \times & 173 & 4\end{array}$

$1.4 \times 187 \quad 5$ $1.9 \times \quad 76 \quad 3$ $1.5 \times \quad 84 \quad 3$ $1.6 \times \quad 83 \quad 2$ $1.5 \times \quad 83 \quad 1$

$1.5 \times \quad 74 \quad 4$ $1.5 \times 136 \quad 5$ $1.5 \times \quad 94 \quad 6$ $1.1 \times 140 \quad 4$ $2.0 \times 110 \quad 0$

$1.1 \times 194 \quad 2$ 1.6X $114 \quad 5$ $1.3 \times \quad 86 \quad 9$ $1.5 \times 166$

$1 \times 41$

$\begin{array}{lll}1.8 \times & 49 & 3\end{array}$ $\begin{array}{llll}1.1 \times & 82 & 2\end{array}$ $\begin{array}{lll}1.4 \times & 84 & 2\end{array}$ $\begin{array}{lll}1.1 \times & 183 & 8 \\ 1.5 x & 143 & 1\end{array}$ $1.0 \times 210$ $1.7 \times 124$ $\begin{array}{lll}1.5 \times & 121 & 7\end{array}$ $1.2 \times 210 \quad 9$ $1.2 \times 205 \quad 8$

$1.1 \times 202 \quad 7$ $1.4 \times \quad 77 \quad 2$ $\begin{array}{lll}1.2 \times & 52 & 2\end{array}$ $.9 \mathrm{X} \quad 89 \quad 5$ $\begin{array}{lll}1.5 \times & 153 \quad 4\end{array}$

$1.0 \times \quad 94 \quad 7$ $\begin{array}{llll}1.5 \times & 101 & 6\end{array}$ $\begin{array}{lll}1.3 \times & 60 & 5\end{array}$ $\begin{array}{lll}1.8 \times & 134 & 7\end{array}$ 1.4024212 $\begin{array}{llll}1.6 \times 152 & 4\end{array}$ $1.2 \times 2205$ $1.3 \times 195 \quad 5$
作 YEAR MON DA HRMN SEC DEG MIN DEG MIN KM RD SEC KM KM REMKS

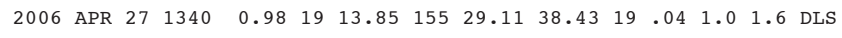

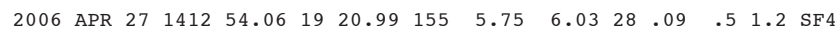

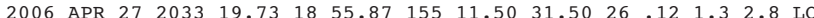

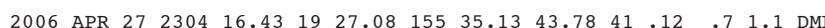

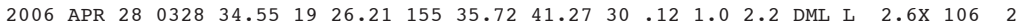

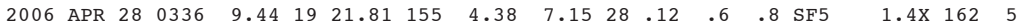

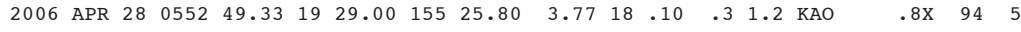

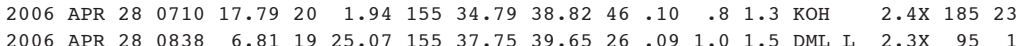

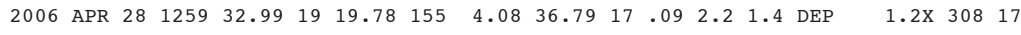
$\begin{array}{llllllllllllllllll}2006 & \text { APR } & 28 & 1408 & 6.54 & 19 & 24.94 & 155 & 38.00 & 3.13 & 41 & .15 & .3 & .5 & \text { MLO } & 2.6 \mathrm{X} & 98 & 1\end{array}$

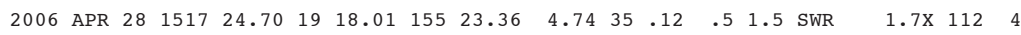
$\begin{array}{lllllllllllllllll}2006 & \text { APR } 28 & 1526 & 59.77 & 19 & 18.01 & 155 & 23.35 & 3.51 & 28 & .11 & .4 & .8 & \text { SWR } & 1.3 \times & 150 & 4\end{array}$

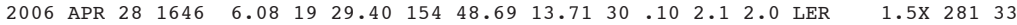

$\begin{array}{lllllllllllllllll}2006 & \text { APR } 28 & 1903 & 18.02 & 19 & 15.59 & 155 & 29.56 & 9.20 & 36 & .11 & .3 & .8 & \text { LSW } & 1.6 \mathrm{X} & 80 & 1\end{array}$

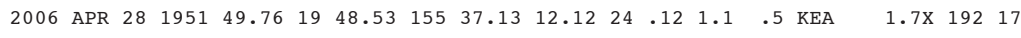

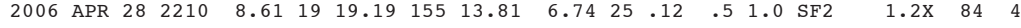

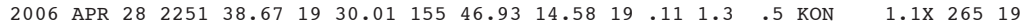

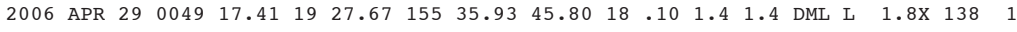

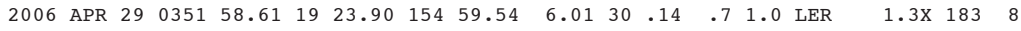

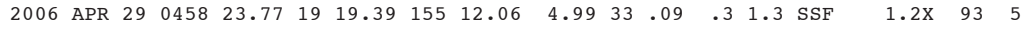

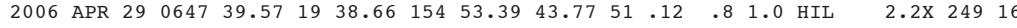

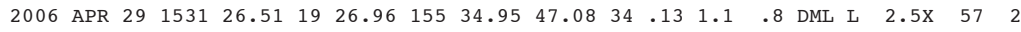

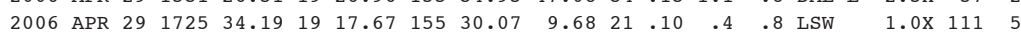

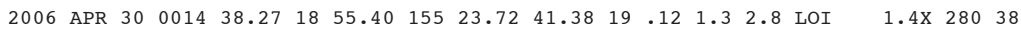

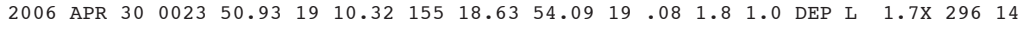

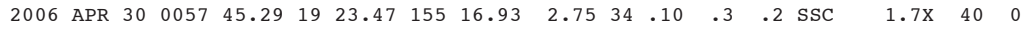

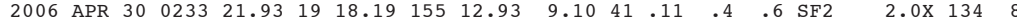

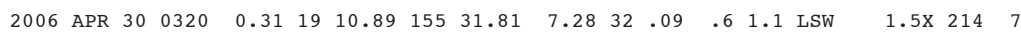

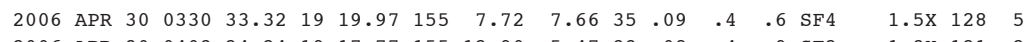

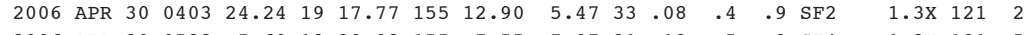

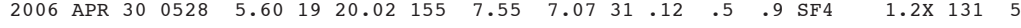

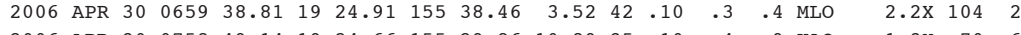

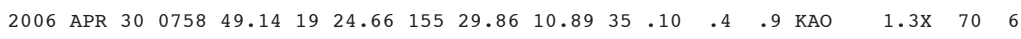

$\begin{array}{lllllllllllllllll}2006 & \text { APR } 30 & 0905 & 7.54 & 19 & 27.20 & 155 & 34.77 & 50.91 & 22 & .11 & 1.3 & 1.5 & \text { DML L } & 1.8 \times & 62 & 2\end{array}$

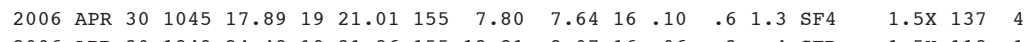

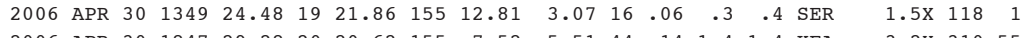

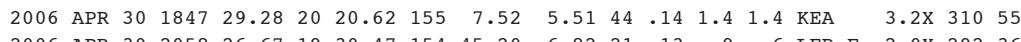

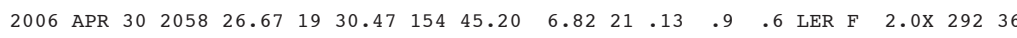

2006 APR $30215950.912023 .95 \quad 155 \quad 50.5522 .6140 .11 \quad 1.26 .2$ кон $\quad 2.3 \times 317 \quad 77$

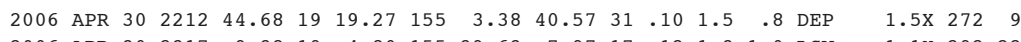

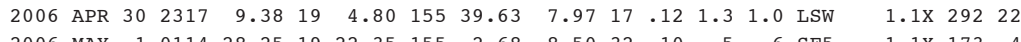

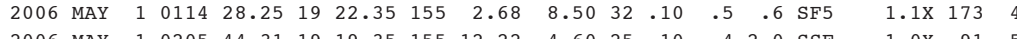
$\begin{array}{llllllllllllllllll}2006 & \text { MAY } & 1 & 0205 & 44.31 & 19 & 19.35 & 155 & 12.22 & 4.60 & 25 & .10 & .4 & 2.0 & \text { SSF } & 1.0 \mathrm{X} & 91 & 5\end{array}$ 
---ORIGIN TIME (HST)-- -LAT N-- --LON W-- DEPTH N RMS ERH ERZ LOC

$\begin{array}{lllllllllllllll}2006 & \text { MAY } & 1 & 0211 & 14.38 & 19 & 21.78 & 155 & 17.51 & 2.91 & 20 & .07 & .3 & .5 & \text { SWR }\end{array}$ $\begin{array}{lllllllllllllll}2006 & \text { MAY } & 1 & 0439 & 57.57 & 19 & 21.32 & 155 & 18.60 & 2.74 & 24 & .08 & .3 & .6 & \text { SWR }\end{array}$ $\begin{array}{llllllllllllllll}2006 & \text { MAY } & 1 & 0527 & 7.25 & 19 & 24.71 & 155 & 19.31 & 5.23 & 20 & .10 & .4 & 1.0 & \text { KAO }\end{array}$ $\begin{array}{lllllllllllllll}2006 & \text { MAY } & 1 & 0857 & 48.68 & 19 & 18.04 & 155 & 23.26 & 2.92 & 18 & .07 & .5 & .7 & \text { SWR }\end{array}$

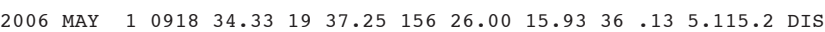

$\begin{array}{lllllllllllllll}2006 & \text { MAY } & 1 & 1252 & 41.64 & 19 & 23.11 & 155 & 17.02 & 3.08 & 20 & .06 & .3 & .3 & \text { SSC }\end{array}$ $\begin{array}{llllllllllllllll}2006 & \text { MAY } & 1 & 1334 & 42.81 & 19 & 10.32 & 155 & 29.19 & 9.25 & 31 & .10 & .6 & 1.0 & \text { LSW }\end{array}$ $\begin{array}{lllllllllllllll}2006 & \text { MAY } & 1 & 1540 & 32.19 & 19 & 14.27 & 155 & 26.35 & 7.91 & 31 & .11 & .4 & 1.4 & \text { LSW }\end{array}$ $\begin{array}{lllllllllllllll}2006 & \text { MAY } & 1 & 1636 & 50.38 & 19 & 23.80 & 155 & 15.27 & 1.33 & 16 & .07 & .2 & .4 & \text { SEC }\end{array}$

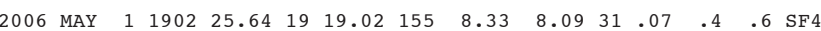

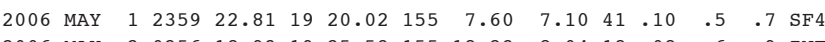
$\begin{array}{lllllllllllllll}2006 & \text { MAY } & 2 & 0256 & 18.93 & 19 & 25.53 & 155 & 18.23 & 8.04 & 18 & .08 & .6 & .9 & \text { INT }\end{array}$ $\begin{array}{lllllllllllllll}2006 & \text { MAY } & 2 & 0547 & 29.71 & 19 & 21.96 & 155 & 2.35 & 6.23 & 27 & .12 & .7 & 1.3 & \text { SF5 }\end{array}$

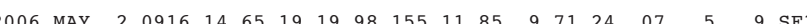

$\begin{array}{lllllllllllllll}2006 & \text { MAY } & 2 & 1028 & 41.78 & 19 & 26.63 & 155 & 28.58 & 9.14 & 24 & .08 & .4 & 1.1 & \text { KAO }\end{array}$ $\begin{array}{lllllllllllllll}2006 & \text { MAY } & 2 & 1117 & 28.93 & 19 & 21.68 & 155 & 12.90 & 2.76 & 16 & .06 & .3 & .4 & \text { SER }\end{array}$ $\begin{array}{llllllllllllllll}2006 & \text { MAY } & 2 & 1249 & 11.80 & 19 & 19.94 & 155 & 7.43 & 7.12 & 34 & .09 & .4 & .7 & \mathrm{SF} 4 \\ 2006 & \text { MAY } & 2 & 1620 & 11.47 & 19 & 23.29 & 155 & 17.03 & 2.90 & 16 & 07 & .3 & .3 & \mathrm{SSC}\end{array}$ $\begin{array}{lllllllllllll}2006 & & & \end{array}$

$\begin{array}{lllllllllllllll}2006 & \text { MAY } & 2 & 1839 & 14.98 & 19 & 18.12 & 155 & 12.74 & 7.13 & 29 & .10 & .5 & .7 & \mathrm{SF} 2\end{array}$ $\begin{array}{lllllllllllllll}2006 & \text { MAY } & 2 & 2212 & 2.57 & 19 & 19.36 & 155 & 9.13 & 6.89 & 21 & .08 & .5 & 1.0 & \text { SF } 3\end{array}$

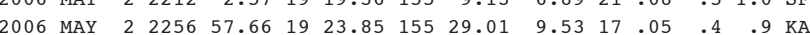

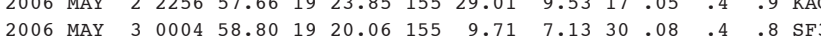

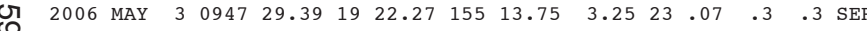

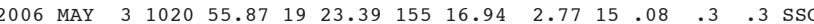
$\begin{array}{lllllllllllllll}2006 & \text { MAY } & 3 & 1059 & 39.72 & 19 & 23.35 & 155 & 17.10 & 2.67 & 17 & .07 & .2 & .3 & \text { SSC }\end{array}$

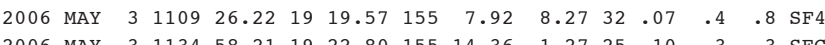

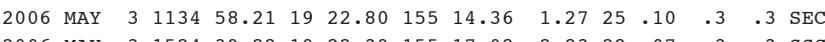

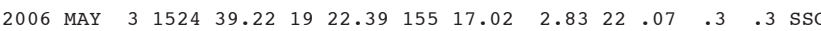

$\begin{array}{lllllllllllllll}2006 & \text { MAY } & 3 & 1654 & 37.90 & 19 & 21.60 & 155 & 18.14 & 2.83 & 18 & .08 & .3 & .5 & \text { SWR }\end{array}$

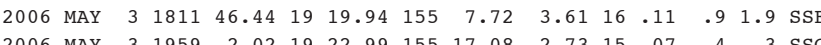
$\begin{array}{llllllllllllllll}2006 & \text { MAY } & 3 & 1959 & 2.02 & 19 & 22.99 & 155 & 17.08 & 2.73 & 15 & .07 & .4 & .3 & \text { SSC }\end{array}$

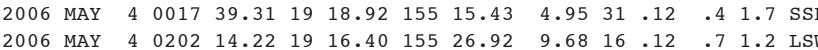

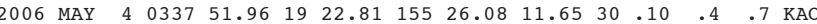
$\begin{array}{llllllllllllllll}2006 & \text { MAY } & 4 & 0916 & 59.17 & 19 & 11.59 & 155 & 21.54 & 46.73 & 29 & .10 & 1.0 & 1.7 & \text { DEP }\end{array}$

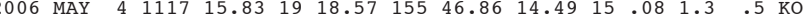
$\begin{array}{lllllllllllllll}2006 & \text { MAY } & 4 & 1218 & 1.27 & 19 & 20.03 & 155 & 6.34 & 4.77 & 15 & .11 & .7 & 2.9 & \text { SSE }\end{array}$ $\begin{array}{lllllllllllllll}2006 & \text { MAY } & 4 & 1303 & 50.33 & 19 & 19.99 & 155 & 7.22 & 9.38 & 28 & .09 & .6 & .6 & \mathrm{SF}\end{array}$

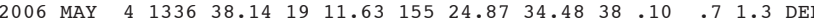

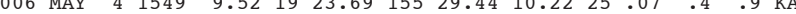
$\begin{array}{lllllllllllllll}2006 & \text { MAY } & 4 & 1554 & 23.98 & 19 & 23.46 & 155 & 16.92 & 2.62 & 18 & .07 & .3 & .3 & \text { SSC }\end{array}$ $\begin{array}{llllllllllllll}2006 & \text { MAY } & 4 & 1608 & 26.11 & 19 & 19.78 & 155 & 26.28 & 9.02 & 19 & .10 & .5 & 1.0\end{array}$ $\begin{array}{lllllllllllllll}2006 & \text { MAY } & 4 & 1852 & 4.53 & 19 & 19.71 & 155 & 8.52 & 6.04 & 26 & .09 & .5 & 1.3 & \mathrm{SF}^{4}\end{array}$ $1.4 \times \quad 61$ $.4 \times \quad 75 \quad 5$ $.9 \times 102-2$ $2.6 \times 30956$

$\begin{array}{lll}1.3 \times & 47 \quad 1 \\ 1.7 \times & 195 & 3\end{array}$ $.7 \times 195$ $1.3 \times \quad 99$ $3 \times 130-3$

$2.4 \times 131 \quad 5$ $.9 \times 145 \quad 1$ $2.3 \times 146 \quad 1$ $\begin{array}{rrr}2.3 \times & 146 & 1 \\ 1.4 \times & 83 & 5\end{array}$

$1.4 \times \quad 87 \quad 8$ $1.5 \times 116 \quad 2$ $\begin{array}{rrr}1.5 \times & 135 & 5 \\ .9 \times & 66 & 0\end{array}$ $\begin{array}{rrr}.9 \times & 66 & 0 \\ 1.3 \times & 48 & 0\end{array}$

1.3X $114 \quad 2$ $1.0 \times 107$ $\begin{array}{lll}1.2 \times & 70 & 3\end{array}$ $\begin{array}{lll}1.5 \mathrm{X} & 97 & 5\end{array}$ $\begin{array}{lll}1.4 \mathrm{X} & 72 & 1\end{array}$

$\begin{array}{lll}1.2 \mathrm{x} & 80 & 0\end{array}$ $\begin{array}{lll}1.5 \mathrm{x} & 70 & 0\end{array}$ $\begin{array}{lll}1.5 \times & 144 & 4\end{array}$ $\begin{array}{lll}1.8 \times & 80 & 2\end{array}$ $1.4 \times \quad 50 \quad 2$

$\begin{array}{lll}1.4 \times \quad 67 \quad 3 \\ .9 \times & 147 & 5\end{array}$ $\begin{array}{rrrr}.9 \times & 147 & 5 \\ .9 \times & 63 & 1\end{array}$ $1.4 \times \quad 97 \quad 5$ $.7 \times 169 \quad 6$

$\begin{array}{lll}1.2 \times \quad 73 & 3\end{array}$ $1.4 \times \quad 170 \quad 12$ $.7 \times 262 \quad 13$ $1.1 \times 184 \quad 6$ $1.5 \times 161 \quad 5$

$1.9 \times 160 \quad 7$ $1.1 \times \quad 73 \quad 4$ $1.2 \times \quad 51 \quad 0$ $1.3 \times 114 \quad 5$ $1.1 \times 125$
--ORTGIN TIME (HST)-- -LAT N-- --LON W-- DEPTH N RMS ERH ERZ LOC YEAR MON DA HRMN SEC DEG MIN DEG MIN KM RD SEC KM KM REMKS

$\begin{array}{lllllllllllllll}2006 & \text { MAY } & 4 & 1903 & 18.47 & 19 & 19.76 & 155 & 8.31 & 6.00 & 24 & .10 & .4 & 1.1 & \mathrm{SF} 4\end{array}$

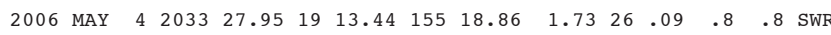

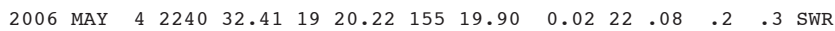
$\begin{array}{lllllllllllllll}2006 & \text { MAY } & 4 & 2338 & 54.69 & 19 & 24.61 & 155 & 19.40 & 7.66 & 44 & .10 & .3 & .5 & \text { KAO }\end{array}$

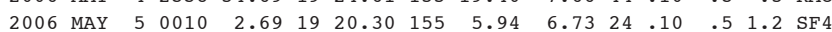

$\begin{array}{lllllllllllllll}2006 & \text { MAY } & 5 & 0014 & 13.33 & 19 & 20.23 & 155 & 13.07 & 7.68 & 23 & .08 & .5 & .8 & \text { SF2 }\end{array}$ $\begin{array}{lllllllllllllll}2006 & \text { MAY } & 5 & 0154 & 54.87 & 19 & 22.31 & 155 & 14.46 & 2.75 & 14 & .08 & .6 & .4 & \text { SEC }\end{array}$ $\begin{array}{llllllllllllll}2006 & \text { MAY } & 5 & 0212 & 24.66 & 19 & 24.52 & 155 & 19.55 & 6.24 & 25 & .10 & .4 & 1.0\end{array}$ $\begin{array}{lllllllllllllll}2006 & \text { MAY } & 5 & 0241 & 41.45 & 19 & 20.42 & 155 & 7.14 & 8.22 & 28 & .09 & .5 & .8 & \mathrm{SF}\end{array}$ $\begin{array}{llllllllllllll}2006 & \text { MAY } & 5 & 0244 & 47.31 & 19 & 24.54 & 155 & 19.47 & 6.14 & 19 & .09 & .4 & 1.1 \\ \text { KAO }\end{array}$

$\begin{array}{lllllllllllllll}2006 & \text { MAY } & 5 & 0452 & 55.62 & 19 & 19.93 & 155 & 7.80 & 6.07 & 22 & .10 & .6 & 1.3 & \text { SF } 4\end{array}$

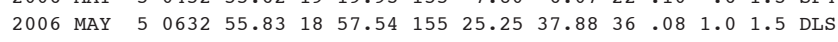
$\begin{array}{lllllllllllll}2006 & & 5\end{array}$

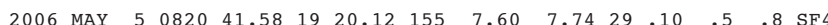

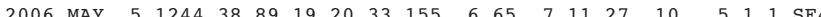

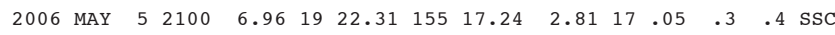

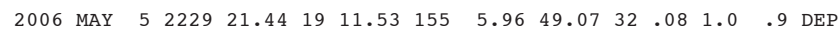

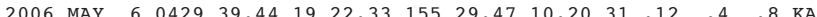
2006 MAY $6 \begin{array}{llllllllllllll}6 & 0436 & 25.75 & 19 & 18.84 & 155 & 13.57 & 5.58 & 15 & 09 & 6 & 1.6 & \text { SF }\end{array}$

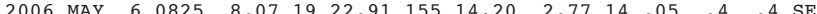

$\begin{array}{lllllllllllllll}2006 & \text { MAY } & 6 & 0901 & 56.89 & 19 & 15.36 & 155 & 33.10 & 6.95 & 29 & .18 & .6 & 1.2 & \text { LSW }\end{array}$

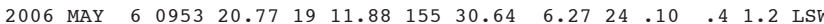

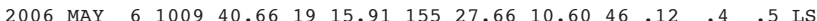

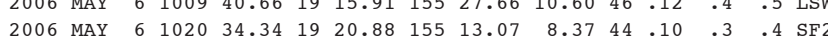

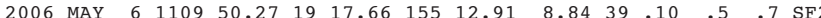

$\begin{array}{llllllllllllll}2006 & \text { MAY } & 6 & 1140 & 23.30 & 19 & 23.12 & 155 & 17.18 & 2.71 & 17 & .08 & .3 & .3 \\ \text { SSC }\end{array}$ 2006 MAY 6 \begin{tabular}{lllllllllll}
1248 & 52.36 & 19 & 20.02 & 155 & 19.42 & 3.82 & 22 & 08 & 3 & .9 \\
\hline
\end{tabular}

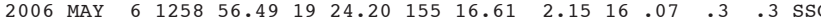
$\begin{array}{lllllllllllllll}2006 & \text { MAY } & 6 & 1349 & 17.03 & 19 & 19.80 & 155 & 11.91 & 7.11 & 19 & .09 & .5 & 1.2 & \text { SF } 3\end{array}$ $\begin{array}{llllllllllllll}2006 & \text { MAY } & 6 & 1351 & 34.92 & 19 & 24.98 & 155 & 39.30 & 3.26 & 24 & .09 & .6 & .5\end{array}$

$\begin{array}{lllllllllllllll}2006 & \text { MAY } & 6 & 1726 & 44.62 & 19 & 18.07 & 155 & 22.88 & 3.78 & 32 & .10 & .5 & .9 & \text { SWR }\end{array}$ $\begin{array}{lllllllllllllll}2006 & \text { MAY } & 7 & 0214 & 26.66 & 19 & 19.50 & 155 & 10.30 & 8.52 & 22 & .07 & .5 & .8 & \text { SF } 3\end{array}$

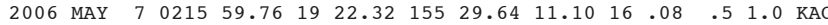

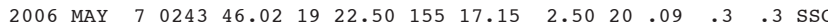
$\begin{array}{lllllllllllllll}2006 & \text { MAY } & 7 & 0606 & 47.40 & 19 & 18.32 & 155 & 12.98 & 8.22 & 29 & .07 & .5 & .9 & \text { SF2 }\end{array}$

$\begin{array}{lllllllllllllll}2006 & \text { MAY } & 7 & 0655 & 20.28 & 19 & 18.98 & 155 & 13.31 & 5.99 & 29 & .08 & .4 & 1.1 & \text { SF } 2\end{array}$

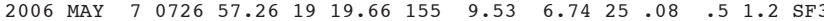
$\begin{array}{lllllllllllllll}2006 & \text { MAY } & 7 & 0825 & 0.79 & 19 & 30.15 & 155 & 29.05 & 6.66 & 20 & .10 & .4 & 1.7 & \text { MLO }\end{array}$

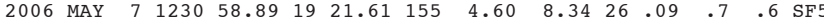
$\begin{array}{lllllllllllllll}2006 & \text { MAY } & 7 & 1253 & 22.90 & 19 & 41.01 & 155 & 21.04 & 14.55 & 41 & .10 & .6 & .4 & \text { KEA }\end{array}$

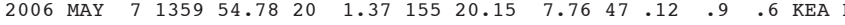
$\begin{array}{llllllllllllllll}2006 & \text { MAY } & 7 & 1549 & 6.34 & 19 & 24.76 & 155 & 18.69 & 6.86 & 27 & .11 & .4 & .8 & \text { INT }\end{array}$ $\begin{array}{llllllllllllllll}2006 & \text { MAY } & 7 & 1812 & 26.35 & 19 & 21.80 & 155 & 12.77 & 2.92 & 17 & .08 & .4 & .5 & \text { SER }\end{array}$ $\begin{array}{rllllllllllllll}2006 & \text { MAY } & 7 & 1812 & 38.93 & 19 & 21.92 & 155 & 12.44 & 3.12 & 17 & .05 & .5 & .3 & \text { SER } \\ 2006 & \text { MAY } & 8 & 0302 & 51.19 & 19 & 32.91 & 155 & 26.79 & 32.83 & 20 & .07 & .8 & 1.2 & \text { DML }\end{array}$
REF AZ MIN 50

$1.4 \times 130$ $\begin{array}{lll}1.3 \times & 206 & 8 \\ 1.1 \times & 119 & 5\end{array}$ $\begin{array}{llll}2.4 \times \quad 45 \quad 2 & \end{array}$ $1.2 \times 190 \quad 6$

$\begin{array}{ccc}1.0 \times \quad 67 & 4\end{array}$ $\begin{array}{rrr}1.5 \times & 105 \quad 2 \\ 1.0 \times & 97 & 2\end{array}$ $.4 \times \quad 138 \quad 5$

$1.1 \times 146 \quad 5$ $2.0 \times \quad 233 \quad 23$ $1.6 \times 130 \quad 5$ $\begin{array}{llll}1.5 \times 172 & 6\end{array}$

$1.3 \times \quad 59 \quad 2$ $\begin{array}{lll}1.9 \times & 247 & 11 \\ 1.1 \times & 73 & 3\end{array}$ $\begin{array}{rrr}1.1 \times & 73 & 3 \\ .8 \times & 84 & 3\end{array}$ $4 \times \quad 95 \quad 2$

$\begin{array}{lll}1.5 \times 105 & 5 \\ 1.7 \times & 128 & 6\end{array}$ $\begin{array}{rrr}1.7 \times & 128 & 6 \\ 2.3 \times & 97 & 5 \\ 2.5 x & 61 & 3\end{array}$ $2.5 \mathrm{X} \quad 61 \quad 3$ $\begin{array}{lll}1.8 \times & 127 \quad 2\end{array}$

$\begin{array}{lll}1.2 \mathrm{X} & 67 & 1\end{array}$ $\begin{array}{rrr}1.3 \times & 114 & 4 \\ .9 \times & 104 & 1\end{array}$ $\begin{array}{rrr}.9 \times & 104 & 1 \\ 1.2 \times & 86 & 6\end{array}$ $1.8 \times 204 \quad 3$

$\begin{array}{lll}1.5 \times & 113 & 4\end{array}$ $\begin{array}{rrr}1.1 \mathrm{X} & 98 & 6 \\ .8 \mathrm{X} & 81 & 4\end{array}$ $\begin{array}{lll}1.2 \mathrm{X} & 53 \quad 2\end{array}$ $\begin{array}{lll}1.3 \times & 99 & 3\end{array}$

$\begin{array}{lll}1.4 \times \quad 78 \quad 4 & 4\end{array}$ $\begin{array}{lll}1.2 \times & 99 & 5\end{array}$ $\begin{array}{lll}1.5 x & 97 & 4\end{array}$ $\begin{array}{rrr}.5 \times & 186 & 5 \\ .9 x & 84 & 16\end{array}$

$3.2 \times 25430$ $\begin{array}{lll}1.2 \mathrm{X} & 98 & 2\end{array}$ $\begin{array}{lll}.7 \times & 89 & 2\end{array}$ $.6 \times 132 \quad 2$ 
---ORIGIN TIME (HST)-- -LAT N-- --LON W-- DEPTH N RMS ERH ERZ LOC PREF AZ MIN 51 YEAR MON DA HRMN SEC DEG MIN DEG MIN KM RD SEC KM KM REMKS MAG GAP DS

$\begin{array}{lllllllllllllll}2006 & \text { MAY } & 8 & 0632 & 43.61 & 19 & 27.42 & 155 & 26.15 & 7.80 & 32 & .12 & .4 & 1.2 & \text { KAO }\end{array}$ $\begin{array}{llllllllllllllll}2006 & \text { MAY } & 8 & 0701 & 41.48 & 19 & 20.55 & 155 & 4.91 & 8.56 & 37 & .12 & .5 & .6 & \text { SF5 }\end{array}$ $\begin{array}{lllllllllllllll}2006 & \text { MAY } & 8 & 1014 & 31.43 & 19 & 18.58 & 155 & 13.07 & 7.95 & 38 & .10 & .4 & .7 & \text { SF2 }\end{array}$ $\begin{array}{lllllllllllllll}2006 & \text { MAY } & 8 & 1014 & 42.92 & 19 & 18.60 & 155 & 12.56 & 6.73 & 45 & .11 & .4 & .7 & \text { SF2 }\end{array}$ $\begin{array}{lllllllllllllll}2006 & \text { MAY } & 8 & 1106 & 25.52 & 19 & 18.34 & 155 & 13.00 & 5.45 & 30 & .10 & .4 & 1.1 & \text { SF2 }\end{array}$

2006 MAY $8 \begin{array}{lllllllllllll}1111 & 10.54 & 19 & 19.84 & 155 & 12.36 & 4.81 & 23 & .10 & .5 & 1.8 & \text { SSF }\end{array}$ 2006 MAY $8 \begin{array}{llllllllllllll}1149 & 55.90 & 19 & 18.26 & 155 & 12.83 & 5.07 & 21 & .09 & .6 & 1.4 & \text { SF2 }\end{array}$ 2006 MAY $8 \begin{array}{llllllllllllll}1415 & 40.63 & 19 & 18.35 & 155 & 12.99 & 8.71 & 38 & .09 & .4 & .4 & \mathrm{SF} 2\end{array}$ 2006 MAY $8 \begin{array}{lllllllllllll}8 & 1420 & 51.22 & 19 & 18.23 & 155 & 12.92 & 8.10 & 36 & .09 & .4 & .6 & \mathrm{SF} 2\end{array}$ $\begin{array}{llllllllllllllll}2006 & \text { MAY } & 8 & 1510 & 32.08 & 19 & 17.42 & 155 & 28.28 & 29.93 & 22 & .08 & .7 & 1.3 & \text { DLS }\end{array}$

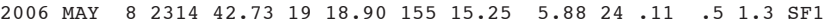

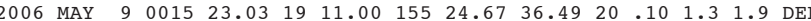

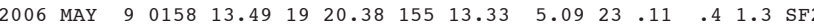

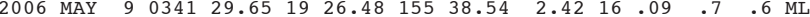

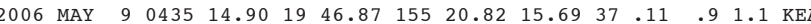

$\begin{array}{lllllllllllllll}2006 & \text { MAY } & 9 & 0544 & 52.71 & 19 & 39.75 & 156 & 11.23 & 39.21 & 14 & .13 & 8.5 & 7.9 & \text { HUA }\end{array}$ $\begin{array}{lllllllllllllll}2006 & \text { MAY } & 9 & 0633 & 30.13 & 19 & 18.47 & 155 & 28.89 & 11.58 & 17 & .09 & .5 & 1.1 & \text { LSW }\end{array}$

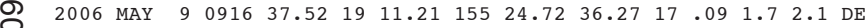
$\begin{array}{llllllllllllllll}2006 & \text { MAY } & 9 & 0941 & 5.40 & 19 & 16.46 & 155 & 25.91 & 8.91 & 20 & .11 & .5 & 1.3 & \text { LSW }\end{array}$ $\begin{array}{lllllllllllllll}2006 & \text { MAY } & 9 & 1148 & 41.70 & 19 & 19.32 & 155 & 15.38 & 6.22 & 21 & .11 & .6 & 1.1 & \text { SF }\end{array}$

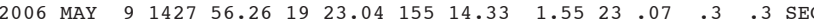

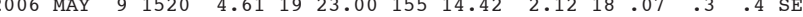

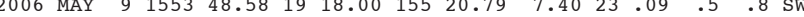

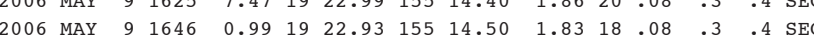

$\begin{array}{lllllllllllllll}2006 & \text { MAY } & 9 & 1646 & 52.21 & 19 & 22.96 & 155 & 14.43 & 2.51 & 16 & .07 & .3 & .4 & \text { SEC }\end{array}$

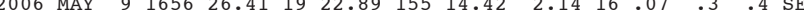

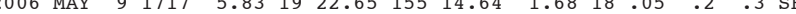

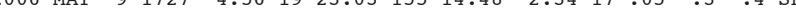
$\begin{array}{lllllllllllllll}2006 & \text { MAY } & 9 & 1728 & 3.96 & 19 & 23.01 & 155 & 14.42 & 2.10 & 11 & .06 & .4 & .6 & \text { SE }\end{array}$

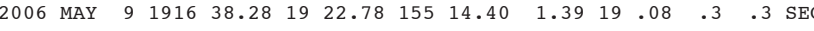

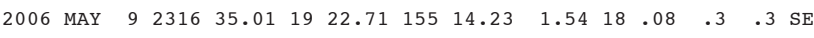

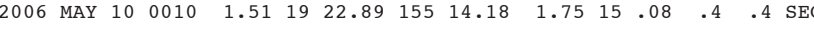

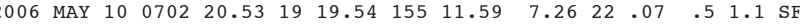

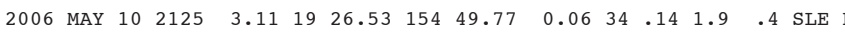

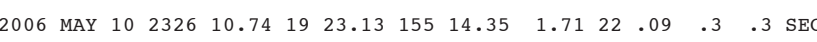

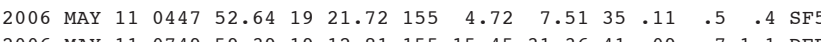

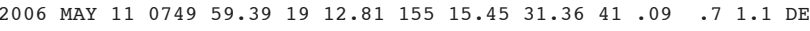

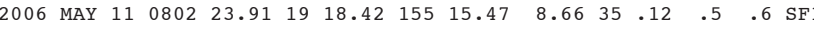

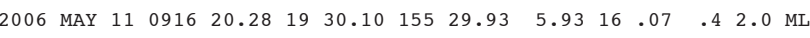

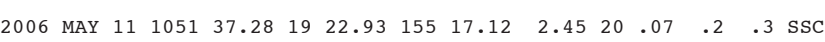
$\begin{array}{llllllllllllll}2006 & \text { MAY } & 11 & 1421 & 24.06 & 20 & 6.75 & 156 & 13.53 & 38.93 & 22 & .10 & 9.0 & 4.3 \\ \text { KOH }\end{array}$

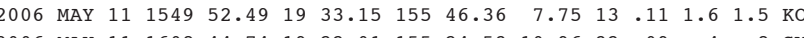
$\begin{array}{lllllllllllllll}2006 & \text { MAY } & 11 & 1608 & 44.74 & 19 & 22.01 & 155 & 24.53 & 10.96 & 32 & .09 & .4 & .8 & \text { SWR } \\ 2006 \text { MAY } & 11 & 1820 & 49.08 & 19 & 28.42 & 155 & 25.89 & 8.94 & 26 & .09 & .4 & 1.0 & \text { KAO }\end{array}$ $\begin{array}{rrr}1.5 \mathrm{X} & 61 & 7 \\ 1.6 \mathrm{X} & 171 & 7\end{array}$ $.6 \times 171$ $2.4 \times 104 \quad 3$ $1.1 \times \quad 98 \quad 3$

$1.0 \times 149 \quad 5$ $.8 \times 105 \quad 3$ $2.1 \times \quad 98 \quad 3$ $1.6 \times 104 \quad 2$ $1.5 \times \quad 86 \quad 5$

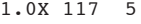
.2x $226 \quad 12$ $.9 \times \quad 63 \quad 4$ $1.4 \times 204 \quad 4$ 1.8X 10312

$1.6 \times 292 \quad 64$ $.1 \times 104 \quad 7$ $1.2 \times 247 \quad 12$ $.9 \times 108 \quad 8$ $.9 \times 150 \quad 4$

$\begin{array}{lll}1.7 \mathrm{X} & 82 & 2\end{array}$ $\begin{array}{lll}1.5 x & 85 & 2\end{array}$ $1.2 \times 121$ $1.5 \times \quad 85$

$\begin{array}{lll}1.3 \times & 87 \quad 3\end{array}$

$\begin{array}{lll}1.2 \times \quad 83 & 2\end{array}$ $1.2 \mathrm{X} \quad 84$ $\begin{array}{lll}1.5 \times & 77 \quad 2\end{array}$ $1.3 \times \quad 88$ $1.2 \times 118 \quad 2$

$1.9 \times \quad 79$ $\begin{array}{lll}1.7 \times & 82 & 2 \\ 1.6 \times & 90 & 2\end{array}$ $1.6 \mathrm{X} \quad 90 \quad 2$ $\begin{array}{lll}1.4 \times & 94 \quad 6\end{array}$

$\begin{array}{lll}2.0 \mathrm{X} & 82 & 2\end{array}$ $\begin{array}{llll}1.8 \times & 159 & 5\end{array}$ $2.0 \times 183 \quad 9$ $\begin{array}{lll}1.5 \times & 107 & 4\end{array}$ $\begin{array}{rrr}1.5 \times & 49 & 1\end{array}$ $\begin{array}{rrr}1.8 \times & 306 & 62 \\ .9 \times & 268 & 5\end{array}$ $.9 \times 2685$ $\begin{array}{lll}1.5 \times & 54 & 4 \\ 1.5 \times & 81 & 5\end{array}$
--ORIGIN TIME (HST)-- -LAT N-- --LON W-- DEPTH N RMS ERH ERZ LOC YEAR MON DA HRMN SEC DEG MIN DEG MIN KM RD SEC KM KM REMKS

$\begin{array}{lllllllllllll}2006 & \text { MAY } 11 & 2027 & 43.04 & 19 & 23.58 & 155 & 28.48 & 10.18 & 27 & .10 & .4 & .7\end{array}$

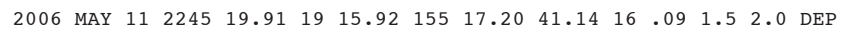

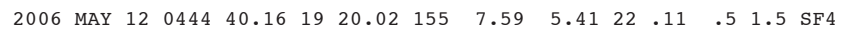

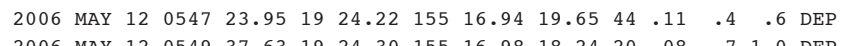

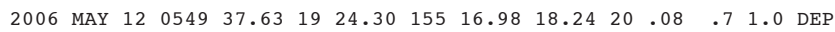

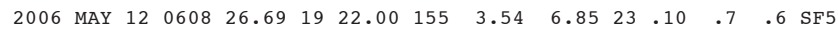

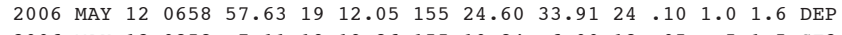

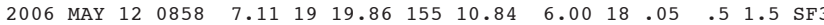

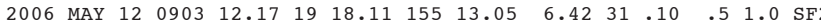
$\begin{array}{lllllllllllllll}2006 & \text { MAY } & 12 & 0910 & 56.24 & 19 & 18.23 & 155 & 13.10 & 8.21 & 36 & .08 & .5 & .6 & \text { SF2 }\end{array}$

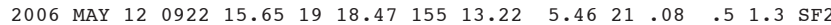

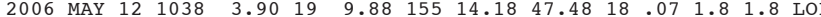

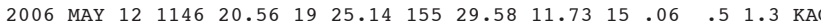

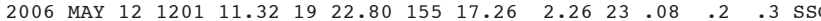
$\begin{array}{lllllllllllllll}2006 & \text { MAY } & 12 & 1201 & 58.64 & 19 & 22.79 & 155 & 17.25 & 2.38 & 25 & .08 & .2 & .3 & \text { SSC }\end{array}$

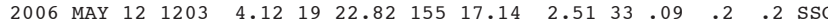

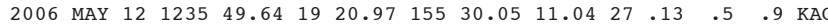

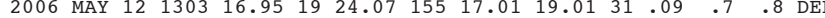

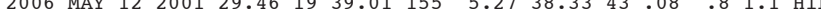

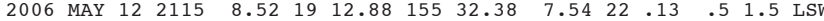

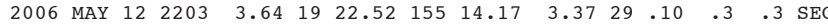

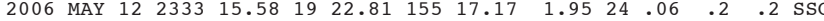

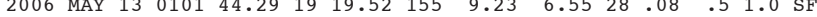

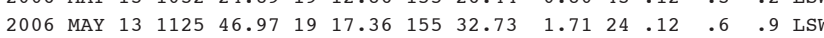

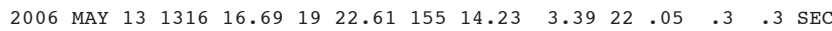

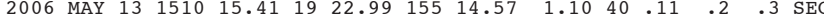

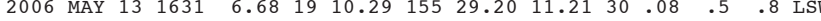

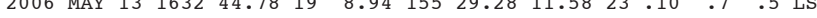

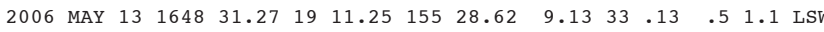

$\begin{array}{llllllllllllll}2006 & \text { MAY } & 13 & 1714 & 22.19 & 19 & 27.23 & 155 & 26.17 & 7.06 & 28 & .11 & .4 & 1.3 \\ \text { KAO }\end{array}$

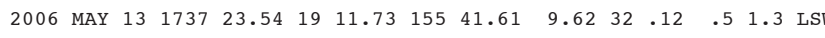

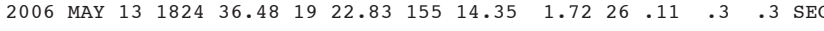

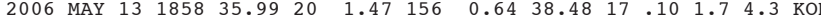

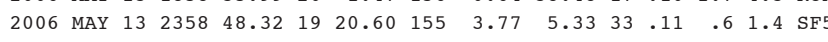

$\begin{array}{lllllllllllllll}2006 & \text { MAY } 14 & 0552 & 15.87 & 19 & 8.44 & 155 & 38.43 & 0.93 & 32 & .16 & 2.7 & 1.3 & \text { LSW }\end{array}$

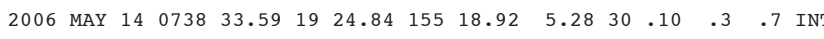

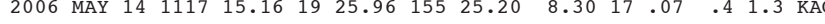

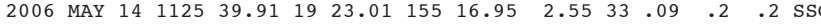

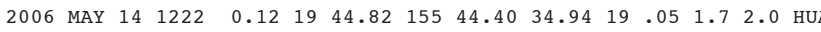
$\begin{array}{lllllllllllllll}2006 & \text { MAY } 14 & 1411 & 22.24 & 19 & 27.14 & 155 & 29.22 & 10.51 & 22 & .08 & .4 & 1.0 & \text { KAO }\end{array}$ $\begin{array}{llllllllllllll}2006 & \text { MAY } & 14 & 1510 & 55.82 & 19 & 22.34 & 155 & 49.21 & 12.69 & 24 & .12 & .6 & .6 \\ \text { KON }\end{array}$

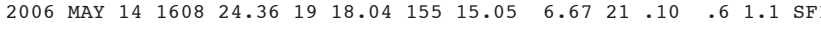
$\begin{array}{llllllllllllllll}2006 & \text { MAY } & 14 & 2100 & 46.98 & 19 & 47.31 & 155 & 35.22 & 14.43 & 21 & .13 & .7 & .6 & \text { KEA }\end{array}$
PREF AZ MIN 52 $\begin{array}{rrr}2 \times & 61 & 3 \\ 2 \times & 269 & 5\end{array}$ $1.1 \mathrm{U} 151 \quad 5$ $\begin{array}{lll}2.8 \times & 42 & 1\end{array}$ $1.5 \times 126 \quad 2$

$\begin{array}{lll}1.1 \times \quad 168 \quad 4 & \end{array}$ $\begin{array}{lll}1.1 \times & 167 \quad 7\end{array}$ $.1 \times \quad 91 \quad 6$ $\begin{array}{rrr}1.3 \times & 102 & 2 \\ 1.7 x & 97 & 2\end{array}$

$\begin{array}{lll}.8 \mathrm{X} & 88 \quad 3\end{array}$ $1.8 \times \quad 236 \quad 13$ $1.1 \times 106 \quad 6$ $1.6 \mathrm{X} \quad 50 \quad 1$ $1.7 \times \quad 50 \quad 1$

2.0X $41 \quad 1$ $1.5 \mathrm{x} \quad 64 \quad 5$ $\begin{array}{lll}1.4 \mathrm{X} & 82 & 1\end{array}$ $\begin{array}{lll}1.9 \times \quad 116 & 8\end{array}$ $1.4 \times 127 \quad 6$

$\begin{array}{lll}1.9 \times & 77 \quad 2\end{array}$ $\begin{array}{lll}1.6 \mathrm{X} & 50 & 1\end{array}$ $1.1 \times \quad 105 \quad 5$ $2.2 \times 128 \quad 7$

$\begin{array}{lll}1.6 \mathrm{X} & 85 & 2\end{array}$ $\begin{array}{lll}1.9 \times & 48 & 3\end{array}$ $1.1 \times 221$ $\begin{array}{lll}1.9 \times & 75 & 9\end{array}$ $\begin{array}{lll}2.2 \mathrm{x} & 81 \quad 2\end{array}$

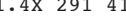
$1.2 \times 182 \quad 7$ $2.0 \times 279 \quad 19$ $1.1 \times 104 \quad 3$ $\begin{array}{lll}1.1 \mathrm{X} & 88 & 8 \\ 2.1 \mathrm{X} & 40 & 1\end{array}$ $\begin{array}{lll}1.1 \times & 40 & 1\end{array}$ $1.5 \times \quad 71 \quad 8$ $\begin{array}{lll}1.5 \times & 117 \quad 13 \\ 1.5 \times & 135 & 3\end{array}$ $\begin{array}{lll}1.1 \times & 135 & 3\end{array}$ $\begin{array}{rrr}1.4 \mathrm{X} & 55 & 6 \\ 1.5 \mathrm{X} & 110 & 11\end{array}$ 
--ORIGIN TIME (HST)-- -LAT N-- --LON W-- DEPTH N RMS ERH ERZ LOC YEAR MON DA HRMN SEC DEG MIN DEG MIN KM RD SEC KM KM REMKS

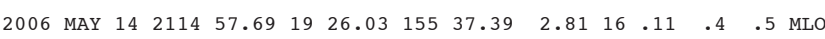

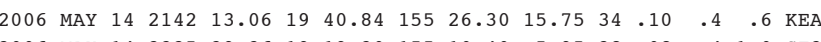
$\begin{array}{llllllll} & \end{array}$ $\begin{array}{lllllll} & 0\end{array}$

$\begin{array}{lllllllllllllll}2006 & \text { MAY } 15 & 0206 & 13.66 & 19 & 26.17 & 155 & 18.88 & 7.70 & 27 & .08 & .5 & .7 & \text { INT }\end{array}$

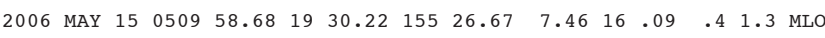

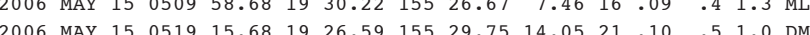

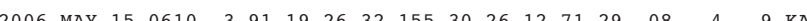

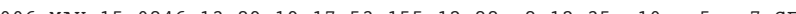

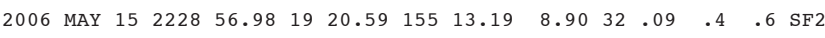

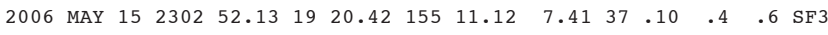

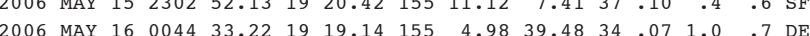

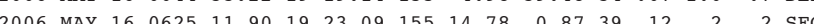

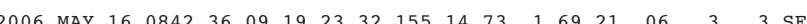

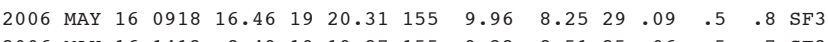

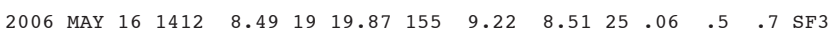

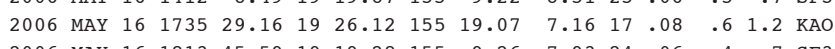

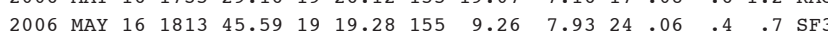
$\begin{array}{lllllllllllllll}2006 & \text { MAY } & 16 & 2351 & 33.73 & 19 & 15.50 & 155 & 33.82 & 8.16 & 26 & .14 & .5 & 1.6 & \text { LSW }\end{array}$

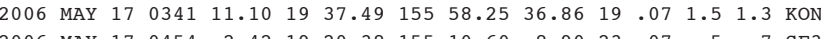

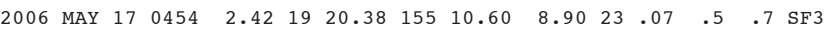
$\begin{array}{lllllllllllllll}2006 & \text { MAY } & 17 & 0811 & 59.82 & 19 & 24.61 & 154 & 58.86 & 0.87 & 22 & .10 & .8 & .6 & \text { SLE } \\ 2006 \text { MAY } & 17 & 1013 & 22.55 & 19 & 20.19 & 155 & 12.85 & 7.93 & 17 & .05 & .5 & 1.1 & \text { SF2 }\end{array}$

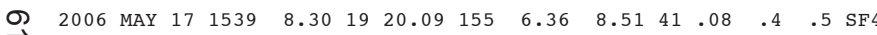

$\begin{array}{lllllllllllll}2006 & \text { MAY } 17 & 1834 & 33.72 & 19 & 29.19 & 155 & 28.14 & 6.20 & 19.10 & .4 & 1.9 & \text { KAO }\end{array}$

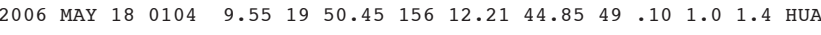

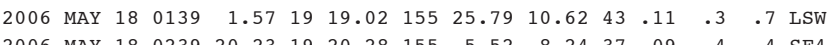
$\begin{array}{llllllllllllll}2006 & \text { MAY } 18 & 0239 & 20.23 & 19 & 20.28 & 155 & 5.52 & 8.24 & 37 & .09 & .4 & .4 & \text { SF } 4\end{array}$ $\begin{array}{lllllllllllllll}2006 & \text { MAY } & 18 & 0330 & 47.24 & 19 & 24.31 & 155 & 28.89 & 10.35 & 32 & .10 & .4 & .6 & \text { KA0 }\end{array}$

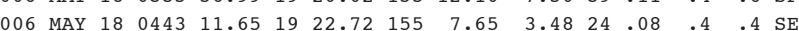

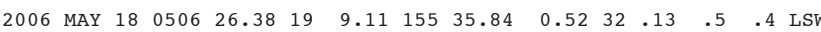
$\begin{array}{lllllllllllll}2006 & \text { MAY } 18 & 0547 & 5.40 & 19 & 24.64 & 155 & 20.24 & 6.82 & 33 & .09 & .3 & .8 \\ \text { KAO }\end{array}$

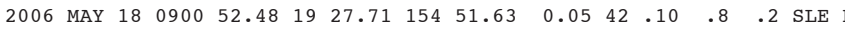

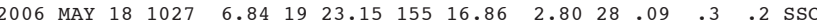

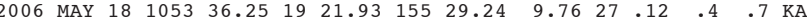

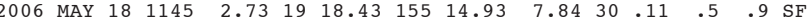

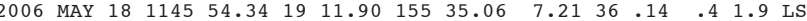

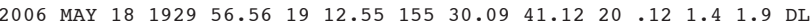

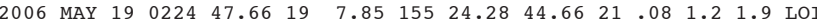

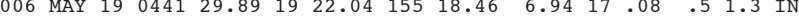

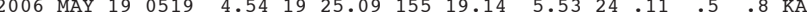

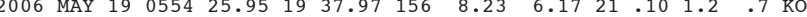

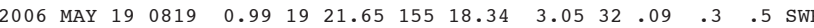

PREF AZ MIN 53 MAG GAP DS $1.5 \times \quad 91 \quad 3$ $\begin{array}{rrr}1.8 \times & 78 & 10 \\ 1.5 \times & 102 & 6 \\ 2.0 \times & 84 & 5\end{array}$ $1.5 \times 1020$ $2.0 \times 48 \quad 3$

$1.5 \times 152 \quad 3$ $\begin{array}{rrr}.6 \times & 117 \quad 4 \\ .9 \times & 68 & 6\end{array}$ $\begin{array}{rrr}.9 \times & 68 & 6 \\ 1.7 \times & 67 & 8 \\ 1.4 \times & 336 & 1\end{array}$ $1.7 \times 136$ $1.3 \times \quad 62 \quad 4$ $\begin{array}{lll}1.5 \times \quad 79 & 5\end{array}$ $\begin{array}{llll}1.9 \times & 269 & 6\end{array}$ $2.3 \times \quad 48 \quad 2$ $\begin{array}{lll}2.3 \mathrm{X} & 48 & 2 \\ 1.7 \mathrm{x} & 67 & 3\end{array}$

$\begin{array}{lll}1.6 \times & 93 \quad 5\end{array}$ $\begin{array}{lll}1.5 \times & 107 \quad 5\end{array}$ $1.3 \times 154 \quad 3$ $\begin{array}{lll}1.1 \times & 103 & 4\end{array}$ $1.2 \mathrm{X} \quad 65$

$1.2 \times 287 \quad 16$ $1.1 \times \quad 80 \quad 4$ $1.3 \times 1818$ $.8 \times \quad 70 \quad 5$ 2.0X 1556

1. $3 \times \quad 82 \quad 5$ 2.6X $263 \quad 42$ $1.5 \mathrm{X} \quad 93 \quad 5$ $1.4 \times \quad 166 \quad 7$ $\begin{array}{lll}1.3 \mathrm{X} & 41 & 4\end{array}$

$\begin{array}{lll}1.5 \times & 80 & 5\end{array}$ $1.6 \times 117 \quad 1$

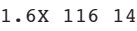
$\begin{array}{lll}1.6 \times & 73 \quad 2\end{array}$

$1.2 \times 251 \quad 5$
-ORTGIN TIME (HST)-- -LAT N-- - TON W-- DEPTH N RMS ERH ERZ LOC YEAR MON DA HRMN SEC DEG MIN DEG MIN DM RD SEC KM KM REMES

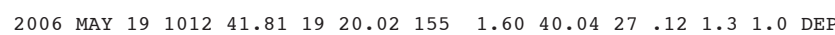

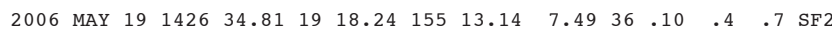

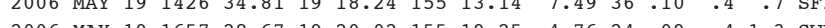

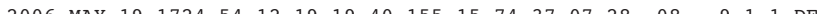

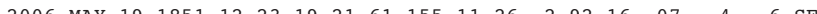

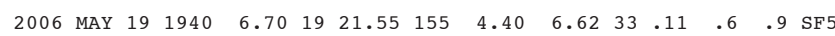

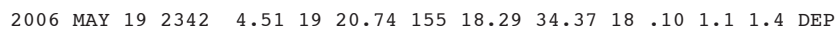

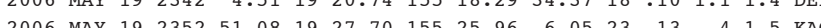

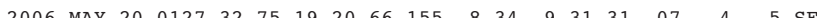

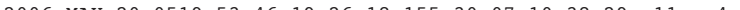

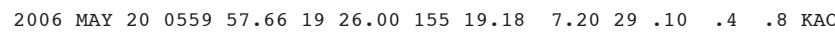

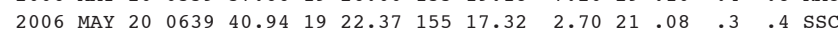
$\begin{array}{llllllllllll}2006 & 0 & .8 & \text { KAO }\end{array}$ $\begin{array}{llllllllll}2006 & & \end{array}$ $\begin{array}{lllllllllllll}2006 & 0 & 0\end{array}$

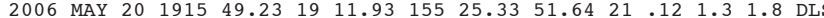

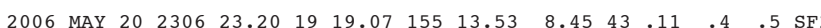

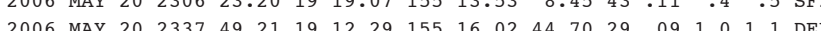

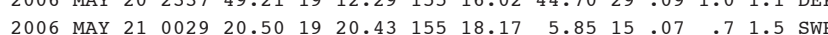
$\begin{array}{llllllllllll}2006 & 0 & 0\end{array}$

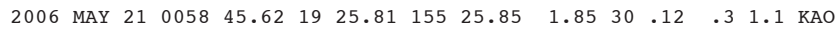

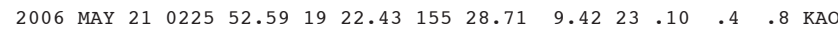

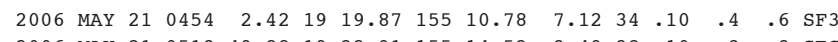

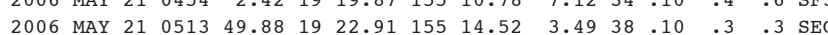

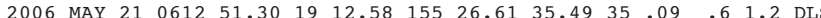

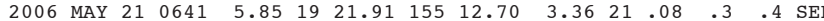

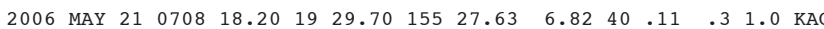

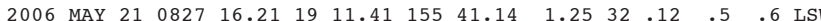

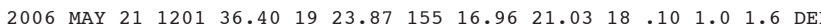

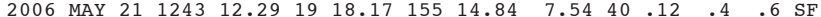

$\begin{array}{lllllllllllllll}2006 & \text { MAY } & 21 & 1430 & 44.85 & 19 & 22.58 & 155 & 2.52 & 8.41 & 28 & .10 & .7 & .5 & \text { SF5 }\end{array}$

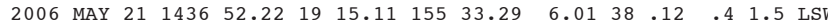

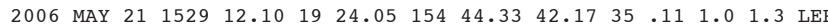

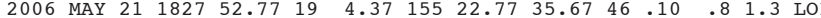
$\begin{array}{llllllllllllll}2006 & \text { MAY } 21 & 1855 & 43.34 & 19 & 3.37 & 155 & 22.97 & 36.52 & 28 & .10 & 1.0 & 1.4 & \text { LOI }\end{array}$

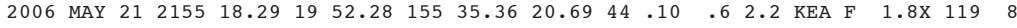

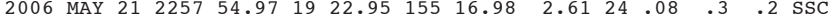

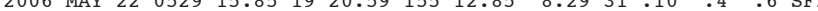

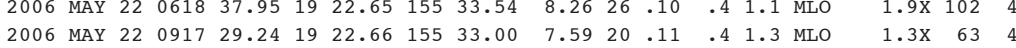
$\begin{array}{lllllllllllllllll}2006 & \text { MAY } 22 & 1007 & 53.65 & 19 & 23.01 & 155 & 26.65 & 11.34 & 50 & .12 & .3 & .4 & \text { KAO } & 2.3 \times & 54 & 2\end{array}$

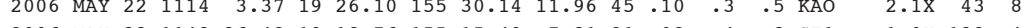

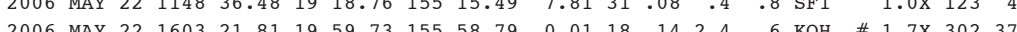

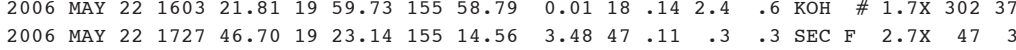

$1.7 \times 27512$ $1.7 \times 195020$ $.4 x+106$ $1.3 \mathrm{X} \quad 84 \quad 3$

$1.6 \times 164 \quad 5$ $\begin{array}{llll}1.5 \times & 122 & 5 \\ 1.2 \times & 60 & 6\end{array}$

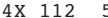

$\begin{array}{lll}1.3 \times & 113 \quad 3\end{array}$ $\begin{array}{lrl}1.4 \times \quad 57 \quad 2 \\ 1.4 \times & 120 & 6\end{array}$ $\begin{array}{lll}1.4 \times & 120 & 6 \\ 1.3 \times & 90 & 2\end{array}$ $1.1 \times \quad 96 \quad 2$

$\begin{array}{lll}1.5 \times 160 & 6\end{array}$ $\begin{array}{rrr}1.8 \times & 71 & 4 \\ 1.6 \times & 208 & 10\end{array}$ $\begin{array}{rrr}1.6 \times & 208 & 10 \\ 1.8 \times & 146 & 5\end{array}$ $1.3 \times \quad 82 \quad 1$

$\begin{array}{lll}1.3 \mathrm{X} & 61 & 7 \\ 1.5 \mathrm{X} & 63 & 2\end{array}$ $\begin{array}{lll}1.5 \mathrm{X} & 89 & 5\end{array}$ $2.4 \times \quad 49 \quad 3$ $\begin{array}{lll}1.5 \mathrm{X} & 98 \quad 1\end{array}$ $\begin{array}{rrr}1.5 \times & 75 & 4 \\ 1.6 x & 147 & 9\end{array}$ $1.2 \times 196 \quad 5$ $1.4 \times 105 \quad 3$

$\begin{array}{lll}.3 \times 188 & 4 \\ .5 \times 107 & 6\end{array}$ $1.9 \times 29620$ $2.1 \times 20413$ $\begin{array}{rrr}.8 \times & 119 & 8 \\ .5 \times & 48 & 1\end{array}$ $\begin{array}{lll}.3 \times & 66 & 4\end{array}$ $.9 \times 102 \quad 4$ 
---ORIGIN TIME (HST)-- -LAT N-- --LON W-- DEPTH N RMS ERH ERZ LOC PREF AZ MIN 55 YEAR MON DA HRMN SEC DEG MIN DEG MIN KM RD SEC KM KM REMKS MAG GAP DS

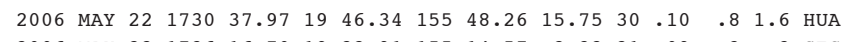

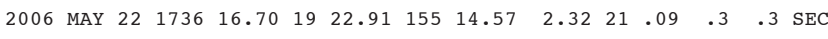

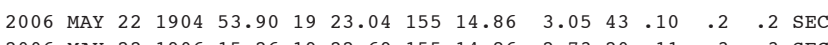

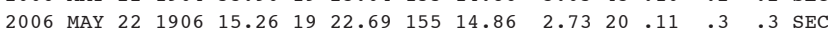

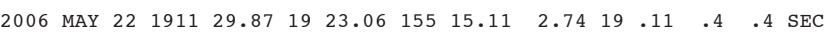

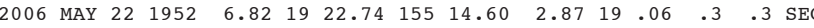

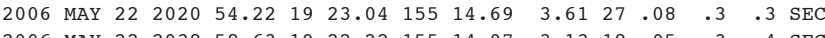

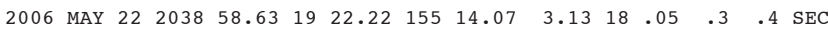

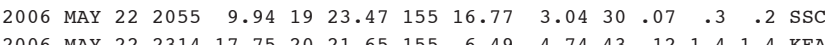

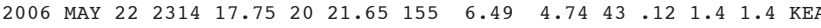

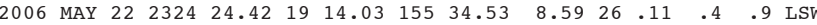

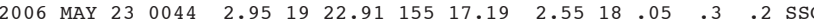

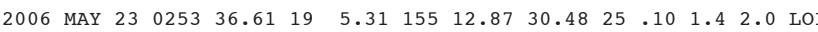

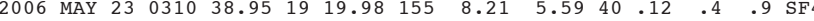

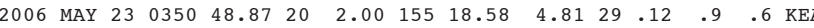

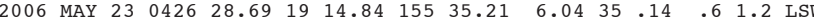

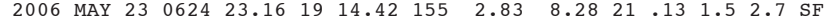

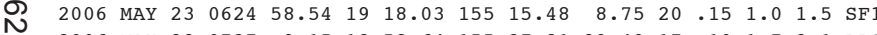

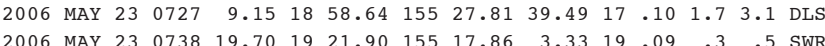

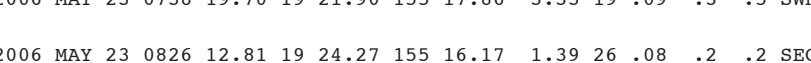

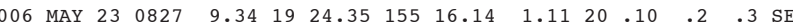

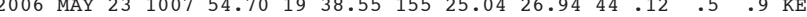

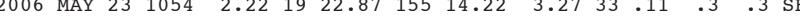

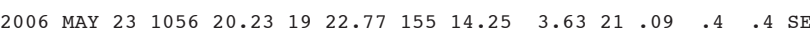

$\begin{array}{llllllllllllll}2006 & \text { MAY } 23 & 1331 & 12.50 & 19 & 24.92 & 155 & 19.24 & 7.06 & 40 & .09 & .4 & .6 & \text { KAO }\end{array}$

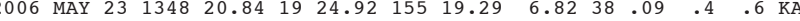

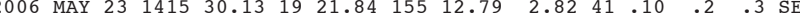

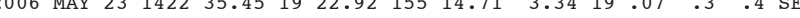

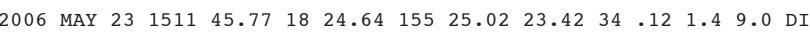

$\begin{array}{llllllllllllll}2006 & \text { MAY } 23 & 1534 & 23.42 & 19 & 24.94 & 155 & 19.12 & 7.15 & 35 & .09 & .4 & .6 & \text { KAO }\end{array}$

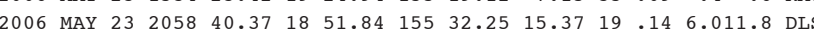
$\begin{array}{llllllllllllll}2006 & \text { MAY } 23 & 2106 & 48.00 & 19 & 26.20 & 155 & 29.89 & 7.39 & 29 & .13 & .4 & 1.3 & \text { KAO }\end{array}$

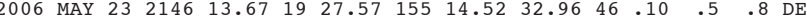

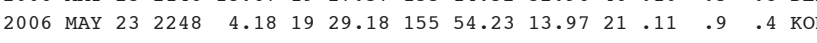

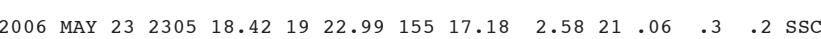
$\begin{array}{llllllllllllll}2006 & \text { MAY } 24 & 0423 & 42.53 & 19 & 16.08 & 155 & 27.68 & 9.96 & 41 & .11 & .3 & .6 & \text { LS }\end{array}$

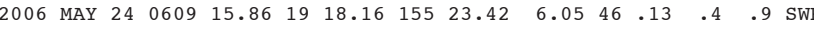

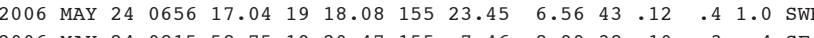

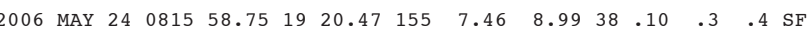

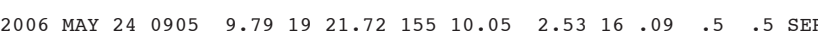

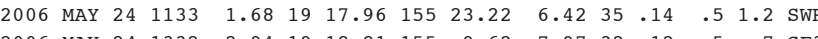

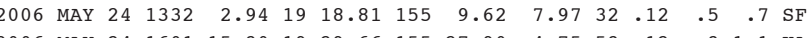

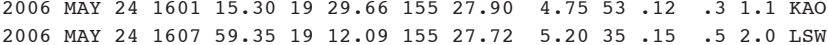

$1.7 \times 16210$ $1.5 \times \quad 79 \quad 3$ $\begin{array}{lll}.2 \mathrm{X} & 49 & 2 \\ .3 \mathrm{X} & 74 & 2\end{array}$ $1.4 \mathrm{X} \quad 69 \quad 2$

$\begin{array}{lll}1.5 x & 78 \quad 2\end{array}$ $1.8 \mathrm{X} \quad 65$ $1.2 \mathrm{X} \quad 92 \quad 2$ $\begin{array}{lll}1.6 \mathrm{X} & 44 & 0\end{array}$ $2.5 \times 30258$

$\begin{array}{lll}.4 \times \quad 75 & 8\end{array}$ $\begin{array}{lll}1.0 \times \quad 49 & \end{array}$ $1.4 \times 287 \quad 22$ $1.9 \times 116 \quad 5$ $1.5 \times 26416$

$2.2 \mathrm{U} 155 \quad 9$ $1.6 \times 323 \quad 20$ $1.3 \times 243 \quad 6$ $1.6 \times 310 \quad 39$ $\begin{array}{lll}1.3 \mathrm{X} & 61 \quad 3\end{array}$

$1.8 \times 126$ $1.5 \times 130$ $\begin{array}{lll}1.5 x & 65 & 8\end{array}$ $1.8 \mathrm{X} \quad 84$ $1.5 \times \quad 87 \quad 2$

$1.9 \times \quad 75$ $1.9 \times \quad 75 \quad 2$ $2.1 \mathrm{x} \quad 61$ $\begin{array}{lll}1.3 \times & 75 & 2\end{array}$ 2.0X 31783

$\begin{array}{lll}1.7 \times & 74 \quad 2\end{array}$ $1.3 \times 29643$ $\begin{array}{lll}1.3 \times & 62 & 8\end{array}$ $1.6 \times \quad 51 \quad 5$ $1.2 \times 106 \quad 2$ $\begin{array}{ccc}1.2 \mathrm{X} & 49 & 1 \\ 1.3 \mathrm{X} & 97 & 5\end{array}$ $\begin{array}{lll}1.3 \mathrm{X} & 97 & 5 \\ 2.5 \times & 111 & 4\end{array}$ $2.5 \times 1114$ $1.5 \times 1114$ $\begin{array}{lll}1.3 \times & 90 & 2 \\ 1.5 x & 113 & 4\end{array}$ $\begin{array}{lll}1.5 \times & 113 & 4 \\ 1.2 x & 112 & 4\end{array}$ $\begin{array}{llll}1.2 \times & 112 & 4 \\ 2.5 \times \quad 47 & 4\end{array}$ $\begin{array}{rrr}2.5 \times & 47 & 4 \\ 1.2 \times & 115 & 5\end{array}$
--ORIGIN TIME (HST)-- -LAT N-- --LON W-- DEPTH N RMS ERH ERZ LOC PREF AZ MIN 56 YEAR MON DA HRMN SEC DEG MIN DEG MIN $\quad$ KM RD SEC KM KM REMKS MAG GAP DS

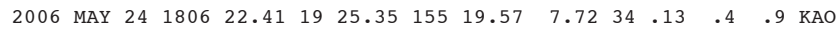
$\begin{array}{lllllllllllll}2006 & \text { MAY } 24 & 1808 & 44.07 & 19 & 53.68 & 155 & 55.21 & 13.86 & 20 & .09 & 2.9 & 1.5\end{array}$ HUA

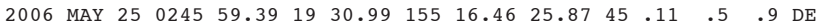

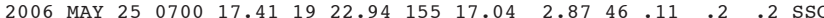

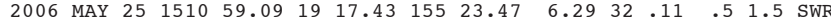

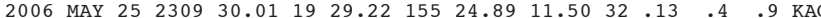

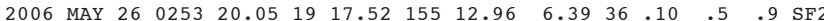

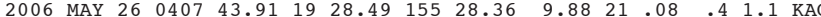
$\begin{array}{lllllllllllllll}2006 & \text { MAY } & 26 & 0556 & 12.11 & 19 & 19.31 & 155 & 29.68 & 12.05 & 19 & .11 & .6 & 1.4 & \text { KAO }\end{array}$

$\begin{array}{llllllllllllll}2006 & \text { MAY } 26 & 1157 & 30.79 & 19 & 19.75 & 155 & 17.18 & 8.46 & 26 & .08 & .4 & .8 & \text { SWR }\end{array}$

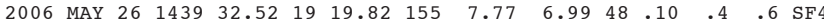

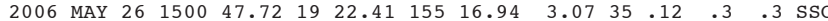

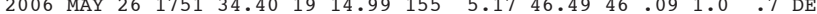
$\begin{array}{llllllllllllll}2006 & \text { MAY } 26 & 1827 & 10.82 & 19 & 23.91 & 155 & 26.57 & 10.69 & 53 & .11 & .3 & .4 & \text { KAO }\end{array}$

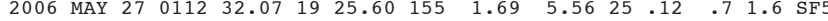

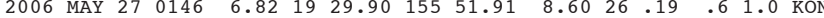

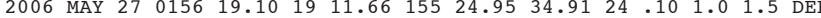

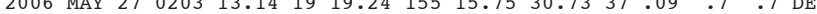
$\begin{array}{llllllllllllll}2006 & \text { MAY } & 27 & 0701 & 2.15 & 19 & 59.24 & 155 & 35.67 & 19.95 & 17 & .10 & 1.5 & 2.2\end{array}$

$\begin{array}{llllllllllllll}2006 & \text { MAY } 27 & 0744 & 39.64 & 19 & 18.31 & 155 & 30.37 & 10.54 & 28 & .11 & .4 & 1.1 & \text { LSW }\end{array}$

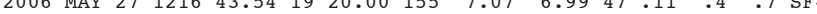

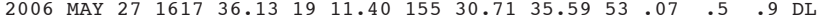

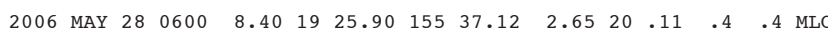

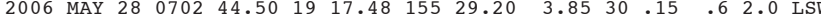

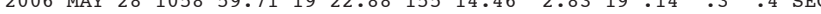

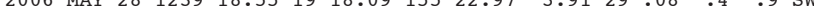

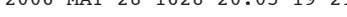

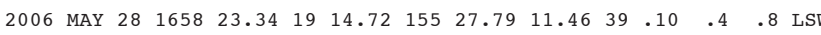

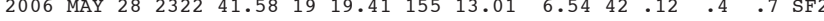

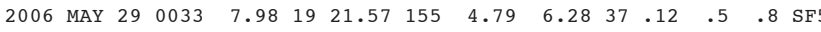

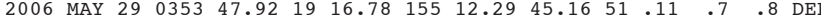

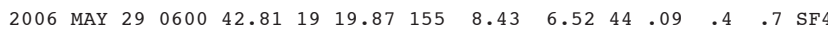

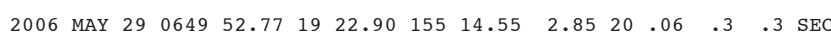

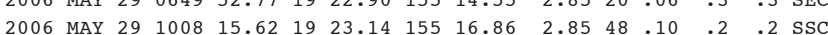
$\begin{array}{lllllllllllllll}2006 & \text { MAY } & 29 & 1008 & 15.62 & 19 & 23.14 & 155 & 16.86 & 2.85 & 48 & .10 & .2 & .2 & \text { SSC } \\ 2006 \text { MAY } & 29 & 1024 & 57.63 & 19 & 23.01 & 155 & 17.07 & 2.34 & 17 & .09 & .3 & .2 & \text { SSC }\end{array}$ $\begin{array}{lllllllllll}2006 & .3 & .2 & \text { SSC }\end{array}$

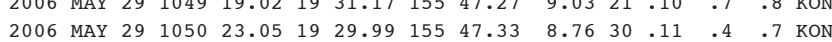

$\begin{array}{lllllllllllllll}2006 & \text { MAY } 29 & 1241 & 54.66 & 19 & 28.35 & 155 & 24.73 & 11.61 & 24 & .08 & .5 & 1.0 & \text { KAO }\end{array}$

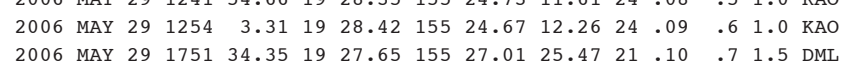

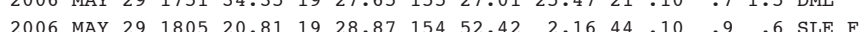

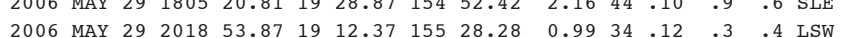

$1.4 \times \quad 89 \quad 3$ $.5 \times 23024$ $.4 \times 30651$ $\begin{array}{rrr}1.7 x & 60 & 11 \\ 2.5 \times & 37 & 1\end{array}$

$1.2 \times 158 \quad 5$ $\begin{array}{lll}1.2 \times & 59 & 3\end{array}$ $\begin{array}{lll}1.2 \times \quad 132 & 1\end{array}$ $\begin{array}{lll}1.2 x & 78 & 7 \\ 1.4 \mathrm{X} & 89 & 7\end{array}$

$\begin{array}{rrr}1.2 \times & 91 & 1 \\ 1.7 \times & 126 & 5\end{array}$ $\begin{array}{rrr}1.7 \times & 126 & 5 \\ 1.9 \times & 58 & 2\end{array}$ 2.0X 2097 $2.1 \times \quad 45 \quad 3$

$1.3 \times 139 \quad 5$ $\begin{array}{lll}1.3 \times & 108 \quad 6\end{array}$ $1.6 \times 186 \quad 6$ $\begin{array}{lll}1.6 \times 110 & 6\end{array}$ $1.4 \times 26616$

$\begin{array}{lll}.3 \times & 89 & 6\end{array}$ $\begin{array}{lll}1.7 \times & 141 \quad 5\end{array}$ $\begin{array}{lll}.4 \times \quad 92 & 6\end{array}$ $1.2 \times 1190$ $\begin{array}{lll}2 \times & 88 \quad 3\end{array}$

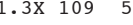
$\begin{array}{lll}1.2 \times \quad 83 & 2\end{array}$ $\begin{array}{lll}1.5 \times & 151 & 4\end{array}$ $\begin{array}{lll}1.5 \times & 57 \quad 3\end{array}$ $\begin{array}{llll}1.8 \times & 100 & 4\end{array}$

$\begin{array}{lll}1.6 \times & 78 & 4\end{array}$ $\begin{array}{lll}1.5 \times & 158 & 5\end{array}$ $\begin{array}{lll}2.5 \times & 155 & 2 \\ 1.4 \times & 166 & 5\end{array}$ $1.4 \times 166 \quad 5$

$\begin{array}{lll}1.3 \mathrm{X} & 80 & 3\end{array}$ $\begin{array}{lll}2.5 \mathrm{X} & 38 & 0 \\ 1.3 \mathrm{X} & 89 & 1\end{array}$ $\begin{array}{lll}1.3 \times & 89 & 1\end{array}$ $\begin{array}{llll}1.7 \times & 89 & 3\end{array}$ $1.2 \times \quad 70 \quad 4$ $\begin{array}{ll}1.1 \times & 71\end{array}$ $2.2 \times \quad 66 \quad 8$ $\begin{array}{rrr}1.5 \times & 97 & 5\end{array}$ 
---ORIGIN TIME (HST)-- -LAT N-- --LON W-- DEPTH N RMS ERH ERZ LOC YEAR MON DA HRMN SEC DEG MIN DEG MIN

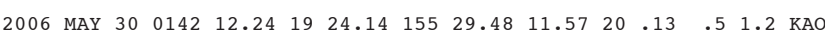

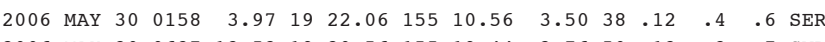
$\begin{array}{llllllllll} & .6 & \end{array}$

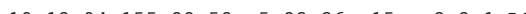

$\begin{array}{llllllllllllll}2006 & \text { MAY } 30 & 1612 & 7.54 & 19 & 23.56 & 155 & 29.87 & 10.91 & 19 & .08 & .4 & 1.0 & \text { KAO }\end{array}$

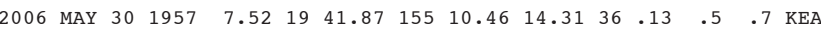

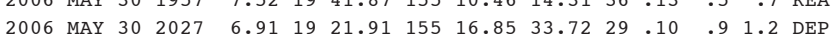

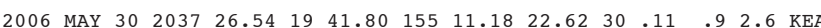
$\begin{array}{llllllllllllll}2006 & \text { MAY } 30 & 2112 & 26.09 & 19 & 42.12 & 155 & 10.28 & 14.66 & 27 & .12 & .7 & .9 & \text { KEA }\end{array}$

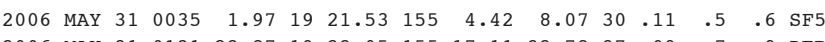

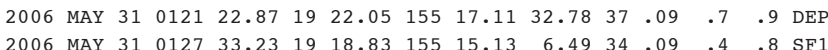

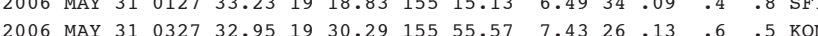

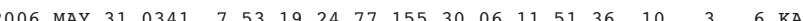

$\begin{array}{llllllllllllll}2006 & \text { MAY } 31 & 0442 & 13.04 & 19 & 41.30 & 155 & 9.94 & 14.40 & 22 & .14 & .6 & .9 & \text { KEA }\end{array}$

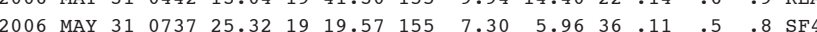
2006 MAY $310946 \begin{array}{llllllllllll}50.85 & 19 & 20.48 & 155 & 6.90 & 8.80 & 36 & .08 & .5 & .8 & \text { SF }\end{array}$

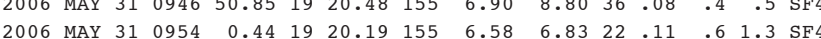

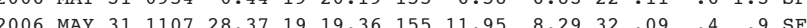

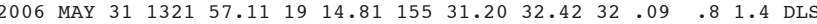

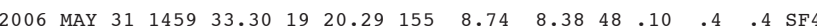
$\begin{array}{lllllllllllllll}2006 & \text { MAY } & 31 & 1503 & 38.83 & 19 & 19.02 & 155 & 8.06 & 3.02 & 37 & .12 & .6 & 1.0 & \text { SSE }\end{array}$

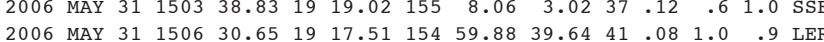

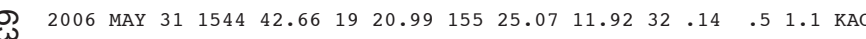

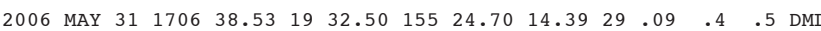
$\begin{array}{lllllllllllllll}2006 & \text { MAY } & 31 & 1755 & 12.46 & 19 & 19.99 & 155 & 11.28 & 8.01 & 43 & .10 & .4 & .6 & \text { SF } 3\end{array}$

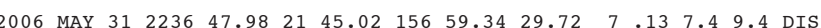
2006 JUN $110110 \quad \begin{array}{llllllllllll}2 & 0110 & 19 & 23.31 & 155 & 16.97 & 2.64 & 24 & .08 & .3 & .2 & \text { SSC }\end{array}$

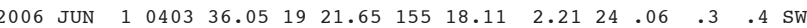

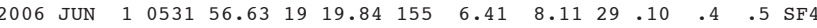

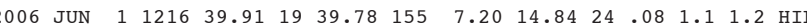

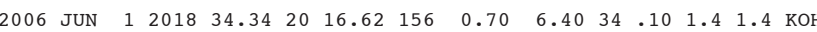

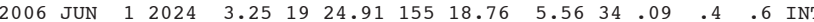

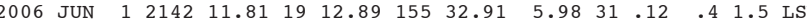

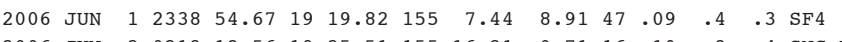
2006 JUN $2 \begin{array}{lllllllllllll}0213 & 12.56 & 19 & 25.51 & 155 & 16.21 & 0.71 & 16 & .10 & .3 & .4 & \text { SNC }\end{array}$

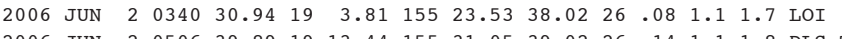
$\begin{array}{llllllllllllllll}2006 & \text { JUN } & 2 & 0506 & 39.89 & 19 & 13.44 & 155 & 31.05 & 39.02 & 26 & .14 & 1.1 & 1.8 & \text { DLS }\end{array}$

2006 JUN $2 \begin{array}{lllllllllllll}0911 & 45.04 & 19 & 15.67 & 155 & 22.74 & 34.52 & 33 & .10 & 1.1 & 1.4 & \text { DEP }\end{array}$

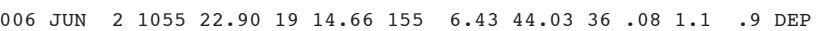

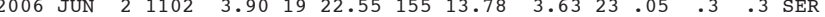

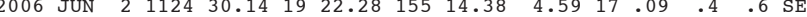
$\begin{array}{lllllllllllllll}2006 & \text { JUN } & 2 & 1127 & 22.66 & 19 & 22.72 & 155 & 13.95 & 3.62 & 20 & .07 & .3 & .4 & \text { SER }\end{array}$
PREF AZ MIN 57 MAG GAP DS

$1.3 \times \quad 71 \quad 4$ $\begin{array}{lll}1.6 \mathrm{X} & 78 & 1 \\ 2.3 \mathrm{X} & 53 & 5 \\ 1.9 \mathrm{x} & 40 & 0\end{array}$

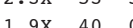
$1.7 \times 202-7$

$1.3 \times \quad 76 \quad 4$ $1.6 \times \quad 64 \quad 2$ $\begin{array}{lrrr}1.6 \times & 64 & 2 \\ 1.6 \times & 170 & 27\end{array}$ $1.7 \times 129 \quad 15$

1.3X $163 \quad 5$ $1.7 \times \quad 55$ $\begin{array}{lll}1.7 \times & 55 & 2 \\ 1.1 \times & 118 & 4\end{array}$ $1.2 \times 217 \quad 2$ 1. $5 \times \quad 44$

$1.3 \times 122 \quad 14$ $\begin{array}{lll}1.7 \times & 141\end{array}$ $\begin{array}{lll}1.6 \times & 141 & 5\end{array}$ $\begin{array}{lll}1.3 \times & 176 & 6 \\ 1.2 \times & 95 & 5\end{array}$ $1.2 \times \quad 95$

$1.5 \times 125 \quad 2$ $2.2 \times 103 \quad 5$ $\begin{array}{llll}1.5 \times & 121 & 3\end{array}$ $1.7 \times \quad 237 \quad 14$ $1.3 \times \quad 78 \quad 3$

$1.4 \times 115 \quad 5$ $1.6 \times \quad 87 \quad 5$ $4.1 \times 25262$ $\begin{array}{lll}1.5 \mathrm{X} & 46 & 0 \\ 1.2 \mathrm{x} & 66 & 4\end{array}$

$1.2 \times 157 \quad 5$

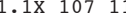
1.9X 30859 $1.6 \times 106 \quad 2$ $\begin{array}{lll}1.8 \mathrm{X} & 82 & 6\end{array}$

$2.3 \times 135 \quad 5$ $1.5 \times 199 \quad 2$ $1.3 \times 21913$ $\begin{array}{lll}1.7 \times & 172 & 3 \\ 1.5 \times & 244 & 3\end{array}$

1.5X 1838 $1.7 \times 217 \quad 6$ $\begin{array}{lll}1.4 \times & 95 & 1\end{array}$ $\begin{array}{lll}1.6 \mathrm{X} & 87 \quad 2\end{array}$

$\begin{array}{lll}1.4 \mathrm{X} & 94 & 2\end{array}$
--ORTGIN TTME (HST) -- -LAT N-- --LON W-- DEPTH N RMS ERH ERZ LOC YEAR MON DA HRMN SEC DEG MIN DEG MIN DEM RD SEC KM KM PEMKS

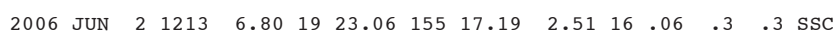

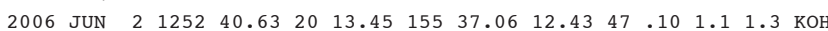

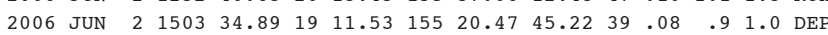
$\begin{array}{llllllllllllllll}2006 & \text { JUN } & 2 & 1544 & 27.93 & 19 & 21.75 & 155 & 2.69 & 5.56 & 17 & .14 & 1.1 & 2.1 & \text { SF5 }\end{array}$

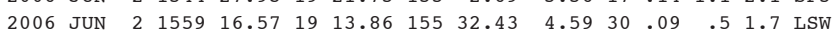

$\begin{array}{llllllllllllll}2006 & \text { JUN } & 2 & 1902 & 42.97 & 19 & 27.94 & 155 & 23.70 & 10.18 & 47 & .13 & .3 & .6 \\ \text { KAO }\end{array}$

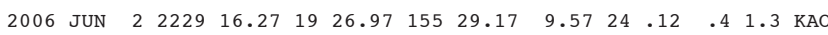

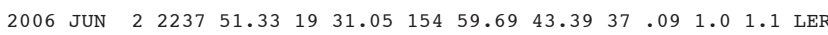

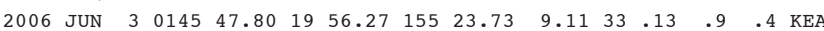

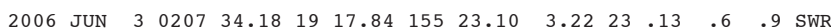

$\begin{array}{lllllllllllllll}2006 & \text { JUN } & 3 & 0316 & 53.35 & 19 & 22.51 & 155 & 29.96 & 10.84 & 25 & .07 & .4 & .9 & \text { KAO }\end{array}$

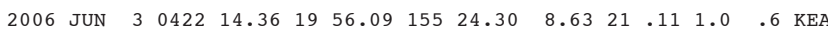

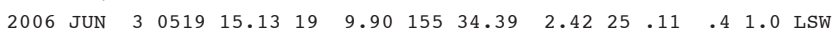

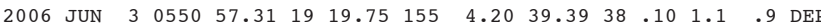

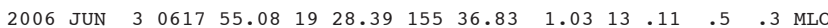

$\begin{array}{lllllllllllllll}2006 & \text { JUN } & 3 & 0635 & 46.21 & 19 & 16.10 & 155 & 29.92 & 9.68 & 31 & .16 & .5 & 1.1 & \text { LSW }\end{array}$

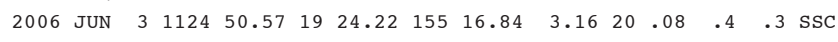

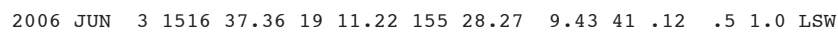

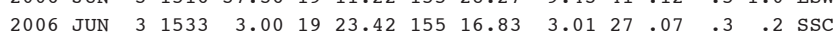

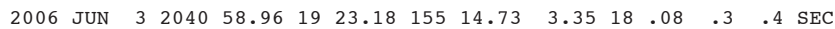

$\begin{array}{lllllllllllllll}2006 & \text { JUN } & 3 & 2104 & 5.58 & 19 & 23.35 & 155 & 14.97 & 2.60 & 20 & .09 & .3 & .4 & \text { SEC }\end{array}$ $\begin{array}{lllllllllllllll}2006 & \text { JUN } & 3 & 2224 & 58.74 & 19 & 22.64 & 155 & 17.18 & 2.87 & 18 & .09 & .3 & .4 & \text { SSC }\end{array}$ $\begin{array}{lllllllllllllll}2006 & \text { JUN } & 3 & 2246 & 42.53 & 19 & 20.00 & 155 & 10.66 & 8.53 & 46 & .11 & .4 & .5 & \mathrm{SF} 3\end{array}$ 2006 JUN $3 \begin{array}{llllllllllll}2307 & 5.92 & 19 & 19.86 & 155 & 7.36 & 8.25 & 40 & .08 & .4 & .5 & \text { SF4 }\end{array}$

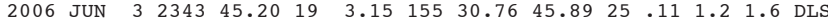

$\begin{array}{llllllllllllll}2006 & \text { JUN } & 4 & 0439 & 44.48 & 19 & 24.49 & 155 & 19.73 & 7.04 & 37 & .10 & .4 & .7 \\ \text { KAO }\end{array}$ $\begin{array}{lllllllllllllll}2006 & \text { JUN } & 4 & 0612 & 16.26 & 19 & 22.03 & 155 & 17.43 & 1.79 & 19 & .12 & .3 & .4 & \text { SSC }\end{array}$

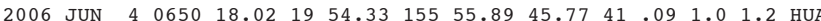

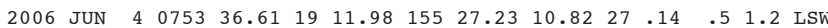
$\begin{array}{lllllllllllllll}2006 & \text { JUN } & 4 & 0835 & 16.84 & 19 & 21.57 & 155 & 4.71 & 9.00 & 47 & .09 & .5 & .4 & \text { SF5 }\end{array}$

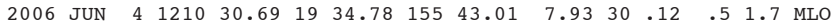

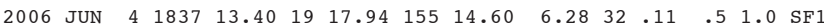

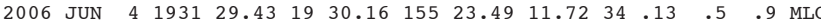

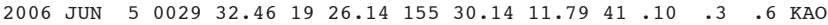

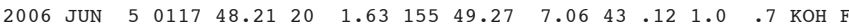

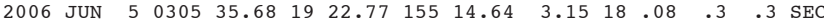

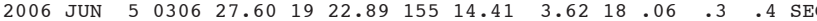

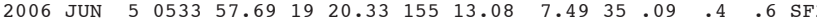
2006 JUN $5 \begin{array}{llllllllllllll}5 & 0700 & 3.13 & 19 & 19.48 & 155 & 9.94 & 7.57 & 34 & .09 & .4 & .5 & \text { SF } 3\end{array}$ 2006 JUN $5 \begin{array}{lllllllllllll}1209 & 43.06 & 19 & 19.72 & 155 & 4.04 & 5.00 & 31 & .10 & .6 & 1.8 & \text { SSF }\end{array}$

2006 JUN $5 \begin{array}{lllllllllllll}1230 & 35.62 & 19 & 31.84 & 155 & 59.68 & 22.96 & 26 & .14 & .9 & 2.4 \\ \text { KON }\end{array}$

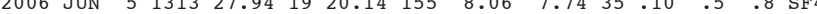
$\begin{array}{lllllllllllllll}2006 & \text { JUN } & 5 & 1409 & 5.29 & 19 & 22.26 & 155 & 10.26 & 2.81 & 17 & .08 & .6 & .3 & \text { SER }\end{array}$

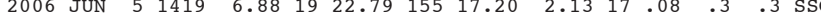
2006 JUN $5 \begin{array}{lllllllllllll}1553 & 39.28 & 19 & 11.67 & 155 & 39.11 & 6.82 & 49 & .14 & .4 & 1.1 & \text { LSW }\end{array}$
PREF AZ MIN 58 $.2 \mathrm{x} \quad 66 \quad 1$ 2.6X 29141 $1.7 \times 1912$ $\begin{array}{lll}.2 \times & 206 & 5\end{array}$

$1.9 \times \quad 44 \quad 4$ $\begin{array}{rrr}.3 \times & 70 & 9 \\ .7 \times & 171 & 10\end{array}$ $\begin{array}{llll}.7 \times & 171 & 10\end{array}$ $9 \times 1534$ $1.3 \times \quad 71 \quad 4$ $\begin{array}{lll}1.4 \times \quad 234 & 8\end{array}$ $\begin{array}{llll}1.8 \times & 189 & 8\end{array}$ $1.9 \times 198 \quad 2$

$\begin{array}{lll}1.4 \mathrm{X} & 77 & 2 \\ 1.5 \mathrm{x} & 90 & 1\end{array}$ $\begin{array}{lll}1.5 \mathrm{X} & 90 & 1\end{array}$ $\begin{array}{lll}1.6 x & 95 & 3 \\ 1.7 x & 45 & 0\end{array}$ $2 \times 812$ $\begin{array}{lll}1.1 \mathrm{X} & 74 & 2 \\ 1.0 \mathrm{X} & 58 & 1\end{array}$ $\begin{array}{lll}1.0 \mathrm{X} & 58 & 1 \\ .8 \mathrm{x} & 87 & 5 \\ 1.7 \mathrm{x} & 136 & 5\end{array}$ $1.7 \times \quad 136 \quad 5$ $1.6 \times 209 \quad 13$ $\begin{array}{lll}1.4 \mathrm{X} & 66 & 1 \\ 1.9 \mathrm{X} & 60 & 3\end{array}$ 1.9X 21826 $1.2 \times 126 \quad 5$ $2.3 \times 160 \quad 5$

1.3X 1019 $\begin{array}{lll}1.2 \times & 141 & 3 \\ 1.4 \times & 108 & 1\end{array}$ $\begin{array}{lll}1.6 \mathrm{X} & 65 & 8\end{array}$

$\begin{array}{lll}1.1 x & 77 \quad 2\end{array}$ $\begin{array}{lll}1.3 x & 84 & 2\end{array}$ $\begin{array}{lll}1.3 x & 66 & 4\end{array}$ $\begin{array}{rrr}.2 \times & 96 & 5 \\ .2 \times & 191 & 8\end{array}$

1.4X $247 \quad 24$ $1.2 \times 136 \quad 5$ $\begin{array}{lll}1.5 \times 141 & 1\end{array}$ $\begin{array}{rrr}.1 x & 84 & 13\end{array}$ 
---ORIGIN TIME (HST)-- -LAT N-- --LON W-- DEPTH N RMS ERH ERZ LOC PREF AZ MIN 59 YEAR MON DA HRMN SEC DEG MIN DEG MIN KM RD SEC KM KM REMKS MAG GAP DS

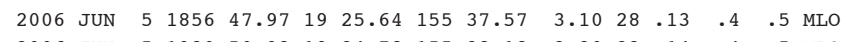

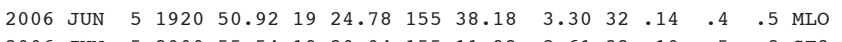
$\begin{array}{llllllllllllllll}2006 & \\ \text { JUN } & 5 & 2000 & 55.54 & 19 & 20.04 & 155 & 11.92 & 8.61 & 32 & .10 & .5 & .8 & \text { SF3 }\end{array}$

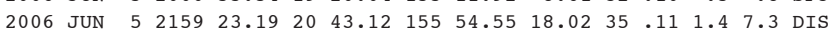
2006 JUN $5 \begin{array}{llllllllllll}2300 & 41.77 & 19 & 21.61 & 155 & 18.00 & 3.13 & 19 & .07 & .3 & .6 & \text { SWR }\end{array}$

2006 JUN $6 \begin{array}{lllllllllllll}6 & 0025 & 25.82 & 19 & 25.39 & 155 & 18.85 & 6.80 & 21 & .09 & .5 & 1.0 & \text { INT }\end{array}$

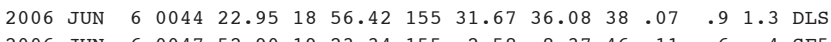
$\begin{array}{lllllllllllllll}2006 & \text { JUN } & 6 & 0047 & 52.90 & 19 & 23.34 & 155 & 2.58 & 8.37 & 46 & .11 & .6 & .4 & \text { SF5 }\end{array}$ $\begin{array}{lllllllllllllll}2006 & \text { JUN } & 6 & 0616 & 37.12 & 19 & 26.45 & 155 & 20.56 & 13.86 & 48 & .11 & .3 & .3 & \text { DML }\end{array}$

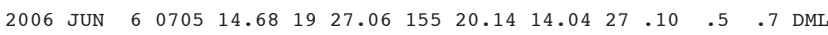

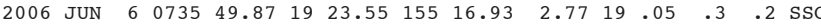
2006 JUN $6 \begin{array}{lllllllllllll}6 & 0820 & 56.75 & 19 & 20.24 & 155 & 10.41 & 7.53 & 42 & .10 & .4 & .6 & \text { SF } 3\end{array}$

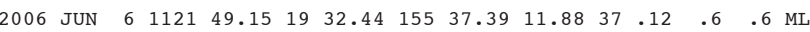
2006 JUN $6 \begin{array}{lllllllllllll}1447 & 30.63 & 19 & 22.63 & 155 & 13.46 & 4.01 & 17 & .09 & .4 & .4 & \text { SER }\end{array}$ 2006 JUN $\quad \begin{array}{lllllllllllll}6 & 1514 & 25.11 & 19 & 20.27 & 155 & 12.67 & 8.49 & 44 & .09 & .4 & .4 & \mathrm{SF} 2\end{array}$

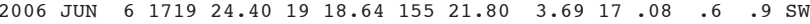

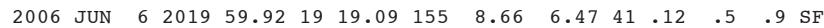

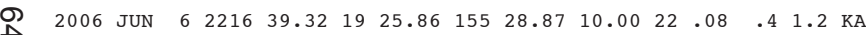
$\begin{array}{llllllllllllllll}2006 & \text { JUN } & 6 & 2331 & 18.41 & 19 & 12.22 & 155 & 37.13 & 10.72 & 25 & .11 & .4 & 1.5 & \text { LS }\end{array}$

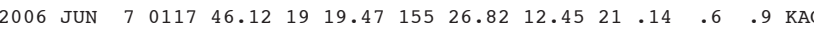

2006 JUN $7 \begin{array}{lllllllllllll}7 & 0153 & 52.18 & 19 & 23.53 & 155 & 16.97 & 2.77 & 22 & .07 & .3 & .2 & \text { SSC }\end{array}$

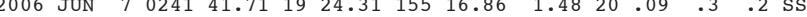

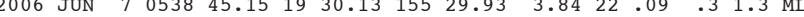

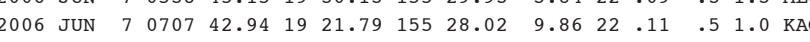

$\begin{array}{lllllllllllllll}2006 & \text { JUN } & 7 & 0923 & 10.47 & 19 & 22.96 & 155 & 17.00 & 2.82 & 18 & .08 & .3 & .3 & \text { SSC }\end{array}$

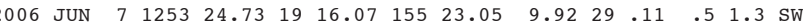

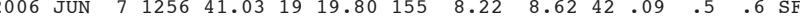

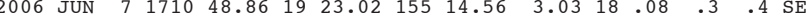

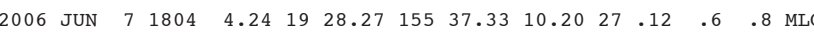

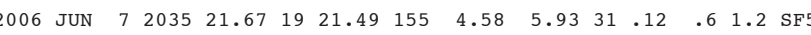
$\begin{array}{lllllllllllllll}2006 & \text { JUN } & 8 & 0041 & 55.59 & 19 & 57.69 & 155 & 23.01 & 8.20 & 38 & .12 & .6 & .5 & \mathrm{KEA}\end{array}$ 2006 JUN $8 \begin{array}{lllllllllllll}0 & 0055 & 57.34 & 19 & 53.96 & 155 & 22.50 & 8.80 & 23 & .13 & .9 & .5 & \text { KEA }\end{array}$ 2006 JUN $8 \begin{array}{lllllllllllll}0 & 0152 & 46.83 & 19 & 19.38 & 155 & 2.57 & 40.28 & 17 & .08 & 2.4 & 1.7 & \text { DE }\end{array}$

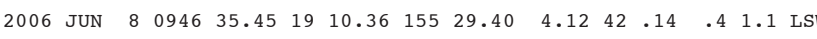

$\begin{array}{lllllllllllllll}2006 & \text { JUN } & 8 & 1257 & 50.80 & 19 & 19.84 & 155 & 8.88 & 5.71 & 43 & .11 & .4 & .6 & \text { SF4 }\end{array}$

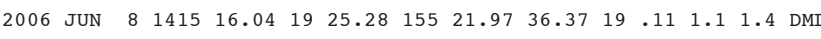
2006 JUN $8 \begin{array}{lllllllllllll}1735 & 54.81 & 19 & 18.70 & 155 & 15.19 & 7.82 & 47 & .11 & .4 & .5 & \text { SF }\end{array}$

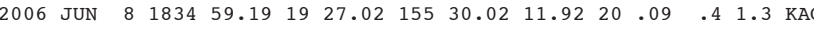
$\begin{array}{lllllllllllllll}2006 & \text { JUN } & 8 & 2008 & 32.96 & 19 & 20.44 & 155 & 8.31 & 9.06 & 39 & .12 & .4 & .5 & \text { SF }\end{array}$

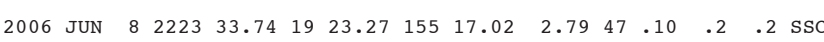

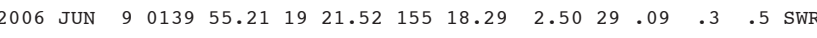

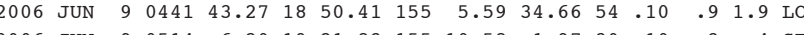
$\begin{array}{lllllllllllllll}2006 & \text { JUN } & 9 & 0514 & 6.20 & 19 & 21.83 & 155 & 10.58 & 1.97 & 30 & .10 & .3 & .4 & \text { SER } \\ 2006 & \text { JUN } & 9 & 0514 & 50.37 & 19 & 21.83 & 155 & 10.49 & 1.87 & 19 & .08 & .3 & .4 & \text { SER }\end{array}$ $\begin{array}{lll}1.5 \times & 80 & 2 \\ 1.8 \times & 75 & 1\end{array}$

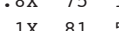
$2.2 \times 322 \quad 67$ $\begin{array}{rrr}1.0 \times & 68 & 4\end{array}$

$1.0 \times 130 \quad 2$ $2.0 \times 24116$ $1.9 \times 158 \quad 3$ $1.9 \times \quad 48 \quad 5$ $1.2 \times 113 \quad 5$

$\begin{array}{lll}.4 \mathrm{X} & 67 & 0\end{array}$ $\begin{array}{lll}1.6 \times & 82 & 5\end{array}$ $\begin{array}{lll}1.6 \mathrm{X} & 97 \quad 6\end{array}$ $\begin{array}{lll}1.3 \times & 108 & 1\end{array}$ 1.8X $\quad 71 \quad 4$

$1.3 \times 203 \quad 4$ $1.3 \times 101$ $\begin{array}{lll}1.1 \mathrm{x} & 62 & 7\end{array}$ $1.4 \times \quad 86 \quad 13$ 1.3X $116 \quad 6$

$\begin{array}{lll}1.5 \times \quad 46 & 0\end{array}$ $1.5 \mathrm{X} \quad 65 \quad 3$ $1.2 \times \quad 93$ $1.4 \times 104$ $\begin{array}{lrl}1.2 \mathrm{X} & 84 & 1\end{array}$

$1.1 \times \quad 70 \quad 1$ $1.3 \times 131 \quad 8$ $1.6 \times 115 \quad 5$ $\begin{array}{lll}1.2 \times & 86 & 3\end{array}$ $1.6 \times 118 \quad 3$

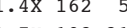
$1.7 \times 19221$ $1.2 \times 205$ $1.3 \times 31420$ $1.6 \times 103 \quad 3$ $\begin{array}{lll}1.7 \times \quad 99 & 5\end{array}$ $\begin{array}{lll}1.7 \times & 103 & 5\end{array}$ $\begin{array}{lll}1.7 \mathrm{X} & 98 & 4 \\ 1.2 \times & 73 & 6\end{array}$ $\begin{array}{lll}1.2 \times & 73 & 6\end{array}$ $\begin{array}{lll}2.2 \mathrm{x} & 46 & 0 \\ 1.3 \mathrm{x} & 55 & 4\end{array}$ $\begin{array}{lll}1.3 \times & 55 & 4 \\ 2.9 \times & 271 & 50 \\ 1.5 X & 77 & \end{array}$ $\begin{array}{lll}2.9 \times & 27150 \\ 1.5 \times & 77 & 2\end{array}$ $\begin{array}{lll}1.5 \mathrm{x} & 77 & 2 \\ 1.2 \mathrm{x} & 78 & 2\end{array}$
--ORIGIN TIME (HST)-- -LAT N-- --LON W-- DEPTH N RMS ERH ERZ LOC YEAR MON DA HRMN SEC DEG MIN DEG MIN KM RD SEC KM KM REMKS

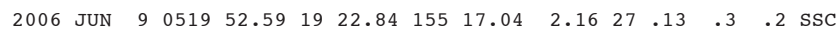

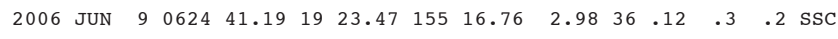

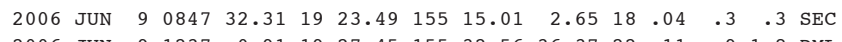

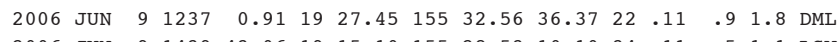

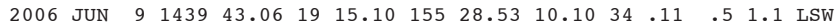

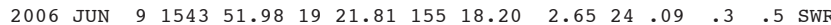
$\begin{array}{lllllllllllllll}2006 & \text { JUN } & 9 & 1805 & 8.50 & 19 & 19.52 & 155 & 8.57 & 7.88 & 39 & .09 & .4 & .6 & \text { SF } 4\end{array}$

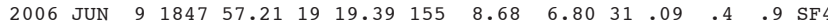
$\begin{array}{lllllllllllllll}2006 & \text { JUN } & 9 & 1958 & 46.26 & 19 & 29.66 & 155 & 42.21 & 8.11 & 34 & .12 & .5 & 1.0 & \text { MLO }\end{array}$

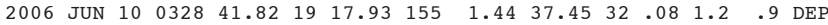

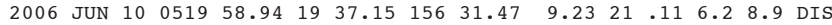

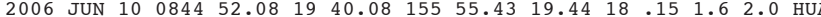

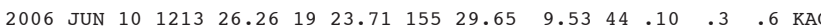

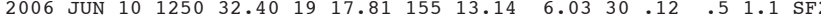
$\begin{array}{llllllllllllll}2006 & \text { JUN } 10 & 1310 & 11.01 & 19 & 22.95 & 155 & 15.58 & 12.45 & 43 & .10 & .4 & .3 & \text { INT }\end{array}$

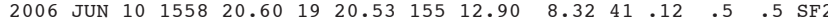

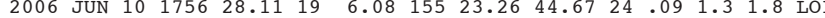

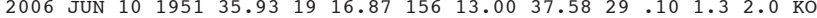

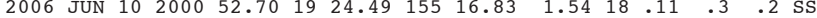

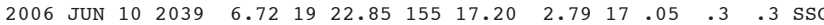

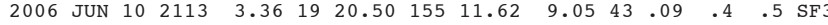

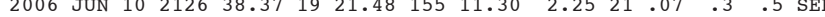
$\begin{array}{llllllllll}2006 & & \end{array}$

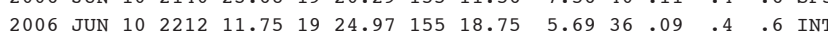

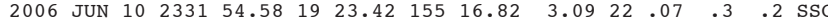

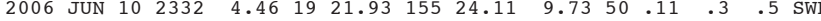

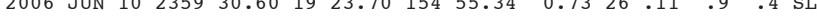

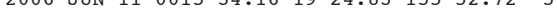
$\begin{array}{llllllllllllll}2006 & \text { JUN } & 11 & 0211 & 24.45 & 19 & 23.35 & 155 & 16.72 & 3.21 & 43 & .11 & .3 & .2 \\ \text { SSC }\end{array}$

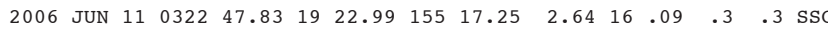

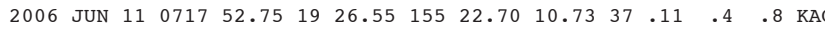

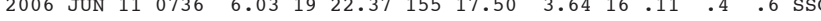

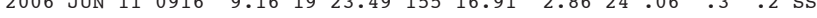

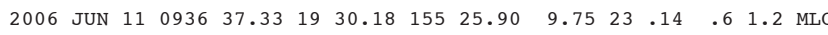

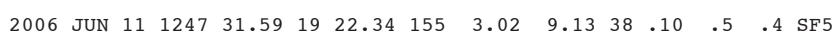

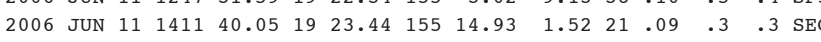

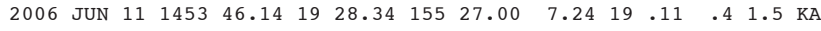

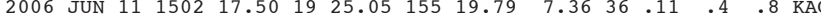
$\begin{array}{llll}11 & .4 & .7 & \text { IN }\end{array}$

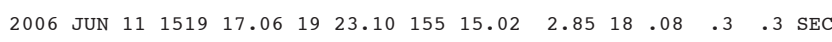

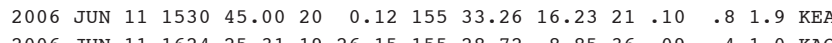

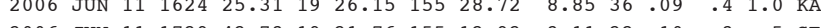

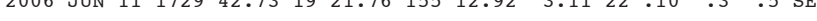

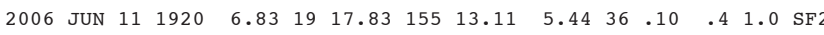

PREF AZ MIN 60 $\begin{array}{rrr}.5 \mathrm{X} & 49 & 1 \\ 2.0 \mathrm{X} & 44 & 0\end{array}$ $1.2 \times \quad 80 \quad 3$ $1.5 \times \quad 80 \quad 5$ $1.5 \times \quad 97 \quad 3$ $\begin{array}{rrr}1.3 \times & 65 & 3 \\ 1.4 x & 105 & 4 \\ 1.2 x & 103 & 4\end{array}$ $\begin{array}{lll}.2 \times & 103 & 4 \\ 1.6 \times & 81 & 7\end{array}$ $\begin{array}{lll}1.6 \times & 81 \quad 7\end{array}$ $1.4 \times 234 \quad 11$

1.6X 31179 $1.4 \times \quad 271 \quad 9$ $\begin{array}{lll}1.6 \mathrm{X} & 51 & 4\end{array}$ $1.3 \times 107 \quad 2$ $\begin{array}{lll}1.7 \times & 68 \quad 1\end{array}$

$\begin{array}{lll}1.3 \mathrm{X} & 65 \quad 4\end{array}$ $1.2 \times 250 \quad 10$ $1.8 \times 294 \quad 36$ $\begin{array}{lll}1.1 \mathrm{x} & 106 \quad 1\end{array}$ $1.1 \times \quad 50 \quad 1$

$\begin{array}{lll}1.6 \times & 75 \quad 5\end{array}$ $1.2 \mathrm{X} \quad 81 \quad 3$ $\begin{array}{lll}1.7 x & 74 & 4\end{array}$ $\begin{array}{lll}1.4 \times & 80 \quad 5\end{array}$ $\begin{array}{lll}.4 \times & 71 & 2\end{array}$

$\begin{array}{lll}1.3 \mathrm{X} & 59 & 0\end{array}$ $\begin{array}{lll}1.5 x & 42 & 3\end{array}$ $\begin{array}{lll}.6 \times 242 & 6\end{array}$ $\begin{array}{ll}1.8 \times 157 & 9\end{array}$

$1.1 \times \quad 81 \quad 1$ $\begin{array}{lll}1.4 \times 111 & 6\end{array}$ $\begin{array}{lll}1.3 \times \quad 89 & 2\end{array}$ $\begin{array}{lll}1.3 \mathrm{X} & 45 & 0\end{array}$ $\begin{array}{lll}1.1 \times & 124 & 5\end{array}$

$\begin{array}{lll}1.4 \times \quad 169 \quad 4 & 4\end{array}$ $1.4 \times \quad 76 \quad 3$ $\begin{array}{lll}1.1 \mathrm{X} & 69 & 7\end{array}$ $1.5 \times 108 \quad 3$

$1.3 \times 113 \quad 2$ $\begin{array}{lrrr}1.4 \times & 178 & 19 \\ 1.4 \times & 63 & 7\end{array}$ $\begin{array}{lll}1.4 \mathrm{X} & 63 & 7 \\ 1.5 \mathrm{X} & 79 & 2\end{array}$ $\begin{array}{lll}1.3 \times & 108 & 2\end{array}$ 
---ORIGIN TIME (HST)-- -LAT N-- --LON W-- DEPTH N RMS ERH ERZ LOC PREF AZ MIN 61 YEAR MON DA HRMN SEC DEG MIN DEG MIN KM RD SEC KM KM REMKS MAG GAP DS

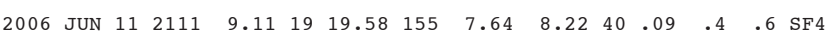

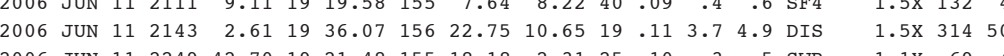

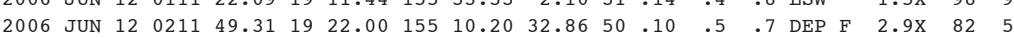

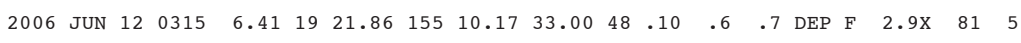

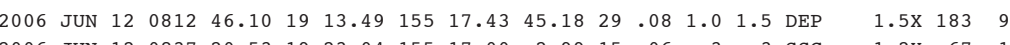

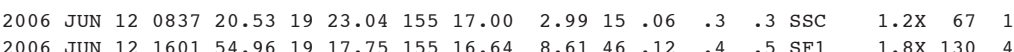

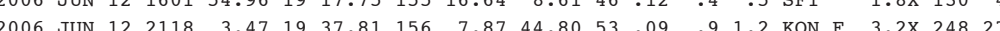

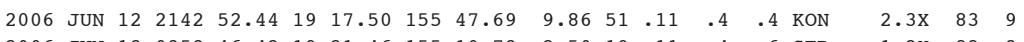

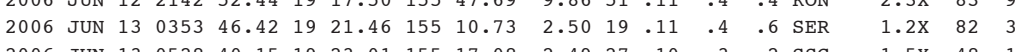

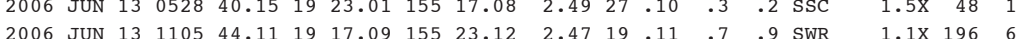

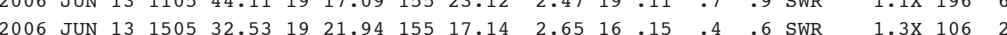

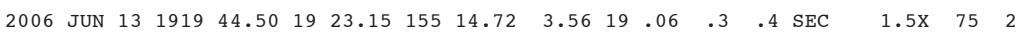

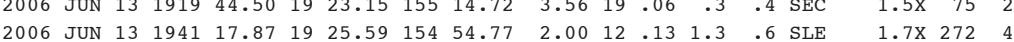

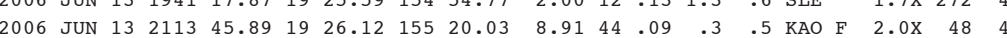
$\begin{array}{llllllllllll}2006 & & \end{array}$

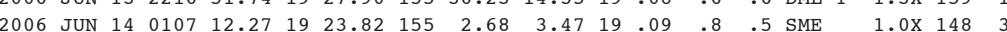

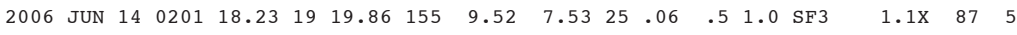

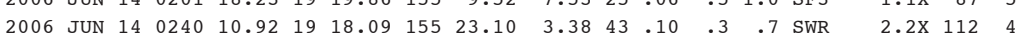

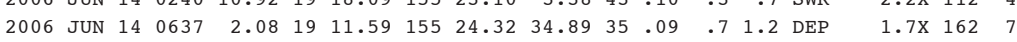

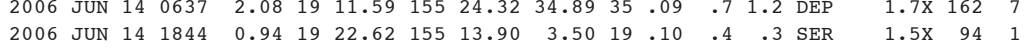

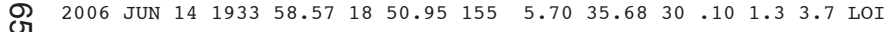

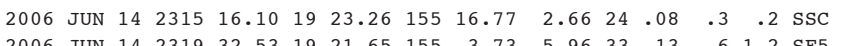

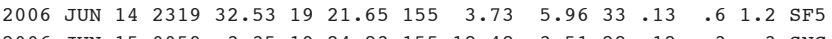

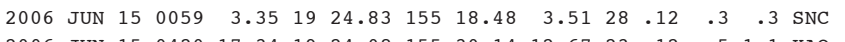

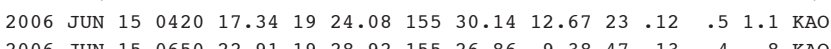

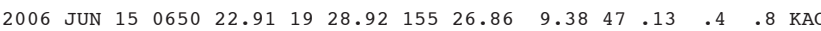

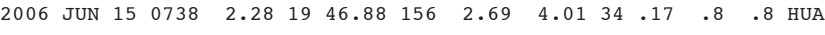

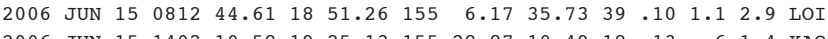

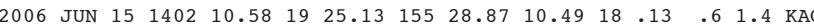

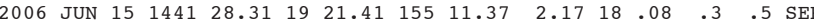

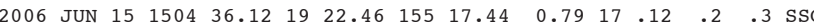

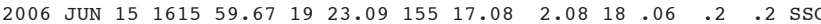

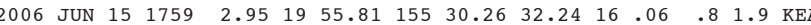

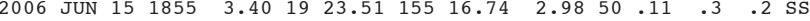

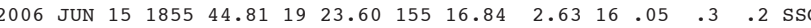

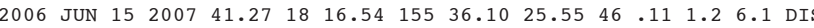

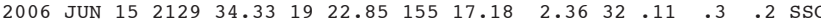

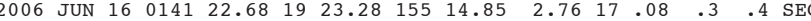

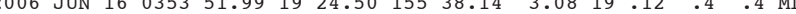

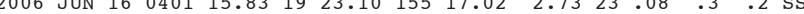

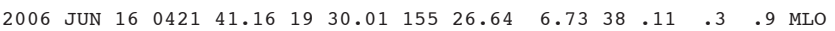

--ORTGIN TIME (HST)-- -LAT N-- --LON W-- DEPTH N RMS ERH ERZ LOC YEAR MON DA HRMN SEC DEG MIN DEG MIN KM RD SEC KM KM REMKS

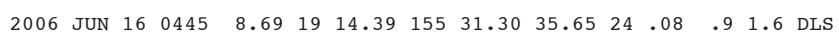

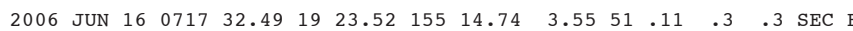

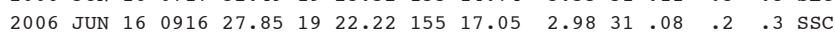
$\begin{array}{llllllllllllll}2006 \text { JUN } 16 & 1233 & 37.83 & 19 & 22.94 & 155 & 17.09 & 2.17 & 21 & .05 & .2 & .2 & \text { SSC }\end{array}$

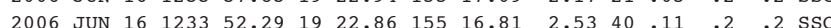

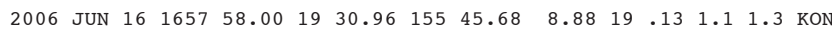

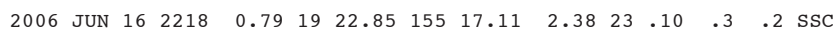

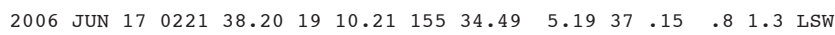

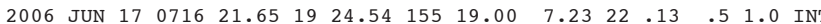

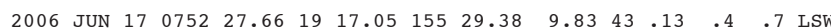

$\begin{array}{llllllllllllll}2006 & \text { JUN } 17 & 1029 & 34.83 & 19 & 23.31 & 155 & 14.75 & 3.60 & 27 & .08 & .3 & .4 & \text { SEC }\end{array}$

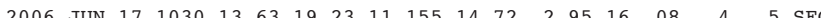
$\begin{array}{lllllllllllll}2006 & .4 & \text { SEC }\end{array}$

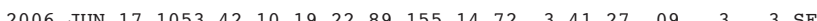
$\begin{array}{llllllllllll}2006 & & 3 & \end{array}$

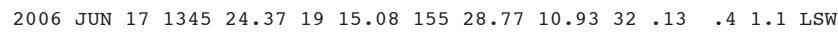

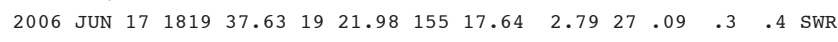

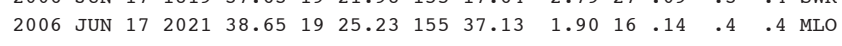

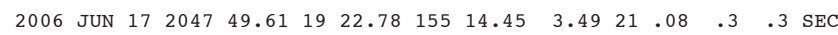

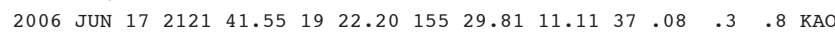

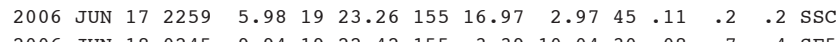

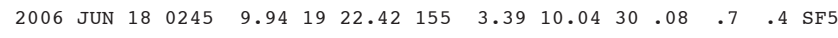

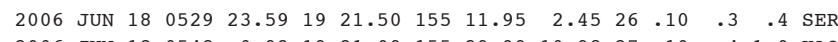
2006 JUN $18 \quad 0548 \quad 0.931921 .09 \begin{array}{lllllllll}155 & 29.99 & 10.98 & 27 & .10 & .4 & 1.0 & \text { KAO }\end{array}$

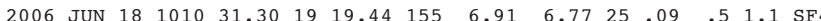

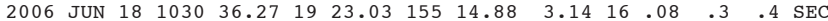
$\begin{array}{llllllllllllll}2006 & \text { JUN } 18 & 1138 & 5.08 & 19 & 23.20 & 155 & 2.78 & 2.75 & 45 & .11 & .5 & .5 & \text { SME }\end{array}$

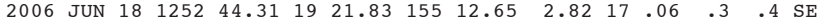
$\begin{array}{llllllllllllll}2006 & \text { JUN } 18 & 1443 & 40.27 & 19 & 21.10 & 155 & 3.21 & 7.99 & 34 & .13 & .6 & .8 & \text { SF } 5\end{array}$

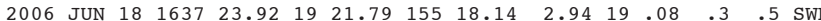

$\begin{array}{llllllllllllll}2006 & \text { JUN } 19 & 0132 & 30.04 & 19 & 21.56 & 155 & 11.25 & 2.19 & 19 & .07 & .4 & .4 & \text { SER }\end{array}$

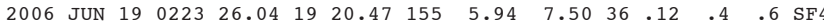

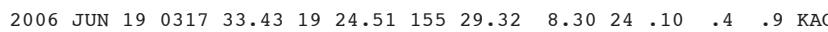

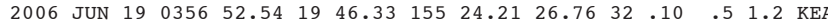

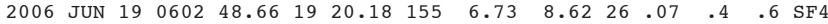

$\begin{array}{llllllllllllll}2006 & \text { JUN } 19 & 1621 & 38.05 & 18 & 59.15 & 155 & 16.54 & 27.80 & 15 & .12 & 1.7 & 3.5 & \text { LOI }\end{array}$ 2006 JUN $19 \begin{array}{llllllllllll}1628 & 38.71 & 19 & 1.33 & 155 & 26.06 & 37.52 & 42 & .11 & .9 & 1.3 & \text { DLS }\end{array}$

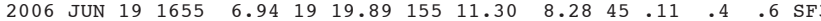
2006 JUN $19 \begin{array}{llllllllllll}1655 & 37.71 & 19 & 18.25 & 155 & 10.32 & 16.45 & 24 & .13 & 1.0 & 1.1 & \text { DEP }\end{array}$ $\begin{array}{lllllllllllllll}2006 & \text { JUN } & 19 & 1818 & 24.22 & 19 & 22.23 & 155 & 14.07 & 3.22 & 22 & .10 & .3 & .4 & \text { SEC }\end{array}$

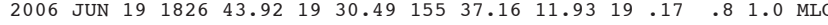
2006 JUN $19 \begin{array}{llllllllllll}1855 & 52.26 & 19 & 22.43 & 155 & 14.24 & 3.36 & 22 & .10 & .3 & .4 & \text { SEC }\end{array}$

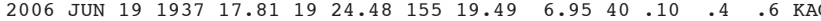

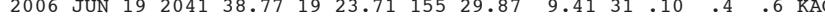

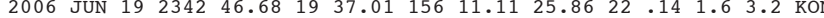

PREF AZ MIN 62 AAC DS $\begin{array}{lll}.5 \times & 104 \quad 2\end{array}$ $\begin{array}{lll}3.4 \mathrm{U} & 45 & 2 \\ 1.4 \mathrm{X} & 61 & 2\end{array}$ $\begin{array}{lll}1.4 \mathrm{X} & 61 & 2 \\ 1.2 \mathrm{X} & 71 & 1\end{array}$ $1.9 \times \quad 42 \quad 1$ $1.3 \times 223 \quad 1$ $\begin{array}{lll}1.4 \times & 49 & 1\end{array}$ $\begin{array}{lll}.8 \times 127 & 12\end{array}$ $1.6 \times \quad 79 \quad 4$

$2.0 \times \quad 68 \quad 3$ $\begin{array}{lll}1.4 \mathrm{x} & 94 & 2 \\ 1.3 \times & 80 & 3\end{array}$ $\begin{array}{lll}1.3 \mathrm{X} & 80 & 3 \\ 1.9 \mathrm{X} & 70 & 2\end{array}$ $\begin{array}{lll}1.4 \times \quad 61 \quad 4 & \end{array}$

$1.5 \times \quad 89 \quad 2$ $\begin{array}{lll}1.4 \times \quad 58 & 3 \\ 1.1 \times & 110 & 2\end{array}$ $\begin{array}{rrr}1.7 \times & 82 & 2\end{array}$ $1.5 \mathrm{X} \quad 60 \quad 4$

$\begin{array}{rrr}2.4 \times & 46 & 0 \\ 1.3 \times & 163 & 4\end{array}$ $\begin{array}{rrr}1.3 \times & 163 & 4 \\ 1.6 x & 88 & 3\end{array}$ $\begin{array}{lll}1.6 \mathrm{X} & 88 & 3 \\ 1.4 \mathrm{X} & 64 & 5\end{array}$ 1.3X $153 \quad 4$ $\begin{array}{lll}1.5 \times & 76 \quad 2\end{array}$ $2.2 \times 158 \quad 3$ $\begin{array}{rrr}1.6 \mathrm{X} & 96 & 2 \\ 1.8 \mathrm{x} & 182 & 6\end{array}$ $1.2 \times \quad 713$

$1.6 \times \quad 83 \quad 3$ $\begin{array}{rrr}1.9 \times & 157 & 6 \\ 1.3 \times & 64 & 5\end{array}$ $1.5 \times \quad 95 \quad 6$ $\begin{array}{lll}1.5 \times & 147 \quad 6\end{array}$

$1.4 \times 28133$ $1.9 \times 213 \quad 15$ $\begin{array}{lll}1.8 \times & 88 & 6\end{array}$ $\begin{array}{rrr}1.5 \times & 137 & 4 \\ 1.6 \times & 71\end{array}$

$1.3 \times 187 \quad 3$ $\begin{array}{lll}1.5 \mathrm{X} & 81 \quad 2\end{array}$ $\begin{array}{lll}1.9 \mathrm{X} & 64 & 1\end{array}$ $\begin{array}{lll}1.3 \mathrm{X} & 69 & 5\end{array}$ $1.9 \times 28431$ 
---ORIGIN TIME (HST)-- -LAT N-- --LON W-- DEPTH N RMS ERH ERZ LOC PREF AZ MIN 63 YEAR MON DA HRMN SEC DEG MIN DEG MIN KM RD SEC KM KM REMKS MAG GAP DS

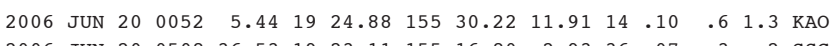

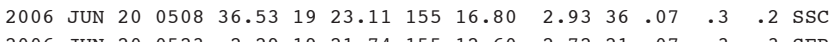

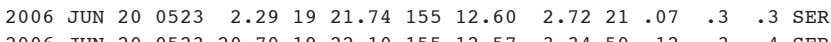

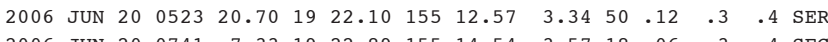

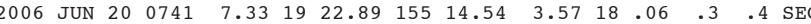

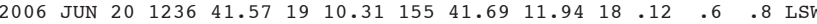

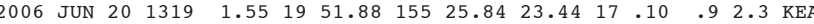

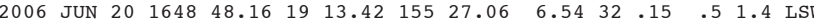

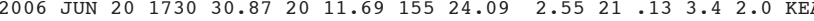

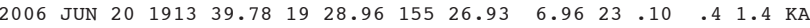

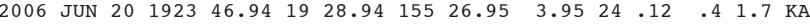

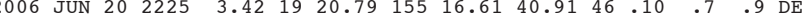

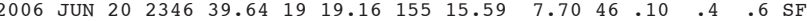

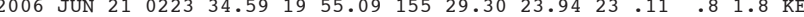

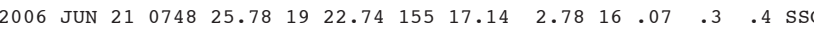

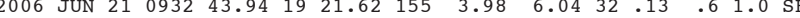

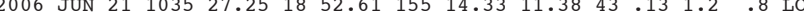

S 2006 JUN 21

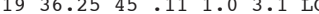
$\begin{array}{llllllllllllll}2006 & \text { JUN } 21 & 1355 & 40.77 & 19 & 19.83 & 155 & 6.94 & 7.76 & 43 & .12 & .4 & .7 & \text { SF } 4\end{array}$

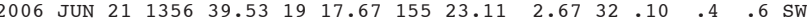

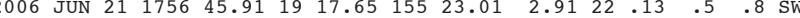

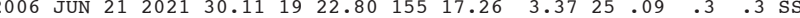

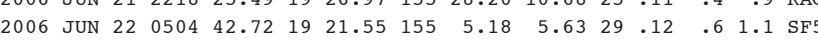

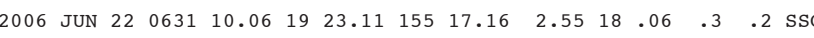

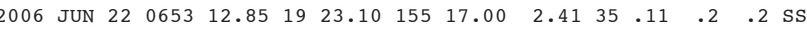

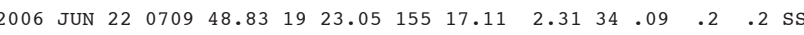

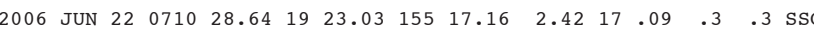

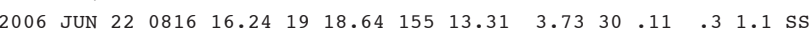

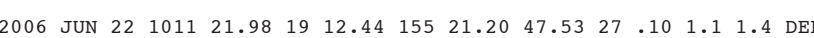

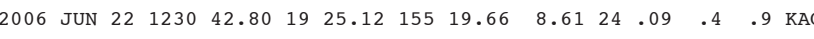

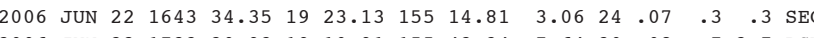

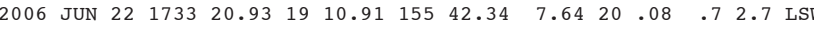

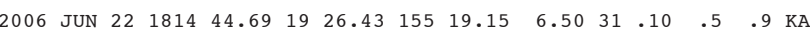

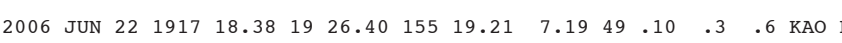

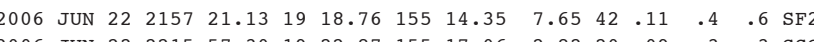

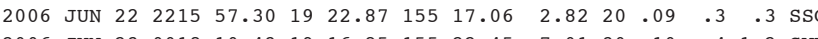

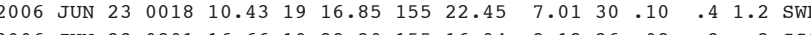

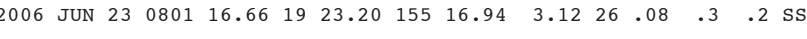

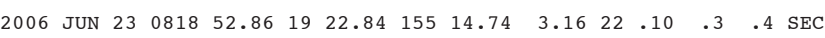

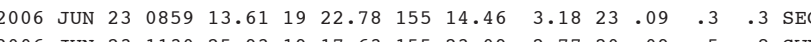

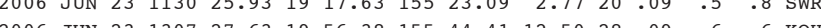
$\begin{array}{llllllllllllll}2006 & \text { JUN } 23 & 1445 & 20.76 & 19 & 18.23 & 155 & 14.97 & 5.11 & 25 & .11 & .6 & 1.7 & \text { SF1 }\end{array}$
$1.1 \times \quad 92 \quad 5$ $\begin{array}{lll}.1 \times & 47 & 1 \\ 1.3 \mathrm{x} & 93 & 2\end{array}$ $2.7 \times \quad 61 \quad 1$ $1.3 \times \quad 85 \quad 3$

$1.5 \times 1628$ $1.3 \times 12410$ $1.4 \times 115 \quad 6$ $1.5 \times 30734$ $1.5 \times \quad 72 \quad 6$

$\begin{array}{lll}1.3 \mathrm{X} & 88 & 6\end{array}$ $\begin{array}{lll}1.8 \mathrm{X} & 74 & 3\end{array}$ $\begin{array}{lll}1.6 \times & 95 & 5\end{array}$

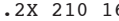
$\begin{array}{lll}1.1 \times & 81 & 1\end{array}$

$1.7 \times 168 \quad 5$ $2.4 \times 261 \quad 39$ $3.1 \times 29373$ $\begin{array}{lll}2.7 \times & 147 \quad 5\end{array}$

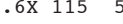
$1.3 \times 117 \quad 5$ $\begin{array}{lll}.4 \times & 50 & 1\end{array}$ $\begin{array}{lll}1.4 \times & 55 & 8\end{array}$ $1.4 \times \quad 154$

$\begin{array}{lll}.4 \times \quad 66 & 1\end{array}$ $1.9 \mathrm{X} \quad 41$ $1.9 \mathrm{X} \quad 42$ $1.1 \mathrm{x} \quad 68$ .3X 82

.6X 18811 $.3 \times 117 \quad 3$ $1.9 \times \quad 67 \quad 2$ $\begin{array}{llll}1.1 \times 149 & 7\end{array}$ $\begin{array}{lll}2.6 \times & 48 & 3 \\ 1.5 \mathrm{X} & 81 & 4\end{array}$ $\begin{array}{lll}1.2 \mathrm{X} & 49 & 1 \\ 1.3 \mathrm{X} & 128 & 6\end{array}$ $\begin{array}{lll}1.3 \times & 128 & 6\end{array}$

$1.5 \times \quad 75 \quad 2$ $\begin{array}{lll}1.7 X & 82 & 2 \\ 1.0 X & 157 & 5 \\ 1.5 x & 148 & 2\end{array}$ $\begin{array}{lll}1.0 \times & 157 & 5 \\ 1.5 \times 148 & 12\end{array}$ $\begin{array}{rrr}1.5 \times & 148 & 12 \\ 1.2 \times & 136 & 3\end{array}$
-ORIGIN TIME (HST)-- -LAT N-- --LON W-- DEPTH N RMS ERH ERZ LOC YEAR MON DA HRMN SEC DEG MIN DEG MIN KM RD SEC KM KM REMKS $\begin{array}{llllllllllllll}2006 & \text { JUN } 23 & 1558 & 17.20 & 19 & 21.98 & 155 & 17.70 & 3.59 & 17 & .10 & .3 & .7 & \text { SWR }\end{array}$ $\begin{array}{lllllllllllll}2006 & \text { JUN } 23 & 1654 & 31.06 & 19 & 56.58 & 155 & 34.30 & 11.87 & 21 & .11 & .9 & 1.0 \\ \text { кон }\end{array}$

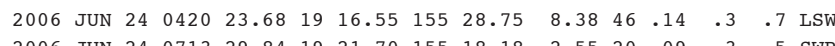

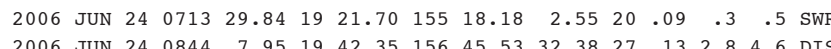
2006 JUN $24 \quad 0844 \quad 7.95 \quad 1942.35 \quad 156 \quad 45.53 \quad 32.38 \quad 27 \quad \begin{array}{lllllll}13 & 2.8 & 4.6 & \text { DIS }\end{array}$

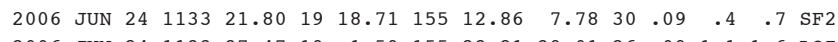

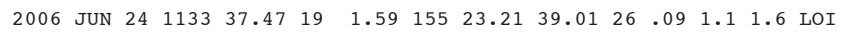

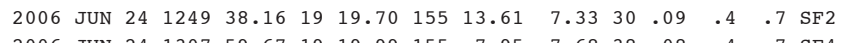

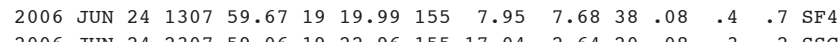

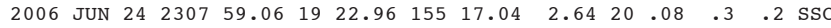

$\begin{array}{llllllllllllll}2006 & \text { JUN } 24 & 2332 & 32.81 & 19 & 19.63 & 155 & 7.97 & 8.70 & 25 & .06 & .4 & .5 & \mathrm{SF} 4\end{array}$

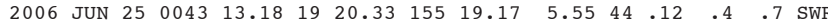

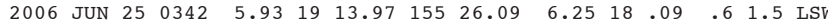

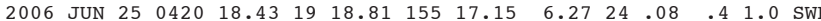

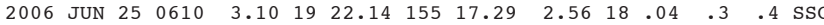

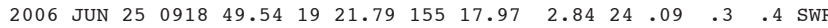

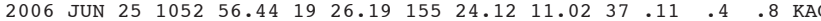

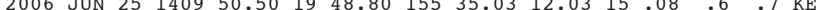

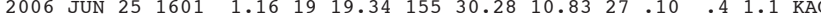
$\begin{array}{lllllllllllllll}2006 & \text { JUN } 25 & 2308 & 18.55 & 19 & 20.86 & 155 & 12.98 & 7.76 & 43 & .10 & .5 & .4 & \text { SF2 }\end{array}$

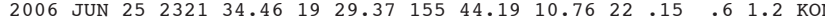

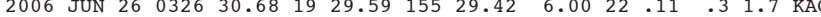

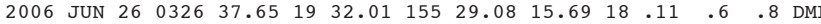

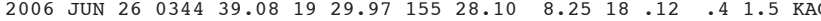

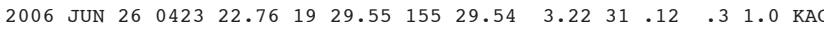

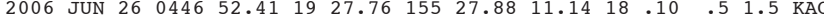

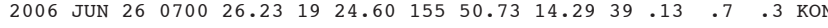

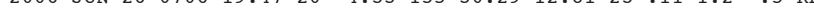
$\begin{array}{llllll}22 & .12 & .4 & .3 & \text { SER }\end{array}$

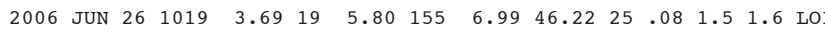

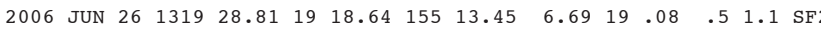

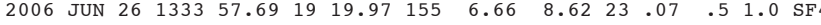

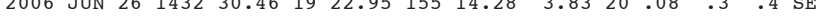

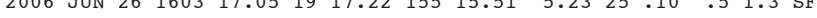
$\begin{array}{lllllllllllll}2006 & \text { JUN } 26 & 2237 & 19.08 & 19 & 24.88 & 155 & 19.08 & 6.30 & 34 & .07 & .4 & .6 \\ \text { KAO }\end{array}$

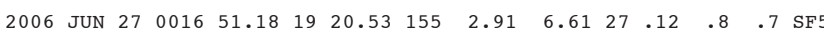

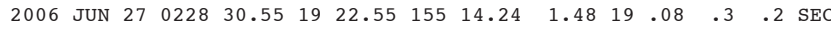
\begin{tabular}{lllllllllll}
2006 & 006 \\
\hline
\end{tabular}

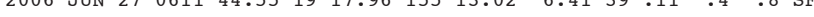
2006 JUN 270612

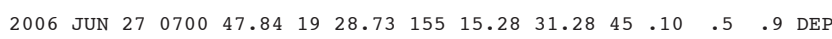

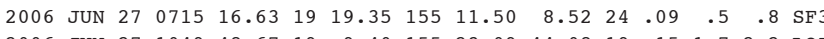

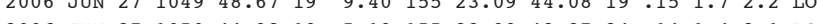

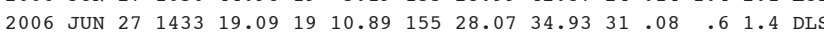

PREF AZ MIN 64 $\begin{array}{rrr}.1 \times & 59 & 3 \\ .5 \times & 149 & 25\end{array}$ $\begin{array}{rrr}1.5 \times & 149 & 25 \\ 1.6 \times & 85 & 4\end{array}$ $1.1 \times \quad 66 \quad 3$ $2.3 \times 32897$ $\begin{array}{lll}1.3 \times & 93 \quad 3\end{array}$ $\begin{array}{rrr}1.6 \times & 231 & 17 \\ 1.6 \times & 73 & 5\end{array}$ $1.8 \times 120 \quad 5$ $1.3 \mathrm{X} \quad 80 \quad 1$

$1.2 \times 122 \quad 4$ $\begin{array}{lll}2.3 \mathrm{X} & 97 & 6\end{array}$ $1.1 \times 126 \quad 7$ \begin{tabular}{rr}
$.1 \times \quad 60 \quad 2$ \\
\hline
\end{tabular}

$\begin{array}{lll}1.4 \times \quad 91 & 3\end{array}$ $\begin{array}{lll}1.5 \mathrm{X} & 80 & 7\end{array}$ $\begin{array}{lll}.1 \times & 128 & 9\end{array}$ $\begin{array}{lll}.5 \times & 160 & 3\end{array}$

$\begin{array}{lll}1.0 \times \quad 95 \quad 4 & 4\end{array}$ $1.3 \mathrm{X} \quad 94 \quad 5$ $\begin{array}{lll}2.0 \times \quad 90 & 2\end{array}$ $\begin{array}{llll}1.4 \times & 87 & 4\end{array}$ $\begin{array}{lll}1.7 \mathrm{X} & 52 \quad 6\end{array}$

$\begin{array}{lll}1.4 \mathrm{X} & 71 \quad 8\end{array}$ $\begin{array}{lll}2.2 \times 129 & 12\end{array}$ $1.6 \times 21626$ $\begin{array}{lll}1.7 \mathrm{X} & 99 & 1\end{array}$ $1.8 \times 30032$

\begin{tabular}{lll}
$1.5 \times \quad 99 \quad 3$ \\
\hline
\end{tabular} $1.2 \times 151 \quad 5$ $\begin{array}{lll}1.6 \times & 87 & 2\end{array}$ $\begin{array}{lll}1.3 \times & 166 & 4\end{array}$ $\begin{array}{lll}1.5 \mathrm{X} & 71 \quad 2\end{array}$

$1.5 \times 200 \quad 7$ $\begin{array}{lrr}1.6 \mathrm{X} & 85 & 2 \\ 1.07 \times & 176 & 1\end{array}$ $\begin{array}{lll}1.0 \times 176 & 1\end{array}$ $\begin{array}{lll}1.7 \times 108 & 2\end{array}$ $\begin{array}{lll}1.9 \mathrm{X} & 54 \quad 7\end{array}$ $\begin{array}{lll}1.3 \times & 99 & 6 \\ 1.1 \times & 261 & 16\end{array}$ $\begin{array}{lll}1.1 \times & 261 \quad 16 \\ 1.5 \times & 266 \quad 10\end{array}$ $\begin{array}{lll}1.5 \times \quad 266 & 10\end{array}$ 
---ORIGIN TIME (HST)-- -LAT N-- --LON W-- DEPTH N RMS ERH ERZ LOC

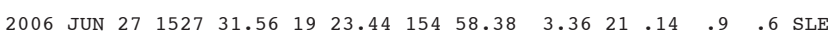
$\begin{array}{llllllllllllll}2006 & \text { JUN } 27 & 1848 & 51.07 & 19 & 6.43 & 154 & 49.13 & 46.87 & 33 & .11 & 1.3 & 1.4 & \text { DIS }\end{array}$

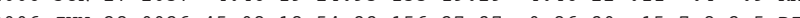

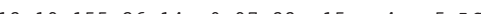

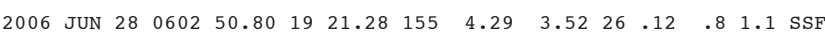
$\begin{array}{lllllllll}2006 & 0 & 1.1 \text { SSP }\end{array}$

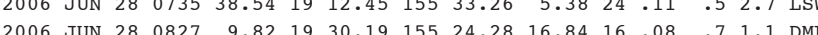

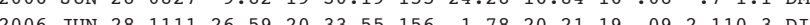
$\begin{array}{llllllllll}2006 & & \end{array}$

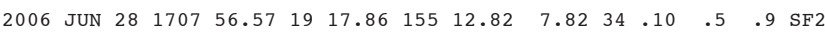

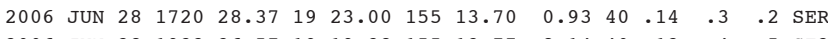

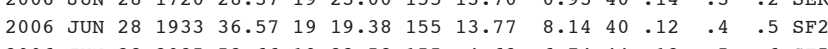

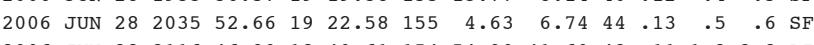

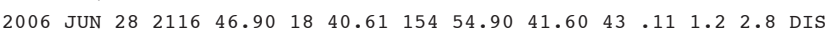

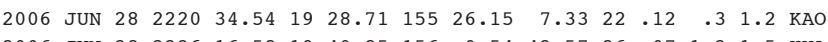

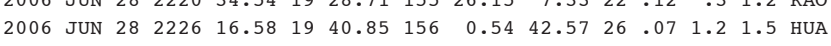

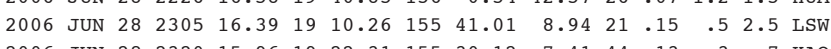

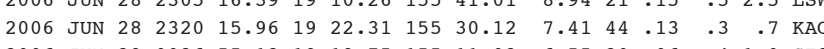

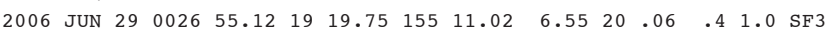

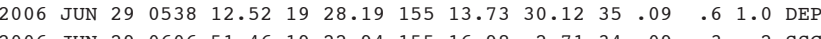

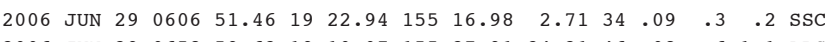
$\begin{array}{llllllllllllll}2006 & \text { JUN } 29 & 0658 & 59.63 & 19 & 10.07 & 155 & 27.91 & 34.21 & 46 & .08 & .6 & 1.1 & \text { DLS } \\ 2006 & \text { JUN } 29 & 0811 & 14.75 & 19 & 25.82 & 155 & 37.31 & 2.85 & 18 & .14 & .4 & .5 & \text { MLO }\end{array}$

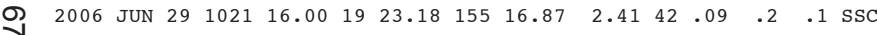

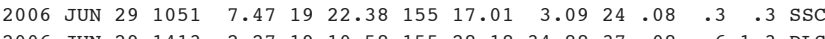
$\begin{array}{llllllllllllll}2006 & \text { JUN } 29 & 1413 & 2.27 & 19 & 10.58 & 155 & 28.18 & 34.88 & 37 & .08 & .6 & 1.3 & \text { DLS }\end{array}$

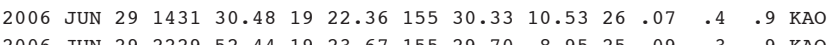

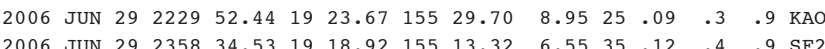

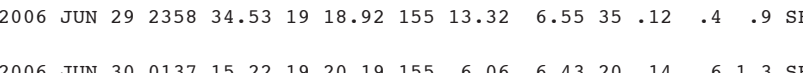
$\begin{array}{llllllllllllll}2006 & \text { JUN } 30 & 0137 & 15.22 & 19 & 20.19 & 155 & 6.06 & 6.43 & 20 & .14 & .6 & 1.3 & \mathrm{SF}\end{array}$

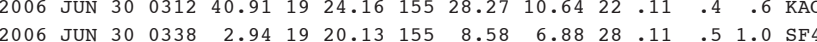

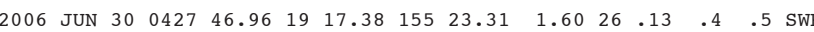

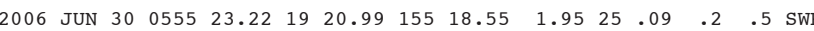

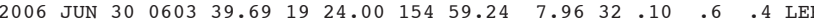

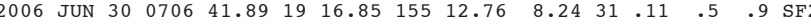

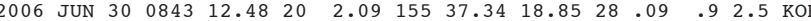

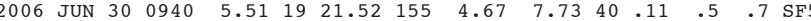

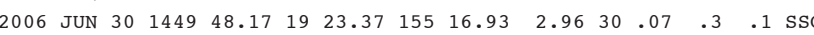

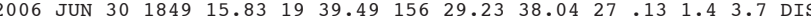

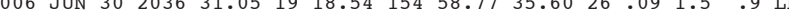

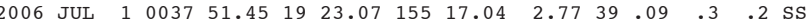

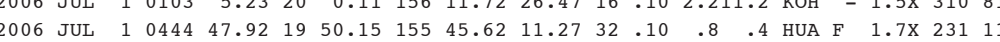

--ORTGIN TIME (HST)-- -LAT N-- --LON W-- DEPTH N RMS ERH ERZ LOC YEAR MON DA HRMN SEC DEG MIN DEG MIN DM RD SEC KM KM PEMKS

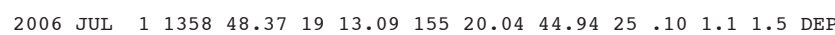

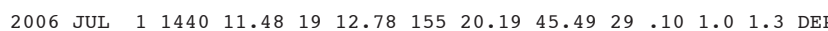

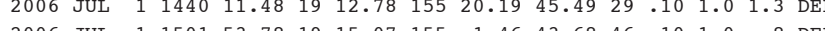

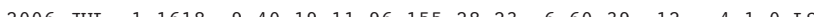

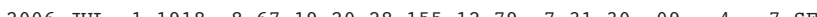

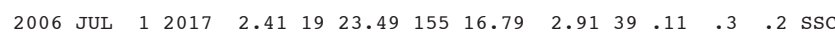
$\begin{array}{llllllllll}2006 & .2 & \text { SSC }\end{array}$ $\begin{array}{lllllllllll}2006 & 0 & 0 & .8 & \text { SEP }\end{array}$

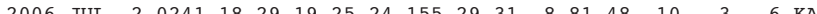

$\begin{array}{lllllllllllllll}2006 & \text { JUL } & 2 & 0729 & 22.80 & 19 & 20.45 & 155 & 11.67 & 9.25 & 53 & .11 & .3 & 3 & \text { SF } 3\end{array}$

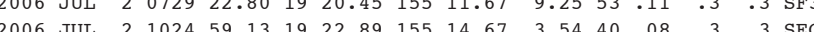

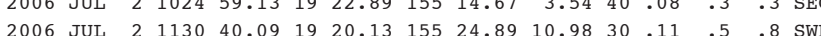

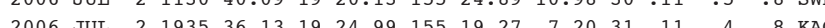
$\begin{array}{lllllllllll}2006 & & & \end{array}$

$\begin{array}{llllllllllllll}2006 & \text { JUL } & 2 & 2042 & 34.47 & 19 & 24.70 & 155 & 28.96 & 10.24 & 20 & .08 & .4 & 1.1 \\ \text { KAO }\end{array}$

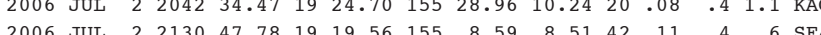

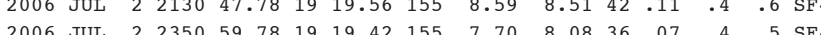

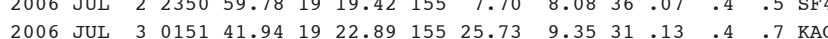

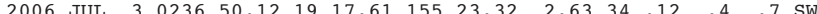

$\begin{array}{lllllllllllllll}2006 & \text { JUL } & 3 & 0614 & 5.25 & 19 & 29.30 & 155 & 26.68 & 10.52 & 30 & .11 & .4 & .9 & \text { KAO }\end{array}$

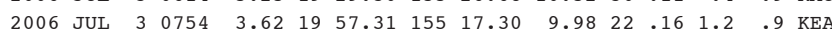
2006 JUL $3 \quad \begin{array}{lllllllllll}1042 & 53.72 & 19 & 30.40 & 155 & 23.57 & 4.12 & 19 & .15 & .8 & .6\end{array}$

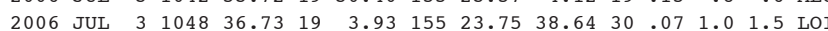

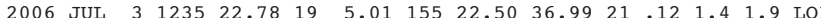

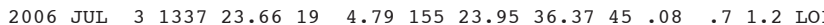

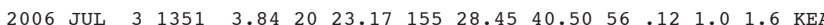

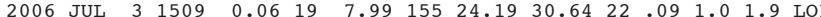

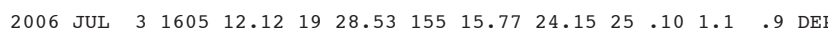
2006 JUL $3 \begin{array}{lllllllllllll}2016 & 5.15 & 19 & 18.73 & 155 & 6.20 & 10.29 & 35 & .08 & .6 & .4 & \mathrm{SF} 4\end{array}$

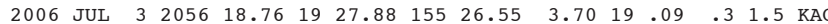

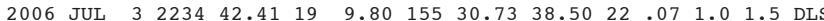
$\begin{array}{lllllllllllllll}2006 & \text { JUL } & 3 & 2313 & 28.88 & 19 & 27.64 & 155 & 25.92 & 8.59 & 43 & .14 & .4 & .9 & \text { KAO }\end{array}$ $\begin{array}{lllllllllllllll}2006 & \text { JUL } & 4 & 0135 & 49.08 & 19 & 12.03 & 155 & 28.03 & 7.31 & 29 & .13 & .4 & 1.0 & \text { LSW }\end{array}$ $\begin{array}{lllllllllllllll}2006 & \text { JUL } & 4 & 0331 & 15.29 & 19 & 21.33 & 155 & 6.35 & 7.75 & 29 & .09 & .4 & .5 & \mathrm{SF} 4\end{array}$

$\begin{array}{lllllllllllllll}2006 & \text { JUL } & 4 & 0612 & 49.27 & 19 & 19.72 & 155 & 8.88 & 7.62 & 37 & .09 & .4 & .7 & \mathrm{SF} 4\end{array}$ 2006 JUL $4 \begin{array}{lllllllllllll}0 & 0638 & 1.03 & 19 & 20.17 & 155 & 12.04 & 7.52 & 45 & .11 & .4 & .5 & \text { SF3 }\end{array}$

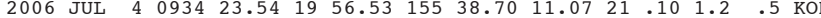
2006 JUL $4 \begin{array}{lllllllllll}1002 & 57.35 & 19 & 11.45 & 155 & 28.49 & 8.65 & 30 & .12 & .4 & 1.0\end{array}$ $\begin{array}{llllllllllllllll}2006 & \text { JUL } & 4 & 1341 & 57.08 & 19 & 18.70 & 155 & 11.48 & 6.71 & 44 & .10 & .4 & .5 & \mathrm{SF} 3\end{array}$

$\begin{array}{lllllllllllllll}2006 & \text { JUL } & 4 & 2332 & 41.97 & 19 & 17.58 & 155 & 37.49 & 10.75 & 18 & .06 & .4 & 1.4 & \text { LSW }\end{array}$

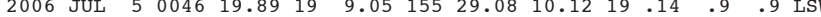

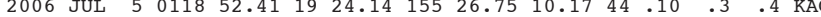

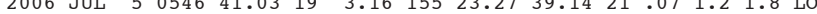

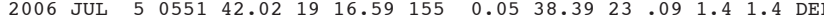

PREF AZ MIN 66 CAP DS

$1.5 \times 226 \quad 14$ $\begin{array}{lll}.6 \times 190 & 14\end{array}$

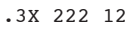

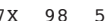

2.2X $44 \quad 0$ $\begin{array}{lll}2.6 \times & 245 & 9 \\ 1.6 \times & 164 & 5\end{array}$ $1.6 \times \quad 46 \quad 6$ $2.3 \times \quad 56 \quad 2$

$2.7 \times \quad 76 \quad 5$ $\begin{array}{lrr}2.2 \times & 50 & 2 \\ 1.3 \times & 135 & 3\end{array}$ $\begin{array}{lll}1.3 \times & 135 & 3 \\ 1.3 \times & 113 & 2 \\ 1.5 \times & 90 & 2\end{array}$ $1.5 \times \quad 90 \quad 2$

$1.4 \times \quad 66 \quad 5$ $\begin{array}{lll}.6 \mathrm{X} & 105 & 4 \\ .2 \mathrm{x} & 131 & 4\end{array}$ $\begin{array}{lrr}1.2 \times & 131 & 4 \\ 1.4 \times & 63 & 3\end{array}$ $1.5 \mathrm{X} 116 \quad 5$

$\begin{array}{rrr}1.7 X & 71 & 5 \\ 1.5 \times & 240 & 39\end{array}$ $\begin{array}{llll}1.5 \times & 240 & 39\end{array}$ $\begin{array}{llll}1.5 \times & 172 & 1 \\ 1.5 \times & 247 & 13 \\ 1.4 \times & 297 & 13\end{array}$ $1.4 \times 29713$

2.0x 20011 $\begin{array}{lll}2.6 \times & 27943 \\ 1.6 \times & 267 & 7\end{array}$ $\begin{array}{lll}1.6 \times 267 & 7 \\ 1.4 \times 182 & 7\end{array}$ $1.3 \times 184 \quad 4$

$\begin{array}{lll}1.4 \mathrm{X} & 65 & 7\end{array}$ $\begin{array}{rrr}1.3 \times & 124 & 5 \\ 1.9 \times & 48 & 6\end{array}$ $1.5 \times 104 \quad 5$ 1.3X $139 \quad 4$

$\begin{array}{lll}1.5 \times & 99 & 5\end{array}$ $\begin{array}{lll}1.7 \times & 78 & 5\end{array}$ $1.7 \times 244 \quad 9$ $\begin{array}{lll}.5 \times & 119 & 4 \\ 2.0 \times & 118 & 5\end{array}$

$\begin{array}{lll}1.1 \mathrm{X} & 93 & 8\end{array}$ $\begin{array}{lll}1.2 \times & 159 & 2\end{array}$ $2.0 \times \quad 45 \quad 3$ $1.6 \times 27014$ $1.3 \times 224 \quad 16$ 
---ORIGIN TIME (HST)-- -LAT N-- --LON W-- DEPTH N RMS ERH ERZ LOC PREF AZ MIN 67 YEAR MON DA HRMN SEC DEG MIN DEG MIN KM RD SEC KM KM REMKS MAC GAP DS

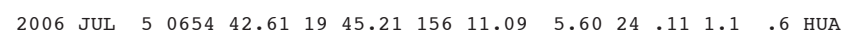

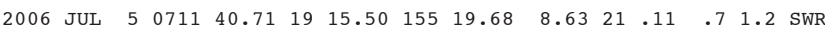
2006 JUL $5 \begin{array}{lllllllllllll}1238 & 28.26 & 19 & 18.65 & 155 & 15.21 & 7.96 & 23 & .10 & .5 & .9 & \text { SF1 }\end{array}$

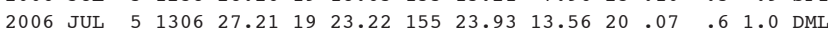
$\begin{array}{lllllllllllllll}2006 & \text { JUL } & 5 & 1344 & 9.20 & 19 & 22.49 & 155 & 30.23 & 10.25 & 20 & .07 & .4 & .9 & \text { KAO }\end{array}$

2006 JUL $5 \begin{array}{lllllllllllll}1717 & 32.70 & 19 & 17.66 & 155 & 23.18 & 2.39 & 20 & .09 & .5 & .6 & \text { SWR }\end{array}$ 2006 JUL $6 \begin{array}{llllllllllllll}6 & 0408 & 7.12 & 19 & 29.23 & 155 & 26.66 & 7.34 & 20 & .10 & .4 & 1.2 & \text { KAO }\end{array}$

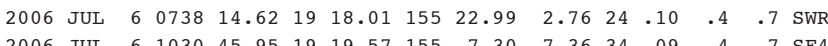
$\begin{array}{lllllllllllllll}2006 & \text { JUL } & 6 & 1030 & 45.95 & 19 & 19.57 & 155 & 7.30 & 7.36 & 34 & .09 & .4 & .7 & \text { SF4 }\end{array}$ 2006 JUL $6 \begin{array}{llllllllllll}1035 & 12.00 & 19 & 22.34 & 155 & 17.18 & 2.96 & 18 & .08 & .3 & .4 & \mathrm{SSC}\end{array}$

$\begin{array}{lllllllllllllll}2006 & \text { JUL } & 6 & 1451 & 36.27 & 19 & 19.55 & 155 & 15.20 & 7.13 & 33 & .07 & .3 & .7 & \text { SF1 }\end{array}$ 2006 JUL $6 \begin{array}{lllllllllllll}1531 & 21.94 & 19 & 20.67 & 155 & 4.55 & 2.43 & 21 & .12 & .7 & 1.2 & \text { SSF }\end{array}$ $\begin{array}{lllllllllllllll}2006 & \text { JUL } & 6 & 1926 & 36.31 & 18 & 56.68 & 155 & 10.70 & 47.03 & 42 & .10 & 1.1 & 1.6 & \text { LOI }\end{array}$ $\begin{array}{llllllllllllllll}2006 & \text { JUL } & 6 & 2134 & 36.58 & 19 & 24.75 & 155 & 19.14 & 5.35 & 25 & .11 & .4 & .9 & \text { KAO }\end{array}$

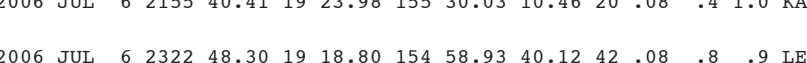

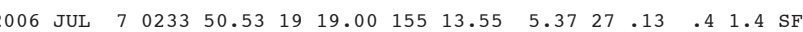

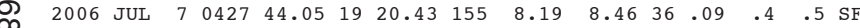
$\begin{array}{lllllllllllllll}2006 & \text { JUL } & 7 & 0512 & 18.53 & 19 & 22.09 & 155 & 30.11 & 9.98 & 39 & .09 & .3 & .7 & \text { KAO }\end{array}$

2006 JUL $7 \quad 0616 \quad 55.9017 \quad 43.51 \quad 154 \quad 53.76 \quad 21.16 \quad 50 \begin{array}{llllll}.12 & 1.7 & 6.4 & \text { DIS }\end{array}$

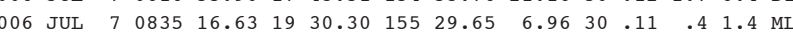

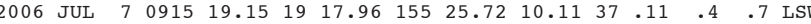

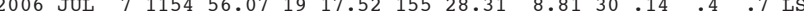
2006 JUL $\quad \begin{array}{lllllllllllll}7 & 1208 & 48.79 & 19 & 20.00 & 155 & 7.75 & 8.07 & 41 & .10 & .5 & .7 & \text { SF } 4\end{array}$

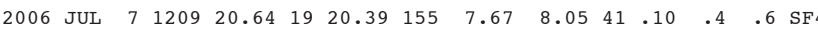
2006 JUL $71657 \begin{array}{lllllllllll}163.81 & 19 & 12.78 & 155 & 35.15 & 6.70 & 50 & .15 & .4 & 1.0 & \text { LSW }\end{array}$

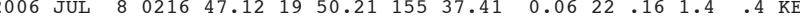

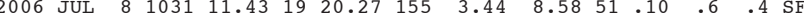
$\begin{array}{lllllllllllllll}2006 & \text { JUL } & 8 & 2041 & 46.35 & 19 & 19.81 & 155 & 10.82 & 9.42 & 32 & .07 & .5 & .7 & \text { SF } 3\end{array}$

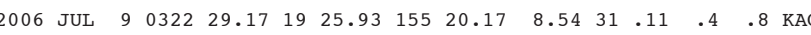

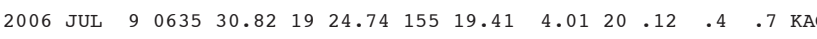

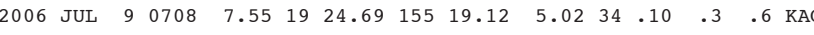

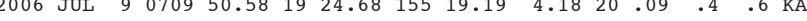
2006 JUL $9 \begin{array}{llllllllllll}1108 & 35.20 & 19 & 19.30 & 155 & 10.06 & 5.82 & 30 & .10 & .5 & 1.1 & \text { SF3 }\end{array}$

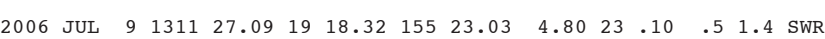

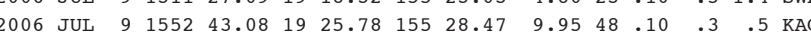
2006 JUL $9 \begin{array}{llllllllllll}1559 & 22.89 & 19 & 25.82 & 155 & 28.26 & 9.93 & 20 & .12 & .5 & 1.2 & \text { KAO }\end{array}$

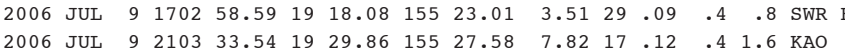

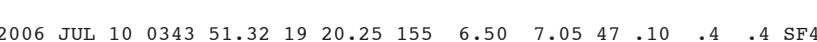

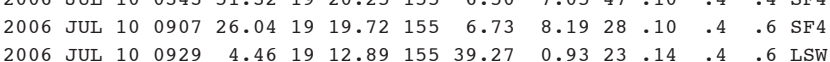

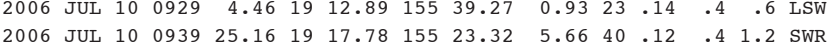

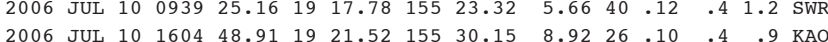

$2.0 \times 28952$ $\begin{array}{rrr}.9 \times & 181 & 5 \\ .8 \times & 125 & 4\end{array}$ $.9 \times \quad 91 \quad 6$ $\begin{array}{rrr}1.6 \times & 67 & 5\end{array}$

$1.2 \times 156 \quad 5$ $\begin{array}{llll}1.4 \times \quad 81 & 5\end{array}$ $1.2 \times 113 \quad 4$ $1.6 \times 164$ $1.4 \times \quad 99 \quad 2$

$\begin{array}{lll}1.3 \times & 97 & 4\end{array}$ $1.3 \times 174 \quad 7$ $2.1 \times 25038$ $1.2 \times 103 \quad 2$ $\begin{array}{lll}1.3 \times & 69 & 5\end{array}$

1.9X 23112 $1.0 \times 81$ $1.4 \times 1135$ $1.6 \times \quad 73 \quad 4$ $1.6 \times 153 \quad 4$

$3.1 \times 330161$ 1.6X $92 \quad 5$ $1.3 \times 105 \quad 6$ $1.6 \times \quad 86$ $1.5 \times 126 \quad 5$

$2.2 \times 125 \quad 5$ $2.5 \times 121 \quad 10$ $1.5 \times 23518$ $\begin{array}{lll}2.5 \times & 189 & 8\end{array}$ $1.3 \times 158 \quad 6$

1.5X 102 $1.1 \times 105 \quad 2$ $\begin{array}{lll}1.7 \times & 65 & 2\end{array}$ $1.0 \times 101$ $1.5 \times 101 \quad 5$

$1.4 \times 147$ $2.0 \times \quad 35 \quad 6$ $\begin{array}{rrr}1.4 \mathrm{X} & 60 & 6\end{array}$ $\begin{array}{rrr}1.2 \times & 151 & 4 \\ 1.3 \times & 92 & 4\end{array}$

$2.2 \times 149 \quad 6$ $1.6 \times 179 \quad 5$ $\begin{array}{lll}1.6 \times & 159 & 13\end{array}$ $\begin{array}{lrrr}2.1 \times & 114 & 4 \\ 1.3 \times & 71 & 5\end{array}$
--ORIGIN TIME (HST)-- -LAT N-- --LON W-- DEPTH N RMS ERH ERZ LOC YEAR MON DA HRMN SEC DEG MIN DEG MIN KM RD SEC KM KM REMKS

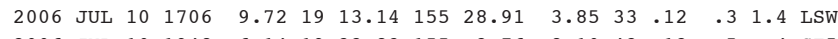

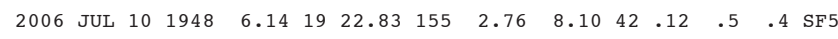

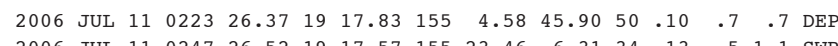

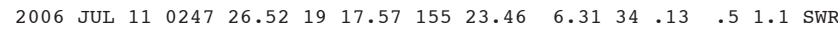
2006 JUL $11 \quad \begin{array}{lllllllllllll}11 & 0357 & 39.18 & 19 & 21.59 & 155 & 11.96 & 2.94 & 21 & .10 & .3 & .5 & \text { SER }\end{array}$

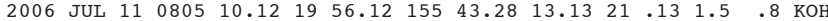

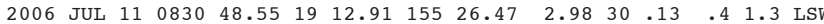

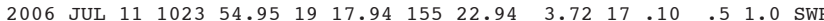

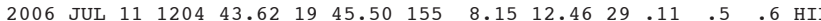

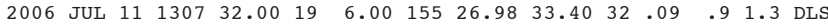

$\begin{array}{llllllllllllll}2006 & \text { JUL } 11 & 1448 & 48.84 & 19 & 20.51 & 155 & 8.65 & 7.99 & 21 & .09 & .5 & .9 & \text { SF } 4\end{array}$

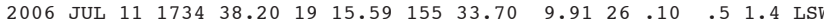

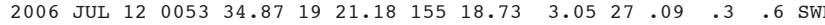

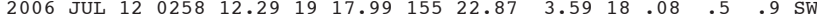
2006 JUL $12 \quad \begin{array}{llllllllllllll}12 & 40.20 & 19 & 25.41 & 155 & 29.98 & 14.77 & 25 & .10 & .4 & .7 & \text { DML }\end{array}$

$\begin{array}{llllllllllllll}2006 & \text { JUL } 12 & 0635 & 47.24 & 19 & 14.21 & 155 & 32.47 & 6.17 & 20 & .18 & .6 & 2.0 & \text { LSW }\end{array}$

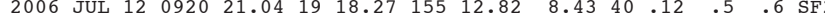

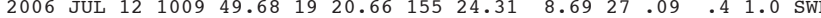

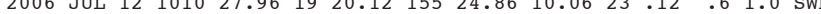

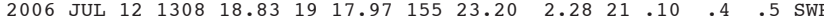

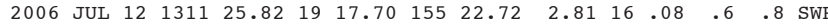
2006 JUL $12 \quad 1349 \begin{array}{lllllllllll}10.84 & 19 & 15.46 & 155 & 30.69 & 7.85 & 27 & .13 & .4 & 1.1 & \text { LSW }\end{array}$

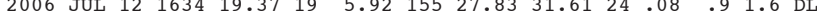

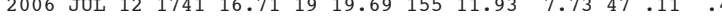

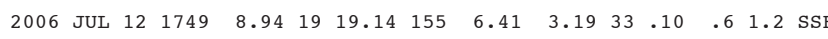

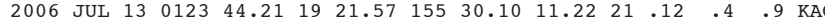

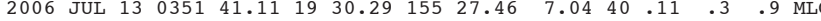

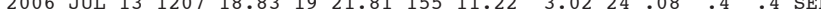

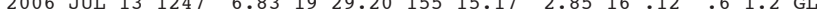

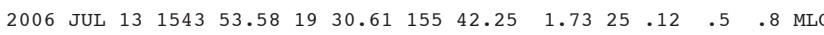

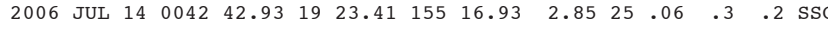

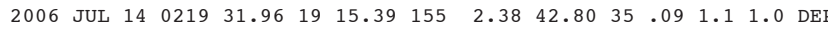

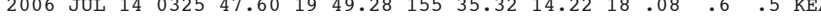

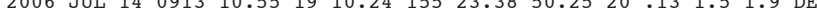

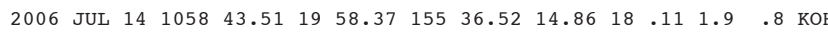

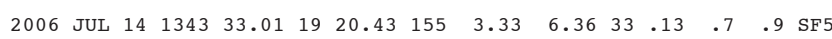

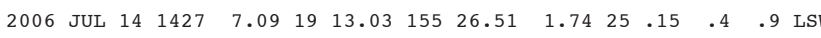

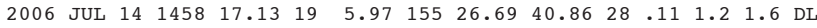
2006 JUL $14 \quad 1500 \begin{array}{llllllllllll}148.17 & 19 & 6.78 & 155 & 27.11 & 38.07 & 23 & .12 & 1.6 & 1.9 & \text { DLS }\end{array}$ $\begin{array}{llllllllllllll}2006 & \text { JUL } 14 & 1701 & 19.33 & 19 & 20.37 & 155 & 19.61 & 3.34 & 15 & .07 & .3 & .7 & \text { SWR }\end{array}$

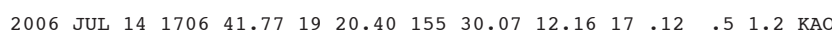

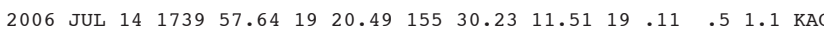

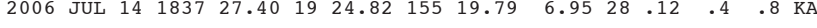
$\begin{array}{llllllllllllll}2006 & \text { JUL } 14 & 1906 & 27.52 & 19 & 19.83 & 155 & 11.41 & 8.27 & 21 & .07 & .5 & 1.0 & \text { SF3 }\end{array}$ $\begin{array}{rrr}.6 \mathrm{X} & 87 & 7 \\ .9 \mathrm{x} & 163 & 4 \\ 2.5 \times & \end{array}$ $2.5 \times 199 \quad 6$ $\begin{array}{lll}1.5 \times & 115 & 5\end{array}$ $1.4 \times \quad 90 \quad 3$

$1.6 \times 25211$ $\begin{array}{lll}1.3 \times & 127 & 7 \\ 1.2 x & 115 & 4\end{array}$ $1.5 \times 20126$ $1.8 \times 273 \quad 7$

$1.3 \times 120 \quad 5$ $\begin{array}{llll}1.5 \times & 178 & 6\end{array}$ $\begin{array}{lll}.4 \times \quad 63 & 5\end{array}$ $\begin{array}{rrr}.3 \times & 152 & 4 \\ .5 x & 67 & 5\end{array}$

$\begin{array}{lll}1.5 \times 139 & 4\end{array}$ $1.6 \times 106 \quad 3$ $\begin{array}{lll}1.3 \mathrm{X} & 81 \quad 2\end{array}$ $1.4 \times 120 \quad 3$ $1.5 \times 179$

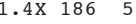
$1.3 \times 117$ $1.6 \times 206 \quad 7$ $\begin{array}{lll}1.9 \times & 116 & 6\end{array}$

$\begin{array}{lll}1.5 \mathrm{X} & 62 & 5\end{array}$ $\begin{array}{lll}1.0 \times & 61 & 3\end{array}$ $1.6 \mathrm{X} \quad 913$ $\begin{array}{lll}1.7 \times & 219 & 8 \\ 1.6 \times & 100 & 6\end{array}$

$\begin{array}{lll}1.9 \times & 46 & 0\end{array}$ $\begin{array}{lll}1.7 \times & 228 & 17\end{array}$ $1.1 \times 133 \quad 9$ $\begin{array}{lll}1.6 \times & 197 \quad 8\end{array}$ 1.5x 26414

$\begin{array}{lll}1.5 \times & 188 \quad 7\end{array}$ $\begin{array}{lll}1.4 \times & 125 \quad 7\end{array}$ $\begin{array}{lll}1.9 \times & 233 & 7\end{array}$ $\begin{array}{lll}2.0 \times & 270 & 16\end{array}$ $\begin{array}{ccc}1.2 \mathrm{X} & 96 & 6 \\ 1.0 \mathrm{x} & 86 & 6\end{array}$ $\begin{array}{lll}1.0 \times & 86 & 6 \\ 1.5 \times & 75 & 2\end{array}$ $\begin{array}{lll}1.5 \times & 75 & 2 \\ 2.1 \times & 77 & 2\end{array}$ $\begin{array}{lll}2.1 \times & 77 & 2 \\ 1.2 x & 89 & 6\end{array}$ 
---ORIGIN TIME (HST)-- -LAT N-- --LON W-- DEPTH N RMS ERH ERZ LOC $\begin{array}{llllllllllllll}2006 & \text { JUL } 14 & 2259 & 23.89 & 19 & 19.82 & 155 & 8.71 & 8.24 & 26 & .08 & .4 & .7 & \text { SF4 }\end{array}$

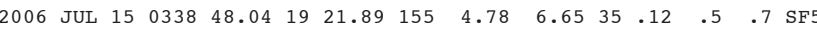

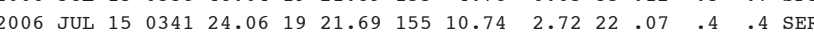
$\begin{array}{lllllllllllll}2006 & \text { JUL } 15 & 0526 & 19.28 & 19 & 18.30 & 155 & 21.82 & 3.36 & 20 & .09 & .5 & .8\end{array}$

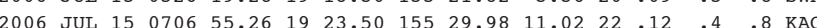

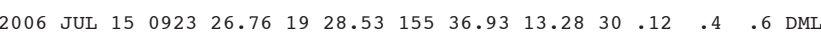

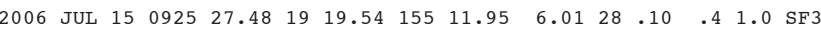

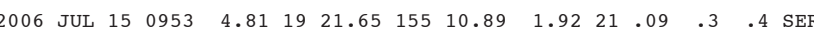
$\begin{array}{lllllllllllll}2006 & \text { JUL } 15 & 1243 & 55.87 & 19 & 21.69 & 155 & 18.39 & 2.68 & 35 & .10 & .2 & .4\end{array}$

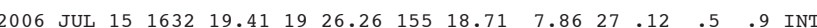

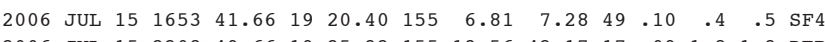

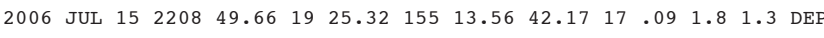

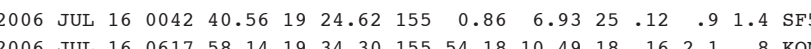
$\begin{array}{llllllllllll}2006 & & 0\end{array}$

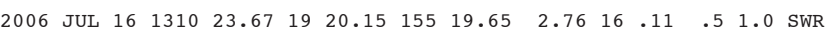
$\begin{array}{llllllllllllll}2006 & \text { JUL } 16 & 1356 & 23.80 & 19 & 15.97 & 155 & 29.01 & 8.93 & 25 & .14 & .4 & 1.1 & \text { LSW }\end{array}$

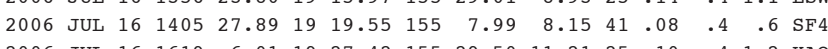

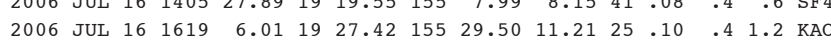
$\begin{array}{lllllllllllllll}2006 & \text { JUL } & 16 & 1737 & 33.83 & 19 & 19.21 & 155 & 3.67 & 40.77 & 37 & .10 & 1.1 & .7 & \text { DEP }\end{array}$

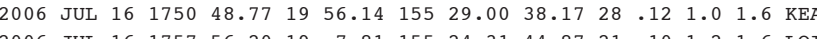

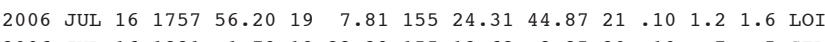
$\begin{array}{llllllllllllll}2006 & \text { JUL } 16 & 1821 & 1.79 & 19 & 22.29 & 155 & 13.68 & 3.85 & 20 & .10 & .7 & .5 & \text { SER }\end{array}$

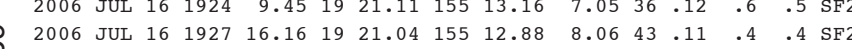

$\begin{array}{llllllllllllll}2006 & \text { JUL } 16 & 2238 & 18.18 & 19 & 17.26 & 155 & 23.00 & 3.28 & 19 & .11 & .8 & 1.1 & \text { SWR }\end{array}$

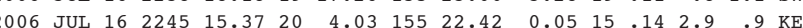

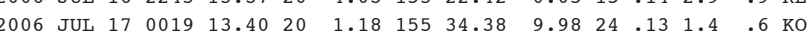

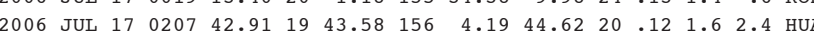

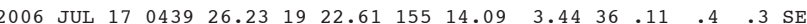

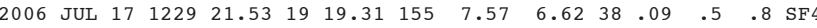

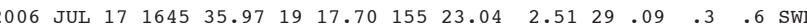
2006 JUL $17 \quad \begin{array}{llllllllllll}1807 & 18.28 & 19 & 18.95 & 155 & 12.05 & 2.71 & 29 & .11 & .4 & 1.0 & \text { SSF }\end{array}$

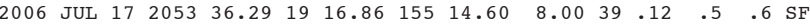

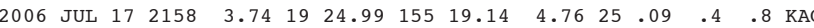

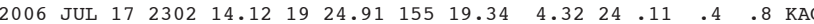

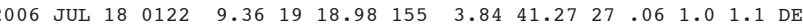

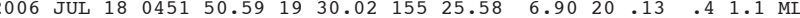

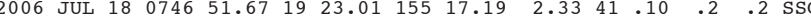

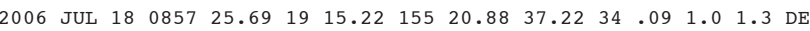

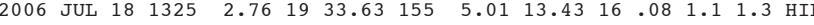

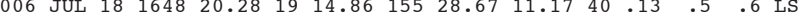

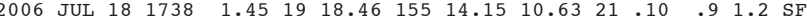

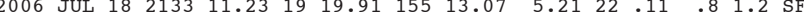

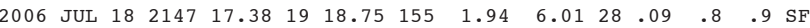

PREF AZ MIN $1.1 \times 103 \quad 5$ $1.5 \times 154 \quad 5$ $1.0 \times 149$ $1.0 \times 14040$

$1.8 \times \quad 69 \quad 2$ $1.4 \times \quad 91 \quad 5$ $1.8 \times \quad 56 \quad 3$ $1.2 \times 154=30$

2.3X $142 \quad 6$ $1.3 \times 24516$ $2.2 \times 315 \quad 22$ $1.0 \times 255 \quad 9$ $1.0 \times 255$
$1.3 \times \quad 80$

$\begin{array}{lll}1.3 \times & 115 \quad 4\end{array}$ $\begin{array}{lll}1.3 \times & 85 & 3\end{array}$ $\begin{array}{lll}1.3 \times & 85 & 3 \\ 1.7 \times & 122 & 4\end{array}$ $\begin{array}{lrr}1.5 \times & 75 & 9\end{array}$ $1.8 \times 208 \quad 8$

1.7X 23516 $1.9 \times 249 \quad 7$ $1.6 \times 177$ $1.4 \times 123 \quad 3$ $\begin{array}{lll}1.5 \times & 63 & 3\end{array}$

$1.4 \times 221 \quad 5$ \# 1.4X 29520 $1.6 \times 26220$ $1.3 \times 28225$

$1.5 \times 136 \quad 4$ $1.3 \times 116 \quad 5$ $1.1 \times 165$ 1.5X $179 \quad 2$ $1.1 \times 113 \quad 3$

$\begin{array}{lll}1.1 \times 110 \quad 2 & \end{array}$ $1.5 \times 2058$ $\begin{array}{rrr}2.1 \times & 42 & 1 \\ 1.5 \times & 150 & 6\end{array}$

1.3X 20617 $2.1 \times 109 \quad 3$ 1.0X 185 $1.1 \times 216 \quad 5$ $1.3 \times 2401$
-ORTGIN TIME (HST)-- -LAT N-- --LON W-- DEPTH N RMS ERH ERZ LOC YEAR MON DA HRMN SEC DEG MIN DEG MIN KM RD SEC KM KM REMKS $\begin{array}{llllllllllllll}2006 & \text { JUL } 18 & 2244 & 3.96 & 19 & 17.60 & 155 & 23.08 & 2.76 & 17 & .11 & .5 & .8 & \text { SWR }\end{array}$

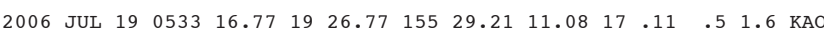

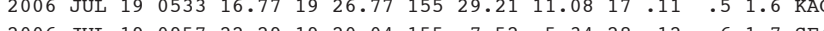
$\begin{array}{lllllllll}2006 & 006 \\ 0\end{array}$

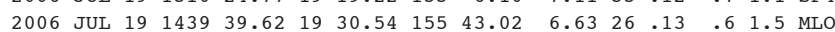

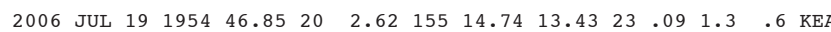

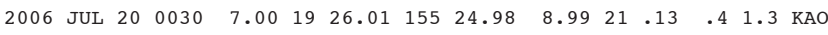

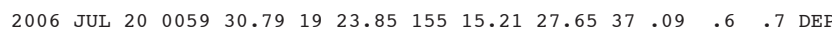

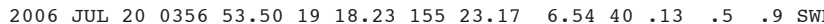

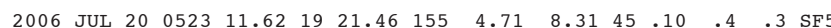

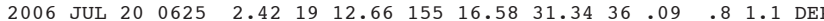

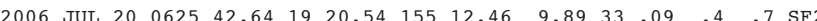

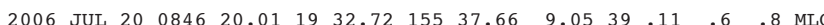

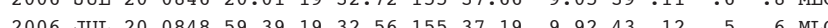

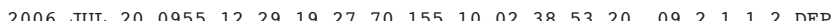

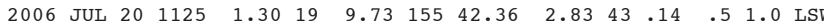

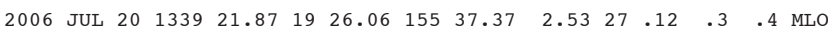

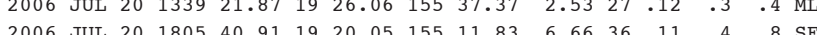

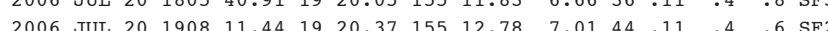

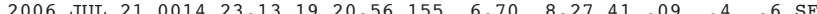

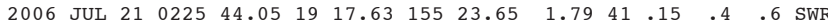

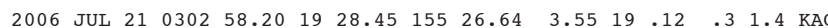

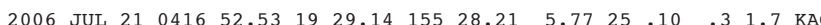

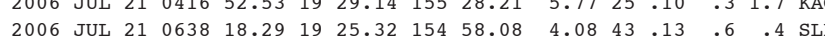

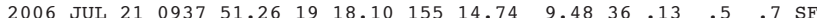

$\begin{array}{llllllllllllll}2006 & \text { JUL } 21 & 1246 & 37.21 & 19 & 20.34 & 155 & 6.05 & 7.70 & 33 & .12 & .5 & .9 & \mathrm{SF} 4\end{array}$

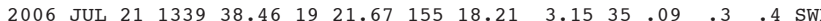

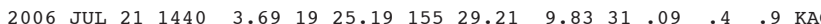
$\begin{array}{lllllllllllll}2006 & \text { JUL } 21 & 1449 & 50.58 & 19 & 25.05 & 155 & 39.16 & 3.13 & 22 & .10 & .7 & .6\end{array}$

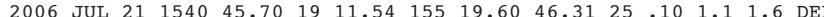

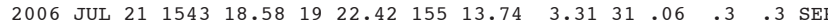

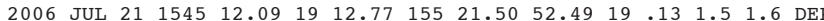

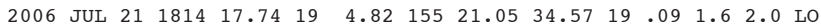

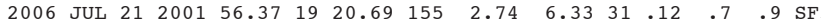

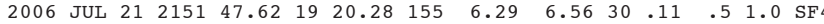

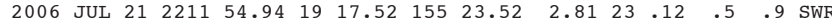

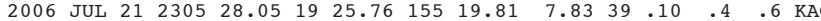

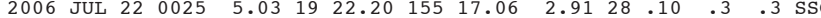

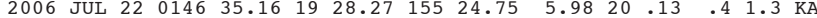

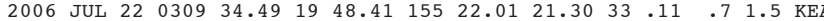

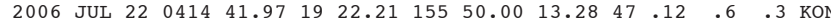

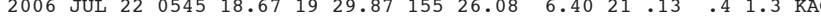

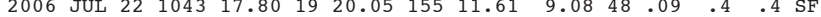

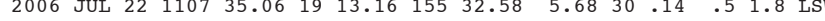

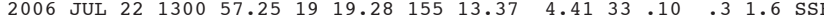

PREF AZ MIN 70 HAG GAP DS

$1.3 \mathrm{U} 165 \quad 5$ $.2 \mathrm{U} 83 \quad 7$ $1.5 \times 1830$ $1.4 \times \quad 98 \quad 5$

$1.7 \times 27220$ $1.0 \times \quad 72 \quad 8$ $.6 \times \quad 84 \quad 2$ $8 \times 160 \quad 5$

1.6x 19410 $1.4 \times \quad 70 \quad 4$ $1.9 \times \quad 97 \quad 6$ $\begin{array}{rrr}1.9 \times & 97 & 6 \\ 1.4 \times & 273 & 14\end{array}$

2.5X 19420 $\begin{array}{lll}1.9 \mathrm{X} & 91 & 3 \\ 1.3 \mathrm{x} & 82 & 5\end{array}$ $\begin{array}{lll}1.3 \mathrm{X} & 82 & 5 \\ 1.4 \mathrm{x} & 69 & 4 \\ 1.5 \mathrm{n} & 43 & 5\end{array}$ $1.5 \times 143 \quad 5$

$\begin{array}{lll}1.5 \times \quad 114 & 5\end{array}$ $\begin{array}{lll}1.4 \mathrm{X} & 81 & 6\end{array}$ $\begin{array}{rrr}1.4 \mathrm{X} & 71 & 5 \\ 2.2 \mathrm{x} & 174 & 1\end{array}$ $1.3 \times 105 \quad 3$

$\begin{array}{lll}1.3 \times & 157 \quad 6\end{array}$ $\begin{array}{lll}1.9 \mathrm{X} & 54 & 3 \\ 1.4 \mathrm{X} & 51 & 6\end{array}$ $1.5 \times 202 \quad 3$ $1.4 \times \quad 210 \quad 15$

$\begin{array}{lll}.9 \times & 93 & 1\end{array}$ $1.4 \times 232 \quad 16$ $1.1 \times 278 \quad 15$ $\begin{array}{lll}1.2 \times & 190 & 7 \\ 1.3 \times & 154 & 6\end{array}$

1.3X $157 \quad 5$ $\begin{array}{lll}1.4 \times & 86 & 4\end{array}$ $\begin{array}{llll}1.5 \times & 167 & 2\end{array}$ $\begin{array}{rrr}.2 \mathrm{X} & 71 & 4 \\ .5 \mathrm{X} & 114 & 10\end{array}$

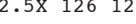
$\begin{array}{lll}1.4 \mathrm{X} & 95 \quad 5\end{array}$ $2.3 \times \quad 84 \quad 5$ $1.4 \times 124 \quad 6$ $1.1 \mathrm{X} \quad 73 \quad 4$ 
---ORIGIN TIME (HST)-- -LAT N-- --LON W-- DEPTH N RMS ERH ERZ LOC YEAR MON DA HRMN SEC DEG MIN DEG MIN KM RD SEC KM KM REMKS PREF AZ MIN

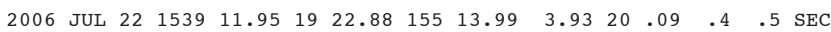

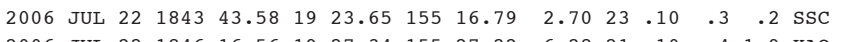

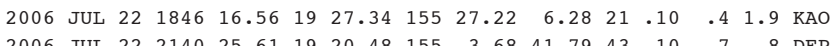

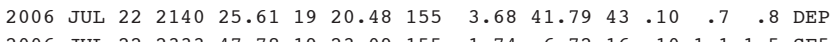

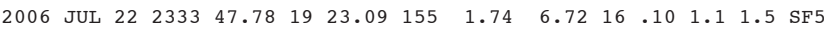

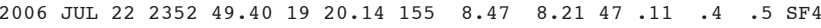

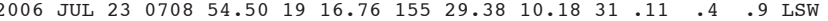

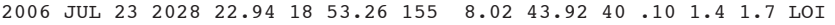

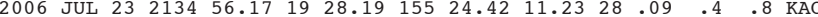

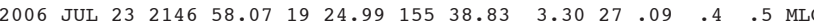

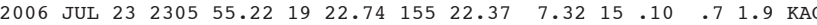

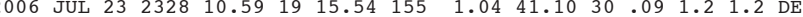

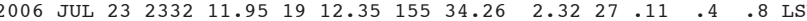

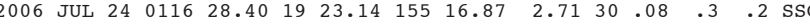

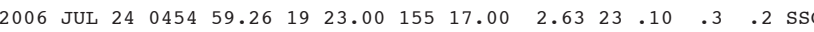

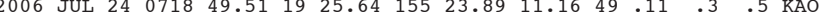

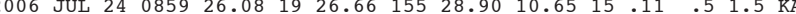

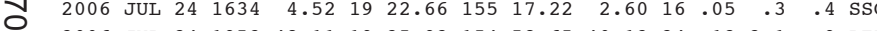

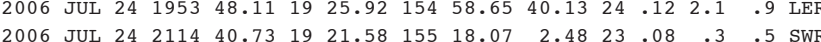

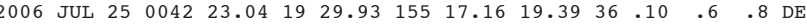
$\begin{array}{llllllllll} & & \end{array}$ $\begin{array}{lllllllllll} & 006 & & \end{array}$

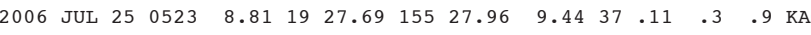

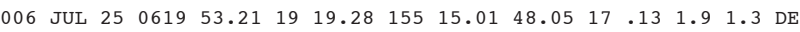

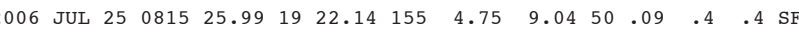

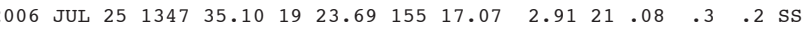

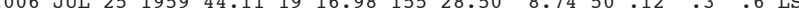

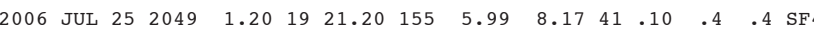

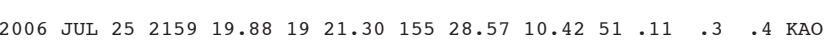

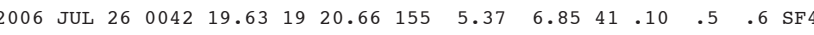

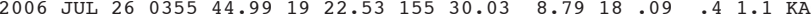

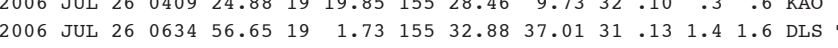

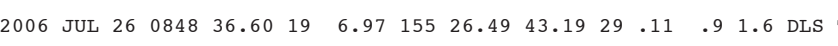

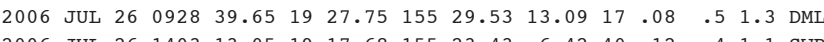

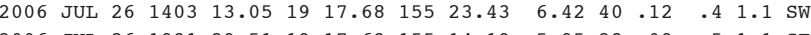

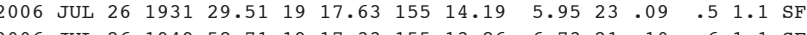

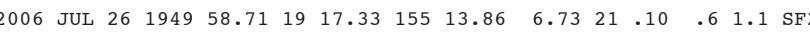

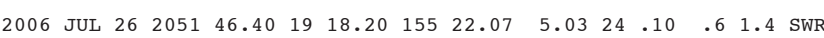
$\begin{array}{llllllllllllll}2006 & \text { JUL } 26 & 2328 & 6.87 & 19 & 21.38 & 155 & 5.25 & 5.80 & 21 & .08 & .5 & 1.1 & \text { SF } 5\end{array}$

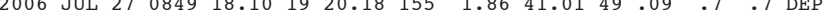

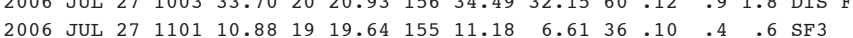

$1.5 \times \quad 95 \quad 2$ $1.5 \mathrm{X} \quad 53$ $1.9 \times 185$ $1.1 \times 199 \quad 5$

$1.9 \times 109$ $1.5 \times \quad 80 \quad 3$ $2.4 \times 26644$ $1.9 \times 106 \quad 2$

$1.1 \times 132 \quad 5$ $1.5 \times 23318$ $1.6 \times 125$ $1.9 \times \quad 46 \quad 0$ $\begin{array}{lll}1.5 \mathrm{X} & 48 & 1\end{array}$

$\begin{array}{lll}2.4 \mathrm{X} & 44 & 8\end{array}$ $1.1 \mathrm{x} \quad 83 \quad 8$ $\begin{array}{ll}1.1 \times \quad 83 & 83\end{array}$ $\begin{array}{lll}1.7 \times & 308 & 21\end{array}$ $1.1 \times \quad 66$

$\begin{array}{lll}1.4 \times 126 \quad 8 & \end{array}$ $1.1 \times \quad 83$ $1.6 \times \quad 45 \quad 3$ $1.4 \times 22318$ $\begin{array}{lll}1.5 \times & 50 & 8\end{array}$

$1.8 \times 297 \quad 12$ $2.5 \times 151$ $1.4 \times \quad 73 \quad 1$ $2.2 \mathrm{X} \quad 86 \quad 5$ $1.8 \times 146 \quad 5$

$\begin{array}{lll}2.0 \times & 67 \quad 3\end{array}$ $\begin{array}{lll}1.7 \times & 163 & 6\end{array}$ $1.0 \times \quad 72 \quad 4$ $\begin{array}{lll}1.3 \times & 75 & 5\end{array}$

$2.0 \times 189 \quad 5$ $\begin{array}{lll}1.4 \times & 77 \quad 8\end{array}$ $1.9 \times 115 \quad 5$ $1.0 \times 130$

$.2 x+150$ .2x 150 $2.4 \times 2009$ $4.4 \mathrm{U} 15987$ $\begin{array}{lll}1.5 \mathrm{X} & 94 \quad 6\end{array}$
---ORIGIN TIME (HST)-- -LAT N-- --LON W-- DEPTH N RMS ERH ERZ LOC YEAR MON DA HRMN SEC DEG MIN DEG MIN KM RD SEC KM KM REMKS

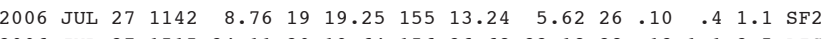

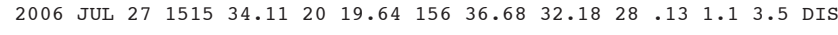

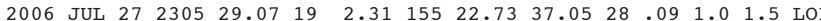

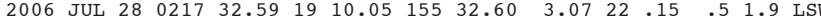
$\begin{array}{llllllllllllll}2006 & \text { JUL } 28 & 0232 & 20.01 & 19 & 19.73 & 155 & 8.86 & 6.70 & 43 & .09 & .4 & .6 & \text { SF } 4\end{array}$

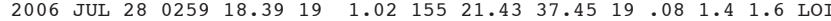

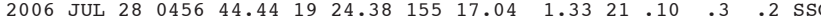

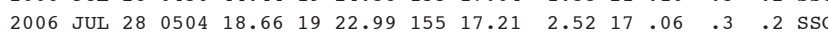

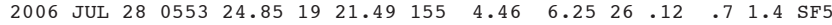

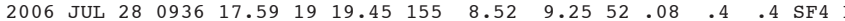

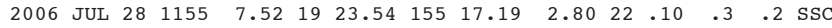

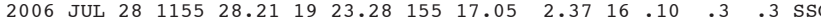

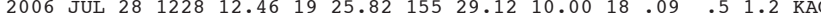

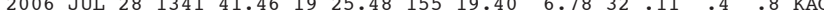
2006 JUL $28 \quad 1409 \begin{array}{lllllllllll}52.70 & 19 & 8.97 & 155 & 32.96 & 0.03 & 19 & .13 & 1.1 & .4 & \text { LSW }\end{array}$

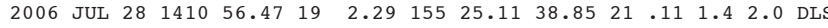

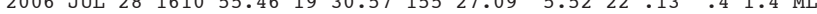
$\begin{array}{llllllllll} & \end{array}$

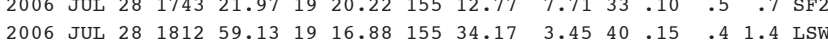

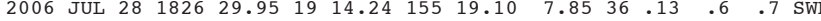

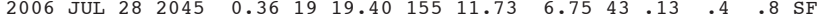

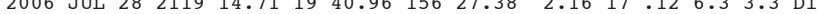

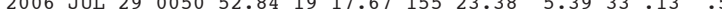
$\begin{array}{llllllllllllll}2006 & \text { JUL } 29 & 0207 & 9.61 & 19 & 56.37 & 155 & 22.25 & 8.36 & 43 & .13 & .8 & .6 & \text { KEA }\end{array}$

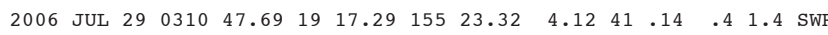

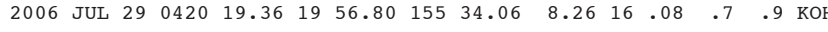

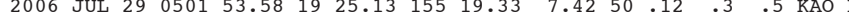

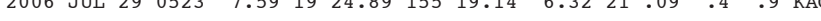

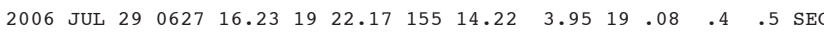

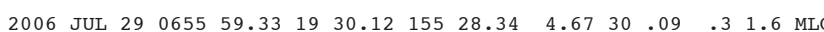

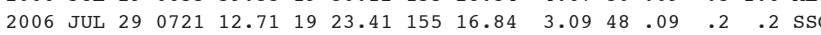

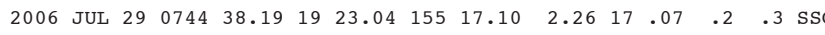
$\begin{array}{llllllllllllll}2006 & & 0\end{array}$ 2006 JUL $29 \quad 0758 \quad 14.7919 \quad 29.98 \quad 155 \quad 28$

$\begin{array}{llllllllllllll}2006 & \text { JUL } 29 & 0930 & 35.24 & 19 & 25.05 & 155 & 19.55 & 6.61 & 38 & .11 & .4 & .8 & \text { KAO }\end{array}$

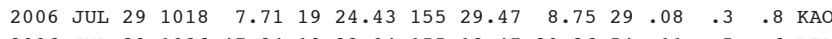

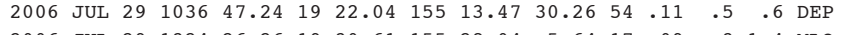

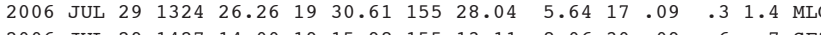
$\begin{array}{llllllllllllll}2006 & \text { JUL } 29 & 1427 & 14.00 & 19 & 15.98 & 155 & 13.11 & 8.06 & 30 & .09 & .6 & .7 & \mathrm{SF} 2\end{array}$

$\begin{array}{llllllllllllll}2006 & \text { JUL } 29 & 1530 & 36.80 & 19 & 23.22 & 155 & 17.08 & 2.88 & 21 & .07 & .2 & .2 & \text { SSC }\end{array}$

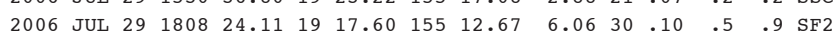

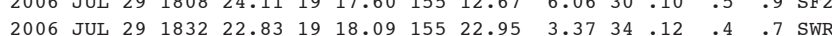

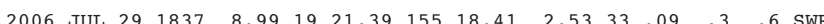
$\begin{array}{lllllllllllll}2006 & & 0\end{array}$ $\begin{array}{rrr}.3 \times & 76 & 4 \\ 6 \times & 169 & 90 \\ .3 \times & 229 & 6\end{array}$ $\begin{array}{lll}1.6 \times & 169 & 90 \\ 1.3 \times 229 & 16\end{array}$ $1.1 \times 118 \quad 8$ $1.5 \times 100 \quad 5$

.5X 25619 $\begin{array}{rrr}.5 \mathrm{X} & 79 & 1 \\ .4 \mathrm{X} & 81 & 1 \\ 1.3 \mathrm{x} & 163 & 5\end{array}$ $\begin{array}{lll}1.3 \times & 163 \quad 5\end{array}$ $.0 \times 1074$

$\begin{array}{lll}.3 \times & 50 & 1\end{array}$ $\begin{array}{lll}1.1 x & 67 & 0\end{array}$ $\begin{array}{lll}1.5 \times & 208 & 9\end{array}$

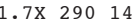
$\begin{array}{llll}1.4 \times & 120 & 3\end{array}$ $\begin{array}{lll}.7 \times \quad 282 & 14\end{array}$ $1.3 \times \quad 70 \quad 4$ $1.7 \times \quad 96 \quad 8$

$\begin{array}{lll}1.4 \times 179 & 7\end{array}$ $\begin{array}{lll}1.4 \times \quad 96 & 5\end{array}$ $1.7 \times 31265$ $1.1 \times \quad 156 \quad 5$ $1.7 \times 232 \quad 20$

$1.6 \times 119 \quad 5$ $1.3 \times 23814$ $2.4 \times \quad 46 \quad 3$ $1.6 \times 108{ }^{2}$ $\begin{array}{lll}2.5 \mathrm{U} & 82 & 4\end{array}$ $\begin{array}{lll}2.4 \mathrm{X} & 37 & 0 \\ 1.2 \times & 67 & 1\end{array}$ $\begin{array}{lll}1.2 \mathrm{X} & 67 & 1\end{array}$ $1.3 \times 1590$ $\begin{array}{lll}.4 \times & 80 \quad 2\end{array}$ $\begin{array}{lll}1.4 \mathrm{X} & 50 & 5 \\ 2.8 \mathrm{X} & 54 & 1\end{array}$ $\begin{array}{lll}.5 \times & 89 & 3\end{array}$ $1.5 \times \quad 223 \quad 2$

$\begin{array}{lll}1.5 \mathrm{X} & 47 & 0\end{array}$ $\begin{array}{lll}1.0 \times \quad 140 & 2\end{array}$ $\begin{array}{rrr}1.6 \times & 151 & 4 \\ 1.7 \times & 57 & 4\end{array}$ $\begin{array}{lll}1.4 \mathrm{X} & 65 & 4\end{array}$ 
---ORIGIN TIME (HST)-- -LAT N-- --LON W-- DEPTH N RMS ERH ERZ LOC

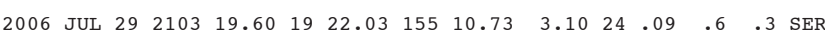
2006 JUL $292346 \begin{array}{lllllllllll}30.90 & 19 & 22.61 & 155 & 17.13 & 2.36 & 19 & .07 & .3 & .3 & \text { SSC }\end{array}$

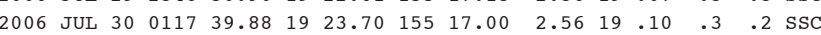
$\begin{array}{lllllllllllll}2006 & \text { JUL } 30 & 0319 & 13.65 & 19 & 23.17 & 155 & 16.97 & 3.04 & 24 & .05 & .3 & .2 \\ \text { SSC }\end{array}$

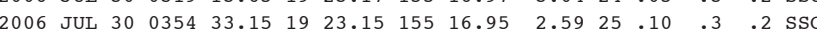

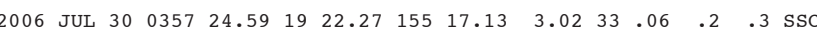

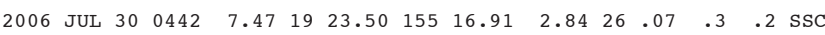
2006 JUL $30 \quad 0653 \quad 48.41 \quad 19 \begin{array}{llllllllll}19.61 & 155 & 9.04 & 7.67 & 38 & .08 & .5 & .6 & \mathrm{SF} 4\end{array}$

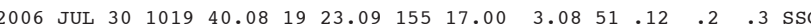

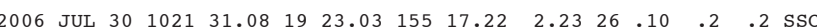

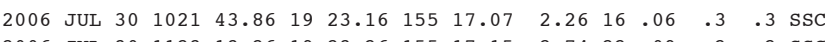

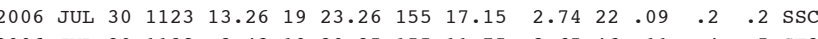

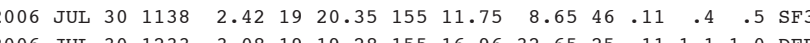
$\begin{array}{llllllllllll} & 2.506 & & \end{array}$

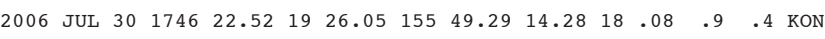

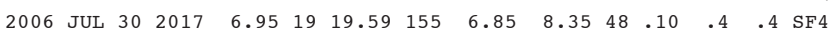

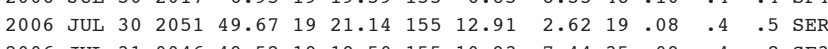

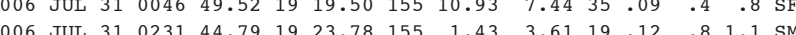

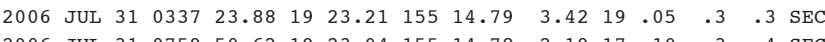

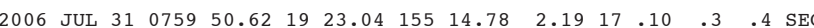

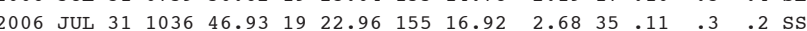

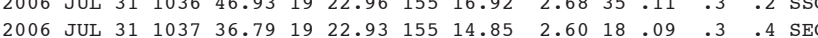

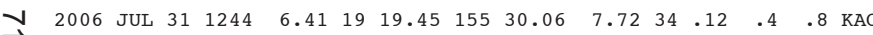

2006 JUL $\begin{array}{lllllllllllll}31 & 1350 & 28.02 & 19 & 18.90 & 155 & 30.12 & 11.88 & 27 & .09 & .4 & 1.0 & \text { LSW }\end{array}$

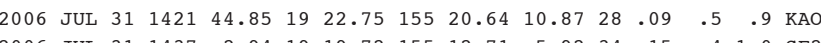

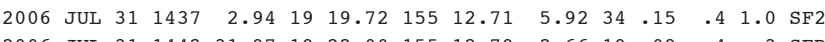

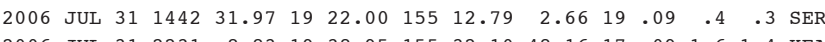

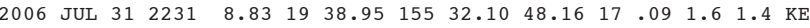

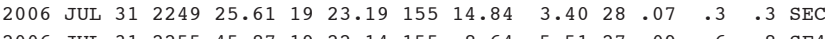
2006 JUL $\begin{array}{lllllllllllll}31 & 2255 & 45.87 & 19 & 22.14 & 155 & 8.64 & 5.51 & 27 & .09 & .6 & .8 & \text { SF4 }\end{array}$

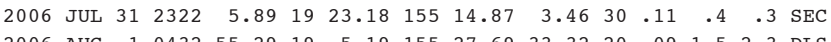

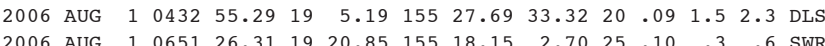

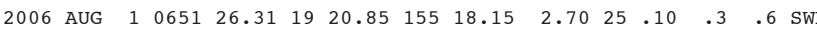

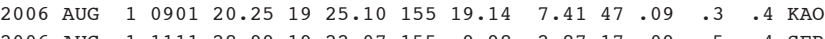

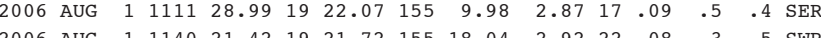

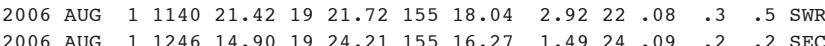

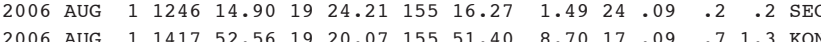

2006 AUG $111659 \begin{array}{llllllllllll}17.73 & 19 & 31.28 & 155 & 27.62 & 4.98 & 12 & .11 & .5 & 1.0 & \text { MLO }\end{array}$

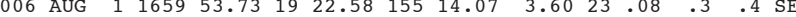

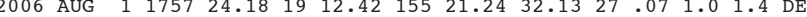

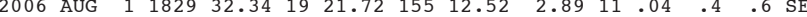

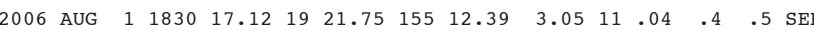

PREF AZ MIN $1.5 \times 134 \quad 2$ $\begin{array}{ll}1.3 \times & 50 \\ 1.1 \times & 70\end{array}$ $\begin{array}{lll}1.1 \times & 47 & 0 \\ 1.5 x & 47 & 0\end{array}$ $\begin{array}{lll}1.5 \times \quad 47 & 0\end{array}$

$1.5 \times \quad 80 \quad 2$ $\begin{array}{lll}1.5 \times & 57 & 0 \\ 1.2 x & 95 & 5\end{array}$ $\begin{array}{lll}1.2 \mathrm{X} & 95 & 5 \\ 2.7 \mathrm{x} & 37\end{array}$ $1.6 \times \quad 68$ $1.3 \mathrm{X} \quad 65 \quad 0$ $\begin{array}{lll}1.3 \times & 65 & 0 \\ 1.5 \times & 48 & 0\end{array}$ $\begin{array}{lll}1.6 \times & 139 & 5\end{array}$ $\begin{array}{lll}1.6 \times & 152 \quad 2\end{array}$ $\begin{array}{llll}1.4 \times & 152 & 2 \\ 2 & 214 & 9\end{array}$

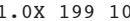
2.0X $152 \quad 5$ $1.4 \times 112 \quad 3$ $\begin{array}{rrr}1.4 \times & 112 & 3 \\ 1.3 \times & 98 & 6\end{array}$ $\begin{array}{lll}1.1 \times 162 & 5\end{array}$

$\begin{array}{rrr}1.5 \times & 73 & 2 \\ 1.2 \times & 112 & 2\end{array}$ $\begin{array}{lll}1.2 \times & 112 & 2 \\ 1.9 \times & 47 & 12\end{array}$ $\begin{array}{lll}1.9 \mathrm{X} & 47 & 1 \\ 1.4 \mathrm{x} & 71 & 2\end{array}$ $\begin{array}{lll}2.0 \mathrm{X} & 83 & 7\end{array}$

$\begin{array}{lll}1.1 \mathrm{x} & 82 \quad 7\end{array}$ $1.2 \times \quad 78 \quad 2$ $2.2 \times \quad 78 \quad 5$ $\begin{array}{lll}1.7 \times & 114 & 1\end{array}$ $1.4 \times 110 \quad 7$

$\begin{array}{lll}1.7 \mathrm{X} & 65 \quad 2\end{array}$ $2.0 \times 184 \quad 3$ $\begin{array}{lll}1.8 \times & 64 & 2\end{array}$ $\begin{array}{rrr}1.4 \times & 262 & 19 \\ 1.3 \times & 73 & 5\end{array}$

$2.0 \times \quad 46 \quad 3$ $\begin{array}{lll}1.8 \mathrm{X} & 90 & 1\end{array}$ $1.7 \times \quad 65 \quad 3$ $2.1 \times 120$ 1.2X $194 \quad 8$

1.5X 112 $\begin{array}{lll}1.6 \mathrm{X} & 89 & 2\end{array}$ $1.4 \times 18811$ $2.1 \times 120$ $1.4 \times 123 \quad 2$
- ORTGIN TIME (HST)-- -LAT N-- --LON W-- DEPTH N RMS ERH ERZ LOC YEAR MON DA HRMN SEC DEG MIN DEG MIN DM RD SEC KM KM REMKS PREF AZ MIN

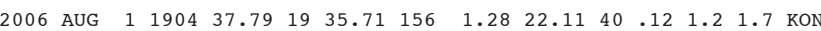

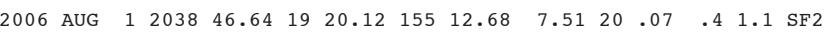

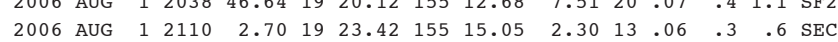

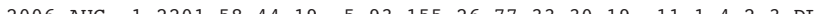

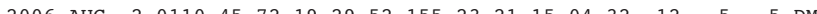

$\begin{array}{llllllllllllllll}2006 & \text { AUG } & 2 & 0228 & 57.02 & 19 & 23.36 & 155 & 16.98 & 2.88 & 18 & 08 & 3 & 3 & .2 & \text { SSC }\end{array}$ $\begin{array}{lllllllll}2006 & & \end{array}$

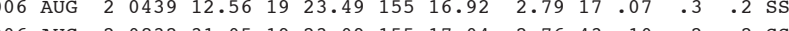

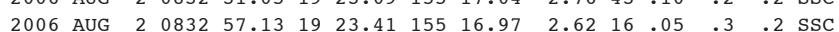

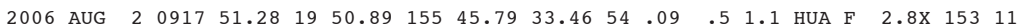

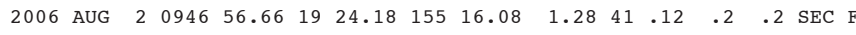

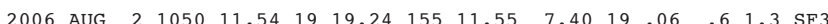
\begin{tabular}{llllllllllllll}
2006 & AUG & 2 & 1157 & 1.80 & 19 & 20.68 & 155 & 26.50 & 9.78 & 44 & .12 & 3 & .6 \\
\hline
\end{tabular}

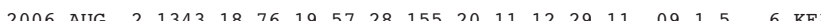
2006 AUG $2 \quad 141928.601922 .04 \quad 155 \quad 10.96 \quad 2.47 \quad 18 \quad 08 \quad 6 \quad .3$ KER

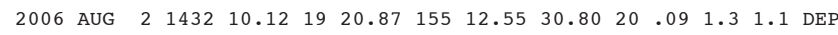

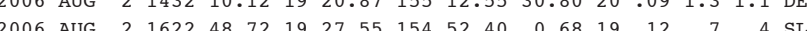

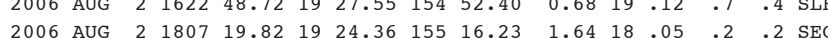
2006 AUG $2 \begin{array}{llllllllllll}1826 & 8.26 & 19 & 13.69 & 155 & 25.72 & 7.24 & 18 & .09 & .6 & .2 & \text { SEC }\end{array}$

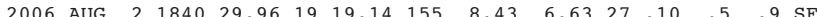

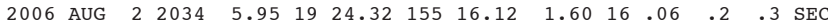

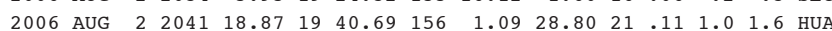

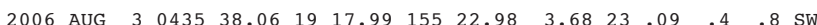

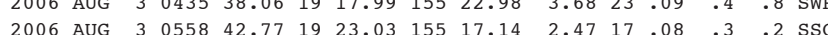
$\begin{array}{lllllllllllllll}2006 & \text { AUG } & 3 & 0807 & 3.86 & 19 & 22.98 & 155 & 14.27 & 2.64 & 19 & .07 & .3 & .4 & \text { SEC }\end{array}$

$\begin{array}{lllllllllllllll}2006 & \text { AUG } & 3 & 0851 & 18.60 & 19 & 17.40 & 155 & 23.07 & 2.79 & 27 & .09 & .5 & .7 & \text { SWR }\end{array}$

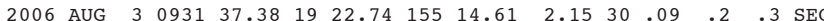

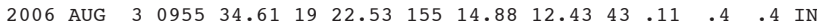
2006 AUG $3 \begin{array}{llllllllllll}0958 & 26.18 & 19 & 22.86 & 155 & 14.55 & 2.65 & 17 & .06 & .4 & .3 & \text { SEC }\end{array}$ 2006 AUG $3 \begin{array}{llllllllllllll}1033 & 46.00 & 19 & 22.65 & 155 & 14.44 & 2.58 & 21 & .13 & .3 & .3 & \text { SEC }\end{array}$

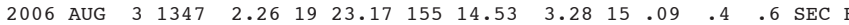
2006 AUG $3 \begin{array}{lllllllllllll}1405 & 24.75 & 19 & 45.57 & 155 & 34.10 & 15.44 & 17 & .07 & .6 & .7 & \text { KEA }\end{array}$ 2006 AUG $3 \begin{array}{llllllllllll}1514 & 14.75 & 19 & 21.28 & 155 & 30.10 & 8.21 & 45 & .10 & .3 & .7 & \text { KAO }\end{array}$ 2006 AUG $3 \begin{array}{llllllllllll}1603 & 22.65 & 19 & 23.17 & 155 & 15.12 & 1.91 & 22 & .10 & .3 & .3 & \text { SEC }\end{array}$ 2006 AUG $3 \begin{array}{llllllllllllll}1734 & 3.69 & 19 & 23.03 & 155 & 14.59 & 3.30 & 23 & .08 & .3 & .4 & \text { SEC }\end{array}$

2006 AUG $3 \begin{array}{llllllllllll}1749 & 20.38 & 19 & 35.87 & 155 & 19.30 & 13.66 & 53 & .13 & .3 & .4 & \text { KEA }\end{array}$

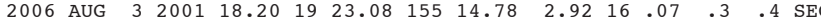
2006 AUG $3 \begin{array}{lllllllllllll}2234 & 37.29 & 19 & 22.65 & 155 & 14.95 & 3.57 & 17 & .13 & .4 & .5 & \text { SEC }\end{array}$

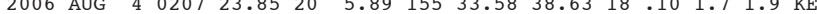
2006 AUG $4 \quad \begin{array}{lllllllllllll}0230 & 19.52 & 19 & 22.49 & 155 & 13.61 & 3.29 & 20 & .11 & .4 & .3 & \text { SER }\end{array}$

$\begin{array}{lllllllllllllll}2006 & \text { AUG } & 4 & 0645 & 29.06 & 19 & 10.44 & 155 & 31.46 & 37.11 & 48 & .08 & .6 & 1.0 & \text { DLS }\end{array}$

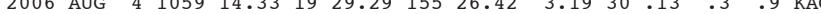

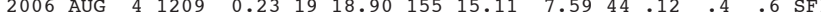
2006 AUG $4 \begin{array}{llllllllllll}1209 & 45.63 & 19 & 18.44 & 155 & 15.30 & 7.58 & 24 & .08 & .4 & 1.0 & \text { SF } 1\end{array}$ 2006 AUG $4 \quad \begin{array}{llllllllllll}1810 & 50.15 & 19 & 22.42 & 155 & 29.66 & 9.49 & 17 & .06 & .4 & .9 & \text { KAO }\end{array}$
$2.0 \times 26916$ $1.3 \times \quad 73 \quad 5$ $1.1 \times 106 \quad 2$ . $5 \times 114$ 190

$1.3 \times \quad 64 \quad 0$ $\begin{array}{lll}1.0 \times & 67 & 0\end{array}$ $\begin{array}{lll}.4 \mathrm{X} & 45 \quad 1\end{array}$ $\begin{array}{rrr}1.2 \times & 102 & 5 \\ 1.8 \times & 77 & 4\end{array}$ $1.6 \times 288 \quad 7$ $1.4 \times \quad 78 \quad 2$

$\begin{array}{lrr}1.3 \times & 78 & 3 \\ 1.8 \times & 201 & 5\end{array}$ $\begin{array}{lll}.8 \times & 201 & 5 \\ .8 \times & 130 & 1\end{array}$ $1.0 \times 153 \quad 8$ $1.3 \times 109 \quad 3$

$\begin{array}{rrr}1.7 \times & 129 & 1 \\ 1.5 \times & 225 & 19\end{array}$ $\begin{array}{lll}1.5 \times & 225 & 19\end{array}$ $\begin{array}{lll}1.6 \times & 151 & 4 \\ 1.3 \times & 127 & 1\end{array}$ $\begin{array}{rrrr}1.3 \times & 127 & 1 \\ 1.7 \times \quad 88 & 2\end{array}$

$1.5 \times 159 \quad 5$ $\begin{array}{lll}2.3 \times & 73 & 2 \\ 1.7 \times & 53 & 2\end{array}$ $\begin{array}{rrr}1.7 \mathrm{X} & 53 & 2 \\ 1.4 \mathrm{X} & 139 & 3 \\ 1.6 \mathrm{X} & 79 & 2\end{array}$ $\begin{array}{rrr}1.6 \times & 79 & 2\end{array}$

$\begin{array}{lll}1.5 \times 111 \quad 3 & 3\end{array}$ $\begin{array}{rrr}.4 \mathrm{X} & 103 & 15 \\ .8 \mathrm{X} & 63 & 5\end{array}$ $\begin{array}{lll}1.5 \mathrm{X} & 71 & 2\end{array}$ $\begin{array}{lll}1.8 \mathrm{X} & 74 & 3\end{array}$

$2.7 \times \quad 72 \quad 13$ $\begin{array}{rrr}1.1 X & 133 & 2 \\ 1.3 \mathrm{X} & 75 & 2 \\ 1.9 \mathrm{X} & 293 & 28\end{array}$ 1.9x 29328 $\begin{array}{lll}1.5 \times & 92 & 1\end{array}$

2.1X $112 \quad 7$ $1.9 \times 100 \quad 5$ $2.1 \times \quad 91 \quad 5$ $\begin{array}{rrr}1.2 \times & 74 & 4\end{array}$ 
---ORIGIN TIME (HST)-- -LAT N-- --LON W-- DEPTH N RMS ERH ERZ LOC PREF AZ MIN 75 YEAR MON DA HRMN SEC DEG MIN DEG MIN $\quad$ KM RD SEC KM KM RFMYS MAG GAP DS

2006 AUG $4 \begin{array}{lllllllllllll}1824 & 23.64 & 19 & 29.47 & 155 & 25.75 & 6.51 & 18 & .11 & .4 & 1.4 & \text { KAO }\end{array}$

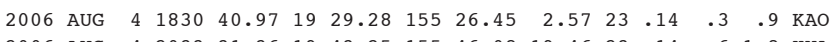
2006 AUG $4 \begin{array}{llllllllllll}2033 & 31.36 & 19 & 43.25 & 155 & 46.08 & 19.46 & 23 & .14 & .6 & 1.8 & \text { HUA }\end{array}$

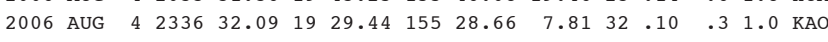

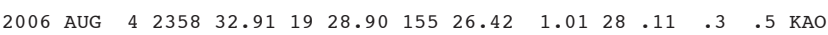

2006 AUG $5 \quad \begin{array}{lllllllllllll}0 & 033 & 31.83 & 18 & 53.57 & 155 & 54.66 & 44.27 & 52 & .10 & .9 & 1.2 & \text { DIS }\end{array}$ 2006 AUG $5 \begin{array}{llllllllllllll}5 & 0034 & 46.14 & 19 & 21.37 & 155 & 52.64 & 7.10 & 37 & .14 & .7 & .5 & \text { KON }\end{array}$

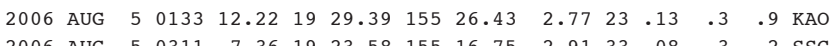
$\begin{array}{lllllllllllllll}2006 & \text { AUG } & 5 & 0311 & 7.36 & 19 & 23.58 & 155 & 16.75 & 2.91 & 33 & .08 & .3 & .2 & \text { SSC }\end{array}$

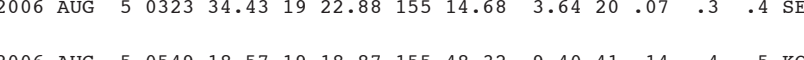
$\begin{array}{llllllllllllll}2006 & \text { AUG } & 5 & 0549 & 18.57 & 19 & 18.87 & 155 & 48.32 & 9.40 & 41 & .14 & .4 & .5 \\ \text { KON }\end{array}$

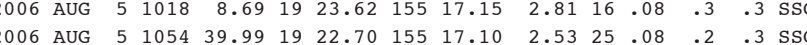
2006 AUG $5 \begin{array}{lllllllllllll}1159 & 11.16 & 19 & 12.45 & 155 & 37.23 & 6.07 & 48 & .15 & .4 & 1.0 & \text { LSW }\end{array}$ 2006 AUG $5 \begin{array}{llllllllllllll}1412 & 33.82 & 19 & 23.75 & 155 & 9.85 & 33.31 & 17 & .13 & 3.3 & 1.3 & \text { DEP }\end{array}$

2006 AUG $5 \begin{array}{llllllllllll}1759 & 20.35 & 19 & 22.35 & 155 & 14.11 & 3.09 & 19 & .10 & .3 & .4 & \mathrm{SEC}\end{array}$

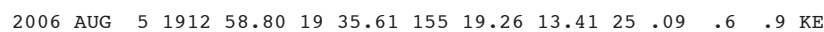

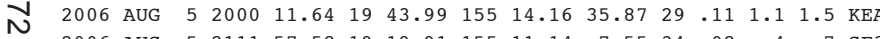

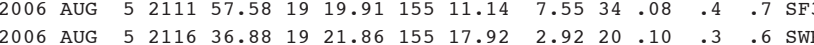

2006 AUG $5 \begin{array}{lllllllllllll}2 & 2249 & 46.37 & 19 & 21.83 & 155 & 12.52 & 2.66 & 23 & .07 & .3 & .3 & \text { SER }\end{array}$

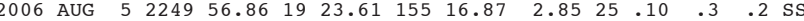

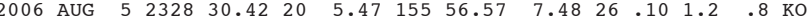
2006 AUG $6 \begin{array}{lllllllllllll}6 & 0105 & 58.79 & 20 & 1.56 & 155 & 28.48 & 34.87 & 15 & .08 & 1.2 & 1.9 & \text { KEA }\end{array}$ 2006 AUG $6 \begin{array}{lllllllllllll}6 & 0236 & 43.84 & 19 & 24.94 & 155 & 19.25 & 8.46 & 45 & .13 & .4 & .5 & \text { KAO }\end{array}$

2006 AUG $6 \begin{array}{lllllllllllll} & 0440 & 36.36 & 19 & 26.45 & 155 & 23.63 & 10.16 & 49 & .12 & .3 & .6 & \text { KAO }\end{array}$

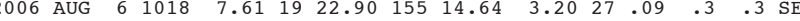

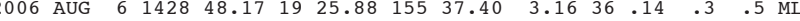

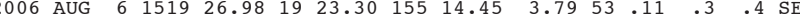
2006 AUG $6 \begin{array}{llllllllllllll}6 & 1521 & 26.26 & 19 & 23.03 & 155 & 14.44 & 3.53 & 31 & .12 & .4 & .4 & \text { SEC }\end{array}$

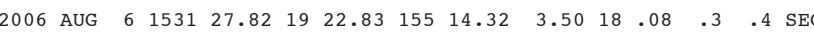

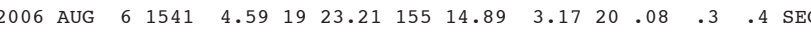
2006 AUG $6 \begin{array}{lllllllllllll}6 & 1600 & 12.69 & 19 & 15.54 & 155 & 28.86 & 10.78 & 27 & .14 & .5 & 1.1 & \text { LSW }\end{array}$

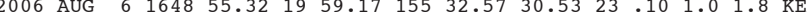

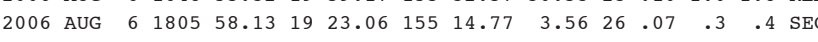

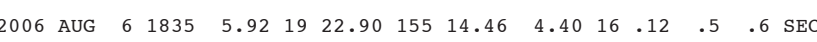

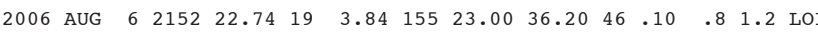
2006 AUG $6 \begin{array}{lllllllllllll}6 & 2223 & 55.72 & 19 & 2.38 & 155 & 21.29 & 38.22 & 28 & .12 & 1.3 & 1.9 & \text { LOI }\end{array}$

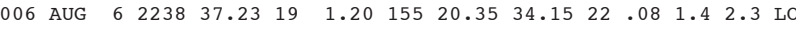

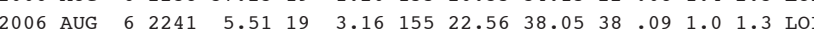

2006 AUG $6 \begin{array}{llllllllllll}2243 & 21.25 & 19 & 3.65 & 155 & 22.85 & 35.83 & 44 & .09 & .8 & 1.2 & \text { LOI }\end{array}$

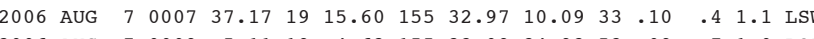

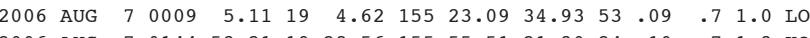
$\begin{array}{lllllllllllllll}2006 & \text { AUG } & 7 & 0144 & 53.21 & 19 & 28.56 & 155 & 55.51 & 21.30 & 24 & .10 & .7 & 1.3 & \text { KOI } \\ 2006 & \text { AUG } & 7 & 1019 & 24.71 & 19 & 21.76 & 155 & 4.55 & 8.76 & 36 & .09 & .5 & .4 & \text { SF5 }\end{array}$
$1.2 \times 106 \quad 4$ $.8 \times \quad 99 \quad 5$ $1.5 \times 1528$ $1.6 \times \quad 76 \quad 6$

$3.0 \times 29227$ $1.8 \times 16810$ $\begin{array}{lrr}1.8 \times & 85 & 5\end{array}$ $2.0 \times \quad 44 \quad 0$ $\begin{array}{lll}2.8 \times & 71 & 2\end{array}$

$1.8 \times \quad 96 \quad 9$ $1.2 \times \quad 75$ - 10 $1.6 \times \quad 50 \quad 1$ $2.4 \times \quad 84 \quad 13$ $1.9 \times 252 \quad 2$

$\begin{array}{lll}.3 \times & 90 & 2\end{array}$ $\begin{array}{lll}1.1 \times \quad 160 & 13\end{array}$ $1.5 \times 20121$ $\begin{array}{lll}1.7 \mathrm{X} & 88 & 6\end{array}$ $1.5 \times \quad 63 \quad 3$

$\begin{array}{lll}1.3 \times & 98 \quad 2\end{array}$ $1.7 \times \quad 54 \quad 0$ $1.9 \times 29239$ $1.4 \times 27020$

$\begin{array}{lll}1.7 \times & 45 \quad 2\end{array}$

$2.1 \mathrm{x} \quad 46 \quad 6$ $1.8 \times \quad 72$ $2.0 \times \quad 80 \quad 3$ $\begin{array}{lll}3.0 \times \quad 46 & 3\end{array}$ $2.2 \times \quad 78 \quad 2$ $\begin{array}{lll}1.6 \times \quad 85 & 2\end{array}$ $\begin{array}{lll}1.6 \times & 70 & 2\end{array}$ $\begin{array}{lll}1.4 \mathrm{X} & 87 \quad 2\end{array}$ $1.7 \times 25219$ $\begin{array}{lll}1.8 \mathrm{X} & 68 \quad 2\end{array}$

$\begin{array}{lll}1.4 \times & 88 & 2\end{array}$ 2.0X $206 \quad 13$ $1.3 \times 2331$ 1.6X 2501

$1.9 \times 20814$ $\begin{array}{llll}1.5 \times & 102 \quad 5\end{array}$ 2.4X 20212 $\begin{array}{lll}1.3 \times & 215 & 2 \\ 1.9 \times & 159 & 5\end{array}$
--ORIGIN TIME (HST)-- -LAT N-- --LON W-- DEPTH N RMS ERH ERZ LOC YEAR MON DA HRMN SEC DEG MIN DEG MIN KM RD SEC KM KM REMKS

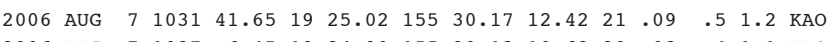
$\begin{array}{lllllllllllllll}2006 & \text { AUG } & 7 & 1037 & 6.45 & 19 & 24.90 & 155 & 30.12 & 10.68 & 20 & .08 & .4 & 1.1 & \text { KAO }\end{array}$ 2006 AUG $7 \begin{array}{lllllllllllll}1119 & 44.11 & 19 & 19.49 & 155 & 12.01 & 5.69 & 31.10 & .4 & 1.2 & \mathrm{SF} 3\end{array}$

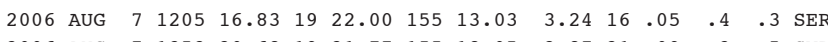

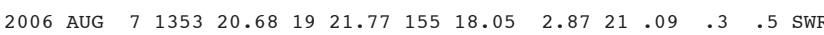

2006 AUG $7 \begin{array}{llllllllllll}1443 & 43.67 & 19 & 19.57 & 155 & 6.65 & 7.72 & 29 & .09 & .5 & .6 & \mathrm{SF} 4\end{array}$

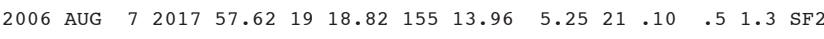
2006 AUG $7 \begin{array}{llllllllllllll}2140 & 11.62 & 19 & 25.05 & 155 & 8.60 & 42.19 & 49 & .09 & .6 & .8 & \text { DEP }\end{array}$

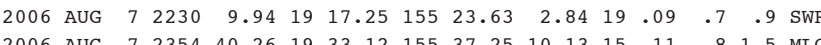
$\begin{array}{lllllllllllllll}2006 & \text { AUG } & 7 & 2354 & 40.26 & 19 & 33.12 & 155 & 37.25 & 10.13 & 15 & .11 & .8 & 1.5 & \text { MLO }\end{array}$

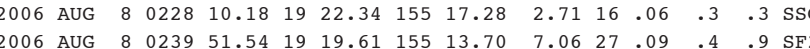

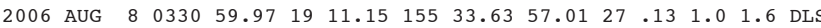
2006 AUG $8 \begin{array}{lllllllllllll}8 & 0439 & 49.87 & 19 & 8.97 & 155 & 33.69 & 51.05 & 32 & .11 & 1.0 & 1.3 & \text { DLS }\end{array}$ 2006 AUG $8 \begin{array}{lllllllllllll}0 & 0617 & 46.95 & 19 & 22.96 & 155 & 17.15 & 2.55 & 16 & .09 & .3 & .2 & \text { SSC }\end{array}$

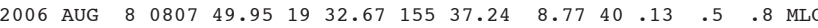
2006 AUG $8 \begin{array}{llllllllllllll}8 & 0837 & 57.96 & 19 & 17.88 & 155 & 23.01 & 3.50 & 21 & .08 & .5 & .9 & \text { SWR }\end{array}$

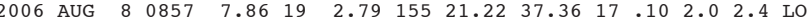

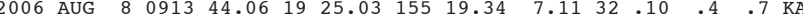
2006 AUG $8 \begin{array}{llllllllllll}1227 & 13.85 & 19 & 20.35 & 155 & 11.24 & 9.36 & 40 & .08 & .4 & .6 & \mathrm{SF} 3\end{array}$

2006 AUG $81409 \begin{array}{llllllllllll}34.83 & 19 & 19.03 & 155 & 18.65 & 8.93 & 31 & .10 & .5 & .8 & \text { SWR }\end{array}$

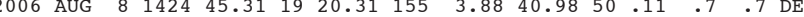

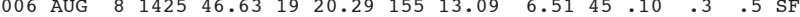
2006 AUG $881843 \quad 18.0419 \begin{array}{lllllllllll}48.62 & 155 & 36.61 & 13.61 & 19 & .10 & 1.3 & .5 & \text { KEA }\end{array}$ 2006 AUG $8 \begin{array}{lllllllllllll}2306 & 39.70 & 19 & 17.09 & 155 & 23.61 & 2.51 & 21 & .13 & .6 & .9 & \text { SWR }\end{array}$

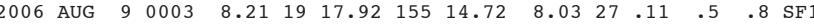

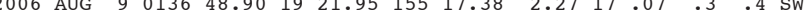

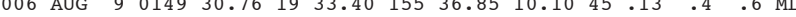

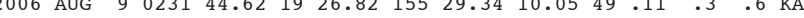
$\begin{array}{lllllllllllllll}2006 & \text { AUG } & 9 & 0233 & 48.88 & 19 & 19.74 & 155 & 24.02 & 31.97 & 46 & .10 & .5 & .8 & \text { DEP }\end{array}$

2006 AUG $90454 \quad 39.80 \quad 18 \quad 55.30 \quad 155 \quad 32.35 \quad 36.29 \quad 24 \quad .09 \quad 1.3 \quad 2.4$ DLS

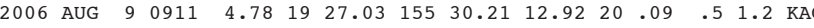

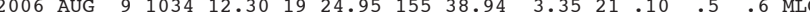

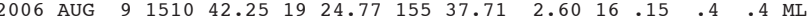
2006 AUG $9 \begin{array}{llllllllllll}1617 & 14.36 & 19 & 5.01 & 155 & 29.61 & 38.87 & 24 & .11 & 1.2 & 2.0 & \text { DLS }\end{array}$

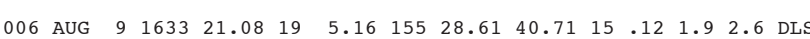

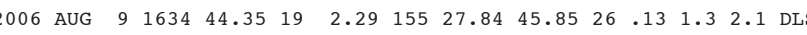

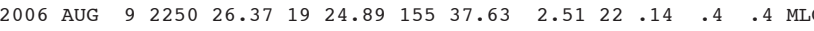
$\begin{array}{lllllllllll} & & \end{array}$

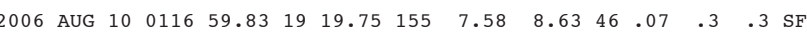

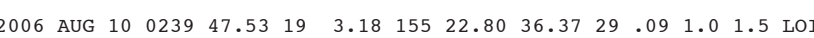

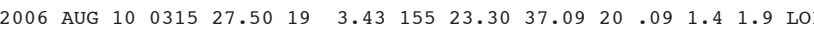

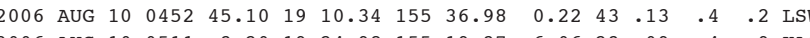

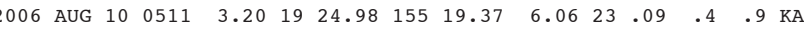

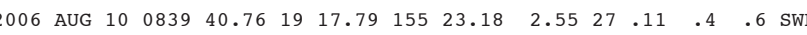

PREF AZ MIN 76 $\begin{array}{rrr}.3 \times & 70 & 5 \\ .9 \times & 71 & 6\end{array}$ $\begin{array}{rrr}.9 \mathrm{x} & 71 & 6 \\ 1.4 \mathrm{x} & 91 & 5\end{array}$ $1.5 \times 110 \quad 1$ $1.5 \times \quad 64 \quad 3$

$1.4 \times \quad 157 \quad 5$ $.9 \times \quad 95 \quad 3$ $2.0 \times \quad 59 \quad 4$ $\begin{array}{lll}.4 \times & 205 & 5 \\ 10 & 140 & 7\end{array}$ $1.2 \times 110 \quad 2$ $1.4 \times \quad 76 \quad 5$ $1.9 \times 180 \quad 10$ $\begin{array}{lll}1.7 \times \quad 128 & 10\end{array}$ $1.5 \mathrm{X} \quad 92 \quad 1$

$\begin{array}{lll}1.8 \mathrm{X} & 98 \quad 7\end{array}$ $\begin{array}{lll}.9 \times & 153 \quad 4\end{array}$ $1.4 \times 29728$ $1.8 \times 115 \quad 3$ $1.6 \times \quad 80 \quad 5$

$1.2 \times \quad 98 \quad 2$ 2.0X $185 \quad 7$ $\begin{array}{lll}1.5 \mathrm{X} & 66 & 4\end{array}$ $1.1 \times 26526$ 1.3X $207 \quad 6$

$1.1 \times 133 \quad 3$ $\begin{array}{lll}1.2 \times 114 & 3\end{array}$ $1.9 \times \quad 95 \quad 8$ $1.7 \times \quad 479$ $\begin{array}{lll}.7 \times & 912\end{array}$ $\begin{array}{lll}1.6 \times & 289 & 27\end{array}$ $\begin{array}{lll}1.0 \times & 73 \quad 9\end{array}$ $1.6 \times 114 \quad 2$ $\begin{array}{lll}1.3 \times & 93 & 1\end{array}$ $2.1 \times 30018$ $\begin{array}{lll}1.8 \times & 287 \quad 13\end{array}$ $\begin{array}{lll}1.3 \mathrm{X} & 73 & 1 \\ 1.4 \mathrm{X} & 74 & 2\end{array}$ $\begin{array}{lll}1.6 \times 21015 & 15 \\ 1.5 \times & 239 & 14\end{array}$ $\begin{array}{lll}1.5 \times & 239 & 14 \\ 1.7 \times & 101 & 15\end{array}$ $1.7 \times 101 \quad 15$ $\begin{array}{lll}1.1 \times & 112 & 2 \\ 1.4 \times & 115 & 4\end{array}$ $1.4 \times 1154$ 
---ORIGIN TIME (HST)-- -LAT N-- --LON W-- DEPTH N RMS ERH ERZ LOC YEAR MON DA HRMN SEC DEG MIN DEG MIN

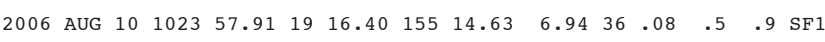

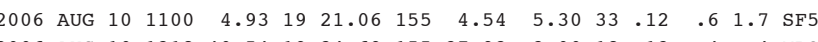

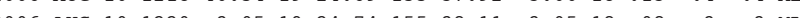

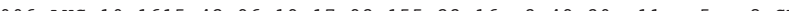

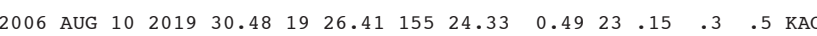

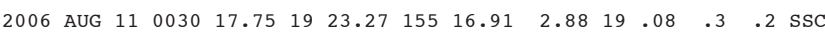

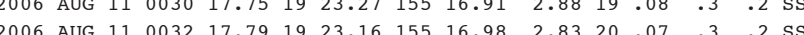

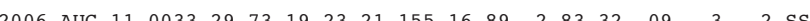

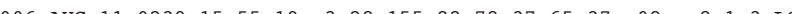

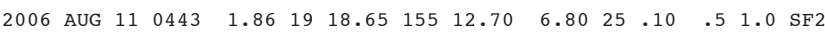

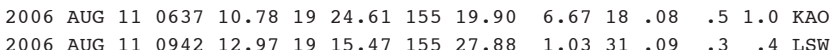
$\begin{array}{lllllllllll}2006 & \end{array}$

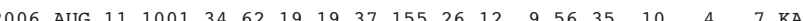

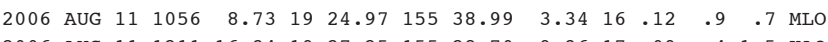

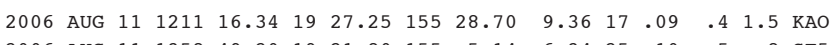

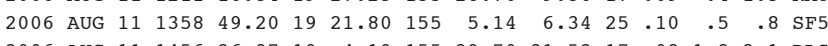

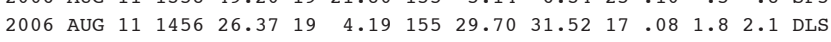

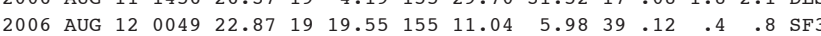

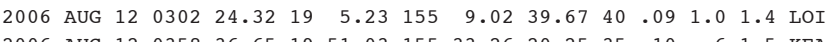

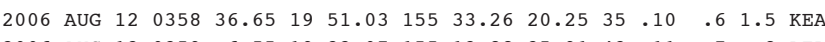

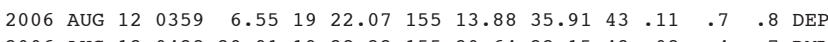
2006 AUG $120428 \begin{array}{lllllllllll}29.91 & 19 & 28.22 & 155 & 30.64 & 22.15 & 43 & 08 & .4 & .7 & \text { DM }\end{array}$

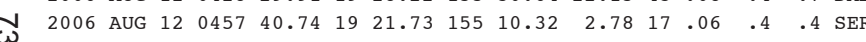

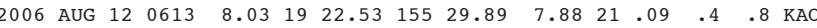

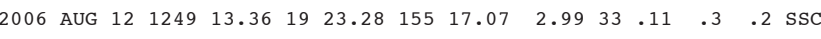

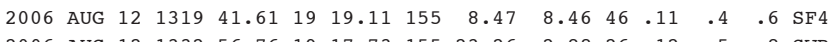

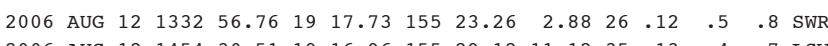
2006 AUG $12 \quad \begin{array}{llllllllllll}1454 & 30.51 & 19 & 16.96 & 155 & 29.12 & 11.12 & 35 & .13 & .4 & .7 & \text { LSW }\end{array}$

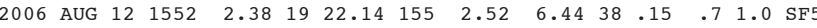

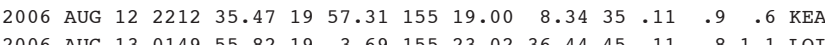

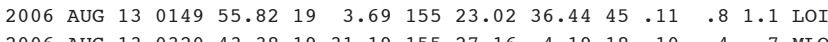

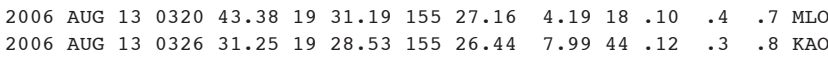

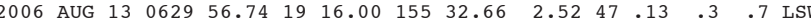

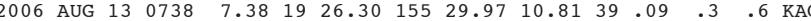

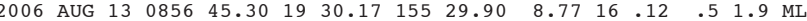

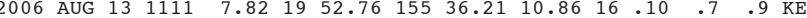
2006 AUG $13 \quad 142138.53 \quad 19 \quad 51.03 \quad 155 \quad 54.75 \quad 35.03 \quad 19.11 \quad 2.0 \quad 3.4$ HUA

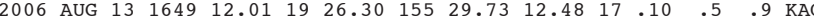

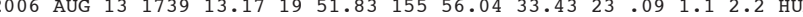

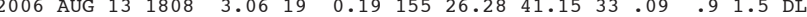

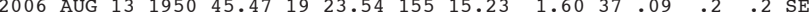

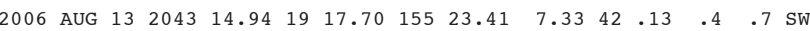

PREF AZ MIN MAG GAP DS $1.3 \times 184 \quad 2$ $\begin{array}{rrr}1.4 \times 169 & 6 \\ 1.5 \times \quad 96 & 1\end{array}$ $1.5 \times \quad 96$ $1.3 \times 100$ $1.2 \times \quad 82 \quad 7$ $\begin{array}{lll}1.4 \times & 66 & 0\end{array}$ $\begin{array}{lll}1.6 x & 71 & 0 \\ 1.8 x & 67 & 0\end{array}$ $1.6 \times 210 \quad 15$

$1.3 \mathrm{U} \quad 99 \quad 3$ $1.0 \times 100$ $1.4 \times \quad 96 \quad 4$ $1.5 \times 895$

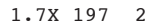
$\begin{array}{rrr}1.3 \times & 73 & 9 \\ 1.6 x & 150 & 5\end{array}$ $\begin{array}{lll}1.5 \times 269 & 20\end{array}$ $\begin{array}{lll}1.5 \times 269 & 20 \\ 1.7 \times \quad 97 & 6\end{array}$

1.8X 22922 $1.7 \times \quad 115 \quad 11$ $2.0 \times \quad 62$ $\begin{array}{lll}2.0 \times & 62 & 2 \\ 1.8 x & 87 & 7\end{array}$ $\begin{array}{lll}1.5 \times & 80 & 2\end{array}$ $1.4 \times \quad 58 \quad 4$ $\begin{array}{lll}1.8 \mathrm{X} & 47 & 0\end{array}$ $1.9 \times 108 \quad 3$ $1.4 \times 154 \quad 5$ $\begin{array}{lrr}1.5 \mathrm{X} & 81 & 4\end{array}$

1.4X $176 \quad 5$ $1.6 \times 250 \quad 24$ $1.8 \times 207 \quad 14$ $1.3 \times 1112$ $\begin{array}{lll}1.8 \mathrm{X} & 55 & 6\end{array}$

$1.9 \times \quad 59 \quad 15$ $\begin{array}{lll}1.4 \times & 62 & 8\end{array}$

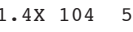
$1.0 \times 197 \quad 6$ $1.7 \times 32441$

$1.4 \times \quad 92 \quad 8$ $1.6 \times 214 \quad 33$ $1.7 \times 22017$ $\begin{array}{lll}1.7 \times & 81 & 2\end{array}$ $1.8 \times 114 \quad 5$
--ORTGIN TIME (HST)-- -LAT N-- --LON W-- DEPTH N RMS ERH ERZ LOC YEAR MON DA HRMN SEC DEG MIN DEG MIN KM RD SEC KM KM REMRS

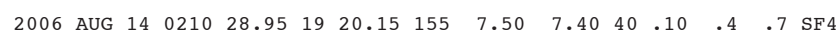

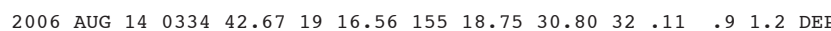
2006 AUG $14 \quad \begin{array}{llllllllllll}0438 & 22.30 & 19 & 11.39 & 155 & 29.02 & 5.88 & 46 & .13 & .4 & .7 & \text { LSW }\end{array}$

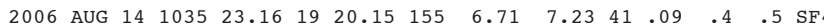
2006 AUG $14 \quad 1900 \quad 41.501921 .59 \begin{array}{llllllllll}155 & 10.48 & 2.03 & 15 & .06 & .4 & .5 & \text { SER }\end{array}$

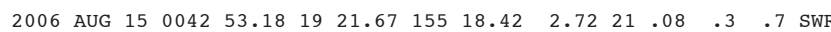

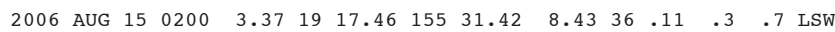

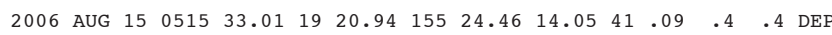

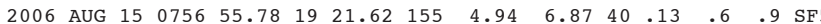

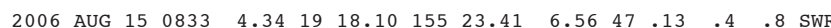

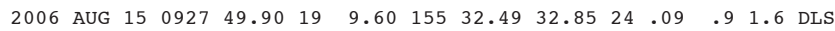

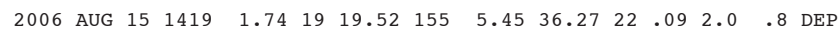

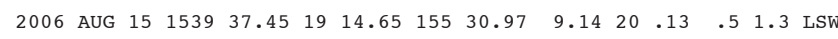
$\begin{array}{lllllllllll}2006 & \end{array}$

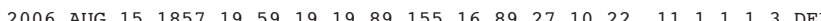

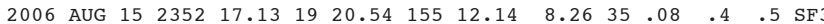

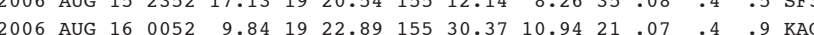
2006 AUG $\begin{array}{llllllllllllll}16 & 0151 & 28.59 & 19 & 23.78 & 155 & 15.35 & 1.48 & 36 & .10 & .4 & .9 & \text { KAO }\end{array}$

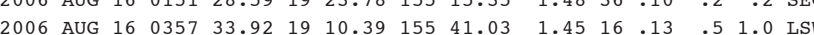

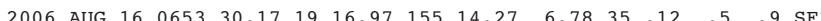

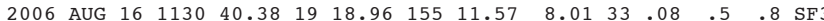
$\begin{array}{llllllllllllll}2006 & \text { AUG } & 16 & 1905 & 28.71 & 19 & 22.27 & 155 & 28.29 & 3.68 & 18 & .11 & 4 & 4 \\ 2006 & \text { KAO }\end{array}$

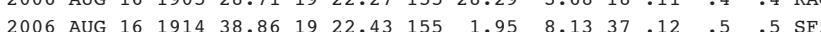

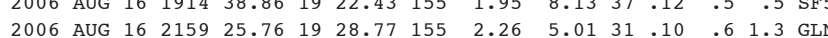

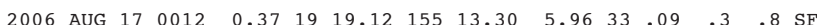

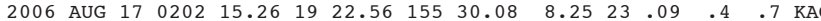
2006 AUG $17 \quad \begin{array}{llllllllllll}0206 & 49.48 & 19 & 23.49 & 15 & 15.50 & 1.36 & 30 & .10 & .2 & .2 & \text { SEC }\end{array}$ 2006 AUG $17 \begin{array}{lllllllllllll}0220 & 42.41 & 19 & 10.38 & 155 & 32.84 & 37.14 & 33 & .12 & .7 & 1.9 & \text { DLS T }\end{array}$

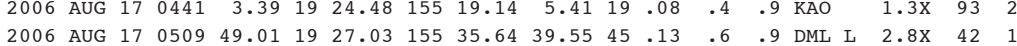

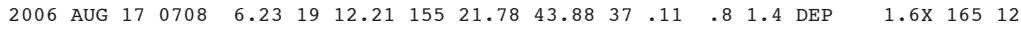

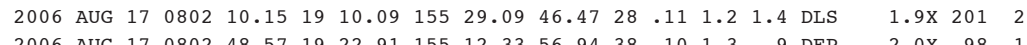

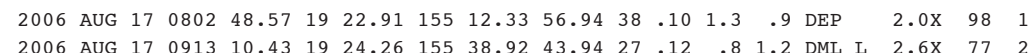

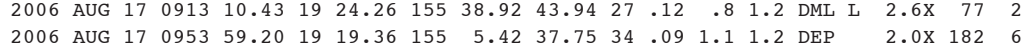

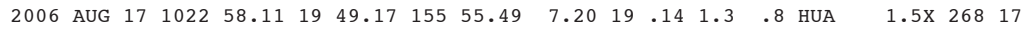

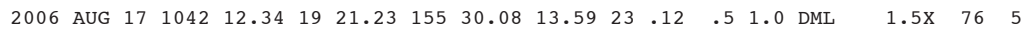

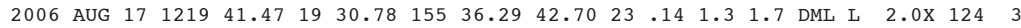
2006 AUG $17 \quad 1226 \begin{array}{lllllllllllllll}38.09 & 19 & 23.05 & 155 & 16.72 & 1.01 & 31 & .13 & .2 & .1 & \text { SSC L } & 1.3 \times & 64 & 1\end{array}$

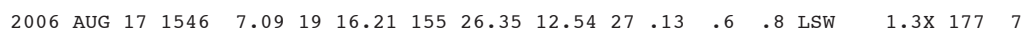

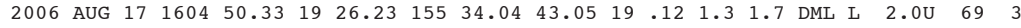
2006 AUG $17 \quad \begin{array}{lllllllllllllllllll}1918 & 42.81 & 19 & 28.95 & 155 & 34.91 & 53.43 & 20 & .12 & 1.7 & 1.2 & \text { DML L } & 2.0 \times & 107 & 1\end{array}$

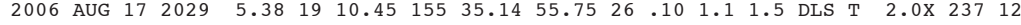

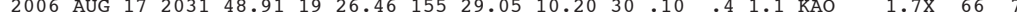

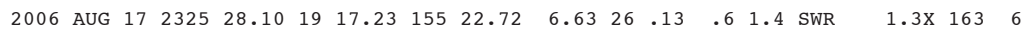


---ORIGIN TIME (HST)-- -LAT N-- --LON W-- DEPTH N RMS ERH ERZ LOC PREF AZ MIN 79 YEAR MON DA HRMN SEC DEG MIN DEG MIN KM RD SEC KM KM REMKS MAG GAP DS

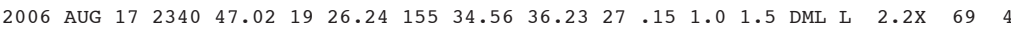

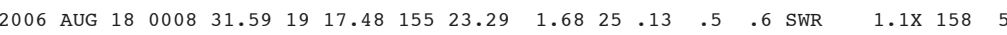

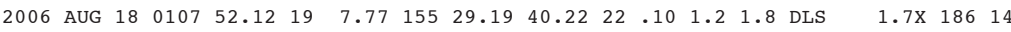

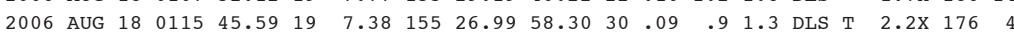

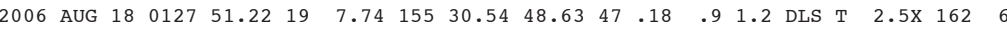

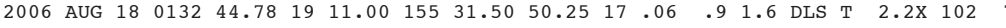

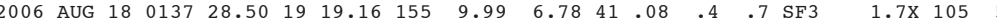

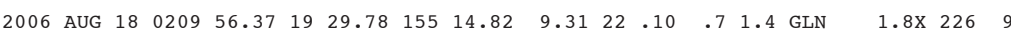

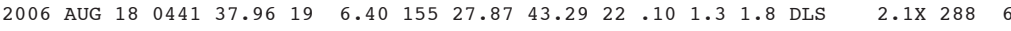

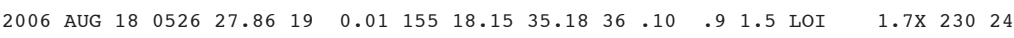

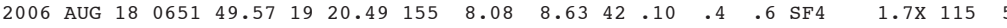
2006 AUG $18 \quad 0744 \begin{array}{llllllllllllll}53.94 & 19 & 26.19 & 155 & 35.50 & 43.07 & 25 & .19 & 1.5 & 2.0 & \text { DML L } & 2.0 X & 68\end{array}$

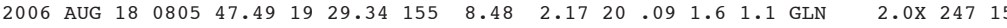

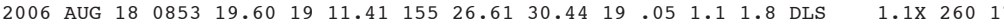

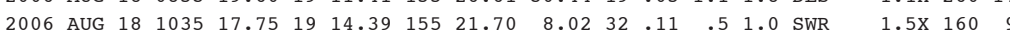

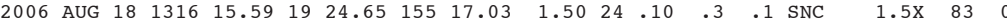

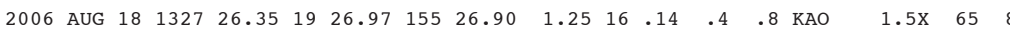

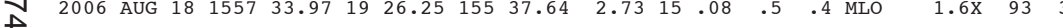

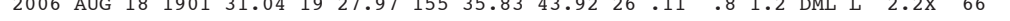

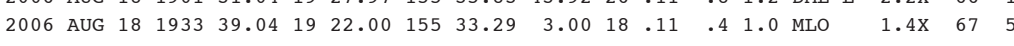

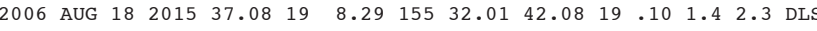

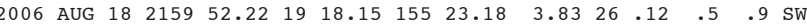

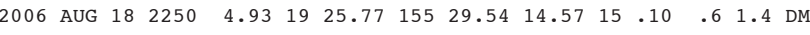
2006 AUG $19025023.102014 .93 \quad 155 \quad 40.27 \quad 33.92 \quad 23 \quad .11 \quad 1.6 \quad 1.5$ KO

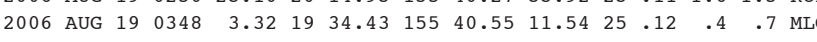

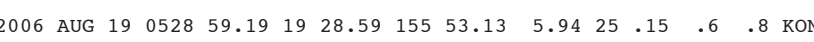

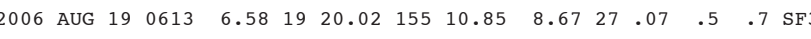

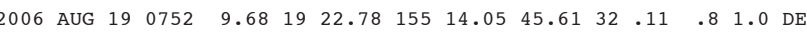

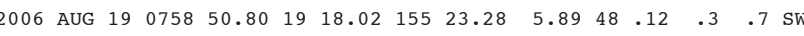

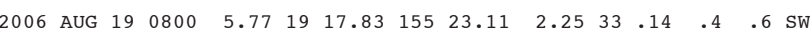

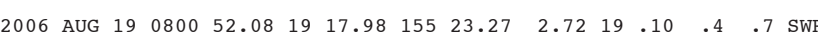

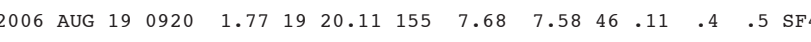

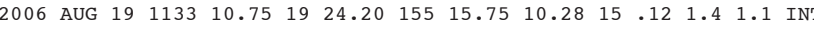

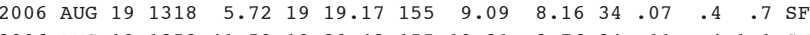
2006 AUG $19 \begin{array}{lllllllllllllll}1853 & 41.59 & 19 & 20.43 & 155 & 19.21 & 3.76 & 24 & .11 & .4 & 1.1 & \text { SWR }\end{array}$

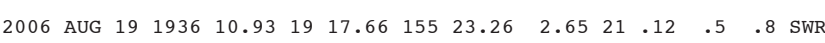

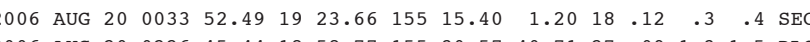

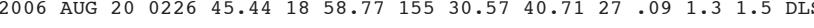

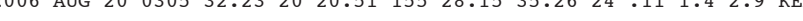

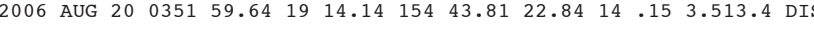

2006 AUG $20 \quad 0402 \quad 28.712011 .83 \quad 155 \quad 39.72 \quad 30.79 \quad 35.12 \quad 1.1 \quad 2.1$ кон

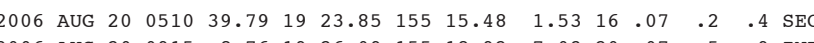

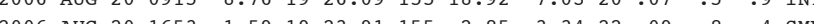

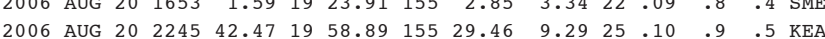

---ORIGIN TIME (HST)-- -LAT N-- --LON W-- DEPTH N RMS ERH ERZ LOC YEAR MON DA HRMN SEC DEG MIN DEG MIN $\quad$ KM RD SEC KM KM REM

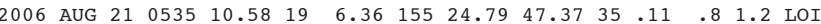

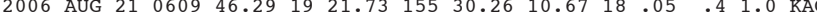

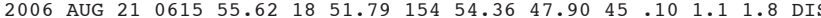

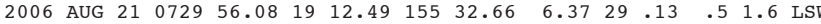

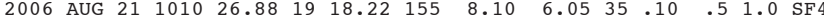

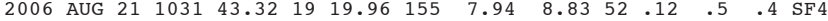
2006 AUG $21 \quad 1449 \begin{array}{lllllllllll}37.73 & 19 & 28.52 & 155 & 14.70 & 10.77 & 42 & .13 & .3 & .7 & \text { GLN }\end{array}$ 2006 AUG $21 \quad \begin{array}{llllllllllllllll}1516 & 6.60 & 19 & 25.69 & 155 & 18.88 & 5.28 & 33 & .08 & .4 & .7 & .7 & \text { IN }\end{array}$

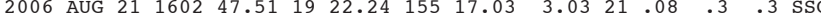

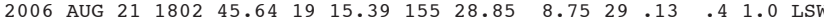

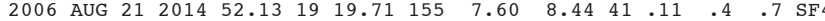

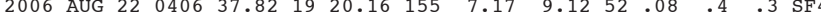

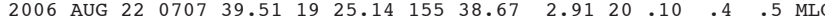

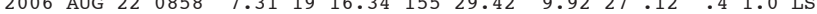

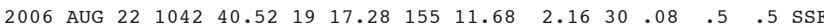

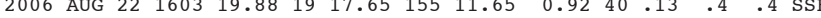

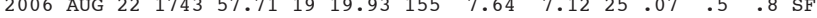

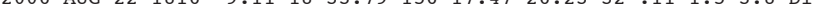

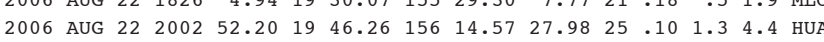

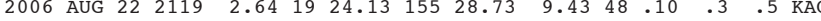

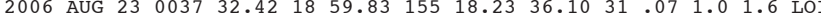

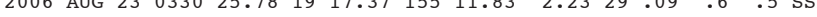

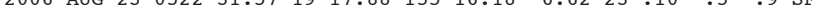

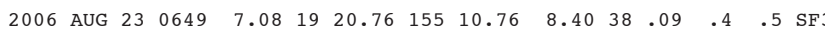

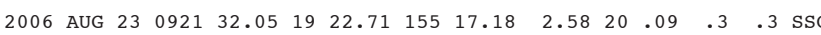
2006 AUG $23 \quad 1117 \quad 46.0920 \quad 9.61 \quad 155 \quad 41.13 \quad 28.12 \quad 41.12 \quad 1.0 \quad 1.9$ кон

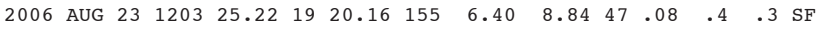

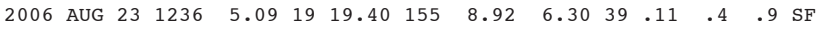

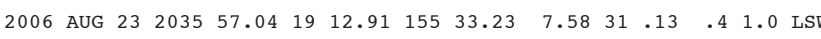
$\begin{array}{llllllllllllll}2006 & \text { AUG } 23 & 2102 & 41.09 & 19 & 17.61 & 155 & 22.83 & 3.23 & 29 & .11 & .5 & .9 & \text { SWR }\end{array}$

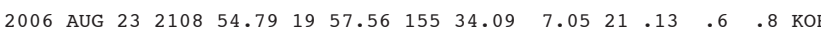

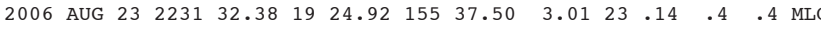

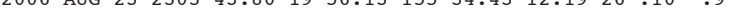

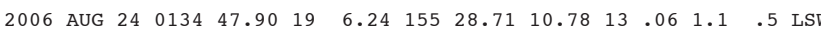

2006 AUG $24 \quad \begin{array}{lllllllllllll}0355 & 23.23 & 19 & 19.67 & 155 & 8.79 & 5.55 & 19 & .10 & .6 & 1.7 & \mathrm{SF} 4\end{array}$

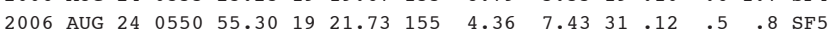

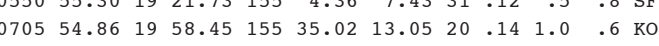

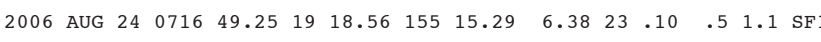

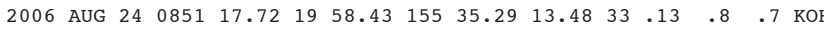
$\begin{array}{llllllllllllll}2006 & \text { AUG } 24 & 1045 & 51.14 & 20 & 3.24 & 155 & 35.75 & 12.33 & 20 & .09 & 1.3 & .9 & \text { кон }\end{array}$

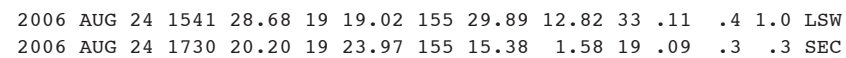

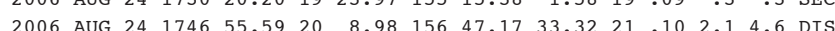

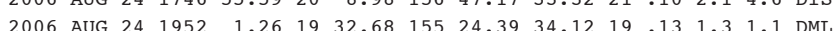

$1.8 \times \quad 224 \quad 8$ $1.4 \mathrm{U} \quad 70 \quad 5$ $2.1 \times 28153$ $\begin{array}{rrr}1.4 \times & 86 & 7 \\ 1.4 \times & 118 & 2\end{array}$

$.7 \mathrm{U} 122 \quad 5$ $1.6 \times \quad 54 \quad 7$ $1.6 \times 144 \quad 2$ $\begin{array}{rrr}1.53 & 105 & 2 \\ 1.3 \times & 87 & 2\end{array}$

$1.4 \times \quad 131 \quad 4$ $2.3 \times 137 \quad 5$ $1.1 \times 109 \quad 2$ $1.3 \mathrm{X} \quad 80 \quad 3$ $1.4 \times 156 \quad 3$

1.9X $149 \quad 3$ $1.2 \times 129 \quad 5$ $2.4 \times \quad 330 \quad 80$

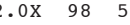
$1.8 \times 29546$

$\begin{array}{lll}1.8 \mathrm{X} & 30 & 4\end{array}$

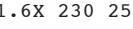
$\begin{array}{lll}1.2 \times 168 & 3\end{array}$ $\begin{array}{lll}1.2 \times & 165 & 4\end{array}$ $\begin{array}{lll}1.7 \mathrm{X} & 74 & 4\end{array}$

$\begin{array}{lll}1.3 \times & 50 & 1\end{array}$ $2.2 \times \quad 235 \quad 10$ $\begin{array}{lll}2.1 \times & 153 & 6\end{array}$ $\begin{array}{lll}1.3 \times & 96 & 4\end{array}$ $\begin{array}{lll}1.5 \times & 83 & 7\end{array}$ $1.2 \times 157 \quad 5$ $\begin{array}{lll}1.6 \times & 157 & 15\end{array}$ $\begin{array}{lll}1.6 \times & 72 & 1\end{array}$ $\begin{array}{llll}1.6 \times & 233 \quad 12\end{array}$ $1.10100 \quad 5$ $1.2 \times 161 \quad 5$ $\begin{array}{lll}1.8 \times 160 & 15\end{array}$ $\begin{array}{lll}1.0 \times \quad 129 \quad 4 & 4\end{array}$

$1.3 \times 27123$ $\begin{array}{lll}1.5 \times & 71 & 7\end{array}$ $\begin{array}{llll}1.4 \times & 109 \quad 2\end{array}$ $1.7 \times \quad 177 \quad 6$ 
---ORIGIN TIME (HST)-- -LAT N-- --LON W-- DEPTH N RMS ERH ERZ LOC YEAR MON DA HRMN SEC DEG MIN DEG MIN KM RD SEC KM KM REMKS

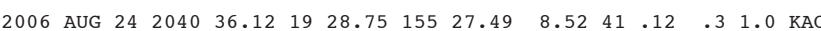

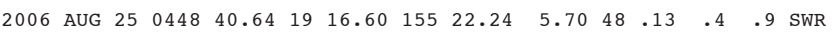

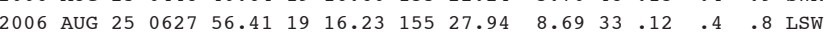
$\begin{array}{lllllllllllll} & 006 \text { AUG } 25 & 0703 & 46.08 & 19 & 24.97 & 155 & 19.13 & 6.71 & 36 & .11 & .4 & .7 \\ \text { KAO }\end{array}$ 2006 AUG $25 \quad \begin{array}{llllllllllll}0 & 39.02 & 19 & 20.10 & 154 & 59.73 & 5.91 & 22 & .13 & .9 & 1.4 & \text { LER }\end{array}$

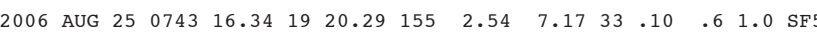

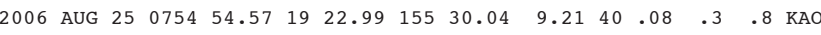

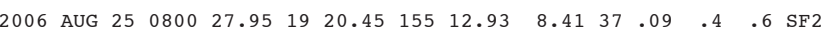

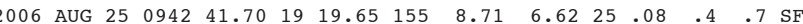

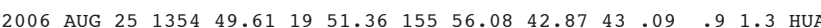

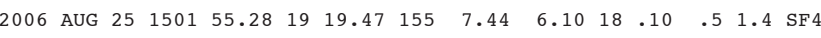

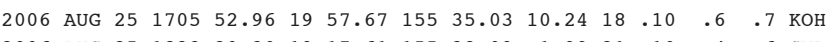

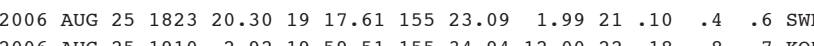

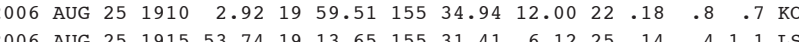

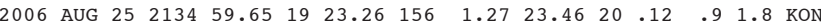

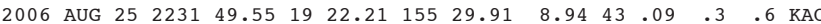

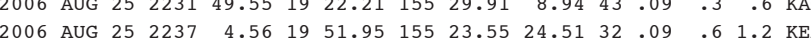
2006 AUG 26 \begin{tabular}{lllllllllllll}
0 & 0108 & 32.42 & 19 & 58.39 & 155 & 35.44 & 13.06 & 43 & .11 & .6 & .5 & \\
\hline
\end{tabular}

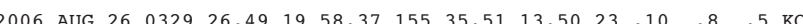

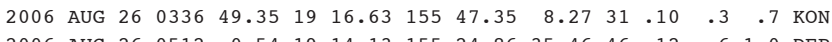

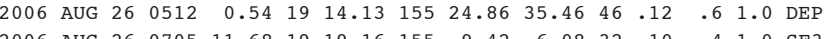

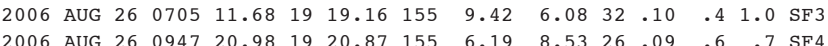

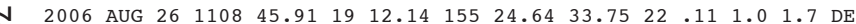

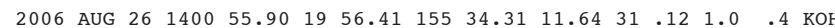
2006 AUG $\begin{array}{lllllllllllll}26 & 1519 & 9.19 & 19 & 26.87 & 155 & 29.60 & 12.55 & 21 & .12 & .5 & 1.6 & \text { KAO }\end{array}$

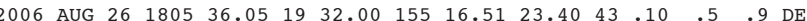

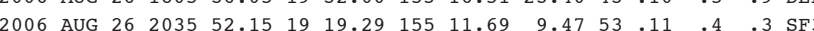

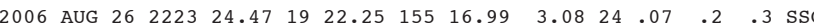

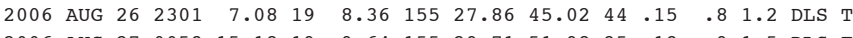

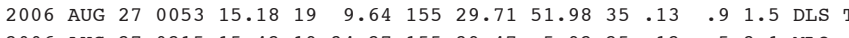

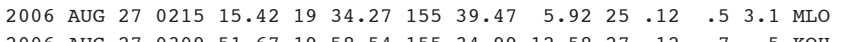

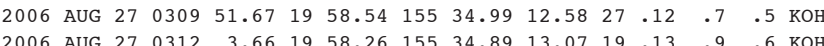
2006 AUG $27 \quad \begin{array}{llllllllllllll}0312 & 3.66 & 19 & 58.26 & 155 & 34.89 & 13.07 & 19 & .13 & .9 & .6 & \text { KOH }\end{array}$

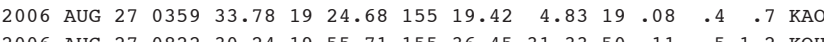

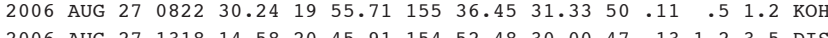

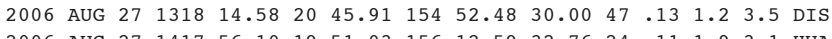

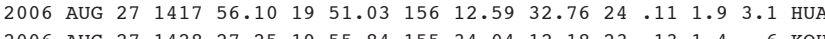

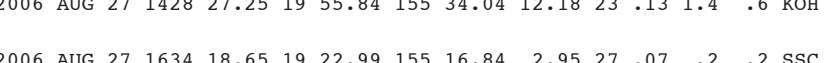

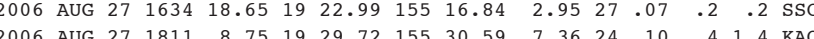

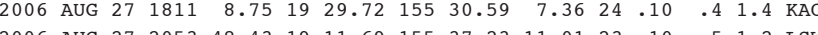

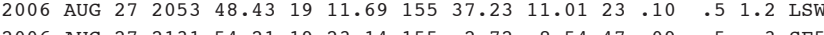

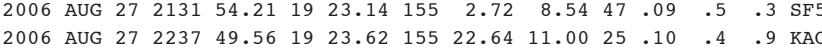

PREF AZ MIN 81 MAG GAP DS $1.4 \times \quad 73 \quad 6$ $1.5 \times 131$ $\begin{array}{lll}1.3 \times & 93 & 4 \\ 1.7 \times & 75 & 3\end{array}$ $1.3 \times 228$ (1. $\begin{array}{rrr}1.5 \times & 198 & 8 \\ 1.4 \times & 57 & 4\end{array}$ $1.4 \times \quad 57 \quad 4$ $1.3 \times 5 \times 103 \quad 4$ $\begin{array}{llll}1.5 \times & 103 & 4\end{array}$

$.7 \times 138 \quad 4$ $2.0 \times 154 \quad 14$ $.9 \times 157$ $\begin{array}{llll}1.5 \times & 167 & 17\end{array}$ $1.4 \times \quad 69 \quad 3$ $1.4 \times 25616$ $1.6 \times \quad 60$ $\begin{array}{lll}1.6 \times & 60 & 4 \\ 1.1 \times & 116 & 6\end{array}$ $2.3 \times 157 \quad 15$

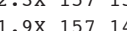

$\begin{array}{rrr}1.7 \times & 94 & 9 \\ 1.7 \times & 134 & 9 \\ 1.3 \times & 99 & 4\end{array}$ $\begin{array}{lll}1.7 \times & 134 & 9\end{array}$ $\begin{array}{rrr}1.3 \times & 99 & 4 \\ 1.2 \times & 148 & 5\end{array}$ $1.3 \times 165 \quad 7$

1.8X 23613 $1.2 \times \quad 83$ $\begin{array}{lll}1.4 \mathrm{X} & 64 & 12\end{array}$ $\begin{array}{lll}2.7 x & 99 & 5\end{array}$ 1.6X $62 \quad 2$

$2.3 \times 168 \quad 2$ $\begin{array}{lll}2.2 \times & 130 & 3 \\ 1.4 \times & 66 & 11\end{array}$ $\begin{array}{rrr}1.4 \times & 66 & 11 \\ 1.9 \times & 160 & 15\end{array}$ $\begin{array}{lll}1.7 \times & 158 & 15\end{array}$

$1.4 \mathrm{U} 102 \quad 2$ $2.0 \times 137 \quad 9$ $3.0 \times 308109$ $1.5 \times 31843$ 1.8X $228 \quad 12$
--ORTGIN TIME (HST)-- -LAT N-- --LON W-- DEPTH N RMS ERH ERZ LOC YEAR MON DA HRMN SEC DEG MIN DEG MIN KM RD SEC KM KM REMKS

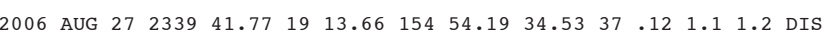

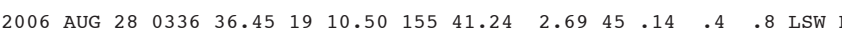

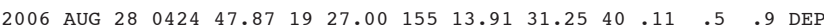

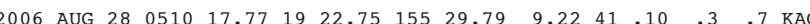
2006 AUG $28 \quad \begin{array}{lllllllllllll}0824 & 28.16 & 19 & 16.65 & 155 & 20.53 & 29.78 & 23 & .10 & .8 & 1.5 & \text { DEP }\end{array}$

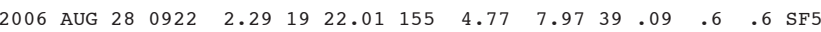

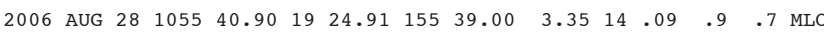
$\begin{array}{llllllllllllll}2006 & \text { AUG } 28 & 1122 & 44.43 & 19 & 18.23 & 155 & 13.23 & 5.62 & 24 & .10 & .4 & 1.1 & \mathrm{SF} 2\end{array}$

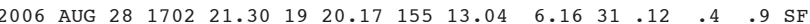

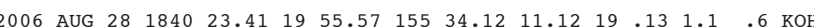

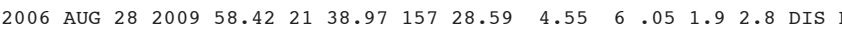

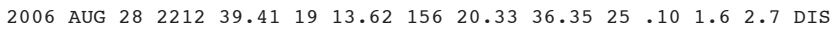

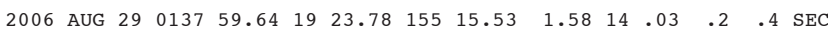

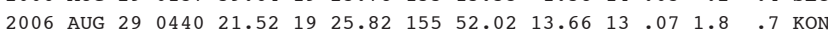

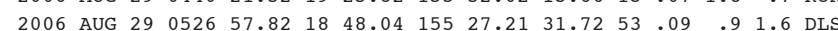

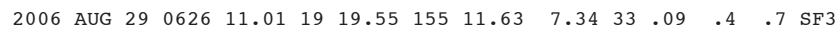

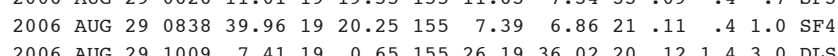

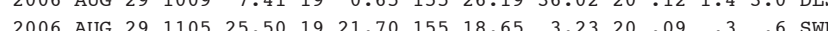
$\begin{array}{lllllllllllll}2006 & .0 & .6 & \text { SWR }\end{array}$

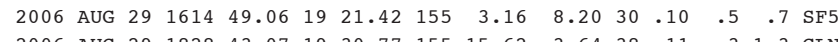

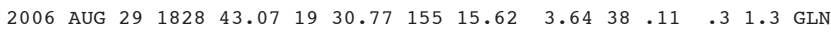
$\begin{array}{llllllllllllll}2006 & \text { AUG } 29 & 1848 & 42.75 & 19 & 22.07 & 155 & 2.25 & 9.73 & 29 & .07 & .8 & .6 & \text { SF5 }\end{array}$

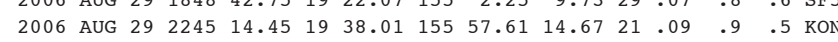

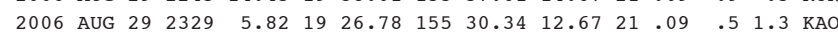

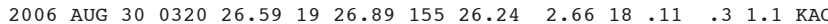

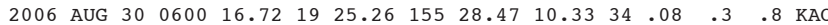

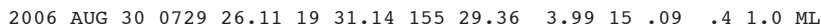

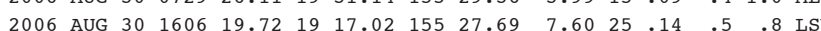

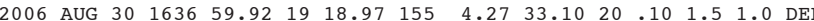

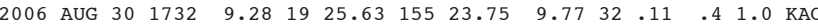

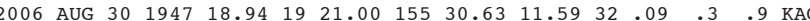

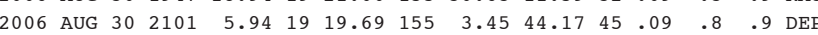

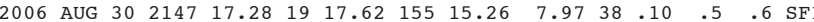

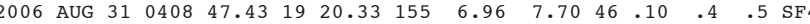

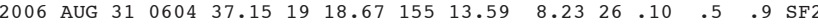

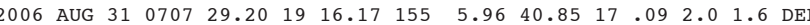

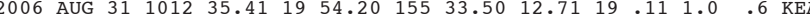

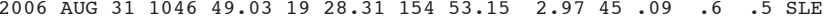

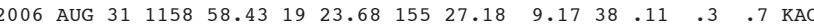

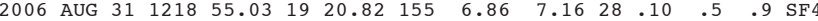

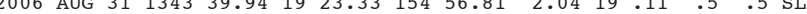

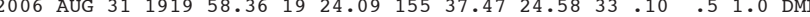

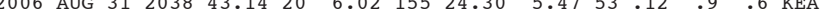

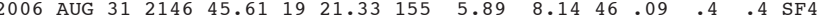

PREF AZ MIN 82 PRAB

$1.6 \times 27723$ $\begin{array}{lll}1.8 \times & 81 & 9\end{array}$ $\begin{array}{lll}1.6 x & 51 & 4 \\ 1.4 x & 70 & 4\end{array}$ $1.7 \times 139$ 1.8X $178 \quad 4$ $\begin{array}{lll}.3 \times 197 \quad 2 \\ .0 \times & 92 & 2\end{array}$ $\begin{array}{lll}1.2 \mathrm{X} & 68 & 5\end{array}$ $1.8 \times 226 \quad 12$

$4.0 \times 24342$ $1.4 \times 30449$ $\begin{array}{lll}1.3 \times & 101 & 2 \\ 1.3 \times & 242 & 18\end{array}$ $\begin{array}{llll}1.3 \times & 242 & 18 \\ 2.7 \times & 275 & 30\end{array}$

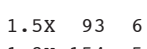
$\begin{array}{lll}1.3 \times & 154 & 5 \\ 1.5 \times & 287 & 35\end{array}$ $\begin{array}{lll}1.5 \times & 287 & 35 \\ 1.5 \times & 73 & 4\end{array}$ $1.5 \times 124 \quad 2$

$\begin{array}{lll}1.4 \times & 181 & 6 \\ 1.5 \times & 60 & 11\end{array}$ $\begin{array}{lrr}1.5 x & 60 & 11 \\ 1.7 x & 206 & 5\end{array}$ $\begin{array}{lll}1.7 \times & 206 & 5 \\ 1.8 \times & 244 & 17\end{array}$ $\begin{array}{rrr}1.8 \times & 244 & 17 \\ 1.2 x & 70 & 6\end{array}$

$1.2 \times \quad 59 \quad 7$ $1.4 \times \quad 62 \quad 5$ $1.1 \times \quad 94 \quad 3$ $\begin{array}{lll}1.0 \times 141 & 6 \\ 1.2 \times & 206 & 7\end{array}$

$\begin{array}{lll}1.5 \mathrm{X} & 87 & 8\end{array}$ $\begin{array}{lll}1.5 \times & 87 & 8 \\ 1.5 x & 71 & 6\end{array}$ $2.5 \times 196 \quad 9$ $\begin{array}{lll}.6 \times & 123 & 3 \\ .7 \times & 140 & 6\end{array}$

\begin{tabular}{lll}
$1.1 \times \quad 86 \quad 3$ \\
\hline
\end{tabular} $1.4 \times 315 \quad 22$

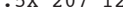
$\begin{array}{rrr}1.5 \times & 178 & 4 \\ 1.4 \times & 66 & 2\end{array}$

$1.3 \times 137 \quad 5$ $1.7 \times 226 \quad 5$ $\begin{array}{lll}1.5 \mathrm{X} & 68 & 1\end{array}$ $\begin{array}{lll}1.4 \times & 223 & 24 \\ 1.9 \times & 147 & 5\end{array}$ 
---ORIGIN TIME (HST)-- -LAT N-- --LON W-- DEPTH N RMS ERH ERZ LOC PREF AZ MIN 8 YEAR MON DA HRMN SEC DEG MIN DEG MIN KM RD SEC KM KM REMKS MAG GAP DS

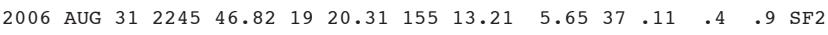

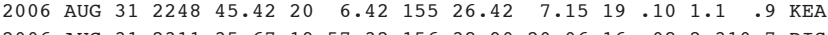

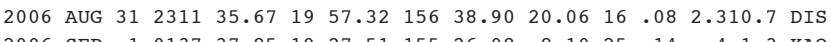
$\begin{array}{lllllllllllllll}2006 & \text { SEP } & 1 & 0137 & 37.85 & 19 & 27.51 & 155 & 26.08 & 8.10 & 25 & .14 & .4 & 1.3 & \text { KAO }\end{array}$

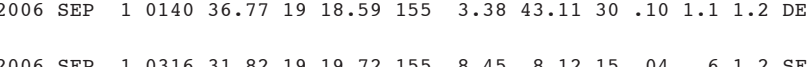
$\begin{array}{lllllllllllllll}2006 & \text { SEP } & 1 & 0316 & 31.82 & 19 & 19.72 & 155 & 8.45 & 8.12 & 15 & .04 & .6 & 1.2 & \text { SF } 4 \\ 2006 & \text { SEP } & 1 & 0602 & 52.84 & 19 & 17.06 & 155 & 23.32 & 2.06 & 20 & .10 & .5 & .8 & \text { SWR }\end{array}$ $\begin{array}{lllllllllllllll}2006 & \text { SEP } & 1 & 0631 & 46.17 & 19 & 29.13 & 156 & 19.49 & 38.14 & 32 & .12 & 1.1 & 2.6 & \text { KON }\end{array}$ $\begin{array}{llllllllllllllll}2006 & \text { SEP } & 1 & 0650 & 24.80 & 19 & 26.87 & 155 & 14.99 & 28.95 & 43 & .11 & .6 & .7 & \text { DEP }\end{array}$ $\begin{array}{llllllllllllllll}2006 & \text { SEP } & 1 & 0804 & 2.42 & 19 & 20.31 & 155 & 12.94 & 7.12 & 46 & .11 & .4 & .6 & \mathrm{SF} 2\end{array}$

$\begin{array}{lllllllllllllll}2006 & \text { SEP } & 1 & 0809 & 6.57 & 19 & 22.11 & 155 & 13.70 & 34.76 & 36 & .08 & 1.0 & .9 & \text { DEP }\end{array}$

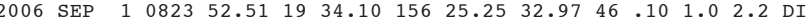

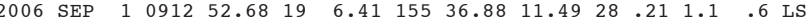

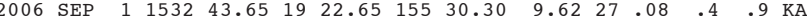
$\begin{array}{lllllllllllllll}2006 & \text { SEP } & 1 & 1547 & 33.60 & 19 & 2.07 & 155 & 24.48 & 39.55 & 25 & .11 & 1.2 & 2.3 & \text { LOI }\end{array}$

$\begin{array}{lllllllllllllll}2006 & \text { SEP } & 1 & 1652 & 29.56 & 19 & 37.81 & 155 & 55.49 & 15.49 & 20 & .09 & 1.1 & .5 & \text { KON }\end{array}$

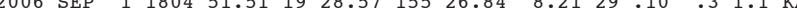

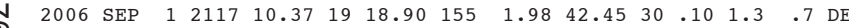

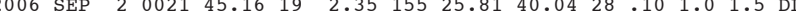
$\begin{array}{lllllllllllllll}2006 & \text { SEP } & 2 & 0219 & 26.52 & 19 & 17.59 & 155 & 23.02 & 2.93 & 35 & .11 & .3 & .7 & \text { SWR }\end{array}$

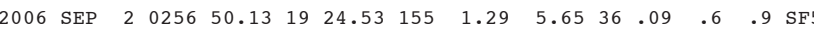

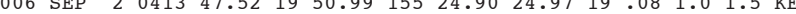
$\begin{array}{llllllllll} & & \end{array}$

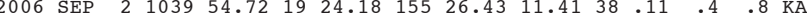
$\begin{array}{lllllllllllllll}2006 & \text { SEP } & 2 & 1110 & 9.46 & 19 & 25.33 & 155 & 16.47 & 2.17 & 14 & .11 & .6 & .3 & \text { SNC }\end{array}$

$\begin{array}{lllllllllllllll}2006 & \text { SEP } & 2 & 1546 & 5.47 & 19 & 24.90 & 155 & 19.20 & 6.70 & 26 & .09 & .4 & .8 & \text { KAO }\end{array}$ $\begin{array}{llllllllllllll}2006 & \text { SEP } & 2 & 1739 & 15.83 & 19 & 24.88 & 155 & 19.28 & 7.41 & 33 & .11 & .4 & .7 \\ \mathrm{KAO}\end{array}$ $\begin{array}{lllllllllllllll}2006 & \text { SEP } & 2 & 2009 & 53.67 & 19 & 2.29 & 155 & 24.74 & 40.19 & 29 & .07 & 1.0 & 1.2 & \text { LO }\end{array}$

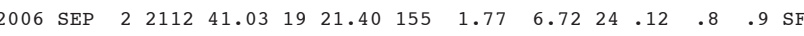
$\begin{array}{llllllllllllll}2006 & \text { SEP } & 2 & 2159 & 15.93 & 19 & 2.21 & 155 & 24.70 & 40.50 & 39 & .08 & .8 & 1.2\end{array}$

$\begin{array}{lllllllllllllll}2006 & \text { SEP } & 2 & 2223 & 21.01 & 19 & 19.12 & 155 & 4.01 & 42.37 & 41 & .08 & .9 & 1.0 & \text { DEP }\end{array}$ $\begin{array}{lllllllllllllll}2006 & \text { SEP } & 2 & 2334 & 27.99 & 19 & 26.14 & 155 & 28.17 & 10.52 & 30 & .10 & .4 & .8 & \text { KAO }\end{array}$ $\begin{array}{lllllllllllllll}2006 & \text { SEP } & 2 & 2349 & 8.30 & 19 & 18.95 & 155 & 15.32 & 3.57 & 23 & .11 & .3 & 1.3 & \text { SS }\end{array}$ $\begin{array}{lllllllllllllll}2006 & \text { SEP } & 3 & 0057 & 52.39 & 19 & 8.08 & 155 & 27.57 & 49.37 & 25 & .13 & 1.0 & 1.6 & \text { DLS } \\ 2006 & \text { SEP } & 3 & 0159 & 46.74 & 19 & 27.03 & 155 & 29.23 & 12.70 & 18 & .11 & .5 & 1.5 & \text { KAO }\end{array}$

$\begin{array}{lllllllllllllll}2006 & \text { SEP } & 3 & 0220 & 29.47 & 19 & 18.76 & 155 & 13.53 & 9.14 & 46 & .11 & .4 & .4 & \text { SF2 }\end{array}$

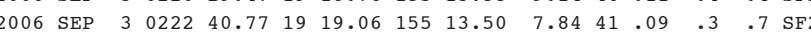

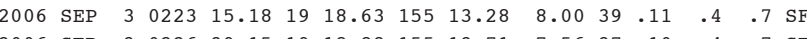

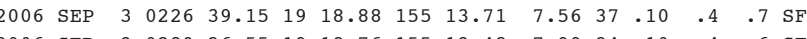
$\begin{array}{lllllllllllllll}2006 & \text { SEP } & 3 & 0239 & 26.55 & 19 & 18.76 & 155 & 13.48 & 7.99 & 34 & .10 & .4 & .6 & \mathrm{SF} 2\end{array}$

$\begin{array}{lllllllllllllll}2006 & \text { SEP } & 3 & 0243 & 5.54 & 19 & 19.12 & 155 & 13.52 & 8.18 & 43 & .10 & .4 & .5 & \text { SF2 }\end{array}$ $\begin{array}{llllllllllllll}2006 & \text { SEP } & 3 & 0405 & 45.15 & 19 & 32.24 & 155 & 45.90 & 10.40 & 26 & .13 & .6 & .6 \\ \text { KON }\end{array}$ $\begin{array}{lllllllllllllll}2006 & \text { SEP } & 3 & 0432 & 2.22 & 19 & 29.13 & 155 & 28.67 & 10.48 & 39 & .11 & .3 & .6 & \mathrm{KAO}\end{array}$

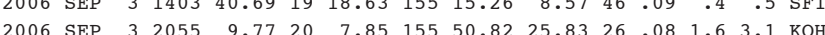

$\begin{array}{rrr}1.1 \times & 65 & 4 \\ 1.8 \times & 298 & 36\end{array}$ $\begin{array}{lll}1.8 \times & 29836 \\ 1.8 \times 335105\end{array}$ $1.4 \times \quad 61 \quad 6$ $1.7 \times 221 \quad 8$

$2.0 \times 109 \quad 4$ $1.4 \times 163 \quad 6$ $2.1 \times 27043$ $1.8 \times 142$ $1.7 \mathrm{X} \quad 68 \quad 4$

$\begin{array}{lll}1.5 \mathrm{X} & 61 & 1\end{array}$ $2.4 \times 278 \quad 53$ $1.4 \times 21917$ $1.4 \times \quad 58 \quad 5$ 1.5X 26426

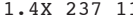
$1.5 \times 219 \quad 1$ $1.3 \times 117 \quad 5$

$1.5 \times 146 \quad 5$ $1.5 \times 2169$ $1.4 \times \quad 95$ $\begin{array}{lll}1.6 \times & 54 \quad 4\end{array}$ $1.5 \times 108 \quad 2$ $1.5 \times 21114$ $2.0 \times 21115$

2.0x 2018 $\begin{array}{lll}1.5 X & 47 & 7 \\ .9 x & 116 & 5\end{array}$ $.9 \times 116 \quad 5$ $1.9 \times 256$ $\begin{array}{lll}1.3 \mathrm{X} & 72 & 4 \\ 1.2 \mathrm{x} & 83 & 3\end{array}$ $\begin{array}{lll}1.2 \mathrm{x} & 83 & 3 \\ 1.5 \mathrm{x} & 85 & 3\end{array}$ $\begin{array}{lll}1.5 \times & 85 & 3 \\ 1.3 \times & 78 & 3\end{array}$ $1.7 \times \quad 70$ $\begin{array}{llll}1.7 \times & 70 & 4\end{array}$ $\begin{array}{lll}1.5 X & 84 & 3 \\ 1.7 X & 64 & 6\end{array}$ $\begin{array}{rrr}1.7 \times & 64 & 6 \\ 2.0 \times & 100 & 4\end{array}$ $\begin{array}{lrr}2.0 \times & 100 & 4 \\ 1.6 \times & 307 & 36\end{array}$
---ORIGIN TIME (HST)-- -LAT N-- --LON W-- DEPTH N RMS ERH ERZ LOC YEAR MON DA HRMN SEC DEG MIN DEG MIN KM RD SEC KM KM REMKS

PREF AZ MIN
MAG GAP DS

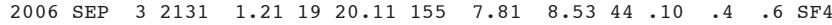

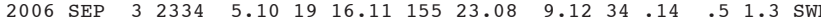

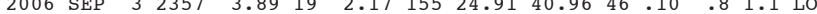

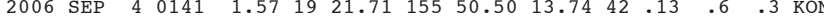
$\begin{array}{llllllllllllllllll} & \end{array}$

$\begin{array}{llllllllllllllllll}2006 & \text { SEP } & 4 & 0753 & 7.98 & 19 & 24.99 & 155 & 19.16 & 6.30 & 29 & .09 & .4 & .8 & \text { KAO } & 1.1 \times & 113 & 3\end{array}$

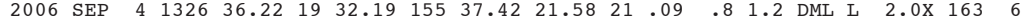

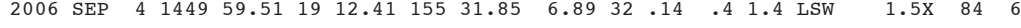

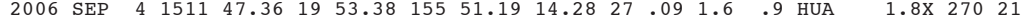

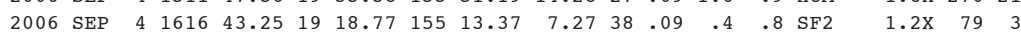

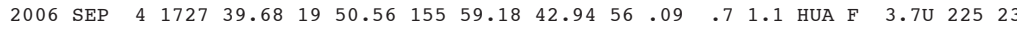

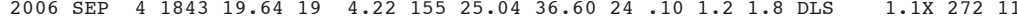

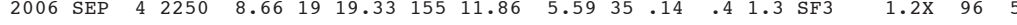

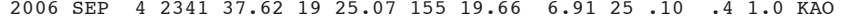

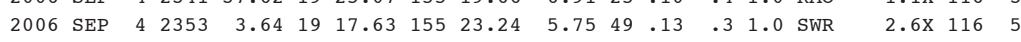

$\begin{array}{llllllllllllllll}2006 & \text { SEP } & 5 & 0136 & 24.30 & 19 & 10.86 & 155 & 20.19 & 47.21 & 31 & .09 & .8 & 1.1 & \text { DEP } & 1.4 \times \\ 2006 & 13\end{array}$ $\begin{array}{lllllllllllllllllll}2006 & \text { SEP } & 5 & 0140 & 33.68 & 19 & 23.62 & 155 & 16.77 & 2.74 & 15 & .09 & .4 & .3 & \text { SSC } & 1.0 x & 65 & 1\end{array}$

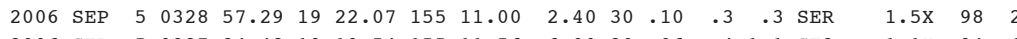

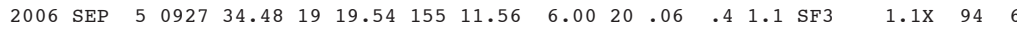

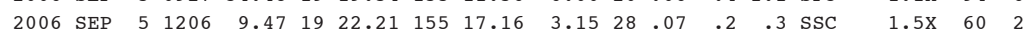

$\begin{array}{llllllllllllllllll}2006 & \text { SEP } & 5 & 1525 & 54.77 & 19 & 21.68 & 155 & 18.02 & 3.17 & 26 & .10 & .3 & .5 & \text { SWR } & 1.5 \mathrm{X} & 65 & 3\end{array}$

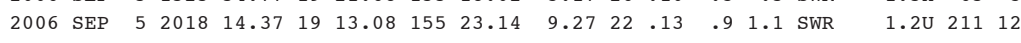
$\begin{array}{lllllllllllllllllll}2006 & \text { SEP } & 5 & 2334 & 35.50 & 19 & 23.29 & 155 & 17.16 & 2.64 & 15 & .08 & .3 & .3 & \text { SSC } & 1.4 \mathrm{x} & 69 & 0\end{array}$

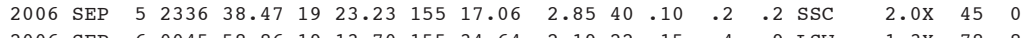
$\begin{array}{lllllllllllllll}2006 & \text { SEP } & 6 & 0045 & 58.86 & 19 & 13.70 & 155 & 34.64 & 2.19 & 22 & .15 & .4 & .9 & \text { LSW }\end{array}$

$\begin{array}{lllllllllllllll}2006 & \text { SEP } & 6 & 0057 & 18.60 & 19 & 15.49 & 155 & 24.78 & 9.17 & 19 & .08 & .6 & .8 & \text { SWR }\end{array}$

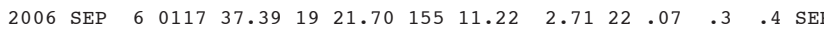

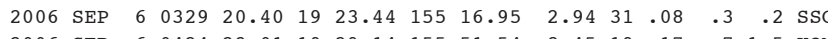

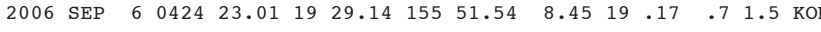
$\begin{array}{lllllllllllllll}2006 & \text { SEP } & 6 & 0606 & 6.11 & 19 & 18.30 & 155 & 14.83 & 5.75 & 27 & .12 & .5 & 1.4 & \text { SF } 1\end{array}$

$\begin{array}{lllllllllllllll}2006 & \text { SEP } & 6 & 0626 & 47.77 & 19 & 18.51 & 155 & 15.48 & 7.65 & 26 & .07 & .4 & .9 & \mathrm{SF} 1\end{array}$ $\begin{array}{lllllllllllllll}2006 & \text { SEP } & 6 & 0816 & 0.23 & 19 & 20.30 & 155 & 2.90 & 3.42 & 20 & .13 & .8 & 2.0 & \text { SSF }\end{array}$

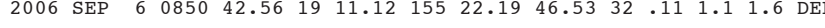

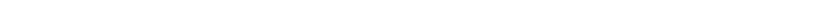

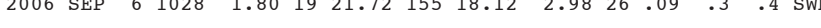

$\begin{array}{lllllllllllllll}2006 & \text { SEP } & 6 & 1300 & 51.27 & 19 & 19.54 & 155 & 7.52 & 7.00 & 33 & .09 & .5 & .8 & \text { SF } 4\end{array}$

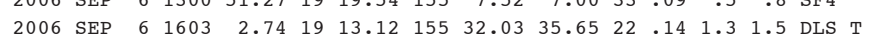
$\begin{array}{lllllllllllll}2006 & \text { SEP } 6 & 1645 & 5.46 & 19 & 30.35 & 155 & 29.20 & 7.24 & 16 & .08 & .4 & 1.8 \\ 2006 & \text { MLO }\end{array}$

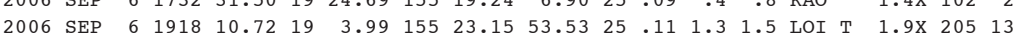

$\begin{array}{lllllllllllllllllll}2006 & \text { SEP } & 6 & 1934 & 9.20 & 19 & 7.28 & 155 & 30.70 & 36.95 & 20 & .12 & 1.2 & 2.0 & \text { DLS T } & 1.9 \times & 244 & 6\end{array}$

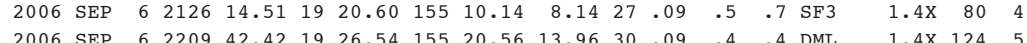

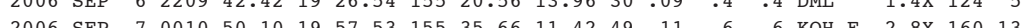

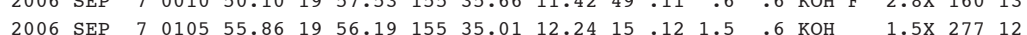


---ORIGIN TIME (HST)-- -LAT N-- --LON W-- DEPTH N RMS ERH ERZ LOC YEAR MON DA HRMN SEC DEG MIN DEG MIN

$\begin{array}{lllllllllllllll}2006 & \text { SEP } & 7 & 0114 & 6.97 & 20 & 0.45 & 155 & 33.75 & 12.62 & 24 & .14 & 1.4 & .6 & \text { KEA }\end{array}$ $\begin{array}{lllllllllllllll}2006 & \text { SEP } & 7 & 0142 & 17.47 & 19 & 59.26 & 155 & 34.49 & 12.93 & 22 & .13 & 1.3 & .6 & \text { кон }\end{array}$ $\begin{array}{lllllllll} & \end{array}$ $\begin{array}{lllllllllllllll}2006 & \text { SEP } & 7 & 0510 & 41.56 & 19 & 47.02 & 155 & 51.07 & 14.62 & 32 & .11 & .9 & .6 & .6\end{array}$

$\begin{array}{lllllllllllllll}2006 & \text { SEP } & 7 & 0529 & 34.35 & 19 & 58.00 & 155 & 35.47 & 9.50 & 22 & .09 & 1.0 & .8 & \text { кон }\end{array}$ $\begin{array}{lllllllllllllll}2006 & \text { SEP } & 7 & 0640 & 58.19 & 20 & 0.14 & 155 & 32.41 & 9.81 & 16 & .09 & 1.3 & .8 & \mathrm{KEA}\end{array}$ $\begin{array}{lllllllllllllll}2006 & \text { SEP } & 7 & 0701 & 36.33 & 19 & 13.95 & 155 & 37.26 & 7.43 & 22 & .10 & .4 & 1.8 & \text { LSW }\end{array}$

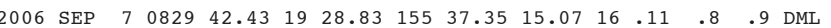
$\begin{array}{lllllllllllllll}2006 & \text { SEP } & 7 & 0843 & 9.07 & 19 & 11.37 & 155 & 26.11 & 8.60 & 26 & .13 & .5 & .8 & \text { LSW }\end{array}$

$\begin{array}{lllllllllllllll}2006 & \text { SEP } & 7 & 0927 & 44.14 & 19 & 18.34 & 155 & 14.79 & 6.37 & 23 & .10 & .5 & 1.1 & \text { SF } 1\end{array}$ $\begin{array}{lllllllllllllll}2006 & \text { SEP } & 7 & 1148 & 12.49 & 19 & 25.57 & 155 & 39.02 & 2.89 & 17 & .09 & .5 & .6 & \text { MLO }\end{array}$ $\begin{array}{lllllllllllllll}2006 & \text { SEP } & 7 & 1243 & 48.28 & 19 & 25.99 & 155 & 19.33 & 6.65 & 26 & .09 & .4 & .9 & \text { KAO }\end{array}$

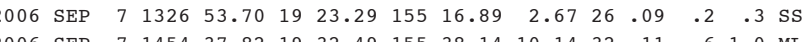
$\begin{array}{llllllllllllllll}2006 & \text { SEP } & 7 & 1454 & 37.82 & 19 & 32.49 & 155 & 38.14 & 10.14 & 32 & .11 & .6 & 1.0 & \text { MLO }\end{array}$

$\begin{array}{lllllllllllllll}2006 & \text { SEP } & 7 & 1459 & 0.03 & 19 & 23.48 & 155 & 16.74 & 3.08 & 35 & .07 & .2 & .2 & \text { SSC }\end{array}$ $\begin{array}{lllllllllllllll}2006 & \text { SEP } & 7 & 1508 & 49.90 & 19 & 23.50 & 155 & 16.89 & 2.95 & 35 & .08 & .2 & .1 & \text { SSC }\end{array}$ $\begin{array}{lllllllllllllll}2006 & \text { SEP } & 7 & 1510 & 49.57 & 19 & 23.51 & 155 & 16.78 & 2.97 & 36 & .08 & .2 & .1 & \text { SSC } \\ 2006 & \text { SEP } & 7 & 1646 & 10.76 & 19 & 14.60 & 155 & 17.58 & 58.41 & 19 & .13 & 2.0 & 1.2 & \text { DEP }\end{array}$ $\begin{array}{lllllllllllllll}2006 & \text { SEP } & 7 & 1723 & 14.87 & 19 & 19.52 & 155 & 3.65 & 42.28 & 42 & .08 & 1.1 & .9 & \text { DEP }\end{array}$

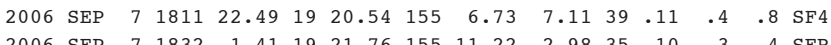
$\begin{array}{llllllllllllllll}2006 & \text { SEP } & 7 & 1832 & 1.41 & 19 & 21.76 & 155 & 11.22 & 2.98 & 35 & .10 & .3 & .4 & \text { SER }\end{array}$ $\begin{array}{lllllllllllllll}2006 & \text { SEP } & 7 & 2117 & 32.95 & 19 & 59.95 & 155 & 33.19 & 3.58 & 26 & .21 & 1.2 & 1.3 & \text { KEA }\end{array}$ 2006 SEP $7 \begin{array}{llllllllllll}2242 & 37.55 & 19 & 21.79 & 155 & 7.94 & 40.48 & 23 & .11 & 1.2 & 1.3 & \text { DEP }\end{array}$ $\begin{array}{llllllllllllllll} & 2006 & \mathrm{SEP} & 8 & 0458 & 35.13 & 19 & 26.12 & 155 & 30.35 & 11.26 & 17 & .08 & .5 & 1.3 & \mathrm{KAO}\end{array}$

$\begin{array}{llllllllllllll}2006 & \text { SEP } & 8 & 0538 & 41.60 & 19 & 25.70 & 155 & 37.41 & 2.49 & 18 & .11 & .4 & .5 \\ \text { MLO }\end{array}$ $\begin{array}{lllllllllllllll}2006 & \text { SEP } & 8 & 1303 & 56.86 & 19 & 18.60 & 155 & 15.13 & 5.52 & 32 & .11 & .4 & 1.2 & \text { SF } 1\end{array}$

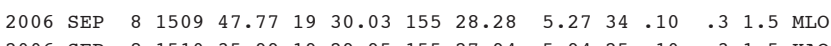

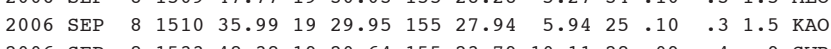
$\begin{array}{llllllllllllll}2006 & \text { SEP } & 8 & 1533 & 48.38 & 19 & 20.64 & 155 & 23.79 & 10.11 & 28 & .09 & .4 & .9\end{array}$

$\begin{array}{llllllllllllll}2006 & \text { SEP } & 8 & 1740 & 3.23 & 19 & 56.67 & 155 & 34.96 & 10.93 & 46 & .13 & .9 & .5 \text { кон } \mathrm{F}\end{array}$ $\begin{array}{lllllllllllllll}2006 & \text { SEP } & 8 & 1758 & 36.32 & 19 & 23.24 & 155 & 30.11 & 11.20 & 22 & .08 & .4 & 1.0 & \text { KAO }\end{array}$ $\begin{array}{lllllllllllllll}2006 & \text { SEP } & 8 & 1840 & 43.74 & 19 & 19.88 & 155 & 7.70 & 8.68 & 40 & .09 & .4 & .6 & \text { SF4 }\end{array}$ $\begin{array}{llllllllllllllll}2006 & \text { SEP } & 8 & 2002 & 3.31 & 19 & 28.29 & 155 & 0.72 & 43.78 & 46 & .11 & .7 & .8 & \text { DEP }\end{array}$ $\begin{array}{lllllllllllllll}2006 & \text { SEP } & 8 & 2302 & 14.39 & 19 & 12.11 & 155 & 22.85 & 36.72 & 22 & .10 & 1.2 & 1.8 & \text { DEP }\end{array}$

$\begin{array}{lllllllllllllll}2006 & \text { SEP } & 9 & 0023 & 0.90 & 19 & 22.64 & 155 & 14.46 & 2.86 & 20 & .07 & .3 & .3 & \text { SEC }\end{array}$ $\begin{array}{lllllllllllllll}2006 & \text { SEP } & 9 & 1103 & 18.55 & 19 & 19.71 & 155 & 9.63 & 7.21 & 20 & .09 & .5 & 1.1 & \text { SF } 3\end{array}$

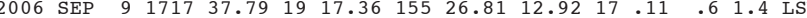

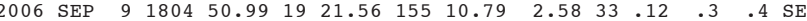

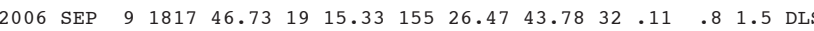

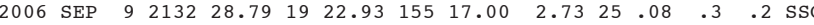

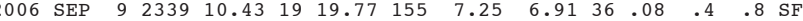

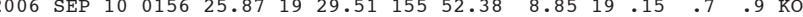

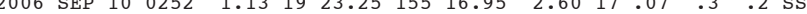

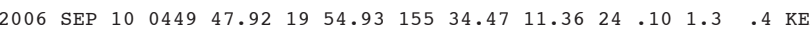

PREF AZ MIN 85 MAG GAP DS

$1.7 \times 20819$ $.6 \times 20017$ $.8 \times 199 \quad 16$ $2.2 \times 178$ $\begin{array}{lll}1.9 \times & 163 & 25 \\ 1.4 x & 221 & 20\end{array}$ $.4 \times 22120$
$.3 \times \quad 75 \quad 13$ $1.3 \times 206$ $1.4 \times 158 \quad 5$

$1.2 \times 129 \quad 3$ $1.2 \times 114$ $\begin{array}{lll}1.2 \times & 114 & 3 \\ 1.2 \times 150 & 150\end{array}$ $\begin{array}{rrr}1.2 \times & 150 & 3 \\ 1.7 \times & 46 & 1\end{array}$ $\begin{array}{rrr}1.7 \times & 46 & 1 \\ 1.5 \times & 169 & 7\end{array}$ $2.0 \times \quad 45 \quad 0$ $2.0 \times \quad 38 \quad 0$ $2.2 \times \quad 40 \quad 0$ $1.9 \times 288$ $2.1 \times 199$ $1.4 \times 142 \quad 5$ $\begin{array}{lll}1.6 \mathrm{X} & 89 \quad 3\end{array}$ $\begin{array}{lll}1.5 \times & 288 & 19\end{array}$ $1.4 \times 286 \quad 4$ $\begin{array}{lll}1.4 \times & 113 & 8\end{array}$

$1.2 \times \quad 91 \quad 3$ $1.3 \times 122 \quad 4$ $\begin{array}{lll}1.5 \mathrm{X} & 76 & 4\end{array}$ $1.6 \mathrm{X} \quad 84 \quad 4$ $1.1 \mathrm{x} \quad 95 \quad 1$

$2.2 \times \quad 250 \quad 12$ $\begin{array}{rrr}1.3 \mathrm{X} & 79 & 5 \\ 1.6 \mathrm{X} & 128 & 5 \\ 1.9 \times & 101 & 7\end{array}$ 1.9X $101 \quad 7$ 1.3X $181 \quad 10$ $\begin{array}{lll}1.6 \mathrm{X} & 81 & 2 \\ 1.2 \mathrm{x} & 88 & 5\end{array}$ $1.7 \times 150$ $\begin{array}{lll}1.7 \times & 75 \quad 2\end{array}$ $1.6 \times 111 \quad 6$

$\begin{array}{lll}1.8 \times & 48 & 1\end{array}$ $1.5 \times 140 \quad 5$ $1.4 \mathrm{U} 147 \quad 5$ $1.2 \mathrm{X} \quad 64$ $1.6 \mathrm{X} 264 \quad 11$
--ORTGIN TTME (HST)-- -LAT N-- --LON W-- DEPTH N RMS ERH ERZ LOC YEAR MON DA HRMN SEC DEG MIN DEG MIN DM RD SEC KM KM REMKS PREF AZ MIN 86

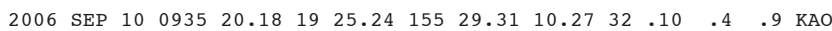

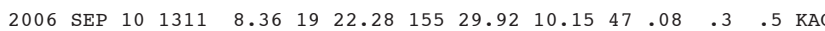

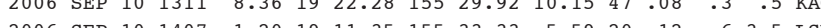

2006 SEP $110117 \quad 39.49 \quad 19 \quad 10.49 \quad 155 \quad 20.0047 .16 \quad 29 \quad 14 \quad 1.0 \quad 1.6$ DEP

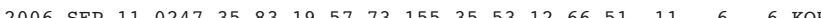

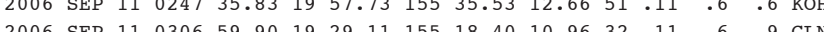

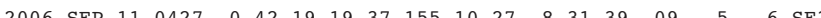

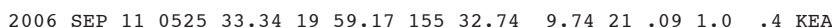

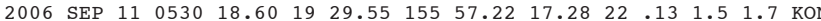

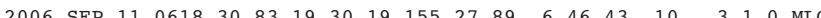

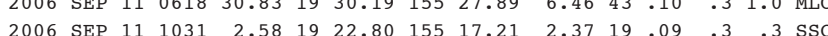

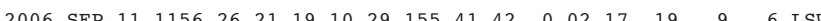

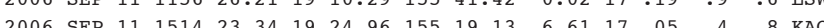

$\begin{array}{llllllllllllll}2006 & \text { SEP } 11 & 1726 & 16.38 & 19 & 19.59 & 155 & 7.26 & 4.67 & 20 & .11 & .5 & 2.0 & \text { SSF }\end{array}$

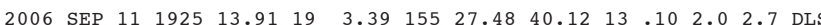

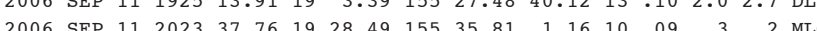
$\begin{array}{lllllllllllllll}2006 & \text { SEP } 11 & 2134 & 41.51 & 20 & 0.60 & 155 & 34.58 & 3.76 & 18 & 13 & 1.0 & .2 & 5 & \text { KO }\end{array}$

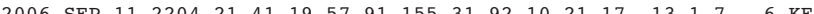

$\begin{array}{llllllllllllll}2006 & \text { SEP } & 11 & 2237 & 25.61 & 19 & 4.26 & 155 & 23.72 & 38.66 & 31 & .08 & .9 & 1.5\end{array}$

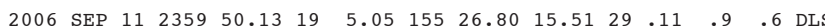

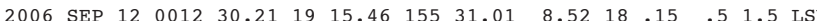

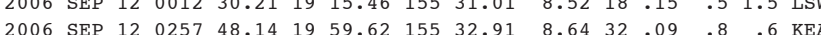

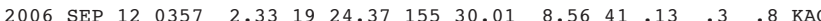

$\begin{array}{llllllllllllll}2006 & \text { SEP } 12 & 0554 & 24.34 & 19 & 20.37 & 155 & 19.41 & 1.68 & 18 & .10 & .3 & .6 & \text { SWR }\end{array}$

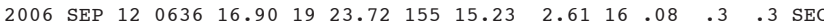

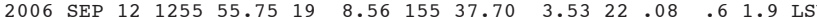

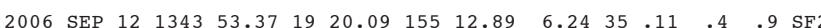
$\begin{array}{lllllllllllll}2006 & \text { SEP } 12 & 1609 & 27.52 & 19 & 56.77 & 155 & 34.36 & 11.90 & 20 & .10 & 1.3 & .6 \\ \text { KOH }\end{array}$

$\begin{array}{llllllllllllll}2006 & \text { SEP } 12 & 1850 & 24.98 & 19 & 23.21 & 155 & 30.43 & 10.01 & 48 & .08 & .3 & .5 & \text { KAO }\end{array}$

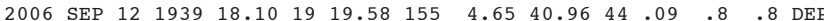

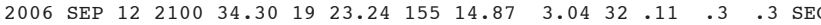

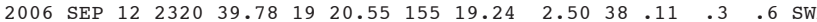
$\begin{array}{lllllllllllllll}2006 & \text { SEP } & 12 & 2348 & 6.34 & 19 & 31.63 & 155 & 15.53 & 36.15 & 51 & .09 & .6 & .8 & \text { DEP }\end{array}$

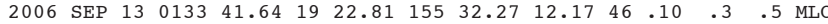

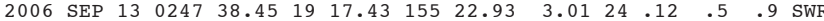

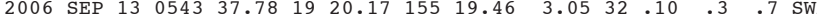

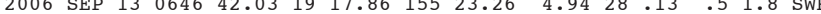
$\begin{array}{lllllllllllllll}2006 & \text { SEP } & 13 & 0716 & 36.27 & 19 & 11.06 & 155 & 3.83 & 47.50 & 44 & .10 & .9 & 1.1 & \text { DEP }\end{array}$

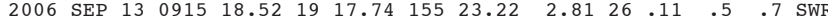

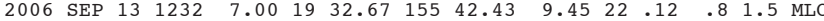

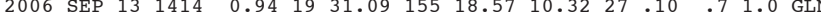
$\begin{array}{lllllllllllllll}2006 & \text { SEP } & 13 & 1531 & 37.41 & 20 & 1.43 & 155 & 17.08 & 2.05 & 25 & .12 & 1.3 & .6 & \text { KEA }\end{array}$

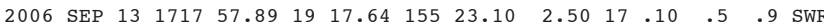

\begin{tabular}{ll}
$.1 x \quad 57$ \\
\hline $.1 \times$
\end{tabular} $\begin{array}{rr}2.1 \times & 60 \\ 1.4 \times & 151\end{array}$ $1.4 \times 151$ $3.3 \times \quad 91$

$1.3 \times \quad 22214$ $.2 \times 16113$ $1.3 \times 101 \quad 6$ $3 \times 1018$

$1.6 \times 258 \quad 4$ $2.1 \mathrm{x} \quad 61 \quad 3$ $\begin{array}{rrr}.4 \times & 61 & 1 \\ 1.4 \times & 163 & 8\end{array}$ $1.1 \times 112 \quad 3$

$\begin{array}{lll}1.1 \mathrm{U} & 142 \quad 4\end{array}$ $\begin{array}{lll}1.4 \times \quad 287 & 22\end{array}$ $1.7 \times 29619$ $1.4 \times \quad 287 \quad 18$

$\begin{array}{lll}1.4 \times \quad 203 \quad 12 \\ 1.4 \times \quad 190 & 8\end{array}$ $\begin{array}{rrr}1.4 \times & 57 & 2\end{array}$ $1.8 \times 178 \quad 25$ $\begin{array}{rrrr}1.8 \times & 178 & 25 \\ 1.6 \times & 43 \quad 5\end{array}$

$1.1 \times 106 \quad 5$ $1.4 \times \quad 94 \quad 2$ $\begin{array}{rrr}1.4 \times & 191 & 14 \\ 1.3 \times & 71 & 5\end{array}$ $1.6 \times 29213$

$2.0 \times \quad 54 \quad 5$ $\begin{array}{rrr}2.0 \times & 54 & 5 \\ 1.9 \times & 64 & 2\end{array}$ $\begin{array}{lll}1.7 \times \quad 50 & 5\end{array}$ $2.3 \times \quad 129 \quad 12$

$\begin{array}{lll}1.8 \times & 52 \quad 8\end{array}$ $\begin{array}{llll}1.3 \times & 159 \quad 5\end{array}$ $.4 \times 1114$ $\begin{array}{rrr}1.3 \times & 113 & 4 \\ 1.9 \times & 225 & 14\end{array}$

1.0X $161 \quad 5$ $1.3 \times 1957$ $1.4 \times 188 \quad 9$ $1.6 \times 25251$ $1.2 \mathrm{U} 164 \quad 5$ 
---ORIGIN TIME (HST)-- -LAT N-- --LON W-- DEPTH N RMS ERH ERZ LOC PREF AZ MIN 87 YEAR MON DA HRMN SEC DEG MIN DEG MIN KM RD SEC KM KM REMKS MAG GAP DS

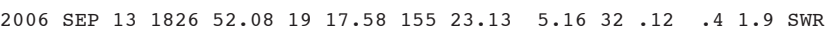

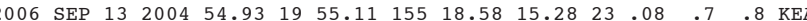

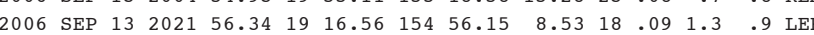

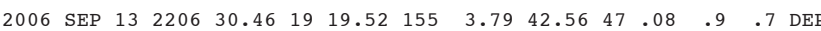

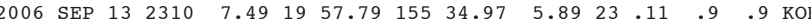

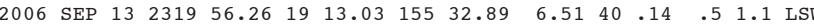
2006 SEP $132328 \begin{array}{llllllllllll}13 & 232.61 & 19 & 10.20 & 155 & 34.40 & 0.52 & 27 & .11 & .7 & .3 & \text { LSW }\end{array}$

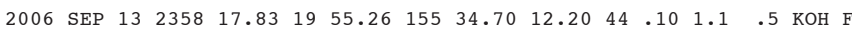

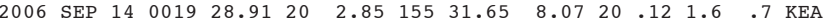

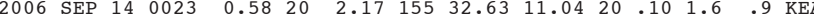

$\begin{array}{lllll}4 \times & 81 & 6\end{array}$ $1.5 \times 19312$ $2.3 \times 24011$ $1.7 \times 30025$

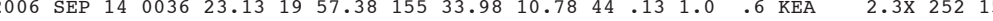

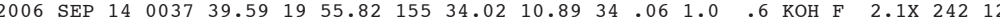

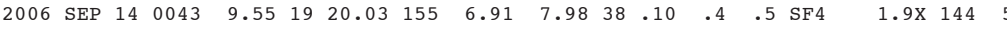

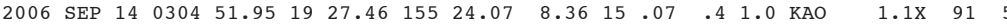

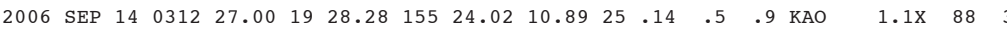

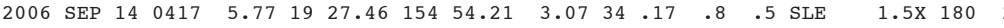

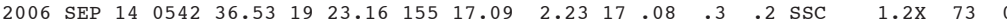

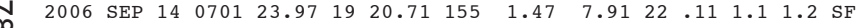

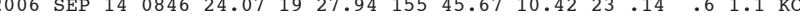

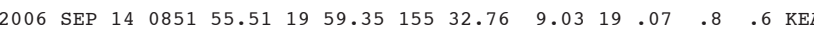

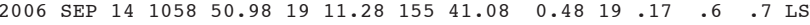

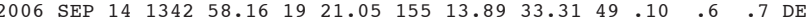

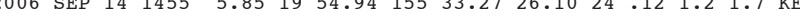

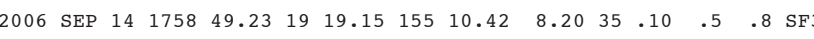

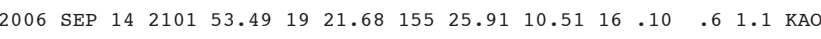

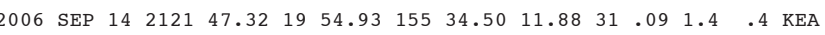

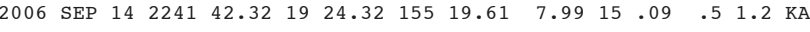

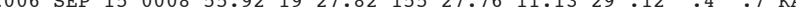

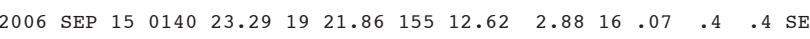

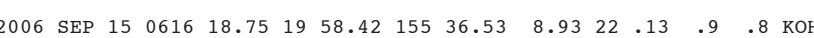

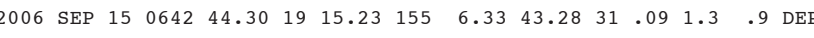

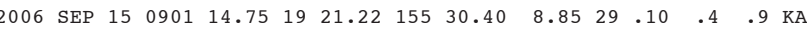

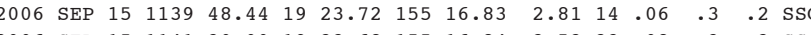

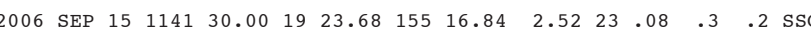

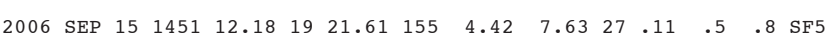
$\begin{array}{llllllllllllll}2006 & \text { SEP } 15 & 1617 & 31.40 & 19 & 23.50 & 155 & 17.01 & 2.80 & 16 & .05 & .3 & .2 & \text { SSC }\end{array}$

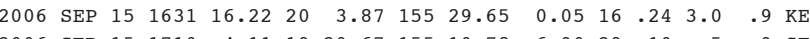

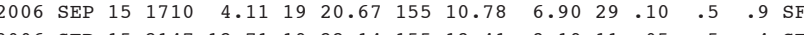

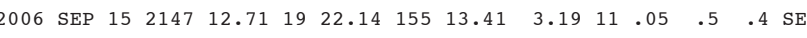

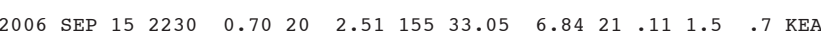

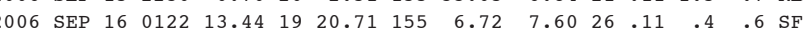
$\begin{array}{lllllllllllll}2006 & \text { SEP } 16 & 0345 & 12.23 & 19 & 22.70 & 155 & 56.51 & 12.75 & 25 & .14 & 1.0 & .5 \\ 2006 & \text { KON }\end{array}$

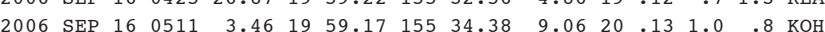

---ORIGIN TIME (HST)-- -LAT N-- --LON W-- DEPTH N RMS ERH ERZ LOC YEAR MON DA HRMN SEC DEG MIN DEG MIN KM RD SEC KM KM REMKS

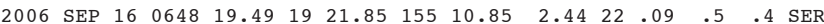

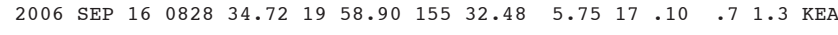

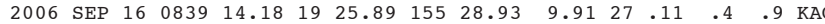

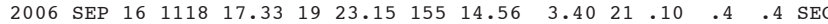
$\begin{array}{lllllllllllllll}2006 & \text { SEP } & 16 & 1122 & 22.10 & 19 & 24.35 & 155 & 30.20 & 10.39 & 24 & .11 & .5 & 1.1 & \text { KAO }\end{array}$

$\begin{array}{llllllllllllll}2006 & \text { SEP } 16 & 1203 & 27.20 & 19 & 24.63 & 155 & 19.77 & 6.18 & 34 & .09 & .4 & .8 & \text { KAO }\end{array}$ $\begin{array}{llllllllllllll}2006 & \text { SEP } 16 & 1403 & 36.84 & 19 & 25.46 & 155 & 24.39 & 9.98 & 26 & .11 & .5 & 1.2 & \text { KAO }\end{array}$ $\begin{array}{llllllllllllll}2006 & \text { SEP } 16 & 1423 & 14.30 & 19 & 23.17 & 155 & 14.91 & 2.97 & 20 & .11 & .3 & .4 & \text { SEC }\end{array}$

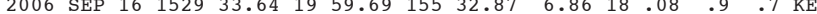
$\begin{array}{lllllllllllllll}2006 & \text { SEP } 16 & 1616 & 6.00 & 19 & 23.38 & 155 & 14.75 & 3.29 & 43 & .11 & .2 & .3 & \text { SEC }\end{array}$

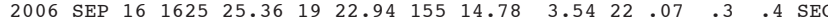

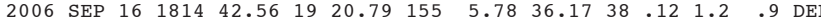

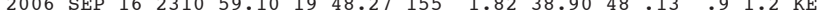

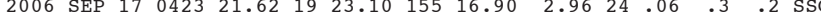
$\begin{array}{llllllllllllll}2006 & \text { SEP } 17 & 0503 & 50.59 & 20 & 2.83 & 155 & 34.57 & 11.04 & 22 & .12 & 1.3 & .6 & \text { кон }\end{array}$

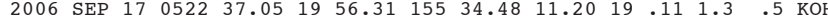

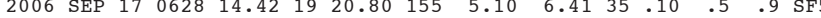

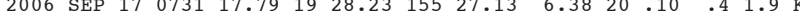

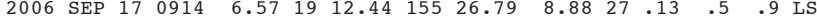

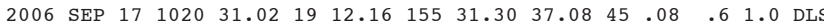

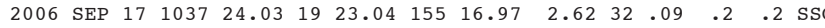
$\begin{array}{llllllllllllll}2006 & \text { SEP } 17 & 1057 & 46.85 & 19 & 11.05 & 155 & 22.19 & 36.21 & 32 & .09 & .8 & 1.2 & \text { DE }\end{array}$

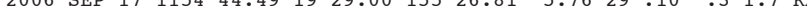

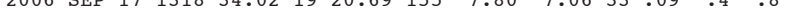

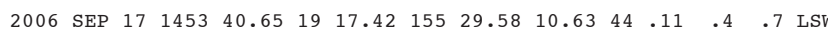

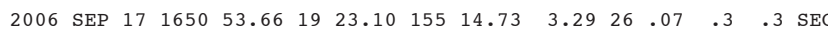
$\begin{array}{llllllllllllll}2006 & \text { SEP } 17 & 2058 & 32.53 & 19 & 20.78 & 155 & 18.67 & 30.67 & 35 & .11 & .8 & 1.1 & \text { DEP }\end{array}$

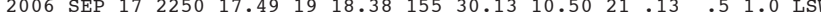

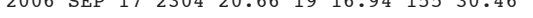

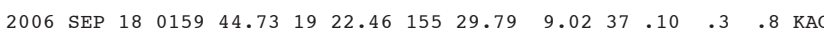

$\begin{array}{llllllllllllll}2006 & \text { SEP } 18 & 0526 & 27.88 & 19 & 11.32 & 155 & 21.29 & 36.65 & 43 & .10 & .8 & 1.0 & \text { DEP }\end{array}$

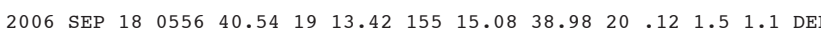

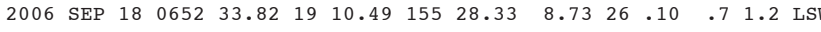
$\begin{array}{lllllllllllll}2006 & \text { SEP } 18 & 1006 & 19.09 & 19 & 28.82 & 155 & 28.33 & 8.48 & 37 & .12 & .3 & 1.0\end{array}$

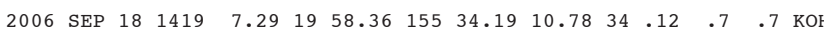

$\begin{array}{lllllllllllll}2006 & \text { SEP } 18 & 1429 & 21.07 & 19 & 23.34 & 155 & 16.75 & 2.90 & 35 & .10 & .3 & .2 \\ \text { SSC }\end{array}$

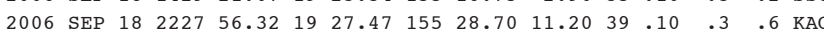

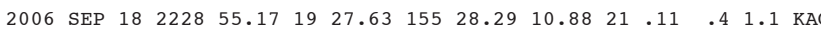

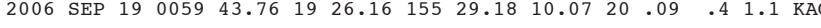
$\begin{array}{llllllllllllll}2006 & \text { SEP } & 19 & 0240 & 34.64 & 19 & 23.21 & 155 & 26.65 & 9.09 & 24 & .10 & .4 & .9 \\ \text { KAO }\end{array}$

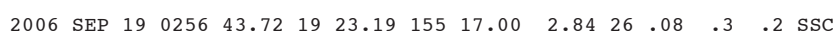

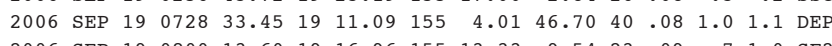

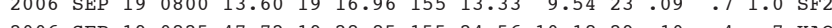

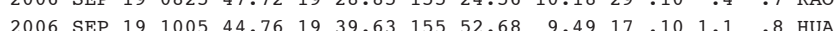
$\begin{array}{lll}.5 \times & 84 & 2\end{array}$ $1.1 \times \quad 517$ $1.5 \times \quad 80 \quad 3$ $1.3 \times \quad 74 \quad 6$

$1.7 \times 101 \quad 2$ $\begin{array}{lll}1.6 \times & 77 & 8\end{array}$ $\begin{array}{lll}1.5 \mathrm{X} & 69 & 2\end{array}$ $\begin{array}{rrrr}1.5 x & 69 & 2 \\ .5 \times & 304 & 19 \\ .3 \times & 54 & 3\end{array}$

$\begin{array}{lll}1.6 \times & 73 \quad 2\end{array}$ $\begin{array}{lll}1.8 \times & 155 & 6\end{array}$ $\begin{array}{llll}2.6 \times & 242 \quad 11\end{array}$ $\begin{array}{lll}1.5 \mathrm{X} & 47 & 1\end{array}$ $1.6 \times 30623$

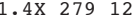
$\begin{array}{lll}1.2 \times 165 & 6\end{array}$ $\begin{array}{lll}1.3 \times & 73 & 7\end{array}$ $\begin{array}{llll}1.3 \times & 130 & 6\end{array}$ $\begin{array}{lll}1.8 \mathrm{x} & 84 & 6\end{array}$

$\begin{array}{lll}1.7 \times & 47 & 1\end{array}$ $\begin{array}{lll}1.5 \times \quad 190 & 10\end{array}$ $\begin{array}{lll}1.5 x & 91 & 6\end{array}$ $\begin{array}{llll}1.5 \times & 121 & 5\end{array}$ $1.9 \times \quad 77 \quad 4$

$\begin{array}{lll}1.8 \times & 69 & 2\end{array}$ $\begin{array}{lll}1.6 \times \quad 52 & 5\end{array}$ $\begin{array}{lll}1.3 \mathrm{X} & 85 & 6\end{array}$ $1.6 \times 127 \quad 3$ $\begin{array}{lll}1.5 \mathrm{X} & 71 & 4\end{array}$

2.0x 19012 $1.4 \times \quad 267$ $\begin{array}{lll}1.2 \times & 159 & 2 \\ 1.8 \times & 79 & 6\end{array}$ $\begin{array}{lll}1.8 \times & 79 & 6\end{array}$ $\begin{array}{lll}2.3 \times & 45 & 0\end{array}$ $\begin{array}{lll}1.7 \times & 53 & 9 \\ 1.1 \times & 58 & 8\end{array}$ $1.1 \times 58 \quad 5$ $\begin{array}{lll}1.2 \mathrm{x} & 52 & 2\end{array}$

$1.5 \times \quad 70 \quad 0$ $.0 \times \quad 22513$ $\begin{array}{lll}1.0 \times 196 & 0 \\ 1.6 \times & 81 & 3\end{array}$ $\begin{array}{lrr}1.6 x & 81 & 3 \\ 1.2 \times & 268 & 5\end{array}$ 
---ORIGIN TIME (HST)-- -LAT N-- --LON W-- DEPTH N RMS ERH ERZ LOC YEAR MON DA HRMN SEC DEG MIN DEG MIN

$\begin{array}{llllllllllllll}2006 & \text { SEP } 19 & 1126 & 20.12 & 19 & 28.37 & 154 & 55.20 & 25.78 & 27 & .18 & 1.5 & 2.2 & \text { LER }\end{array}$ $\begin{array}{lllllllllllll}2006 & \text { SEP } 19 & 1310 & 44.02 & 19 & 33.58 & 155 & 52.30 & 27.63 & 27 & .11 & 1.0 & 1.6\end{array}$ KON $\begin{array}{llllllllllllll}2006 & \text { SEP } 19 & 1352 & 47.51 & 19 & 5.90 & 155 & 24.21 & 43.84 & 20 & .14 & 2.0 & 2.3 & \text { LOI }\end{array}$ $\begin{array}{llllllllllllll}2006 & \text { SEP } 19 & 1526 & 53.62 & 20 & 11.73 & 156 & 5.87 & 34.78 & 40 & .12 & 1.1 & 2.9 & \text { KO }\end{array}$

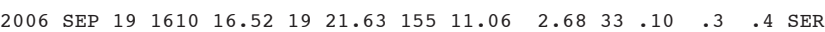

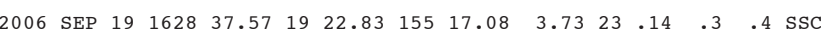

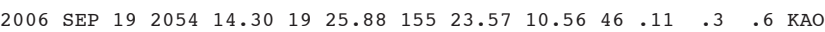

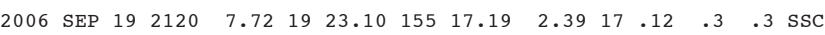

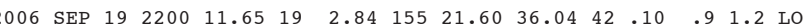

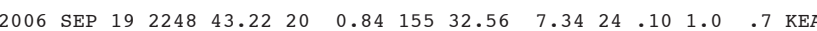

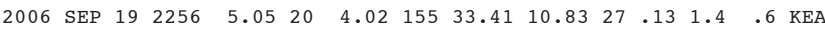

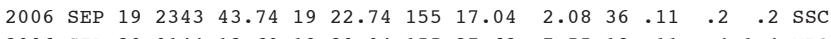

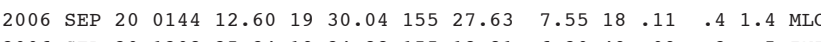
$\begin{array}{llllllllllll} & & \end{array}$

$\begin{array}{llllllllllllll}2006 & \text { SEP } 20 & 1218 & 11.71 & 19 & 24.83 & 155 & 18.63 & 5.82 & 27 & .09 & .4 & .7 & \text { INT }\end{array}$

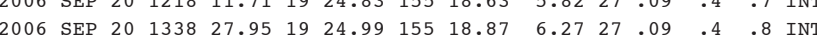
$\begin{array}{llllllllllllll}2006 & \text { SEP } 20 & 1448 & 48.61 & 19 & 21.62 & 155 & 11.18 & 2.90 & 20 & .07 & .4 & .8 & \text { IN }\end{array}$

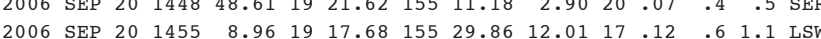

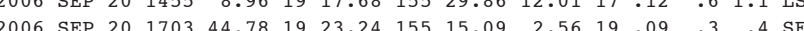

$\begin{array}{lllllllllllllll}2006 & \text { SEP } 20 & 2056 & 18.18 & 19 & 17.72 & 155 & 23.22 & 3.93 & 17 & .13 & .6 & 1.5 & \text { SWR }\end{array}$ $\begin{array}{llllllllllllll}2006 & \text { SEP } 20 & 2248 & 47.59 & 19 & 9.44 & 155 & 40.78 & 28.66 & 25 & .09 & .7 & 1.8 & \text { DLS }\end{array}$

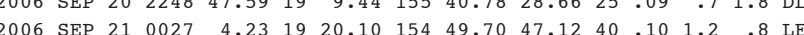

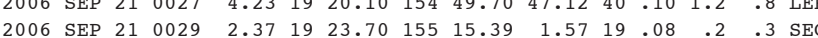

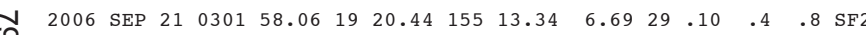

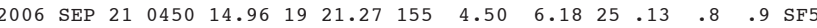

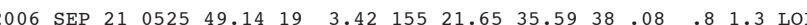

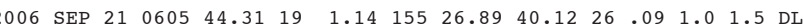

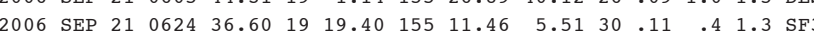

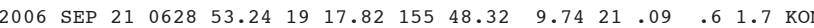

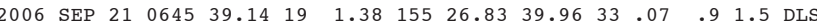

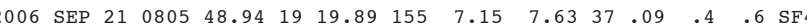

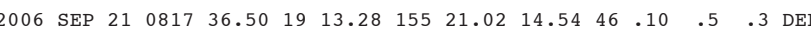

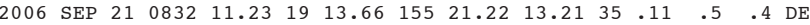

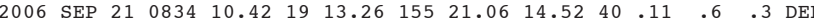

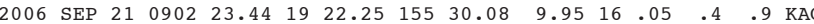

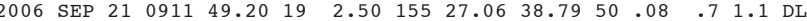
$\begin{array}{llllllllllllll}2006 & \text { SEP } 21 & 0927 & 8.63 & 19 & 22.60 & 155 & 30.44 & 10.37 & 19 & .07 & .4 & 1.0 & \text { KAO }\end{array}$

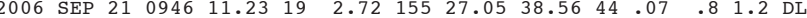

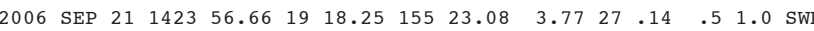

$\begin{array}{llllllllllllll}2006 & \text { SEP } & 21 & 1437 & 25.14 & 19 & 25.04 & 155 & 30.16 & 10.13 & 17 & .09 & .4 & 1.2 \\ \text { KAO }\end{array}$

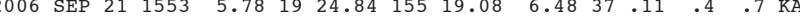

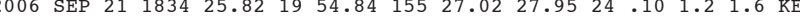

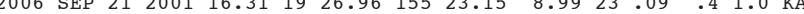

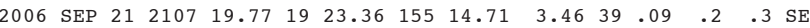

PREF AZ MIN 89 MAG GAP DS $1.5 \times 295 \quad 17$ $\begin{array}{llr}1.8 \times & 161 & 9 \\ 1.3 \times & 257 & 20\end{array}$ $2.5 \times 30962$ $2.0 \times \quad 81$ $\begin{array}{lll}1.6 \mathrm{X} & 49 & 1\end{array}$ $\begin{array}{lll}2.2 \mathrm{X} & 48 & 7 \\ 1.5 \mathrm{X} & 66 & 1\end{array}$ $\begin{array}{llr}1.5 \times & 66 & 1 \\ 1.9 \times & 213 & 16\end{array}$ $1.7 \times \quad 28921$

2.0X 29525 $2.2 \mathrm{x} \quad 49 \quad 1$ $\begin{array}{lll}1.3 \times & 93 & 4 \\ 2.7 \times & 79 & 2\end{array}$ $\begin{array}{lll}1.4 \times & 102 \quad 2\end{array}$ 1.6X $99 \quad 2$ $1.4 \times 111 \quad 2$ $\begin{array}{lrl}1.6 \times & 84 & 3\end{array}$ $\begin{array}{lll}1.6 \mathrm{X} & 84 & 3 \\ 1.3 \mathrm{U} & 98 & 5\end{array}$ $1.5 \mathrm{X} \quad 73 \quad 2$

$1.2 \mathrm{U} \quad 163 \quad 5$ $1.4 \mathrm{X} \quad 91$ 2.0X $286 \quad 17$ $1.8 \mathrm{x} \quad 95$ $1.2 \mathrm{X} \quad 62 \quad 4$

$1.2 \times 166 \quad 6$ $1.9 \times 21016$ $1.4 \times 22316$ $\begin{array}{lll}1.3 \times & 98 \quad 6\end{array}$ $\begin{array}{lll}1.6 \times & 125 & 8\end{array}$

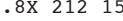
$\begin{array}{llll}2.2 \times & 141 & 5 \\ 2.2 \times & 161 & 10\end{array}$ $1.6 \times 158$ $\begin{array}{lll}1.8 \times & 161 & 10\end{array}$

$\begin{array}{lll}.4 \times & 84 & 4\end{array}$ $\begin{array}{llll}2.5 \times & 205 & 13 \\ 1.5 \times & 86 & 5\end{array}$ $1.5 \times \quad 86 \quad 5$ 2.0x $250 \quad 13$ $1.4 \times 148 \quad 4$
-ORTGIN TIME (HST)-- -LAT N-- --LON W-- DEPTH N RMS ERH ERZ LOC YEAR MON DA HRMN SEC DEG MIN DEG MIN DM RD SEC KM KM REMKS $\begin{array}{llllllllllllll}2006 & \text { SEP } 21 & 2314 & 38.85 & 19 & 28.08 & 154 & 50.84 & 7.16 & 21 & .09 & .8 & .4 & \text { LER }\end{array}$ $\begin{array}{llllllllllllll}2006 & \text { SEP } 22 & 0037 & 16.59 & 19 & 14.65 & 155 & 32.99 & 13.79 & 21 & .14 & .6 & .9 & \text { DLS }\end{array}$

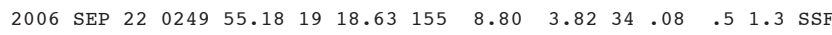
$\begin{array}{lllllllllllll}2006 & \text { SEP } 22 & 0924 & 3.52 & 19 & 10.38 & 155 & 35.25 & 0.88 & 26 & .10 & .6 & .5\end{array}$

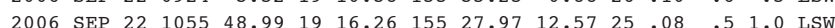

$\begin{array}{llllllllllllll}2006 & \text { SEP } 22 & 1115 & 16.58 & 19 & 19.53 & 155 & 10.92 & 5.55 & 29 & .09 & .4 & 1.3 & \mathrm{SF} 3\end{array}$

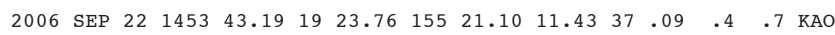

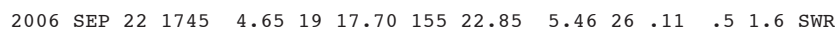

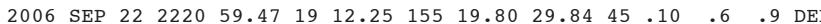

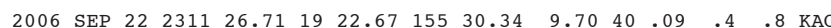

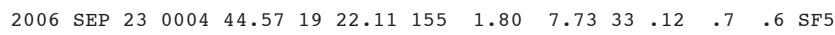

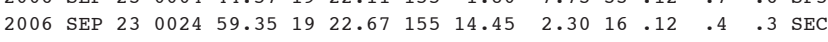

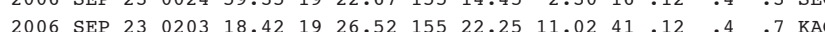

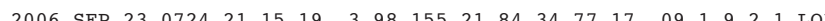

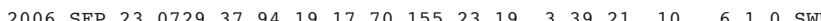

$\begin{array}{lllllllllllll}2006 & \text { SEP } 23 & 1201 & 8.73 & 19 & 57.36 & 155 & 34.21 & 11.24 & 33 & .12 & .9 & .5 \\ \text { KOH }\end{array}$

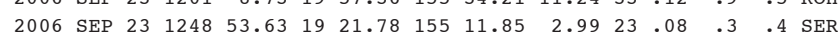

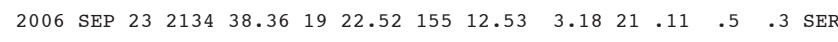

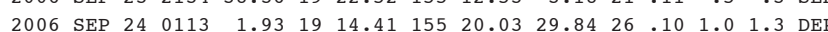

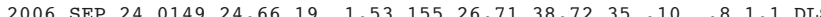

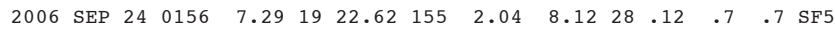

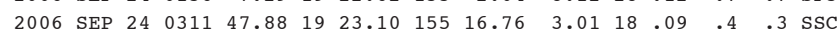

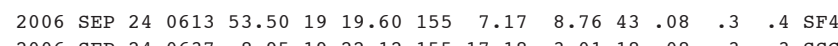

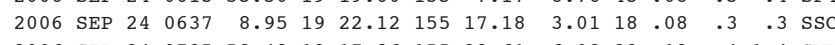

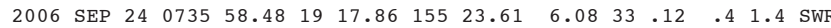

$\begin{array}{llllllllllllll}2006 & \text { SEP } 24 & 0751 & 48.26 & 19 & 12.47 & 155 & 19.98 & 55.22 & 16 & .09 & 1.5 & 1.0 & \text { DEP }\end{array}$

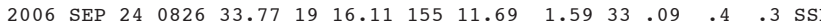

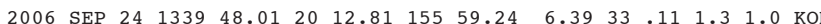

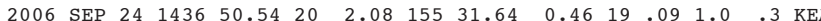

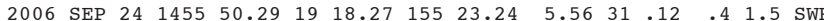

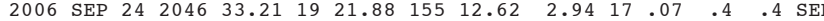

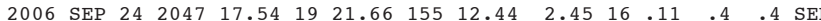

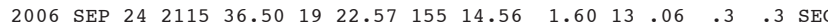

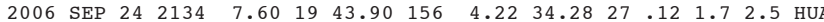

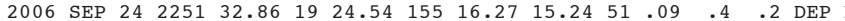

$\begin{array}{llllllllllllll}2006 & \text { SEP } 24 & 2357 & 4.00 & 19 & 12.53 & 155 & 42.56 & 7.82 & 26 & .10 & .4 & 1.1 & \text { LSW }\end{array}$

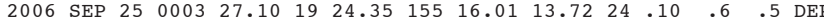

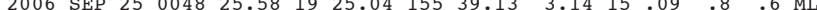

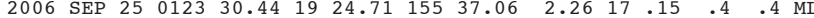

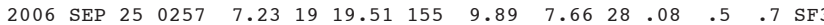

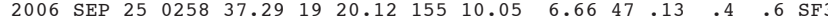

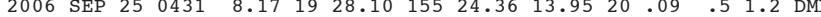

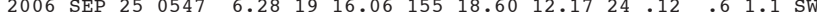

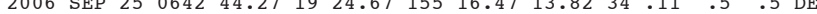

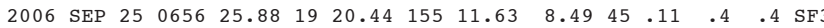

PREF AZ MIN 90

$2.0 \times 228 \quad 4$ $1.6 \times \quad 71 \quad 5$ $1.3 \times 1043$ $1.3 \times 155 \quad 4$

$\begin{array}{lll}1.3 \mathrm{X} & 97 & 6\end{array}$ $\begin{array}{lll}1.7 \times & 82 & 2\end{array}$ $1.8 \times 17111$ 1.5X $75 \quad 5$

$\begin{array}{lll}1.5 \times 182 & 6\end{array}$

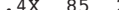
$\begin{array}{lll}1.6 \mathrm{X} & 64 \quad 6\end{array}$ $1.3 \times \quad 304 \quad 25$ 1. $2 \times 155 \quad 5$

$\begin{array}{lll}1.9 \times \quad 252 \quad 14 & 14\end{array}$ $\begin{array}{lll}1.4 \times & 97 & 3 \\ 1.7 \times & 118 & 1\end{array}$ $\begin{array}{lll}1.7 \mathrm{x} & 118 & 1 \\ 1.1 \mathrm{x} & 207 & 7\end{array}$ 1.6x $211 \quad 15$

$1.2 \times 175 \quad 5$ $\begin{array}{lll}1.2 \times & 70 & 1\end{array}$ $2.1 \times 144 \quad 4$ $\begin{array}{llll}1.3 \times & 112 & 4\end{array}$

1.5X 22918 $\begin{array}{llll}1.4 \times & 177 & 4\end{array}$ $2.7 \times 300 \quad 24$ $\begin{array}{rrr}1.3 \times & 233 & 24 \\ 1.6 \times & 110 & 4\end{array}$

$2.0 \times \quad 99 \quad 2$ $\begin{array}{rrr}.3 \mathrm{X} & 92 & 2 \\ .4 \mathrm{X} & 134 & 2\end{array}$ $1.8 \times 237 \quad 25$

$\begin{array}{lll}.6 \mathrm{X} & 68 \quad 8\end{array}$ $1.2 \mathrm{U} 124 \quad 1$ $1.3 \times 2013$ $1.7 \mathrm{U} \quad 76 \quad 1$ $\begin{array}{lll}1.5 \times & 95 & 5\end{array}$

$\begin{array}{lll}.1 \times \quad 83 & 5\end{array}$ $\begin{array}{lll}1.2 \mathrm{X} & 82 & 4\end{array}$ $\begin{array}{lll}.2 \times 183 & 4\end{array}$ $\begin{array}{lll}1.5 \times & 77 & 5\end{array}$ 
---ORIGIN TIME (HST)-- -LAT N-- --LON W-- DEPTH N RMS ERH ERZ LOC PREF AZ MIN YEAR MON DA HRMN SEC DEG MIN DEG MIN KM RD SEC KM KM REMRS MAG GAP DS

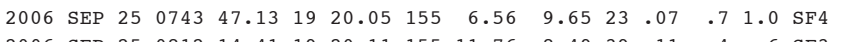

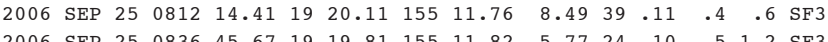

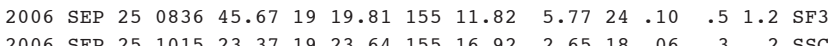

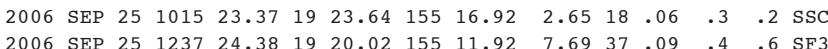

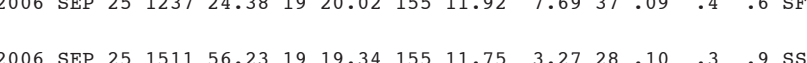

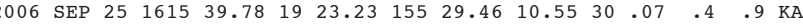

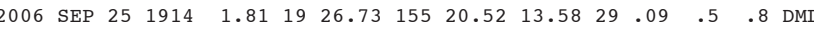

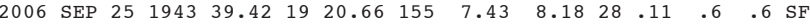
$\begin{array}{llllllllllllllll}2006 & \text { SEP } & 25 & 2120 & 8.46 & 19 & 25.73 & 155 & 24.32 & 11.24 & 21 & .10 & .4 & 1.0 & \mathrm{KAO}\end{array}$

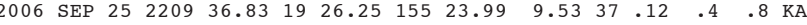

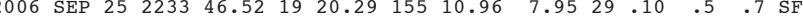

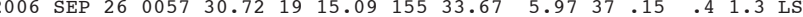

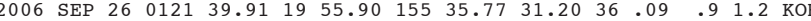

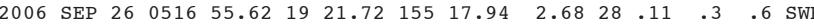

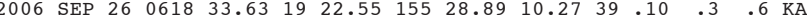

○ 2006 SEP 26

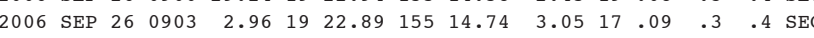

$\begin{array}{llllllllllllll}2006 & \text { SEP } 26 & 1218 & 36.57 & 19 & 19.56 & 155 & 7.55 & 6.18 & 22 & .12 & .5 & 1.4 & \text { SF }\end{array}$

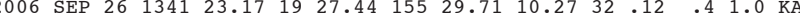

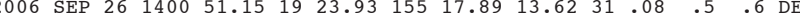

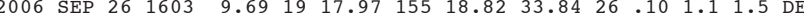
2006 SEP $26 \quad 1654 \quad 45.57 \quad 19 \quad 37.33 \quad 155 \quad 45.57 \quad 34.07 \quad 16 \quad 07 \quad 1.2 \quad 1.5$ KON

$\begin{array}{llllllllllllll}2006 & \text { SEP } & 26 & 1655 & 53.77 & 19 & 21.87 & 155 & 11.04 & 2.26 & 29 & .09 & .3 & .3\end{array}$ SER

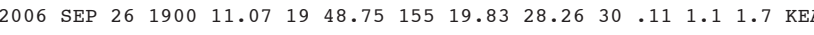
$\begin{array}{llllllllllllll}2006 & \text { SEP } 26 & 1904 & 2.99 & 19 & 25.08 & 155 & 39.18 & 3.34 & 18 & .09 & .8 & .7 & \mathrm{MLO}\end{array}$

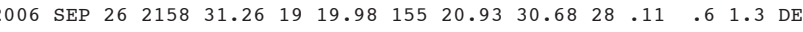

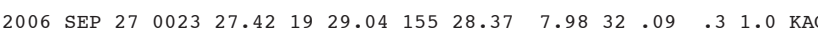

$\begin{array}{llllllllllllll}2006 & \text { SEP } 27 & 0638 & 2.61 & 19 & 17.23 & 155 & 13.91 & 8.03 & 26 & .10 & .6 & .9 & \text { SF } 2\end{array}$

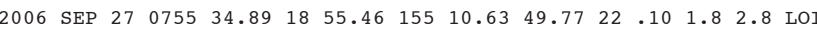

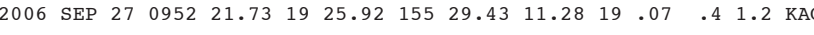
$\begin{array}{llllllllllllll}2006 & \text { SEP } 27 & 1001 & 16.44 & 18 & 56.59 & 155 & 15.54 & 34.04 & 15 & .08 & 1.8 & 4.4 & \text { LOI }\end{array}$

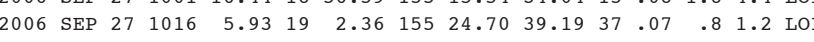

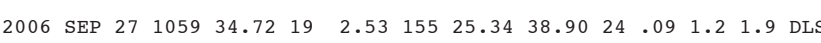
$\begin{array}{llllllllllllll}2006 & \text { SEP } 27 & 1221 & 29.95 & 19 & 18.22 & 155 & 23.52 & 4.61 & 35 & .12 & .4 & 1.3 & \text { SWR }\end{array}$

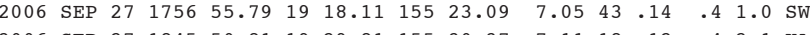

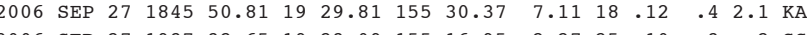

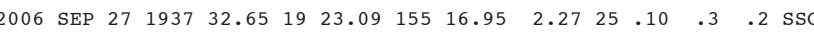

$\begin{array}{llllllllllllll}2006 & \text { SEP } 27 & 2255 & 47.53 & 19 & 20.79 & 155 & 11.28 & 8.55 & 35 & .09 & .5 & .6 & \text { SF3 }\end{array}$ $\begin{array}{llllllllllllll}2006 & \text { SEP } 28 & 0154 & 43.37 & 19 & 19.15 & 155 & 9.85 & 7.74 & 35 & .08 & .4 & .7 & \text { SF } 3\end{array}$

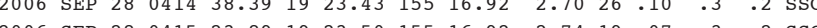

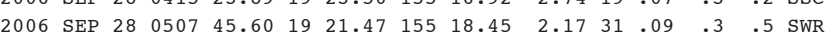

$1.8 \times 152 \quad 6$ $\begin{array}{lll}.81 & 81 & 5 \\ 1.18 & 87 & 6\end{array}$ $1.5 \times \quad 68 \quad 0$ $1.8 \times \quad 82 \quad 5$

$\begin{array}{rrrr}2 \times & 98 & 5\end{array}$ $\begin{array}{rrr}.5 \times & 75 & 3 \\ .4 \times & 127 & 6\end{array}$ $1.6 \times 128 \quad 5$ $1.2 \times \quad 79 \quad 8$

$\begin{array}{llll}4 \times & 77 & 7\end{array}$ $1.5 \times \quad 82 \quad 5$ $1.7 \times 1120$ $1.8 \times 24910$ $.5 \times \quad 56 \quad 4$

$\begin{array}{lll}7 \times \quad 60 & 2\end{array}$ .5X 28835 $.9 \times \quad 75 \quad 3$ $1.9 \times \quad 79 \quad 3$ $\begin{array}{lll}1.5 \mathrm{X} & 69 & 2\end{array}$

$1.3 \times 156 \quad 4$ $\begin{array}{lll}1.7 \times & 57 \quad 9\end{array}$ $1.6 \mathrm{X} \quad 50$ $1.4 \times 155 \quad 0$ 1.1X 14111

$\begin{array}{lll}1.8 \times & 90 & 2\end{array}$ $1.4 \times 237 \quad 28$ $1.6 \times 202 \quad 3$ $1.6 \times 814$ $\begin{array}{lll}1.6 \mathrm{X} & 68 & 6\end{array}$ $\begin{array}{lll}1.2 \times & 147 \quad 1\end{array}$ $1.8 \times 29340$ $\begin{array}{lll}1.3 \mathrm{X} & 62 \quad 7\end{array}$ $1.6 \times 31748$ $1.9 \times 21214$

$1.8 \times 252 \quad 14$ $\begin{array}{lll}1.5 \times & 109 & 4\end{array}$ $2.2 \times 112 \quad 4$ $\begin{array}{lll}1.3 \times & 105 & 6 \\ 1.9 \times & 115 & 1\end{array}$

$1.5 \times 143$ $1.5 \times 143 \quad 4$ $\begin{array}{llll}1.6 \times & 105 & 5 \\ 1.6 \times & 45 & 0\end{array}$ $\begin{array}{lll}1.6 \mathrm{X} & 45 & 0 \\ 1.5 \mathrm{X} & 52 & 0\end{array}$ $\begin{array}{lll}1.6 \mathrm{X} & 58 \quad 4\end{array}$
---ORIGIN TIME (HST)-- -LAT N-- --LON W-- DEPTH N RMS ERH ERZ LOC YEAR MON DA HRMN SEC DEG MIN DEG MIN KM RD SEC KM KM REMKS

$\begin{array}{llllllllllllll}2006 & \text { SEP } 28 & 0533 & 12.50 & 19 & 23.47 & 155 & 16.86 & 2.93 & 26 & .06 & .3 & .2 & \text { SSC }\end{array}$

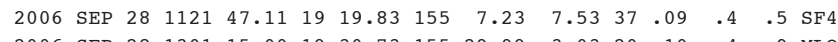
$\begin{array}{llllllllllllll}2006 & \text { SEP } 28 & 1301 & 15.00 & 19 & 30.73 & 155 & 29.99 & 3.03 & 20 & .10 & .4 & .9 & \text { MLO }\end{array}$ $\begin{array}{llllllllllllll}2006 & \text { SEP } 28 & 1551 & 58.38 & 19 & 6.49 & 155 & 28.14 & 30.23 & 38 & .08 & .6 & 1.3 & \text { DLS }\end{array}$

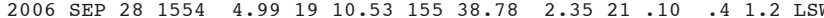

$\begin{array}{lllllllllllll}2006 & \text { SEP } 28 & 1637 & 44.48 & 19 & 23.42 & 155 & 16.88 & 2.84 & 23 & .08 & .3 & .2\end{array}$ SSC

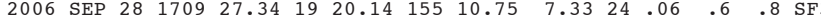

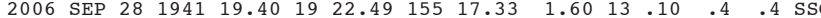

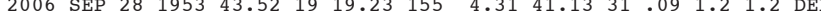

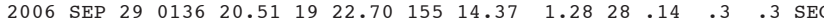

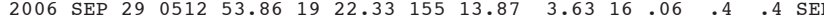
$\begin{array}{llllllllllllll}2006 & \text { SEP } 29 & 0541 & 1.16 & 19 & 29.57 & 155 & 25.92 & 4.41 & 18 & .10 & .3 & 1.5 & \text { KAO }\end{array}$

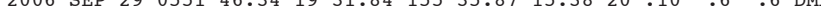
$\begin{array}{llllllllllllll}2006 & \text { SEP } 29 & 0618 & 49.58 & 19 & 20.54 & 155 & 48.08 & 10.97 & 36 & .13 & .4 & .4 & \text { KON }\end{array}$ $\begin{array}{llllllllllllll}2006 & \text { SEP } 29 & 0656 & 11.30 & 19 & 21.98 & 155 & 25.30 & 10.21 & 19 & .09 & .4 & 1.0 & \text { KAO }\end{array}$

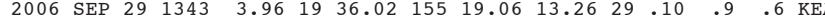

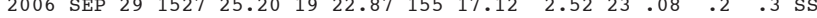
$\begin{array}{llllllll}18.32 & 3.70 & 22 & .09 & .3 & .6 & \text { SWR }\end{array}$

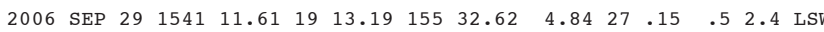

$\begin{array}{llllllllllllll}2006 & \text { SEP } 29 & 1615 & 3.81 & 19 & 2.94 & 155 & 22.56 & 37.42 & 24 & .09 & 1.4 & 1.7 & \text { LOI }\end{array}$

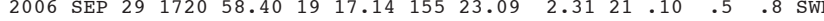

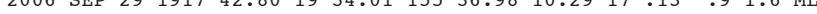

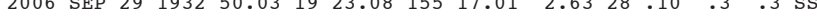

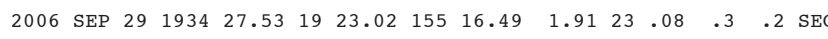

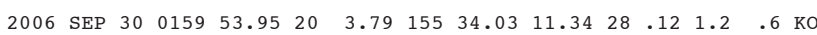

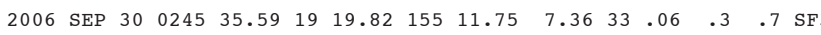

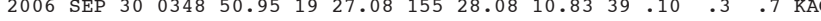

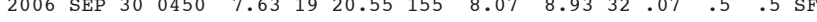

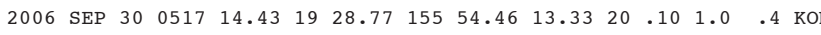

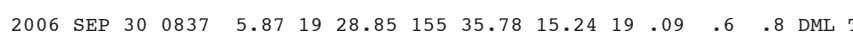

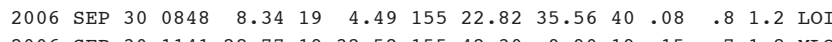

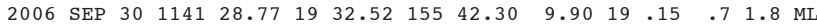

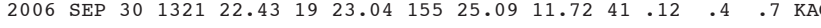
$\begin{array}{lllll}11 & .4 & .4 & \text { SME }\end{array}$ $\begin{array}{llllllllllllll}2006 & \text { SEP } 30 & 1631 & 57.58 & 19 & 20.38 & 155 & 7.48 & 7.87 & 48 & .11 & .4 & .5 & \text { SF } 4\end{array}$ $\begin{array}{lllllllllllll}2006 & \text { SEP } 30 & 1739 & 16.33 & 19 & 24.81 & 155 & 19.21 & 6.96 & 27 & .09 & .4 & .8 \\ \text { KAO }\end{array}$

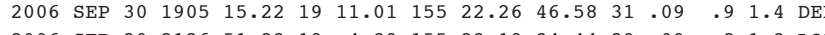

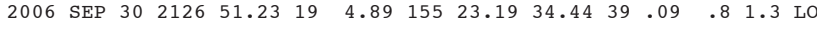
$\begin{array}{lllllllllllllll}2006 & \text { OCT } & 1 & 0431 & 43.71 & 19 & 21.01 & 155 & 18.33 & 31.84 & 39 & .10 & .7 & .9 & \text { DEP }\end{array}$

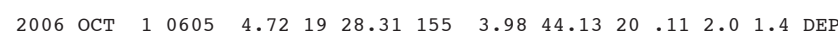

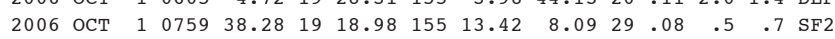

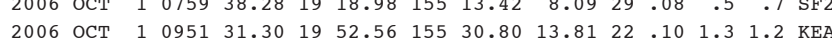

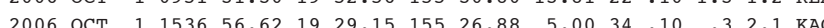
$\begin{array}{lllllllllllllll}2006 & \text { OCT } & 1 & 1541 & 47.52 & 19 & 29.44 & 155 & 26.16 & 7.32 & 18 & .09 & .4 & 1.3 & \text { KAO }\end{array}$
PREF AZ MIN 92 $.5 \times \quad 44 \quad 0$ $.0 \times 163 \quad 5$ $\begin{array}{rrr}.0 \times & 185 & 6 \\ .4 \times \quad 92 & 13\end{array}$

$1.6 \times \quad 520$ $1.5 \times 164 \quad 5$ $1.2 \times 156 \quad 2$ $\begin{array}{rrr}.7 \times & 79 & 2\end{array}$

$\begin{array}{lll}1.6 \mathrm{X} & 78 & 1\end{array}$ $\begin{array}{lll}1.4 \mathrm{X} & 88 \quad 5\end{array}$ $1.1 \times 124 \quad 5$ $1.8 \times 101 \quad 11$ $1.1 \times \quad 81 \quad 4$

$\begin{array}{lll}1.5 \times & 227 & 14\end{array}$ $1.2 \times 184$ $1.4 \mathrm{X} \quad 49 \quad 1$ $\begin{array}{lll}1.3 \times & 68 & 3\end{array}$ $\begin{array}{lll}1.5 \mathrm{x} & 78 \quad 6\end{array}$

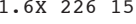
$1.6 \mathrm{U} 163 \quad 6$ $\begin{array}{lll}1.3 \times & 184 & 9\end{array}$ $\begin{array}{lll}1.7 \mathrm{X} & 46 & 1 \\ 1.6 \mathrm{X} & 57 & 1\end{array}$

2.0X 27925 $\begin{array}{lll}1.2 \mathrm{X} & 87 & 6\end{array}$ $1.5 \times \quad 55 \quad 8$ $1.3 \times 115 \quad 5$

$\begin{array}{lll}.3 \times \quad 72 \quad 1 \\ 19 x & 205 & 13\end{array}$ $1.9 \times 20513$
$1.4 \times 113$ $\begin{array}{llll}1.4 \times 113 \quad 7 & 7\end{array}$ $\begin{array}{lrr}1.7 \times & 57 & 5 \\ 2.0 \times & 133 & \end{array}$ .18 $105 \quad 2$ $\begin{array}{lll}1.1 \times & 105 \quad 2 \\ 1.5 \times & 171 & 10\end{array}$ $1.5 \times 17110$ $1.6 \times 565$ $1.6 \times 28611$ $\begin{array}{ll}.6 \times & 286 \\ 11 & 75 \quad 4\end{array}$ $\begin{array}{lll}1.7 \times & 75 & 4\end{array}$ $\begin{array}{rrr}7 x & 280 & 30 \\ 6 x & 93 & 5\end{array}$ $1.3 \times 104 \quad 5$ 
---ORIGIN TIME (HST)-- -LAT N-- --LON W-- DEPTH N RMS ERH ERZ LOC

$\begin{array}{lllllllllllllll}2006 & \text { OCT } & 1 & 1723 & 29.73 & 19 & 19.83 & 155 & 7.13 & 8.39 & 34 & .07 & .5 & .6 & \mathrm{SF} 4\end{array}$ $\begin{array}{lllllllllllllll}2006 & \text { ост } & 1 & 1846 & 57.83 & 19 & 23.11 & 155 & 16.87 & 2.86 & 21 & .07 & .3 & .2 & \text { SSC }\end{array}$ $\begin{array}{llllllllllllllll}2006 & \text { OCT } & 1 & 2342 & 58.77 & 19 & 18.72 & 155 & 15.52 & 5.45 & 31 & .12 & .5 & 1.5 & \text { SF } 1\end{array}$

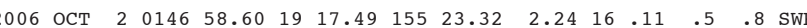
$\begin{array}{llllllllllllllll}2006 & \text { ОСТ } & 2 & 0323 & 9.80 & 19 & 24.59 & 155 & 19.14 & 5.71 & 22 & .07 & .4 & .8 & \text { KAO }\end{array}$

$\begin{array}{lllllllllllllll}2006 & \text { OCT } & 2 & 0758 & 47.05 & 19 & 23.16 & 155 & 14.99 & 3.13 & 19 & .08 & .3 & .4 & \text { SEC }\end{array}$ $\begin{array}{llllllllllllllll}2006 & \text { OCT } & 2 & 0831 & 44.14 & 19 & 1.54 & 155 & 28.68 & 40.49 & 25 & .07 & 1.1 & 1.7 & \text { DLS }\end{array}$ $\begin{array}{lllllllllllllllll}2006 & \text { ОСт } & 2 & 0943 & 16.27 & 19 & 22.77 & 155 & 17.11 & 2.78 & 17 & .05 & .2 & .3 & \text { SSC }\end{array}$ $\begin{array}{lllllllllllllll}2006 & \text { оCT } & 2 & 1434 & 13.49 & 19 & 23.08 & 155 & 17.01 & 2.83 & 34 & .10 & .2 & .2 & \text { SSC }\end{array}$ $\begin{array}{lllllllllllllll}2006 & \text { ост } & 2 & 1616 & 34.65 & 19 & 19.35 & 155 & 26.42 & 10.22 & 48 & .13 & .4 & .6 & \text { KAO }\end{array}$

$\begin{array}{lllllllllllllll}2006 & \text { ОСТ } & 2 & 1805 & 17.38 & 19 & 19.29 & 155 & 8.76 & 7.58 & 23 & .08 & .5 & 1.0 & \text { SF4 }\end{array}$ $\begin{array}{lllllllllllllll}2006 & \text { OCT } & 2 & 1808 & 27.71 & 19 & 22.99 & 155 & 15.02 & 1.13 & 22 & .11 & .2 & .3 & \text { SEC }\end{array}$ $\begin{array}{lllllllllllllll}2006 & \text { OCT } & 2 & 1808 & 58.88 & 19 & 22.99 & 155 & 15.01 & 1.28 & 18 & .06 & .2 & .3 & \text { SEC }\end{array}$

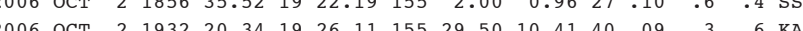

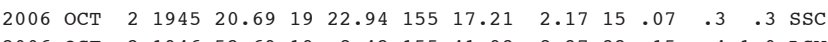
$\begin{array}{lllllllllllllll}2006 & \text { OCT } & 2 & 1946 & 52.69 & 19 & 8.42 & 155 & 41.93 & 8.37 & 32 & .15 & .4 & 1.0 & \text { LSW }\end{array}$

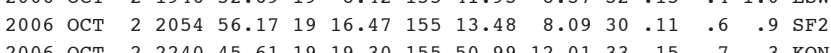

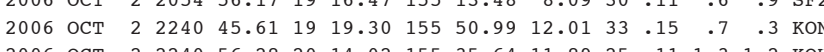

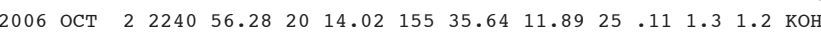

$\begin{array}{lllllllllllllll}2006 & \text { ост } & 2 & 2255 & 35.74 & 19 & 11.35 & 155 & 42.49 & 8.82 & 30 & .12 & .4 & 1.4 & \text { LSW }\end{array}$ $\begin{array}{llllllllllllllll}2006 & \text { ост } & 3 & 0001 & 34.96 & 19 & 13.06 & 155 & 35.85 & 1.62 & 22 & .13 & .4 & .9 & \text { LSW }\end{array}$ $\begin{array}{lllllllllllllll}2006 & \text { ОСТ } & 3 & 0409 & 47.06 & 19 & 21.65 & 155 & 4.72 & 7.13 & 27 & .10 & .5 & .6 & \text { SF5 } \\ 2006 & \text { ОСт } & 3 & 0614 & 37.85 & 19 & 21.33 & 155 & 4.37 & 8.64 & 30 & .12 & .6 & .4 & \text { SF5 }\end{array}$

$\begin{array}{lllllllllllllll}2006 & \text { оСт } & 3 & 1534 & 26.79 & 19 & 19.11 & 155 & 10.18 & 8.77 & 32 & .07 & .4 & .8 & \text { SF3 }\end{array}$

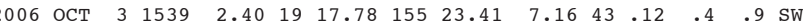

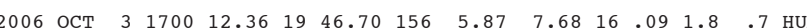
$\begin{array}{llllllllllllllll}2006 & \text { ОСТ } & 3 & 1735 & 37.45 & 19 & 17.49 & 155 & 15.30 & 9.82 & 31 & .11 & .5 & .8 & \text { SF } 1\end{array}$ $\begin{array}{llllllllllllll}2006 & \text { ОСТ } & 3 & 2330 & 51.64 & 19 & 22.57 & 155 & 17.42 & 2.19 & 22 & .07 & 2 & 3\end{array}$

$\begin{array}{lllllllllllllll}2006 & \text { ОСт } & 3 & 2331 & 28.23 & 19 & 22.79 & 155 & 13.40 & 11.80 & 17 & .13 & 1.2 & .7 & \mathrm{SF} 2\end{array}$

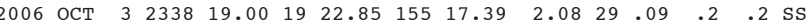

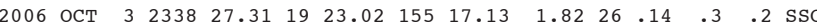
$\begin{array}{llllllllllllllll}2006 & \text { OCT } & 4 & 0213 & 30.72 & 19 & 17.51 & 155 & 23.54 & 1.76 & 21 & .13 & .5 & .7 & \text { SWR }\end{array}$ $\begin{array}{llllllllllllll}2006 & \text { OCT } & 4 & 0221 & 51.49 & 19 & 17.46 & 155 & 23.29 & 3.31 & 24 & .13 & .6 & 1.1\end{array}$

$\begin{array}{lllllllllllllll}2006 & \text { ОСт } & 4 & 0227 & 33.87 & 19 & 23.02 & 155 & 17.14 & 2.71 & 17 & .10 & .4 & .3 & \text { SSC }\end{array}$

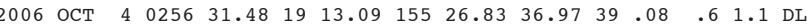
$\begin{array}{lllllllllllllll}2006 & \text { OCT } & 4 & 0427 & 0.51 & 19 & 13.22 & 155 & 27.50 & 34.44 & 20 & .06 & .9 & 1.7 & \text { DLS }\end{array}$ $\begin{array}{llllllllllllllll}2006 & \text { OCT } & 4 & 0511 & 4.36 & 19 & 12.95 & 155 & 27.21 & 35.62 & 27 & .07 & .9 & 1.4 & \text { DLS }\end{array}$ $\begin{array}{lllllllllllllll}2006 & \text { ост } & 4 & 0616 & 26.45 & 19 & 10.35 & 155 & 27.90 & 9.01 & 22 & .11 & .7 & 1.2 & \text { LSW }\end{array}$

$\begin{array}{lllllllllllllll}2006 & \text { OCT } & 4 & 0719 & 36.29 & 19 & 56.44 & 155 & 32.68 & 42.46 & 29 & .12 & 1.8 & 2.3 & \text { KEA }\end{array}$

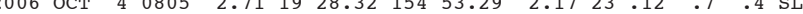
$\begin{array}{lllllllllllllll}2006 & \text { OCT } & 4 & 1051 & 51.51 & 19 & 19.53 & 155 & 10.32 & 5.58 & 29 & .11 & .5 & 1.5 & \mathrm{SF} 3\end{array}$

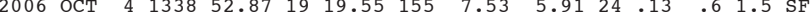
$\begin{array}{lllllllllllllll}2006 & \text { ОСТ } & 4 & 1512 & 51.28 & 19 & 22.96 & 155 & 17.19 & 2.35 & 15 & .06 & .3 & .3 & \text { SSC }\end{array}$ $1.5 \times 142$ $\begin{array}{rrr}1.5 \times & 47 & 1 \\ 1.2 \times & 125 & 5\end{array}$

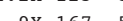
$.0 \times 167 \quad 5$

$1.6 \times \quad 69 \quad 2$ $1.6 \times 260-15$ $1.5 \times \quad 59 \quad 1$ $2.0 \times \quad 41 \quad 1$ $1.8 \times \quad 88 \quad 6$ $1.0 \times 100 \quad 4$ $\begin{array}{llll}1.7 \times \quad 69 & 2\end{array}$ $\begin{array}{lll}1.7 \times & 69 & 2 \\ 1.1 \times & 135 & 2\end{array}$ 2.0X $206 \quad 5$ $\begin{array}{lll}1.0 \times & 206 \\ 1.7 \mathrm{X} & 41\end{array}$ $1.4 \times \quad 64 \quad 1$ $\begin{array}{lll}1.8 \times & 112 & 7 \\ 1.7 \times & 186 & 1\end{array}$ $\begin{array}{lll}1.7 \times & 186 & 1 \\ 1.6 \times & 138 & 7\end{array}$ $2.2 \times 301 \quad 42$ $\begin{array}{rrr}1.7 \mathrm{X} & 73 & 7 \\ 1.3 \mathrm{x} & 82 & 11\end{array}$ $\begin{array}{lll}.3 \times & 82 & 11\end{array}$ $\begin{array}{lll}1.4 \times & 158 & 5 \\ 1.7 \times & 167 & 6\end{array}$ $\begin{array}{rrr}1.7 \times & 167 & 6 \\ 1.8 \times & 92 & 6\end{array}$

$1.5 \times 108 \quad 5$ $2.5 \times 114$ $1.5 \times 317 \quad 29$ $1.2 \times 132$ $\begin{array}{rrr}1.2 \times & 53 & 2\end{array}$

$\begin{array}{lll}1.8 \times & 173 & 1\end{array}$ $\begin{array}{lll}1.5 \times & 47\end{array}$ $\begin{array}{lll}1.8 \mathrm{X} & 47 & 1\end{array}$ $1.0 \times 165 \quad 5$ 1.2X $167 \quad 5$

$\begin{array}{lll}1.2 \times \quad 79 & 1\end{array}$ $\begin{array}{lll}1.7 \times & 120 \quad 7\end{array}$ $1.4 \times 126 \quad 6$ $1.5 \times 115 \quad 6$ $1.2 \times 136 \quad 2$
--ORTGIN TIME (HST)-- -LAT N-- --LON W-- DEPTH N RMS ERH ERZ LOC YEAR MON DA HRMN SEC DEG MIN DEG MIN DM RD SEC KM KM REMKS $\begin{array}{lllllllllllllll}2006 & \text { OCT } & 4 & 1605 & 24.12 & 19 & 19.83 & 155 & 7.87 & 6.34 & 40 & .12 & .4 & .8 & \text { SF4 }\end{array}$ $\begin{array}{llllllllllllll}2006 & \text { ОСТ } & 4 & 1830 & 39.83 & 19 & 25.39 & 154 & 57.62 & 3.97 & 29 & .12 & 1.1 & .5\end{array}$ $\begin{array}{lllllllllllllll}2006 & \text { OCT } & 4 & 1952 & 44.22 & 19 & 20.59 & 155 & 10.24 & 7.39 & 30 & .08 & .5 & .7 & \mathrm{SF} 3\end{array}$ $\begin{array}{llllllllllllllll}2006 & \text { OCT } & 4 & 2235 & 26.69 & 19 & 17.84 & 155 & 23.41 & 5.42 & 15 & .14 & .8 & 2.2 & \text { SWR }\end{array}$ $\begin{array}{lllllllllllllll}2006 & \text { ОСТ } & 5 & 0042 & 54.39 & 19 & 3.93 & 155 & 23.68 & 39.31 & 22 & .09 & 1.5 & 1.3 & \text { LOI }\end{array}$

$\begin{array}{llllllllllllll}2006 & \text { ОСТ } & 5 & 0117 & 9.34 & 19 & 22.25 & 155 & 27.55 & 9.92 & 43 & .12 & .4 & .5 \\ \text { КАО }\end{array}$

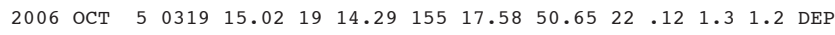
$\begin{array}{lllllllllllllll}2006 & \text { ОСТ } & 5 & 0342 & 6.06 & 19 & 25.02 & 155 & 29.54 & 11.86 & 15 & .07 & .5 & 1.1 & \text { KAO }\end{array}$ $\begin{array}{lllllllllllllll}2006 & \text { ОСТ } & 5 & 0420 & 14.19 & 19 & 29.53 & 155 & 25.65 & 6.39 & 16 & .13 & .7 & 2.0 & \text { КАО }\end{array}$

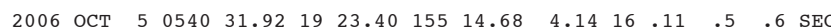

$\begin{array}{lllllllllllllll}2006 & \text { Ост } & 5 & 0616 & 43.62 & 19 & 0.69 & 155 & 28.36 & 42.40 & 24 & .07 & 1.0 & 1.9 & \text { DLS }\end{array}$

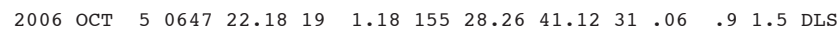

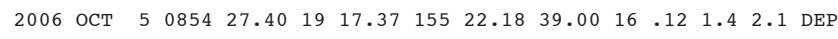
$\begin{array}{lllllllllllllll}2006 & \text { ОСт } & 5 & 1139 & 38.88 & 19 & 20.32 & 155 & 12.93 & 7.99 & 30 & .09 & .5 & .8 & \mathrm{SF} 2\end{array}$

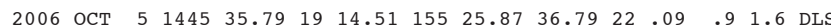

$\begin{array}{lllllllllllllll}2006 & \text { ост } & 5 & 1625 & 45.36 & 19 & 24.84 & 155 & 18.84 & 6.30 & 20 & .11 & .5 & 1.0 & \text { INT }\end{array}$

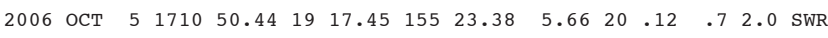

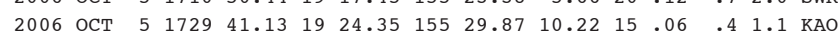
$\begin{array}{lllllllllllllll}2006 & \text { OCT } & 5 & 2020 & 10.17 & 19 & 12.45 & 155 & 27.65 & 8.86 & 40 & .12 & .4 & .8 & \text { LSW }\end{array}$

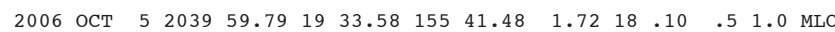

$\begin{array}{llllllllllllll}2006 & \text { OCT } & 5 & 2354 & 53.14 & 19 & 28.09 & 155 & 24.89 & 10.03 & 19 & .13 & .5 & 1.1 \\ \text { KAO }\end{array}$ $\begin{array}{lllllllllllllll}2006 & \text { OCT } & 6 & 0335 & 9.87 & 19 & 17.59 & 155 & 13.13 & 7.86 & 31 & .11 & .6 & .8 & \text { SF2 }\end{array}$ $\begin{array}{lllllllllllllll}2006 & \text { OCT } & 6 & 0402 & 24.26 & 19 & 16.75 & 155 & 24.03 & 1.08 & 15 & .09 & .5 & .8 & \text { SWR }\end{array}$ $\begin{array}{lllllllllllllll}2006 & \text { OCT } & 6 & 0446 & 55.17 & 19 & 22.69 & 155 & 17.18 & 2.44 & 21 & .05 & .3 & .3 & \text { SSC }\end{array}$ $\begin{array}{lllllllllllllll}2006 & \text { ОСТ } & 6 & 0639 & 24.46 & 19 & 19.48 & 155 & 8.72 & 7.21 & 30 & 09 & .5 & .9 & \end{array}$

$\begin{array}{lllllllllllllll}2006 & \text { ОСт } & 6 & 0709 & 7.96 & 19 & 19.67 & 155 & 8.98 & 7.42 & 31 & .11 & .5 & .8 & \mathrm{SF} 4\end{array}$ $\begin{array}{lllllllllllllll}2006 & \text { OCT } & 6 & 1009 & 31.07 & 19 & 29.68 & 155 & 25.88 & 5.78 & 17 & .10 & .4 & 1.6 & \text { KAO }\end{array}$

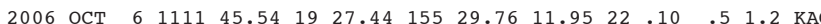

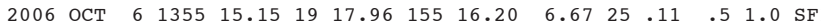
$\begin{array}{llllllllllllllll}2006 & \text { ОСТ } & 6 & 2027 & 5.56 & 19 & 18.64 & 155 & 12.94 & 6.80 & 41 & .11 & .4 & .7 & \text { SF2 }\end{array}$

$\begin{array}{lllllllllllllll}2006 & \text { ОСТ } & 6 & 2051 & 15.29 & 19 & 18.72 & 155 & 13.09 & 6.68 & 37 & .11 & .4 & .8 & \mathrm{SF} 2\end{array}$

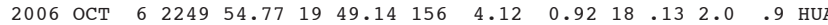
$\begin{array}{lllllllllllllll}2006 & \text { ОСТ } & 6 & 2327 & 6.29 & 19 & 10.65 & 155 & 35.72 & 9.84 & 27 & .14 & .8 & 1.9 & \text { LSW }\end{array}$

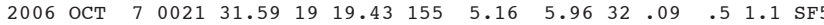
$\begin{array}{lllllllllllllll}2006 & \text { ОСТ } & 7 & 0654 & 51.57 & 19 & 17.19 & 155 & 23.34 & 2.92 & 21 & .15 & .7 & 1.3 & \text { SWR }\end{array}$

$\begin{array}{lllllllllllllll}2006 & \text { ОСТ } & 7 & 0719 & 7.52 & 19 & 23.17 & 155 & 2.75 & 6.97 & 32 & .12 & .6 & .6 & \text { SF5 }\end{array}$

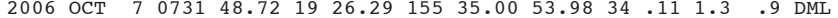

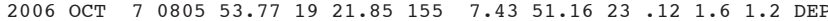
$\begin{array}{lllllllllllllll}2006 & \text { ОСТ } & 7 & 1037 & 57.50 & 19 & 24.96 & 155 & 18.86 & 6.83 & 33 & .11 & .4 & .6 & \text { IN } 1\end{array}$ $\begin{array}{llllllllllllllll}2006 & \text { ОСТ } & 7 & 1427 & 47.13 & 19 & 26.33 & 155 & 29.20 & 11.69 & 24 & .07 & .4 & 1.0 & \text { KAO }\end{array}$

$\begin{array}{llllllllllllll}2006 & \text { OCT } & 8 & 0014 & 35.94 & 19 & 26.01 & 155 & 28.14 & 10.19 & 29 & .09 & .3 & .8 \\ \text { KAO }\end{array}$

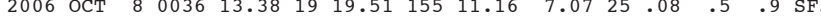

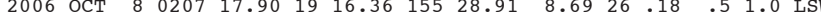

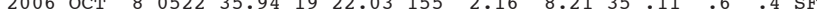
$\begin{array}{lllllllllllllll}2006 & \text { ОСТ } & 8 & 0537 & 36.94 & 19 & 23.18 & 155 & 27.12 & 5.17 & 19 & .12 & .4 & .9 & \text { KАО }\end{array}$
PREF AZ MIN 94 IAC GAP DS

$2.0 \times 124 \quad 5$ $\begin{array}{rrr}1.8 \times & 185 & 2 \\ 1.6 \times & 79 & 4\end{array}$ $1.6 \times \quad 1594$

$2.1 \times \quad 66 \quad 0$ $.8 \times \quad 247 \quad 15$ $1.0 \times 107 \quad 4$ .2x 84

1.8X 22416 $\begin{array}{lll}1.5 \times 210 & 15\end{array}$ $\begin{array}{rrr}.2 \times & 174 & 6 \\ 3 \times & 67 & 4\end{array}$ $1.2 \times 178 \quad 8$

1.0x $104 \quad 2$ $\begin{array}{rrr}1.4 \mathrm{X} & 158 & 5 \\ 1.3 \mathrm{U} & 72 & 5\end{array}$ $\begin{array}{rrrr}1.30 & 72 & 5 \\ 1.8 \times & 147 & 6\end{array}$ $1.3 \mathrm{U} 123 \quad 10$

$\begin{array}{lll}.2 \times \quad 70 \quad 4 & 4\end{array}$ $\begin{array}{lll}1.5 \times & 118 \quad 1\end{array}$ $1.1 \mathrm{U} 178 \quad 6$ $\begin{array}{rrr}.4 \times \quad 50 & 1 \\ 6 \times & 102 & 4\end{array}$ $1.6 \times 1024$

$\begin{array}{lll}1.6 \times & 97 \quad 5\end{array}$ $\begin{array}{rrr}1.4 \times & 111 & 5 \\ 1.4 \times & 75 & 7\end{array}$ $\begin{array}{lll}1.2 \times 149 & 4\end{array}$ $\begin{array}{lll}1.7 \times & 93 & 3\end{array}$

$\begin{array}{lll}1.5 \mathrm{X} & 87 \quad 3\end{array}$ $\begin{array}{lll}.4 \times & 282 & 28 \\ .6 \times & 237 & 13\end{array}$ $1.3 \times 186 \quad 6$ 1.2X $161 \quad 6$

$\begin{array}{lll}1.3 \times & 159 \quad 3\end{array}$ $\begin{array}{lll}2.8 x & 67 & 3\end{array}$ $2.1 \times 166 \quad 10$ $\begin{array}{rrr}.5 \times & 109 & 2 \\ .3 \times & 87 & 7\end{array}$

$\begin{array}{lll}1.6 \mathrm{X} & 46 & 7\end{array}$ $\begin{array}{lll}1.3 \times & 97 & 6\end{array}$ $\begin{array}{rrr}.8 \times & 84 & 3 \\ .3 \times & 180 & 5\end{array}$ $\begin{array}{lll}1.4 \mathrm{X} & 71 & 2\end{array}$ 
---ORIGIN TIME (HST)-- -LAT N-- --LON W-- DEPTH N RMS ERH ERZ LOC PREF AZ MIN 95 YEAR MON DA HRMN SEC DEG MIN DEG MIN KM RD SEC KM KM REMKS MAC GAP DS

$\begin{array}{lllllllllllllll}2006 & \text { OCT } & 8 & 0954 & 35.05 & 19 & 20.53 & 155 & 19.49 & 5.71 & 44 & .12 & .4 & .7 & \text { SWR }\end{array}$ $\begin{array}{lllllllllllllll}2006 & \text { ОСТ } & 8 & 0955 & 19.71 & 19 & 20.21 & 155 & 19.33 & 3.86 & 34 & .12 & .3 & .9 & \text { SWR }\end{array}$

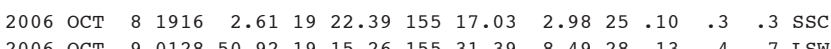
$\begin{array}{llllllllllllllll}2006 & \text { ОСт } & 9 & 0128 & 50.92 & 19 & 15.26 & 155 & 31.39 & 8.49 & 28 & .13 & .4 & .7 & \text { LSW }\end{array}$ $\begin{array}{llllllllllllllll}2006 & \text { ост } & 9 & 0132 & 6.11 & 19 & 18.06 & 155 & 23.28 & 3.89 & 15 & .11 & .7 & 1.4 & \text { SWR }\end{array}$

$\begin{array}{llllllllllllllll}2006 & \text { ОСТ } & 9 & 0458 & 8.29 & 19 & 20.07 & 155 & 11.75 & 6.98 & 34 & .10 & .4 & .8 & \mathrm{SF} 3\end{array}$ $\begin{array}{llllllllllllll}2006 & \text { ост } 9 & 0516 & 27.38 & 19 & 57.10 & 155 & 38.04 & 11.27 & 19 & .13 & .9 & .6 & \text { кон }\end{array}$ $\begin{array}{lllllllllllllll}2006 & \text { ОСТ } & 9 & 0550 & 2.69 & 19 & 25.33 & 155 & 25.85 & 2.01 & 33 & .12 & .3 & .9 & \text { КАO }\end{array}$ $\begin{array}{lllllllllllllll}2006 & \text { ост } & 9 & 0628 & 56.70 & 19 & 18.75 & 155 & 26.30 & 10.17 & 15 & .13 & .6 & 1.4 & \text { LSW }\end{array}$

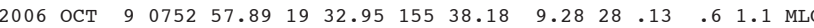

2006 ост $91013 \quad 58.75 \quad 1920.23 \quad 155 \quad 25.79 \quad 52.28 \quad 28.101 .11 .8$ DML

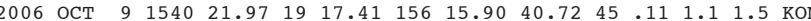
$\begin{array}{lllllllllllllll}2006 & \text { ост } & 9 & 1643 & 17.54 & 19 & 16.96 & 155 & 11.91 & 1.56 & 43 & .10 & .5 & .3 & \text { SS }\end{array}$

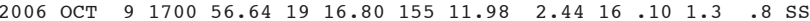
$\begin{array}{lllllllllllllll}2006 & \text { ОСТ } & 9 & 1711 & 32.76 & 19 & 17.44 & 155 & 11.70 & 1.00 & 28 & .12 & .6 & .5 & \text { SS }\end{array}$

$\begin{array}{lllllllllllllll}2006 & \text { ОСТ } & 9 & 2034 & 32.08 & 19 & 18.70 & 155 & 8.43 & 7.55 & 37 & .10 & .5 & .6 & \mathrm{SF} 4\end{array}$

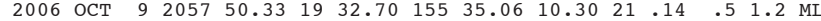

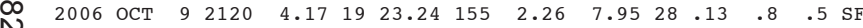
$\begin{array}{llllllllllllll}2006 & \text { OCT } 10 & 0644 & 58.11 & 19 & 20.11 & 155 & 16.59 & 33.24 & 22 & .10 & 1.0 & 1.2 & \text { DEP }\end{array}$ $\begin{array}{lllllllllllll}2006 & \text { OCT } 10 & 0648 & 54.51 & 19 & 24.01 & 155 & 8.50 & 39.32 & 31 & .09 & .8 & 1.2\end{array}$

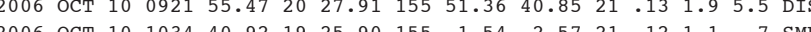
$\begin{array}{llllllllllllll}2006 & \text { OCT } 10 & 1034 & 40.92 & 19 & 25.90 & 155 & 1.54 & 2.57 & 21 & .12 & 1.1 & .7 & \text { SME }\end{array}$ $\begin{array}{llllllllllllll}2006 & \text { OCT } 10 & 1329 & 51.42 & 19 & 19.82 & 155 & 8.66 & 8.96 & 25 & .06 & .5 & 1.0 & \text { SF } 4\end{array}$ $\begin{array}{llllllllllllll}2006 & \text { OCT } 10 & 1839 & 20.16 & 19 & 17.78 & 155 & 14.00 & 6.14 & 32 & .12 & .4 & 1.1 & \text { SF2 } \\ 2006 & \text { OCT } & 10 & 1950 & 14.19 & 19 & 26.13 & 155 & 38.03 & 3.37 & 19 & .09 & .5 & .6\end{array}$

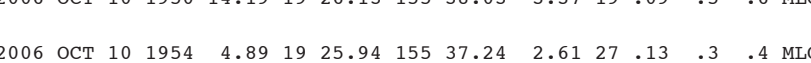

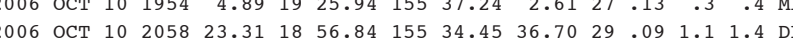

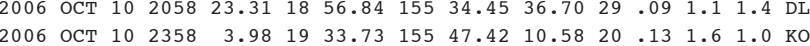

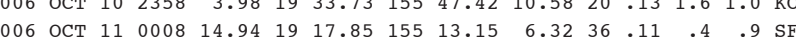

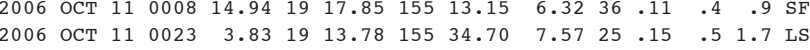

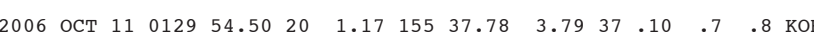
$\begin{array}{llllllllllllll}2006 & \text { ОСТ } 11 & 0453 & 29.43 & 19 & 26.07 & 155 & 37.41 & 2.76 & 44 & .11 & .3 & .4 & \text { MLO }\end{array}$

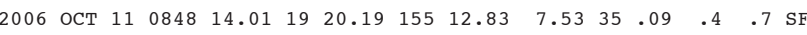

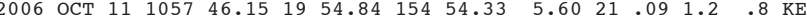

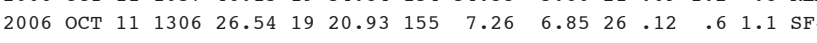

$\begin{array}{llllllllllllll}2006 & \text { OCT } 11 & 1530 & 5.88 & 19 & 29.70 & 155 & 27.73 & 7.94 & 16 & .13 & .5 & 1.7 & \text { KAO }\end{array}$ $\begin{array}{llllllllllllll}2006 & \text { OCT } 11 & 1746 & 46.92 & 19 & 24.36 & 155 & 37.83 & 2.73 & 16 & .15 & .4 & .3 & \text { MLO }\end{array}$ $\begin{array}{lllllllllllllll}2006 & \text { ост } 11 & 1759 & 50.31 & 19 & 19.76 & 155 & 8.67 & 6.66 & 30 & .08 & .4 & .9 & \mathrm{SF}\end{array}$

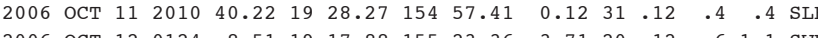

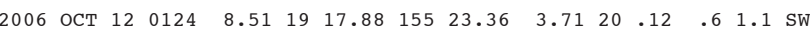

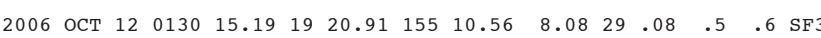

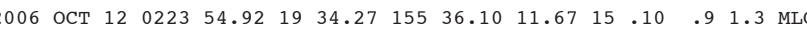

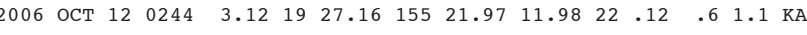
$\begin{array}{lllllllllllllll}2006 & \text { ост } 12 & 0325 & 23.86 & 19 & 23.64 & 155 & 2.13 & 2.95 & 20 & .11 & .6 & .6 & \text { SME } \\ 2006 & \text { ост } & 12 & 0649 & 59.15 & 19 & 22.34 & 155 & 4.13 & 7.73 & 38 & .16 & .5 & .6 & \text { SF5 }\end{array}$
$2.3 \times \quad 61 \quad 4$

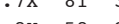
$\begin{array}{lll}1.8 \times & 58 & 2 \\ 1.6 \mathrm{x} & 59 & 2 \\ 1.30 & 55 & 4\end{array}$ $1.3 \mathrm{U} \quad 155 \quad 4$

$1.5 \times \quad 83 \quad 5$ $1.1 \times 17211$ $1.7 \times \quad 61 \quad 6$ $1.4 \mathrm{U} 1296$ $1.6 \times 144 \quad 8$

$1.8 \times \quad 83 \quad 4$ $2.4 \times 27041$ $2.2 \times 153 \quad 3$ $1.1 \times 198$ $1.3 \times 166 \quad 3$

$1.6 \times 106 \quad 3$ $.1 x \quad 76 \quad 7$ $\begin{array}{lll}1.2 \times & 163 \quad 4\end{array}$ $1.5 \times 157$ $\begin{array}{lll}1.4 \times & 76 \quad 2\end{array}$

$2.3 \times 31686$ $1.6 \times 166$ $\begin{array}{lll}1.9 \times & 121 \quad 5\end{array}$ $1.2 \times \quad 92$ $\begin{array}{lll}1.6 \times & 98 \quad 3\end{array}$

$\begin{array}{lll}1.7 \times \quad 79 & 3\end{array}$

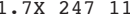
$1.4 \times 246$ $1.2 \times 106 \quad 2$ 1.3X $124 \quad 8$ $1.9 \times 27132$ $\begin{array}{lll}2.8 \mathrm{X} & 65 & 35\end{array}$ $1.4 \times \quad 70$ $2.0 \times 28127$ $1.1 \times 1504$ $\begin{array}{lll}1.4 \mathrm{X} & 87 & 4\end{array}$ $\begin{array}{lll}1.0 \times & 94 & 0\end{array}$ $\begin{array}{lll}1.4 \times & 104 & 5\end{array}$ $1.5 \times 127 \quad 4$ $\begin{array}{lll}1.5 \mathrm{X} & 85 & 4\end{array}$ $\begin{array}{lll}1.1 \times & 210 & 9 \\ 1.2 \times & 133 & 5\end{array}$ $1.2 \times 1335$ $1.5 \times 157 \quad 4$
---ORIGIN TIME (HST)-- -LAT N-- --LON W-- DEPTH N RMS ERH ERZ LOC $\begin{array}{lllllll}\text { YEAR MON DA HRMN } & \text { SEC DEG MIN DEG MIN } & \text { KM } & \text { RD SEC } & \text { KM } & \text { KM REMKS }\end{array}$

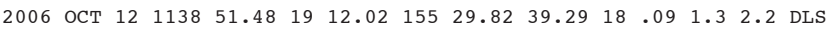
$\begin{array}{lllllllllllllll}2006 & \text { OCT } 12 & 1323 & 12.91 & 19 & 20.21 & 155 & 8.07 & 5.75 & 22 & .14 & .5 & 1.4 & \text { SF } 4\end{array}$

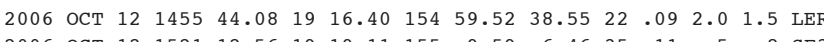
$\begin{array}{lllllllllllllll}2006 & \text { OCT } 12 & 1521 & 12.56 & 19 & 19.11 & 155 & 9.59 & 6.46 & 35.11 & .5 & .8 & \mathrm{SF} 3\end{array}$

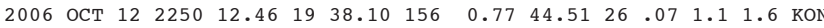

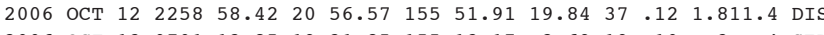
$\begin{array}{llllllllllllll}2006 & \text { ОСТ } 13 & 0701 & 12.85 & 19 & 21.85 & 155 & 13.17 & 2.69 & 19 & .10 & .3 & .4 & \text { SER }\end{array}$

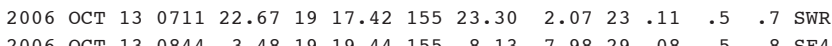

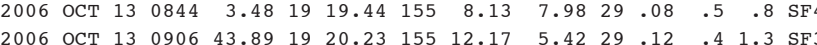

$\begin{array}{llllllllllllll}2006 & \text { ост } 13 & 0933 & 5.76 & 19 & 20.18 & 155 & 11.97 & 6.48 & 33 & .09 & .4 & .8 & \mathrm{SF} 3\end{array}$

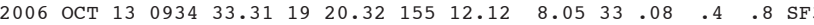

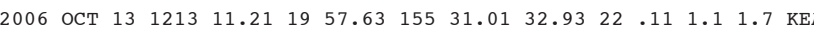
$\begin{array}{llllllllllllll}2006 & \text { OCT } & 13 & 1304 & 6.87 & 19 & 46.47 & 155 & 49.55 & 22.44 & 21 & .13 & 1.2 & 2.1\end{array}$ $\begin{array}{llllllllllllll}2006 & \text { ОСТ } 13 & 1306 & 46.40 & 19 & 22.22 & 155 & 26.20 & 10.51 & 41 & .13 & .4 & .8 & \text { KAO }\end{array}$

$\begin{array}{llllllllllllll}2006 & \text { ОСТ } 13 & 1451 & 14.64 & 19 & 27.96 & 155 & 27.04 & 10.83 & 26 & .13 & .4 & 1.1 & \text { KAO }\end{array}$ $\begin{array}{lllllllllllllll}2006 & \text { ОСТ } 13 & 1557 & 23.96 & 19 & 20.28 & 155 & 8.64 & 6.43 & 43 & .12 & .4 & .7 & \text { SF } 4\end{array}$ $\begin{array}{lllllllllllllll}2006 & \text { OCT } 13 & 1857 & 39.40 & 19 & 19.97 & 155 & 12.86 & 6.20 & 27 & .11 & .5 & 1.1 & \text { SF2 }\end{array}$

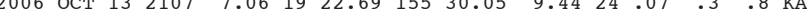

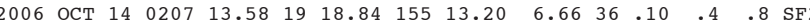

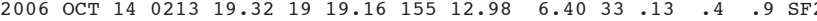

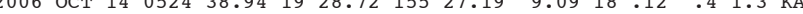

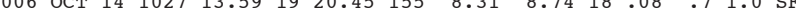

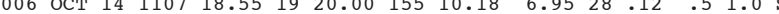
$\begin{array}{llllllllllllll}2006 & \text { оСт } 14 & 1122 & 16.41 & 19 & 22.46 & 155 & 14.05 & 3.69 & 14 & .08 & .7 & .4 & \text { SEC }\end{array}$

$\begin{array}{lllllllllllllll}2006 & \text { OCT } 14 & 1330 & 31.56 & 19 & 25.87 & 155 & 25.12 & 7.43 & 14 & .11 & .5 & 1.7 & \text { KAO }\end{array}$ $\begin{array}{llllllllllllll}2006 & \text { ОСТ } 14 & 1505 & 2.46 & 19 & 24.72 & 155 & 29.84 & 11.08 & 17 & .12 & .5 & 1.3 & \text { KAO }\end{array}$

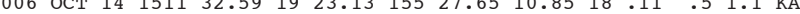

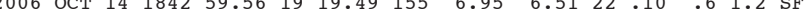
$\begin{array}{llllllllllllll}2006 & \text { ОСт } 14 & 1938 & 19.12 & 19 & 19.38 & 155 & 8.70 & 5.23 & 23 & .09 & .5 & 1.6 & \mathrm{SF} 4\end{array}$

$\begin{array}{llllllllllllll}2006 & \text { OCT } 14 & 2030 & 38.33 & 19 & 20.06 & 155 & 13.09 & 6.04 & 26 & .12 & .4 & 1.1 & \text { SF }\end{array}$ $\begin{array}{lllllllllllllll}2006 & \text { ОСТ } 14 & 2259 & 4.07 & 19 & 19.43 & 155 & 10.92 & 6.74 & 27 & .12 & .5 & .9 & \text { SF } 3\end{array}$

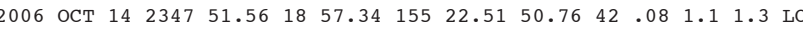

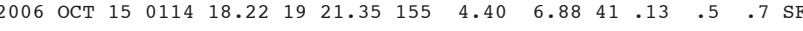
$\begin{array}{llllllllllllll}2006 & \text { ОСТ } 15 & 0226 & 14.69 & 19 & 20.61 & 155 & 12.99 & 8.76 & 43 & .10 & .4 & .4 & \mathrm{SF} 2\end{array}$

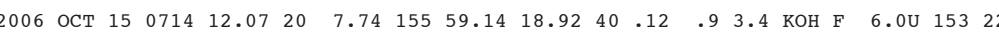

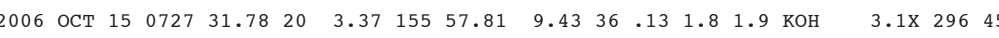

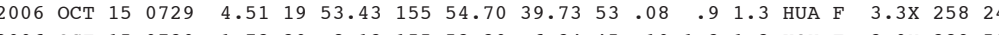

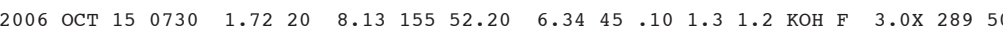

$\begin{array}{llllllllllll}2006 & \text { ост } 15 \quad 0733 & 51.64 & 19 & 53.08 & 156 & 12.65 & 37.51 & 49 & .10 & 1.0 & 1.7\end{array}$ HUA $\begin{array}{llllllllllllll}2006 & \text { OCT } 15 & 0735 & 56.08 & 19 & 52.93 & 155 & 55.84 & 41.96 & 50 & .11 & .9 & 1.2 & \text { HUA }\end{array}$

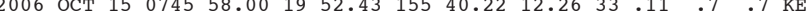
$\begin{array}{llllllll} & \end{array}$

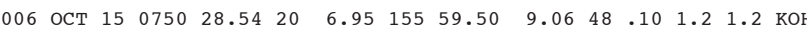

AZ MIN

$1.3 \times 117 \quad 6$ $\begin{array}{llll}.9 \times & 135 & 5 \\ 1.5 \times 24822 & 248\end{array}$

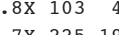

$2.4 \mathrm{U} 326153$ $\begin{array}{lll}1.7 \times & 68 & 1 \\ 1.0 \times & 168 & 5\end{array}$ $\begin{array}{rrr}.5 \times & 118 & 4 \\ .5 & 77 & 5\end{array}$

$\begin{array}{lll}1.3 \times & 79 & 5\end{array}$ $\begin{array}{rrr}1.2 \mathrm{X} & 76 & 5 \\ 1.5 \mathrm{X} & 174 & 19 \\ 1.5 \times & 179\end{array}$ $\begin{array}{llll}1.5 \times \quad 179 & 10\end{array}$ $\begin{array}{lll}.6 \times & 62 \quad 3\end{array}$

$\begin{array}{lll}.3 \times \quad 68 \quad 7 & \end{array}$ $1.1 \times \quad 73 \quad 5$ $\begin{array}{lll}.6 \mathrm{X} & 60 & 4 \\ .5 \mathrm{x} & 83 & 3\end{array}$

$\begin{array}{lll}1.9 \times & 83 & 4\end{array}$ $\begin{array}{lll}1.3 x & 73 & 6 \\ 1.3 x & 111 & 5\end{array}$

$1.3 \times 1115$

$1.6 \times 183 \quad 2$

$\begin{array}{lll}1.0 \times & 70 & 8\end{array}$ $\begin{array}{rrr}.9 \mathrm{x} & 86 & 5 \\ 1.2 \mathrm{x} & 65 & 1\end{array}$ $\begin{array}{llll}1.1 \times 150 & 150\end{array}$ $.8 \times 102$

$\begin{array}{lll}.2 \times \quad 68 & 5\end{array}$ $\begin{array}{lll}.2 \times 100 & 6\end{array}$ $3.3 \times 30645$ $\begin{array}{lll}2.4 \times 189 & 24 \\ 2.1 \times & 161 & 27\end{array}$ $\begin{array}{llll}2.1 \times & 161 & 27\end{array}$ $2.3 \times 23313$ 
---ORIGIN TIME (HST)-- -LAT N-- --LON W-- DEPTH N RMS ERH ERZ LOC YEAR MON DA HRMN SEC DEG MIN DEG MIN KM RD SEC KM KM REMKS

$\begin{array}{lllllllllllll}2006 & \text { OCT } 15 & 0753 & 39.31 & 19 & 52.32 & 155 & 58.21 & 38.32 & 54 & .10 & .8 & 1.2\end{array}$ HUA

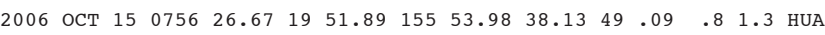
$\begin{array}{lllllllllllll}2006 & \text { ост } 15 & 0759 & 54.07 & 19 & 53.40 & 156 & 0.30 & 38.04 & 39 & .10 & .8 & 1.4\end{array}$ $\begin{array}{lllllllllllllll}2006 & \text { OCT } 15 & 0804 & 30.81 & 19 & 55.07 & 156 & 0.07 & 36.32 & 36 & .11 & 1.1 & 1.3 & \text { KOH }\end{array}$ $\begin{array}{lllllllllllll}2006 & \text { ост } 15 & 0809 & 3.77 & 20 & 2.87 & 155 & 36.80 & 12.66 & 22 & .14 & 2.3 & .9 \\ \text { кон }\end{array}$

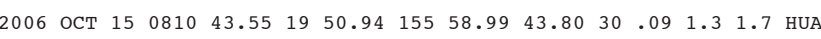
$\begin{array}{lllllllllllll}2006 & \text { ОСТ } & 15 & 0811 & 2.72 & 19 & 53.35 & 155 & 55.18 & 39.30 & 30.11 & 1.4 & 1.5\end{array}$ $\begin{array}{llllllllllllll}2006 & \text { ОСТ } & 15 & 0814 & 52.42 & 19 & 46.20 & 155 & 46.29 & 30.90 & 52 & .09 & .7 & 1.1\end{array}$

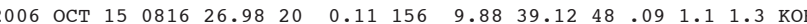

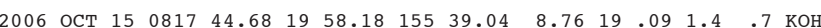

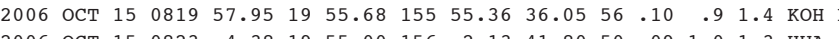
$\begin{array}{lllllllllllll}2006 & \text { ОСТ } 15 & 0823 & 4.38 & 19 & 55.00 & 156 & 2.13 & 41.80 & 50 & .09 & 1.0 & 1.3\end{array}$

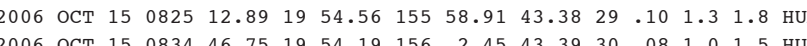

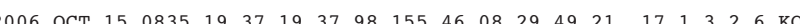

$\begin{array}{lllllllllllll}2006 & \text { ОСТ } 15 & 0839 & 9.97 & 19 & 48.64 & 156 & 5.77 & 40.93 & 39 & .11 & 1.1 & 1.4\end{array}$

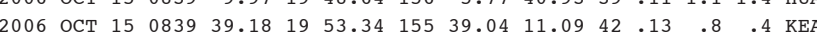
$\begin{array}{lllllllllllllll}2006 & \text { OCT } & 15 & 0841 & 58.25 & 19 & 56.29 & 156 & 4.99 & 42.33 & 39 & .13 & .8 & .4 & \text { KEA }\end{array}$

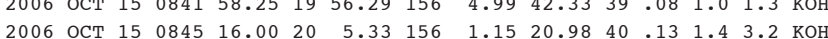

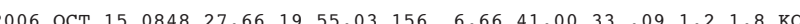

$\begin{array}{llllllllllllll}2006 & \text { OCT } & 15 & 0851 & 37.54 & 19 & 54.54 & 155 & 53.93 & 37.96 & 52 & .11 & .9 & 1.2\end{array}$ HUA

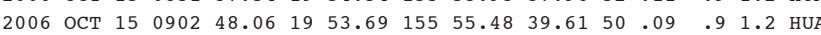
$\begin{array}{lllllllllllll}2006 & \text { ост } 15 & 0908 & 18.79 & 19 & 52.64 & 156 & 1.40 & 32.48 & 48 & .09 & .8 & 1.3\end{array}$

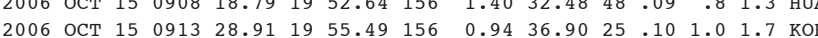

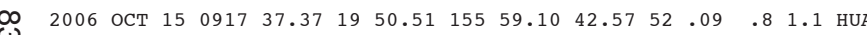

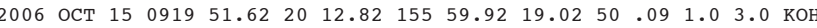

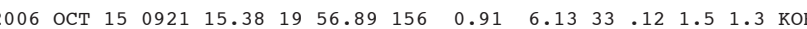

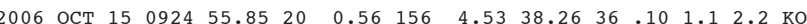

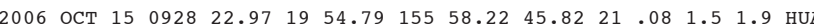
$\begin{array}{lllllllllllllll}2006 & \text { OCT } 15 & 0930 & 2.14 & 20 & 4.61 & 155 & 56.29 & 10.00 & 51 & .12 & 1.1 & 1.2 & \text { KO }\end{array}$

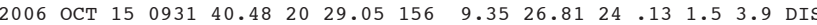

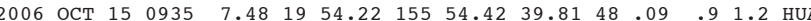

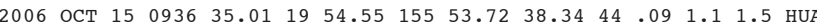

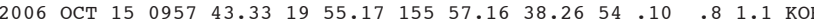
$\begin{array}{llllllllllllll}2006 & \text { ОСТ } & 15 & 0959 & 6.27 & 19 & 53.38 & 155 & 59.75 & 41.18 & 33 & .10 & 1.1 & 1.7\end{array}$

$\begin{array}{lllllllllllll}2006 & \text { ОСт } 15 & 1003 & 7.82 & 19 & 51.23 & 155 & 51.35 & 40.17 & 19 & .09 & 1.9 & 2.7\end{array}$

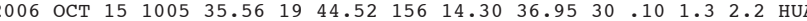
$\begin{array}{lllllllllllll}2006 & \text { ОСТ } 15 & 1008 & 19.78 & 19 & 54.35 & 155 & 48.07 & 35.23 & 21 & .08 & 1.2 & 1.4\end{array}$ $\begin{array}{lllllllllllll}2006 & \text { OCT } 15 & 1011 & 13.28 & 19 & 53.30 & 155 & 56.52 & 40.64 & 52 & .10 & .8 & 1.1\end{array}$

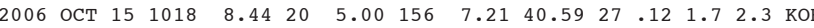

$\begin{array}{llllllllllllll}2006 & \text { ОСТ } 15 & 1020 & 8.44 & 19 & 17.94 & 155 & 15.04 & 7.32 & 43 & .14 & .4 & .8 & \text { SF } 1\end{array}$

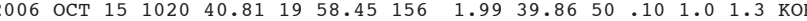

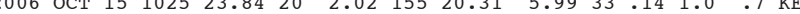

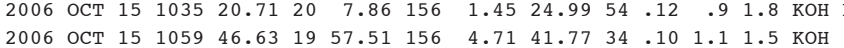

PREF AZ MIN 97 MAG GAP DS $3.3 \times 20324$ $\begin{array}{llll}2.7 \times & 174 \quad 21\end{array}$ $2.2 \times 217 \quad 26$ $2.0 \times 27621$

$2.2 \times 26722$ $2.3 \times 282 \quad 24$ $2.4 \times 114 \quad 12$ $2.5 \times 271 \quad 41$ $2.1 \times 28329$

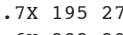
$2.6 \times 232 \quad 29$ $2.0 \times 29129$ $2.6 \times 232 \quad 27$ $2.0 \times 113 \quad 10$

$2.2 \times 245 \quad 19$ $2.3 \times 23023$ $2.6 \times 249 \quad 32$ 2.6x 26125 $2.6 \times 300 \quad 30$

$2.5 \times 182 \quad 25$ $\begin{array}{lll}2.6 \times & 189 & 25\end{array}$ $2.3 \times 224 \quad 24$ $2.1 \times 22630$ 2.6X 20421

$2.9 \times 29025$ $2.3 \times 2993$ $2.5 \times 30643$ $1.8 \times 29229$ $2.7 \times 28244$

$2.7 \times \quad 166 \quad 27$ $2.4 \times 184 \quad 25$ $2.0 \times 28125$ 3.3X 20428 2.0X 29228

$1.3 \times 303 \quad 39$ 2.3X $302 \quad 42$ $1.9 \times 175 \quad 24$ 2.9X 19425 2.6X 27936

$1.7 \times 115$ $2.6 \times 240 \quad 32$ $\begin{array}{llll}1.8 \times & 293 \quad 31\end{array}$ $4.3 \times 2712$ $2.4 \times 24934$
--ORTGin TIME (HST)-- -LAT N-- --LON W-- DEPTH N RMS ERH ERz LOC YEAR MON DA HRMN SEC DEG MIN DEG MIN KM RD SEC KM KM REMKS $\begin{array}{llllllllllllll}2006 & \text { ост } 15 & 1108 & 9.43 & 19 & 56.24 & 156 & 0.05 & 37.92 & 46 & .10 & 1.0 & 1.6 & \text { кон }\end{array}$

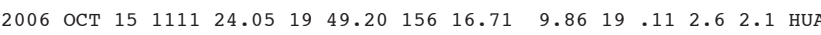

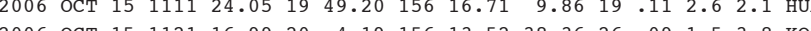
2006 OCT $15112316.9520 \quad 4.193015613 .5233 .36 \quad 26.091 .5 \quad 3.8$ kOH

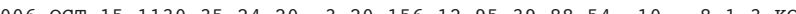
$\begin{array}{llllllllllllll}2006 & \text { ост } 15 & 1145 & 37.34 & 20 & 8.34 & 155 & 50.56 & 8.58 & 46 & .10 & 1.2 & 1.3 & \text { кон }\end{array}$

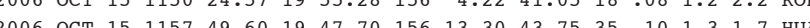

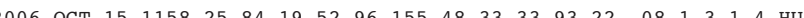

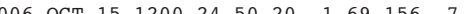

$\begin{array}{lllllllllllllll}2006 & \text { ОСТ } & 15 & 1209 & 43.45 & 19 & 57.07 & 155 & 37.03 & 1.01 & 32 & .14 & .6 & .4 & \text { кон }\end{array}$

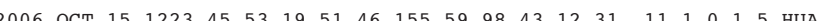

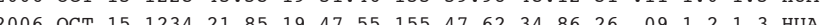

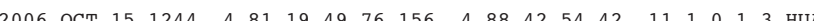

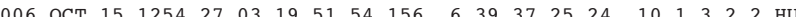

$\begin{array}{llllllllllllll}2006 & \text { OCT } 15 & 1310 & 37.81 & 19 & 56.38 & 155 & 57.02 & 36.95 & 19 & .10 & 1.6 & 2.4 & \text { кон }\end{array}$

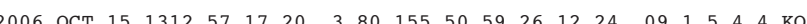

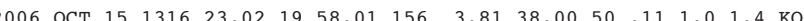

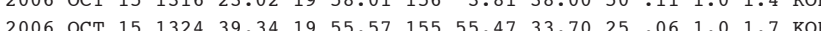

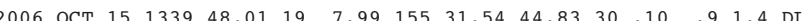

$\begin{array}{lllllllllllllll}2006 & \text { OCT } & 15 & 1341 & 30.79 & 19 & 18.12 & 155 & 15.07 & 6.18 & 41 & .12 & .4 & .9 & \mathrm{SF} 1\end{array}$ $\begin{array}{llllllllllllll}2006 & \text { ост } & 15 & 1343 & 42.83 & 20 & 8.41 & 155 & 49.45 & 24.94 & 29 & .09 & .9 & 1.1\end{array}$

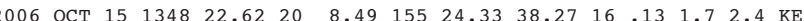

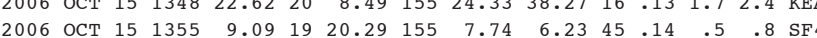

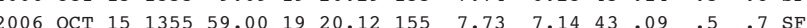

$\begin{array}{llllllllllllll}2006 & \text { OCT } 15 & 1406 & 3.08 & 19 & 55.04 & 155 & 33.77 & 8.29 & 13 & .13 & 1.6 & 1.1 & \text { KEA }\end{array}$ $\begin{array}{llllllllllllll}2006 & \text { OCT } 15 & 1413 & 43.93 & 19 & 57.86 & 156 & 6.68 & 42.86 & 24 & .11 & 1.7 & 2.6 & \text { Kон }\end{array}$

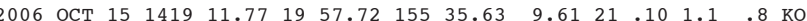
2006 OCT $151424 \quad 36.93 \quad 2010.96155 \quad 48.05 \quad 33.7518$.12 1.94 .8 KOH

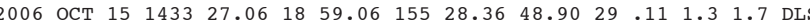

$\begin{array}{llllllllllllll}2006 & \text { OCT } 15 & 1452 & 35.19 & 19 & 28.58 & 155 & 25.74 & 0.32 & 17 & .13 & .4 & .5 & \text { KAO }\end{array}$

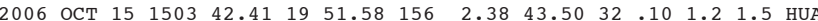
$\begin{array}{lllllllllllll}2006 & \text { OCT } 15 & 1511 & 3.70 & 19 & 53.24 & 155 & 56.07 & 36.32 & 53 & .09 & .7 & 1.2\end{array}$ HUA

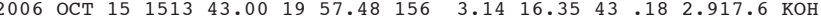
$\begin{array}{llllllllllllll}2006 & \text { ОСТ } 15 & 1542 & 11.76 & 19 & 54.99 & 155 & 56.12 & 38.48 & 45 & .11 & .9 & 1.4\end{array}$

$\begin{array}{llllllllllllll}2006 & \text { OCT } 15 & 1546 & 30.81 & 19 & 27.22 & 155 & 25.78 & 11.27 & 26 & .11 & .4 & 1.0 & \text { KAO }\end{array}$

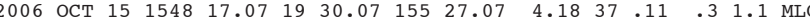

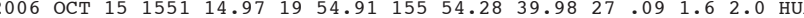

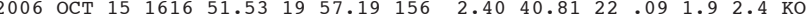
$\begin{array}{lllllllllllllll}2006 & \text { ОСТ } & 15 & 1626 & 25.64 & 19 & 50.99 & 156 & 4.17 & 47.42 & 51 & .11 & 1.0 & 1.2\end{array}$

$\begin{array}{llllllllllllll}2006 & \text { OCT } 15 & 1636 & 19.03 & 19 & 18.20 & 155 & 15.15 & 4.68 & 32 & .12 & .4 & 1.5 & \text { SSF }\end{array}$

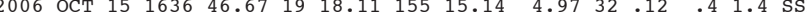

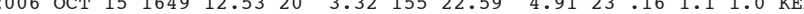

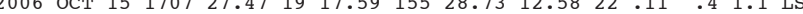

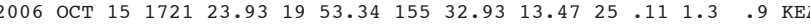

PREF AZ MIN 98

$2.5 \times 28231$ $1.8 \times 32048$ $.9 \times 31459$ $.8 \times 27423$ $2.9 \times 20046$

2.5x 27950 $1.8 \times 24938$ $2.2 \times 31842$

1.9 15425 .3X 22925 .1X 24913 $2.5 \times 24320$ $2.3 \times 24934$

$1.5 \times 306 \quad 30$ $\begin{array}{lll}1.6 \times & 314 \quad 42 \\ 2.5 \times & 246 & 35\end{array}$ $2.5 \times 24635$ $2.0 \times 157 \quad 7$

$\begin{array}{lll}1.5 \times \quad 110 \quad 3 & 3\end{array}$ $2.3 \times 282 \quad 5$ $2.2 \times 23239$ $2.0 \times 124 \quad 5$ $1.7 \times 126 \quad 5$

$1.8 \times 29636$ $1.8 \times 320 \quad 42$ $1.7 \times \quad 190 \quad 25$ $.7 \mathrm{U} 316 \quad 57$

$\begin{array}{lll}1.2 \times \quad 90 & 5\end{array}$ $2.2 \times 23828$ $\begin{array}{lll}2.2 \times & 238 & 28 \\ .4 \times & 19124 \\ -7 \times 298 & 54 \\ 2.3 \times & 217 & 22\end{array}$ $\begin{array}{lll}2.3 \times & 217 & 27\end{array}$

$\begin{array}{lll}1.7 \mathrm{X} & 68 & 6\end{array}$ $\begin{array}{lll}1.8 \times & 71 \quad 4\end{array}$ $2.0 \times 28526$ $\begin{array}{lll}2.0 \times & 30136 \\ 2.5 \times & 239 & 22\end{array}$

$\begin{array}{lll}1.2 \times 109 \quad 4 & 4\end{array}$ $\begin{array}{lll}1.6 \times & 108 \quad 4\end{array}$ $1.6 \times 21532$ $1.1 \mathrm{X} \quad 87 \quad 5$ $2.0 \times 23632$ 


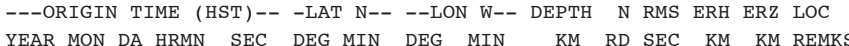
$\begin{array}{llllllllllll}\text { YEAR MON DA HRMN } & \text { SEC } & \text { DEg MIN } & \text { DEG } & \text { MIN } & \text { KM } & \text { RD } & \text { SEC } & \text { KM } & \text { KM REMIS } \\ & & & & & & & & & & \end{array}$

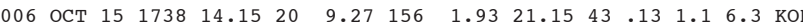

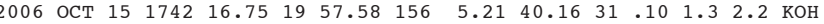

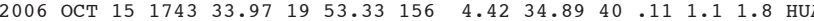

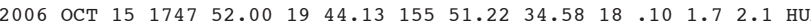

$\begin{array}{llllllllllllll}2006 & \text { OCT } 15 & 1755 & 45.47 & 19 & 58.89 & 156 & 4.28 & 37.84 & 26 & .07 & 1.6 & 2.3 & \text { кон }\end{array}$ $\begin{array}{lllllllllllll}2006 & \text { ост } 15 & 1803 & 7.57 & 19 & 54.06 & 156 & 0.57 & 36.30 & 22 & .09 & 1.4 & 1.8\end{array}$

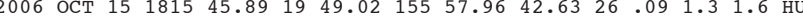

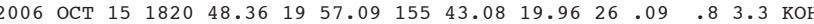
$\begin{array}{llllllllllllll}2006 & \text { ост } & 15 & 1822 & 34.55 & 19 & 55.61 & 155 & 55.94 & 36.71 & 50 & .11 & .9 & 1.7\end{array}$

$\begin{array}{lllllllllllll}2006 & \text { ОСТ } 15 & 1830 & 35.80 & 19 & 53.46 & 156 & 2.71 & 39.88 & 26 & .10 & 1.6 & 2.1\end{array}$ HUA $\begin{array}{lllllllllll}19 & 52.28 & 156 & 3.85 & 42.47 & 48 & .11 & .9 & 1.4 & \text { HUA }\end{array}$

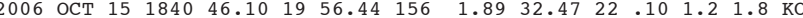

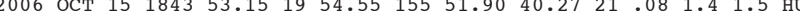

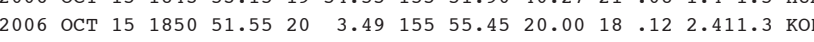

$\begin{array}{lllllllllllll}2006 & \text { OCT } 15 & 1859 & 53.30 & 19 & 53.36 & 156 & 0.56 & 42.14 & 33 & .08 & 1.3 & 1.5\end{array}$ $\begin{array}{llllllllllllll}2006 & \text { ОСТ } 15 & 1925 & 28.29 & 19 & 55.44 & 155 & 38.06 & 0.60 & 45 & .12 & .5 & .3 & \text { ко }\end{array}$

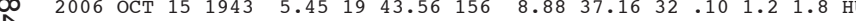

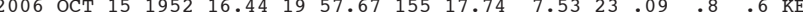
$\begin{array}{lllllllllllll}2006 & \text { ост } 15 & 1952 & 51.75 & 19 & 58.63 & 156 & 7.20 & 36.20 & 28 & .11 & 1.6 & 2.5 \\ \text { кон }\end{array}$

$\begin{array}{llllllllllll}2006 & \text { ост } 15 & 2013 & 0.97 & 19 & 59.34 & 155 & 14.31 & 17.75 & 18 & .11 & 1.812 .1\end{array}$ $\begin{array}{lllllllllllll}2006 & \text { ОСТ } 15 & 2016 & 1.38 & 19 & 47.39 & 156 & 0.41 & 34.85 & 47 & .10 & .9 & 1.2\end{array}$ $\begin{array}{lllllllllllllll}2006 & \text { ост } 15 & 2038 & 6.90 & 20 & 1.68 & 156 & 4.57 & 36.45 & 29 & .10 & 1.4 & 2.5 & \text { кон }\end{array}$

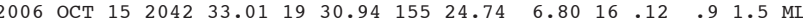

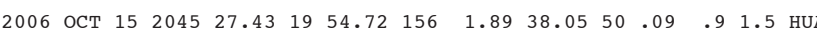

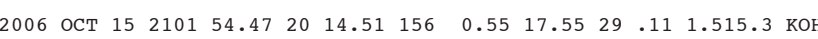
$\begin{array}{llllllllllllll}2006 & \text { OCT } 15 & 2122 & 53.13 & 20 & 9.44 & 155 & 59.88 & 23.84 & 19 & .10 & 1.8 & 8.4 & \text { ко }\end{array}$ $\begin{array}{lllllllllllll}2006 & \text { ОСТ } 15 & 2132 & 11.59 & 19 & 50.66 & 156 & 7.61 & 44.58 & 26 & .12 & 1.5 & 2.0\end{array}$ $\begin{array}{llllllllllllll}2006 & \text { ОСТ } & 15 & 2148 & 24.52 & 19 & 52.78 & 155 & 50.31 & 37.65 & 18 & .08 & 1.8 & 1.5\end{array}$

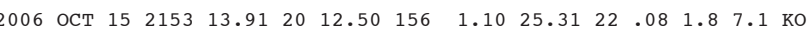

$\begin{array}{llllllllllllll}2006 & \text { ост } 15 & 2212 & 39.53 & 20 & 1.64 & 155 & 39.67 & 11.88 & 19 & .11 & 2.4 & 2.9 & \text { ко }\end{array}$ $\begin{array}{lllllllllllll}2006 & \text { ОСТ } 15 & 2217 & 32.08 & 19 & 51.80 & 155 & 58.68 & 39.64 & 29 & .10 & 1.3 & 1.8\end{array}$ HUA

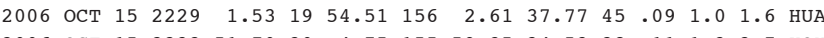

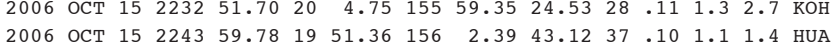

$\begin{array}{llllllllllll}2006 & & & \end{array}$ $\begin{array}{rlllllrllllllll}2006 & \text { OCT } & 15 & 2249 & 39.04 & 20 & 5.35 & 155 & 50.66 & 32.56 & 21 & .11 & 1.9 & 5.5 & \text { KOH } \\ 2006 & \text { ОСт } & 15 & 2300 & 14.56 & 19 & 45.73 & 156 & 31.39 & 30.38 & 46 & .11 & 1.4 & 2.4 & \text { DIS }\end{array}$ 2006 OCT $15232747.42 \quad 1948.06 \quad 15611.43 \quad 40.76 \quad 30.10 \quad 1.42 .40$ HUA $\begin{array}{llllllllllllll}2006 & \text { ост } 15 & 2341 & 47.35 & 20 & 11.28 & 155 & 53.90 & 20.99 & 43 & .10 & 1.1 & 7.0 & \text { кон }\end{array}$

$\begin{array}{lllllllllllll}2006 & \text { ОСт } 15 & 2348 & 3.09 & 19 & 49.68 & 155 & 53.22 & 34.95 & 19.10 & 2.1 & 1.5 & \text { HUA }\end{array}$

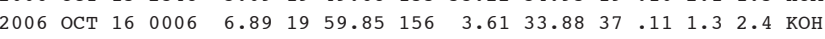
$\begin{array}{llllllllllllll}2006 & \text { OCT } & 16 & 0011 & 8.16 & 19 & 29.58 & 155 & 24.91 & 7.58 & 18 & .11 & .4 & 1.1 \\ \text { KAO }\end{array}$

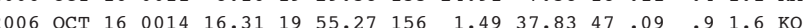
$\begin{array}{llllllllllllll}2006 & \text { OCT } & 16 & 0032 & 11.41 & 19 & 49.92 & 156 & 4.95 & 44.63 & 31 & .08 & 1.2 & 1.6\end{array}$
PREF AZ MIN 99 GAP DS

$2.3 \times 33174$ $2.5 \times 30655$ $.2 \times 29934$ $2.2 \times 24733$ $1.8 \times 3076$

$1.9 \times 31941$ $2.0 \times 31830$ $1.5 \times 30220$ $1.5 \times 14921$ 2.8X $286 \quad 28$

$2.2 \times 2963$ $2.6 \times 2953$ $1.7 \times 24434$ $1.5 \times 30325$ $1.8 \times 32142$

$2.0 \times 29329$ $1.9 \times 144 \quad 24$ $1.9 \times 25033$ $1.7 \times 252 \quad 26$ $2.2 \times 31344$

$1.9 \times 32033$ $2.5 \times 226 \quad 21$ 2.3X 31245 $.9 \times 167 \quad 3$ $3.0 \times 2963$ $2.2 \times 31864$ $1.9 \times 32676$ $1.8 \times 252 \quad 35$ $\begin{array}{llll}1.3 \times & 308 & 21\end{array}$ $2.0 \times 32761$

1.6x 29042 $\begin{array}{lll}1.9 \times & 307 & 24\end{array}$ $\begin{array}{ll}2.2 \times \quad 296 & 33 \\ 2.0 \times 2633 & 23\end{array}$ $2.0 \times 26323$

- $.8 \times 29649$ $\begin{array}{lll}1.8 \times & 320 & 65 \\ 2.5 \times & 315 & 70\end{array}$ $2.1 \times 25939$ $2.3 \times 29856$

$1.5 \times 29516$ $2.2 \times 300 \quad 41$ $\begin{array}{lll}1.4 \times & 89 & 3 \\ 2.5 \times & 295 & 32\end{array}$ $2.5 \times 29532$
---ORIGIN TIME (HST)-- -LAT N-- --LON W-- DEPTH N RMS ERH ERZ LOC YEAR MON DA HRMN SEC DEG MIN DEG MIN KM RD SEC KM KM RA $\begin{array}{lllllllllllllll}2006 & \text { OCT } 16 & 0049 & 10.40 & 19 & 52.52 & 155 & 33.60 & 25.36 & 26 & .12 & 1.3 & 1.9 & \text { KEA }\end{array}$

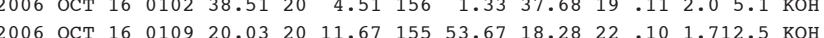

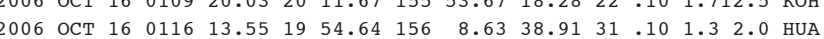
$\begin{array}{lllllllllllll}2006 & \text { OCT } 16 & 0129 & 34.04 & 19 & 53.79 & 156 & 0.33 & 36.40 & 48 & .10 & .9 & 1.4\end{array}$

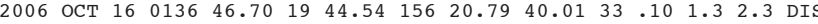

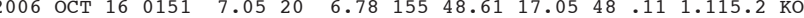

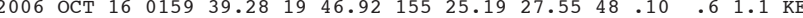

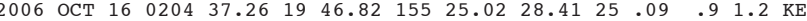

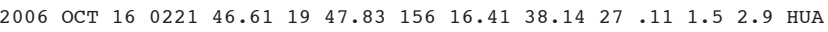

$\begin{array}{llllllllllllll}2006 & \text { OCT } 16 & 0226 & 33.69 & 19 & 53.44 & 155 & 55.49 & 38.61 & 29 & .09 & 1.3 & 1.7 & \text { HUA }\end{array}$

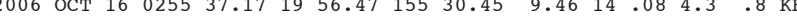

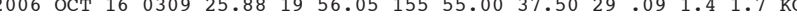

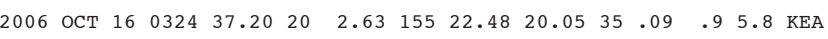

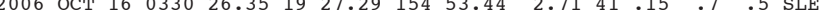

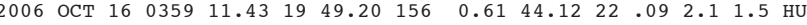

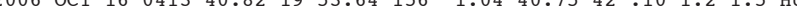
$\begin{array}{lllllllllllll}2006 & \text { ост } 16 & 0459 & 24.22 & 19 & 55.94 & 156 & 5.35 & 39.30 & 47 & .11 & 1.2 & 1.4\end{array}$

$\begin{array}{llllllllllllll}2006 & \text { ОСт } 16 & 0527 & 55.59 & 20 & 2.09 & 156 & 7.58 & 39.06 & 20 & .10 & 2.3 & 3.2 & \text { кон }\end{array}$ $\begin{array}{lllllllllllll}2006 & \text { OCT } 16 & 0531 & 8.23 & 19 & 53.86 & 156 & 4.71 & 44.42 & 22 & .09 & 1.9 & 2.6\end{array}$ 2006 ОСТ $16 \quad 055941.76 \quad 1952.39156 \quad 3.60 \quad 40.74 \quad 17.10 \quad 2.8 \quad 4.0$ HU

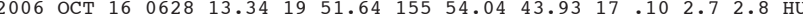

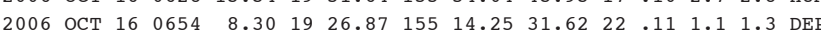

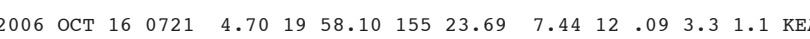

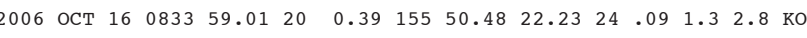

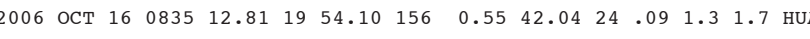

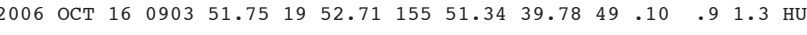

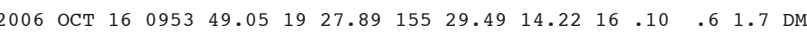

$\begin{array}{lllllllllllll}2006 & \text { OCT } 16 \quad 1022 & 0.66 & 19 & 53.68 & 155 & 55.36 & 43.43 & 17 & 09 & 1.1 & 1.5 & \text { HUA }\end{array}$ $\begin{array}{lllllllllllllll}2006 & \text { ОСТ } & 16 & 1052 & 26.05 & 19 & 48.33 & 156 & 8.87 & 44.67 & 28 & .08 & 1.7 & 1.9 \text { нUA } \\ 2006 & \text { ОСТ } & 16 & 1102 & 20.22 & 19 & 55.43 & 156 & 2.20 & 45.95 & 18 & .09 & 2.3 & 3.8 & \text { кон }\end{array}$

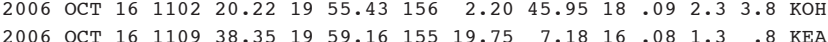

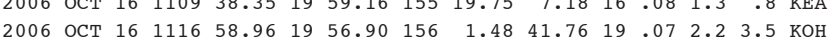

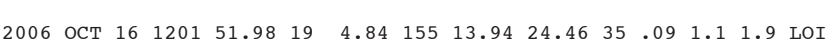
$\begin{array}{llllllllllllll}2006 & \text { OCT } & 16 & 1308 & 46.82 & 19 & 52.97 & 155 & 59.28 & 41.45 & 22 & .10 & 2.2 & 2.7\end{array}$ $\begin{array}{lllllllllllllll}2006 & \text { OCT } 16 & 1308 & 46.82 & 19 & 52.97 & 155 & 59.28 & 41.45 & 22 & .10 & 2.2 & 2.7 & \text { HUA }\end{array}$

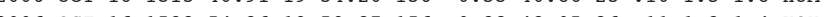

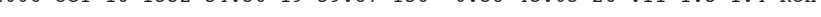

2006 OCT $16161949.001955 .99-156$

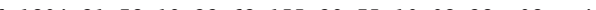
$\begin{array}{llllllllllllll}2006 & \text { OCT } 16 & 1804 & 21.78 & 19 & 23.62 & 155 & 29.75 & 10.08 & 28 & .08 & .4 & .9 & \text { KAO }\end{array}$

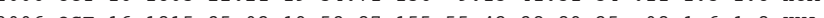

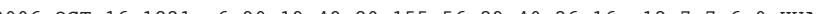

PREF AZ MIN 100 .8X 25915 $1.7 \times 31765$ $\begin{array}{lll}2.0 \times & 303 & 40 \\ 2.5 \times & 234 & 29\end{array}$

$1.9 \times 30853$ $2.3 \times 28847$ $2.0 \times 146 \quad 4$ $1.7 \times 243 \quad 4$ $1.9 \times 30747$

$1.9 \times 28424$ $2.1 \times 31631$ $1.6 \times 28318$ $1.9 \times 28829$ $2.2 \times 213 \quad 4$

$2.2 \times 190$ $1.9 \times 30738$ $2.6 \times 29430$ $1.5 \times \quad 90 \quad 5$ $2.6 \times 25031$

$2.1 \times 323 \quad 49$ $1.9 \times 31548$ $1.7 \times 31351$ $1.5 \times 30541$ $1.5 \times 143$ 1.5X 26922 $\begin{array}{lll}2.5 \times & 197 \quad 15\end{array}$ $2.2 \times 23530$ $2.7 \times 16121$ $\begin{array}{lll}2.4 \times 313 \quad 43 \\ 1.9 \times & 315 & 54\end{array}$ $1.9 \times 31554$ $1.8 \times 30426$

$2.0 \times 22222$ $2.0 \times 31147$ 2.2x 23530 $2 \times 248$ $2.3 \times 313 \quad 47$ 2.2X 31651 $\begin{array}{lrr}1.5 \times & 53 & 4 \\ 2.2 x & 303 & 41\end{array}$ $\begin{array}{lll}2.2 \times & 303 & 41\end{array}$ $1.7 \times \quad 305 \quad 19$ 
---ORIGIN TIME (HST)-- -LAT N-- --LON W-- DEPTH N RMS ERH ERZ LOC PREF AZ MIN 101 YEAR MON DA HRMN SEC DEG MIN DEG MIN KM RD SEC

$\begin{array}{llllllllllllll}2006 & \text { ОСТ } 16 & 1900 & 17.92 & 19 & 36.51 & 155 & 52.28 & 28.76 & 31 & .08 & .7 & 1.2 & \text { коN }\end{array}$

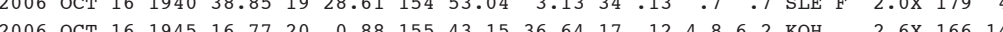

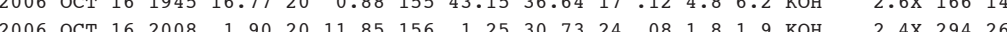

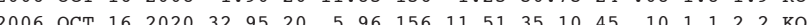

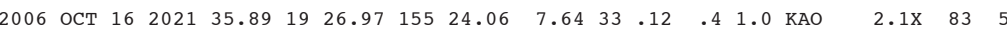

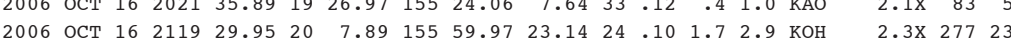

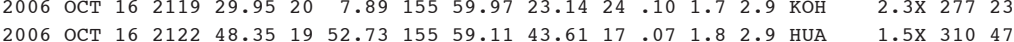

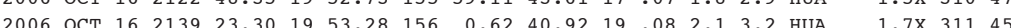

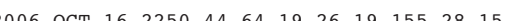

$\begin{array}{lllllllllllllllll}2006 & \text { ост } 16 & 2300 & 59.37 & 19 & 18.87 & 155 & 14.91 & 3.93 & 24 & .13 & .5 & 1.8 & \mathrm{SSF} & 1.2 \mathrm{X} & 113 & 4\end{array}$

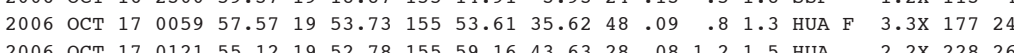

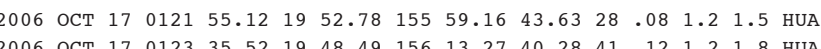

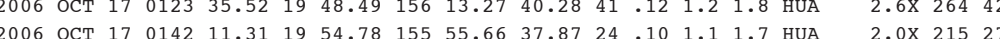

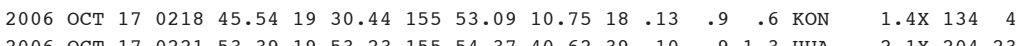

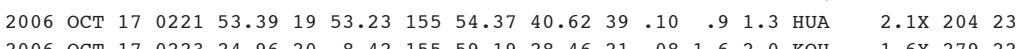

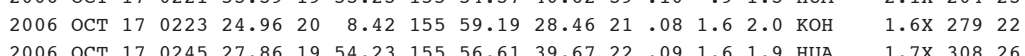
2006 ОСТ 170245

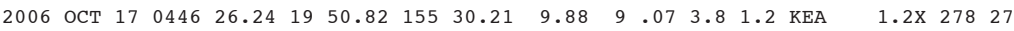

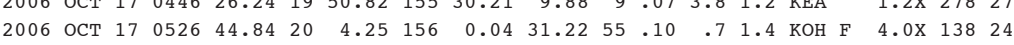

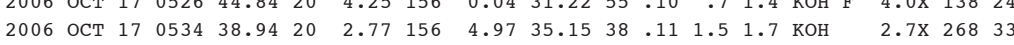

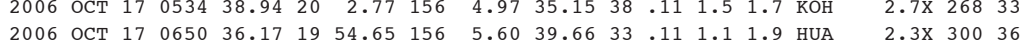

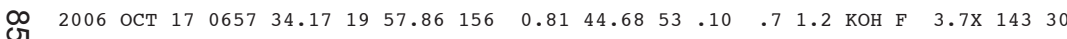

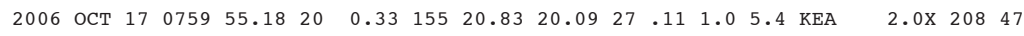

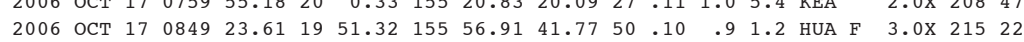

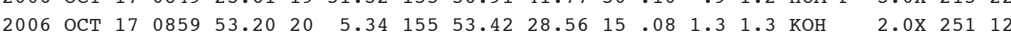

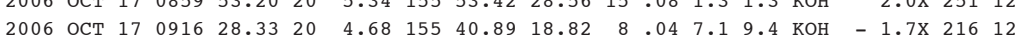

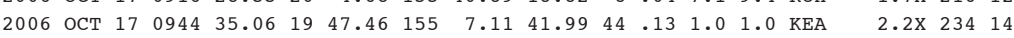

$\begin{array}{llllllllllllll}2006 & \text { ост } 17 & 1024 & 45.51 & 20 & 1.04 & 156 & 1.94 & 45.58 & 20 & .09 & 2.0 & 3.0 & \text { кон }\end{array}$

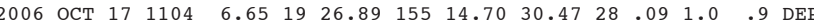
$\begin{array}{lllllllllllll}2006 & \text { OCT } 17 & 1208 & 22.50 & 19 & 28.86 & 155 & 22.24 & 12.48 & 39 & .12 & .4 & .4 \\ \text { KAO }\end{array}$ $\begin{array}{lllllllllllllll}2006 & \text { ост } 17 & 1255 & 44.82 & 19 & 19.54 & 155 & 10.31 & 6.99 & 32 & .12 & .4 & 1.0 & \text { SF } 3\end{array}$

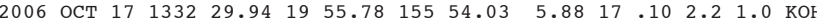

$\begin{array}{lllllllllllllll}2006 & \text { OCT } & 17 & 1432 & 1.98 & 20 & 1.60 & 155 & 51.69 & 30.61 & 51 & .11 & .8 & 1.4 & \text { кон } \mathrm{F}\end{array}$ $\begin{array}{llllllllllllll}2006 & \text { OCT } 17 & 1433 & 23.54 & 19 & 59.67 & 155 & 15.57 & 1.82 & 29 & .11 & 1.3 & .7 & \text { KEA }\end{array}$ $\begin{array}{llllllllllllll}2006 & \text { OCT } 17 & 1654 & 11.33 & 19 & 53.42 & 156 & 0.18 & 41.86 & 24 & .12 & 1.0 & 1.7 & \text { HUA }\end{array}$ $\begin{array}{lllllllllllll}2006 & \text { OCT } 17 & 1804 & 51.38 & 19 & 52.97 & 156 & 3.50 & 41.99 & 26 & .09 & 1.5 & 2.2 \\ 200 A\end{array}$ $\begin{array}{lllllllllllll}2006 & \text { ОСТ } 172020 & 48.71 & 19 & 40.54 & 156 & 5.73 & 37.46 & 28 & .08 & 1.4 & 1.8 & \text { HUA }\end{array}$

$\begin{array}{llllllllllllllll}2006 & \text { OCT } 17 & 2021 & 23.66 & 19 & 57.27 & 156 & 2.39 & 33.09 & 21 & .11 & 1.3 & 2.3 & \mathrm{KOH}\end{array}$ $\begin{array}{llllllllllllll}2006 & \text { OCT } 17 & 2036 & 59.26 & 20 & 7.23 & 156 & 11.91 & 33.83 & 20 & .06 & 1.8 & 3.7 & \text { KOH }\end{array}$ $\begin{array}{llllllllllllll}2006 & \text { OCT } 17 & 2113 & 36.77 & 19 & 27.54 & 154 & 52.67 & 1.66 & 15 & .12 & .8 & .5 & \text { SLE }\end{array}$ $\begin{array}{lllllllllllll}2006 & \text { OCT } 17 & 2201 & 18.17 & 19 & 53.72 & 155 & 56.52 & 39.41 & 44 & .10 & 1.0 & 1.3\end{array}$
$1.7 \times 32542$ $1.6 \times 164 \quad 4$ $1.6 \times \quad 96 \quad 3$ $1.5 \times 33656$

$2.5 \times 21114$ $2.0 \times 28132$ $2.0 \times 23335$ $2.0 \times 31432$ $2.3 \times 24128$

$.9 \mathrm{U} 24833$ 1.6032761 $2.5 \times 196 \quad 5$ $1.9 \times 26335$ $2.3 \times 21725$
---ORTGIN TIME (HST)-- -LAT N-- --LON W-- DEPTH N RMS ERH ERZ LOC $\begin{array}{llllllllllllll}2006 & \text { OCT } 17 & 2205 & 4.27 & 19 & 51.35 & 155 & 24.73 & 12.72 & 21 & .15 & 1.0 & .5 & \text { KEA }\end{array}$ $\begin{array}{lllllllllllll}2006 & \text { ОСт } 18 & 0012 & 40.14 & 19 & 53.37 & 156 & 0.36 & 48.35 & 21 & .06 & 1.9 & 2.0\end{array}$ HUA $\begin{array}{lllllllllllll}2006 & \text { ОСТ } 18 & 0118 & 1.59 & 20 & 0.27 & 155 & 51.87 & 30.75 & 22 & .10 & 1.3 & 3.6 \\ \text { кон }\end{array}$

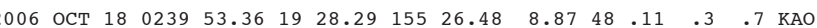
$\begin{array}{lllllllllllll}2006 & \text { OСТ } 18 & 0358 & 21.47 & 19 & 49.73 & 156 & 0.38 & 40.89 & 48 & .12 & .9 & 1.2\end{array}$

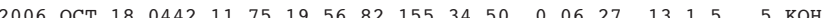

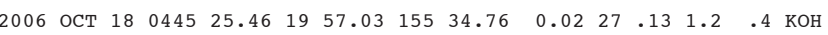

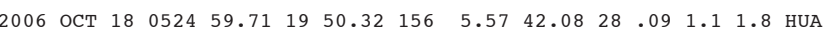

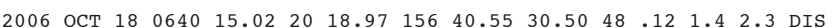

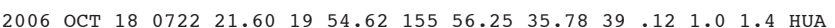

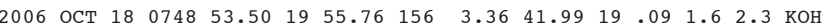

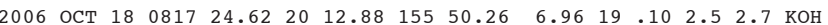

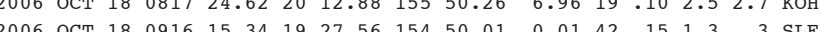

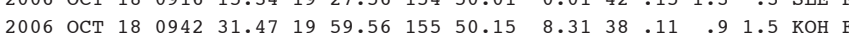

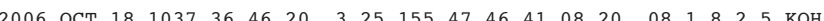

$\begin{array}{llllllllllllll}2006 & \text { ОСт } 18 & 1118 & 8.77 & 20 & 2.03 & 156 & 0.19 & 29.42 & 37 & .11 & 1.2 & 4.2 & \text { кон }\end{array}$ $\begin{array}{lllllllllllllll}2006 & \text { ОСТ } 18 & 1128 & 38.52 & 19 & 55.44 & 155 & 58.79 & 34.66 & 20 & .09 & 1.5 & 4.6 & \text { кон } \\ 2006 & \text { ОСТ } 18 & 1141 & 40.89 & 19 & 29.38 & 155 & 27.89 & 8.43 & 31 & .09 & .3 & 1.1 & \text { KAO }\end{array}$

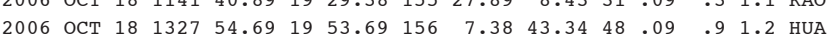

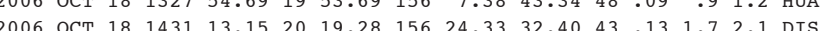

$\begin{array}{lllllllllllll}2006 & \text { ОСТ } 18 & 1627 & 27.81 & 19 & 47.52 & 155 & 43.88 & 23.51 & 19 & .13 & 1.4 & 2.1\end{array}$ HUA $\begin{array}{lllllllllllll}2006 & \text { OCT } 18 & 1825 & 53.66 & 19 & 52.50 & 156 & 5.45 & 42.98 & 23 & .09 & 1.5 & 2.3 \\ & \text { HUA }\end{array}$

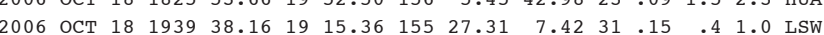
$\begin{array}{llllllllllllll}2006 & \text { OCT } 18 & 1940 & 8.52 & 19 & 15.23 & 155 & 27.26 & 7.39 & 27 & .12 & .4 & 1.2 & \text { LSW }\end{array}$ $\begin{array}{lllllllllllll}2006 & \text { OCT } 18 & 2001 & 40.98 & 19 & 54.54 & 156 & 0.59 & 39.03 & 47 & 10 & 1.0 & 1.4\end{array}$

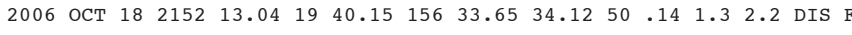
$\begin{array}{llllllllllllll}2006 & \text { ост } 18 & 2254 & 18.45 & 19 & 52.85 & 155 & 54.90 & 40.75 & 51 & .09 & .8 & 1.1 & \text { HUA }\end{array}$ $\begin{array}{llllllllllllll}2006 & \text { ОСТ } 18 & 2300 & 4.67 & 19 & 53.86 & 155 & 58.60 & 35.54 & 42 & .09 & 1.0 & 1.3 & \text { HUA }\end{array}$

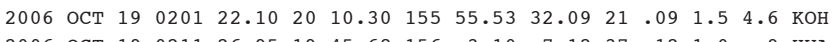

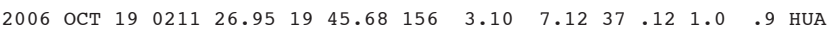

$\begin{array}{llllllllllllll}2006 & \text { ОСТ } 19 & 0257 & 8.81 & 19 & 52.40 & 156 & 5.06 & 48.32 & 24 & .11 & 1.4 & 2.3 & \text { HUA }\end{array}$

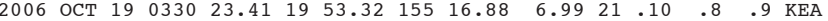

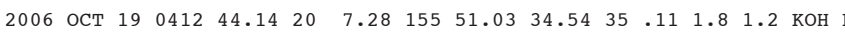

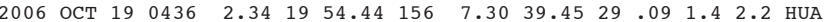
$\begin{array}{llllllllllllll}2006 & \text { OCT } 19 & 0436 & 42.87 & 19 & 25.08 & 155 & 37.76 & 2.60 & 25 & .13 & .3 & .4 & \text { MLO }\end{array}$

$\begin{array}{llllllllllllll}2006 & \text { ОСт } 19 & 0520 & 49.15 & 19 & 18.01 & 155 & 23.10 & 3.14 & 26 & .11 & .4 & .9 & \text { SWR }\end{array}$

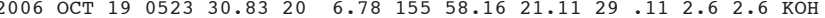

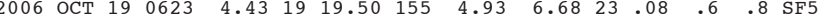

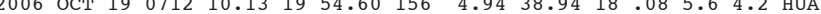
$\begin{array}{lllllllllllllll}2006 & \text { ОСТ } & 19 & 0748 & 16.62 & 19 & 29.18 & 155 & 26.81 & 8.35 & 43 & .12 & .4 & .9 & \text { KAO }\end{array}$

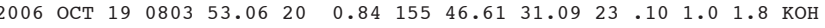

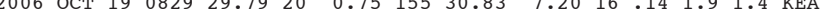

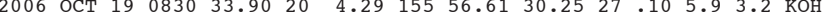

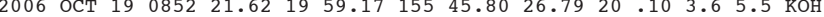

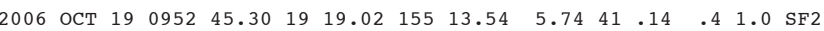

PREF AZ MIN

$1.3 \times 224 \quad 10$ $1.6 \times 31128$ $2.1 \times 30535$

$1.8 \times 23922$ $2.1 \times 30931$ $3.4 \times 31896$ $2.2 \times 22027$

$1.6 \times 31535$ $1.8 \times 32758$ $2.3 \times 2819$ $2.6 \times 28754$ $1.6 \times 31041$

$2.4 \times 298 \quad 42$ $\begin{array}{lll}1.7 \times & 295 & 51 \\ 1.6 \times \quad 80 & 5\end{array}$ $2.5 \times 25529$ $3.0 \times 31369$

$1.5 \times 26316$ $2.0 \times 248 \quad 34$ $1.5 \times 103$ $1.5 \times 103$ $2.4 \times 23630$

$2.8 \times 29070$ $\begin{array}{llll}3.1 \times & 183 & 23 \\ 2.2 \times & 228 & 27\end{array}$ $2.2 \times \quad 228 \quad 27$ $\begin{array}{lll}1.9 \times & 315 & 54 \\ 2.287 & 24\end{array}$

$2.0 \times 29733$ $1.4 \times 24922$ $2.3 \times 258$ 1.5030638 $2.4 \times \quad 75$

1.6X 113 $2.4 \times 270 \quad 20$ $1.6 \times 18607$ $\begin{array}{rrr}2.1 \times & 250 & 35 \\ 2.2 \times & 68 & 5\end{array}$ $\begin{array}{llll}1.7 \times \quad 164 & 13\end{array}$ $1.5 \times 22131$ $\begin{array}{lll}2.3 \times & 252 \quad 18\end{array}$ $1.7 \times 157 \quad 16$ $1.6 \times \quad 71$ 
---ORIGIN TIME (HST)-- -LAT N-- --LON W-- DEPTH N RMS ERH ERZ LOC PREF AZ MIN 103 YEAR MON DA HRMN SEC DEG MIN DEG MIN KM RD SEC KM KM REMKS MAG GAP DS

$\begin{array}{llllllllllllll}2006 & \text { OCT } 19 & 1433 & 59.15 & 20 & 2.06 & 155 & 54.57 & 13.13 & 14 & .11 & 2.0 & 1.3 & \text { кон }\end{array}$

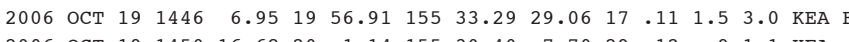

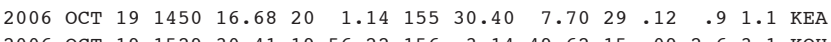
$\begin{array}{llllllllllllll}2006 & \text { ОСТ } 19 & 1529 & 30.41 & 19 & 56.22 & 156 & 3.14 & 49.62 & 15 & .09 & 2.6 & 3.1 & \text { кон }\end{array}$ 2006 ост $191722 \quad 18.3619 \quad 54.65 \quad 155 \quad 50.08 \quad 41.96 \quad 12 \quad .10 \quad 2.4 \quad 4.2$ HUA

$\begin{array}{lllllllllllllll}2006 & \text { OCT } 19 & 1850 & 49.87 & 19 & 52.79 & 156 & 3.20 & 41.05 & 47 & .09 & .9 & 1.3 & \text { HUA }\end{array}$ $\begin{array}{llllllllllllll}2006 & \text { ОСТ } 19 & 1855 & 37.60 & 20 & 1.90 & 155 & 22.84 & 8.86 & 8 & .06 & 2.7 & 1.9 & \mathrm{KEA}\end{array}$ $\begin{array}{lllllllllllll}2006 & \text { ОСТ } 19 & 2030 & 13.18 & 19 & 52.37 & 156 & 5.08 & 43.10 & 40 & .10 & 1.0 & 1.4\end{array}$

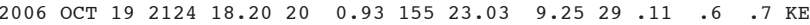

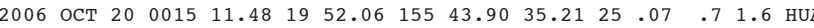

$\begin{array}{lllllllllllll}2006 & \text { OCT } 20 & 0116 & 19.49 & 19 & 52.51 & 156 & 2.44 & 38.23 & 25 & .09 & 4.1 & 3.3\end{array}$ HUA

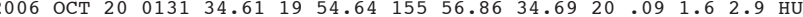

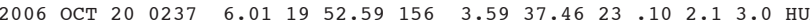
$\begin{array}{llllllllllllll}2006 & \text { OСТ } 20 & 0416 & 49.35 & 19 & 27.81 & 155 & 30.36 & 10.32 & 35 & .11 & .3 & .7 & \text { KAO }\end{array}$

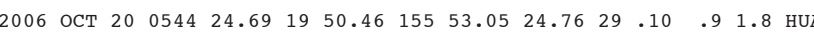

$\begin{array}{llllllllllllll}2006 & \text { OCT } 20 & 0604 & 3.76 & 19 & 8.54 & 155 & 37.39 & 0.66 & 33 & .12 & .4 & .3 & \text { LSW }\end{array}$ $\begin{array}{lllllllllllll}2006 & \text { ОСТ } 20 & 0720 & 14.20 & 20 & 5.03 & 155 & 48.91 & 9.31 & 21 & .10 & 1.4 & .6 \\ \text { Kо }\end{array}$

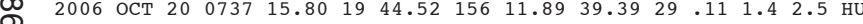

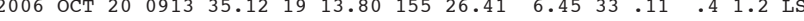

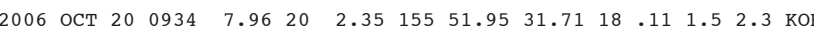

$\begin{array}{lllllllllllll}2006 & \text { ОСТ } 20 & 1203 & 43.37 & 19 & 52.61 & 155 & 53.98 & 38.47 & 50 & .10 & .7 & 1.2\end{array}$

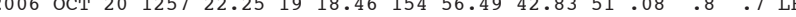

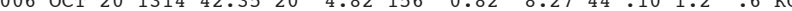

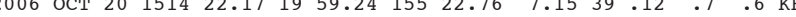

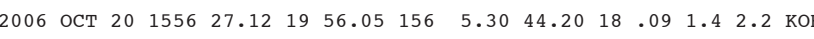

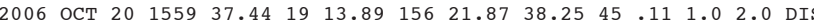

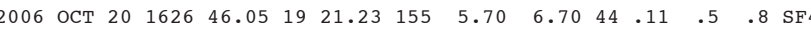
$\begin{array}{llllllllllllll}2006 & \text { ОСТ } 20 & 1628 & 3.30 & 19 & 21.01 & 155 & 5.62 & 8.11 & 43 & .09 & .4 & .5 & \text { SF } 4\end{array}$

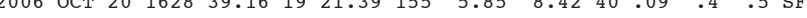
$\begin{array}{llllllllllllll}2006 & \text { ОСТ } 20 & 1840 & 49.62 & 19 & 54.45 & 155 & 53.84 & 37.89 & 29 & .10 & 1.3 & 1.6 & \text { HUA }\end{array}$

$\begin{array}{lllllllllllll}2006 & \text { ОСТ } 20 & 1850 & 42.28 & 19 & 52.90 & 155 & 53.80 & 39.82 & 47 & .09 & .7 & 1.2\end{array}$ $\begin{array}{lllllllllllll}2006 & \text { ОСТ } 20 & 1932 & 10.18 & 19 & 53.96 & 155 & 54.79 & 41.07 & 29 & .09 & 1.2 & 1.5\end{array}$

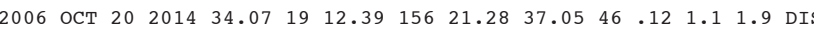

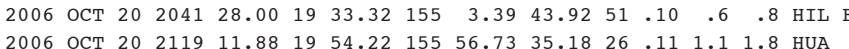

$\begin{array}{llllllllllllll}2006 & \text { ОСт } 20 & 2226 & 4.73 & 19 & 17.54 & 155 & 23.29 & 2.18 & 26 & .13 & .5 & .7 & \text { SWR }\end{array}$

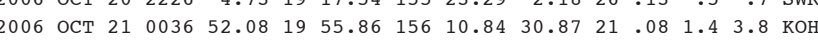

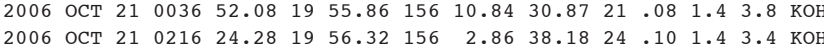

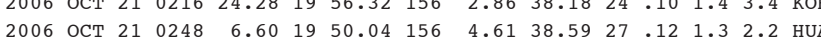

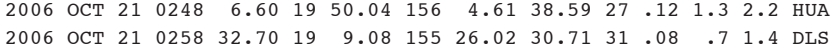

$\begin{array}{llllllllllll}2006 & \text { ОСт } 21 & 0349 & 6.34 & 19 & 52.00 & 156 & 0.14 & 40.43 & 49.11 & .9 & 1.3\end{array}$ $\begin{array}{lllllllllllll}2006 & \text { OCT } 21 & 0428 & 7.88 & 19 & 47.20 & 156 & 11.27 & 40.73 & 19 & .09 & 1.5 & 2.7\end{array}$ $\begin{array}{lllllllllllllll}2006 & \text { OCT } 21 & 0429 & 45.91 & 19 & 53.35 & 156 & 11.57 & 0.03 & 47 & .13 & 2.0 & .4 & \text { HUA } \\ 2006 & \text { OCT } & 21 & 0630 & 45.27 & 20 & 26.22 & 156 & 4.12 & 31.18 & 29 & .11 & 1.7 & 3.7 & \text { DTS }\end{array}$

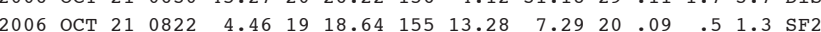

$1.5 \times 231 \quad 17$ $1.8 \times 20315$ $1.7 \times 19024$ $.9 \times 24836$ $2.8 \times 24231$ $1.6 \mathrm{U} 25635$ $2.3 \times 24733$ $1.8 \times 20527$ $1.7 \times 141 \quad 8$

$1.4 \mathrm{U} 23930$ $\begin{array}{lll}1.1 \mathrm{U} & 22027\end{array}$ $2.1 \times 2433$ $1.9 \times \quad 54 \quad 8$

$1.9 \times 20018$

$1.7 \times 113 \quad 15$ $1.9 \times 307 \quad 44$ $2.1 \times 290 \quad 52$ $1.6 \times 120 \quad 7$

$1.6 \times 227 \quad 13$

$2.8 \times 176 \quad 22$ $2.3 \times 21913$ $2.8 \times 301 \quad 44$ $1.5 \times 25439$

$2.4 \times 28152$ $2.0 \times 150 \quad 5$ $1.7 \times 155$ $2.0 \times 146$ 1.9X 20925

2.7x 20122 $1.6 \times 28325$ $2.4 \times 2825$ $\begin{array}{lll}2.8 \mathrm{X} & 95 \quad 16\end{array}$ $2.0 \times 2182$

$1.3 \times 157 \quad 5$ 1.6X 30645 $1.8 \times 29642$ $1.7 \times 28530$ $1.5 \times 29238$ $\begin{array}{lll}1.3 \times 265 \quad 43 \\ 2.5 \times & 321 & 86\end{array}$ $2.5 \times 32186$
---ORIGIN TIME (HST)-- -LAT N-- --LON W-- DEPTH N RMS ERH ERZ LOC PREF AZ MIN 104

$\begin{array}{llllllllllllll}2006 & \text { OCT } 21 & 1000 & 40.73 & 19 & 13.58 & 156 & 21.77 & 35.95 & 50 & .12 & 1.0 & 1.8 & \text { DIS }\end{array}$

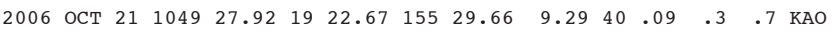
$\begin{array}{lllllllllllll}2006 & \text { OCT } 21 & 1051 & 28.43 & 19 & 22.59 & 155 & 26.69 & 10.37 & 32 & .13 & .4 & .8\end{array}$

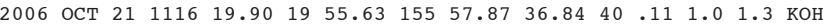
$\begin{array}{llllllllllllll}2006 & \text { ОСт } 21 & 1230 & 8.95 & 19 & 13.37 & 155 & 34.66 & 3.16 & 24 & .12 & .5 & 1.5 & \text { LSW }\end{array}$

$\begin{array}{lllllllllllll}2006 & \text { OCT } 21 & 1304 & 50.49 & 19 & 53.72 & 156 & 8.30 & 38.28 & 16 & .09 & 2.0 & 3.1\end{array}$ HUA 2006 ОСТ $21 \quad 1416 \quad 31.00 \quad 19 \quad 50.51 \quad 155 \quad 54.32 \quad 40.05 \quad 16.12 \quad 2.3 \quad 3.2$ HUA

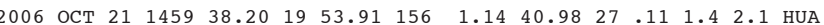
$\begin{array}{lllllllllllll}2006 & \text { OCT } 21 & 1533 & 33.77 & 19 & 20.30 & 155 & 30.16 & 12.04 & 33 & .06 & .3 & .9\end{array}$

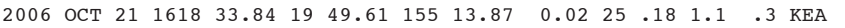

$\begin{array}{llllllllllllll}2006 & \text { ОСт } 21 & 1719 & 16.74 & 19 & 36.30 & 155 & 51.81 & 15.21 & 27 & .09 & .8 & .5 & \text { коN }\end{array}$

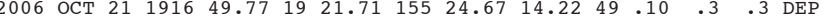

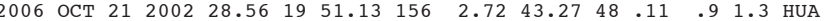

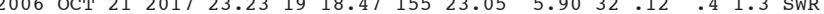
$\begin{array}{llllllllllllllll}2006 & \text { OCT } 21 & 2100 & 44.90 & 19 & 17.99 & 155 & 23.32 & 5.85 & 37 & .14 & .4 & 1.4 & \text { SWR }\end{array}$

$\begin{array}{lllllllllllll}2006 & \text { ОСТ } 21 & 2129 & 46.95 & 19 & 51.97 & 156 & 15.38 & 6.60 & 21 & .11 & 2.5 & 2.7\end{array}$ HUA

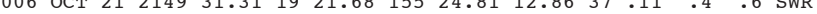

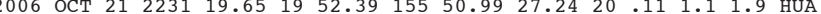

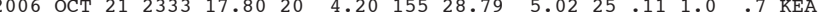

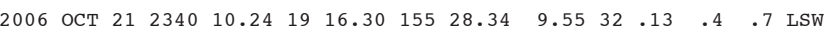

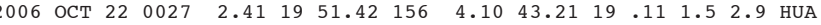

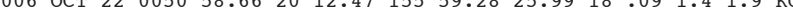

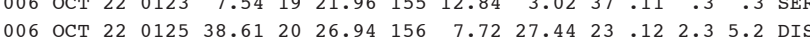

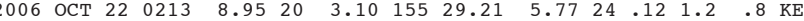

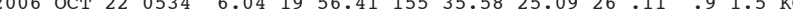

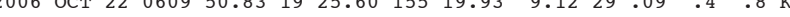

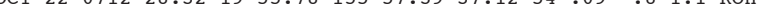

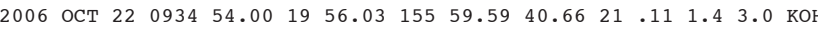
$\begin{array}{lllllllllllll}2006 & \text { ОСТ } 22 & 1116 & 39.86 & 19 & 48.47 & 156 & 2.95 & 34.23 & 21 & .12 & 1.5 & 1.8\end{array}$

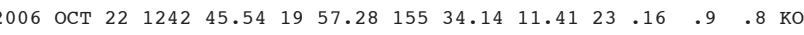

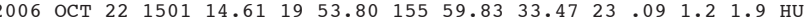

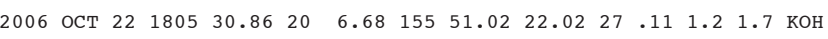

$\begin{array}{lllllllllllll}2006 & \text { ОСт } 22 & 1931 & 48.89 & 19 & 26.37 & 155 & 52.66 & 7.45 & 44 & .15 & .5 & .5 \\ \text { KоN }\end{array}$ $\begin{array}{llllllllllllll}2006 & \text { OCT } & 22 & 2013 & 2.04 & 20 & 2.53 & 155 & 30.07 & 2.31 & 38 & .12 & .8 & .5 \\ 2006\end{array}$

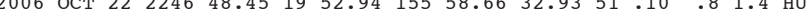

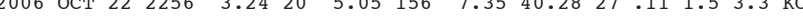
2006 OCT

$\begin{array}{llllllllllll}2006 & \text { OCT } 23 \quad 0058 & 23.73 & 19 & 52.60 & 156 & 3.78 & 44.51 & 47 & .10 & 1.0 & 1.2 \text { HUA F }\end{array}$

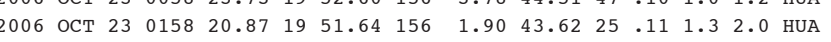

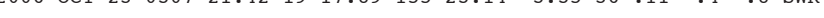

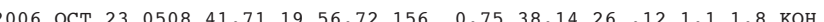

$2.7 \times 28152$ $1.5 \times \quad 58 \quad 4$ $1.4 \times \quad 5902$ $1.5 \times 176 \quad 9$

$1.4 \times 31939$ $1.3 \times 29818$ $1.9 \times 28930$ $1.5 \times \quad 66$

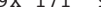
$2.1 \times \quad 55 \quad 4$ $2.4 \times 23828$ $\begin{array}{lll}1.7 \times & 109 & 3 \\ 1.7 \times & 113 & 4\end{array}$

$1.9 \times 30255$ $1.4 \times \quad 59$ $1.5 \times 272 \quad 20$ $1.9 \times 24230$ $1.4 \mathrm{X} \quad 89 \quad 4$

$1.5 \times 28731$ $1.7 \times 308 \quad 24$ $1.5 \times 28125$ $2.0 \times \quad 91$ $1.9 \times 32882$

$1.5 \times 237 \quad 28$ $\begin{array}{lll}1.8 \times 250 & 11\end{array}$ $1.5 \times 113$ $1.4 \times 123 \quad 3$ $1.7 \times 31326$ $1.6 \times 16414$ $1.9 \times 253 \quad 8$

2.5X 1447 $\begin{array}{lll}1.9 \times 198 & 26 \\ 2.5 \times & 207 & 25\end{array}$ 2.5X 20725 $2.2 \times 31253$ $\begin{array}{lll}1.6 \times & 293 \quad 28\end{array}$ $\begin{array}{lll}1.7 \times & 114 & 4 \\ 2.5 \times & 253 & 36\end{array}$ $\begin{array}{lll}2.5 \times & 253 & 36 \\ 1.7 x & 242 & 32\end{array}$ 
---ORIGIN TIME (HST)-- -LAT N-- --LON W-- DEPTH N RMS ERH ERZ LOC

$\begin{array}{lllllllllllllll}2006 & \text { OCT } 23 & 0543 & 12.16 & 19 & 17.50 & 155 & 23.50 & 4.30 & 24 & .13 & .6 & 2.0 & \text { SWR }\end{array}$

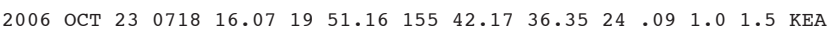
$\begin{array}{lllllllllllll}2006 & \text { OCT } 23 & 0751 & 16.32 & 19 & 22.48 & 155 & 27.21 & 9.64 & 31 & .13 & .4 & .8 \\ \text { KAO }\end{array}$ $\begin{array}{lllllllllllllll}2006 & \text { OCT } 23 & 0809 & 37.48 & 19 & 18.26 & 155 & 22.09 & 3.67 & 24 & .14 & .6 & 1.1 & \text { SWR }\end{array}$ $\begin{array}{llllllllllllll}2006 & \text { ОСТ } 23 & 0844 & 4.84 & 19 & 54.71 & 156 & 5.01 & 39.18 & 37 & .08 & .9 & 1.3 & \text { HUA }\end{array}$

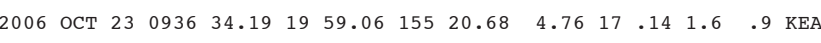
$\begin{array}{llllllllllllll}2006 & \text { OCT } 23 & 1112 & 36.28 & 19 & 53.38 & 156 & 9.04 & 1.97 & 15 & .14 & 2.9 & 1.3 & \text { HUA }\end{array}$ $\begin{array}{lllllllllllll}2006 & \text { ост } 23 & 1137 & 30.32 & 19 & 51.83 & 155 & 56.80 & 40.75 & 25 & .08 & 1.0 & 1.8\end{array}$ $\begin{array}{llllllllllllll}2006 & \text { OCT } 23 & 1842 & 23.58 & 19 & 42.39 & 156 & 2.96 & 0.43 & 22 & .09 & 2.0 & .5 & \text { HUA }\end{array}$ $\begin{array}{llllllllllllll}2006 & \text { ОСт } 23 & 1910 & 50.98 & 19 & 19.60 & 155 & 6.98 & 7.81 & 40 & .09 & .4 & .6 & \text { SF } 4\end{array}$

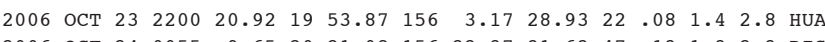

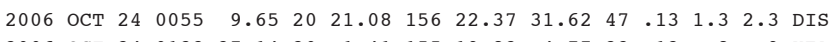
$\begin{array}{lllllllllllll}2006 & \text { ОСТ } 24 & 0123 & 37.14 & 20 & 1.41 & 155 & 19.83 & 4.77 & 23 & .12 & .8 & .9 \\ \text { KEA }\end{array}$ $\begin{array}{llllllllllllllll}2006 & \text { OCT } 24 & 0337 & 48.67 & 19 & 46.50 & 156 & 9.71 & 35.09 & 20 & .11 & 2.2 & 2.7 & \text { HUA }\end{array}$

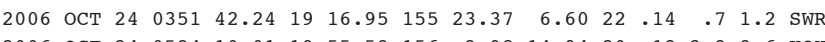

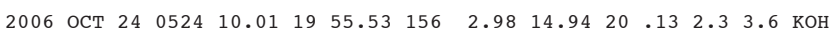

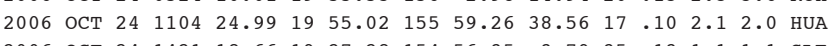

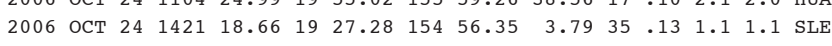
$\begin{array}{lllllllllllllll}2006 & \text { Ост } 24 & 1441 & 34.42 & 19 & 20.24 & 155 & 11.90 & 8.78 & 46 & .11 & .4 & .5 & \text { SF3 }\end{array}$

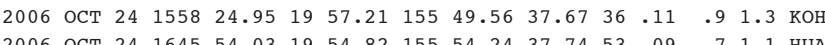
$\begin{array}{lllllllllllll}2006 & \text { ОСТ } 24 & 1645 & 54.03 & 19 & 54.82 & 155 & 54.24 & 37.74 & 53 & .09 & .7 & 1.1\end{array}$ $\begin{array}{llllllllllllll}2006 & \text { ОСТ } 24 & 1802 & 16.82 & 19 & 17.69 & 155 & 13.98 & 5.65 & 27 & .12 & .5 & 1.2 & \text { SF2 }\end{array}$

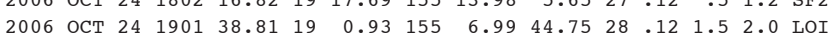

$\begin{array}{llllllllllllllll}\infty & 2006 & \text { ОСТ } 24 & 2059 & 36.88 & 19 & 20.19 & 155 & 3.66 & 6.34 & 28 & .14 & .7 & .8 & \text { SF5 }\end{array}$

$\begin{array}{llllllllllllll}2006 & \text { ОСт } 24 & 2145 & 22.00 & 19 & 58.22 & 155 & 38.01 & 11.62 & 27 & .13 & .9 & .6 & \text { кон }\end{array}$ $\begin{array}{llllllllllllll}2006 & \text { OCT } 24 & 2208 & 15.94 & 19 & 52.11 & 155 & 52.30 & 30.52 & 23 & .09 & 2.5 & 1.4 & \text { HUA }\end{array}$

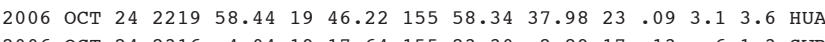

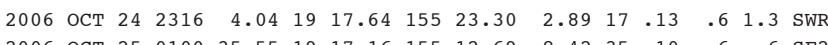

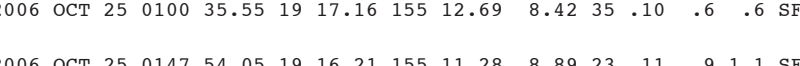
$\begin{array}{lllllllllllllll}2006 & \text { OCT } 25 & 0147 & 54.05 & 19 & 16.21 & 155 & 11.28 & 8.89 & 23 & .11 & .9 & 1.1 & \mathrm{SF} 3\end{array}$

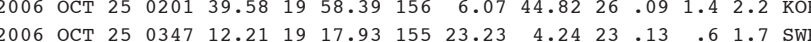
$\begin{array}{llllllllllllll}2006 & \text { OCT } 25 & 0433 & 18.79 & 20 & 8.26 & 155 & 49.70 & 22.96 & 22 & .12 & 2.0 & 1.7 & \text { кон }\end{array}$

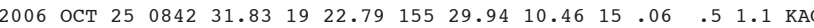

$\begin{array}{lllllllllllllll}2006 & \text { OCT } 25 & 0847 & 54.42 & 19 & 34.76 & 155 & 8.92 & 1.69 & 40 & .14 & .4 & .7 & \text { HIL }\end{array}$

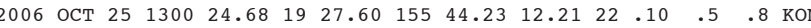
$\begin{array}{lllllllllllll}2006 & \text { ОСТ } 25 & 1434 & 8.81 & 19 & 51.18 & 156 & 11.82 & 43.24 & 19 & .09 & 2.5 & 3.1\end{array}$ $\begin{array}{llllllllllllll}2006 & \text { OCT } 25 & 1439 & 27.65 & 19 & 16.11 & 155 & 31.80 & 29.39 & 30 & .08 & .5 & 1.4 & \text { DLS }\end{array}$

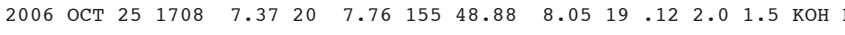

$\begin{array}{llllllllllllll}2006 & \text { ОСт } 25 & 1813 & 8.26 & 19 & 57.26 & 155 & 48.19 & 14.85 & 18 & .11 & 1.7 & .7 & \text { кон }\end{array}$

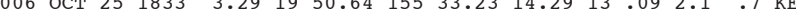
$\begin{array}{lllllllllllllll}2006 & \text { OCT } 25 & 1926 & 7.09 & 20 & 5.67 & 155 & 49.16 & 11.38 & 17 & .11 & 2.4 & .7 & \text { KOH }\end{array}$

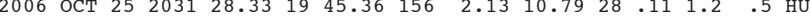

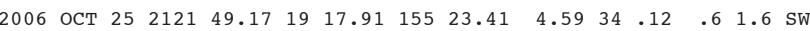

$1.3 \times 157 \quad 5$ $1.5 \times 222 \quad 5$ $\begin{array}{lrr}1.5 \times & 62 & 1 \\ 1.3 \times & 115 & 4 \\ 2.4 x & 246 & 29\end{array}$ $2.4 \times 24629$

$1.4 \times 28925$ $\begin{array}{lll}1.6 \times & 33540 \\ 1.8 \times & 217 & 23\end{array}$ $1.9 \times 29722$ $1.5 \times 149$

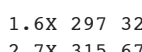
$\begin{array}{llll}2.7 \times & 315 & 67 \\ 1.8 \times & 255 & 30\end{array}$ $\begin{array}{llll}1.8 \times & 255 & 30 \\ 2.7 \times 276 & 23\end{array}$ $\begin{array}{llll}1.7 \times & 276 & 23 \\ 1.4 \times 298 & 35\end{array}$

$1.3 \times 177 \quad 6$ $1.3 \times 24634$ $\begin{array}{llll}1.3 \times & 246 & 34 \\ 1.5 \times & 331 & 30\end{array}$ $\begin{array}{lll}1.5 \times & 331 & 30 \\ 1.8 \times & 149 & 2\end{array}$ $2.7 \times \quad 78 \quad 5$

1.8X 16220 2.5X 20626 $1.2 \times \quad 95 \quad 2$ 1.8X 26132 $1.4 \times 189 \quad 8$

2.0X $187 \quad 13$ $1.8 \times 26520$ $2.0 \times 26432$ $1.1 \times 156 \quad 5$ $\begin{array}{lll}1.6 \times & 156 & 1\end{array}$

$\begin{array}{lll}1.2 \times & 211 \quad 4\end{array}$ $1.9 \times 303 \quad 42$ $1.2 \times 1524$ $1.8 \times 278 \quad 5$ $\begin{array}{lll}1.2 \mathrm{X} & 80 & 4\end{array}$

$\begin{array}{lll}1.8 \times & 97 \quad 19\end{array}$ $1.2 \times 118 \quad 6$ $1.6 \times 32642$ $\begin{array}{lll}.8 \mathrm{X} \quad 62 \quad 4 & 4\end{array}$ .
--ORTGIN TIME (HST)-- -LAT N-- --LON W-- DEPTH N RMS ERH ERZ LOC

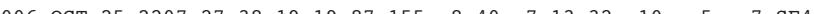

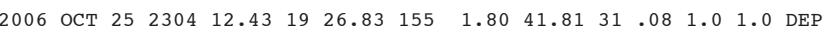

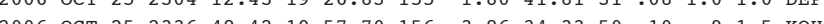

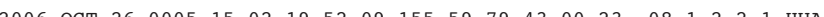

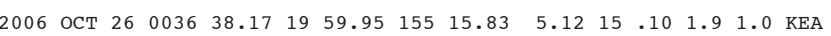

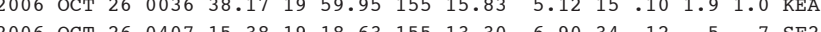

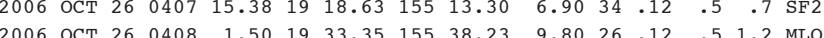

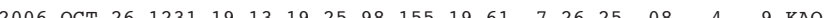

$\begin{array}{llllllllllllll}2006 & \text { ост } 26 & 1341 & 53.07 & 19 & 54.87 & 155 & 51.94 & 36.62 & 51 & .10 & .6 & 1.3 & \text { HUA }\end{array}$

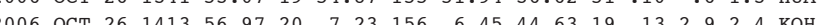

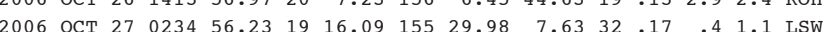

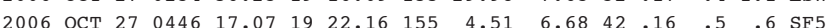

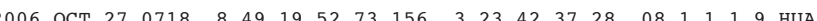

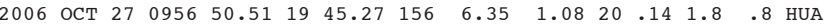

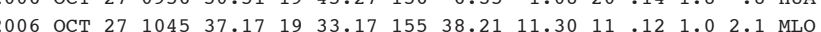

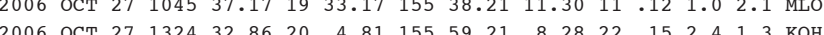

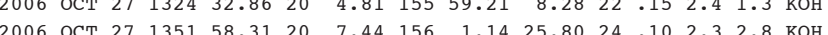

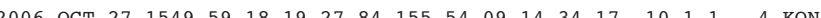

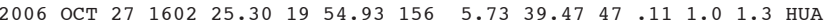

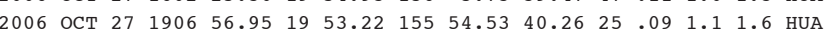

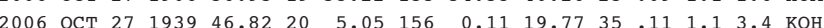

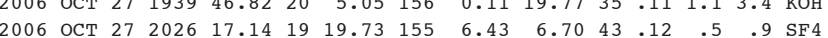
$\begin{array}{lllllllllllll}2006 & \text { OCT } 27 & 2026 & 45.66 & 19 & 20.11 & 155 & 6.27 & 6.23 & 42 & .11 & .5 & 9\end{array}$

$\begin{array}{llllllllllllll}2006 & \text { ОСт } 27 & 2126 & 11.72 & 19 & 20.03 & 155 & 8.71 & 8.31 & 39 & .10 & .4 & .6 & \text { SF } 4\end{array}$ $\begin{array}{lllllllllllll}2006 & \text { ОСТ } 27 & 2309 & 7.03 & 19 & 54.83 & 155 & 58.76 & 27.60 & 24 & .10 & 1.3 & 2.4 \\ 2006\end{array}$

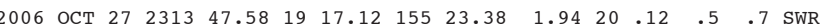

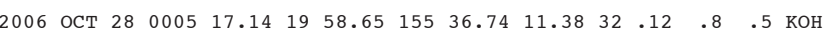

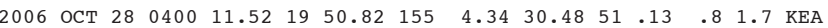

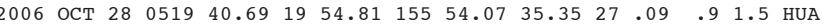
$\begin{array}{lllllllllllll}2006 & \text { OCT } 28 & 0557 & 1.15 & 19 & 24.45 & 155 & 25.80 & 0.88 & 20 & .14 & .3 & .6 \\ \text { KAO }\end{array}$

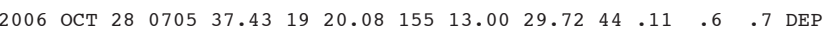

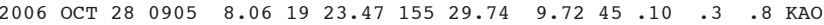
$\begin{array}{lllllllllllll}2006 & \text { ост } 28 & 0910 & 21.19 & 19 & 17.54 & 155 & 23.11 & 2.53 & 31.12 & .4 & .6 & \text { SWR }\end{array}$

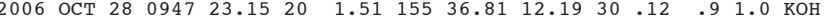

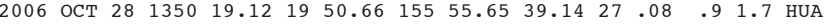

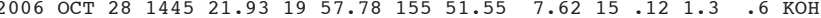

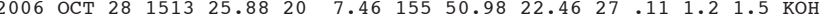
$\begin{array}{llllllllllllll}2006 & \text { ОСТ } 28 & 1706 & 10.48 & 20 & 4.39 & 155 & 34.41 & 32.72 & 24 & .12 & 1.4 & 1.8 & \text { кон }\end{array}$

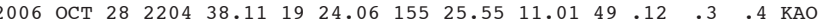

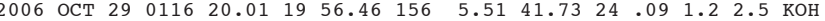

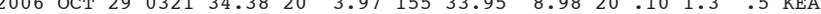
$\begin{array}{llllllllllllll}2006 & \text { OCT } 29 & 0356 & 10.85 & 19 & 17.64 & 155 & 23.47 & 2.66 & 40 & .15 & .4 & .7 & \text { SWR }\end{array}$

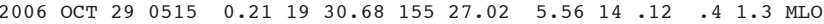

(1)

$1.5 \times 110 \quad 5$ $1.7 \times 133$ $\begin{array}{lll}1.7 \times & 133 & 653 \\ 1.6 \times 230-26\end{array}$ $1.5 \times 24010$

$1.4 \times 30132$ $\begin{array}{lll}1.6 \times & 82 \quad 3\end{array}$ .1X 140 $3.3 \times 29329$

$\begin{array}{lll}2.8 \times & 170 \quad 22\end{array}$ $\begin{array}{llll}1.8 \times & 283 \quad 34\end{array}$ $\begin{array}{lll}1.5 \times \quad 77 & 2\end{array}$ $2.2 \times 2423$

$1.7 \times 30929$ $1.9 \times 145$ $\begin{array}{lll}2.1 \times & 264 & 22 \\ 2.4 \times & 277 & 25\end{array}$ $\begin{array}{lll}2.4 \times 277 & 25 \\ 1.4 \times 156\end{array}$

$2.3 \times 253 \quad 37$ $1.4 \times 20423$ $2.1 \times 267 \quad 24$ $1.8 \times 157$ $1.8 \times 157$

$1.4 \times 103$ $2.0 \times 23029$ $1.0 \times 174$ $\begin{array}{llll}1.7 \times & 162 & 14 \\ 2.7 \times & 214 & 17\end{array}$

1.6X 20526 $1.5 \times \quad 60$ $1.6 \mathrm{X} \quad 70$ $\begin{array}{lll}1.6 \times & 54 \\ 1.1 \times & 117\end{array}$

2.2X 19119 $\begin{array}{lll}1.8 \times 208 & 20\end{array}$ $1.5 \times 3032$ $\begin{array}{lll}2.2 \times & 264 & 72 \\ 2.0 \times 218 \quad 22\end{array}$

$1.8 \mathrm{X} \quad 38 \quad 5$ $1.8 \times \quad 299 \quad 39$ $1.4 \times 303 \quad 25$ $1.7 \times 115$ $1.1 \times 105$ 
---ORIGIN TIME (HST)-- -LAT N-- --LON W-- DEPTH N RMS ERH ERZ LOC PREF AZ MIN 107 YEAR MON DA HRMN SEC DEG MIN DEG MTN KM RD SEC KM KM REMKS MAC GAP DS

$\begin{array}{lllllllllllllll}2006 & \text { ОСТ } 29 & 0518 & 38.98 & 19 & 29.80 & 155 & 28.04 & 6.15 & 18 & .10 & .4 & 1.8 & \text { КАО }\end{array}$ $\begin{array}{lllllllllllll}2006 & \text { ОСТ } 29 & 0522 & 36.05 & 19 & 53.94 & 156 & 4.64 & 38.02 & 30 & .08 & 1.0 & 1.8\end{array}$

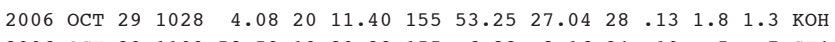
$\begin{array}{llllllllllllll}2006 & \text { ОСТ } 29 & 1109 & 58.59 & 19 & 20.28 & 155 & 6.88 & 8.16 & 34 & .10 & .5 & .7 & \text { SF } 4\end{array}$

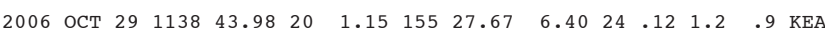

$\begin{array}{lllllllllllllll}2006 & \text { ОСТ } 29 & 1243 & 22.68 & 19 & 19.72 & 155 & 4.10 & 2.58 & 29 & .13 & .7 & .9 & \text { SSP }\end{array}$ $\begin{array}{llllllllllllll}2006 & \text { оСт } 29 & 1317 & 0.67 & 19 & 7.31 & 155 & 30.52 & 43.57 & 23 & .14 & 1.4 & 2.0 & \text { DLS }\end{array}$

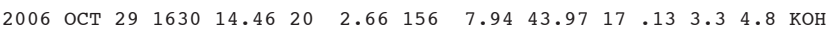

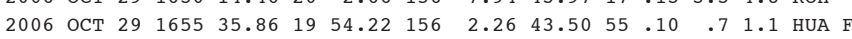

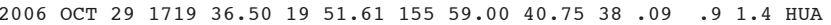

$\begin{array}{llllllllllllll}2006 & \text { ОСТ } 29 & 1848 & 55.26 & 19 & 14.60 & 155 & 31.08 & 33.39 & 31 & .07 & .7 & 1.3 & \text { DLS }\end{array}$

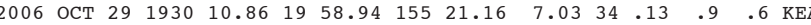
$\begin{array}{llllllllllllll}2006 & \text { ОСТ } 29 & 1950 & 34.98 & 19 & 58.64 & 155 & 20.49 & 6.11 & 29 & .13 & .9 & .5 & \mathrm{KEA}\end{array}$

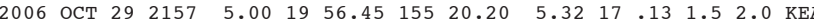
$\begin{array}{lllllllllllllll}2006 & \text { ОСТ } 29 & 2343 & 2.03 & 19 & 56.23 & 155 & 21.89 & 9.76 & 45 & .12 & .7 & .5 & \mathrm{KEA}\end{array}$

2006 Ост $292359 \begin{array}{lllllllllll}16.78 & 19 & 59.64 & 155 & 20.69 & 6.32 & 26 & .14 & 1.1 & .8 & \text { KEA }\end{array}$

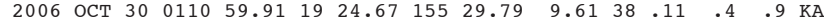

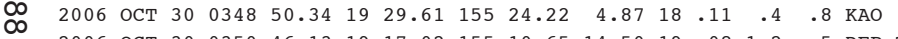

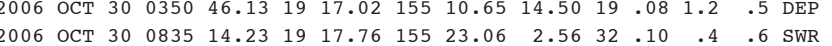

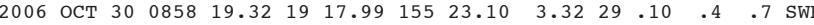

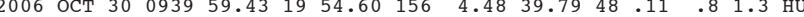

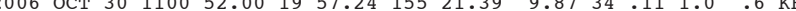

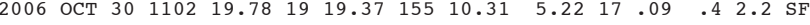
$\begin{array}{lllllllllllll}2006 & \text { ОСТ } 30 & 1110 & 35.29 & 19 & 23.68 & 155 & 27.84 & 10.17 & 47 & .12 & .3 & .5 \\ \text { KAO }\end{array}$

$\begin{array}{llllllllllllll}2006 & \text { ОСТ } 30 & 1113 & 42.31 & 19 & 23.37 & 155 & 28.04 & 10.59 & 22 & .10 & .6 & 1.0 & \text { KAO }\end{array}$

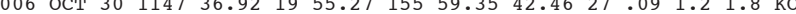

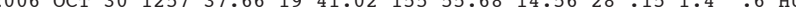

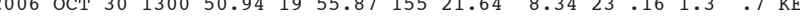

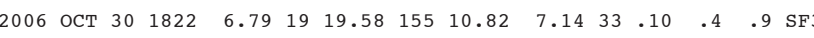

$\begin{array}{llllllllllllll}2006 & \text { ОСт } 30 & 1824 & 31.10 & 19 & 58.07 & 155 & 35.67 & 12.10 & 54 & .10 & .5 & .7 & \text { кон }\end{array}$

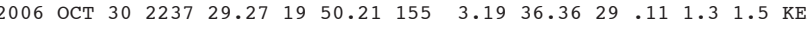

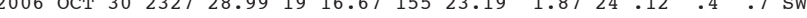

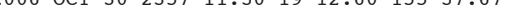

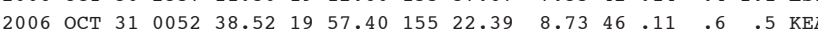

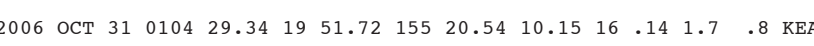
$\begin{array}{lllllllllllll}2006 & \text { ОСТ } 31 & 0438 & 6.37 & 19 & 23.78 & 155 & 25.65 & 9.69 & 44 & .13 & .4 & .6 \\ \text { KAO }\end{array}$ $\begin{array}{llllllllllllll}2006 & \text { ОСТ } 31 & 0612 & 7.30 & 19 & 19.68 & 155 & 8.64 & 4.95 & 37 & .14 & .4 & 1.5 & \text { SSF }\end{array}$

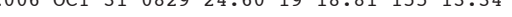
$\begin{array}{llllllllllllll}2006 & \text { ост } 31 & 1105 & 15.71 & 19 & 20.42 & 155 & 12.91 & 7.12 & 40 & .08 & .4 & .6 & \text { SF }\end{array}$

$\begin{array}{llllllllllllll}2006 & \text { OCT } 31 & 1124 & 3.20 & 19 & 17.42 & 155 & 23.07 & 7.11 & 24 & .14 & .6 & 1.5 & \text { SWR }\end{array}$ $\begin{array}{lllllllllllll}2006 & \text { ОСТ } 31 & 1152 & 57.16 & 20 & 2.17 & 156 & 10.23 & 38.11 & 26 & .10 & 1.5 & 2.9 \\ \text { кон }\end{array}$ $\begin{array}{llllllllllllll}2006 & \text { OCT } 31 & 1509 & 25.18 & 19 & 21.78 & 155 & 4.32 & 6.97 & 41 & .12 & .5 & .8 & \text { SF }\end{array}$ $\begin{array}{lllllllllllll}2006 & \text { ОСТ } 31 & 1614 & 11.21 & 19 & 54.01 & 155 & 49.71 & 16.00 & 18 & .09 & 1.4 & 1.2 \\ 2006 & \text { ОСТ } 31 & 1622 & 7.41 & 19 & 49.12 & 155 & 44.78 & 11.73 & 19 & .08 & .7 & .4\end{array}$ $\begin{array}{rrr}1.2 \times & 84 & 4 \\ 2.3 \times & 248 & 34\end{array}$ $2.0 \times 29813$ $1.5 \times 143$ $1.7 \times 29746$

$1.4 \times 2119$ $\begin{array}{lll}1.5 \times & 219 & 14 \\ 1.0 \times & 323 & 50\end{array}$ \begin{tabular}{lll}
$.0 \times$ & 232 & 27 \\
\hline$x$ & 225 & 24
\end{tabular}

$1.5 \times \quad 68 \quad 2$ $1.3 \times 23025$ $1.5 \times 27525$ $1.1 \times 25222$ 1.9X 18920

$1.5 \times 23926$ $1.4 \times \quad 52$ $\begin{array}{lll}1.4 \mathrm{X} & 87 \quad 2\end{array}$ $1.4 \times 188$ $1.6 \times 115 \quad 5$

$\begin{array}{lll}1.5 \times 151 \quad 4 & 4\end{array}$ $\begin{array}{lll}2.7 \times & 243 \quad 29\end{array}$ $2.1 \times 267 \quad 22$ $1.9 \times 100 \quad 6$ $2.0 \times \quad 41 \quad 2$

1.3X $106 \quad 2$ $1.9 \times 23232$ $1.9 \times 270 \quad 9$ $1.6 \times 27920$ $1.7 \times \quad 96 \quad 6$

$\begin{array}{lll}3.4 \times & 163 & 14\end{array}$ $1.6 \times 26815$ $1.1 \times 125$ $\begin{array}{rrr}1.9 \times & 83 & 14 \\ 2.3 \times & 192 & 21\end{array}$ $1.3 \mathrm{U} 27515$ $1.9 \times \quad 58 \quad 4$ $\begin{array}{lll}1.3 \times & 105 & 4\end{array}$ $1.9 \times \quad 79 \quad 3$ $\begin{array}{lll}.5 \times \quad 66 \quad 4 & 4\end{array}$ $1.2 \times 159 \quad 5$ $2.0 \times 27642$ $\begin{array}{lll}1.8 \times 161 & 5\end{array}$ $\begin{array}{lll}1.3 \times & 268 & 18\end{array}$ $1.3 \times 20910$
--ORIGIN TIME (HST)-- -LAT N-- --LON W-- DEPTH N RMS ERH ERZ LOC PREF AZ MIN 108 YEAR MON DA HRMN SEC DEG MIN DEG MIN KM RD SEC KM KM REMKS MAG GAP DS

$\begin{array}{lllllllllllll}2006 & \text { ОСТ } 31 & 1751 & 37.17 & 20 & 2.01 & 155 & 27.60 & 6.69 & 22 & .13 & 1.2 & .8 \\ \text { KEA }\end{array}$ $\begin{array}{lllllllllllllll}2006 & \text { ОСТ } 312302 & 25.07 & 19 & 53.29 & 155 & 58.62 & 32.84 & 19 & .10 & 1.7 & 2.3 & \text { HUA }\end{array}$

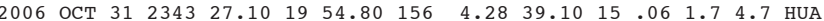

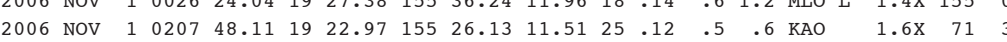

2006 NOV $10307 \quad 29.791923 .60154 \quad 45.63 \quad 44.34 \quad 44 \quad 13 \quad 1.2 \quad 1.0$ LER

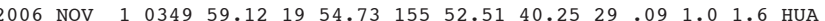

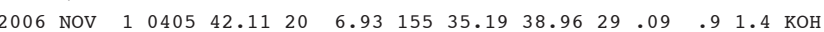

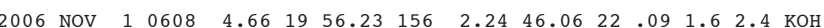
2006 NOV $1065941.93 \quad 19 \quad 18.28 \quad 155 \quad 21.66 \quad 34.86 \quad 18.12 \quad 1.2 \quad 2.1 \quad$ DEP

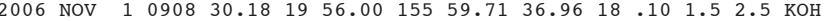

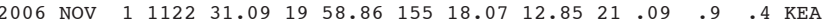

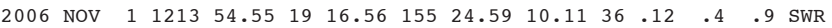
2006 NOV $111334 \quad 3.17 \quad 19 \quad 17.60 \begin{array}{llllllllll}154 & 58.60 & 19.40 & 19 & .11 & 1.6 & 1.9 & \text { LER }\end{array}$

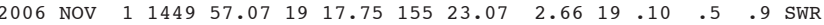

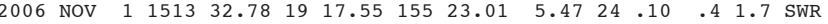

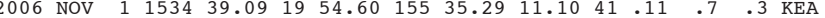

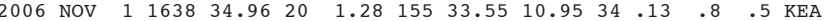

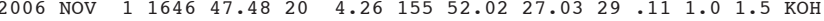

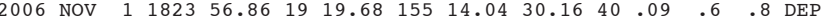

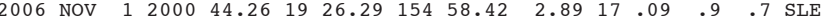

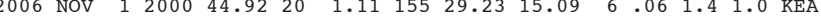
2006 NOV $1201641.601959 .0515529 .9941 .73 \quad 20.101 .31 .9$ KEA

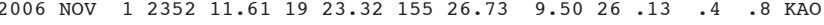
2006 Nov $2 \begin{array}{lllllllllllll}0 & 0127 & 9.60 & 19 & 13.91 & 155 & 31.49 & 6.18 & 32 & .13 & .4 & 1.0 & \text { LSW }\end{array}$

2006 NOV $2 \begin{array}{llllllllllll}0517 & 20.62 & 19 & 21.54 & 155 & 4.59 & 5.91 & 41 & .13 & .5 & .9 & \text { SF5 }\end{array}$

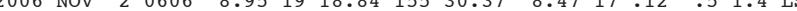

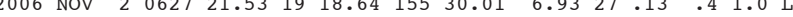

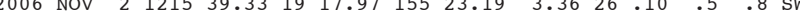
2006 NOV $2 \begin{array}{llllllllllllll}1809 & 22.61 & 19 & 23.14 & 155 & 25.12 & 9.29 & 40 & .14 & .4 & .7 & \text { KAO }\end{array}$

2006 NOV $2 \begin{array}{llllllllllll}1842 & 33.74 & 19 & 20.16 & 155 & 13.31 & 4.97 & 21 & .14 & .5 & 1.7 & \text { SSF }\end{array}$ 2006 NOV $2 \begin{array}{llllllllllll}2012 & 21.06 & 19 & 49.25 & 155 & 56.47 & 38.49 & 28 & .10 & 1.0 & 1.9\end{array}$ HUA

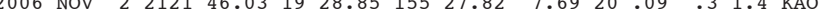

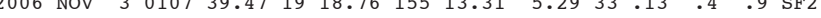
$\begin{array}{lllllllllllllll}2006 & \text { Nov } & 3 & 0138 & 42.17 & 19 & 21.62 & 155 & 4.33 & 7.14 & 34 & .12 & .6 & .7 & \text { SF5 }\end{array}$

$\begin{array}{lllllllllllllll}006 & \text { Nov } & 3 & 0426 & 3.81 & 19 & 24.24 & 155 & 29.52 & 8.71 & 25 & .10 & .3 & .9 & \text { KAO }\end{array}$

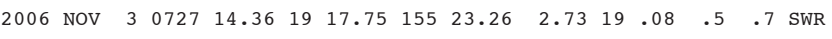

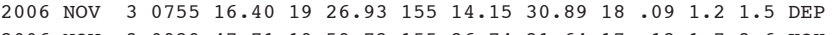

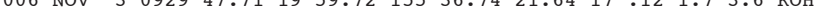
2006 NOV $3 \begin{array}{lllllllllllll}1026 & 59.78 & 19 & 49.02 & 156 & 12.50 & 39.17 & 22 & .12 & 1.6 & 2.5 & \text { HUA }\end{array}$

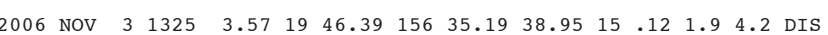

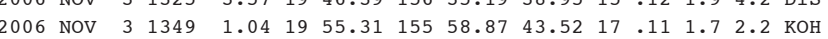
2006 NOV $3 \begin{array}{llllllllllll}3 & 1438 & 40.97 & 19 & 53.11 & 156 & 3.66 & 44.76 & 55 & .10 & .8 & 1.1 \text { HUA }\end{array}$ $\begin{array}{llllrllllllllll}2006 & \text { Nov } & 3 & 1455 & 17.74 & 19 & 28.20 & 154 & 52.82 & 1.77 & 37 & .15 & .8 & .5 & \text { SLE F } \\ 2006 \text { NOV } & 3 & 1826 & 5.12 & 19 & 10.18 & 155 & 31.72 & 30.30 & 50 & .08 & .5 & 1.0 & \text { DLS }\end{array}$
$2.1 \times 28015$ $1.9 \times 196 \quad 23$ $1.9 \times 21220$ $2.0 \times \quad 245 \quad 34$

$1.6 \times \quad 236 \quad 31$ $1.7 \times 20811$ $1.6 \times 117$ $1.1 \times 251 \quad 14$ $1.4 \times 155$

\begin{tabular}{ll}
$.4 \times 117$ \\
\hline
\end{tabular} $2.1 \times 220$ $1.7 \times 18421$ $\begin{array}{lll}2.0 \times & 240 & 11 \\ 1.9 \times & 63 & 5\end{array}$

$1.7 \times 126$ $1.2 \times 2012$ $1.8 \times 25119$ $1.4 \mathrm{X} \quad 69$ $1.8 \times 129 \quad 3$

$1.9 \times 161$ $1.3 \mathrm{U} \quad 71$ $1.5 \times \quad 72$ $1.6 \times 152$ $1.6 \times \quad 43 \quad 5$

$\begin{array}{lll}1.0 \times \quad 65 & 5\end{array}$ $\begin{array}{lll}1.5 \times & 210 & 18 \\ 1.6 \times & 77 & 6\end{array}$ $\begin{array}{llll}1.6 \times & 77 \quad 6\end{array}$ $1.8 \times 163$

1.9X 51 $1.4 \mathrm{U} 154 \quad 5$ $1.2 \times 2228$ $1.5 \times 27316$ 2.0X 2989 $1.6 \times 29930$ $2.9 \times 23726$ $2.2 \times 116$ 
---ORIGIN TIME (HST)-- -LAT N-- --LON W-- DEPTH N RMS ERH ERZ LOC

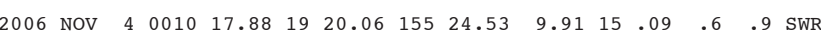

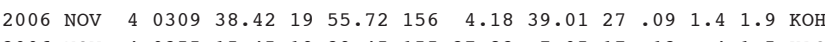
$\begin{array}{llllllllll} & \end{array}$ $\begin{array}{lllll} & \end{array}$

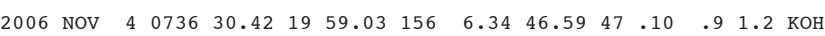

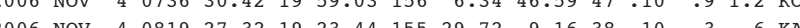
$\begin{array}{lllllllll}.9 & 1.2 \\ 2006 & \text { KAO }\end{array}$

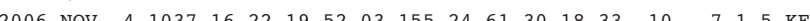

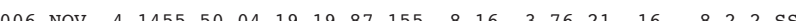

2006 NOV $4 \begin{array}{llllllllllll}1509 & 48.12 & 19 & 57.98 & 155 & 28.67 & 0.02 & 20 & .17 & 1.9 & .6 & \text { KEA }\end{array}$ 2006 NOV $4 \quad \begin{array}{llllllllllll}1518 & 36.18 & 19 & 28.11 & 155 & 32.00 & 33.64 & 17 & .07 & .9 & 1.6 & \text { DML }\end{array}$ 2006 Nov $4 \begin{array}{lllllllllllll}4 & 1532 & 1.58 & 19 & 10.02 & 155 & 29.06 & 6.44 & 22 & .17 & .7 & 1.5 & \text { LSW }\end{array}$

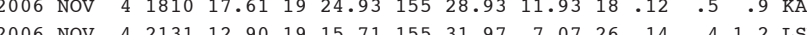

2006 Nov $4 \begin{array}{lllllllllllll}2145 & 51.80 & 20 & 4.04 & 155 & 51.47 & 12.12 & 14 & .13 & 2.0 & 1.0 & \text { кон }\end{array}$

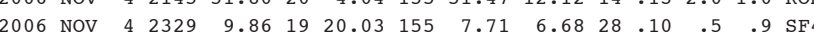

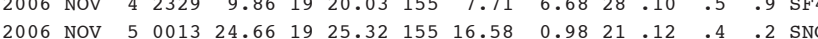

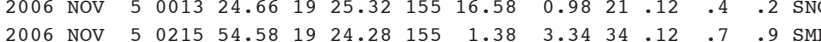
2006 NOV $\begin{array}{llllllllllllll}5 & 0250 & 14.82 & 19 & 21.78 & 155 & 3.99 & 7.57 & 24 & .12 & .7 & .9 & \text { SME } & \end{array}$

2006 NOV $5 \begin{array}{lllllllllllll}5 & 0303 & 30.63 & 19 & 30.27 & 155 & 26.41 & 12.56 & 19 & .12 & .5 & 1.0 & \text { MLO }\end{array}$ 2006 NOV $5 \begin{array}{lllllllllllll}5 & 0657 & 49.41 & 19 & 23.77 & 155 & 29.69 & 8.75 & 34 & .09 & .3 & .8 & \text { KAO }\end{array}$ 2006 NOV $5 \begin{array}{lllllllllllll}5 & 0803 & 1.12 & 19 & 29.02 & 155 & 25.36 & 10.95 & 19 & .11 & .5 & .8 & \text { KAO }\end{array}$

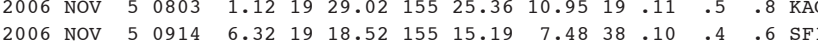

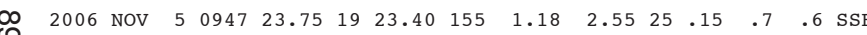

2006 NOV $5 \begin{array}{lllllllllllll}1254 & 39.35 & 19 & 57.72 & 156 & 6.28 & 37.09 & 17 & .10 & 2.0 & 3.3 & \text { кон }\end{array}$ 2006 NOV $5 \begin{array}{llllllllllllll}5 & 1555 & 27.51 & 19 & 18.34 & 155 & 23.18 & 5.43 & 22 & .14 & .5 & 1.6 & \text { SWR }\end{array}$ 2006 NOV $5 \begin{array}{lllllllllllll}5 & 2141 & 33.39 & 19 & 27.82 & 15 & 14.35 & 33.80 & 40 & 14 & .5 & 9 & 9\end{array}$

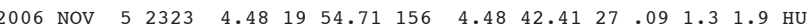
2006 Nov $6 \begin{array}{llllllllllllll}6 & 0350 & 52.12 & 19 & 19.84 & 155 & 8.31 & 7.14 & 32 & .11 & .5 & .9 & \mathrm{SF}\end{array}$

2006 NOV $6 \begin{array}{llllllllllll}6 & 0449 & 18.69 & 19 & 20.93 & 155 & 19.03 & 2.54 & 29 & .10 & .3 & .6\end{array}$

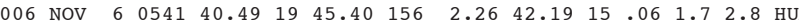
2006 NOV $6 \begin{array}{lllllllllllll}6 & 0642 & 24.28 & 19 & 17.91 & 155 & 23.58 & 2.32 & 20 & .12 & .5 & .7 & \text { SWR }\end{array}$ 2006 Nov $\quad \begin{array}{lllllllllllll}6 & 0810 & 40.87 & 19 & 21.45 & 155 & 5.93 & 6.95 & 36 & .12 & .5 & .8 & \text { SF } 4\end{array}$ 2006 NOV $6 \begin{array}{lllllllllllll}6 & 0859 & 48.82 & 19 & 14.17 & 155 & 1.75 & 40.24 & 26 & .07 & 1.3 & 1.3 & \text { DEP }\end{array}$

2006 NOV $6 \begin{array}{lllllllllllll}6 & 1035 & 52.40 & 19 & 58.54 & 155 & 35.09 & 12.94 & 19 & .11 & .7 & .7 & \text { кон }\end{array}$ 2006 NOV $6 \begin{array}{llllllllllll}1038 & 50.85 & 19 & 58.51 & 155 & 35.56 & 13.48 & 57 & .11 & .6 & .6 & \text { кон }\end{array}$ 2006 NOV $6 \begin{array}{llllllllllll}6 & 1157 & 37.21 & 19 & 52.38 & 155 & 50.75 & 16.36 & 23 & .13 & .9 & 1.9\end{array}$

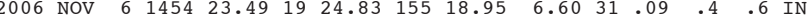
2006 NOV $6 \begin{array}{lllllllllllll}6 & 1717 & 6.82 & 19 & 22.62 & 155 & 29.95 & 9.54 & 17 & .07 & .5 & 1.1 & \text { KAO }\end{array}$

2006 NOV $6 \begin{array}{lllllllllllll}6 & 2142 & 10.75 & 19 & 22.25 & 155 & 29.87 & 9.61 & 31 & .10 & .4 & .8 & \text { KAO }\end{array}$

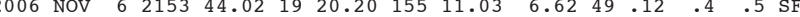

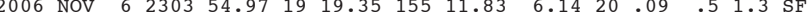

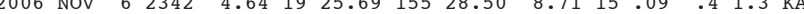
2006 NOV $7 \begin{array}{llllllllllllll}7 & 0032 & 0.53 & 19 & 56.36 & 156 & 2.62 & 38.87 & 22 & .09 & 1.3 & 2.4 & \text { кон }\end{array}$
$1.4 \mathrm{U} 117 \quad 2$ $2.0 \times 24936$ $1.8 \times 26631$ $1.4 \times 182$

$2.5 \times 25937$ $1.3 \times 216 \quad 9$ $1.7 \times 123-7$ $1.2 \times 116$ \# $1.4 \times 24916$ $1.5 \times \quad 90 \quad 6$ $\begin{array}{rrrr}1.1 \times & 154 & 2 \\ 1.0 x & 60 & 5\end{array}$ $\begin{array}{lll}1.0 \mathrm{x} & 60 & 5 \\ 1.2 \mathrm{x} & 97 & 3\end{array}$

$1.3 \times 23911$ $1.3 \times 126 \quad 5$ $\begin{array}{lll}1.3 \times & 126 & 5 \\ 1.5 \times & 153 & 1\end{array}$ $\begin{array}{lll}1.5 \times & 153 & 1 \\ 1.8 \times & 152 & 5\end{array}$ $1.1 \times 165 \quad 5$

1.3X $101 \quad 4$ $1.3 \times \quad 61$ $1.2 \mathrm{x} \quad 58 \quad 4$ $\begin{array}{lll}1.6 \times & 99 \quad 4\end{array}$ $1.6 \times 199 \quad 5$

1.9X 29341 $1.1 \times \quad 109 \quad 3$ $\begin{array}{lll}1.6 \mathrm{X} & 61 & 6\end{array}$ $1.7 \times 25235$ $1.3 \times 113 \quad 5$

$1.0 \times \quad 69 \quad 5$ $1.5 \times 30940$ $1.4 \mathrm{U} 157 \quad 4$ $1.6 \times 145 \quad 5$ $1.6 \times 246 \quad 12$

$1.7 \times 160 \quad 15$ $3.1 \times 158 \quad 15$ 2.0X 19620 $\begin{array}{lll}1.7 x & 69 & 2\end{array}$ $1.1 \times \quad 71 \quad 4$
- ORIGIN TIME (HST)-- -LAT N-- --LON W-- DEPTH N RMS ERH ERz LOC YEAR MON DA HRMN SEC DEG MIN DEG MTN KM RD SEC KM KM REM 2006 NOV $7 \begin{array}{lllllllllllll} & 0120 & 4.87 & 19 & 21.81 & 155 & 10.66 & 2.77 & 21 & .09 & .4 & .5 & \text { SER }\end{array}$

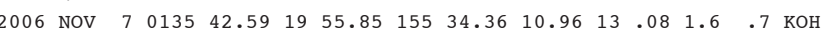

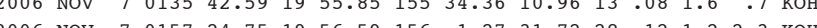

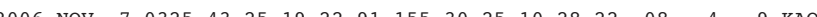

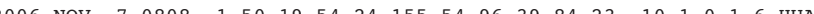

2006 Nov $7 \quad 092153.02 \quad 19 \quad 57.42 \quad 156 \quad 1.13 \quad 37.68 \quad 30.09 \quad 1.1 \quad 1.7$ кон 2006 NOV $\begin{array}{lllllllllllll}7 & 1705 & 57.74 & 19 & 22.43 & 155 & 29.92 & 10.88 & 27 & .09 & .4 & .9 & \text { KAO }\end{array}$ 2006 NOV $7 \begin{array}{lllllllllllll}1820 & 41.82 & 19 & 24.68 & 155 & 29.48 & 10.58 & 25 & .12 & .4 & 1.0 & \text { KAO }\end{array}$ 2006 Nov $8 \begin{array}{lllllllllllll}0 & 0013 & 22.26 & 19 & 20.81 & 155 & 5.61 & 6.49 & 36 & .12 & .5 & .6 & \text { SF } 4\end{array}$ 2006 Nov $8 \begin{array}{lllllllllllll}0254 & 33.53 & 19 & 24.42 & 154 & 58.40 & 3.81 & 33 & .14 & .8 & .5 & \text { SLE }\end{array}$

2006 NOV $8 \begin{array}{lllllllllllll}8 & 0505 & 23.49 & 19 & 24.37 & 154 & 58.73 & 4.49 & 15 & .07 & 1.5 & .6 & \text { SLE }\end{array}$ 2006 NOV $8 \quad \begin{array}{llllllllllllll}0703 & 46.03 & 18 & 52.98 & 155 & 31.87 & 39.16 & 25 & .10 & 1.6 & 1.7 & \text { DLS }\end{array}$

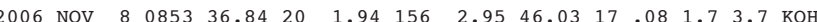

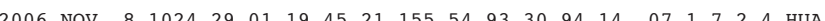

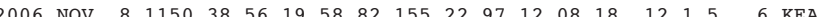

2006 NOV $8 \begin{array}{llllllllllllllll} & 1357 & 23.48 & 19 & 49.74 & 155 & 44.41 & 10.57 & 53 & .12 & .6 & .3 & \text { HUA } & \text { F } & 2.8 \times & 190\end{array}$ 2006 NoV $8 \quad \begin{array}{lllllllllllllll}1717 & 27.97 & 19 & 24.27 & 155 & 34.23 & 49.61 & 35 & .15 & .8 & 1.3 & \text { DML L } & 2.7 \times & 45\end{array}$ 2006 NOV $8 \begin{array}{llllllllllllllll}8 & 1808 & 59.16 & 19 & 25.86 & 15 & 30.78 & 12.27 & 23 & .10 & .4 & .9 & \text { KAO } & 1.5 \times & 68\end{array}$

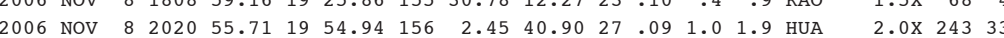

2006 NOV $82058 \quad 58.361922 .43 \quad 155 \quad 39.06 \quad 42.72 \quad 12.12 \quad 1.4 \quad 1.9$ DML L $1.9 \times 108$

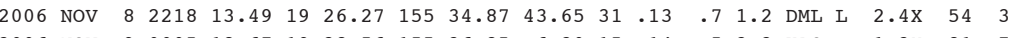

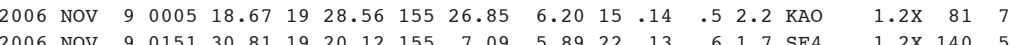

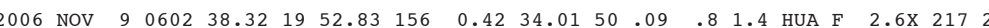

2006 Nov $9 \begin{array}{llllllllllllllll}0647 & 14.65 & 19 & 18.57 & 155 & 14.94 & 6.40 & 39 & .11 & .4 & .6 & \text { SF1 } & 1.7 x & 94 & 4\end{array}$

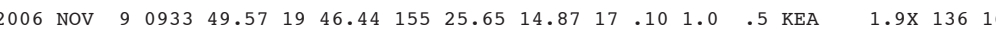

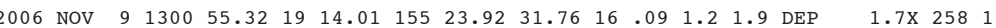
2006 NOV $9 \begin{array}{lllllllllllllll}1843 & 4.44 & 19 & 26.87 & 155 & 35.68 & 53.36 & 32 & .13 & .9 & 1.3 & \text { DML L } & 2.5 \mathrm{X} & 55\end{array}$

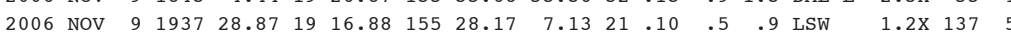

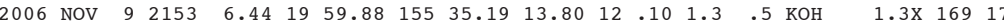

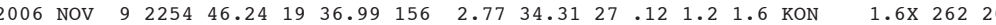

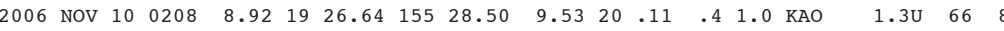

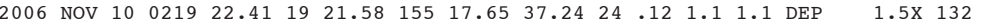

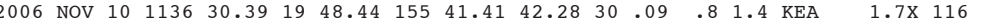

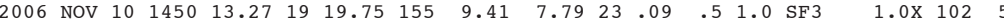

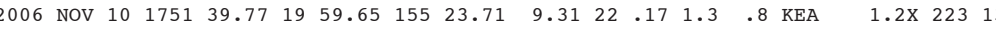

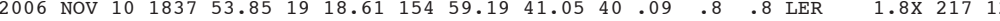

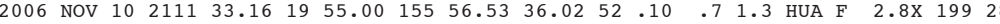

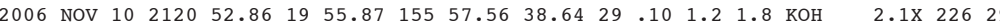

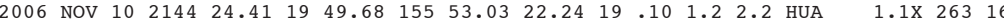

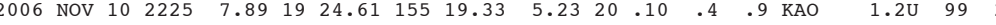

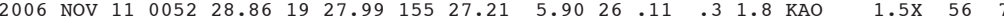

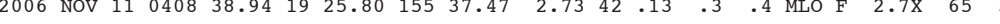

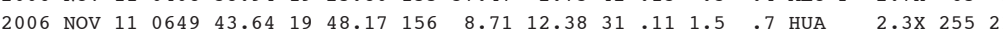


---ORIGIN TIME (HST)-- -LAT N-- --LON W-- DEPTH N RMS ERH ERZ LOC YEAR MON DA HRMN SEC DEG MIN DEG MIN KM RD SEC KM KM REMKS

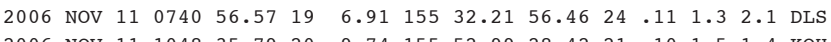

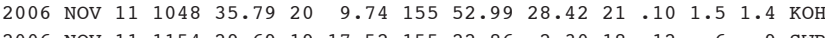

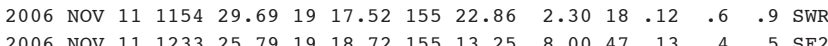

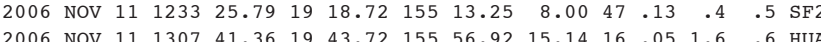

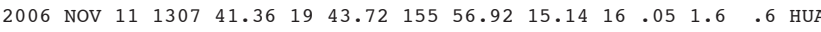

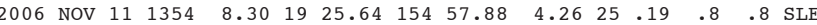

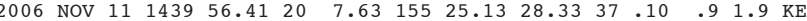

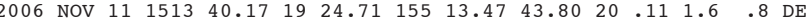

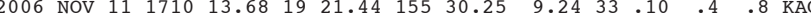

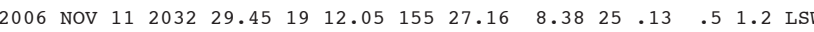

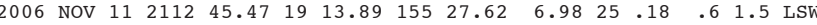

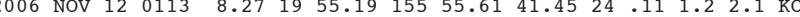

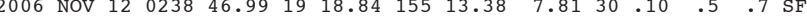

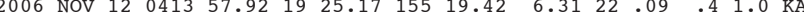

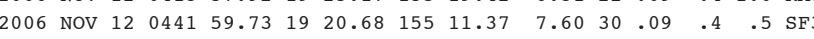

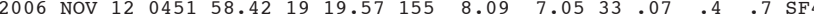

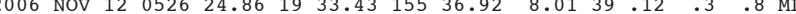

8 2006 NOV $120925 \quad 2.1719 \begin{array}{lllllllllll}124.01 & 155 & 22.96 & 10.21 & 27 & .10 & .4 & .6 & \text { KAO }\end{array}$

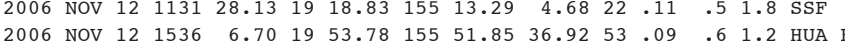

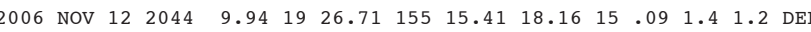

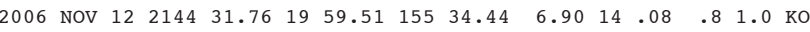

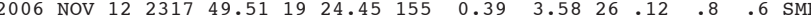
2006 NOV $130218 \quad \begin{array}{llllllllllll} & 4.14 & 19 & 26.92 & 155 & 24.20 & 8.52 & 13 & .12 & .6 & 1.3 & \text { KAO }\end{array}$

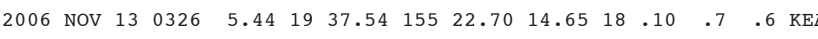

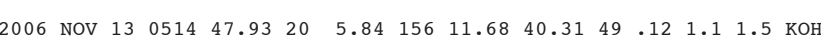

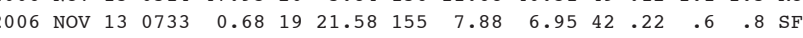

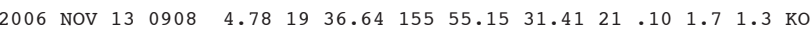

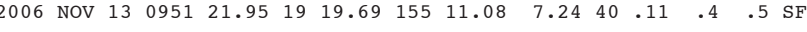

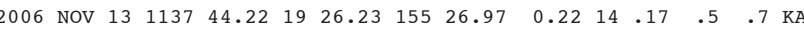

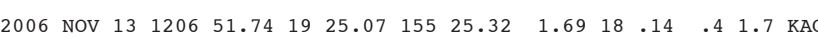

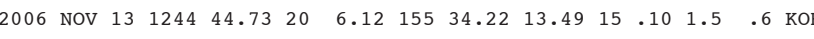

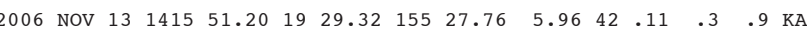

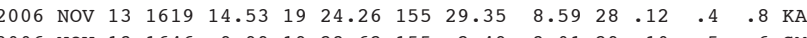

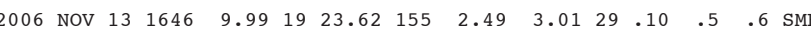

2006 NOV $13 \quad 1712 \quad 14.76 \quad 19 \quad 55.37 \quad 155 \quad 55.05 \quad 36.73 \quad 15 \quad .12 \quad 1.6 \quad 2.5$ кон

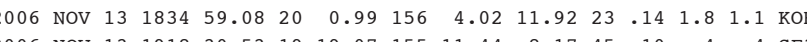

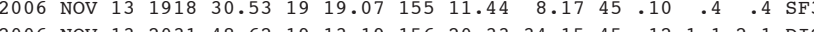

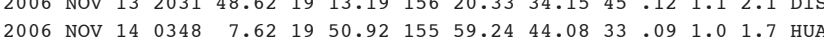

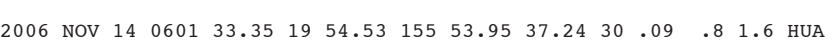
$\begin{array}{llllllllllllll}2006 & \text { NOV } 14 & 1023 & 43.05 & 19 & 11.71 & 155 & 24.90 & 35.27 & 33 & .09 & .8 & 1.3 & \text { DEP } \\ 2006 & \text { NOV } 14 & 1039 & 45.72 & 19 & 49.49 & 155 & 52.78 & 14.34 & 19 & .11 & 1.0 & .6 & \text { HUA }\end{array}$

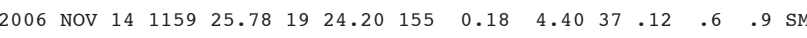

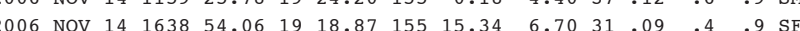

PREF AZ MIN 11 $\begin{array}{lll}.8 \times & 290 & 11 \\ .2 \times & 158 & 5\end{array}$ $2.1 \times \quad 82 \quad 3$ $1.1 \times 29212$

$1.6 \times 173 \quad 2$ $1.9 \times 227 \quad 27$ $1.6 \times 221 \quad 4$ $1.3 \times \quad 62 \quad 5$ $1.4 \times 122 \quad 5$

$1.2 \times 105 \quad 5$ $.9 \times 21528$ $1.1 \times \quad 77 \quad 3$ $\begin{array}{rrr}1.1 \times & 120 & 3 \\ 1.3 \times & 74 & 4\end{array}$

$1.4 \times 119 \quad 4$ $1.9 \times 101$ $1.3 \mathrm{U} \quad 70$ $\begin{array}{lll}1.1 \times & 80 \quad 3\end{array}$ $7 \times 19122$ 1.2X $253 \quad 5$ $1.3 \times 168 \quad 17$ $\begin{array}{lll}1.7 \times & 160 & 3\end{array}$ $\begin{array}{lll}1.7 \times & 87\end{array}$ 1.4X 15111 $2.5 \times 28644$ $1.9 \times 114 \quad 5$ 1.3X 27820 $\begin{array}{lll}.8 \mathrm{x} & 93 & 6 \\ .8 \times & 72 & 7\end{array}$ $.8 \times \quad 72 \quad 7$ $\begin{array}{lrr}1.4 \times & 65 & 6\end{array}$ $\begin{array}{lll}1.5 \times 282 & 28 \\ 1.7 \times & 47 & 5\end{array}$ $\begin{array}{lll}1.7 \mathrm{X} & 47 & 5\end{array}$ $\begin{array}{lll}1.0 \times & 51 & 4\end{array}$

$1.7 \times 277$ $1.7 \times 27728$ $\begin{array}{llll}1.6 \times & 26133 \\ 1.8 \times & 107 & 5\end{array}$ $\begin{array}{llll}1.8 \times & 107 & 5\end{array}$ $2.0 \times 28049$

$2.0 \times 18225$ $1.7 \times 162$ $\begin{array}{lll}1.7 \times & 162 & 7 \\ 1.2 x & 158 & 16\end{array}$ $\begin{array}{lll}1.2 \times 158 & 16 \\ 2.0 \times 171 & 3\end{array}$ $\begin{array}{lll}2.0 \times & 171 & 3 \\ 1.5 \times & 118 & 4\end{array}$
---ORIGIN TIME (HST)-- -LAT N-- --LON W-- DEPTH N RMS ERH ERZ LOC PREF AZ MIN 112 YEAR MON DA HRMN SEC DEG MIN DEG MIN KM RD SEC KM KM REMKS MAG GAP DS

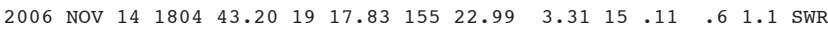

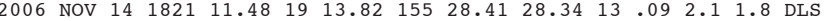

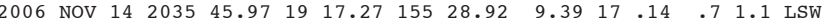

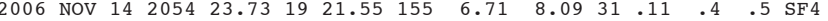

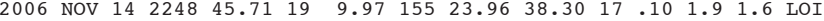

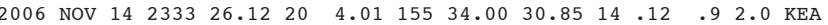

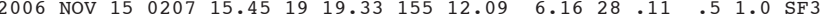

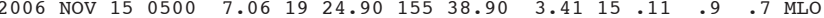

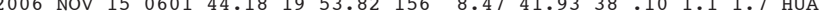
2006 NOV $15 \quad \begin{array}{lllllllllllllll}0624 & 30.07 & 20 & 17.44 & 155 & 37.21 & 27.57 & 11.16 & 1.9 & 2.2 & \text { кон }\end{array}$

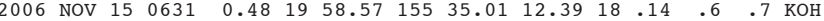

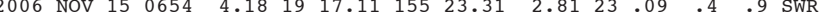

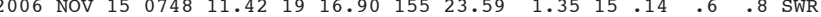
$\begin{array}{llllllll}155 & 23.32 & 1.97 & 19 & .10 & .6 & .9 & \text { SWR }\end{array}$ 2006 NOV $15 \quad \begin{array}{llllllllllllll}0854 & 46.82 & 19 & 26.78 & 155 & 28.45 & 10.71 & 14 & .11 & .6 & 1.8 & \text { KAO }\end{array}$

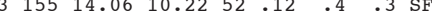

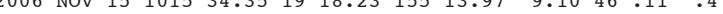

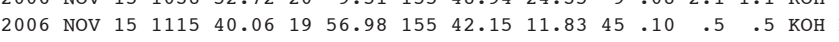

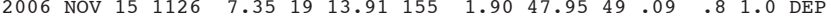

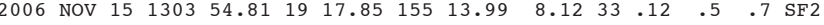

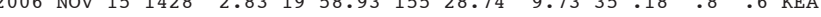

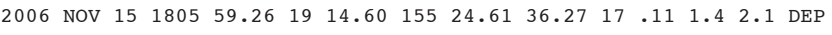

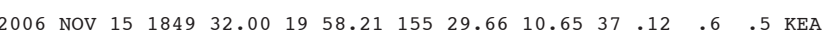

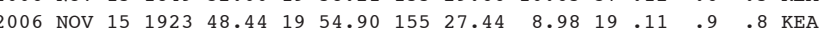

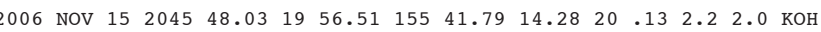

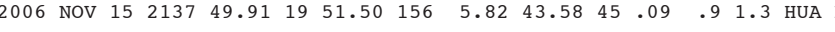

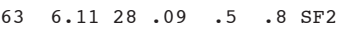

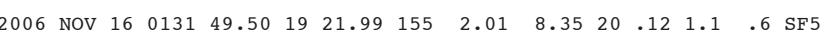

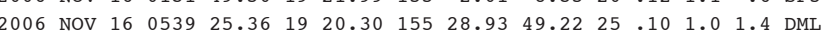

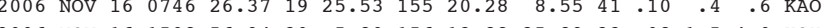
2006 NOV $16 \quad 1508 \quad 56.94 \begin{array}{llllllllll}16 & 15.80 & 156 & 12.88 & 25.89 & 22 & .08 & 1.5 & 4.0 & \text { кон }\end{array}$

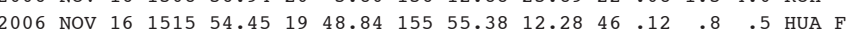

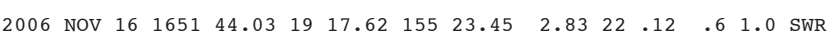
2006 NOV $16 \quad \begin{array}{llllllllllll}1703 & 49.65 & 19 & 24.06 & 155 & 29.11 & 9.93 & 21 & .08 & .4 & 1.0 & \text { KAO }\end{array}$

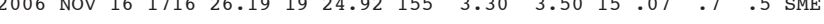

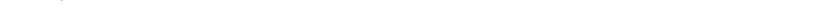

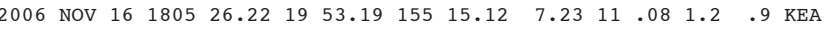

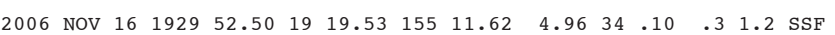

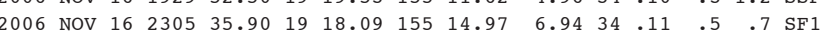
2006 NOV $17 \quad \begin{array}{llllllllllll}0231 & 58.85 & 19 & 11.84 & 155 & 27.45 & 7.58 & 40 & .14 & .4 & .8 & \text { LSW }\end{array}$

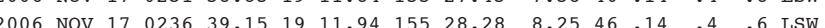
2006 Nov $17 \begin{array}{lllllllllllll}0747 & 1.70 & 19 & 17.60 & 155 & 29.98 & 8.78 & 31 & .11 & .3 & .9 & \text { LSW }\end{array}$
$1.1 \mathrm{U} 160$ $1.4 \mathrm{U} 2361$

$\begin{array}{lll}.4 \times \quad 20123 & 23\end{array}$ $1.2 \times \quad 94$ $2.1 \times 258 \quad 39$ $1.5 \times 28325$

$\begin{array}{lll}1.4 \times \quad 160 & 15\end{array}$ $1.5 \times 120 \quad 6$ $1.2 \times 163$ $\begin{array}{lll}1.0 \times & 78 \quad 8\end{array}$

$2.8 \times 137$ $2.4 \times 135$ $1.8 \times 1360$ $1.7 \times \quad 89$ $\begin{array}{llll}1.7 \times \quad 185 & 18\end{array}$ $1.4 \times 183 \quad 18$ 1.1X 19710

2.2X $178 \quad 1$ $1.1 \times 247 \quad 12$ $1.5 \times 25032$ $\begin{array}{rrr}2.1 \times & 247 & 24 \\ 1.3 \times & 175 & 2\end{array}$

$1.1 \times 202$ $\begin{array}{lll}1.5 \mathrm{x} & 91 & 5 \\ 1.8 \mathrm{x} & 80 & 4\end{array}$ $\begin{array}{lll}1.8 \times & 80 & 4\end{array}$ $2.0 \times 30146$

$1.3 \times 156 \quad 5$ $1.4 \times \quad 51 \quad 4$ $\begin{array}{lll}1.5 \times & 124 & 2 \\ 1.7 \times \quad 55 & 5\end{array}$ $1.2 \times \quad 218 \quad 10$

$1.4 \times \quad 94$ $\begin{array}{llll}1.3 \times & 141 & 3 \\ 1.7 \times & 117 & 4\end{array}$ $\begin{array}{lll}1.7 \times & 117 & 4 \\ 2.1 \times & 97 & 5\end{array}$ $\begin{array}{lll}2.1 \times & 97 & 5 \\ 1.3 \times & 74 & 5\end{array}$ 
---ORIGIN TIME (HST)-- -LAT N-- --LON W-- DEPTH N RMS ERH ERZ LOC

2006 Nov $17 \quad 0929 \quad 6.17 \quad 19 \quad 10.96 \quad 156 \quad 18.19 \quad 37.64 \quad 22 \quad .12 \quad 2.0 \quad 3.2$ коN

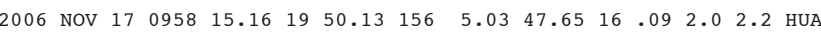

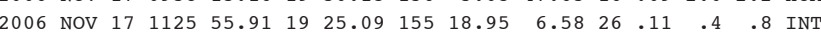

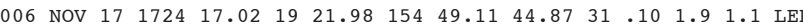

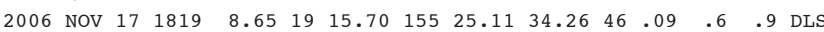

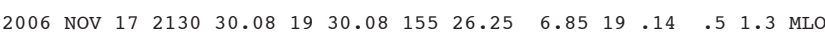
2006 Nov $17 \begin{array}{llllllllllllll}17 & 2334 & 49.52 & 19 & 11.78 & 155 & 27.82 & 6.64 & 39 & .14 & .4 & .7 & \text { LSW }\end{array}$

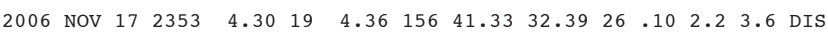
2006 NOV $18 \quad 0129 \begin{array}{lllllllllll}18.20 & 19 & 29.91 & 155 & 27.13 & 7.31 & 21 & .08 & .3 & 1.1 & \text { KAO }\end{array}$

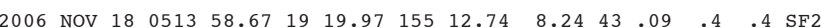

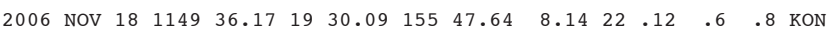

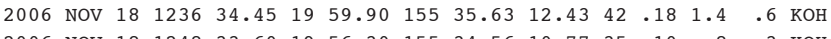

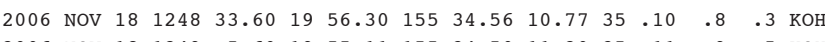
$\begin{array}{llllllllllllll}2006 & \text { NOV } 18 & 1249 & 5.60 & 19 & 55.11 & 155 & 34.50 & 11.20 & 35 & .11 & .9 & .5 & \text { KOH }\end{array}$

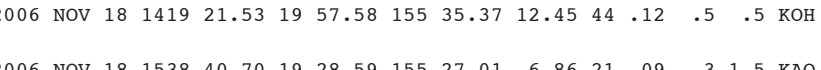

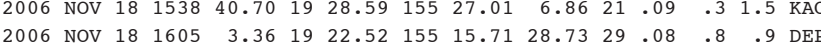

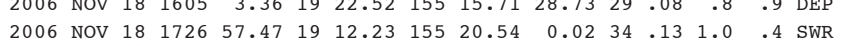

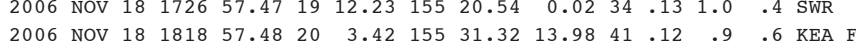

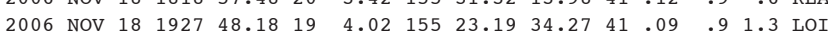

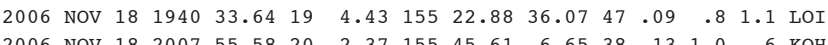

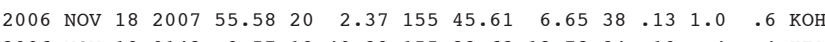
$\begin{array}{llllllllllllll}2006 & \text { Nov } 19 & 0142 & 9.57 & 19 & 40.39 & 155 & 22.63 & 13.78 & 34 & .10 & .4 & .4 & \text { KEA } \\ 2006 & \text { Nov } 19 & 0207 & 45.59 & 19 & 48.62 & 155 & 36.01 & 14.73 & 25 & .11 & .8 & .6 & \text { KEA }\end{array}$

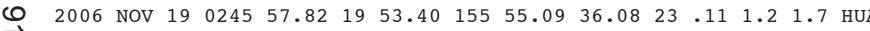

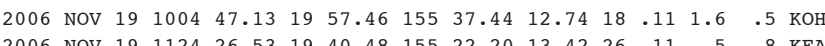

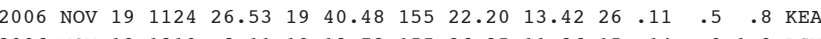

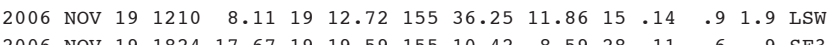
2006 NOV $19 \begin{array}{llllllllllll}1824 & 17.67 & 19 & 19.59 & 155 & 10.42 & 8.59 & 28 & .11 & .6 & .9 & \mathrm{SF} 3\end{array}$

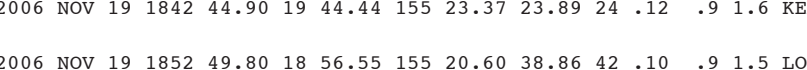

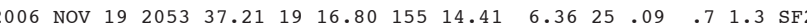

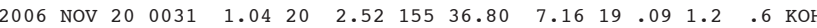

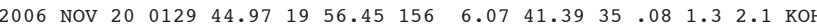

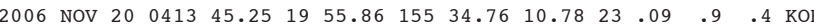

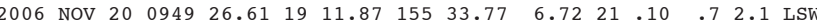

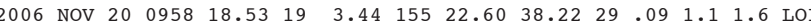
2006 NOV $20103918.38 \quad 19 \quad 3.49155 \quad 22.92 \quad 37.06 \quad 33.091 .1 \quad 1.6 \quad$ LOI

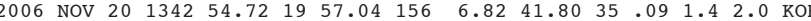

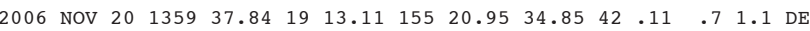

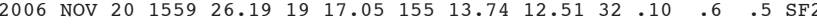

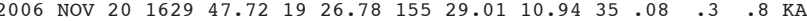

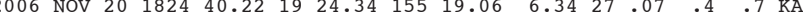

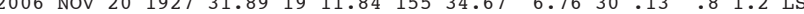
2006 NOV $20 \begin{array}{llllllllllllll}2025 & 34.34 & 19 & 25.01 & 155 & 19.34 & 6.13 & 36 & .10 & .4 & .7 & \text { KAO }\end{array}$
$1.5 \times 317 \quad 46$ $1.4 \times 30930$ $1.7 \times 302 \quad 15$ $1.7 \times 30215$

$1.3 \times \quad 99 \quad 4$ $1.5 \times 109$ 1. $9 \times 32289$ $1.2 \times \quad 92 \quad 4$ $1.5 \times \quad 75$

$1.4 \times \quad 84 \quad 3$ $2.1 \times 257 \quad 17$

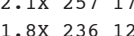
$2.0 \times 223 \quad 11$ $2.0 \times 223 \quad 11$ $\begin{array}{lrl}1.1 \times & 81 & 6 \\ 1.6 \times & 115 & 0\end{array}$ $1.3 \times 22011$ $\begin{array}{lll}1.3 \times & 220 & 11 \\ 2.8 \times & 200 & 26\end{array}$ $\begin{array}{lll}1.8 \times & 206 & 26\end{array}$ $2.5 \times 202 \quad 23$ $2.1 \times 274 \quad 23$ $1.5 \times 115 \quad 14$ $\begin{array}{rrr}1.5 \times & 119 & 8\end{array}$ $1.5 \times \quad 270 \quad 24$

$1.0 \times 24711$ $1.5 \times 11814$ $1.3 \times 216 \quad 12$ 1.3X $194 \quad 6$ $\begin{array}{lll}1.3 \times & 113 & 17\end{array}$

1.9X 24935 $1.0 \times 188$ $1.5 \times 28421$ 2.2X 29154 $1.6 \times \quad 233 \quad 12$

$1.8 \times 217 \quad 9$ $.5 \times \quad 224 \quad 15$ $1.7 \times 282 \quad 14$ 2.6X 29341 $1.9 \times 172 \quad 10$

1.6X $168 \quad 0$ $1.5 \mathrm{X} \quad 57 \quad 8$ $\begin{array}{lll}.9 \mathrm{X} & 88 \quad 2\end{array}$

$1.8 \times \quad 222 \quad 10$

$\begin{array}{lll}1.7 \mathrm{X} & 78 & 2\end{array}$
- ORTGIN TIME (HST)-- -LAT N-- - LON W-- DEPTH N RMS ERH ERZ LOC YEAR MON DA HRMN SEC DEG MIN DEG MIN KM RD SEC KM KM REMKS

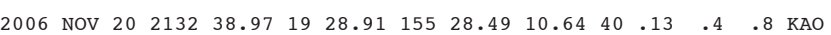

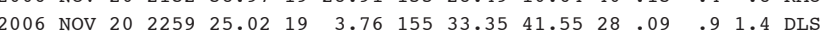
$\begin{array}{llllllllll}2006 & .8 & \text { KAO }\end{array}$

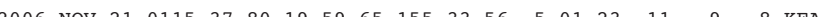

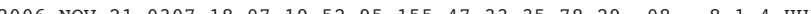

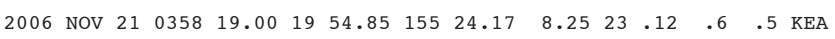

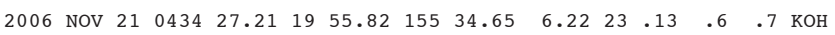
$\begin{array}{lllllllll}2006 & & 0\end{array}$

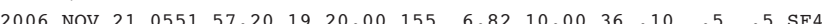

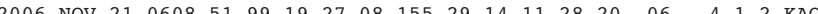

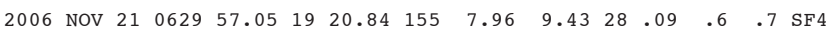

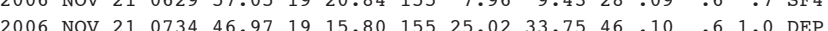

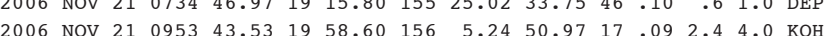

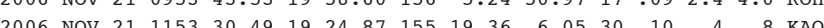

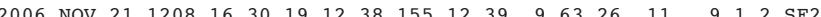

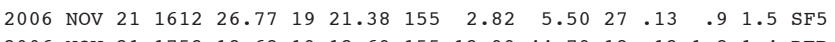

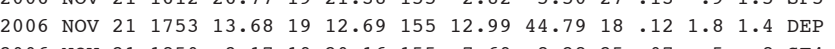

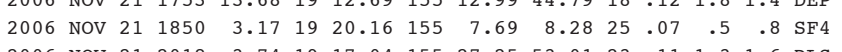

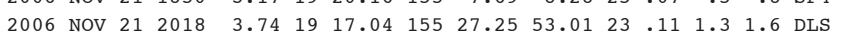

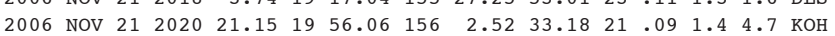

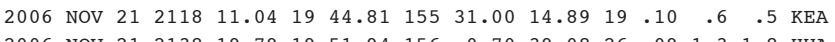

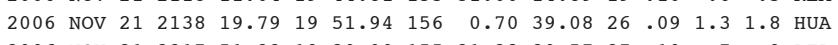

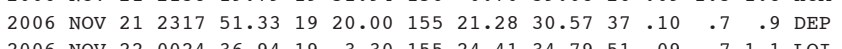

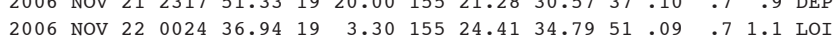
2006 Nov $22 \quad 0709 \begin{array}{lllllllllllll}31.73 & 19 & 58.05 & 156 & 5.18 & 40.97 & 19 & 09 & 2.2 & 3.8 & \text { кон }\end{array}$

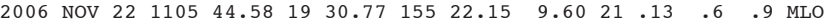

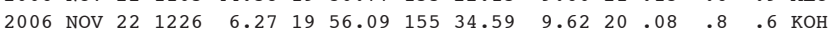

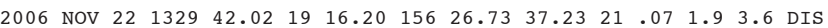

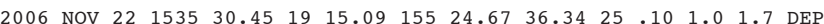

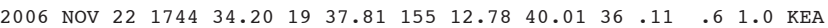

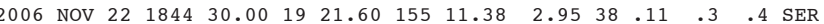

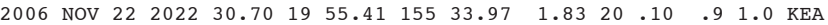

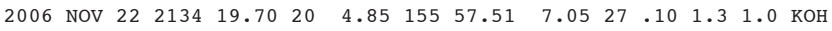

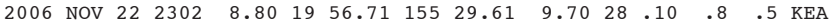

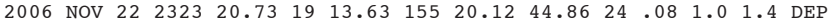

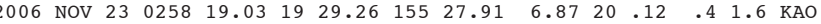

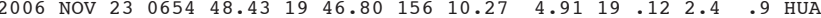

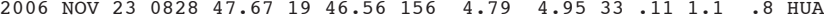

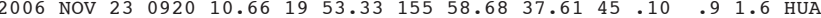

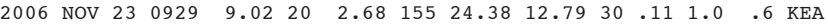

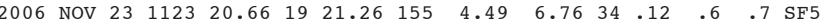

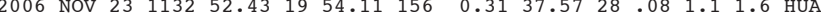

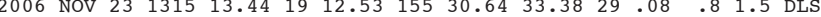

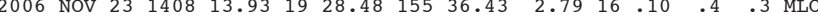

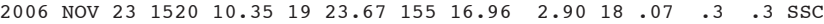

PREF AZ MIN 114 $1.8 \times \quad 63 \quad 6$ $1.5 \times 124$ $1.6 \times 255 \quad 25$

$1.4 \times 215 \quad 7$ $1.4 \times 23212$ $1.6 \times 122$ $1.4 \times \quad 71 \quad 8$

$1.4 \times 133 \quad 5$ $\begin{array}{lll}1.9 \times 121 \quad 9 \\ 1.8 \times & 293 & 62\end{array}$ $1.8 \times 29362$ $1.2 \times 268 \quad 15$

$\begin{array}{lll}1.3 \times & 279 \quad 6 \\ 1.5 \times & 297 & 14\end{array}$ $\begin{array}{llr}1.5 \times & 297 \quad 14 \\ 1.1 \times & 127 & 5\end{array}$ $\begin{array}{llll}1.1 \times & 127 & 5 \\ 1.4 \times & 102 & 6\end{array}$ $1.8 \times 28755$

$\begin{array}{lll}1.6 \times & 148 & 7 \\ 1.7 \times & 280 & 27 \\ 1.4 \times & 83 & 4\end{array}$ $1.4 \times \quad 83$ $2.1 \times \quad 206 \quad 13$ $1.6 \mathrm{X} 323 \quad 40$

$1.1 \times \quad 175 \quad 3$ $1.4 \times \quad 234 \quad 12$ $1.9 \times 31360$ $\begin{array}{rrr}1.0 \times & 130 & 10 \\ 1.6 \times & 92 & 21\end{array}$

$\begin{array}{lll}1.8 \times & 69 & 3\end{array}$ $\begin{array}{llll}1.4 \times & 224 & 12\end{array}$ $\begin{array}{lll}1.9 \times \quad 260 & 19\end{array}$ $\begin{array}{rrr}1.6 \times & 229 & 17 \\ 1.3 \times & 172 & 8\end{array}$

$\begin{array}{lll}1.3 \times & 80 & 5\end{array}$ $1.4 \times 333 \quad 36$ $2.0 \times 24027$ $\begin{array}{lll}5.0 \mathrm{U} & 172 & 27 \\ 2.2 \times & 209 & 18\end{array}$

$\begin{array}{lll}1.5 \times 190 \quad 6 & \end{array}$ 1.6x 23529 $1.5 \times 119 \quad 5$ $1.9 \times 104$ $1.3 \times \quad 70$ 
---ORIGIN TIME (HST)-- -LAT N-- --LON W-- DEPTH N RMS ERH ERZ LOC PREF AZ MIN 115 YEAR MON DA HRMN SEC DEG MIN DEG MIN KM RD SEC KM KM REMKS MAG GAP DS

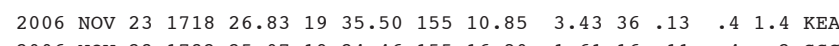

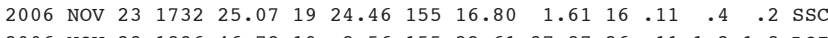

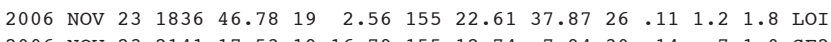

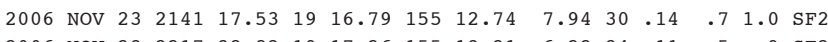

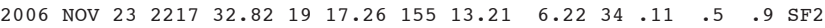

$\begin{array}{llllllllllllll}2006 & \text { NOV } 23 & 2247 & 41.78 & 19 & 57.73 & 155 & 14.19 & 13.15 & 32 & .12 & .8 & .5 & \text { KEA }\end{array}$

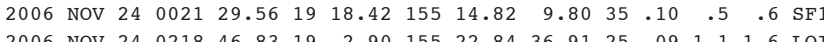

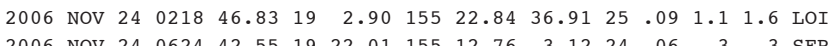

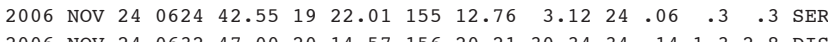
2006 NOV $24 \quad 0632 \quad 47.00 \quad 2014.57 \quad 156 \quad 20.21 \quad 30.34 \quad 34 \quad .14 \quad 1.3 \quad 2.8$ DIS

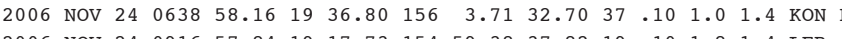
2006 NOV $24 \quad 0916 \quad 57.84 \quad 19 \quad 17.73 \quad 154 \quad 59.38 \quad 37.82 \quad 19.101 .81 .4 \quad$ LER

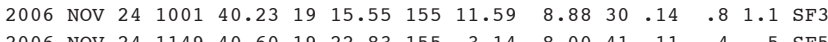

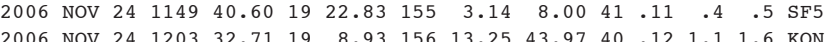
$\begin{array}{llllllllllllll}2006 & \text { NOV } 24 & 1203 & 32.71 & 19 & 8.93 & 156 & 13.25 & 43.97 & 40 & .12 & 1.1 & 1.6 & \text { KON }\end{array}$

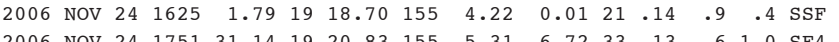

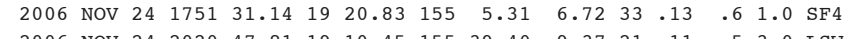

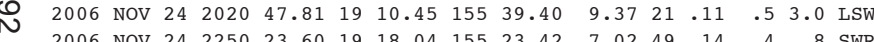
$\begin{array}{llllllllllllll}2006 & \text { NOV } 24 & 2250 & 23.60 & 19 & 18.04 & 155 & 23.42 & 7.02 & 49 & .14 & .4 & .8 & \text { SWR } \\ 2006 & \text { Nov } 25 & 0003 & 33.43 & 19 & 28.84 & 155 & 50.96 & 15.27 & 28 & .11 & .8 & .6 & \text { KON }\end{array}$

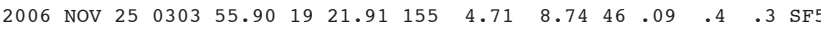

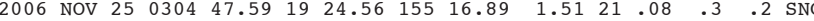
2006 NOV $25 \quad 054923.012013 .86 \quad 155 \quad 33.97 \quad 24.93 \quad 42.10 \quad .92 .15$ KEA 2006 Nov 25009323.68 (1)

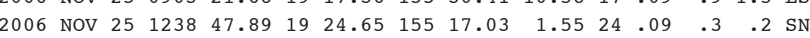

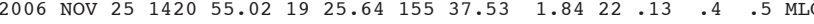

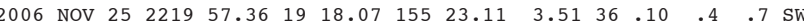

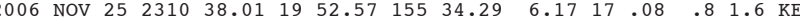

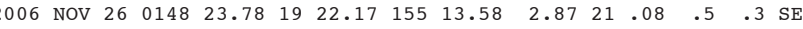

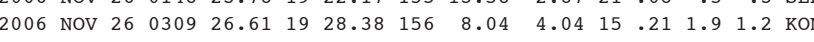

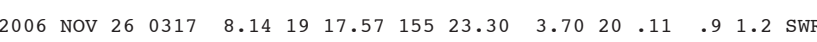

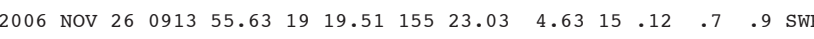

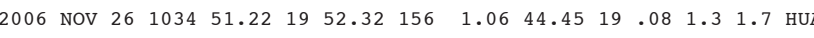

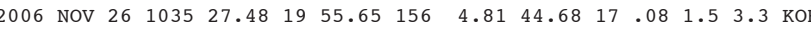

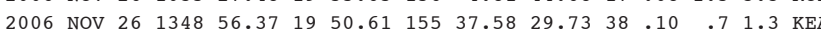

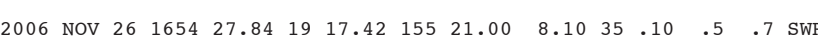

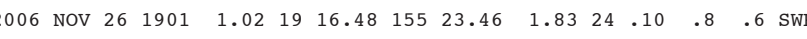

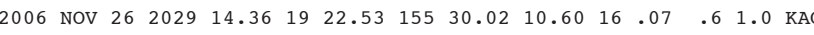

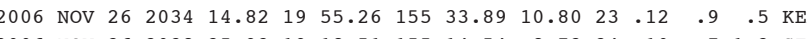

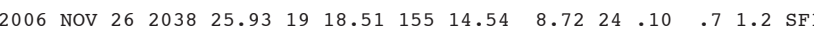

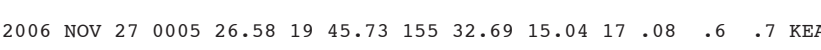

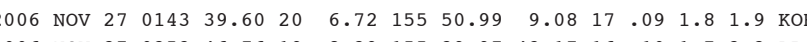

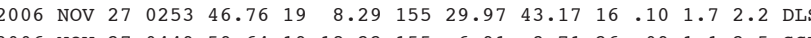
$\begin{array}{llllllllllllll}2006 & \text { NOV } 27 & 0449 & 59.64 & 19 & 18.22 & 155 & 6.91 & 3.71 & 26 & .09 & 1.1 & 2.5 & \text { SSF } \\ 2006 \text { NOV } 27 & 0647 & 59.58 & 19 & 17.35 & 155 & 28.75 & 7.84 & 29 & .12 & .4 & .9 & \text { LSW }\end{array}$
$1.3 \times \quad 7920$ $1.0 \times 107 \quad 1$

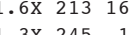
$\begin{array}{llll}1.6 \times & 213 & 16 \\ 1.3 \times & 245 & 1 \\ 137 & 137\end{array}$

1.5X 21314 1.3X $98 \quad 3$ $1.4 \times \quad 213 \quad 15$ $1.7 \times \quad 99 \quad 1$ $2.1 \times 307 \quad 60$

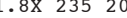
$1.2 \times 24419$ $1.2 \times 218 \quad 4$ $1.9 \times 159 \quad 3$ 2.2X $286 \quad 39$

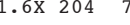
$\begin{array}{lll}1.4 \times & 161 & 6\end{array}$ $\begin{array}{lll}1.2 \times \quad 90 & 12\end{array}$ 2.2x 1114

$1.8 \times 1117$

2.0X 155 $\begin{array}{llll}.4 \times \quad 104 & 1\end{array}$ $3.4 \times 25325$ $1.1 \times 24311$ $1.4 \mathrm{X} \quad 84 \quad 0$

$1.8 \times 111$ $2.4 \times 112$ $1.2 \times 187$ $1.7 \times 179$ 1.3X 30939

$1.4 \times 235 \quad 5$ $1.0 \times 207 \quad 1$ $1.6 \times 24037$ $1.8 \times 30857$ 1.9X 1064

$\begin{array}{lll}1.7 \times \quad 154 \quad 7 & 7\end{array}$ $1.3 \times 236 \quad 7$ $\begin{array}{llr}1.2 \times & 174 & 4 \\ 1.7 \times & 222 & 12\end{array}$ $\begin{array}{lll}1.7 \times \quad 222 & 12\end{array}$

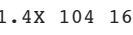
$\begin{array}{lll}1.5 \times \quad 302 & 34 \\ 1.4 \times & 292 & 25\end{array}$ $1.4 \times 29225$ $\begin{array}{rrr}1.4 \times & 230 & 9 \\ 1.5 \times & 100 & 10\end{array}$
---ORIGIN TIME (HST) -- -LAT N-- --LON W-- DEPTH N RMS ERH ERZ LOC YEAR MON DA HRMN SEC DEG MIN DEG MIN PREF AZ MIN 116

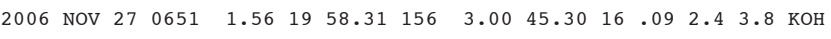

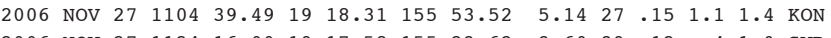

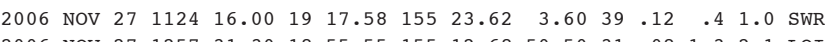

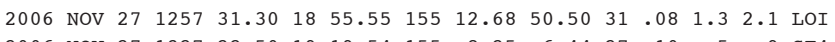
2006 Nov $27 \quad \begin{array}{lllllllllllll}1327 & 22.50 & 19 & 19.54 & 155 & 8.25 & 6.44 & 27 & .10 & .5 & .9 & \text { SF } 4\end{array}$

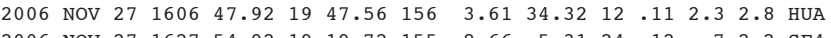

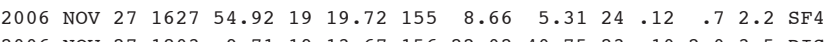

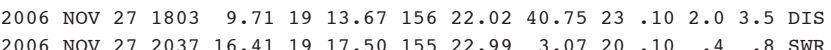

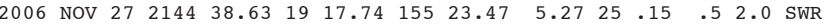

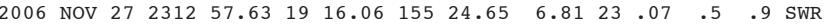

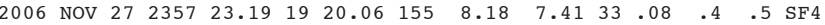

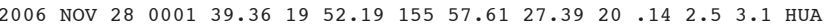

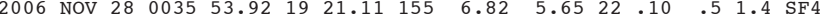

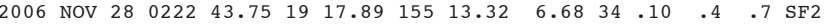

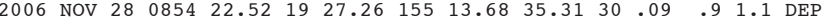

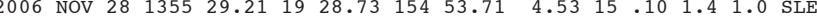

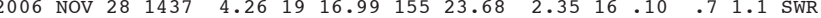

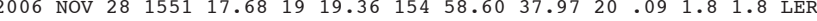

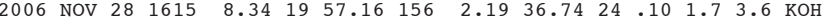

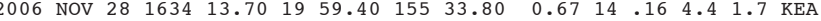

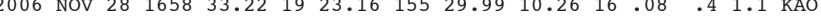

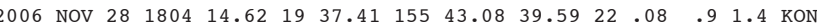

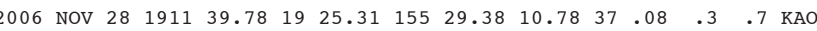

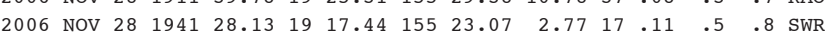

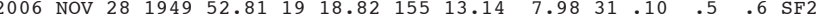

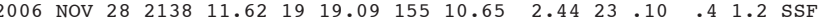

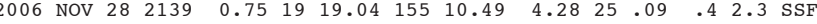

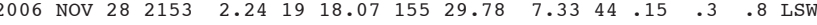

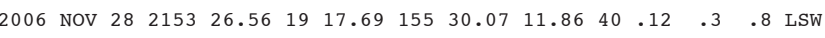

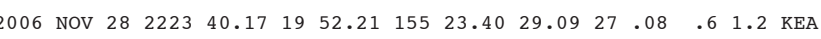

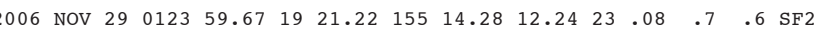

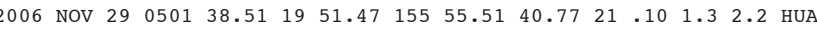

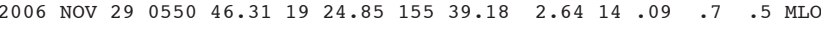

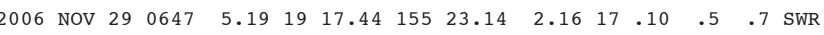

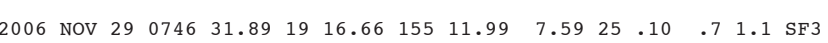

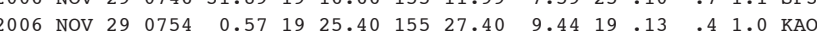

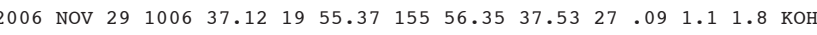

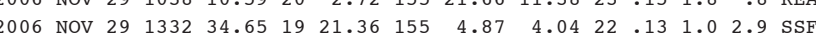

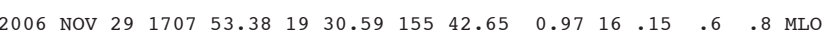

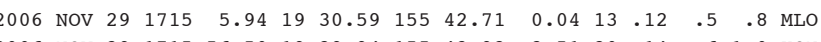

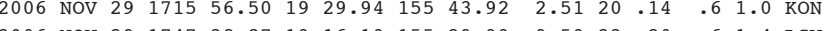

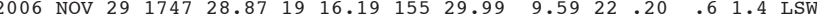
2006 Nov $29 \begin{array}{lllllllllll}1756 & 0.35 & 19 & 43.94 & 155 & 19.79 & 44.23 & 50.09 & .6 & 1.0 & \text { KEA }\end{array}$
$1.7 \times 33559$ $1.5 \times \quad 24921$ $2.3 \times 114$ $2.0 \times 27937$

$1.1 \times 30226$ $.8 \times 121$ $1.6 \times 31055$ $\begin{array}{rrrr}.8 \times & 167 & 5 \\ 1.4 \times 113 & 110\end{array}$

$.7 \times 167 \quad 8$ $1.4 \times 115$ $1.6 \times 24124$ $.9 \times 134$

$1.6 \times \quad 94$

$1.7 \times 138 \quad 5$ $1.6 \times 164$ $1.5 \mathrm{U} 197 \quad 6$ $1.5 \times 293 \quad 17$

$1.6 \times 28836$

$1.9 \times 300 \quad 43$ $1.3 \times \quad 79 \quad 4$ $1.2 \times 113 \quad 14$ $1.4 \mathrm{U} 168$

$\begin{array}{ll}1.4 \times \quad 84 \\ .9 \times & 109\end{array}$ $.9 \times 1096$ $1.2 \times 1115$ $\begin{array}{lll}2.18 & 75 & 6 \\ 1.8 \times & 73 & 5\end{array}$

1.8X 158 $\begin{array}{lll}1.1 \times & 63 & 3\end{array}$ $1.3 \times 2112$ $.9 \times 200$

$1.3 \times 202$ $\begin{array}{llr}1.8 \times & 57 \quad 5\end{array}$ $1.9 \times 222 \quad 28$ $1.3 \times 203$ $1.3 \mathrm{U} 106 \quad 6$ $1.2 \mathrm{U} 1066$ $\begin{array}{lll}1.2 \times & 93 & 4\end{array}$ $2.2 \times 10015$ 
---ORIGIN TIME (HST)-- -LAT N-- --LON W-- DEPTH N RMS ERH ERZ LOC

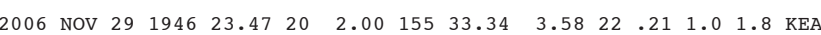

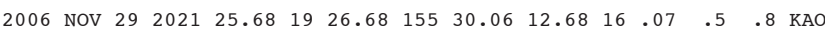

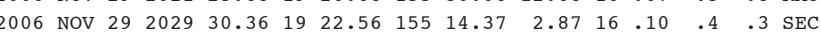

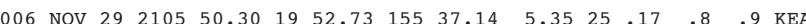

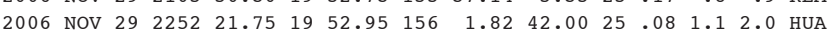

2006 Nov $30 \quad 0109 \quad 22.04 \quad 19 \quad 56.34 \quad 156 \quad 1.30 \quad 51.54 \quad 24 \quad 11 \quad 1.6 \quad 2.0$ кон

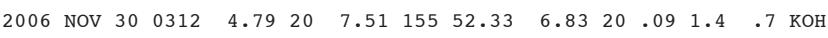

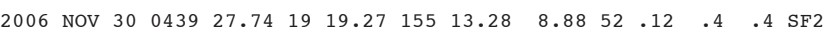

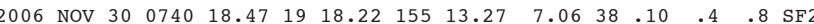

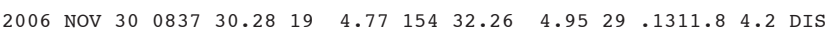

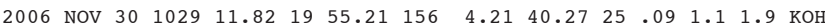

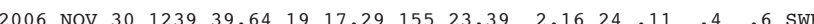

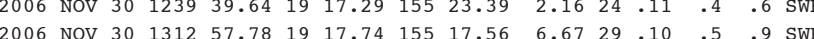

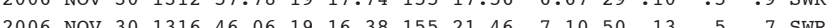

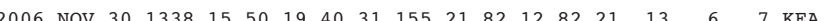

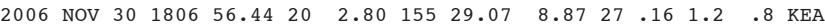

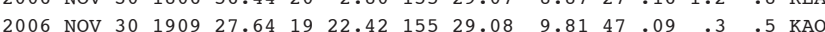

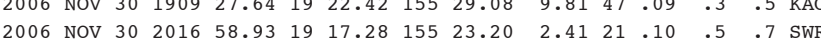

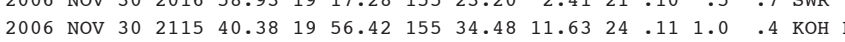

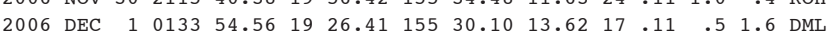

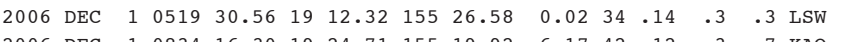

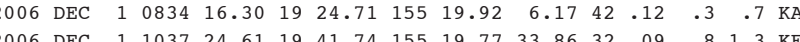
$\begin{array}{lllllllllllllll}2006 & \text { DEC } & 1 & 1037 & 24.61 & 19 & 41.74 & 155 & 19.77 & 33.86 & 32 & .09 & .8 & 1.3 & \text { KEA } \\ 2006 & \text { DEC } & 1 & 1108 & 0.52 & 19 & 20.09 & 155 & 8.57 & 7.34 & 44 & .12 & .4 & .5 & \text { SF }\end{array}$

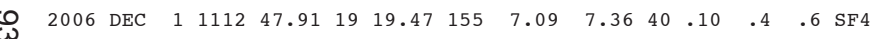

$\begin{array}{lllllllllllllll}2006 & \text { DEC } & 1 & 1256 & 8.09 & 19 & 54.86 & 156 & 6.86 & 39.67 & 26 & .11 & 1.4 & 2.6 & \text { HUA }\end{array}$

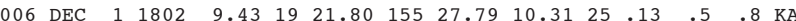

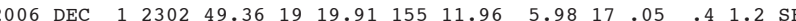
$\begin{array}{lllllllllllllll}2006 & D E C & 1 & 2327 & 27.78 & 19 & 22.99 & 155 & 14.86 & 3.15 & 17 & .07 & .3 & .3 & \text { SEC }\end{array}$ $\begin{array}{llllllllllllll}2006 & \text { DEC } & 1 & 2342 & 22.20 & 20 & 2.09 & 155 & 22.84 & 7.38 & 55 & .12 & .6 & .6 \\ \text { KEA }\end{array}$

$\begin{array}{lllllllllllllll}2006 & \text { DEC } & 2 & 0323 & 33.42 & 19 & 17.78 & 155 & 22.89 & 2.44 & 20 & .11 & .4 & .6 & \text { SWR }\end{array}$

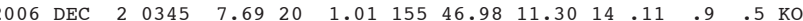

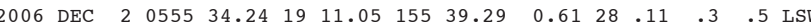
$\begin{array}{llllllllllllllll}2006 & \mathrm{DEC} & 2 & 0605 & 57.02 & 19 & 20.25 & 155 & 10.68 & 6.67 & 23 & .09 & .5 & .9 & \mathrm{SF} 3\end{array}$ $\begin{array}{llllllllllllllll}2006 & \text { DEC } & 2 & 1321 & 47.79 & 19 & 22.15 & 155 & 26.90 & 10.79 & 24 & .12 & .4 & .9 & \mathrm{KAO}\end{array}$

$\begin{array}{lllllllllllllll}2006 & \text { DEC } & 2 & 1332 & 2.48 & 19 & 20.07 & 155 & 7.81 & 6.37 & 28 & .12 & .5 & 1.0 & \mathrm{SF} 4\end{array}$

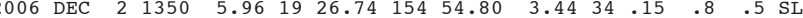

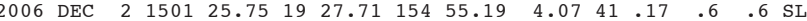

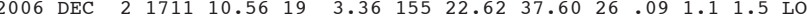
$\begin{array}{lllllllllllllllll}2006 & \text { DEC } & 2 & 1904 & 36.41 & 19 & 18.81 & 155 & 13.21 & 8.97 & 49 & .12 & .4 & .4 & \text { SF } 2\end{array}$

2006 DEC $2 \begin{array}{lllllllllllll}2305 & 6.54 & 19 & 20.06 & 155 & 11.53 & 7.78 & 36 & .12 & .4 & .6 & \mathrm{SF} 3\end{array}$ 2006 DEC $3 \begin{array}{lllllllllllll}0 & 0151 & 15.74 & 19 & 15.37 & 155 & 33.29 & 6.37 & 30 & .17 & .4 & 1.9 & \text { LSW }\end{array}$ $\begin{array}{lllllllllllllll}2006 & \text { DEC } & 3 & 0222 & 21.36 & 19 & 58.82 & 155 & 59.90 & 12.64 & 46 & .11 & 1.1 & .7 & \text { KOH F } \\ 2006 & \text { DEC } & 3 & 0241 & 25.38 & 20 & 3.78 & 156 & 6.51 & 8.24 & 43 & .12 & 1.2 & .6 & \text { KOH F }\end{array}$ 2006 DEC $30318 \begin{array}{llllllllllllllllll} & 51.74 & 20 & 8.24 & 156 & 9.11 & 30.23 & 43 & .11 & 1.2 & 2.0 & \text { кон } & 2.6 \times & 289 & 39\end{array}$
---ORTGIN TIME (HST)-- -LAT N-- --LON W-- DEPTH N RMS ERH ERZ LOC YEAR MON DA HRMN SEC DEG MIN DEG MIN KM RD SEC KM KM REMKS

2006 DEC $3 \begin{array}{lllllllllllll}0342 & 0.60 & 20 & 5.78 & 156 & 8.19 & 28.14 & 26 & .14 & 1.5 & 3.4 & \text { кон }\end{array}$

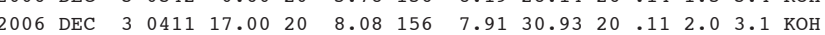

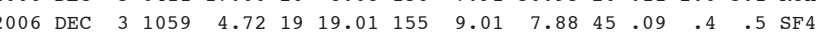
$\begin{array}{llllllllllllll}2006 \text { DEC } & 3 & 1150 & 33.48 & 19 & 25.16 & 155 & 18.49 & 7.60 & 20.10 & .6 & .9 & \text { INT }\end{array}$

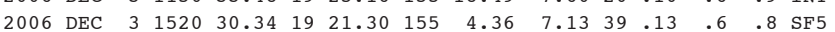

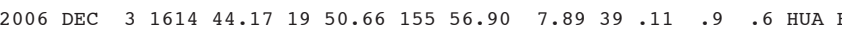
$\begin{array}{lllllllllllllll}2006 & \text { DEC } & 3 & 1815 & 45.11 & 19 & 24.82 & 155 & 19.31 & 6.50 & 32 & .09 & .3 & .7 & \text { KAO }\end{array}$ $\begin{array}{lllllllllllllll}2006 & \text { DEC } & 3 & 1912 & 22.86 & 19 & 22.43 & 155 & 29.82 & 10.37 & 15 & .07 & .5 & 1.1 & \text { KAO }\end{array}$ $\begin{array}{lllllllllllllll}2006 & \text { DEC } & 3 & 2010 & 12.45 & 19 & 48.68 & 155 & 56.64 & 12.82 & 19 & .12 & 1.6 & .8 & \text { HUA }\end{array}$ $\begin{array}{lllllllllllllll}2006 & \text { DEC } & 4 & 0043 & 55.90 & 19 & 28.72 & 155 & 27.34 & 7.94 & 21 & .12 & .4 & 1.2 & \text { KAO }\end{array}$

$\begin{array}{lllllllllllllll}2006 & \text { DEC } & 4 & 0319 & 32.60 & 19 & 57.40 & 155 & 30.84 & 19.75 & 26 & .10 & .9 & 2.0 & \text { KEA }\end{array}$ $\begin{array}{llllllllllllll}2006 & \text { DEC } & 4 & 0649 & 38.91 & 19 & 51.95 & 155 & 58.95 & 42.46 & 53 & .11 & .8 & 1.3\end{array}$ HUA

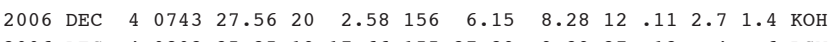
$\begin{array}{llllllllllllll}2006 & \text { DEC } & 4 & 0802 & 35.25 & 19 & 17.66 & 155 & 27.29 & 9.39 & 27 & .12 & .4\end{array}$ $\begin{array}{llllllllllllll}2006 & \text { DEC } & 4 & 0809 & 49.49 & 19 & 25.06 & 155 & 29.56 & 11.45 & 24 & .08 & .4 & \\ & & & & & & \end{array}$

$\begin{array}{lllllllllllllll}2006 & \text { DEC } & 4 & 0826 & 7.37 & 19 & 15.80 & 155 & 7.49 & 44.12 & 51 & .10 & .7 & .9 & \text { DEP }\end{array}$

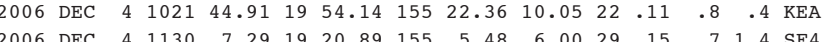
$\begin{array}{lllllllllllllll}2006 & \text { DEC } & 4 & 1130 & 7.29 & 19 & 20.89 & 155 & 5.48 & 6.00 & 29 & .15 & .7 & 1.4 & \text { SF } 4 \\ 2006 & \text { DEC } & 4 & 1130 & 58.81 & 19 & 21.07 & 155 & 5.53 & 6.62 & 36 & .10 & .5 & .6 & \text { SF } 4\end{array}$

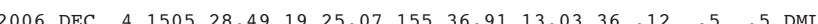

$\begin{array}{lllllllllllllll}2006 & \mathrm{DEC} & 4 & 1535 & 12.01 & 19 & 19.10 & 155 & 15.52 & 5.45 & 31 & .12 & .4 & 1.0 & \mathrm{SF} 1\end{array}$

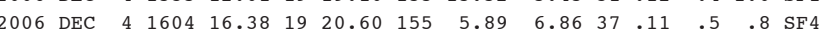

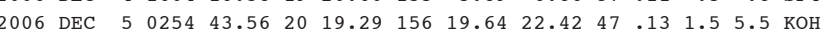

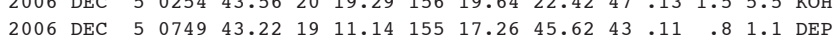
$\begin{array}{lllllllllllllll}2006 & \text { DEC } & 5 & 1528 & 33.02 & 19 & 8.11 & 155 & 37.69 & 0.17 & 36 & .15 & .4 & .3 & \text { LSW }\end{array}$

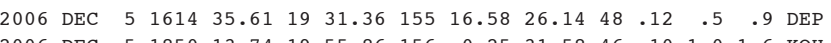

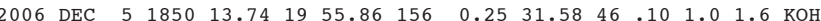

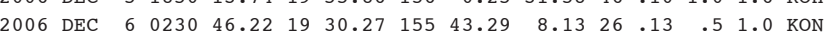
2006 DEC $6 \begin{array}{llllllllllll}0 & 0321 & 40.65 & 19 & 58.59 & 156 & 2.00 & 38.30 & 36 & .12 & 1.1 & 1.8\end{array}$

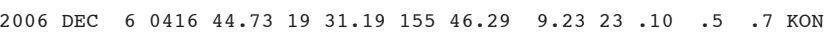

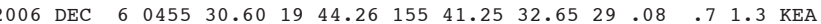
2006 DEC $6 \begin{array}{lllllllllllll}6 & 0505 & 3.16 & 19 & 20.94 & 155 & 58.21 & 43.81 & 35 & .06 & .7 & 1.2 & \text { KON }\end{array}$ 2006 DEC $6 \begin{array}{llllllllllllll}6 & 0510 & 45.13 & 19 & 28.71 & 155 & 51.01 & 7.51 & 37.13 & .4 & .5 & \text { KON }\end{array}$ $\begin{array}{lllllllllllllll}2006 & \text { DEC } & 6 & 0936 & 55.30 & 19 & 22.71 & 155 & 18.94 & 29.14 & 39 & .11 & .7 & .9 & \text { DEP }\end{array}$ 2006 DEC $6 \begin{array}{lllllllllllll}1103 & 5.33 & 19 & 15.29 & 155 & 24.92 & 35.08 & 26 & .09 & .7 & 1.4 & \text { DEP }\end{array}$

2006 DEC $6 \begin{array}{lllllllllllll}1343 & 34.50 & 19 & 19.38 & 155 & 10.99 & 7.95 & 31 & .11 & .5 & .7 & \mathrm{SF} 3\end{array}$ 2006 DEC $6 \begin{array}{lllllllllllll}6 & 1400 & 45.21 & 19 & 22.57 & 155 & 14.00 & 3.49 & 31 & .08 & .3 & .3 & \text { SEC }\end{array}$ 2006 DEC $6 \begin{array}{lllllllllllll} & 1842 & 59.86 & 19 & 13.91 & 155 & 25.17 & 35.28 & 38 & .10 & .7 & 1.1 & \text { DLS }\end{array}$ 2006 DEC $7 \begin{array}{lllllllllllll} & 0608 & 40.96 & 19 & 21.66 & 155 & 4.85 & 7.33 & 28 & .10 & .5 & .6 & \text { SF5 }\end{array}$ $\begin{array}{lllllllllllllll}2006 & \text { DEC } & 7 & 0612 & 57.66 & 19 & 21.95 & 155 & 12.56 & 2.95 & 22 & .11 & .4 & .7 & \text { SER }\end{array}$

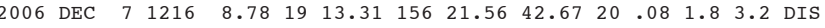
$\begin{array}{lllllllllllllll}2006 & \text { DEC } & 7 & 1559 & 56.63 & 19 & 20.06 & 155 & 8.30 & 5.50 & 17 & .11 & .4 & 1.3 & \text { SF } 4\end{array}$

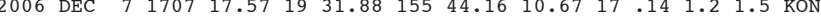

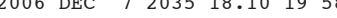
$\begin{array}{llllllllllllll}2006 & \text { DEC } & 7 & 2345 & 2.95 & 19 & 24.70 & 154 & 58.71 & 4.35 & 18 & .15 & 1.0 & .5\end{array}$
PREF AZ MIN 118 $2.0 \times 28137$ $1.9 \times 28737$ $1.9 \times 116$ $2.0 \times 167$

$2.5 \times 27021$ $1.4 \times \quad 72 \quad 2$ $1.7 \times 21418$ $1.3 \times \quad 74$

$1.7 \times \quad 237 \quad 19$ $\begin{array}{llll}2.8 \times & 226 & 25 \\ 1.6 \times & 312 & 48\end{array}$ $\begin{array}{lll}1.4 \times & 97\end{array}$ $1.2 \times \quad 52$

$2.7 \times 194 \quad 3$ $\begin{array}{lll}1.6 \times 211 & 3 \\ 1.5 \times 158 & 6\end{array}$ $\begin{array}{lll}1.5 \times & 158 & 6 \\ 1.8 \times & 155 & 6\end{array}$ $1.7 \mathrm{X} \quad 64$

$\begin{array}{lll}1.5 \times & 113 & 4 \\ 1.7 \times & 156 & 6\end{array}$ $\begin{array}{rrrr}1.7 \times & 156 & 6 \\ 3.5 \times & 317 & 87\end{array}$ $2.2 \times 187 \quad 13$ $1.9 \times 114 \quad 15$ $2.1 \mathrm{x} \quad 61 \quad 11$ $2.5 \times 23832$ $\begin{array}{lll}1.5 \mathrm{U} & 73 & 5\end{array}$ $2.2 \times \quad 25031$

$\begin{array}{lll}1.8 \times & 112 \quad 14\end{array}$ $\begin{array}{lll}1.6 \times & 237 & 14\end{array}$ $1.9 \mathrm{X} \quad 93$ $\begin{array}{llll}2.0 \times & 37 & 2 \\ 1.4 \times & 126 & 9\end{array}$

$\begin{array}{lll}1.7 \times & 100 & 6\end{array}$ $\begin{array}{rrr}1.9 \times & 87 & 2 \\ 1.8 \times & 133 & 9\end{array}$ $\begin{array}{lll}1.8 \times 133 & 13 \\ 1.3 \times \quad 156 & \end{array}$ $1.5 \times 101$

1.9X 31352 $1.2 \times 112$ $1.3 \times 200$ $1.8 \times 205 \quad 19$

$1.7 \times 193$ 
---ORIGIN TIME (HST)-- -LAT N-- --LON W-- DEPTH N RMS ERH ERZ LOC PREF AZ MIN 119 YEAR MON DA HRMN SEC DEG MIN DEG MIN KM RD SEC KM KM REMKS MAG GAP DS

$\begin{array}{lllllllllllllll}2006 & \text { DEC } & 8 & 0233 & 50.19 & 19 & 21.60 & 155 & 28.06 & 14.39 & 24 & .10 & .4 & .6 & \text { DML }\end{array}$

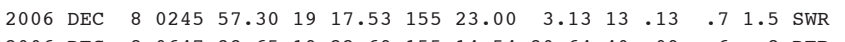

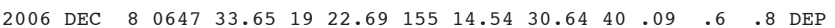

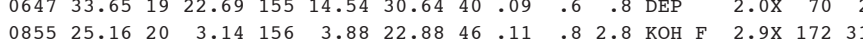

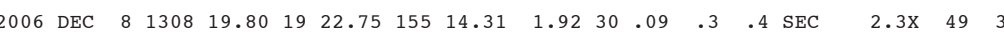

$\begin{array}{lllllllllllllll}2006 & \text { DEC } & 8 & 1312 & 3.92 & 19 & 22.94 & 155 & 14.38 & 2.17 & 16 & .06 & .3 & .5 & \mathrm{SEC}\end{array}$ 2006 DEC $8 \begin{array}{lllllllllllll}1757 & 54.25 & 19 & 21.99 & 155 & 4.46 & 7.89 & 33 & .14 & .5 & .7 & \text { SF5 }\end{array}$

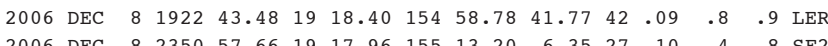

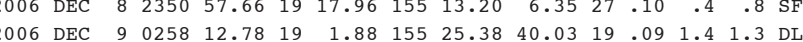

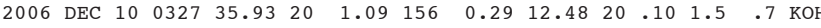

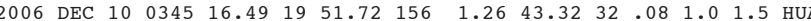

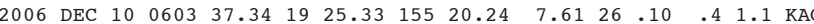

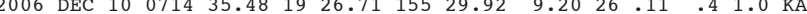

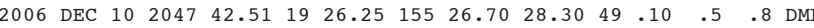

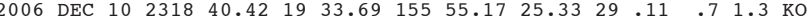

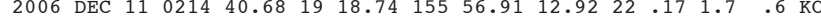

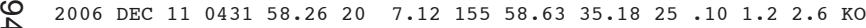

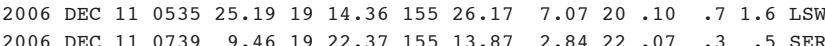

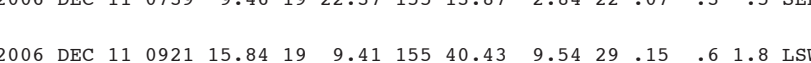

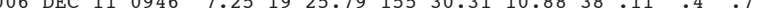

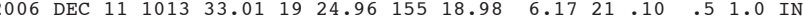

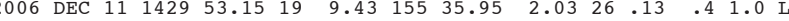

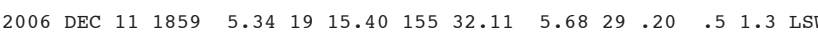

$\begin{array}{llllllllllllll}2006 & \text { DEC } 11 & 1905 & 15.71 & 19 & 56.09 & 155 & 55.70 & 12.24 & 18 & .06 & 1.4 & .8 & \text { KOH }\end{array}$

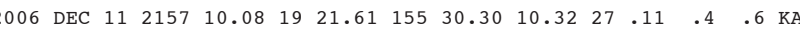

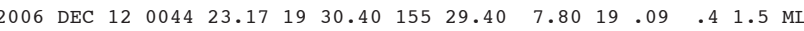

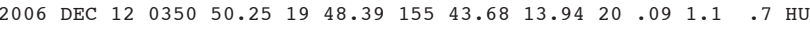

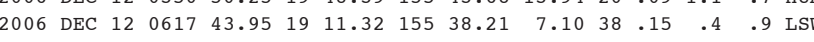

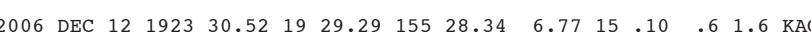

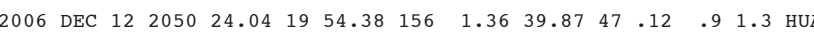
$\begin{array}{llllllllllllll}2006 & D E C & 13 & 0107 & 39.99 & 19 & 42.82 & 155 & 59.03 & 7.50 & 13 & .14 & 1.5 & .9\end{array}$

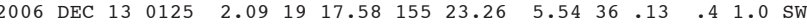

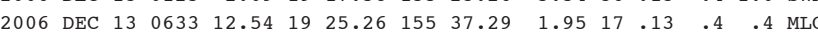

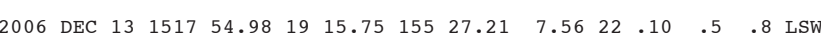

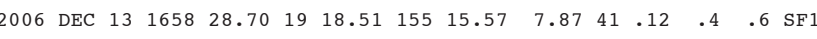

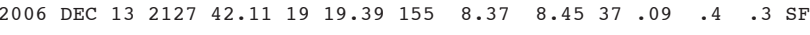

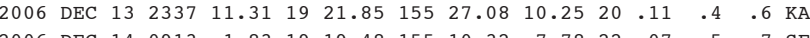

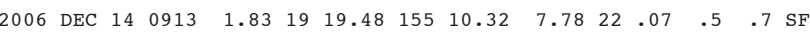

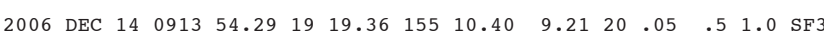

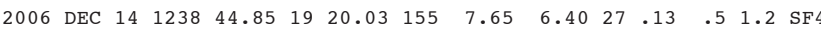

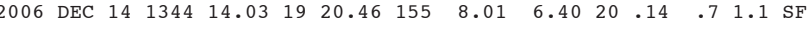

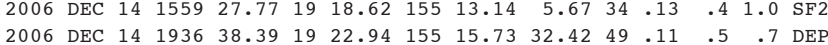

$1.9 \times \quad 85 \quad 3$ $1.8 \times 156 \quad 4$ $2.1 \times 22012$ $1.5 \times 100 \quad 2$ $1.6 \times 23815$

$1.7 \times 304 \quad 40$ $2.2 \times 234 \quad 27$ $\begin{array}{lll}1.2 \times & 121 & 3\end{array}$ $\begin{array}{lll}1.3 \times & 69 & 6\end{array}$ $2.2 \times \quad 36 \quad 7$

$\begin{array}{lll}1.7 \times \quad 212 \quad 8 & 8\end{array}$ $1.1 \times 24310$ $1.9 \times \quad 297 \quad 44$ $1.0 \times 1787$

$1.6 \times \quad 92 \quad 4$

$\begin{array}{lll}1.7 \mathrm{X} & 90 & 10\end{array}$ $1.8 \mathrm{X} \quad 43 \quad 8$ $1.1 \times 111$ $1.5 \times 113 \quad 14$ $1.4 \times \quad 61 \quad 4$

1.9X 1982 $1.2 \times \quad 61$ $\begin{array}{llll}1.4 \times & 102 & 4\end{array}$ $\begin{array}{llll}1.2 \times \quad 215 & 18\end{array}$ $1.8 \mathrm{X} \quad 8914$

$\begin{array}{lll}1.3 \times & 94 \quad 5\end{array}$ 2.8X 22628 $1.6 \times 214$ $1.7 \times 116$ $1.2 \times \quad 95 \quad 2$ $.2 \times 1125$ $1.9 \times 105 \quad 4$ $1.6 \times 1114$ $1.3 \times \quad 69 \quad 2$ $1.6 \mathrm{X} 98$ $1.5 \times 102 \quad 6$ $\begin{array}{llll}1.4 \times & 129 & 5 \\ 1.6 \times & 197 & 5\end{array}$ $1.6 \times 1975$ $\begin{array}{lll}1.8 \mathrm{X} & 88 & 3 \\ 2.9 \mathrm{X} & 42 & 1\end{array}$
---ORIGIN TIME (HST)-- -LAT N-- --LON W-- DEPTH N RMS ERH ERZ LOC PREF AZ MIN 120
MAG GAP DS

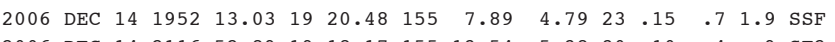

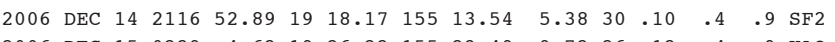

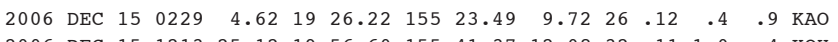

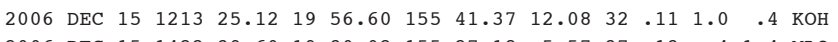

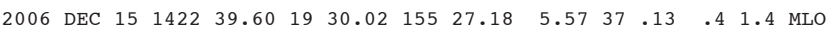

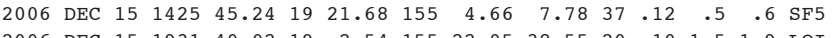

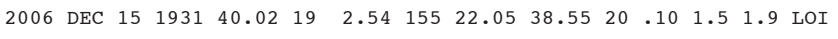

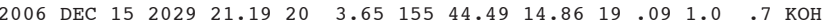

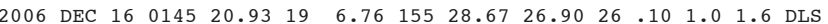

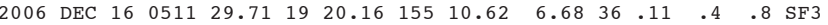

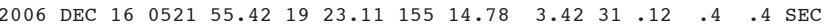

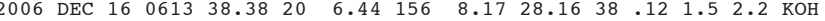

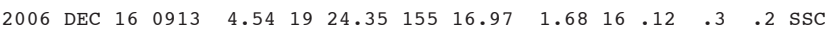

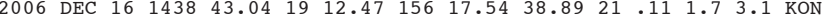

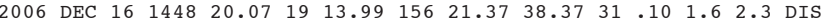

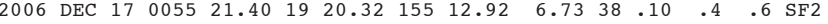

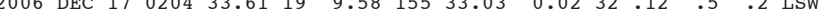

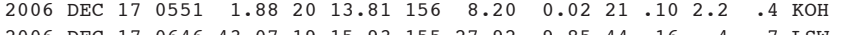
$\begin{array}{llllllllllllll}2006 & \text { DEC } 17 & 0646 & 43.07 & 19 & 15.93 & 155 & 27.92 & 9.85 & 44 & .16 & .4 & .7 & \text { LSW }\end{array}$

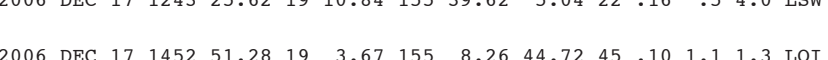

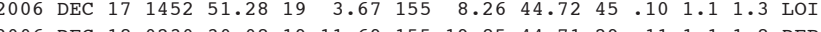

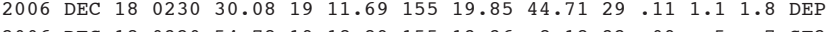

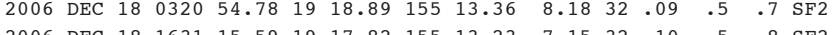

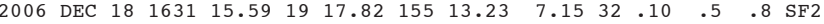

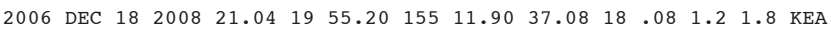

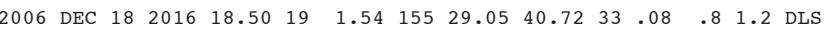

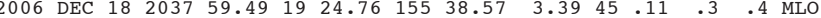

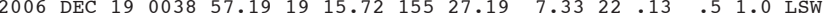

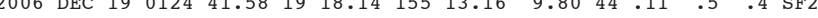
$\begin{array}{lllllllllllllll}2006 & \text { DEC } & 19 & 0624 & 54.59 & 19 & 24.95 & 155 & 18.54 & 5.47 & 33 & .09 & .3 & .6 & \text { INT }\end{array}$

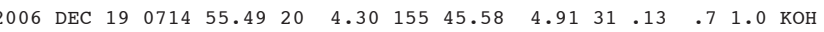

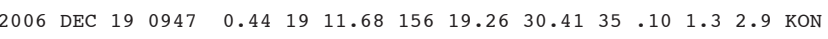

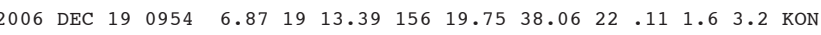

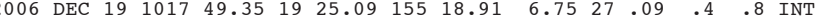

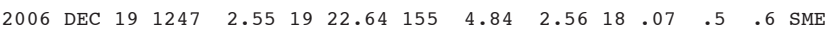

$\begin{array}{llllllllllllll}2006 & \text { DEC } & 19 & 1327 & 25.38 & 19 & 52.78 & 155 & 10.18 & 39.73 & 37.12 & 1.0 & 1.4 & \text { KEA }\end{array}$ 2006 DEC $191459 \begin{array}{lllllllllll}148.48 & 19 & 28.16 & 155 & 14.70 & 32.49 & 51.11 & .5 & .8 & \text { DEP }\end{array}$

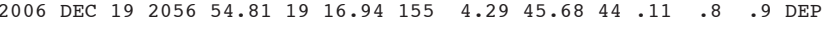

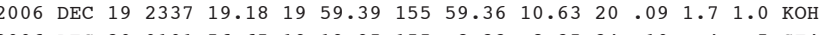

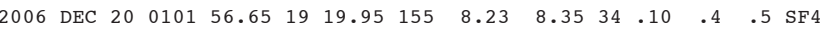

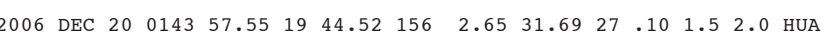

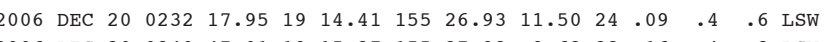

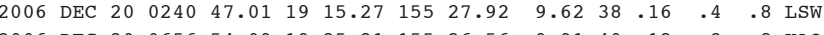

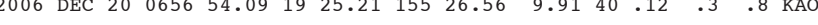

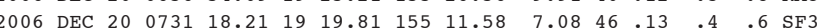

$1.3 \times 121 \quad 5$ $1.4 \times \quad 83$ $1.5 \times \quad 93 \quad 7$ $\begin{array}{lll}2.0 \times & 231 & 10 \\ 2.0 \times \quad 72 & 4\end{array}$

$2.0 \times 158 \quad 5$ $1.8 \times 27616$ $\begin{array}{rrr}1.8 \times & 174 & 5 \\ 1.6 \times & 84 & 5\end{array}$

$\begin{array}{lll}2.1 \times \quad 67 & 2\end{array}$ $\begin{array}{lll}2.6 \times 280 & 37\end{array}$ $1.2 \times \quad 85$ $1.7 \times 30545$ $1.8 \times 3105$

$\begin{array}{ll}1.4 \times \quad 67 & 4\end{array}$ \# $1.5 \times 123$ $2.0 \times 30965$ $\begin{array}{rrr}1.7 \mathrm{X} & 94 & 4 \\ 1.2 \mathrm{X} & 87 & 11\end{array}$

$2.1 \times 23525$ $1.6 \times 188 \quad 15$ $1.5 \times \quad 78$ $1.6 \times 101$ $1.6 \times 24716$

2.0X 20615 $2.8 \times 105$ $1.2 \times 102$ $2.3 \times 135$ $1.7 \times \quad 66$

$\begin{array}{lll}1.7 \times \quad 132 \quad 7 & 7\end{array}$ $\begin{array}{lll}2.2 \times 280 & 48\end{array}$ $1.7 \times 29448$ $1.3 \times 115$ $1.6 \times 144 \quad 3$

$1.7 \times 22918$ $\begin{array}{lrl}2.1 \times \quad 53 & 6\end{array}$ $\begin{array}{lll}2.0 \times 213 & 6\end{array}$ $1.5 \times 114 \quad 5$

$1.5 \times 27222$ $1.6 \times 113 \quad 6$ $1.7 \times \quad 96-4$ $\begin{array}{lll}1.5 \mathrm{X} & 55 & 5 \\ 1.8 \mathrm{X} & 88 & 6\end{array}$ 
---ORIGIN TIME (HST)-- -LAT N-- --LON W-- DEPTH N RMS ERH ERZ LOC YEAR MON DA HRMN SEC DEG MIN DEG MIN KM RD SEC KM KM REMKS

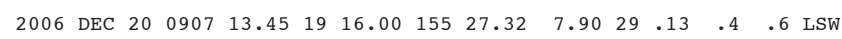

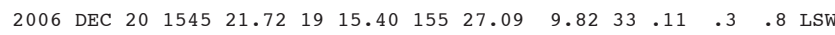

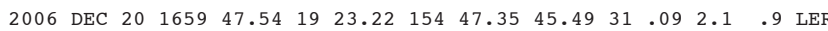

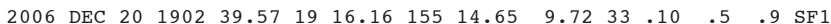

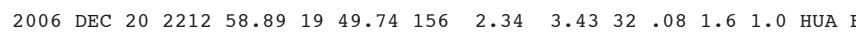

$\begin{array}{llllllllllllll}2006 & \text { DEC } 21 & 0100 & 53.73 & 20 & 4.72 & 156 & 6.49 & 9.19 & 19 & .09 & 1.7 & 1.5 & \text { кон }\end{array}$

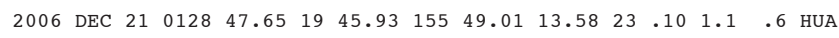

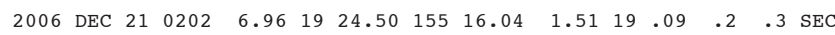

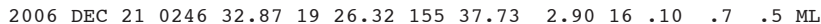

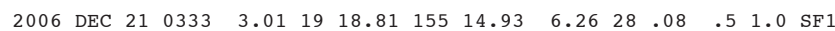

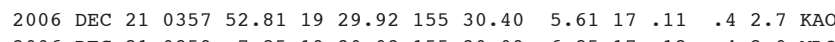

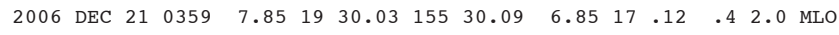

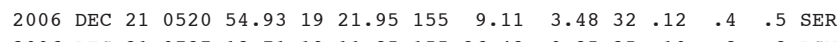

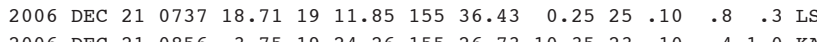

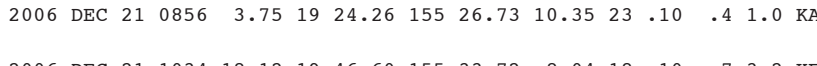

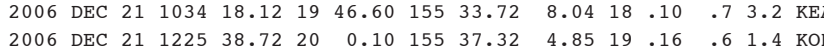

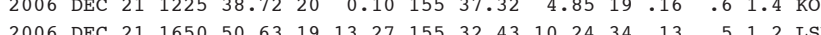

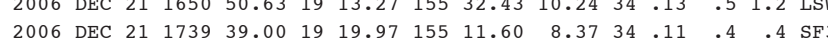

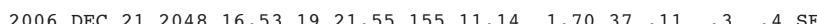

$\begin{array}{lllllllllllll}2006 & \text { DEC } 21 & 2115 & 14.49 & 19 & 25.96 & 155 & 37.35 & 2.80 & 23 & .13 & .4 & .5 \\ \text { MLO }\end{array}$

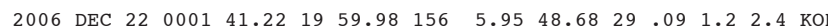

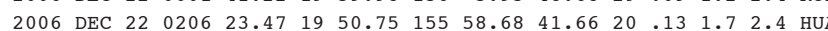

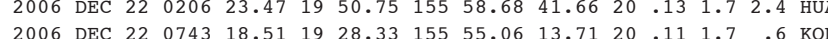

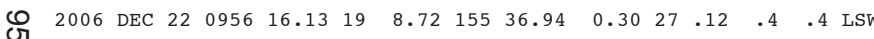

$\begin{array}{lllllllllllll}2006 & \text { DEC } 22 & 1007 & 56.26 & 19 & 25.71 & 155 & 51.96 & 17.30 & 35 & .13 & .8 & 2.2 \\ 200 N\end{array}$

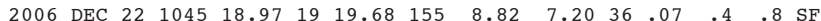

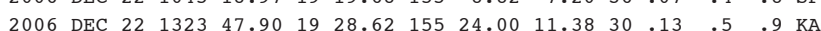

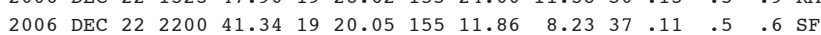

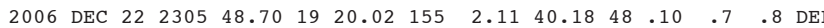

$\begin{array}{lllllllllllll}2006 & \text { DEC } 23 & 0251 & 48.52 & 19 & 54.36 & 155 & 53.47 & 36.71 & 51 & .09 & .7 & 1.1\end{array}$ $\begin{array}{lllllllllllllll}2006 & \text { DEC } & 23 & 0351 & 27.77 & 19 & 23.35 & 155 & 14.42 & 2.33 & 19 & .12 & .3 & .3 & \text { SEC }\end{array}$

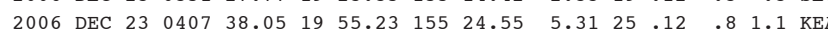

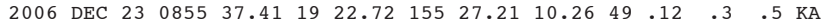

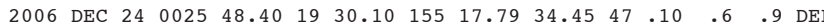

$\begin{array}{lllllllllllllll}2006 & \text { DEC } 24 & 0148 & 52.56 & 19 & 11.04 & 155 & 29.63 & 8.97 & 36 & .11 & .4 & .7 & \text { LSW }\end{array}$

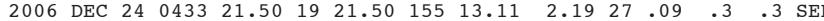

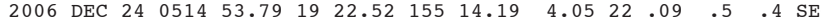

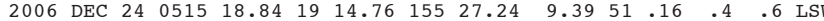

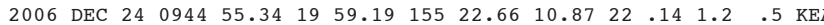

$\begin{array}{llllllllllllll}2006 & \text { DEC } 24 & 1001 & 15.23 & 19 & 23.60 & 155 & 25.37 & 9.87 & 29 & .10 & .4 & .8 & \text { KAO }\end{array}$

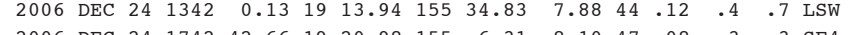

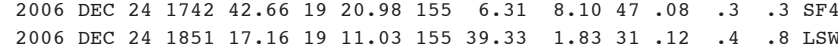

PREF AZ MIN 121 MAG GAP DS

$1.5 \times 100 \quad 5$ $1.5 \times 105 \quad 5$ $\begin{array}{lll}1.6 \times & 311 & 16 \\ 1.3 \times & 187 & 3\end{array}$ $1.3 \times 18703$

$1.8 \times 31452$ $1.5 \times 2229$ $1.3 \times 115$

$1.3 \times 105 \quad 6$ $1.5 \times 105 \quad 6$ $\begin{array}{lrr}1.5 \times & 94 & 2\end{array}$ $\begin{array}{lll}1.0 \times 180 & 13\end{array}$ $1.3 \times 59$

1.3X $109 \quad 14$ $\begin{array}{lll}1.3 \times & 161 & 16\end{array}$ $\begin{array}{rrrr}1.5 \times & 148 & 5 \\ 2.0 \times & 85 & 6\end{array}$ $\begin{array}{lll}1.7 \mathrm{X} & 71 \quad 3\end{array}$

$\begin{array}{lll}1.6 \times \quad 90 & 3\end{array}$ $1.9 \times \quad 295 \quad 44$ $1.2 \times 275 \quad 23$ $1.4 \times 264 \quad 17$ $1.4 \mathrm{X} 114 \quad 16$

$\begin{array}{lll}1.8 \times 139 & 9\end{array}$ $\begin{array}{lll}1.5 \times & 117 \quad 5\end{array}$ $\begin{array}{lll}1.3 \times & 84 & 2\end{array}$ $\begin{array}{rrr}1.3 \times & 82 & 5 \\ 2.1 \times & 199 & 9\end{array}$

$2.3 \times 20125$ $\begin{array}{lll}1.6 \mathrm{X} & 87 \quad 2\end{array}$ $1.4 \times 220 \quad 8$ $2.0 \times \quad 59 \quad 1$ $2.0 \times 1029$

$\begin{array}{lll}.4 \times & 88 & 4\end{array}$ $1.8 \mathrm{X} \quad 65 \quad 2$ 1.6X $92 \quad 2$ $2.4 \times 106 \quad 5$ $1.6 \times 27611$

$\begin{array}{lll}.1 \times & 59 \quad 4\end{array}$ $2.3 \times 29339$ $\begin{array}{lll}2.3 \times & 76 & 9\end{array}$ $\begin{array}{rrr}2.7 \mathrm{X} & 88 & 12\end{array}$
---ORTGIN TIME (HST)-- -LAT N-- --LON W-- DEPTH N RMS ERH ERZ LOC

$\begin{array}{llllllllllllll}2006 & \text { DEC } & 25 & 0359 & 3.28 & 19 & 57.35 & 156 & 12.71 & 42.70 & 36 & .08 & 1.2 & 2.0 \\ \text { кон }\end{array}$

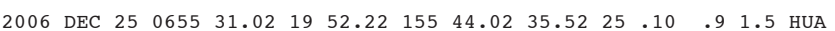

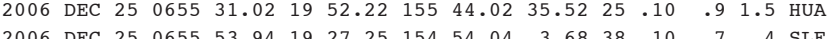

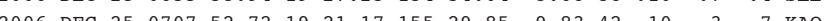

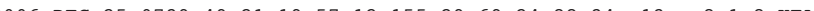

$\begin{array}{lllllllllllll}2006 & \text { DEC } 25 & 0823 & 19.76 & 19 & 21.10 & 155 & 29.48 & 10.16 & 27 & .12 & .4 & .9 \\ \text { KAO }\end{array}$

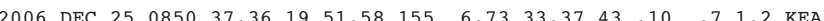

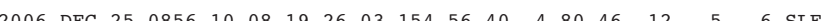

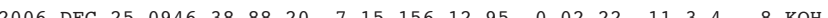

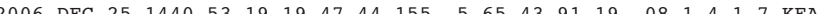

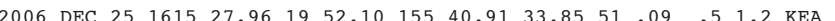

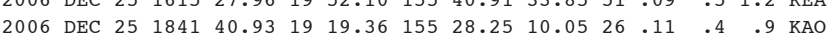

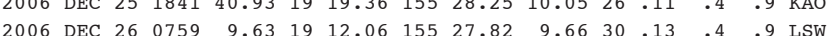

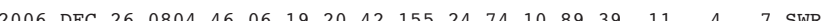

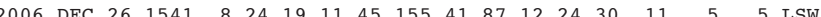

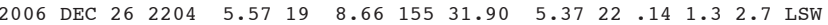

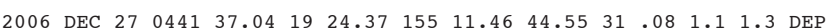

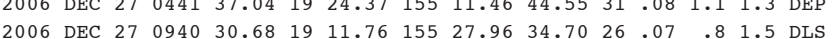

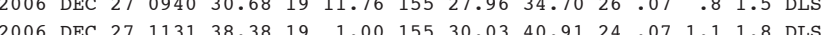

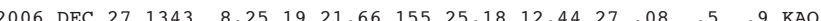

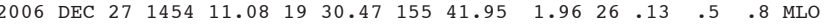

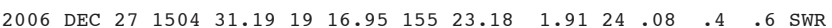

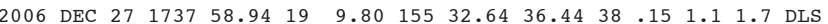

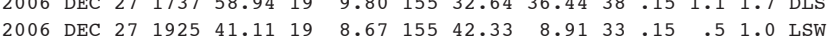

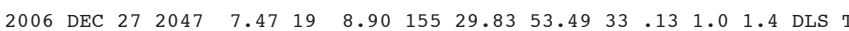

$\begin{array}{llllllllllllll}2006 & \text { DEC } 27 & 2126 & 51.84 & 19 & 57.80 & 155 & 34.95 & 11.84 & 17 & .11 & .6 & .6 & \text { кон }\end{array}$

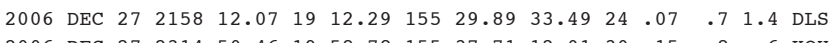
$\begin{array}{llllllllllllll}2006 & \text { DEC } 27 & 2314 & 50.46 & 19 & 58.78 & 155 & 37.71 & 12.01 & 30 & .15 & .8 & .6 & \text { кон }\end{array}$

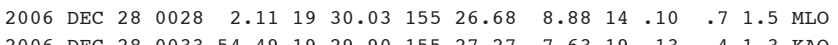

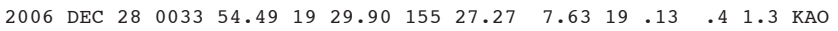

$\begin{array}{lllllllllllllll}2006 & \text { DEC } 28 & 0057 & 34.69 & 19 & 15.70 & 155 & 25.21 & 9.21 & 39 & .12 & .4 & .5 & \text { LSW }\end{array}$

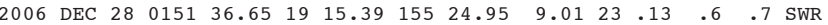
$\begin{array}{lllllllllllll}2006 & \text { DEC } 28 & 0434 & 24.32 & 19 & 23.55 & 155 & 29.52 & 9.47 & 40 & .10 & .3 & .6 \\ \text { KAO }\end{array}$

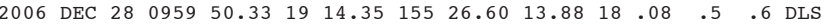

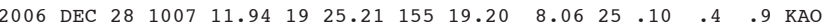

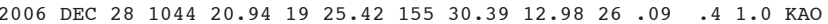

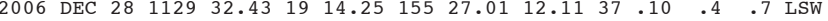

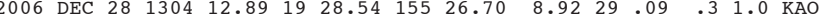

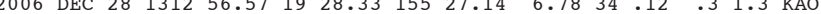

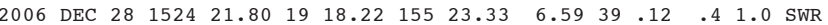

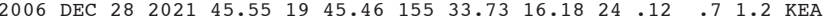

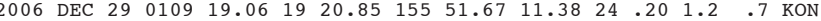

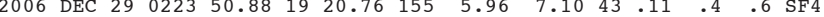

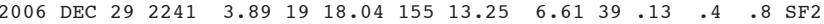

GAP DS

$2.3 \times 30049$ $1.6 \times 237 \quad 8$ $1.6 \times \quad 64 \quad 5$ $1.5 \times 23317$

$\begin{array}{llll}1.0 \times \quad 80 \quad 4 & \end{array}$ $2.0 \times 23520$ 1.6X 23028

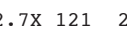
$\begin{array}{lll}1.2 \times \quad 78 & 6\end{array}$ $\begin{array}{lll}1.2 \times 108 & 108\end{array}$ $\begin{array}{lll}1.4 \times & 84 \\ 1.7 \times & 74\end{array}$

$2.4 \mathrm{U} 2527$ $2.4 \mathrm{U} 140$ $\begin{array}{llll}2.4 \mathrm{U} & 140 \\ 1.7 \mathrm{X} & 122 & 4\end{array}$ $1.5 \times 267 \quad 16$ $1.1 \times \quad 87$

$1.2 \times 100$ $1.2 \times 166 \quad 6$ $2.0 \times 175$ $1.8 \times 200$

$1.5 \times 155 \quad 14$ $1.3 \times \quad 72 \quad 5$ $\begin{array}{llll}1.5 \times & 151 & 14\end{array}$ $\begin{array}{lll}1.6 x & 96 & 4 \\ 1.8 x & 91 & 4\end{array}$

$\begin{array}{lll}1.5 \times 120 & 9\end{array}$ 1.50140 $\begin{array}{llll}1.8 \times & 53 & 4 \\ 1.3 \times & 131\end{array}$ $\begin{array}{lll}1.3 \times & 131 \\ 1.2 \times & 84\end{array}$

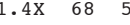
$\begin{array}{llll}1.9 \times & 110 & 6 \\ 1.6 \times & 67 & 6\end{array}$ $\begin{array}{lll}1.6 \times & 67 & 6\end{array}$ $2.1 \times 110$

$\begin{array}{lll}1.6 \times & 161 & 11\end{array}$ $1.3 \times 2029$ $1.9 \times 153$ $\begin{array}{lll}1.2 \mathrm{X} & 63 & 1 \\ 1.5 \mathrm{x} & 95 & 2\end{array}$ 
---ORIGIN TIME (HST)-- -LAT N-- --LON W-- DEPTH N RMS ERH ERZ LOC

PREF AZ MIN 123

YEAR MON DA HRMN SEC DEG MIN DEG MIN KM RD SEC KM KM REMKS MAG GAP DS

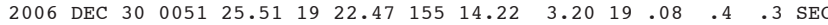

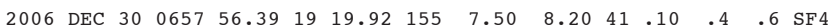
$\begin{array}{llllllllllllll}2006 & \text { DEC } & 30 & 1955 & 10.82 & 19 & 26.44 & 155 & 28.94 & 10.46 & 23 & .13 & .4 & 1.0\end{array}$

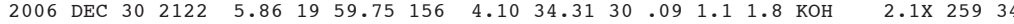

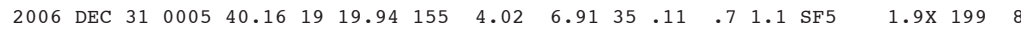

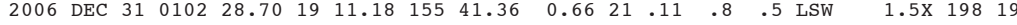

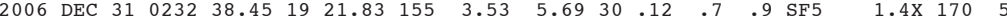

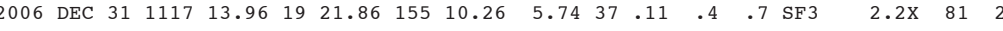

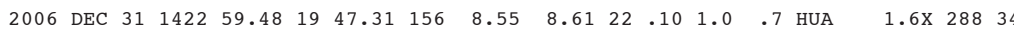

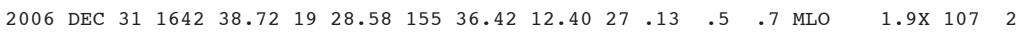

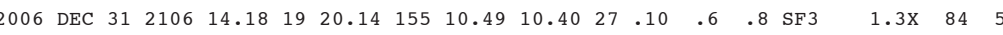

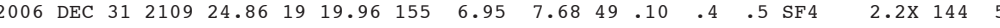

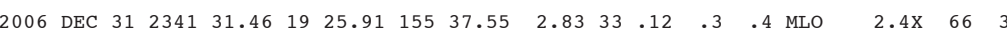


Table 5.

\begin{tabular}{|c|c|c|c|c|c|c|c|c|c|c|c|c|c|c|c|c|c|c|}
\hline-- & GII & & 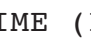 & f & & $1 N--$ & & W-- & DETI & 20 & & LRח & 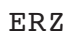 & 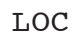 & & PREF & A $\Delta$ & \\
\hline EAR & MON & $\mathrm{DA}$ & HRMN & SEC & $\mathrm{DEG}$ & MIN & $\mathrm{DEG}$ & MIN & $\mathrm{KM}$ & $\mathrm{RD}$ & $\mathrm{SEC}$ & $\mathrm{KM}$ & $\mathrm{KM}$ & REMY & & MAG & GAP & DS \\
\hline 006 & JAN & 2 & 2025 & 6.97 & 9 & 21.38 & 155 & 1.50 & 9.22 & 50 & .11 & .6 & .4 & SF 5 & $\mathrm{~F}$ & $3.1 x$ & 193 & \\
\hline 006 & JAN & 7 & 317 & 47.06 & 9 & 11.27 & 55 & 42.76 & 7.29 & 42 & .13 & .5 & .9 & LSW & $\mathrm{F}$ & $.1 \mathrm{X}$ & 116 & \\
\hline 006 & JAN & 7 & 556 & 5.14 & 9 & 20.33 & 55 & 3.89 & 40.57 & 51 & .10 & .7 & .7 & $E P$ & 1 & $.2 x$ & 85 & \\
\hline 006 & AN & 8 & 313 & 3.95 & 9 & 3.16 & 56 & .66 & 8.73 & 37 & .09 & 4.8 & 3.1 & KON & $\mathrm{F}$ & $.5 x$ & 90 & \\
\hline 006 & AN & 4 & 2304 & 5 & 9 & 9 & 5 & 11 & .65 & 51 & .09 & .7 & .1 & ON & $F$ & $2 x$ & 97 & \\
\hline 006 & AN & 8 & 604 & .34 & 19 & 0.79 & 5 & 7.03 & 41.25 & 49 & .10 & .9 & 1.3 & LS & $\mathrm{F}$ & $.7 \mathrm{U}$ & 214 & \\
\hline 006 & JAN & 23 & 321 & 50.66 & 9 & 25.46 & 55 & 19.23 & 4.62 & 38 & .13 & .4 & .8 & KAO & $\mathrm{F}$ & $3.5 \mathrm{U}$ & 46 & \\
\hline 006 & EB & 11 & 0515 & .26 & 9 & 04 & 5 & .77 & 23.46 & 49 & .11 & .4 & .8 & $\mathrm{DEP}$ & $\mathrm{F}$ & $.0 x$ & 61 & \\
\hline 006 & $\mathrm{FEB}$ & 13 & 2245 & 54.76 & 9 & 12.29 & 155 & 28.14 & 8.37 & 43 & .16 & .6 & .9 & LSW & $\mathrm{F}$ & $3.0 x$ & 150 & \\
\hline 006 & FEB & 14 & 2010 & 18.64 & 21 & 46.30 & 156 & 43.71 & 27.38 & 41 & .11 & 2.7 & 3.7 & DIS & $\mathrm{F}$ & $3.5 x$ & 3422 & \\
\hline 006 & EB & 0 & 135 & .42 & & & & 6.40 & 37.36 & 36 & .10 & 4.0 & 2.4 & HUA & $x^{2}$ & $3.4 x$ & 287 & \\
\hline 2006 & $\mathrm{FEB}$ & 16 & 1522 & 33.05 & 19 & 20.17 & 155 & 12.82 & 10.28 & 48 & .10 & .3 & .3 & $\mathrm{SF} 2$ & F & $4.6 \mathrm{U}$ & 71 & \\
\hline 2006 & MAR & 1 & 857 & 13.68 & 19 & 26.55 & 155 & 19.21 & 5.87 & 46 & .11 & .3 & .6 & KAO & $\mathrm{F}$ & $4.0 \mathrm{U}$ & 48 & \\
\hline 006 & AR & 4 & 41 & 13 & 9 & 26.30 & 55 & 19.16 & 6.32 & 50 & .10 & .3 & .6 & $\mathrm{KAO}$ & $\mathrm{F}$ & $.1 \mathrm{X}$ & 48 & \\
\hline 2006 & MAR & 22 & 0438 & 31.70 & 19 & 57.55 & 155 & 42.67 & 8.93 & 39 & .08 & .9 & .7 & $\mathrm{KOH}$ & $\mathrm{F}$ & $3.5 x$ & 284 & \\
\hline 2006 & APR & 6 & 711 & 43.28 & 9 & 46.13 & 56 & .37 & 60 & 44 & .10 & 1.0 & .7 & HUA & $\mathrm{F}$ & $3.2 x$ & 81 & \\
\hline 2006 & APR & 19 & 1433 & 9.27 & 19 & 11.30 & 155 & 22.04 & 47.70 & 45 & .09 & .8 & 1.1 & DEP & & $3.1 x$ & 170 & \\
\hline 2006 & APR & 30 & 1847 & 29.28 & 20 & 20.62 & 155 & 7.52 & 5.51 & 44 & .14 & 1.4 & 1.4 & $\mathrm{KEA}$ & & $3.2 x$ & 310 & \\
\hline 2006 & MAY & 7 & 1359 & 54.78 & 20 & 1.37 & 155 & 20.15 & 7.76 & 47 & .12 & .9 & .6 & $\mathrm{KEA}$ & $\mathrm{F}$ & $3.2 x$ & 254 & \\
\hline 2006 & IAY & 31 & 2236 & 47.98 & 21 & 45.02 & 156 & 59.34 & 29.72 & 7 & .13 & 7.4 & 9.4 & DIS & $\mathrm{F}$ & $4.1 \mathrm{X}$ & 252 & \\
\hline
\end{tabular}

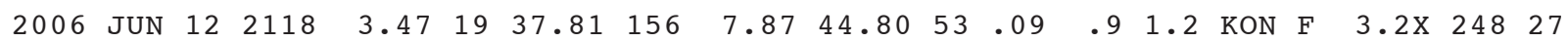

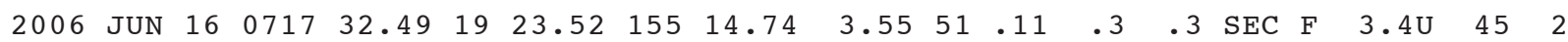

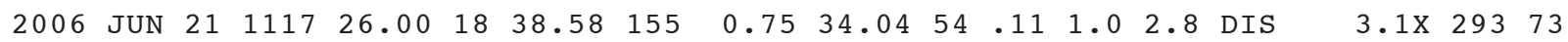

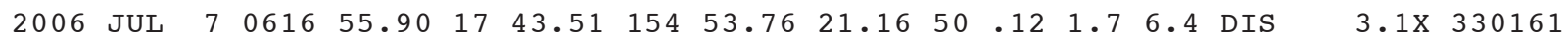

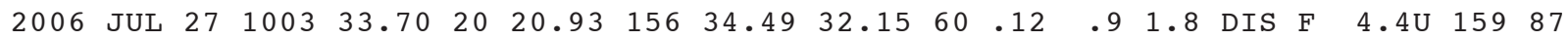

$\begin{array}{lllllllllllllllll}2006 & \text { AUG } 5 & 0033 & 31.83 & 18 & 53.57 & 155 & 54.66 & 44.27 & 52 & .10 & .9 & 1.2 & \text { DIS } & 3.0 \mathrm{X} & 292 & 27\end{array}$

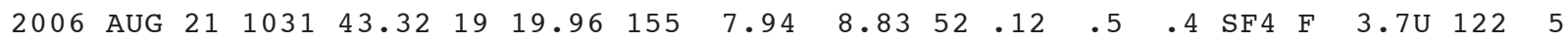

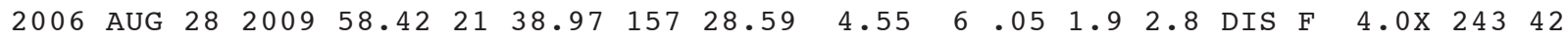

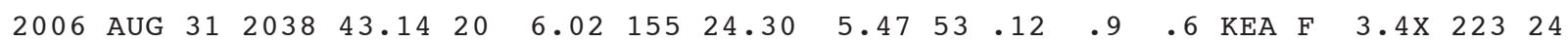
$\begin{array}{lllllllllllllllllll}2006 & \text { SEP } & 4 & 1727 & 39.68 & 19 & 50.56 & 155 & 59.18 & 42.94 & 56 & .09 & .7 & 1.1 & \text { HUA } & \text { F } & 3.7 \mathrm{U} & 225 & 23\end{array}$

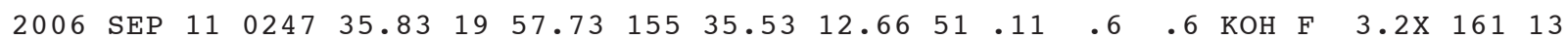

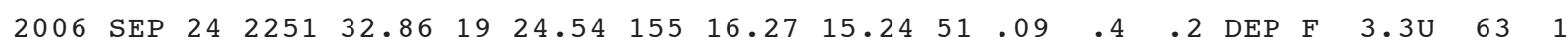

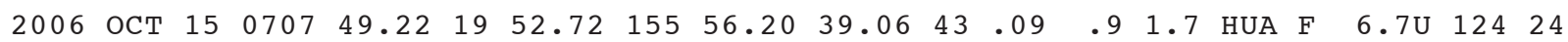

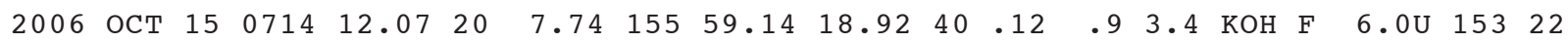
$\begin{array}{llllllllllllllllll}2006 & \text { OCT } & 15 & 0727 & 31.78 & 20 & 3.37 & 155 & 57.81 & 9.43 & 36 & .13 & 1.8 & 1.9 & \mathrm{KOH} & 3.1 \mathrm{X} & 296 & 45\end{array}$

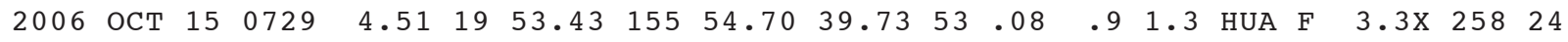
$\begin{array}{llllllllllllllllll}2006 & \text { OСТ } & 15 & 0733 & 51.64 & 19 & 53.08 & 156 & 12.65 & 37.51 & 49 & .10 & 1.0 & 1.7 & \text { HUA } & 3.3 \mathrm{X} & 306 & 45\end{array}$ $\begin{array}{llllllllllllllllll}2006 & \text { OCT } & 15 & 0753 & 39.31 & 19 & 52.32 & 155 & 58.21 & 38.32 & 54 & .10 & .8 & 1.2 & \text { HUA } & 3.3 \mathrm{X} & 203 & 24\end{array}$

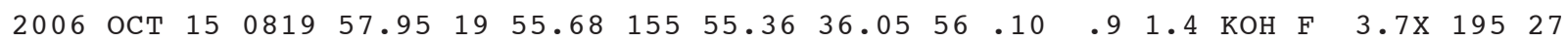

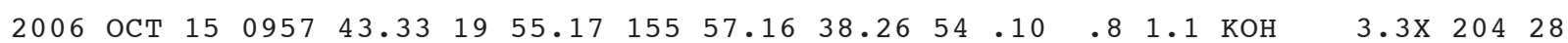

$\begin{array}{lllllllllllllllllll}2006 & \text { ОСТ } & 15 & 1035 & 20.71 & 20 & 7.86 & 156 & 1.45 & 24.99 & 54 & .12 & .9 & 1.8 & \mathrm{KOH} & \mathrm{F} & 4.3 \mathrm{X} & 271 & 26\end{array}$

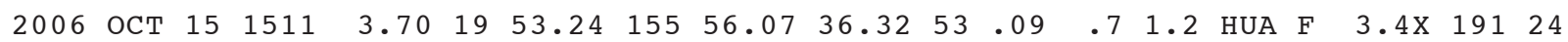

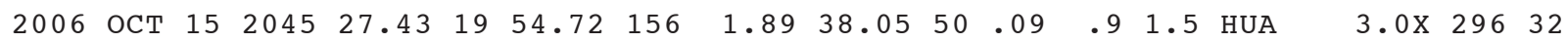

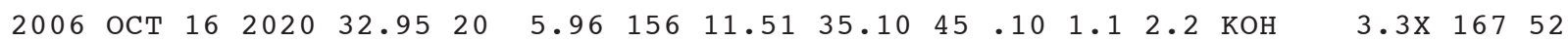
$\begin{array}{lllllllllllllllllllll}2006 & \text { OCT } & 17 & 0059 & 57.57 & 19 & 53.73 & 155 & 53.61 & 35.62 & 48 & .09 & .8 & 1.3 & \text { HUA } & \text { F } & 3.3 \times & 177 & 24\end{array}$ 


\begin{tabular}{|c|c|c|c|c|c|c|c|c|c|c|c|c|c|c|c|c|c|c|}
\hline \multicolumn{2}{|c|}{$---O R I G I N$} & \multicolumn{3}{|c|}{ TIME (HST)-- } & \multicolumn{2}{|c|}{-LAT N-- } & \multicolumn{2}{|c|}{$--\mathrm{LON} \quad \mathrm{W}--$} & \multirow{2}{*}{$\begin{array}{c}\text { DEPTH } \\
\text { KM }\end{array}$} & \multirow{2}{*}{$\begin{array}{r}\mathrm{N} \\
\mathrm{RD}\end{array}$} & \multirow{2}{*}{$\begin{array}{l}\text { RMS } \\
\text { SEC }\end{array}$} & \multirow{2}{*}{$\begin{array}{r}\text { ERH } \\
\text { KM }\end{array}$} & \multicolumn{3}{|c|}{ ERZ LOC } & \multicolumn{3}{|c|}{ PREF AZ MIN } \\
\hline YEAR & MON & $\mathrm{DA}$ & HRMN & $\mathrm{SEC}$ & $\mathrm{DEC}$ & MIN & DEG & MIN & & & & & $\mathrm{KM}$ & REMK & & MAG & GAP & DS \\
\hline 006 & OCT & 17 & 0526 & 44.84 & 20 & 4.25 & 156 & 0.04 & 31.22 & 55 & .10 & .7 & 1.4 & $\mathrm{KOH}$ & $\mathrm{F}$ & $4.0 x$ & 138 & 24 \\
\hline 2006 & OCT & 17 & 0657 & 34.17 & 19 & 57.86 & 156 & 0.81 & 44.68 & 53 & .10 & .7 & 1.2 & $\mathrm{KOH}$ & $\mathrm{F}$ & $3.7 x$ & 143 & 30 \\
\hline 2006 & OCT & 18 & 0640 & 15.02 & 20 & 18.97 & 156 & 40.55 & 30.50 & 48 & .12 & 1.4 & 2.3 & DIS & $\mathrm{F}$ & $3.4 x$ & 318 & 96 \\
\hline 2006 & OCT & 18 & 1431 & 13.15 & 20 & 19.28 & 156 & 24.33 & 32.40 & 43 & .13 & 1.7 & 2.1 & DIS & & $3.0 x$ & 313 & 69 \\
\hline 006 & ост & 18 & 2254 & 18.45 & 19 & 52.85 & 155 & 54.90 & 40.75 & 51 & .09 & .8 & 1.1 & HUA & $\mathrm{F}$ & $3.1 x$ & 183 & 23 \\
\hline 006 & ост & 21 & 0429 & 45.91 & 19 & 53.35 & 156 & 11.57 & 0.03 & 47 & .13 & 2.0 & .4 & HUA & $\mathrm{F} \#$ & $3.3 x$ & 265 & 43 \\
\hline 2006 & OCT & 26 & 1323 & 16.24 & 20 & 11.47 & 156 & 2.89 & 21.62 & 45 & .11 & 1.1 & 3.1 & $\mathrm{KOH}$ & $\mathrm{F}$ & $3.3 x$ & 293 & 29 \\
\hline 2006 & $\mathrm{OCT}$ & 29 & 1655 & 35.86 & 19 & 54.22 & 156 & 2.26 & 43.50 & 55 & .10 & .7 & 1.1 & HUA & F & $3.0 x$ & 232 & 27 \\
\hline 2006 & $\mathrm{OCT}$ & 30 & 1824 & 31.10 & 19 & 58.07 & 155 & 35.67 & 12.10 & 54 & .10 & .5 & .7 & $\mathrm{KOH}$ & $\mathrm{F}$ & $3.4 x$ & 163 & 14 \\
\hline 2006 & NOV & 6 & 1038 & 50.85 & 19 & 58.51 & 155 & 35.56 & 13.48 & 57 & .11 & .6 & .6 & $\mathrm{KOH}$ & $\mathrm{F}$ & $3.1 \mathrm{x}$ & 158 & 15 \\
\hline 2006 & NOV & 12 & 1536 & 6.70 & 19 & 53.78 & 155 & 51.85 & 36.92 & 53 & .09 & .6 & 1.2 & HUA & $\mathrm{F}$ & $3.7 x$ & 191 & 2 \\
\hline 2006 & NOV & 23 & 0920 & 10.66 & 19 & 53.33 & 155 & 58.68 & 37.61 & 45 & .10 & .9 & 1.6 & HUA & $\mathrm{F}$ & $5.0 \mathrm{U}$ & 172 & 27 \\
\hline 2006 & NOV & 25 & 0549 & 23.01 & 20 & 13.86 & 155 & 33.97 & 24.93 & 42 & .10 & .9 & 2.1 & $\mathrm{KEA}$ & $\mathrm{F}$ & $3.4 x$ & 253 & 25 \\
\hline 2006 & $\mathrm{DEC}$ & 1 & 2342 & 22.20 & 20 & 2.09 & 155 & 22.84 & 7.38 & 55 & .12 & .6 & .6 & $\mathrm{KEA}$ & $\mathrm{F}$ & $3.2 x$ & 210 & 29 \\
\hline 2006 & $\mathrm{DEC}$ & 3 & 0222 & 21.36 & 19 & 58.82 & 155 & 59.90 & 12.64 & 46 & .11 & 1.1 & .7 & $\mathrm{KOH}$ & $\mathrm{F}$ & $4.6 \mathrm{U}$ & 288 & 36 \\
\hline 0 & DLE & 5 & 0254 & 43.56 & 20 & 19.29 & 156 & 19.64 & 22.42 & 47 & .13 & 1.5 & 5.5 & $\mathrm{KOH}$ & I & $3.5 x$ & 317 & 87 \\
\hline
\end{tabular}

